\title{
PRISMA: Aspect-Oriented Software Architectures
}

\author{
Jenifer Pérez, Benedí \\ Department of Information Systems and Computation \\ Polytechnic University of Valencia
}

A thesis submitted in partial fulfilment of the requirements for the degree of Doctor of Philosophy in Computer Science

Supervisors: Prof. Dr. Isidro Ramos Salavert

Dr. Jose Ángel Carsí Cubel 
This thesis has been funded by the Department of Science and Technology (Spain) under the National Program for Research, Development and Innovation, DYNAMICA (DYNamic and Aspect-Oriented Modeling for Integrated Component-based Architectures) project, PRISMA (OASIS Platform for Architectural Models) subproject, TIC2003-7804-C05-01. Moreover, it is funded by the Microsoft Research Cambridge, "PRISMA: Model Compiler of aspectoriented component-based software architectures" Project. 


\begin{abstract}
The complexity of current software systems and the fact that their non-functional requirements have become very relevant for the end-user are challenges to be faced in software development. In the last few years, these properties have increased the time and the staff required for the development and maintenance processes of software. As a result, there is greater interest in reducing the costs of these processes in complex software systems. In order to deal with these challenges, to achieve the milestones of software products, and to overcome the competitiveness of the market, this thesis presents a novel approach for developing complex software systems. This approach is called PRISMA.

PRISMA is supported by a framework that consists of a model, a language, a methodology and a Computer-Aided Software Engineering (CASE) tool prototype. The PRISMA model combines two approaches to define software architectures: the Component-Based Software Development (CBSD) and the Aspect-Oriented Software Development (AOSD). The main contributions of the model are the way that it combains both approaches to take their advantages, and its formal language. PRISMA takes into account non-functional requirements from the early stages of the software life cycle and improves the reusability and maintenance of software by decomposing software systems using two different concepts: aspects and architectural elements (components and connectors).

PRISMA provides a formal Aspect-Oriented Architecture Description Language (AOADL) for specifying aspect-oriented software architectures. Its AOADL is independent of technology and is based on formal languages and formalisms to preserve non-ambiguity in order to apply code generation techniques.

The methodology that PRISMA proposes for developing software systems follows the Paradigm of Automatic Programming by applying the Model-Driven Development (MDD) approach. As a result, PRISMA provides traceability from the analysis and design stages of the software life cycle to the implementation stage, and provides mechanisms to improve the time and cost invested in the development and maintenance processes.
\end{abstract}


The PRISMA model and its methodology are supported by a CASE tool prototype called PRISMA CASE. This tool allows the specification of PRISMA architectures using its AOADL in a textual and a graphical way, the verification of PRISMA software architectures, and the automatic C\# code generation thanks to the middleware and the code generation patterns that the CASE tool prototype integrates. Finally, PRISMA CASE provides a generic Graphical User Interface to execute PRISMA applications over .NET platform and to validate them. 


\section{RESUMEN}

Hoy en día, la complejidad de los sistemas software y la gran relevancia que han adquirido los requisitos no funcionales son retos que han de abordarse durante el proceso de desarrollo software. En los últimos años, estas propiedades han provocado un gran incremento en el tiempo y el personal necesario para llevar a cabo los procesos de desarrollo y mantenimiento del software. Por ello, existe un gran interés en mejorar dichos procesos. Esta tesis presenta un nuevo enfoque de desarrollo para sistemas software complejos. Dicho enfoque, llamado PRISMA, da soporte a estos nuevos retos y permite satisfacer la elevada competitividad del mercado.

El enfoque PRISMA se ha materializado en un marco de trabajo formado por un modelo, un lenguaje, una metodología y un prototipo de herramienta CASE (Computer-Aided Software Engineering). El modelo de PRISMA combina dos aproximaciones para definir arquitecturas software: el Desarrollo de Software Basado en Componentes (DSBC) y el Desarrollo de Software Orientado a Aspectos (DSOA). Las principales aportaciones del modelo es la manera en la que integra ambas aproximaciones para obtener sus ventajas y su lenguaje formal. PRISMA tiene en cuenta los requisitos no funcionales desde las primeras etapas del ciclo de vida software y mejora su reutilización y el mantenimiento. Todo ello gracias a la descomposición que realiza de los sistemas software utilizando dos conceptos diferentes: aspectos y elementos arquitectónicos (componentes y conectores).

PRISMA proporciona un Lenguaje de Descripción de Arquitecturas Orientado a Aspectos (LDAOA) formal para la especificación de arquitecturas software orientadas a aspectos. El LDAOA de PRISMA es independiente de cualquier tecnología y está basado en lenguajes formales para evitar la ambigüedad y poder aplicar técnicas de generación automática de código.

La metodología de PRISMA apuesta por el desarrollo de software siguiendo el Paradigma de la Prototipación Automática mediante la aplicación del enfoque de Desarrollo Dirigido por Modelos (DDM). De esta manera, PRISMA proporciona trazabilidad desde las etapas de 
análisis y diseño, a la etapa de implementación del ciclo de vida software, y proporciona mecanismos para mejorar el tiempo y el coste invertido en los procesos de desarrollo y mantenimiento del software.

El modelo de PRISMA y su metodología son soportados mediante una herramienta CASE llamada PRISMA CASE. PRISMA CASE permite la especificación de arquitecturas PRISMA utilizando su LDAOA tanto de forma textual como gráfica, la verificación de arquitecturas PRISMA, y la generación automática de código C\# gracias al middleware y a los patrones de generación de código que la herramienta CASE integra. Finalmente, PRISMA CASE proporciona una Interfaz Gráfica de Usuario (IGU) genérica que permite la ejecución de aplicaciones PRISMA sobre la plataforma .NET y la validación de su comportamiento. 


\section{RESUM}

Avui en dia, la complexitat dels sistemes de programari i la gran rellevància que han adquirit els requisits no funcionals, són reptes que s'han d' abordar durant el procés de desenvolupament del programari. En els últims anys, aquestes propietats han provocat un increment en el temps i del personal necessari per a dur en davant els processos de desenvolupament i manteniment de programari. Per això, hi ha un gran interés de millorar aquestos processos. Aquesta tesi presenta una nova aproximació de desenrotllament per a sistemes programari complexes. La dita aproximació, denominada PRISMA, dóna suport a aquests nou desafiaments i permet satisfer la gran competitivitat del mercat.

L' enfocament PRISMA s'ha materialitzat en un marc de treball format per un model, un llenguatge, una metodologia, i un prototip de ferramenta CASE (Computer-Aided Software Engineering). El model de PRISMA combina dues aproximacions per definir arquitectures de programari: el Desenvolupament de Programari Basat en Components (DPBC) i el Desenvolupament de Programari Orientat a Aspectes (DPOA). Les principals aportacions del model són la manera en la que integra dues aproximacions per obtindre els seus avantatges i el seu llenguatge formal. PRISMA assoleix els requisits no funcionals des de les primeres etapes del cicle de vida de programari i millora la reutilització i manteniment d'aquest. Tot açò, gràcies a la descomposició que fa dels sistemes de programari, tot i utilitzant dos conceptes diferents: aspectes i elements arquitectònics (components i connectors).

PRISMA proporciona un Llenguatge de Descripció d'Arquitectures Orientat a Aspectes (LDAOA) formal per a l'especificació d'arquitectures de programari orientades a aspectes. El LDAOA de PRISMA és independent de tecnologia i està fonamentat en llenguatges formals per tal de evitar l'ambigüitat i poder aplicar tècniques de generació automàtica de codi.

La metodologia de PRISMA aposta pel desenvolupament de programari tot i seguint el Paradigma de la Prototipació Automàtica mitjançant l'aplicació de l'aproximació del Desenvolupament Dirigit per Models (DDM). D'aquesta manera, PRISMA proveïx traçabilitat 
des de les etapes d'anàlisi i disseny del cicle de vida de programari a l' etapa d' implementació. A més a més, proporciona mecanismes per tal de millorar el temps i la despesa invertida en els processos de desenvolupament i manteniment del programari.

El model de PRISMA i la seua metodologia, són suportats mitjançant una eina CASE denominada PRISMA CASE. L'esmentada PRISMA CASE permet l'especificació d'arquitectures PRISMA utilitzant el seu LDAOA, tant de forma textual com gràfica, la verificació d'arquitectures PRISMA, i la generació automàtica de codi C\# gràcies al middleware i als patrons de generació de codi que l'estri CASE integra. Per últim, PRISMA CASE proporciona una Interfície Gràfica d'Usuari (IGU) genèrica que permet l'execució d'aplicacions PRISMA sobre la plataforma .NET i la validació del seu comportament. 


\section{KEY WORDS}

KEY WORDS:

Software Architectures, Aspect-Oriented Software Development (AOSD), Component-Based

Software Development (CBSD), Aspect-Oriented Programming (AOP), Model Driven

Development (MDD), Automatic Code Generation. Tele-Operated Systems.

\section{PALABRAS CLAVE:}

Arquitecturas Software, Desarrollo Software Orientado a Aspectos (DSOA), Desarrollo Software Basado en Componentes (DSBC), Programación Orientada a Aspectos (POA), Desarrollo Dirigido por Modelos (DDM), generación automática de código, sistemas teleoperados

\section{PARAULES CLAU:}

Arquitectures de Programari, Desenvolupament de Programari Orientat a Aspectes (DPOA), Desenvolupament de Programari Basat en Components (DPBC), Programació Orientada a Aspectes (POA), Desenvolupament Dirigit per Models (DDM), generació automàtica de codi, sistemes teleoperats 
A Luís César

A Santiago y Julia 


\section{Acknowledgments/Agradecimientos}

A mi marido, Luis César, por entender mi dedicación a este trabajo que me apasiona, por no permitirme tirar la toalla, por quererme tanto y demostrármelo cada día. Sin tí no lo habría conseguido, gracias mi vida.

A mis padres, Santiago y Julia, por su esfuerzo sin desaliento que me ha permitido llegar hasta aqui, por su confianza, apoyo incondicional, paciencia...... y sobre todo por su cariño.

A mi hermana, Pilar, por su ayuda, consejos y ánimos, por estar siempre pendiente de mí y por ser un ejemplo a seguir en mi vida.

A mi cuñado, José, por ser un hermano para mí, ayudarme y creer desde el principio que éste es mi camino.

A mis sobrinas, María y Alba, por ser tan especiales, por todo lo que significa su presencia en esta vida y por hacer alegres los días tristes y oscuros.

A mis directores de tesis, Isidro Ramos y Jose Ángel Carsí, por compartir conmigo el entusiasmo por el trabajo realizado en esta tesis y por la confianza que han depositado en mí.

A Nour Ali, por ser mi media naranja en el trabajo, por ser una gran amiga y compañera. Por compartir mis inquietudes e ilusiones, por los buenos ratos que hemos pasado debatiendo sobre este trabajo y sobre todo por continuar trabajando en él.

A Isabel Díaz y Javier Jaén, por estar ahí desde el principio, por el cariño y la fuerza que me han dado cuando más lo necesitaba, por compartir tantos buenos y malos momentos conmigo.

A Manoli y Jose Luis, por entender mi trabajo y por apoyar tanto a Luis César como a mí durante mi estancia en Inglaterra.

A Vanessa, por ser una gran amiga, por su confianza y amistad, no cambies nunca. 
A todos mis amigos y amigas con los que no he pasado todo el tiempo que me hubiera gustado o que ellos hubieran necesitado. Especialmente a Montse, Verónica, Mapi, Pablo, Celso, Alvaro, Gonzalo y Nelly por entenderme, por mantener nuestra amistad a pesar de mis largas ausencias y por hacerme pasar tantos buenos ratos.

A Cristóbal Costa, Rafael Cabedo, Ismael Carrascosa, Javier Guillén y Carlos Millán por haber colaborado con tanto entusiasmo y haber trabajado tan duramente en la puesta en marcha de las ideas de esta tesis. Sin ellos PRISMA no se hubiera hecho realidad.

A todos los miembros del grupo ISSI que me han apoyado durante todo este tiempo, por darme su aliento y ánimo. Especialmente a Elena Navarro, Patricio Letelier, Manuel Llavador, Jose Hilario Canós, Ángeles Lorenzo, Ma Eugenia Cabello y Rogelio Limón por haber mostrado interés en colaborar en trabajos conjuntos relacionados con esta tesis que han permitido su mejora.

A Pedro Sánchez, Juan Ángel Pastor y Barbara Álvarez, por enseñarme todo lo necesario sobre los sistemas robóticos teleoperados, por ofrecerme un dominio de aplicación real para PRISMA y sobre todo por prestarme el TeachMover.

A Carlos Cuesta, por compartir conmigo sus conocimientos en formalismos y arquitecturas, por las interesantes discusiones que hemos mantenido juntos y sobre todo por su gran ayuda.

I would like to thank to all of the wonderful people of the Department of Computer Science of the University of Leicester; who made me feel as if I was at home during my stay. I especially want to thank to Jose Luiz Fiadeiro for his hospitality and his helpful advice and comments.

Gracias a todos ellos por su colaboración y cariño.

xii 


\section{TABLE OF CONTENTS}

INDEX OF FIGURES ...................................................................

INDEX OF TABLES .................................................................................

PART I INTRODUCTION ………………………………............................ 27

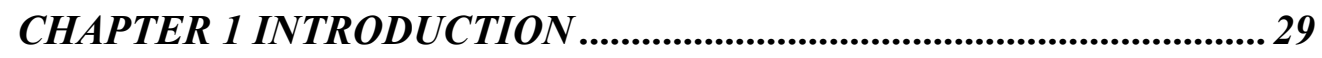

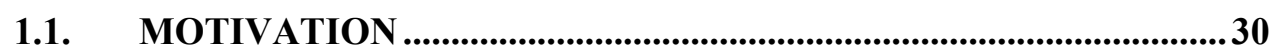

1.2. OBJECTIVES OF THE THESIS ……….......................................... 32

1.3. RESEARCH METHODOLOGY OF THE THESIS............................ 33

1.4. STRUCTURE OF THE THESIS .......................................................34

PART II STATE OF THE ART ................................................................. 37

CHAPTER 2 SOFTWARE ARCHITECTURES.......................................... 39

2.1. SOFTWARE ARCHITECTURES IN THE SOFTWARE LIFE

CYCLE ……...................................................................................40

2.2. PROPERTIES OF SOFTWARE ARCHITECTURES ……................41

2.3. DEFINITION OF SOFTWARE ARCHITECTURE ........................... 44

2.4. MAIN CONCEPTS OF SOFTWARE ARCHITECTURES ..............47

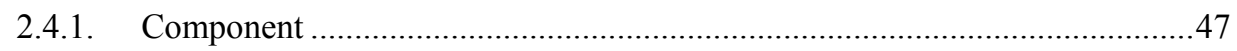

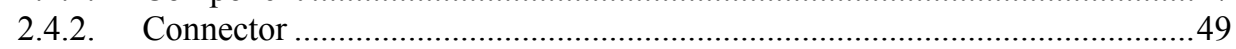

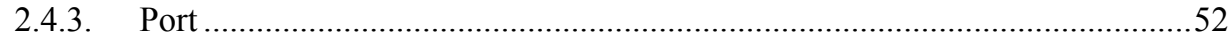

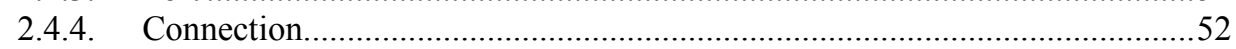

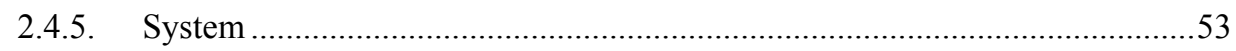

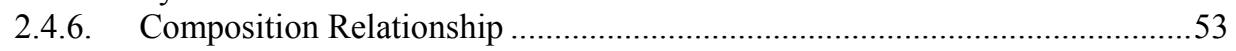

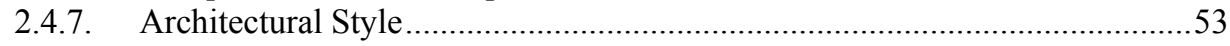

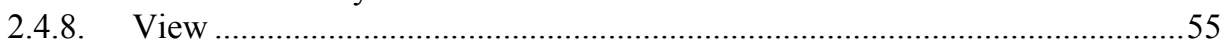

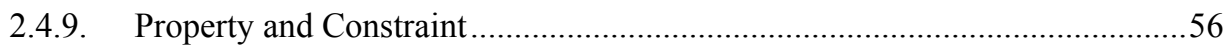

2.5. CONCLUSIONS ............................................................................56

CHAPTER 3 ASPECT-ORIENTED SOFTWARE DEVELOPMENT...... 59

3.1. AOP: ASPECT-ORIENTED PROGRAMMING................................61

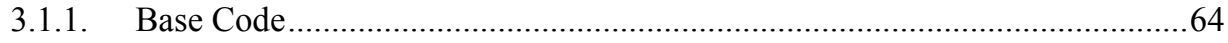

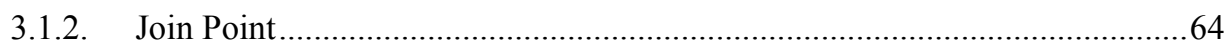

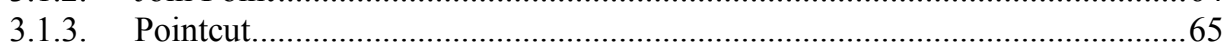

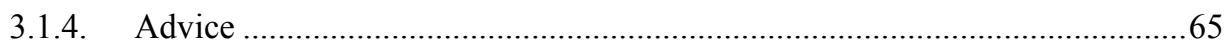




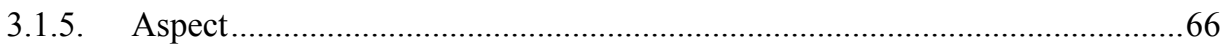

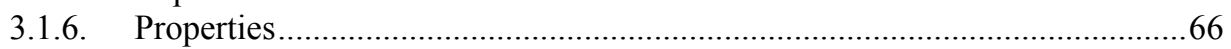

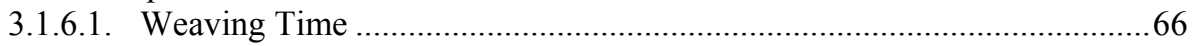

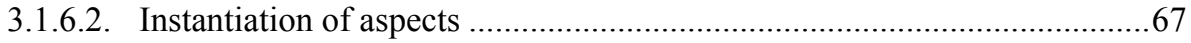

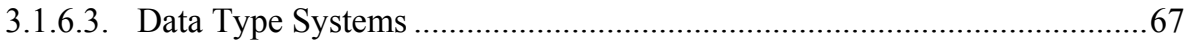

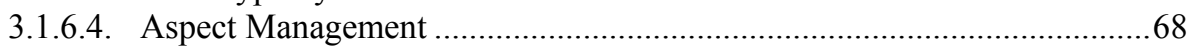

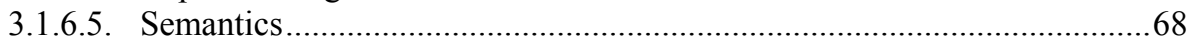

3.2. ASPECT-ORIENTED MODELS ...................................................69

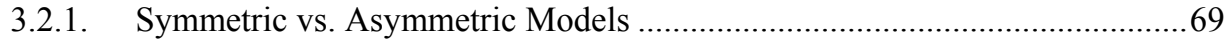

3.2.2. Multi-Dimensional Separation of Concerns (MDSOC) ….............................. 70

3.2.3. Composition Filters (CF) ...................................................................... 72

3.3. ASPECT-ORIENTED DEVELOPMENT IN THE SOFTWARE

LIFE CYCLE ........................................................................................ 74

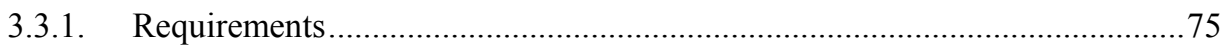

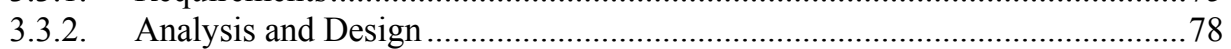

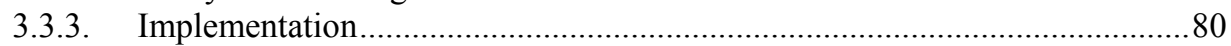

3.4. CONCLUSIONS .................................................................. 82

CHAPTER 4 ASPECT-ORIENTED SOFTWARE ARCHITECTURES... 85

4.1. ASPECT-ORIENTED APPROACHES AT THE

ARCHITECTURAL LEVEL ........................................................8 86

4.1.1. PCS: The Perspectival Concern-Space Framework........................................87

4.1.2. CAM/DAOP: Component-Aspect Model/Dynamic Aspect-Oriented

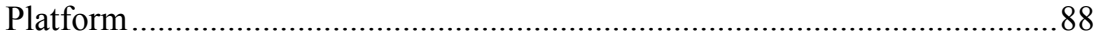

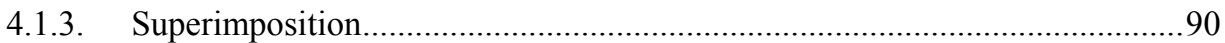

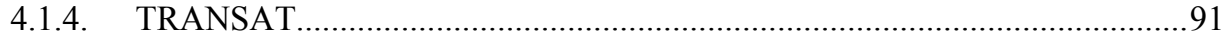

4.1.5. ASAAM: Aspectual Software Architecture Analysis Method ........................92

4.1.6. AVA: Architectural Views of Aspects ......................................................93

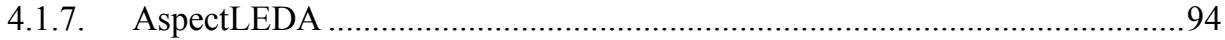

4.1.8. AOCE: Aspect-Oriented Component Engineering ........................................95

4.1.9. Component Views .....................................................................................96

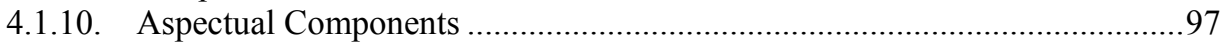

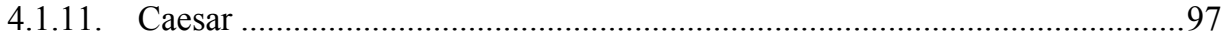

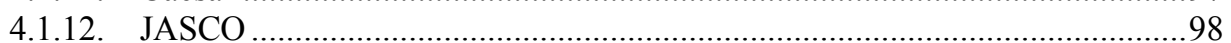

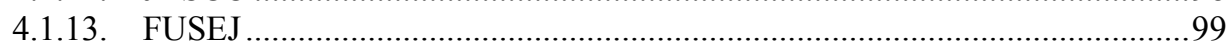

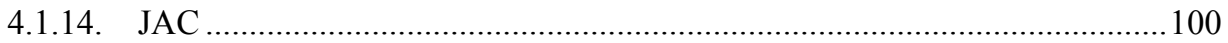

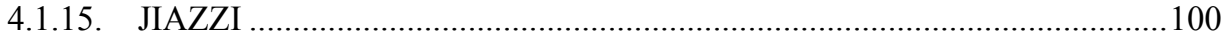

4.2. COMPARISON OF ASPECT-ORIENTED SOFTWARE ARCHITECTURES ............................................................................. 101

4.3. CONCLUSIONS ........................................................................ 107 
5.1. TELEOPERATION SYSTEMS: THE TEACHMOVER ROBOT 111

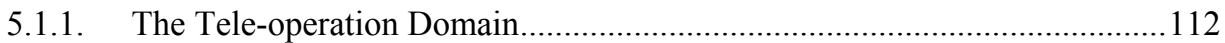

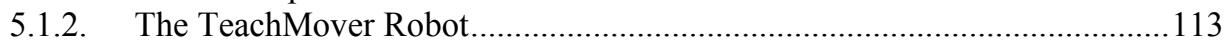

5.1.2.1. The morphology of the TeachMover Robot .............................................113

5.1.2.2. The Software Architecture of the TeachMover Robot.................................115

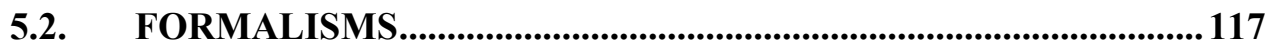

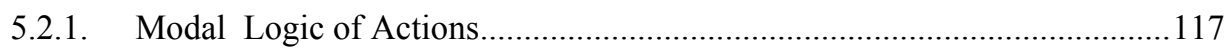

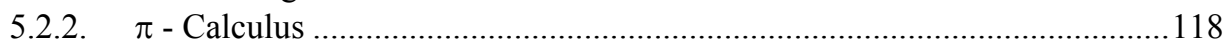

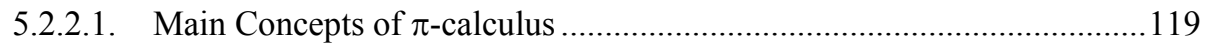

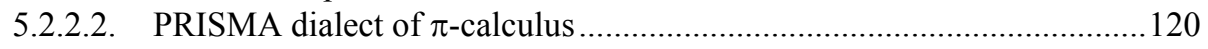

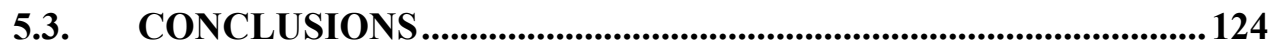

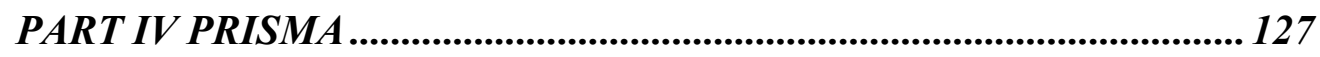

CHAPTER 6 THE PRISMA MODEL ................................................... 129

6.1. INTRODUCTION TO THE PRISMA MODEL ............................... 129

6.2. PRISMA FORMALIZATION ....................................................... 134

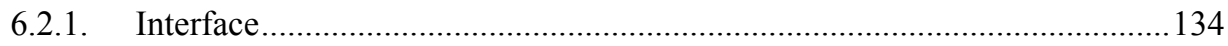

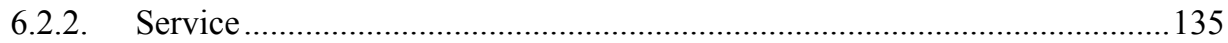

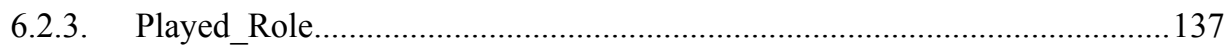

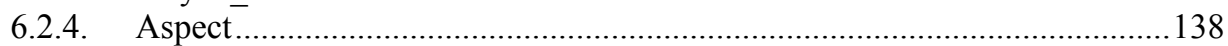

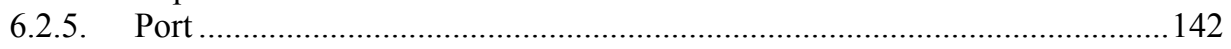

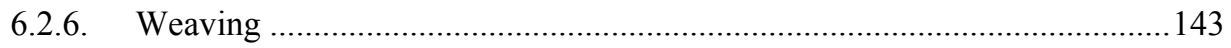

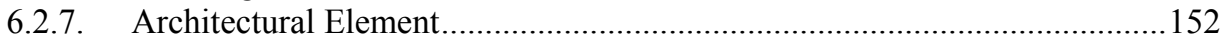

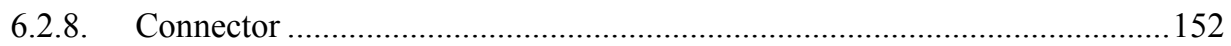

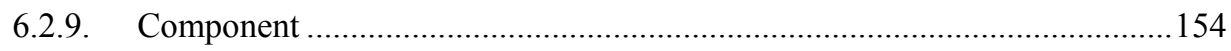

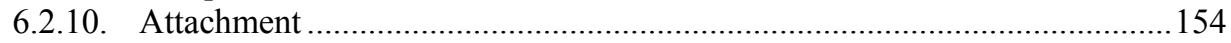

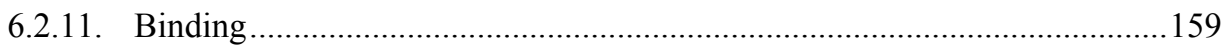

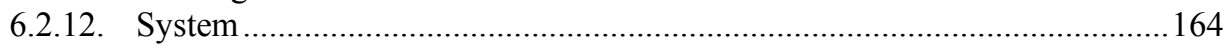

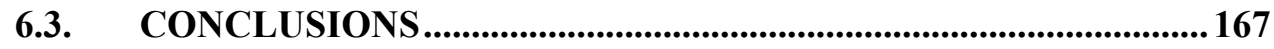

CHAPTER 7 THE PRISMA METAMODEL ....................................... 171

7.1. THE PRISMA METAMODEL ............................................................. 172

7.2. THE PACKAGE “TYPES” ................................................................... 173

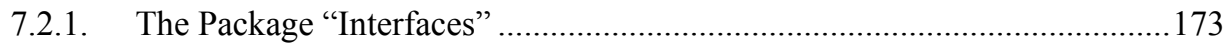

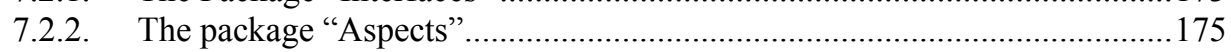

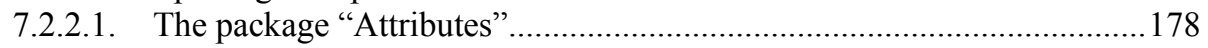

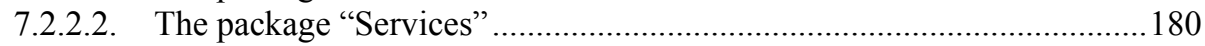

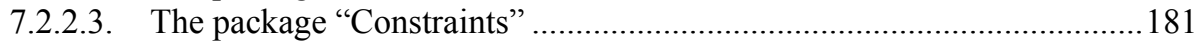

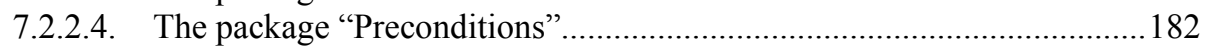

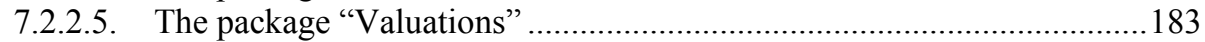

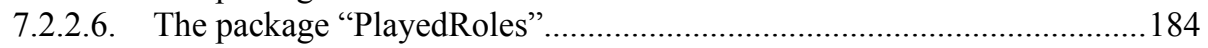




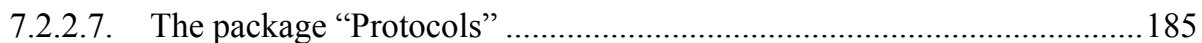

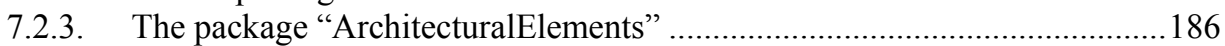

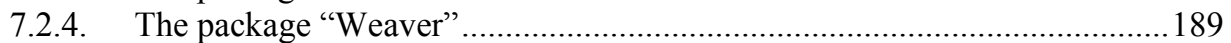

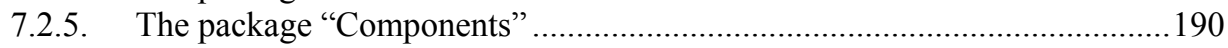

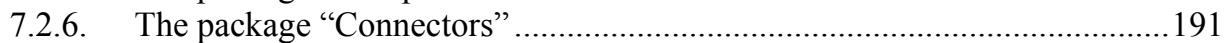

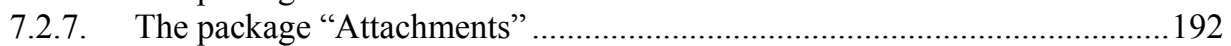

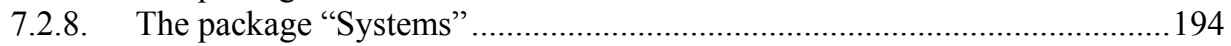

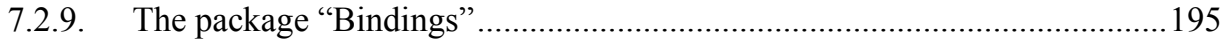

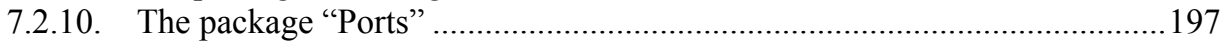

7.3. THE PACKAGE “ARCHITECTURE SPECIFICATION"............. 198

7.4. THE PACKAGE "COMMON" ......................................................... 200

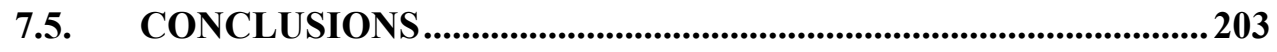

CHAPTER 8 THE PRISMA ASPECT-ORIENTED ARCHITECTURE DESCRIPTION LANGUAGE ...............................................................205

8.1. THE TYPE DEFINITION LEVEL ..................................................206

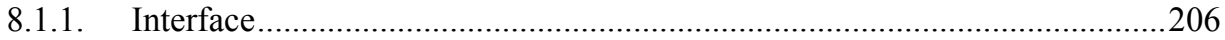

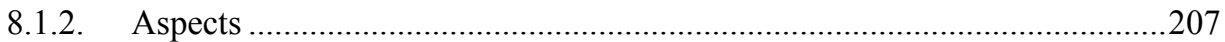

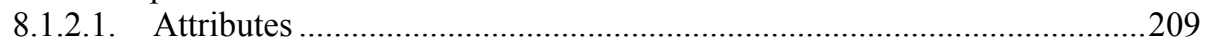

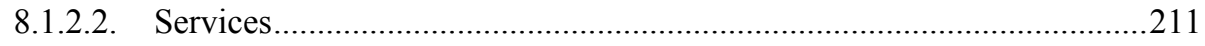

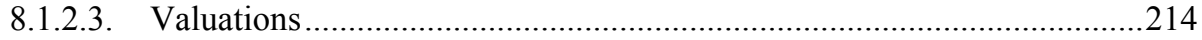

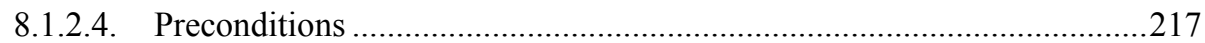

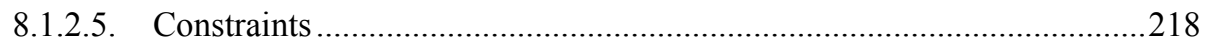

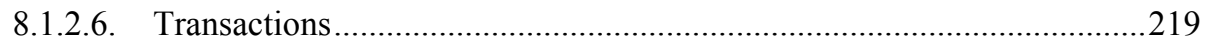

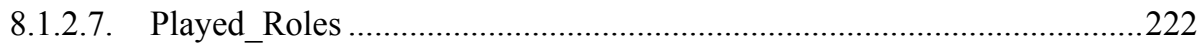

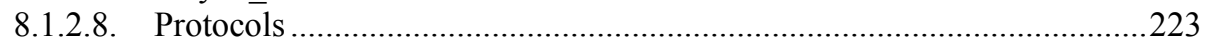

8.1.3. Simple Architectural Elements: Components and Connectors ......................227

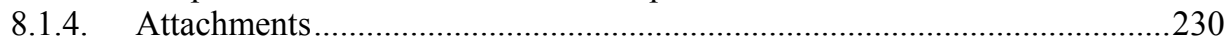

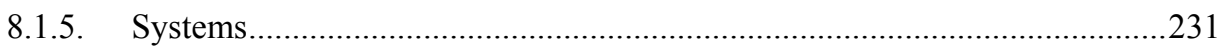

8.2. THE CONFIGURATION LEVEL _................................................ 237

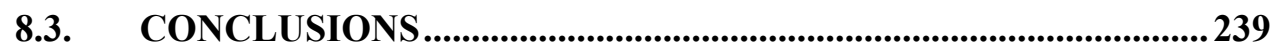

PART V THE PRISMA FRAMEWORK ............................................ 243

CHAPTER 9 THE PRISMA CASE ....................................................... 245

9.1. GRAPHICAL MODELLING TOOL ................................................ 247

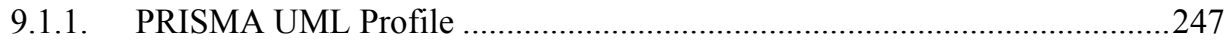

9.1.2. Domain-Specific Language Tools (DSL Tools) ........................................249

9.1.3. PRISMA as a Domain-Specific Language ...................................................252

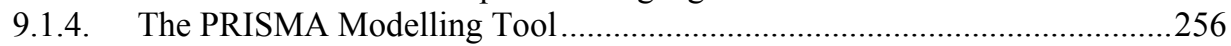

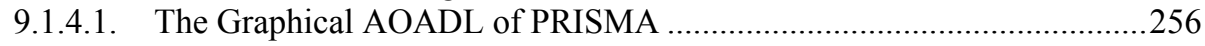

9.1.4.2. Verification of PRISMA Models ..........................................................263

9.2. MODEL COMPILER ............................................................... 264 


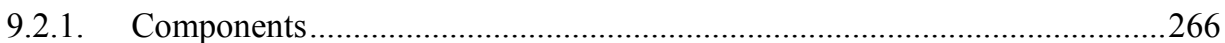

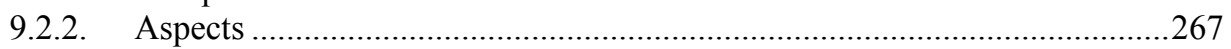

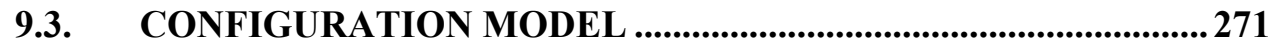

9.4. PRISMANET …....................................................................................2275

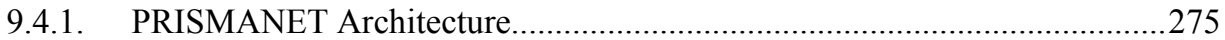

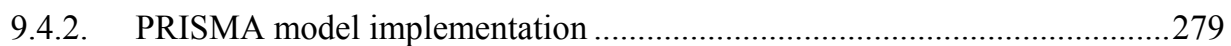

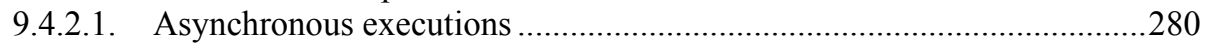

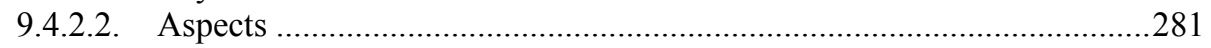

9.4.2.3. Simple Architectural Elements: Components and Connectors .................2.284

9.4.2.4. Communication: Attachments and Bindings ..........................................290

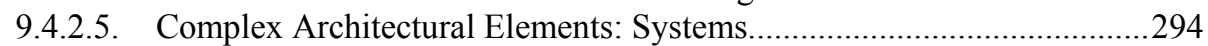

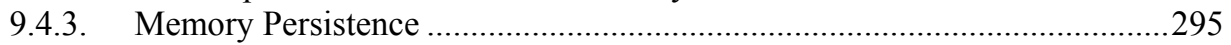

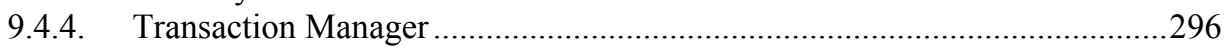

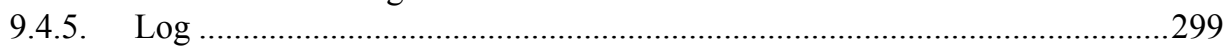

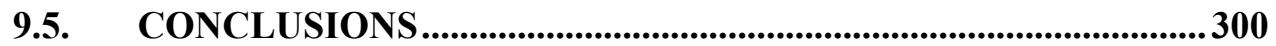

CHAPTER 10 THE PRISMA METHODOLOGY....................................... 303

10.1. STAGES OF THE PRISMA METHODOLOGY ..............................304

10.1.1. First Stage: Detection of Architectural Elements and Aspects .................304

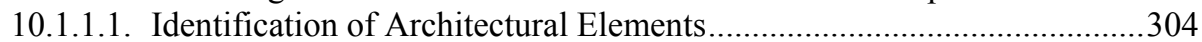

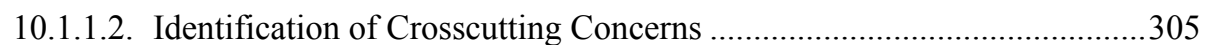

10.1.2. Second Stage: Software Architecture Modelling........................................305

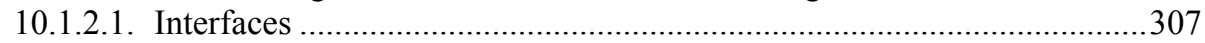

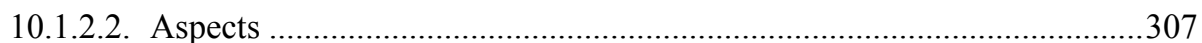

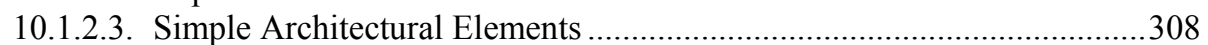

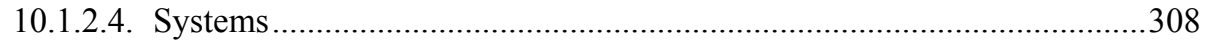

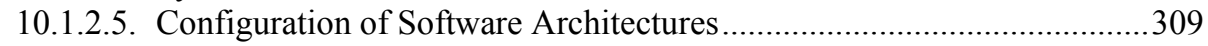

10.1.3. Third Stage: Code Generation and Execution........................................... 310

10.2. INTEGRATION OF COTS IN PRISMA............................................310

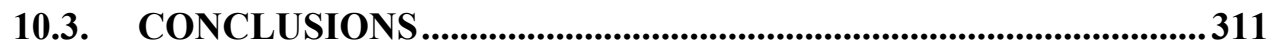

PART VI CONCLUSIONS AND FURTHER RESEARCH.................... 315

CHAPTER 11 CONCLUSIONS AND FURTHER RESEARCH............. 317

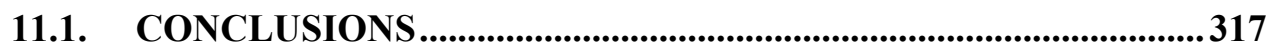

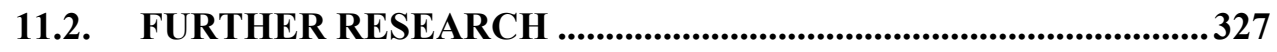

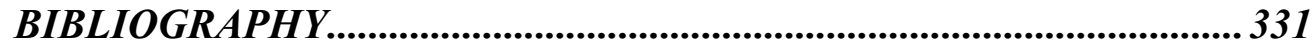

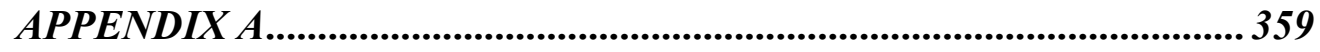

A. PRISMA AOADL SYNTAX.............................................................. 359

A.1. ARCHITECTURAL MODEL........................................................360

xvii 


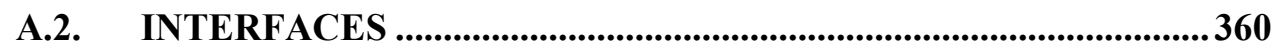

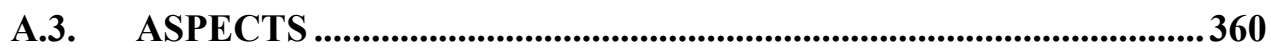

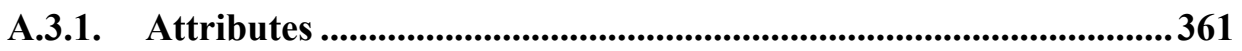

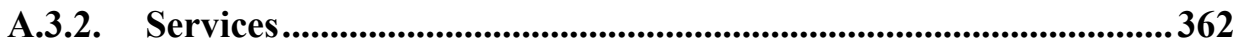

A.3.3. Preconditions .............................................................................................362

A.3.4. Transactions................................................................................................362

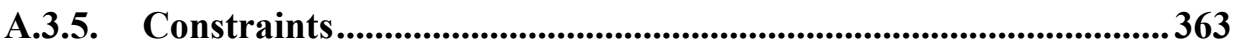

A.3.6. Played_Roles...............................................................................364

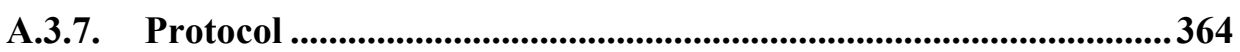

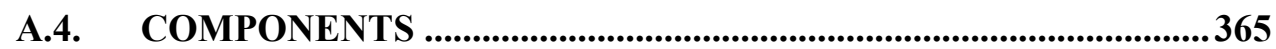

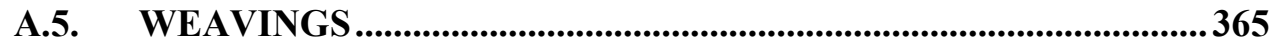

A.6. PORTS.................................................................................................366

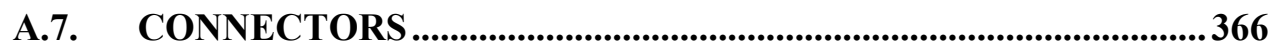

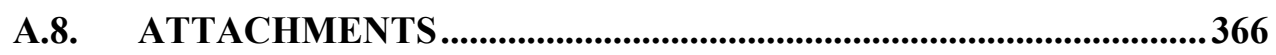

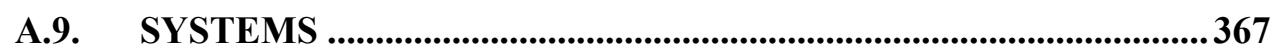

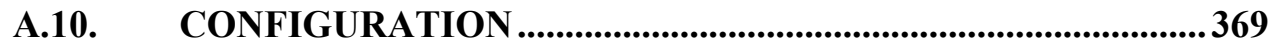

A.11. COMMON ELEMENTS ...........................................................371

A.11.1. DataTypes …………..................................................................................371

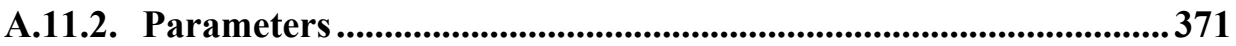

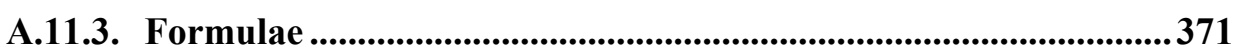

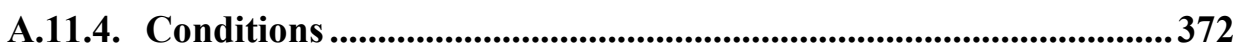

A.11.5. Arithmetic Expressions..................................................................372

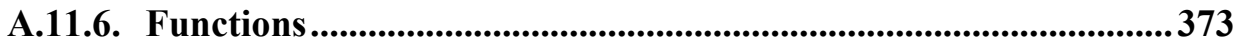

A.11.7. Processes......................................................................................................373

APPENDIX B THE PRISMA UML PROFILE ..................................... 375

B.1. CORRESPONDENCES BETWEEN PRISMA CONCEPTS AND

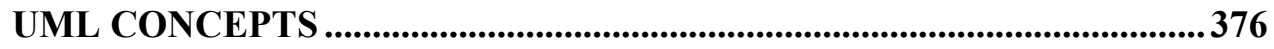

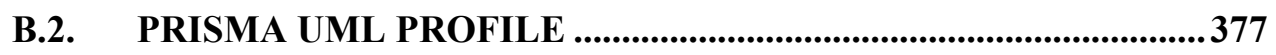

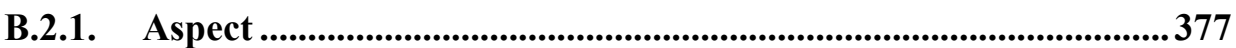

xviii 


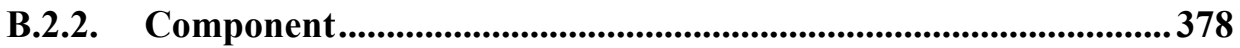

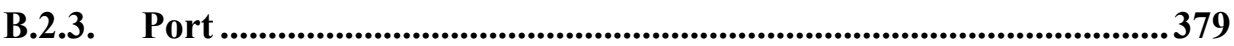

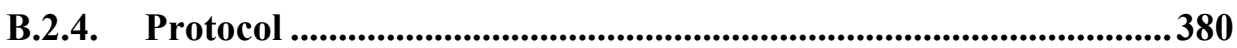

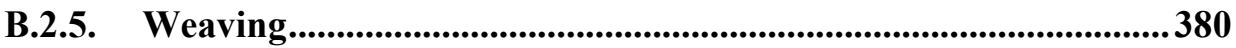

B.2.6. Attachment.................................................................................................. 381

B.2.7. Binding .......................................................................................................381

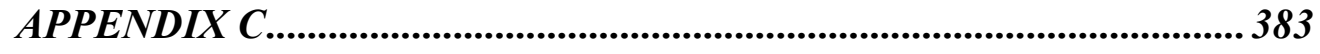

C. PRISMA SOFTWARE ARCHITECTURE OF THE TEACHMOVER 383

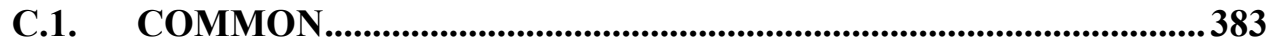

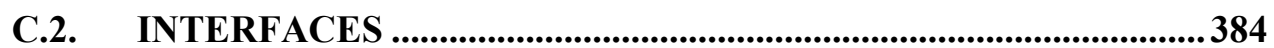

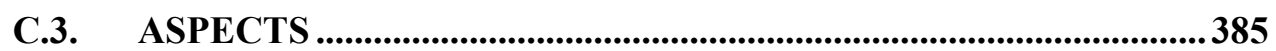

C.4. ARCHITECTURAL ELEMENTS _................................................389

C.5. CONFIGURATION .................................................................................. 392

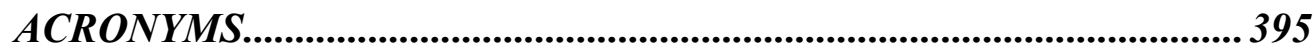

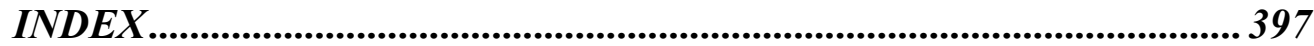




\section{INDEX OF FIGURES}

Figure 1. Software architecture as a bridge between requirements and

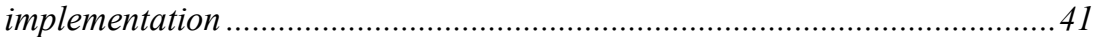

Figure 2. HyperJ/Matrix [Kim02]_........................................................................ 72

Figure 3. The Composition Filter Model with Superimposition [Ber01] .......................73

Figure 4. A Perspectival Concern-Space in Overview [Kan03] …………...................87

Figure 5. Superimposition [Sih03] .............................................................. 90

Figure 6. Concern Diagram of AVA [Kat03].........................................................94

Figure 7. Unified Component Architecture [Suv05b] ………………….....................99

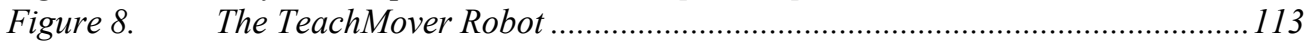

Figure 9. Joints of the TeachMover robot.......................................................... 114

Figure 10. Architectural Elements of the TeachMover Software Architecture ...............116

Figure 11. Simple Kripke structure...................................................................118

Figure 12. Crosscutting-concerns in PRISMA architectures .......................................130

Figure 13. Black box view of an architectural element .................................................131

Figure 14. White box view of an architectural element .............................................. 131

Figure 15. Communication between the white box and the black box views .................132

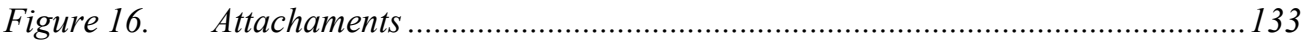

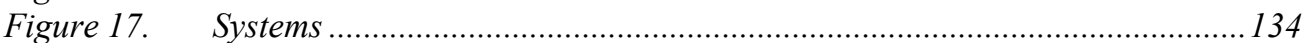

Figure 18. Specification of the interace IMotionJoint …............................................ 135

Figure 19. The interface IMotionJoint ……………….......................................... 135

Figure 20. Formalization of a service.............................................................135

Figure 21. Specification of the played_role ACT ……………………....................... 138

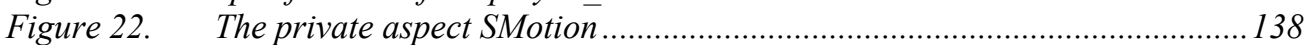

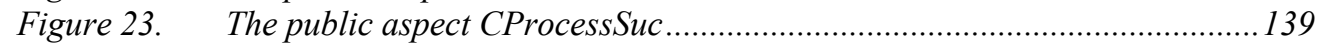

Figure 24. Specificatio of the valuation of the service moveJoint..................................141

Figure 25. Specification of the precondition of the service newPosition .........................141

Figure 26. Specification of the port PAct.................................................................. 143

Figure 27. Formalization of a service controlled by a weaving..................................... 144

Figure 28. Specification of a weaving between moveJoint and

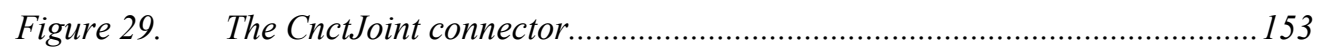

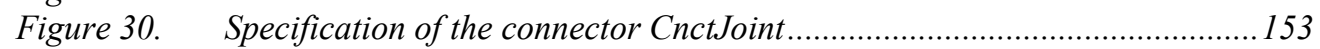

Figure 31. The component Actuator...................................................................... 154

Figure 32. Specification of the component Actuator..................................................154

Figure 33. Formalization of Attachments ................................................................... 155

Figure 34. Specification of an attachment between the Actuator and the CnctJoint......158

Figure 35. The attachment between the Actuator and the CnctJoint ............................158

Figure 36. Specification of played roles $A C T$ and ROBOT …...................................159

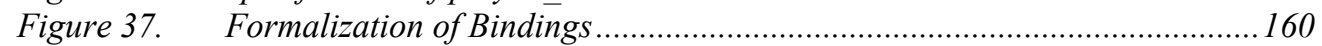

Figure 38. The binding between the CnctJoint and the Joint of the TeachMover..........162

Figure 39. Specification of a binding between the Joint and the CnctJoint ...................163

Figure 40. Specification of the played_role JOINT ……............................................ 163

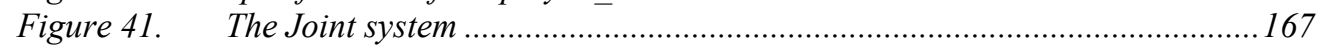


Figure 42. Figure 43. Figure 44. Figure 45. Figure 46. Figure 47. Figure 48. Figure 49. Figure 50. Figure 51. Figure 52.

Figure 53. Figure 54. Figure 55. Figure 56. Figure 57. Figure 58. Figure 59. Figure 60

Figure 61. Figure 62. Figure 63.

Figure 64. Figure 65.

Figure 66. Figure 67.

Figure 68.

Figure 69.

Figure 70.

Figure 71.

Figure 72.

Figure 73.

Figure 74.

Figure 75.

Figure 76.

Figure 77.

Figure 78.

Figure 79.

Figure 80.

Figure 81.

Figure 82.

Figure 83.

Figure 84.

Figure 85.

Figure 86.

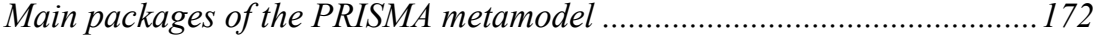

The package Types of the PRISMA metamodel ........................................... 172

The package Common of the PRISMA metamodel ...................................... 173

The package Interfaces of the PRISMA metamodel..................................... 174

The package SignatureOfService of the PRISMA metamodel ..................... 174

The sub-packages of the package Aspects of the PRISMA metamodel.........175

The metaclass Aspect of the package Aspects of the PRISMA metamodel ... 176

Constraints of the metaclass Aspect ........................................................ 177

The package Attributes of the PRISMA metamodel..................................... 178

The package KindsOfAttributes of the PRISMA metamodel.......................... 179

The package Derivations of the PRISMA metamodel.................................... 179

The package Services of the PRISMA metamodel ....................................... 180

The package KindsOfServices of the PRISMA metamodel .......................... 181

The package Constraints of the PRISMA metamodel ................................... 181

The package Preconditions of the PRISMA metamodel ............................. 182

The package Valuations of the PRISMA metamodel ................................... 183

The package PlayedRoles of the PRISMA metamodel................................. 184

The package Protocols of the PRISMA metamodel ..................................... 185

The subpackages of the package ArchitecturalElements of the PRISMA

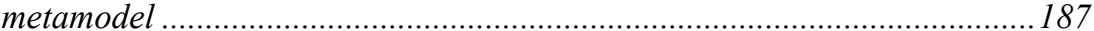

The package ArchitecturalElements of the PRISMA metamodel...................188

The package KindsOfArchitecturalElements of the PRISMA metamodel..... 188

The package Weaver of the PRISMA metamodel ....................................... 189

Constraints of the metaclass Weaving ........................................................ 190

The package Components of the PRISMA metamodel................................ 191

The package Connectors of the PRISMA metamodel .................................. 191

The package Attachments of the PRISMA metamodel................................ 192

The attachment between the Joint and the CnctMUC of the TeachMover ... 192

Different configuration of an architecture depending on the maximum cardinality of an attachment......................................................................... 193

The package Systems of the PRISMA metamodel........................................ 195

The package Bindings of the PRISMA metamodel .................................... 196

The package Ports of the PRISMA metamodel ........................................... 197

Constraints of the metaclass Port................................................................. 198

The package Architecture Specification of the PRISMA metamodel............199

The package DataTypes of the PRISMA metamodel .................................200

The package Parameters of the PRISMA metamodel ...................................2200

The package Constants of the PRISMA metamodel.......................................200

The package Formulae of the PRISMA metamodel..................................... 201

The constraint of the metaclass Postcondition of the Package Formulae....201

The package Processes of the PRISMA metamodel.......................................202

Constraints of the metaclass Transition of the package Processes .............. 202

Specification template of interfaces............................................................207

Specification of the interface IMotionJoint .................................................207

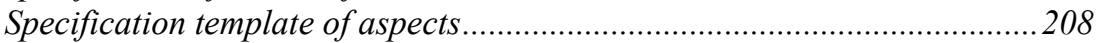

Specification of an aspect head with interfaces (CProcessSuc) ...................208

Specification of an aspect head without interfaces (SMotion).....................208

xxii 
Figure 87. Figure 88.

Figure 89.

Figure 90.

Figure 91.

Figure 92.

Figure 93.

Figure 94.

Figure 95.

Figure 96.

Figure 97.

Figure 98.

Figure 99.

Figure 100.

Figure 101.

Figure 102.

Figure 103.

Figure 104.

Figure 105.

Figure 106.

Figure 107.

Figure 108.

Figure 109.

Figure 110.

Figure 111.

Figure 112.

Figure 113.

Figure 114.

Figure 115

Figure 116.

Figure 117.

Figure 118.

Figure 119.

Figure 120.

Figure 121 Figure 122.

Figure 123.

Figure 124.

Figure 125.

Figure 126.

Figure 127.

Figure 128.

Figure 129.

Figure 130

Figure 131.

Figure 132.
Specification template of attributes ............................209

Specification of the constant attributes of the aspect SMotion .....................210

Specification of the variable and derived attributes of the aspect FJoint....210

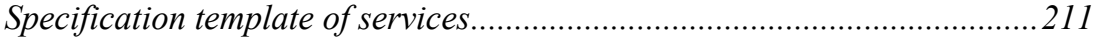

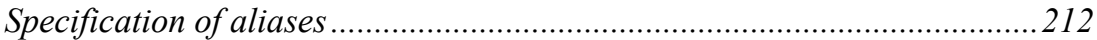

Specification template of valuations...........................................................2 214

Specification of the service check and its valuations..................................216

Specification of the service moveOk and its valuations..............................217

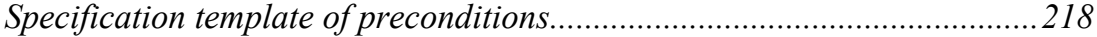

Specification of preconditions ...................................................................218

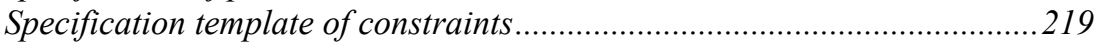

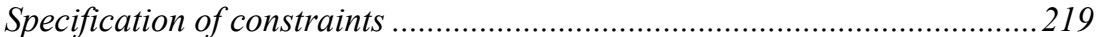

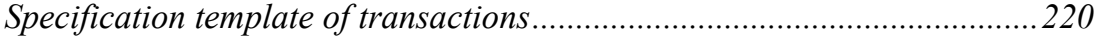

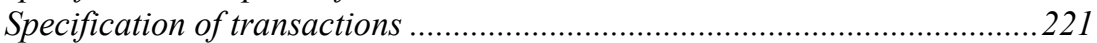

Specification template of played_roles................................................... 222

Specification of played_roles of the aspect CProcessSuc............................222

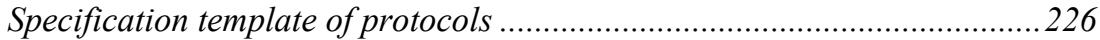

Specification of the protocol of the aspect CProcessSuc..............................226

Specification template of simple architectural elements .............................222

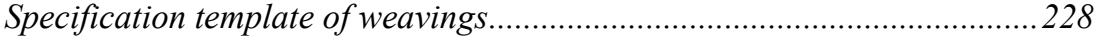

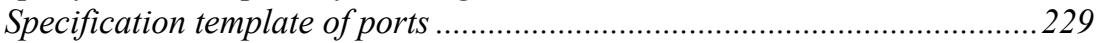

Specification of the connector CnctJoint ...................................................2230

Specification template of attachments ....................................................... 231

Specification of an attachment between the Actuator and the CnctJoint.....2231

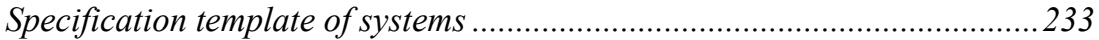

Specification template of bindings............................................................... 233

Specification template of the creation and destruction of systems ...............235

Specification of the system Joint .............................................................2236

Specification template of configurations of architectural models ................2238

The TeachMover architectural model (The Base Configuration).................239

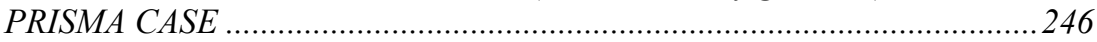

DSLTools Framework: Domain Model of PRISMA …..............................251

Toolbox of the Domain Model ....................................................................... 253

Definition of Architectural Elements and Aspects in the DomainModel of

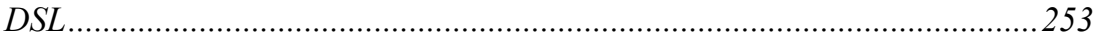

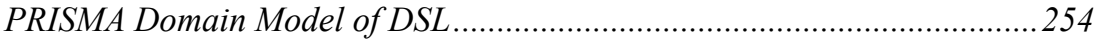

PRISMA Designer of DSL ........................................................................... 255

The Visual Studio Project of PRISMA ......................................................... 255

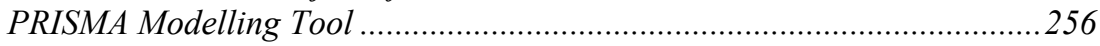

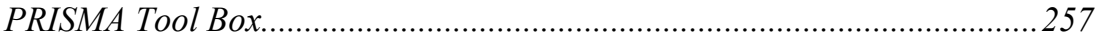

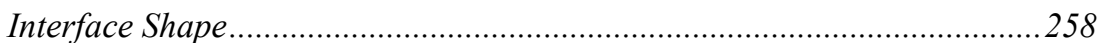

Modelling Services of Interfaces ............................................................... 258

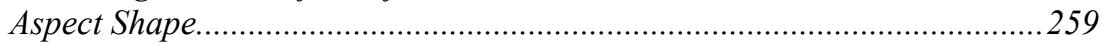

Link Shape between Aspects and Interfaces .............................................2259

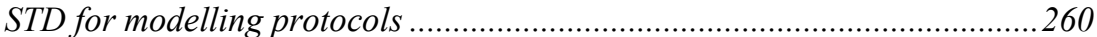

Shapes of Architectural Elements ..............................................................261

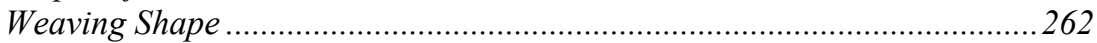

xxiii 
Figure 133. Attachment Shape ................................................................................ 262

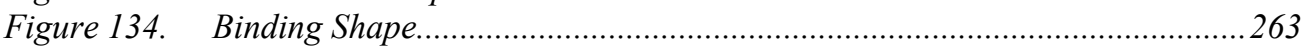

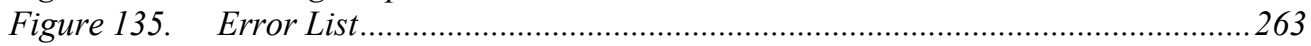

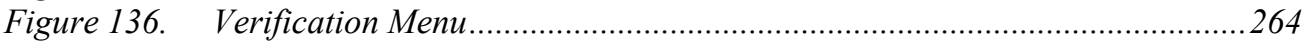

Figure 137. Contextual Menu of an Element ..................................................................2 264

Figure 138. PRISMA Code Generation Templates .........................................................265

Figure 139. The generated C\# code of the component Actuator......................................267

Figure 140. The generated C\# code of the aspect FunctionActuator..............................270

Figure 141. Generation and Execution of the PRISMA Modelling Configuration Tool..272

Figure 142. Model Persistence and Configuration Language Information .......................2 273

Figure 143. Generic GUI of PRISMA Applications .....................................................2 274

Figure 144. Layer classification of technologies that support AOP in .NET framework. 276

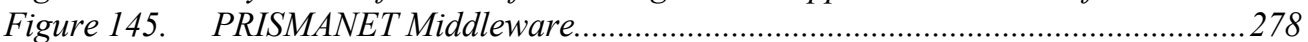

Figure 146. Namespaces of the module PRISMA Execution Model..................................2 279

Figure 147. Main classes of the namespace Aspects of PRISMANET .............................281

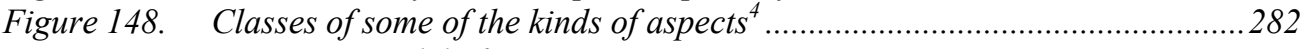

Figure 149. Execution Model of an Aspect ................................................................... 283

Figure 150. Main classes of the namespace Components of PRISMANET ${ }^{4}$....................285

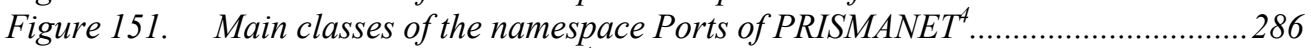

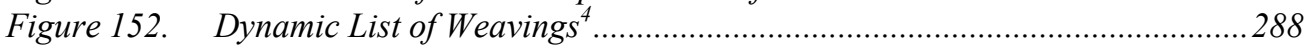

Figure 153. The execution model of an architectural element ........................................28

Figure 154. Main classes of the namespace Attachments of PRISMANET ${ }^{4}$.....................290

Figure 155. The execution model of an attachment .......................................................291

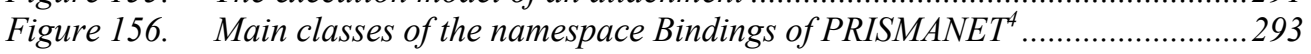

Figure 157. The execution model of bindings ..................................................................294

Figure 158. Main classes of the namespace Systems of PRISMANET ${ }^{4}$............................295

Figure 159. Main classes of the namespace Middleware of PRISMANET ${ }^{4}$.....................296

Figure 160. Main classes of the namespace TransactioManager of PRISMANET ${ }^{4}$..........297

Figure 161. Main classes of the TransactioLog of PRISMANET ${ }^{4}$...................................299

Figure 162. Execution of a PRISMANET Log................................................................299

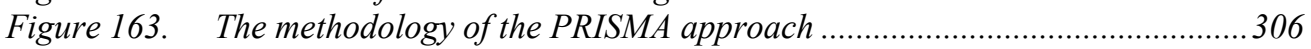

Figure 164. Modelling of the stereotype Aspect..................................................... 378

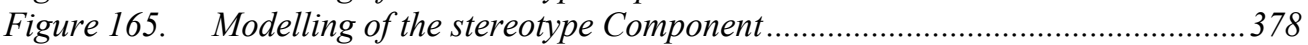

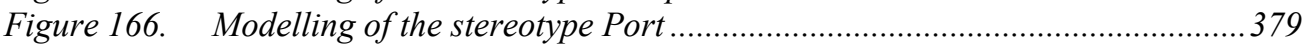

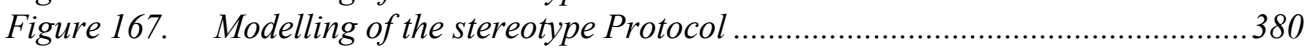

Figure 168. Modelling of the stereotype Weaving ..................................................... 380

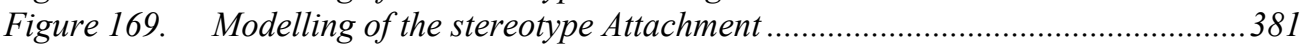

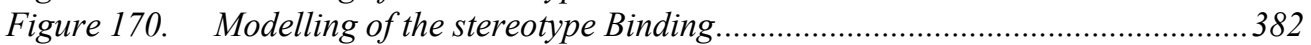




\section{INDEX OF TABLES}

Table 1. First comparison of aspect-oriented software architecture approaches...........105

Table 2. Second comparison of aspect-oriented software architecture approaches ....... 107

Table 3. $\quad$ Polyadic $\pi$-calculus with priorities .................................................................. 122

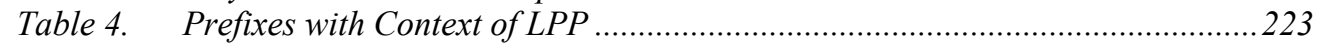

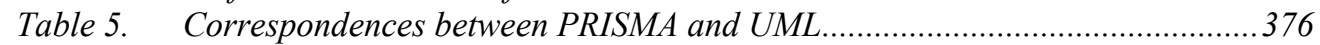

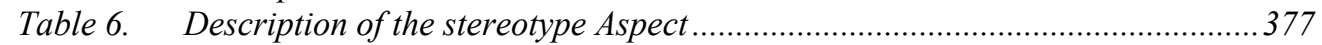

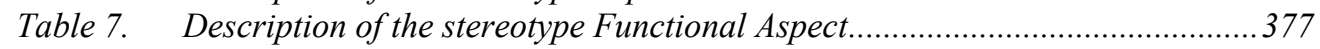

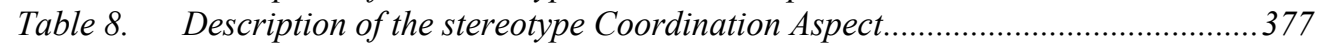

Table 9. Description of the stereotype Safety Aspect ................................................. 377

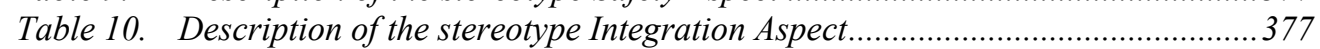

Table 11. Description of the stereotype Component ................................................... 378

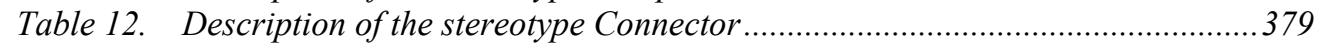

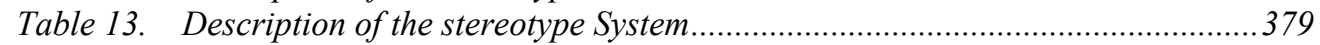

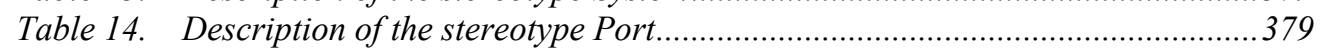

Table 15. Description of the stereotype Protocol ......................................................... 380

Table 16. Description of the stereotype Weaving ......................................................... 380

Table 17. Description of the stereotype Attachment ...................................................... 381

Table 18. Description of the stereotype Binding ......................................................... 381 


\section{PART I}

\section{INTRODUCTION}

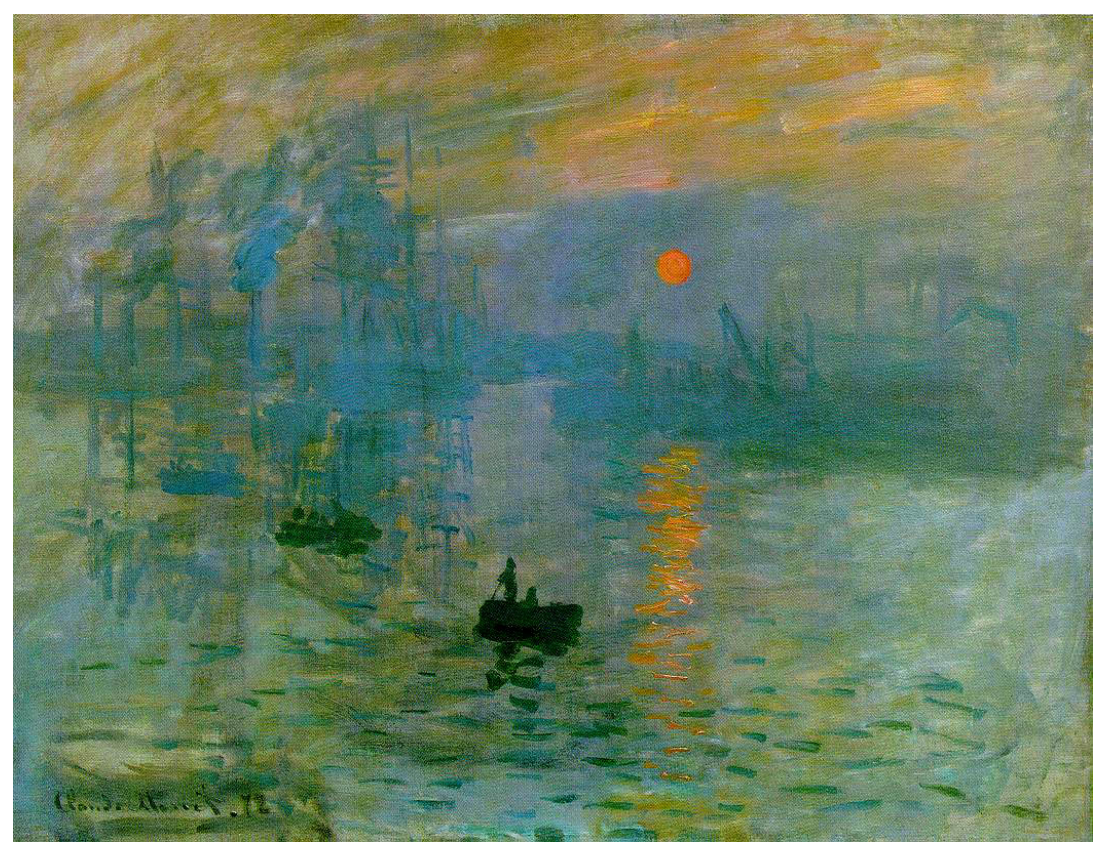

"Soleil Levant”, Claude Monet, 1872 



\section{CHAPTER 1}

\section{INTRODUCTION}

$<<$ There are only two rules for writing:

have something to say and say it >>

Oscar Wilde

The work presented in this thesis is an approach for developing complex software systems that improves the software quality and reduces the time and cost invested in its development and maintenance processes. The approach is supported by a framework that consists of a model, a language, a methodology, and a Computer-Aided Software Engineering (CASE) tool prototype. The model defined in this work is called PRISMA. It combines two approaches to define software architectures: the Component-Based Software Development (CBSD) and the Aspect-Oriented Software Development (AOSD). The main contributions of the model are the way that it integrates both approaches to take their advantages as well as the definition of a formal Aspect-Oriented Architecture Description Language (AOADL). The AOADL is independent of technology and is based on a formal language and formalisms that preserve non-ambiguity for applying code generation techniques.

The methodology proposed in this thesis follows the Paradigm of Automatic Programming [Bal85] by applying the Model-Driven Development (MDD) approach. The PRISMA model and its methodology are supported by the CASE tool prototype called PRISMA CASE. This tool allows the specification of PRISMA architectures using its AOADL in a textual and a 
PRISMA: Aspect-Oriented Software Architectures

graphical way and also allows the automatic code generation thanks to the middleware and the code generation patterns that the CASE tool prototype integrates.

The structure of this chapter is as follows: Section 1 introduces the motivation of this work. Section 2 explains the main goals of the thesis, section 3 presents the research methodology that has been followed during the development of the thesis, and section 4 summarizes the structure of the thesis.

\subsection{MOTIVATION}

Complex structures, non-functional requirements, heterogeneity, scalability, traceability, reusability and maintainability are leading properties that current software systems need to deal with. In the last few years, these properties have increased the time and the staff invested in the development and maintenance processes of software. As a result, there is greater interest in research areas to reduce the time and the cost invested in these software system processes. In order to achieve the milestones of software products and to overcome the competitiveness of the market, models for the software development, techniques to improve reusability, and processes to support automation, traceability and maintainability of software have been proposed.

The complexity, heterogeneity, scalability and reusability properties of current software systems have led to considering the analysis of the software structure as an important phase of the software life cycle. As a result, in the last two decades, a new research area called Software Architectures has emerged. Software architectures are presented as a solution for the design and development of complex software systems. However, there is no a consensus about the different concepts and approaches that should be used in the area.

The Component-Based Software Development (CBSD) approach is used in the field of software architectures. This approach decomposes the software system into reusable entities called components. Components provide services to the rest of the system by encapsulating their functionality (black boxes). As a result, software architectures can be described preserving the reusability of their components. 
The reusability of software allows the same software artefact to be used in different places of the same application or in different applications. The artefact is only programmed one time and can be used more than once. This reusability reduces the development time of software systems. Also, reused software artefacts guarantee their quality and suitable functionality because they have been tested and used before. As a consequence, the COTS (Commercial Off-The-Shelf) importation has acquired relevance, because tools that allow the reuse of their components and the COTS importation achieve the highest reuse and quality code.

Another approach that has emerged to improve reusability is the Aspect-Oriented Software Development (AOSD) approach. This approach allows for the separation of concerns by modularizing crosscutting concerns into a separate entity called aspect. As a result, the same aspect can be reused by different software artefacts, which are usually, objects.

The automatic code generation from models reduces the cost and time of the development process as well. Nowadays, there are many CASE tools that are able to generate applications following the Automatic Programming Paradigm proposed by Balzer [Ba185]. These tools are widely-known as model compilers. They automatically generate the application code and the database schema from the conceptual schema of a software system. The automatic generation can be complete as in Oblog Case [Ser94], OlivaNova ${ }^{\circledR}$ (OO-Method/CASE [Pas97]), or it can be partial, as in Rational Rose [RAT06], System Architect [SYS06], Together [TOG06] and others. However, since these model compilers follow the Object-Oriented Paradigm, the need for developing model compilers that follow the CBSD and/or AOSD approaches has emerged. The combination of the CBSD and AOSD reusability and the automatic code generation achieves higher reduction in the time and cost of the development process than using only one of these approaches.

In the software life cycle, the maintenance process is as important as the development process due to the fact that the requirements of software systems are continuously evolving. The sources of these changes can be caused by several factors. First of all, the requirements specifications are inaccurate and ambiguous and these deficiencies promote misunderstandings from the very beginning of the software life cycle. An incorrect requirements specification can be produced by an inexperienced analyst, by a lack of accuracy in the presentation of the 
PRISMA: Aspect-Oriented Software Architectures

customer's needs or by a misunderstanding between the analyst and the customer because of the semantic gap in their vocabularies. This means that the software product will require continuous changes until the software that the customer really wanted is finally produced. The traceability among the different stages of the software life cycle must be preserved in order to ensure quality maintenance of software products.

An important challenge in the software engineering area is the integration of software architectures, CBSD and AOSD approaches, and automatic code generation and traceability techniques in an unique approach in order to support the development and maintenance of complex software systems in an efficient way.

\subsection{OBJECTIVES OF THE THESIS}

The main goal of this thesis is to provide a framework to develop complex software systems. The framework must integrate the definition of software systems and improve their development and maintenance processes. The development of this framework must be based on approaches and techniques that allow us to obtain the expected results. This is achieved by combining the CBSD, the AOSD, and the MDD approaches together with the Paradigm of Automatic Programming.

The main goal of the thesis can be divided into several specific objectives:

> To study the related works of Architecture Description Languages (ADLs), AspectOriented Languages and the proposals that integrate the aspect-orientation approach and ADLs.

$>$ To define and formalize a model that integrates AOSD and CBSD in the definition of software architectures. This integration must provide mechanisms to reuse aspects and components.

$>$ To define an Aspect-Oriented ADL (AOADL) to specify software architectures based on the defined model. This language must provide the needed expressiveness to completely specify complex software systems and must be based on formalisms that ensure the nonambiguity of specifications. 
$>$ To provide graphical support for the defined AOADL in order to make the analysis and design of aspect-oriented software architectures easier and friendlier.

> To validate the expressiveness of the language by completely specifying an industrial case study using the language.

$>$ To define a metamodel to specify the properties of the model.

$>$ To propose a methodology to guide the analyst throughout the development process of aspect-oriented software architectures.

$>$ To develop a framework that supports the graphical and textual specifications of software architectures, automatic code generation, execution of software architectures, traceability throughout the different stages of the software development, and mechanisms to easily maintain the software product. This framework must integrate a middleware and a catalogue of code generation patterns. The middleware must permit the execution of the software architectures based on the proposed model, and the code generation patterns must provide the rules to automatically generate the source code of a specific programming language from a specification of the proposed ADL.

\subsection{RESEARCH METHODOLOGY OF THE THESIS}

The research methodology that has been applied in order to fulfill the objectives proposed in this thesis follows a classical methodological strategy often called the "feasibility research strategy". This methodology departs from a generic and conceptual hypothesis that is presented as a contribution in the area in which the thesis is developed. This hypothesis is based on a previous analysis of the state of art where the contribution of the thesis is justified. This thesis departs from the following hypothesis: Is it possible to describe and implement software architectures in terms of a symmetric aspect-oriented model?. In addition, the thesis departs from the set of objectives that have been established in order to answer this question as well as the mechanisms and formalisms that are going to be used to reach them. From this starting point, the main goal of this thesis is to reach to a software engineering solution that copes with the set of specific objectives that have been established in section 1.2 . 
PRISMA: Aspect-Oriented Software Architectures

The contributions of the thesis have been developed taken into account the initial hypothesis and the main goal. These contributions are the definition of a model and its corresponding language to describe aspect-oriented software architectures in a formal and pragmatic way. A software engineering solution has been developed from these contributions. This solution must satisfy the feasibility of the hypothesis that makes reference to the implementation of aspectoriented software architectures. As a result, the solution is embodied in a development framework that consists of a model, a language, a middleware and a modelling tool. This framework has permitted the validation of the hypothesis of this thesis by demonstrating the complete description and implementation of a real case study.

\subsection{STRUCTURE OF THE THESIS}

The remainder of this thesis is organized in the following chapters:

$>$ Chapter 2: Software Architectures

This chapter provides an introduction to the role of software architectures in the software life cycle and their main properties. It also establishes a conceptual base for the notion of software architecture and the different concepts of this field.

\section{> Chapter 3. Aspect-Oriented Software Development}

This chapter provides a conceptual base for the different concepts of the aspect-oriented paradigm and presents a review of the different kinds of aspect-oriented models that have been proposed in the field. It also provides an introduction about how the aspect-oriented approach is currently being introduced in the software life cycle.

$>$ Chapter 4. Aspect-Oriented Software Architectures

This chapter analyzes in detail the most relevant approaches that integrate aspects in software architectures. The set of desirable properties that aspect-oriented software architecture approaches should fulfil is also presented. Finally, a comparison of these approaches using this set of properties is presented and discussed. 
Introduction

Chapter 5: Preliminaries

This chapter introduces the case study that has been chosen to demonstrate the PRISMA approach. It introduces the formalisms that have been used to describe software architectures in PRISMA and to formalize the PRISMA model.

$>$ Chapter 6: The PRISMA Model

This chapter defines, formalizes, and exemplifies the main concepts of the PRISMA model.

$>$ Chapter 7: The PRISMA Metamodel

This chapter presents the PRISMA metamodel in detail. Specifically, it presents the packages, metaclasses, relationships, and constraints that the metamodel consists of.

> Chapter 8: The PRISMA Aspect-Oriented Architecture Description Language This chapter presents the structure and syntax of the PRISMA Aspect-Oriented Architecture Description Language in detail.

Chapter 9: The PRISMA CASE

This chapter presents the PRISMA CASE in detail. The chapter explains how the PRISMA CASE supports the metamodel and how the modelling tool supports the graphical PRISMA AOADL. The chapter introduces the PRISMA model compiler and its code generation patterns. Then, the chapter explains how the configuration of software architectures is integrated in PRISMA CASE, and how PRISMA configurations can be executed. Finally, the PRISMANET middleware is presented to show how the execution of PRISMA software architectures is supported by the .NET platform. 
PRISMA: Aspect-Oriented Software Architectures

Chapter 10: The PRISMA Methodology

This chapter presents the PRISMA methodology using the TeachMover robot case study as an example. The different stages of the methodology are described and the integration of COTS in PRISMA software architecture is explained.

> Chapter 11: Conclusions and Further Research

This chapter presents the main contributions of the thesis and future research work.

Appendix A: The PRISMA AOADL Syntax

This appendix presents the BNF of the PRISMA AOADL

Appendix B: The PRISMA UML Profile

This appendix presents the PRISMA UML profile.

Appendix C: The PRISMA Description of the TeachMover Software Architecture This appendix presents the complete specification of a joint of the TeachMover Software Architecture. This specification has been automatically generated using the PRISMA CASE. 


\section{PART II}

\section{STATE OF THE ART}

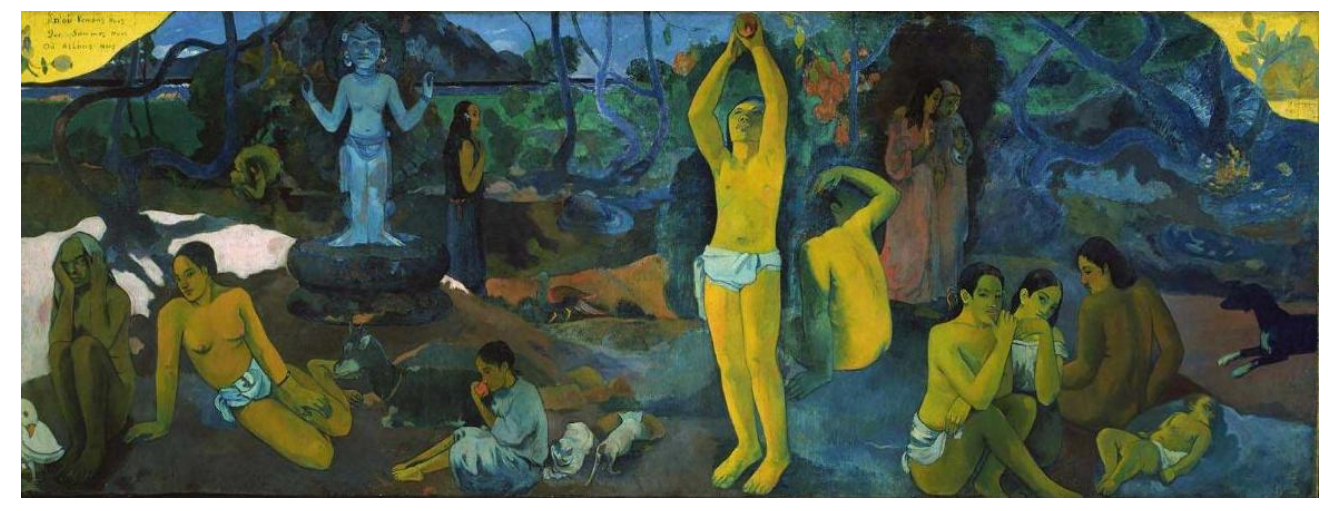

“Where Do We Come From? What Are We? Where Are We Going?”, Paul Gauguin, 1897 



\title{
CHAPTER 2
}

\section{SOFTWARE ARCHITECTURES}

\author{
$<<$ Great souls are not those who have fewer \\ passions and more virtues than others, \\ but only those who have greater designs. $>>$ \\ François de la Rochefoucauld
}

The complexity of current software systems has led computer community to recognize the analysis of software structure as an important phase of the software life cycle. As a result in the last decades, a new research area called Software Architecture has emerged to deal specifically with this phase. The software architecture discipline has emerged due to the natural increase in size and complexity of current software systems. An inaccurate architectural design leads to the failure of large software systems. For this reason, the design, specification, and analysis of the structure of these software systems have become critical issues in software development [Gar01].

Software architectures are presented as a solution for the design and development of large, complex software systems. They allow us to describe the structure of a software system by hiding the low-level details and abstracting the high level important features [Per92]. This structure is usually represented in terms of computational elements and their interactions. As a result, software architectures make software systems simpler and more understandable [Gar95a]. 
PRISMA: Aspect-Oriented Software Architectures

The software architecture discipline is capable of performing the following functions: analyze and describe the properties of systems at a high level of abstraction; validate software requirements; estimate the cost of the development and maintenance processes; reuse software, and establish the bases and guides for the design of large complex software systems [Per92]. At the same time, software architectures should be adaptable and should provide support for the reuse of architectural elements and of partial or complete software architecture descriptions in the new software architecture specifications. Thus, new designs are not started from scratch and only the specific features of the new systems are created from the beginning [Per92]. In this sense, product lines engineering [Cle01], [Dee05] can take advantage of the reuse of the common features of product families, and only customize the specific properties [Gar01].

However, despite the attempt of the IEEE to standardize the software architecture discipline [IEE00], there is no consensus about the definition of software architecture and the different concepts and approaches to be used in this field. Therefore, the main purpose of this chapter is to provide an introduction to the role of software architectures in the software life cycle and their main properties as well as to establish a conceptual base for the notion of architecture and the different concepts of the field.

\subsection{SOFTWARE ARCHITECTURES IN THE SOFTWARE LIFE CYCLE}

The works of Garlan and Perry clearly define the role of software architectures in the software life cycle. The Software Architecture discipline bridges the gap between the requirements phase and implementation phase of the software life cycle (see Figure 1). From the point of view of requirements, software architectures should be the mechanism to ensure that the requirements of the software system are satisfied. In addition, software architecture descriptions not only can help us to study the feasibility of the development of software systems, but also can help to determine which requirements are reasonable and viable [And03].

The software architecture phase should provide a way to describe the outlines of different architectural designs in order to choose one of them based on the advantages and disadvantages of each one [Gar95a]. A good choice and its traceability are the keys to prevent the failure of 
the software development process. For this reason, once a software architecture description has been chosen, it is presented as the guide to follow during the implementation of a software system.

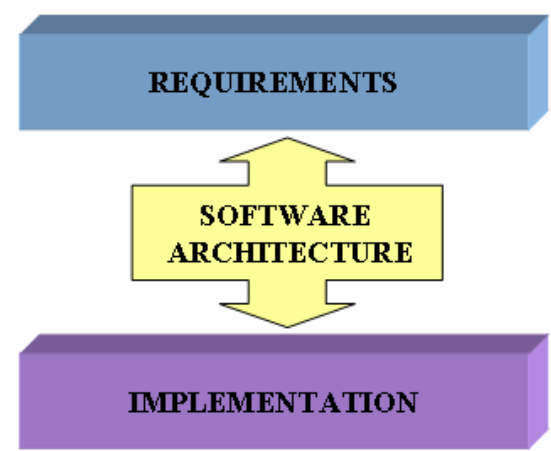

Figure 1. Software architecture as a bridge between requirements and implementation

With regard to implementation, the code should satisfy the architecture and the requirements of the system by defining algorithms and data types that preserve the traceability with the previous stages [Per92].

Finally, it is important to emphasize the role of software architecture in the maintenance phase of the software life cycle. Software architectures clarify the design of software systems and help to determine the impact of changes that occur during the maintenance process of software systems [And03]. As a result, software architectures offer more modifiable designs.

In summary, the software architecture phase has great influence on the other of phases of the software life cycle. For this reason, software architecture has become an important area in the software engineering field.

\subsection{PROPERTIES OF SOFTWARE ARCHITECTURES}

Software architecture descriptions guide the implementation of software systems. The success or failure of the final product depends to a greater extent on the quality of the software architecture specification. Therefore, the specification of software architectures must be done in a formal way. A wide variety of Architecture Description Languages (ADLs) [Med97], 
PRISMA: Aspect-Oriented Software Architectures

[Med00], evaluation methods, and analysis tools have been proposed [And03] to provide a formal support to software architecture descriptions.

ADLs describe the elements that form a software architecture, their interactions, and the needed constraints on these elements and their interactions in order to accurately specify software systems. ADLs should support the hierarchical specification of elements while preserving their encapsulation and showing them as simple elements. They should provide mechanisms that define standard elements or architecture descriptions that can be reused. ADLs should also provide a specific element to describe the coordination process to communicate a set of computational elements [And03].

In his work, Perry [Per92] sets out the need for software architectures to describe design properties in terms of restriction or permission, generality or particularity, necessity or extension, relativity or absolutism. He also addresses the fact that software architectures must clearly separate engineering from aesthetics, and must provide a mechanism of views to show the different aspects of the architecture. Finally, he also describes the traceability between the different levels of the software life cycle and the dependency among the different parts of an architecture description as one of the most important properties of software architectures. Thus, an architectural approach must preserve the forward and backward links of traceability and the dependency of software architecture descriptions in order to keep the consistency of software systems.

Another fundamental property of software architectures is the property of reuse. It is desirable for part of the specification of software architectures to be reused by other descriptions or catalogues [Per92]. This property is especially interesting because reuse at the implementation level is more difficult than at the architectural level. This is due to the fact that the code is so specific and has a lot details that are hidden in a software architecture description. As a result, software architectures have emerged as powerful mechanisms to reduce the time and cost invested in the development process by means of reuse.

From this survey of works, we can determine which properties ADLs should provide: formal specifications, compositional mechanisms, encapsulation, specification of constraints, traceability, reuse, mechanisms of views, analysis of properties, and separation of engineering 42 
and aesthetics. However, there is no consensus about which properties and quality attributes a specific architecture description should have in order to determine its quality and to establish a criterion for selection among several specifications. No much work of the analysis of software architectures has been done, but there are some works [Dob02] that are trying to develop this common analysis criterion. These works propose a set of properties to analyze an architecture description that can be used for any software system.

It is important to emphasize the works of Rick Kazman on the analysis of software architecture properties, the SAAM [Kaz94] and ATAM [Kaz98] methods. The SAAM method introduces three perspectives to analyze software architecture specifications: functionality, structure, and allocation. Functionality is the activity that the system performs; structure refers to the components and connections; and allocation describes how the functionality is reflected on the structure. This method is divided into five steps: the canonical functional partition, the mapping of the functional partition on structure, the selection of quality attributes, the selection of testing tasks, and the evaluation of results. The ATAM method is based on the analysis of scenarios [Kaz96], which are obtained as a refinement of software architecture descriptions. The result of this analysis is a set of risks, non-risks, sensitivity points, and trade-off points in the architecture. In addition, the ARID method [Cle00] emerges to complete the proposal of ATAM with a technique for insuring quality detailed designs in software.

Another work that offers an interesting perspective on the properties that should be analyzed in a software architecture specification is the TOPSA method [Bra99]. TOPSA suggests three properties or dimensions to analyze software architecture descriptions: abstraction level, dynamism, and the aggregation level. The abstraction level dimension determines if the software architecture description is more conceptual (analysis) or realizational (design). The dynamism dimension determines whether the architecture is static or dynamic. Finally, the aggregation dimension establishes to what extent a structure is made from other structures. These three dimensions are represented as a matrix, and the result of the evaluation method is the position of a specific architecture inside the matrix. 
PRISMA: Aspect-Oriented Software Architectures

These methods provide a set of common properties that are interesting to analyze in software architecture descriptions. However, there are specific properties of each software system that are relevant to the analysis and which are not supported by these methods. These specific properties vary depending on the software system. As David Garlan exemplifies in [And03], there are several kinds of structural decomposition that generate different kinds of architectures. Some examples are: code decomposition, run-time structure decomposition, and physical-context decomposition. In the code decomposition architectures, where the primary elements are code modules, the interesting properties to analyze are code dependencies, portability and reuse. However, in the run-time structure decomposition architectures, where the primary elements are the principal components that exist when the software is running, the properties to analyze are deadlocks, race conditions, reliability, performance, and security. Finally, in the physical decomposition architectures, the properties to evaluate are related to processors, networks, etc.

More work should be done in the future to provide mechanisms to analyze and compare software architecture descriptions. These mechanisms should give support, not only to common relevant properties in software architecture descriptions, but also to specific properties of a software system by customizing its analysis criterion.

\subsection{DEFINITION OF SOFTWARE ARCHITECTURE}

There is no single universal or accepted definition of software architecture, and thus, there are a large number of software architecture definitions. The main drawback of this deficiency is the fact that the concept of software architecture is used in different ways and sometimes, it is really difficult to know what the exact meaning is. As a result, it is common to refer to several definitions in order to provide a complete notion of the concept of software architecture.

There are some definitions of software architecture that are general and non-exclusive; however at the same time, they are incomplete, non-explicit, and imprecise definitions. An early definition that was defined by Perry and Wolf is: 
$<<$ A software architecture is a set of architectural (or, if you will, design) elements that have a particular form. $>>$

Another modern definition that it is typically used to define software architecture is the definition presented by Bass, Clements and Kazman.

$<<$ The software architecture of a program or computing system is the structure or structures of the system, which comprise software elements, the externally visible properties of those elements, and the relationships among them.>>

Len Bass, Paul Clements and Rick Kazman [Bass03]

However, this definition is also imprecise and incomplete because of a lot of questions emerge from this definition: For example, What is a structure?, What is an external visible property?, and so forth.

The definition of Garlan and Perry is also used to define software architecture; in fact, this is the definition proposed by the Software Engineering Institute (SEI).

$<<$ The structure of the components of a program/system, their interrelationships, and principles and guidelines governing their design and evolution over time.>>

David Garlan and Dewayne Perry [Gar95a]

The definition that is recommended by the ANSI/IEEE Std 1471-2000 is in essence a small variation of Garlan and Perry's definition.

$<<$ Architecture is defined by the recommended practice as the fundamental organization of a system, embodied in its components, their relationships to each other and the environment, and the principles governing its design and evolution. $>>$ 
PRISMA: Aspect-Oriented Software Architectures

Both definitions are simple and brief, but they lack completeness and accuracy properties. Finally, another general definition is the one proposed by D'Souza:

$<<$ An architecture is an abstraction of a system that describes the design structures and relationships, governing rules, or principles that are (or could be) used across many designs $>>$

Desmond D'Souza [DSo99]

There are other proposals that are not exactly definitions of software architecture but which are more specific and address the issues of the software architecture discipline. Some of them are the following:

$<<$ Beyond algorithms and data structures of the computation; designing and specifying the overall system structure emerge as a new kind of problem. Structural issues include gross organization and global control structure; protocols for communication, synchronization, and data access; assignment of functionality to design elements; physical distribution; composition of design elements; scaling and performance; and selection among design alternatives. >>

David Garlan and Mary Shaw [Gar93]

$<<$ An architecture is the set of significant decisions about the organization of a software system, the selection of the structural elements and their interfaces by which the system is composed, together with their behavior as specified in the collaborations among those elements, the composition of these structural and behavioral elements into progressively larger subsystems, and the architectural style that guides this organization---these elements and their interfaces, their collaborations, and their composition>>

Jan Booch, Rumbaugh and Jacobson [Boo99] 
From all these different definitions, it is possible to conclude that there are two principle kinds of definitions: those that define the concept of software architecture and those that define the specification of software architectures. The former are characterized by being general and non-specific and the latter are characterized by being an enumeration of issues related to the description of software architectures. However, there is a common concept in both kinds of definitions; they are both concerned with the notion of structure and how to organize software.

\subsection{MAIN CONCEPTS OF SOFTWARE ARCHITECTURES}

Software architecture descriptions are specified in a formal way using ADLs. Despite the diversity of the different ADLs that have been proposed to date, all of them share a common conceptual basis. They have a common set of elements to design the structure of software systems. The elements that provide a common foundation for software architecture descriptions are introduced in this section.

\subsubsection{Component}

The concept of component is the basis of software architecture and the concept that ADLs share par excellence. A component is a computational element that permits users to structure the functionality of software systems. It has a high level of encapsulation and it is only possible to interact with it by means of its interfaces. Most ADLs permit the definition of more than one interface for each component. The interface or multiple interfaces of a component define the functionality that the component requires and provides. In this sense, components are considered as black boxes.

The concept of component is not only used in the field of software architecture. For this reason, it is sometimes difficult to know the exact meaning of the concept, and there is no consensus about the definition of component and how to identify the components that make up a software system. There are two tendencies: one is implementation-oriented and the other is more generic. The first one covers definitions that are related to the fact that a component is a package of code [DSo99]; whereas the second one defines a component as an artefact that has been developed to be reused. This second definition is abstract and generic, and a component could be a use case, a class or another element that emerges during the development process. 
PRISMA: Aspect-Oriented Software Architectures

Different criteria to detect components during the software life-cycle have emerge from this abstract notion of component [Ira00]: the use case criterion ([Jac97], [Ber99], [DSo99]), the design pattern criterion ([Fow96], [Gar03], [Hay95], [Lar99]), the business domain criterion [All98], the predicted evolution criterion [Hoo99], the existent component criterion [Voa98], the entity (class or abstract data type) criterion [Jac97], and the functional scene criterion applied to agents [Nor98] and to software architectures [Bac00]. The definition of component in the software architecture field is also in this category. There are a lot of definitions for component; the most widely used definition in the software architecture field is the one proposed by Szyperski.

$<<$ A software component is a unit of composition with contractually specified interfaces and explicit context dependencies only. A software component can be deployed independently and is subject to composition by third parties $>>$

Clemens Szyperski [Szy98]

Another well-known definition is the one of Meyer based on the "seven criteria":

$<<$ A component is a software element that:

1. May be used by other software elements

2. May be used by clients without the intervention of the component's developer

3. Include a specification of all dependencies

4. Include a specification of the functionality it offers

5. Is usable on the sole basis of its specifications

6. Is composable with other components

7. Can be integrated into a system quickly and smoothly >>

Bertran Meyer[MEY] [Szy00]

This "seven criteria" definition of component has been refined over time by the following one: 
$<<A$ component is a software element (modular unit) satisfying the following three conditions:

1. It can be used by other software elements, its "clients".

2. It possesses an official usage description, which is sufficient for a client author to use it.

3. It is not tied to any fixed set of clients. >>

Bertran Meyer [Mey03]

Despite the fact that D'Souza advocates the module of code notion for the definition of component, he also provides a generic definition for component:

$<<A$ component is a coherent package of software artifacts that can be independently developed and delivered as a unit and that can be composed, unchanged, with other components to build something larger $>>$

Desmond D'Souza[DSo99]

\subsubsection{Connector}

The concept of connector emerges from the need to separate the interaction from the computation in order to obtain more reusable and modularized components and to improve the level of abstraction of software architecture descriptions.

Connectors represent the interactions of software systems. They define the coordination process among components, that is, the rules that govern the interaction of components. Interfaces are the way to interact with them and these interfaces represent the roles that each one of the components plays in the coordination process.

In her work, Mary Shaw [Sha94] presents the need for connectors due to the fact that the specification of software systems with complex coordination protocols is very difficult without the notion of connector. From her experience in the software architecture field, she demonstrates that the connector provides not only a high level of abstraction and modularity to software architectures, but also an architectural view of the system instead of the object- 
PRISMA: Aspect-Oriented Software Architectures

oriented view of compositional approaches. She also defends the idea of considering connectors as first-order citizens of ADLs, and she defines the notion of connector as follows:

$<<$ Connectors are the locus of relations among components. They mediate interactions but are not "things" to be hooked up (they are, rather, the hookers-up). Each connector has a protocol specification that defines its properties. These properties include rules about the types of interfaces it is able to mediate for, assurances about properties of the interaction, rules about the order in which things happen, and commitments about the interaction such as ordering, performance, etc. $>>$

Mary Shaw [Sha94]

In their work on the formalization of architectural connections, Allen and Garlan preserve the idea that connectors should be first-order citizens [Al194]. As a result, software architectures can be defined as a collection of computational components together with a collection of connectors, which describe the interactions among components.

This work also emphasizes the expressive power and analysis properties that connectors should have. It establishes that the expressive power of connectors should allow us to specify generic processes of coordination (procedure calls, pipes, broadcasts, etc.), to define complex interactions between components, and provide mechanisms to make fine-grained distinctions between variations of a connector.

With regard to the analysis of connectors, they should allow us to do the following: understand their generic behaviour, independently of the context in which they will be used; check and reason about compositions of components and connectors; provide flexible mechanisms to solve the mismatches detected in this composition analysis.

However, other approaches and their respective ADLs prefer the absence of connectors because they distort the compositional nature of software architectures. Some ADLs, such as Darwin [Mag95], Leda ([Can01], [Can99], [Can00]) and Rapide ([Ken95], [Luc95a], [Luc95b]) do not consider connectors as first-class citizens. Thus, the notion of connector is 50 
sometimes used with a meaning that is different from that of an architectural element; it is used as the connection between two components.

This thesis uses the notion of connector proposed by Shaw and also considers it essential to have connectors as first-class citizens for the reasons that Shaw presents in her work [Sha94]:

> Connectors may be sophisticated: software architecture specifications can require complex and elaborate definitions of coordination among the different components that they connect. In most cases, components are not the appropriate place to define the interaction behaviour. For this reason, software architecture specifications need their own element to specify these complex interactions.

$>$ The definition of a connector should be localized: A good methodology implies that definitions of interactions are localized in elements to improve the design and maintenance of the software system.

> Connectors are potentially abstract: Connectors may be parameterizable and may define generic coordination models that will be specific at the time of instantiation. A connector can be instantiated as many times as necessary. Connectors should also provide mechanisms to specialize and compose themselves from existing connectors.

$>$ Connectors may require distributed system support.

$>$ Components should be independent: Interfaces of components should provide a complete specification of the functionality of components by keeping their internal processes hidden.

> Connectors should be independent: Connectors should be able to mediate interactions for a set of components that are dynamically changing.

> Relations among components are not fixed: Components should be able to participate in more than one coordination process. These processes are specified in different connectors.

Nowadays, the notion of connector has become more accepted and several works on higher-order connectors are emerging [Gar98]. They follow the Mary Shaw's idea about abstract connectors, and they promote the idea that an architectural language must support not only individual connectors, but also high-level compositions that involve a number of connectors in specific relations with each other. Bridget Spitznagel 's compositional approach for constructing connectors ([Spi01], [Spi03]) is presented as a solution to the need for 
PRISMA: Aspect-Oriented Software Architectures

specialized forms of interaction, bridges to solve component mismatches, or extra-functional properties (e.g., security, performance, reliability).

\subsubsection{Port}

The concept of port is related to architectural elements, components, and connectors. Ports are the points through which architectural elements can interact with the rest of a software architecture. They are the parts into which the interface of an architectural element is divided.

Their main function is to preserve the black box view of architectural elements and to publish the behaviour offered and required by architectural elements. They have been used in different ways; some approaches consider a port as a service and other approaches as a process with several services. This last way of defining ports, not only defines the services of ports, but also the conditions of how and when they can be required and provided.

Different names have been used to refer to this concept. Some of them use the name of port to refer to ports of architectural elements, while other approaches use the name of port to refer to the ports of components and the name of role for ports of connectors. Other less frequently used names are players or name of interfaces.

In this thesis, we will use the generic name of port to refer to both component and connectors ports.

\subsubsection{Connection}

Connections are used to constrain the "placement" of architectural elements; that is, they constrain how the different elements may interact and how they are organized with respect to each other in the architecture [Per92].

They establish the communication channels among architectural elements. They connect a component port with a connector port or with a port of another component [Luc95a], depending on whether the connectors are considered first-order citizens or not, respectively. In this thesis, since connectors are considered first-order citizens, a connection is established between a component port and a connector port. These connections are usually called attachments. 


\subsubsection{System}

Most architectural approaches need to provide abstraction mechanisms. These mechanisms permit definition of elements of higher granularity and increase the modularity, composition, and reuse of software systems. Software composition provides flexible support and a reduction in complexity for the development process of software systems [Nie95].

These needs and advantages have led to a wide variety of architectural models and their ADLs to provide the concept of complex component. A complex component is a component that is composed by other architectural elements. An example is the model proposed by Ivar Jacobson [Jac97] that introduces the concept of subsystem as a set of organized components. Other ADLs introduce the concept of composed component such as Durra [Bar01], Darwin [Mag95], ArchWare ADL ([Oqu04a], [Oqu04b]), Wright [All94] and ACME [Gar00]. They are usually called systems.

Systems represent architectural configurations that are made up of connectors and components that can be built in a hierarchical way. For this reason, a system can be composed of other subsystems [And03].

\subsubsection{Composition Relationship}

Compositional relationships emerge with systems due to the fact that it is necessary for systems to communicate with their architectural elements. These connections are different from attachments because they are used to connect architectural elements of different levels of granularity. As a result, the semantics of these connections is compositional, whereas attachments have a communication semantics that is not compositional (the same level of granularity). These relationships are usually called Bindings.

Bindings establish the mappings between the internal and external interfaces of a system [Gar01]. As a result, bindings establish a connection between a system port and a port of one of its architectural elements.

\subsubsection{Architectural Style}

Architectural Style is an important concept in the Software Architecture discipline. Architectural styles are very useful to describe software architectures. They are able to 
PRISMA: Aspect-Oriented Software Architectures

encapsulate design decisions about the architectural elements and to emphasize important constraints on the elements and their relationships [Per92].

$<<$ An architectural style defines a family of systems in terms of a pattern of structural organization. More specifically, an architectural style determines the vocabulary of components and connectors that can be used in instances of that style, together with a set of constraints on how they can be combined. These can include topological constraints on architectural descriptions (e.g., no cycles). Other constraints, having to do with execution semantics, might also be part of the style definition $>>$.

David Garlan and Mary Shaw [Gar93]

There is not a clear difference between the concepts of architectural style and a description of a software architecture. The set of elements that is used to define them is the same. However, there is a difference in the use of these elements. Descriptions of software architectures specify the configuration of a specific software system. However, an architectural style defines constraints about the structure of a family of software architecture descriptions [Gar95a]. As a result, architectural styles are less constrained and less complete than descriptions of software architectures, and they can be used descriptively and prescriptively [Per92] .

Architectural styles represent families of software architecture descriptions that belong to software systems that have something in common [Gar01]. They are patterns that must be followed to be able to specify software architectures. In this sense, the constraints of an architectural style limit the specification and evolution capabilities of the software architecture descriptions that follow this architectural style.

Garlan and Shaw present a partial taxonomy of architectural styles in their work [Gar93]. The taxonomy is the following:

$>$ Pipes and filters

$>$ Data abstraction and object-oriented organization

> Event-based, implicit invocation

$>$ Layered systems 
$>$ Repositories

Table driven interpreters

$>$ Distributed processes

> Main program/subroutine organizations

$>$ Domain-specific software architectures

$>$ State transition systems

Process control systems

$>$ Heterogeneous architectures

\subsubsection{View}

Perry and Wolf were the first authors to recognize the need for multiple views of a software architecture description in order to understand its different aspects. They base their idea on the fact that a software architect needs a number of different views of a software architecture in the same way that a building architect works with different views in which some particular aspect of the building is emphasized [Per92].

The notion of view offers the possibility to analyze a software architecture description from different points of view. As a result, the view concept is presented as a mechanism to analyze software architectures by emphasizing certain properties and to present different architectures depending on which user will use the view. In this last case, the view allows us to hide or present in more detail certain parts of the software architecture.

When a software architecture description is divided into multiple views, the complete software architecture description is obtained by combining these views.

ADLs do not usually provide explicit view mechanisms. The notion of view provides an informal way of analyzing a software architecture. For this reason, a lot of work must be done in this direction.

Other works have proposed defined views of software. One of them is the work by Perry and Wolf, where they present three important views of software architectures: processing, data, and connections [Per92]. Another work is the one of Kruchten, whose 4+1 model has been widely used because of its incorporation in UML [Kru95]. 
PRISMA: Aspect-Oriented Software Architectures

In his work, Garlan [Gar01] emphasizes two of the more important classes of views:

$>$ Code-oriented views: these describe how the software is organized into modules, and what kinds of implementation dependencies exist between those modules.

D Execution views: these describe how the system appears at run time, typically providing one or more snapshots of a system in action. These views are useful for documenting and analyzing execution properties such as performance, reliability, and security.

\subsubsection{Property and Constraint}

Properties define semantics information about software architectures and their architectural elements [And03]. This semantics information is additional to the structural properties of the description of a software architecture. The properties can be associated to any of the architectural elements of a software architecture description (components, connectors, systems, interfaces, ports, bindings or attachments) [Gar01].

Constraints restrict the design of software architecture descriptions or architectural styles throughout their entire execution lives [And03].

\subsection{CONCLUSIONS}

Once the general characteristics of the software architecture area and its main concepts are described, its main advantages and relevance to the development of software systems can be understood. These advantages [Per92], [Gar95a], [Gar93], [Gar01] are the following:

> Understanding: The high level of abstraction of software architectures facilitates the understanding of software systems.

> Reuse: Software architectures allow us to reuse software at different levels of granularity. They permit the reuse of simple components as well as architectural styles. In addition, reuse in software architectures can have different purposes for developing software architectures in general or for product lines. The reuse in software architectures for product lines defines software architectures of the same family by reusing common features and only creating from scratch the specific properties of the new software system. In this sense, software architectures exploit the domain specific field and also provide frameworks specializing in product families. 
Dvolution: Software architecture can help to predict the feasible and possible changes that can occur in a software system as well as the cost associated to these changes.

Analysis: Architectural descriptions provide new opportunities to analyze whether:

- The constraints of an architectural style are consistent

- A software architecture description satisfies an architectural style

- A design satisfies a software architecture description.

- A software architecture description satisfies the requirements of the system

These descriptions can also analyze:

- The consequences of changes on a software architecture description or an architectural style over requirements and design and vice versa

- The conformance of a software architecture description or an architectural style to quality attributes

○ Domain-specific architectures

> Maintenance: Architecture reuse and modularization facilitates the maintenance of software.

> Communication: Software architecture descriptions can be used to facilitate communication with the stakeholders of the system. 
PRISMA: Aspect-Oriented Software Architectures 


\section{CHAPTER 3}

\section{ASPECT-ORIENTED SOFTWARE DEVELOPMENT}

$<<$ Human beings, by changing the inner attitudes of their minds,

can change the outer aspects of their lives. >>

William James

The nature of current software systems has led to software being more complex, its modularity is an essential feature in being more understandable, reusable and maintainable. In addition, non-functional requirements of software systems are acquiring as much relevance as functional requirements. As a result, the support of software modularity and non-functional requirements are essential challenges to be faced in software development. The application of Software Engineering principles is necessary in order to cope with these challenges. A consolidated principle of Software Engineering is Separation of Concerns ( $\mathrm{SoC}$ ), which was introduced in [Par72].

The SoC principle promotes dealing with the different concerns of a software system individually. [Dij76] demonstrated that this division provides better results and offers many advantages. A suitable application of SoC provides a reduction in software complexity and an improvement in the modularity, reuse and maintenance of software artefacts.

In the last decade, Aspect-Oriented Programming (AOP) has emerged as an innovative way of applying SoC in software development [Kiz97], [Elr01]. Its proposal is different from 
PRISMA: Aspect-Oriented Software Architectures

previous ones (packages, modules, classes, interfaces, patterns, etc). The concerns that are dealt with individually in AOP are those that crosscut a software system, instead of those that can be perfectly located as software units of a system. As a result, AOP introduces a new notion of concern. The IEEE defines concerns as:

$<<\ldots$ those interests which pertain to the system's development, its operation or any other aspects that are critical or otherwise important to one or more stakeholders $>>$

ANSI/IEEE Std 1471-2000 [IEE00]

Tarr et al. define concern as a predicate over software units [Tar99]. The crosscuttingconcerns concept comes from this notion of concern. Software systems are usually crosscut by common concerns of a domain system. These crosscutting-concerns are spread throughout the software units of the system. As a result, the crosscutting-concerns are repeated in all the software units that they affect, and these concerns are tangled with the other concerns that also modify the same software unit. The repetition of crosscutting-concerns throughout software systems increases the volume of code and complicates the maintenance that preserves the consistency of changes. Furthermore, tangled concerns make the maintenance of a specific concern more costly because it is so difficult to locate the correct place to introduce the changes. As a result, AOP proposes the separation of the crosscutting-concerns of software systems into separate entities, which are called aspects. This separation avoids the tangled code of software and allows the reuse of the same aspect in different software units (objects, components, modules, etc.).

AOP applies the notion of aspect to cleanly structure software systems in order to easily develop, understand, customize, evolve, and maintain software systems. It was introduced to the research community by the works of Gregor Kizcales [Kiz97], [Kiz01].

The origin of AOP is the programming language AspectJ [ASP06a]. AspectJ was developed by the Palo Alto Research Center (PARC) and was also supported by the National Institute of Standards and Technology (NIST) and the Defence Advanced Research Projects Agency (DARPA). PARC transferred AspectJ to an openly-developed eclipse.org project in 60 
December of 2002 in order to increase the technology and community of AspectJ. As a result, AspectJ is currently the most widely used language for aspect-oriented programming.

Crosscutting-concerns arise throughout the software life cycle. For this reason, despite the fact that AOP emerged from the implementation level, its use is being extended to all the stages of the software life cycle. As a result, Aspect-Oriented Software Development (AOSD) has emerged to gain the advantages that aspects provide in every stage of software development.

The main purpose of this chapter is to provide a conceptual base for the different concepts of the aspect-oriented paradigm and to present other aspect-oriented models. In addition, an introduction about how the aspect-oriented approach is being introduced in the software life cycle is provided.

\subsection{AOP: ASPECT-ORIENTED PROGRAMMING}

Object-Oriented Programming (OOP) has proved to be an important advance in software development. OOP is a programming paradigm that responds to software engineering needs due to the fact that its concepts easily fit real life. In addition, its composition and inheritance mechanisms are very useful for software development. As a result, OOP is "par excellence" the programming paradigm that supports the development of software systems following software engineering principles.

However, OOP has important limitations and it is not able to capture all the important requirements of software systems. OOP has serious difficulties in locating concerns that are related to global constraints or locating behaviours that affect a large number of software artefacts of software systems. Moreover, despite the fact that domain-specific applications need to take into account specialized algorithms for distribution, encryption, safety, and so forth, this specialization is not supported by OOP. These concerns are tangled throughout software artefacts of software systems. As a result, in any object-oriented structure of a software system, there may be concerns that are neatly located in a structural piece and others that cross several structural pieces.

These limitations of OOP are due to the fact that OOP performs a functional decomposition [Par78], [Par74], [Par72]. Each functional unit that is identified during the functional 
PRISMA: Aspect-Oriented Software Architectures

decomposition process is encapsulated in an object, but non-functional requirements are not considered in this procedure. As a result, AOP emerges to deal with the limitations of OOP.

AOP separates the crosscutting concerns into aspects. It introduces the existence of two kinds of software units: components and aspects. These are clearly defined in [Kiz97] from the point of view of a non-aspect-oriented programming language:

$<<$ A component, if it can be cleanly encapsulated in a generalized procedure (i.e. object, method, procedure, API). By cleanly, we mean well localized, and easily accessed and composed as necessary. Components tend to be units of the system's functional decomposition, such as image filters, bank accounts and GUI widgets.

An aspect, if it can not be cleanly encapsulated in a generalized procedure. Aspects tend not to be units of the system's functional decomposition, but rather to be properties that affect the performance or semantics of the components in systemic ways. Examples of aspects include memory access patterns and synchronization of concurrent objects and so forth. $>>$

Kizcales et al. [Kiz97]

AOP promotes the idea that software systems are better programmed using the notion of aspect and considering aspects as first-order citizens of programming languages. Not only AOP offers aspects to encapsulate crosscutting concerns, but it also provides mechanisms to weave aspects with the rest of the software system. In addition, these mechanisms introduce two properties called quantification and obliviousness [Fil00].

$>$ Quantification is the fact that some software units affect many of the other software units of a software system by extending or replacing their code.

$>$ Obliviousness is an implicit invocation, that is, the programmer is unaware of the code extensions or replacements that are going to be produced by implicit invocations at runtime. Implicit invocations are the places where quantifications are going to be applied.

AOP introduces a higher level of modularity of software and simplifies applications. These two properties generate advantages such as: simpler code; easier development, maintenance and evolution; and a higher level of reusability. A demonstration of these advantages is 62 
presented in [Kiz97] by comparing the source code of a non-aspect-oriented application and an aspect-oriented application that were developed for the same software system. In this example, the reduction of code is a clear advantage offered by the aspect-oriented application. The results were the following:

$>$ Size of the non-aspect-oriented application: 35.213 lines of code

$>$ Size of the aspect-oriented application: 4.628 lines of code

$>$ Component program: 756 lines of code

$>$ Three aspects programs: 352 lines of code

$>$ Aspect weaver: 3.520 lines of code

This example shows the reduction of code of aspect-oriented applications taking into account the aspect weaver code. In addition, the aspect weaver code can be reused in other applications. However, different kinds of projects, with different requirements, sizes and programmers should be measured to make a real study about the time and space efficiency of aspect-oriented applications.

The demonstration of [Kiz97] is a starting point for studying the improvement of aspectoriented programming. In fact, it is the basis for formulating an initial measure to analyze how aspect-oriented programming can reduce the code of an application. This measure compares the implementation of the same application using aspect-oriented programming and nonaspect-oriented programming. The result that this measure provides is the percentage of code that has been reduced using aspect-oriented programming. The formula is as follows:

$$
\text { code reduction }=\frac{\text { spread } \text { code size }- \text { component program size }}{\text { sum of aspect program size }}
$$

In the example that has been presented, there is a reduction of $98 \%$ in the number of lines of code:

$$
\text { code reduction }=\frac{35213-756}{352}=98
$$


PRISMA: Aspect-Oriented Software Architectures

AOP introduces a set of new concepts that are essential for correctly understanding this new paradigm. These concepts are introduced in this section to establish a conceptual basis for aspect-oriented programming.

\subsubsection{Base Code}

AOP introduces a clear differentiation between the base and aspect codes. The base code is composed of the software units (modules, objects, components) of an application, which have been obtained as a result of a functional decomposition. However, the aspect code is composed of the aspects that have been implemented to encapsulate the crosscutting-concerns of the same application.

\subsubsection{Join Point}

Join points are situated in the base code of an application. A join point is a semantic concept that defines a well-defined point of the execution of a base code. This point can extend the base code with the aspect code, thereby altering the execution flow of the original application. As a result, join points allow us to suitably coordinate the base code with the aspect code.

In the AOP taxonomy defined by [Dou05], two approaches for specifying join points are detected: one approach marks the join points using labels [Wal03], [Dan04], and the other one uses the language constructors [Col00], [Dou01], [Dou2a], [Dou02b], [Dou04a], [Dou04b]. The former introduces a pre-processing procedure that slows the code injection process down at run-time. The latter is the most widely used way of defining join points. Consequently, this is the approach that has been adopted for defining join point in this thesis. This approach usually matches join points with method calls. The different kinds of join points that this approach uses are presented and classified in [Kiz01].

As [Kiz97] defines, a join point is not an explicit language constructor, it is the semantics of the language constructor. In other words, the join point is associated to a language constructor; however, the different instantiations of this constructor will be different join points at run-time. A clear example is a joint point that is associated to a method call. The different invocations of this method are different join points with different semantics. The semantics is different 
because it depends on the object that has invoked the method, on the instantiation of its arguments, and so forth.

\subsubsection{Pointcut}

From the previous section, it can be deduced that an application has a large quantity of join points in its base code. However, not all join points of the application are interesting or relevant for injecting aspect code. As a result, the relevant join points for this injection of aspect code must be selected. The mechanism that permits this selection is the pointcut.

A pointcut is a set of join points, which are candidates for injecting aspect code into base code at run-time, and their multiple instantiations. Pointcuts perform the weaving between the base code and the aspect code by capturing joinpoints.

The most widely used pointcut model is the AspectJ model [Dan04], [Jag06a], [Jag06b], [Läm02], [Wal03], [Wan04]. There are also other pointcut models that use more general execution patterns, such as stack of events, tree of events, sequence of events, etc [Col00], [Dou2a], [Dou04a], [Mas03].

\subsubsection{Advice}

An advice defines the code that should be executed at the join points of a specific pointcut. Advices define additional code for the join points that have been selected by pointcuts. As [Bru04] cites, the advice code is the profiling code of an aspect-oriented program and the compiler profiles the join points by executing the advice code at run-time. This process by the compiler consists of inserting or replacing the base program code is called weaving. The execution of code depends on the kind of advice. There are three main kinds of advice:

$>$ Before: The before advice adds code to the base program before the join point. As a result, the code of the advice is executed before the code of the join point.

$>$ Around: The around advice substitutes the code of the join point. As a result, the code of the advice is executed instead of the code of the join point.

After: The after advice adds code to the base program after the join point. As a result, the code of the advice is executed after the code of the join point. 
PRISMA: Aspect-Oriented Software Architectures

- After returning: This kind of after advice is executed when the execution of the join point finishes correctly.

- After throwing: This kind of after advice is executed when the join point throws an exception. As a result, the code of the advice is executed after the throwing of the join point.

As [Wan04] concludes, an aspect-oriented program is composed of a base program and some advices.

\subsubsection{Aspect}

An aspect is a language constructor that encapsulates a crosscutting-concern. An aspect is linked to one or more methods of the base code by means of pointcuts. For this reason, an aspect is composed of pointcuts and an advice. As a result, aspects specify whether their execution will be before, after or around a method of the base code by means of the advice that is associated to pointcuts. Despite the fact that aspects are usually pairs of pointcuts and advices, they can have their own state [Dou05]. The definition of aspect proposed by Kizcales is the following:

$<<$ Aspects are units of modular crosscutting implementation, composed of pointcuts, advice, and ordinary Java member declarations. >>

Gregor Kizcales et al. [KizO1]

\subsubsection{Properties}

There are some properties of aspect-oriented programming that are as important as the basic concepts. The most important properties are going to be analyzed in this section.

\subsubsection{Weaving Time}

Weaving is the process that consists of coordinating the base code and the aspect code. It can be static or dynamic. Static weaving is performed at compilation time, whereas dynamic weaving is performed at run-time. Despite the fact that these two kinds of weaving are different, both obtain the same result, that is, the lines of code that are executed and the order in which are executed are the same. 
Most approaches provide a static weaving. Its semantics is to define the final woven program. However, dynamic weaving consists of defining the semantics of the weaver. Since dynamic weaving is only strictly necessary in those approaches in which the dynamic evolution of aspects is permitted, there are not very many approaches that provide dynamic weaving.

The inputs of a static weaving process are the base program and the aspect programs. Thus, the process of a static weaving consists of inserting or replacing the code of aspect advices in the base program [Bru04], depending on whether they are before or after, or around advices, respectively. The result of this process is an output program which contains the integration of the aspects and the base program in a proper way. For example, AspectJ combines its aspects and base code using a static weaving process. It weaves the code at compilation time. The AspectJ compiler transforms advices into standard AspectJ methods, whose calls are inserted into the points of the base code that the pointcuts define.

\subsubsection{Instantiation of aspects}

Aspects can contain a state by means of the definition of attributes. As a result, an aspect can be instantiated, that is, the aspect type is transformed into an aspect instance giving a specific value to its attributes. However, this instantiation has not been formalized and is still pending resolution. As a result, several open questions emerge. One of them is the number of instances that an aspect can have; it is not clear if it should be one, more than one or if it is necessary to establish a specific boundary. In the case of AspectJ, the default behavior for an aspect type is to have a single instance [Kiz01]. Another open question is whether this instantiation should be static, dynamic, or both. In the case of AspectJ, the instantiation is a static process.

This lack of formalization leads aspect-oriented models to define different instantiation strategies depending on their needs. It also generates the need to find a consensus for this question.

\subsubsection{Data Type Systems}

Another important feature to take into account in aspect-oriented models is their data type system. Only a few works take into account this property in their proposals. 
PRISMA: Aspect-Oriented Software Architectures

The data type system is very important for the weavings that are established between the aspects and the base code. This is due to the fact that the data types of an aspect advice must be compliant with the data types of the base code that the weaving links. To do this, aspectoriented models normally define the data type system of advices based on the data type system of the base programs. However, this only ensures that both programs are using the same data type system, or that one is a subset of the other. It does not ensure that the signatures of the advice and the join point match. If they do not match, an error is generated, and it is impossible to execute the weaving. This problem is solved by the weaving process, which pre-processes the arguments. An example of an aspect-oriented proposal that ensures the correctness of the weaving is the typed calculus [Jag06b][Jag06b][Jag06b][Jag06b]. The typed calculus ensures the correctness of the weaving by restricting the correspondence between the types of the pointcut parameters and the pointcut results and the base code methods that are associated to them. As a result, the advice is well-typed if it is consistent with the pointcut definition.

\subsubsection{Aspect Management}

A few aspect-oriented proposals have considered the management of aspects to be an important property to take into account. Some works have started to address this question by providing a mechanism to temporally discard or order aspects for each point of interaction [Dou2a], [Dou04a]. The order of aspects opens up the question of whether all the aspects should be treated as equals or whether should be dominant aspects.

The composition of aspects is also another mechanism to be able to manage aspects in a flexible way. However, the composition property generates a wide variety of open questions about the semantics of aspects, whether they are monotonic or not; or whether they can be an extension of another one or must be completely defined without depending on another aspect, etc.

\subsubsection{Semantics}

Although the aspect-oriented approach is a novel approach for programming, its semantics is not yet formally defined. As a result, a great number of questions must be answered and a lot of research work must be done in order to consolidate the proposal and to find a consensus in the 
area. One of the first works that formalizes the semantics of the join points, pointcuts, and dynamic advices of aspect-oriented programming languages is [Wan04].

\subsection{ASPECT-ORIENTED MODELS}

The most widely used aspect-oriented model is the AspectJ model. However, there are two other aspect-oriented models that are also widely used throughout the aspect-oriented community. They are the multidimensional model that was promoted by the HyperJ programming language and the composition filters model. All three of these generic-models are refined and specialized in specific models. However, since it is important to understand the differences among them, the differences between symmetric and asymmetric models, and the multidimensional and composition filters models are introduced in this section.

\subsubsection{Symmetric vs. Asymmetric Models}

The notion of aspect arises to deal with crosscutting-concerns of software systems. This idea of crosscutting can vary depending on the nature of the model. A model can be symmetric or asymmetric [Har02]. Most widely used aspect-oriented models are asymmetric because of the strong influence of the asymmetric AspectJ model.

An asymmetric model is based on a dominant decomposition, which is usually an objectoriented-like functional decomposition. Models of this kind assume that aspects are nonfunctional concerns that crosscut the functional units of software. However, in symmetric models everything is considered as a concern, and there is not a dominant decomposition. As a result, functionality is considered to be another concern and concerns crosscut each other. Symmetric models rarely use the notion of aspect because they do not need to differentiate between aspect and non-aspect entities, instead, they use modules, and these modules are considered as concerns of the software system. For this reason, symmetric models are considered to be more flexible and abstract. However, asymmetric models are more widely used because they are easier to integrate in current software development approaches and to put into practice. 
PRISMA: Aspect-Oriented Software Architectures

\subsubsection{Multi-Dimensional Separation of Concerns (MDSOC)}

The origin of the Multi-Dimensional Separation of Concerns model (MDSOC) is the subjectoriented programming [Har93] proposed by William Harrison and Harold Ossher. The continuation of this research work by Harol Ossher and Peri Tarr generated the MDSOC model. MDSOC is a symmetric model where aspects are first-order citizens and are considered to be like objects [Oss01]. This model emerges from the need to modularize the code of concerns that are scattered throughout the source code, usually classes. This model opts for flexibility to develop software, and it is based on the idea that the decomposition of software is not always a functional decomposition that is materialized in classes or components. This model presents the modularization of code as a flexible process where the user can choose the criteria for decomposing the software: aspects, classes, components, features, roles, viewpoints, etc. As a result, MDSOC provides support for the clean modularization of multiple kinds of concerns with an "on-demand remodularization". This property is presented by this model as an advantage in comparison with earlier models such as aspect-oriented programming [Kiz97] or subject-oriented programming [Har93]. In addition, another important feature of this model is the dynamic introduction of concerns in the software application as they arise during the software development process. This feature is based on the idea that new concerns can continuously arise during the software life cycle. The approach that puts the MDSOC into practice is called hyperspaces [HYP06].

The hyperspace approach is a language-independent approach that can be applied to any programming language. Specifically, this approach has been applied to the Java programming language, and a tool to support it has been developed. This tool is called Hyper/ $\mathrm{J}^{\mathrm{TM}}$ [Oss00]. As a result, Hyper/J supports MDSOC for Java following the hyperspace approach. This approach introduces a set of concepts that are basic for understanding it [Oss01].

$>$ Concern space: A concern space includes all the units of software in a software body. A unit of software is any language constructor such as a package, interface, state chart, requirement specification, class, method, attribute, etc. A concern space allows the identification, encapsulation, relationship definition and integration of concerns, respectively. 
Hyperspace: A hyperspace is a concern space that is defined for the purpose of supporting the MDSOC model. A hyperspace is the result of the identification task of a concern space. The remarkable feature of a hyperspace is that its units of software are organized in a multi-dimensional matrix, where each axis corresponds to a concern dimension (see Figure 2). A specific concern of a dimension is represented in the matrix by a specific point in an axis that represents this dimension.

> Hyperslice: A hyperslice is a declaratively complete structure that is formed by software units. A declaratively complete structure is a functional element that declares everything that it refers to. Hyperslices permit the encapsulation of code that is related to a specific functionality in an independent structure (encapsulation), that is, they are modules that encapsulate concerns. As a result, the reusability and maintainability are improved because hyperslices are not coupled with each other, and the impact of changes is limited. Each hyperslice can be an aspect or an object of the business logic. For example, the Logging hyperslice in Figure 2 is an aspect, and the Employee hyperslice is an object of the business logic. In addition, Figure 2 illustrates how the different elements of a hyperspace are assembled in a hyperslice.

> Hypermodule: A hypermodule contains a set of hyperslices and uses a set of relationships that specify how the hyperslices are composed (relationship definition). These relationships define how hyperslices are related and how they should be integrated (integration). Hypermodules can be used to encapsulate different kinds of software artefacts (components, classes, fragments, etc) and to nest themselves creating complex hypermodules.

The Hyper/J $\mathrm{J}^{\mathrm{TM}}$ tool supports the hyperspaces approach by providing all the concepts and relationships presented above. It permits the identification, encapsulation, and manipulation of concerns in Java. It encapsulates these concerns into hyperslices and, in turn, encapsulates these into hypermodules. The tool offers mechanisms to define the concepts of the hyperspace approach in a graphical way. 
PRISMA: Aspect-Oriented Software Architectures

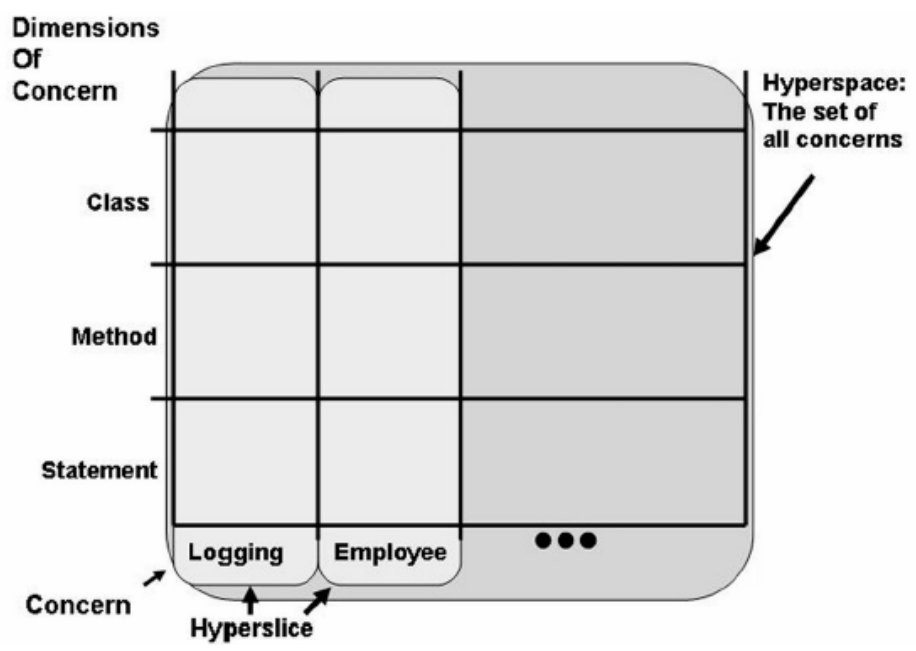

Figure 2. HyperJ/Matrix [Kim02]

In summary, the MDSOC model is a symmetric composition model that is suitable for structural composition. The main difference between Hyper/J and AspectJ is that AspectJ supports the extension of a single model, whereas Hyper/J supports the integration of multiple models [Kiz97], [Kiz01].

\subsubsection{Composition Filters (CF)}

The model of Composition Filters (CF) [Ber94] emerges as another solution to support the definition of crosscutting concerns in an entity that is different from an object. This model, which is proposed by Mehmet Aksit and his research group of the Twente University, uses filters to define crosscutting concerns [Ber01]. As a result, this model also allows the composition, reuse and evolution of multiple concerns.

Filters are used to define complex and crosscutting concerns in a technology-independent way. Filters can be attached to objects that are programmed in different programming languages without modifying the content of the objects. The CF model is an asymmetric model that extends objects in an orthogonal way, that is, the semantics of a filter is independent of other filters. In addition, these filters are specified in modules. The modular and orthogonal properties of filters are the main differences between this model and other asymmetric models. 


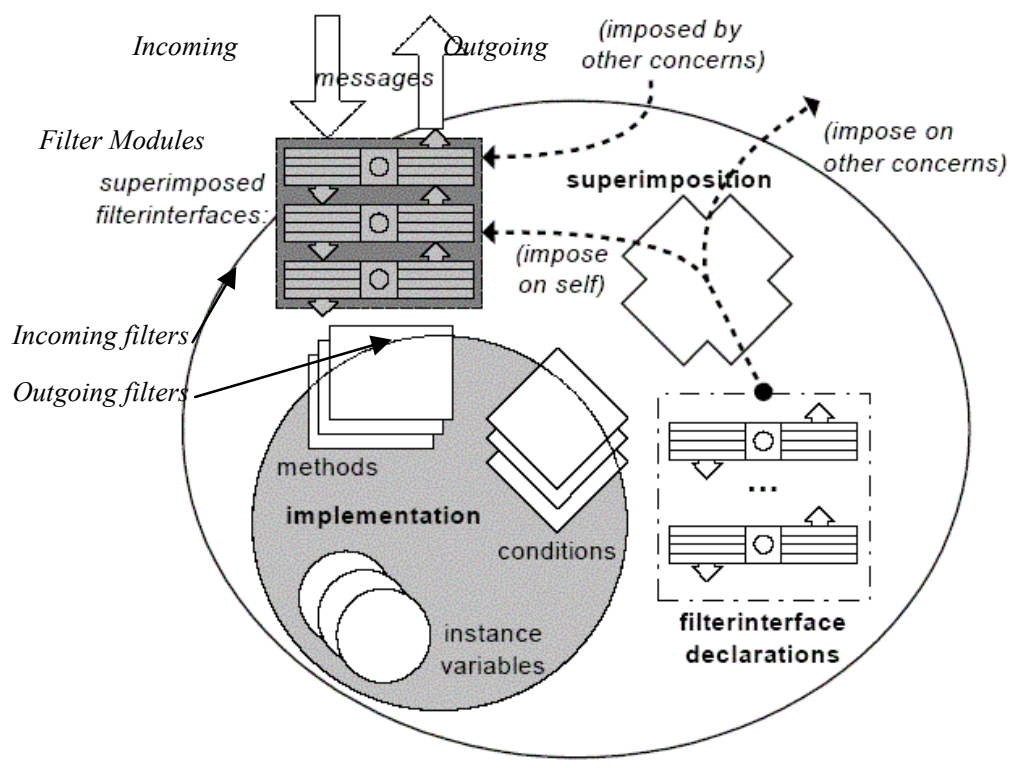

Figure 3. The Composition Filter Model with Superimposition [Ber01]

The CF model allows the management of input and output messages of an object by means of filters [Ber04]. The filters that intercept the incoming to and outgoing messages from an object can express a wide range of changes. These changes generate an adaptation of the behaviour of objects without modifying their implementation. The process of applying filter modules to objects is called superimposition [Car01] (see Figure 3 , extracted from [Ber01]).

Figure 3 illustrates that superimposition introduces a layer of filter instances over the objects, and the input and output messages of the object must pass through this layer in order to be executed in a suitable way. Figure 3 also shows that the declaration of a filter interface separates its declaration from its instantiation.

It is possible to conclude that superimposition allows the multiple composition of crosscutting concerns by superimposing filter interfaces. The concerns are defined as filters and they intercept and transform the messages that arrive to or depart from an object.

A clear advantage of the $\mathrm{CF}$ model specifies its filters in a declarative way without adopting a general purpose language such as AspecJ or Hyper/J. As a result, this model can be applied to different technologies and programming languages. Since the common pattern-matching 
PRISMA: Aspect-Oriented Software Architectures

language, which is used to specify filters, is highly expressive, it provides filters with a concern semantics. However, the interoperability between filters and objects that are programmed in different languages is not addressed in the work.

Moreover, a strong encapsulation of objects is preserved and the specification of filters is modular, facilitating the composition of modules. However, filter modules can only be used to extend the behaviour of objects and the way to extend non-object-oriented models with this model it is not very well-defined.

\subsection{ASPECT-ORIENTED DEVELOPMENT IN THE SOFTWARE LIFE CYCLE}

During the development process of a software system, concerns arise throughout all the stages of the software life cycle. It is important to take into account that concerns are not strictly related to non-functional requirements; they can also be related to functional ones. In addition, the crosscutting-concerns can vary, change their granularity, increase or decrease their number depending on the stage of the software life cycle. This is due to the fact that each stage has different needs. Examples of concerns of early stages are response time, quality, security, safety, etc, whereas concerns introduced by the technology chosen at the design or implementation stages are exception handling, caching, buffering, synchronization, etc. As a result, aspect-oriented development has emerged in order to apply aspect-orientation to every stage of the software life cycle.

Aspect-oriented software development deals with the identification of aspect candidates at early stages of the software life cycle and the maintenance of their traceability throughout the entire cycle. Traceability must be preserved in order to keep the consistency of software systems and their evolution capabilities. Traceability mechanisms should take into account that candidate aspects of early stages may or may not be aspects in later stages, and that aspects of one stage can be refined in its later stages.

An overview of the aspect-oriented software development is provided in this section. The main goal is to provide a notion of the research works that are being developed to apply aspect- 
orientation to the requirements, analysis, design, and implementation stages of the software life cycle.

\subsubsection{Requirements}

One of the first approaches that introduced aspect-orientation in the requirements stage is the Aspect-Oriented Requirements Engineering (AORE) approach [Ras02], [Ara02]. In fact, the authors introduced the notion of early aspects for aspect-oriented works related to requirements engineering and architecture design [EAR06], and they promoted the fact that aspects are primitives of modelling.

AORE is an approach for handling crosscutting concerns at the requirements stage. It is designed to be used by asymmetric models and is based on viewpoint detection, that is, the identification of the points where functional elements or requirements are crossed by nonfunctional aspects or concerns. The approach can be summed up in seven steps[Ras02]:

1. Identification of concerns: Identification of non-functional requirements

2. Specification of concerns

3. Identification of view points: Identification of the functional elements that are affected by non-functional requirements.

4. Identification of candidate aspects: Identification of concerns that crosscut more than one viewpoint

5. Specification of functional requirements using use-case models [Ara02]

6. Composition of functional requirements with aspects using overlapping, overriding and wrapping techniques [Bri02]

7. Specification of Aspects: The aspect is specified and its influence throughout the software life cycle is established

This same process is enriched in [Mor02] by considering quality attributes. The process has been extended with other works on use-case models [Ara03] and interaction diagrams [Whi03]. The work of Araujo and Moreira [Ara03] extends use-case models to support nonfunctional requirements and to identify the crosscutting of functional use cases. The work of Whittle, Araujo and Kim [Whi03] addresses the modelling of aspects in interaction diagrams; 
PRISMA: Aspect-Oriented Software Architectures

aspects are modelled as Interaction Pattern Specifications (IPSs) and are composed with nonaspectual interactions using instantiation. Finally, it is important to mention the ConcernOriented Requirements Engineering Model (CORE) [Mor05a], [Mor05b]. It is an adaptation of the AORE approach designed to be used by symmetric models instead of asymmetric models.

Another approach that applies aspect-oriented techniques to the requirements stage is Aspect-Oriented Software Development with Use Cases (AOSD/UC) [Jac03][Jac05]. This approach is based on the idea that uses cases are crosscutting concerns because their performance affects several classes. This approach extends use-case models by adding pointcuts, use-case slices, and use-case modules. A use-case slice is the specification of a usecase in a specific stage of the software development phase, and a use-case module contains the specification of a use case at all the stages of the software life cycle. For this reason, this approach is applied at all stages of the software life cycle starting from the requirements stage. This approach is a model-driven iterative process that preserves the traceability between the stages of the software development. The aspect modelling of AOSD/UC follows a symmetric approach because there are no dominant functional aspects (use cases). Therefore, the aspects are associated to classes or components in order to build the complete system.

There are other approaches that apply goal-oriented requirements engineering to take into account the $\mathrm{SoC}$ at the requirements stage. Goal-oriented requirements engineering is focused on the analysis and specification of goals, which are the main issues that made up software systems [Myl99], [Lam03]. One of the first approaches that used this methodology for treating aspects at the requirements stage is the Goal-oriented REquirements Methodology founded on the SoC principle (GREMSoC) Model [Sou03]. This approach promotes the reusability and maintainability. It also improves the comprehensibility of requirements specification by using aspect-orientation. This approach specifies functional and non-functional requirements separately. In addition, the relationships between the crosscutting requirements and the requirements that they crosscut are also specified separately. Another approach that combines aspect-oriented techniques with goal-oriented techniques is the Aspects in Requirements Goal Models (ARGM) Model [YuY04]. The ARGM approach proposes the identification of 76 
aspects by means of the relationships between functional and non-functional goals that are represented using a V-Graph. The V-Graph has the shape of the letter V, where the two top vertices are the functional and non-functional goals, and the bottom vertex represents the tasks that must be performed to satisfy both goals. The identification of the candidate aspects of a software system consists of a systematic and iterative process that refines the V-Graphs.

Cosmos is a symmetric approach based on Hyper/J that is applied to the requirements stage [Sut02]. It is a general-purpose concern-space modelling schema. It models concern spaces using concerns, relationships, predicates and topics. Concerns are classified into two categories: physical and logical. The physical concerns are related to hardware components and the logical concerns are related to conceptual software entities. This classification is a new contribution to the analysis of requirements.

An important task at the requirements stage is the analysis of the documents where the requirements are specified. The Theme/Doc approach supports aspect identification and analysis in requirements documentation for symmetric models [Ban04], [THE06]. This approach is based on the theme notion for analyzing the relationships between behaviours of a requirements document to identify aspects. A theme represents a feature of the software system. It classifies the themes into base themes and crosscutting themes. These crosscutting themes are aspects in the Theme/Doc approach. This approach is also supported by the Theme/Doc tool, which provides a set of views to the analyst to analyze the requirements specified in the documents.

Finally, the approach that is being developed to identify PRISMA aspects and protoarchitectures at the requirements stage is the ATRIUM approach (Architecture generaTed from RequIrements applying a Unified Methodology) [Nav03]. This approach combines the goals models, the scenarios models, and the quality attributes (the ISO/IEC 9126 quality model [ISO01]) to obtain aspect-oriented proto-architectures from requirements specifications. ATRIUM proposes an iterative and incremental process to define and refine the different artefacts and allow the analyst to reason about partial views, of both the requirements and the architecture. It consists of five steps: 
PRISMA: Aspect-Oriented Software Architectures

1. Definition of the Goals Model: The different concerns of the software and the crosscutting between them are identified using a Goals Model. These concerns are candidates to be classified as aspects to be realized through aspects that are integrated into components and/or connectors [Nav04a], [Nav04b] .

2. Definition of the Scenarios Model: the set of scenarios is identified. Each scenario describes the elements that interact to satisfy a specific goal and their level of responsibility. These elements are candidates to be components of an ATRIUM proto- architecture.

3. Definition of Collaborations: The collaborations among the candidate components are defined and the previous models are refined. The main purpose is to obtain a skeleton of the architecture.

4. Formalization: A set of derivation rules is provided to generate a formal specification from scenarios, goals, and collaborations. The artefacts, which are defined in the previous steps, are specified in the 3APL language [Hin99], which provides an interpreter to verify its specifications

5. Compilation: An aspect-oriented proto-architecture is obtained using the previous formalization and a set of heuristics.

\subsubsection{Analysis and Design}

There are a great number of works that propose approaches to support aspect-oriented principles at the analysis and design stages of the software life cycle. Since the PRISMA approach proposed in this thesis is applied to these same stages, the approaches that combine software architectures and aspect-oriented software development are analyzed in detail in the next chapter. For this reason, this section presents an overview on the approaches that propose an extension of the object-oriented model by means of the introduction of aspects in a specific object-oriented modelling language.

These approaches usually extend the Unified Modelling Language (UML) because it is one of the most widely used languages in the software engineering community. It is the standard established by the OMG, and it provides easy mechanisms to perform its extensions [UML06]. 
There are two kinds of extension mechanisms: heavy and light. Heavy mechanisms consist of adding new elements to the UML metamodel without modifying the original ones. Light mechanisms (or profiles) use stereotypes, tagged values, and constraints of the UML metamodel to define new derived concepts (metaclasses) from the standard UML metaclasses. An important contribution that addresses the requirements needed for defining a UML profile for AOSD is the work of Aldawud et al [Ald03], who also proposes his own approach for supporting aspect-oriented concepts at the analysis and design stages. This approach is called SUP (State charts and Uml Profile) and defines a UML profile based on state charts for symmetric models [Ald01].

Most approaches use profiles to extend the UML metamodel; however they introduce the notion of aspect in a different way. One of them is the UML extension of the Theme approach, which is called Theme/UML and is presented in section 3.3.1 . It defines a profile where base and aspect themes are modelled using packages. The theme packages are denoted by the $<<$ theme $>>$ stereotype. These packages contain class diagrams to specify the structure and behaviour of aspects. Another approach that models aspects using UML packages is the Aspect-oriented Architecture Modelling (AAM) approach [Fra04], which models aspects for asymmetric models. The main feature of this approach is that it provides two kinds of aspects, context-free and context-specific aspects. The context-free aspects are reusable and are specified at a high abstraction level, whereas the context-specific aspects are instances of the context-free aspects that are specified for use in specific design models.

Aspect-Oriented Design Modelling (AODM) [Ste02b] is another approach for asymmetric models that defines an AO UML profile [Sut02], [Ste03]. It models aspects using UML classes with the stereotype $<<$ aspect $>>$. As a result, the crosscutting is modelled by means of class diagrams and an operation denoted by the $<<$ advice $>>$ stereotype. Another approach that also proposes this kind of extension is the UML for AOSD (UML4AO) approach [Paw01].

UFA (UML for Aspects) [Her02] is an approach that extends UML and that is focused on the extension of the UML collaboration diagrams. It proposes an Aspectual Collaboration Diagram (ACM) for symmetric models. The aspect concept is introduced as a package that contains a class diagram that represents an aspectual part of the software system. 
PRISMA: Aspect-Oriented Software Architectures

Another way of extending UML is by introducing aspects through the classifier element of UML, which is an abstract UML-metaclass that describes structural and behavioural features. One example of this extension is the Uml eXchange Format (UXF) approach, which supports the Aspect-Oriented information exchange between UML case tools [Suz99].

There are other approaches that define their own non-UML language. One example is the CoCompose approach [Wag02], which proposes its own graphical design language to define aspects at the analysis and design stages of symmetric models. Aspects are specified as features with a well-defined semantics. Another example is the Implementation Driven Aspect Modelling (IDAM) approach, which defines its own diagrams called Dynamic Aspect Diagrams (DAD) [Coe04]. This approach proposes a combination of aspect-oriented programming and model-driven development by generating its DADs from AspectJ code.

\subsubsection{Implementation}

Since AOP was developed for Java environments through AspectJ [Kiz01], it is being transferred to other technology platforms and programming languages. Currently, we can find aspect-oriented extensions for original programming languages such as $\mathrm{COBOL}$ (AspectCOBOL [Läm05]), C+ (AspectC+ + [Spi02]), BPEL4WS (AO4BPEL [AO406] ), or C\# (AspectC\# [Kim02], AOP\# [Scü02]). In addition, there are a lot of well-known aspectoriented languages that have been created from scratch. They are CaesarJ [Mez03], FuseJ [Suv05b], HyperJ [Oss00], Jac [Paw04], JBoss [Hil04], Jasco [Suv03], and so forth. Aspectoriented programming languages usually need new execution mechanisms that are not supported by current development platforms. As result, most of them have developed their middlewares to support their execution models [Lou05].

Since the approach proposed in this thesis has been applied to the .NET technology, a detailed study about approaches for this technology has been performed. This study was necessary in order to know whether there was a .NET approach that supported the needs of a dynamic, mobile, distributed, aspect-oriented and component based model. Some of the most important needs are: 
$>$ Reusability of Aspects and Base Code: The weaving should be programmed in another entity

Evolution of aspects: The approach must be able to add and remove aspects at run-time

$>$ Definition of join points using language constructors: The approach allows the definition of join points using the language constructors without adding additional communication mechanisms such as labels (see section 3.1.2).

$>$ Definition of the relation between aspects: The approach should allow the definition of the relationships that can emerge between aspects when they share a join point.

$>$ Aspect-Oriented Mobility: The approach should provide mechanisms to move the base code as well as the aspects that intercept this code.

AspectC\# [Kim02] and SourceWeave.Net [Jac04] support AOP in .NET. These approaches propose joining the base code with the aspects by specifying the weavings in an XML file. Weave.Net [Laf03] and AspectDNG [ASP06b] also define the weavings through an XML file. However, they only use the base code, aspect and weaving assemblies to join the code without the source code being available. Loom.Net [Sch02] is another .NET approach for supporting AOP. It has a graphical interface that allows the addition of defined aspects by means of reusable code templates and allows the performance of weavings.

The approaches mentioned above clearly separate the base code, the aspects, and the weavings in different entities. However, none of them supports mechanisms for dynamically adding or removing aspects. The Rapier-Loom.Net [Sch03]approach does allow dynamic addition and removal of aspects, but it defines the weavings inside the aspects, thereby losing their reusability. SetPoint [SET06]also allows for dynamic addition and removal of aspects. Its weaving is based on the evaluation of logical predicates in which the base code is marked with meta-information that permits the evaluation of such predicates. As a result, the weaving is only performed using additional labels to the code. EOS [Raj03]is another dynamic approach that is able to attach aspects at instance-level by means of events.

None of the approaches mentioned above takes into account the emerging relations that result from the aggregation of various aspects at the same point of the base code (joinpoint). However, JAsCo.Net [Ver03]provides an expressive language that permits the definition of 
PRISMA: Aspect-Oriented Software Architectures

relations among aspects. JAsCo.Net integrates AOP and CBSD. It introduces the concept of connectors for the weaving between the aspects and the base code, which allows for a high level of aspect reusability. One inconvenience of this approach is that the dynamic weaving of aspects to the base code is referential but not inclusive. This requires an execution platform to intercept the application and insert it into the aspects at execution time.

The principal disadvantage of these approaches is that none of them integrates the needed properties at the same time to allow the mobility, the reusability and the evolution of aspectoriented components. These properties are dynamic weaving, the combination of the base code and the aspects inside the same entity, and the reusability of aspects. Therefore, the code mobility is limited because not all the properties of the object code can be moved. However, PRISMA defines a model that combines AOP and the dynamic reconfiguration of the CBSD models. The aspects are separately defined from the weavings and are highly reusable. The components are formed from aspects which can be inclusively and dynamically aggregated. In addition, PRISMA permits the dynamic mobility of its components. The concept of base code does not exist, so the component is solely formed by aspects. The implementation of the PRISMA model in .NET permits the dynamic addition and removal of aspects as well as the dynamic modification of the weavings without stopping the execution of the component.

\subsection{CONCLUSIONS}

The origins of AOP and the main concepts that are necessary to understand this approach has been presented in this chapter. A brief overview about how AOSD is currently being introduced to all the stages of the software life cycle has also been provided. More detailed information about the aspect-oriented works on requirements, analysis and design, middlewares, and aspect-oriented programming languages can be found in the [Chi05], [Lou05], [Bri05] surveys of the AOSD European network, respectively.

Based on the information presented in this chapter, AOSD can be considered as a positive advance in software engineering and a step forward in the application of Separation of Concerns (SoC). AOSD provides important advantages such as the introduction of non- 
Aspect-Oriented Software Development invasive changes, the low impact of changes, improvement in comprehension, reduction of complexity, facilitation of evolution and integration, customizability, and reusability. 
PRISMA: Aspect-Oriented Software Architectures 


\section{CHAPTER 4}

\section{ASPECT-ORIENTED SOFTWARE ARCHITECTURES}

$<<$ One thing only I know, and that is that I know nothing. $>>$

Socrates

The relevance that non-functional requirements have acquired in current software systems has led to the emergence of crosscutting concerns in software architectures. These crosscuttingconcerns are spread throughout software architectures.

There is a wide variety of ADLs that have been proposed in order to specify software architectures, such as ACME [Gar00], Aesop [Gar94], [Gar95b], C2 [Med96], [Med99], Darwin [Mag95], [Mag96], MetaH [Bin96], [Ves96][Bin96], Rapide [Luc95b], [Luc95a], SADL [Mor95], [Mor97], UniCon [Sha95] [Sha96], Weaves [Gor91], [Gor94]and Wright [All97a],[All97b]. An interesting comparison with respect to these ADLs is presented in [Med00]. In addition to this work by Medvidovic and Taylor, there are other interesting surveys on ADLs such as the ones presented in the PhD. Thesis by Cuesta [Cue02], which covers the analysis of other ADLs such as Conic [Kram85], [Mag89], DURRA [Bar01], AML [Wyd01], and Armani [Mon98]. It is also important to mention other approaches that are especially prepared to support evolution in software architectures, such as CommUnity [And03], [Fia04], LEDA [Can00], Pilar [Cue02], GUARANA [Oli98] or R-RIO [Loq00].

These ADLs do not explicitly distinguish the conventional architectural elements from concerns that crosscut multiple architectural elements of software architectures. One of the few approaches that deals with the separation of concerns is the work by Jose Fiadeiro. This work 
PRISMA: Aspect-Oriented Software Architectures

addresses the separation of distribution, mobility [Fia04], contex-awareness [Lop05] and coordination [And03] in software architectures. However, none of these original ADLs supports the separation of concerns by means of the aspect-orientated approach at the architectural level. For this reason, several approaches have emerged to cover this need either by extending original ADLs or by creating new ADLs from scratch.

The combination of AOSD and software architectures has created two new challenges: how to define the concept of aspect at the architectural level and how to integrate aspects and architectural elements in a suitable way. In this chapter, the most relevant approaches that deal with these two questions are analyzed. Starting from the premise that an aspect-oriented software architecture approach should completely support the development and maintenance processes of software, the set of desirable properties that aspect-oriented software architecture approaches should fulfil are also presented. Finally, a comparison of these approaches using this set of properties is presented and discussed.

\subsection{ASPECT-ORIENTED APPROACHES AT THE ARCHITECTURAL LEVEL}

The incorporation of aspects at the architectural level implies considering what an aspect is at this level. An aspect is a new entity for modularizing and encapsulating specifications in software architectures. As a result, it is necessary to define how aspects are related to the rest of the main concepts of software architectures, especially to components and connectors. It is also necessary to define the kind of relationships (reference, connection, composition, etc) that they have with these elements.

In this section, the most important works of the area are analyzed paying special attention to the way that they introduce the notion of aspect in software architectures, how they coordinate aspects and architectural elements, and their main properties. Due to the fact that there has not been much work done at the architectural level, not only are ADL extensions analyzed, but also aspect-oriented component models that could be applied at the architectural level. 


\subsubsection{PCS: The Perspectival Concern-Space Framework}

The Perspectival Concern-Space (PCS) [Kan03] approach is based on the MDSOC model (see section 3.2.2) and IEEE-Std-1471. It uses UML for modelling concerns at the architectural level. PCS describes concerns by means of architectural views. These views consist of one or more models and one or more diagrams. A perspective in PCS is defined as "a way of looking" at a multidimensional space of concerns from a specific viewpoint. As a result, this approach defines a perspectival concern-space as a projection of a concern-space that involves a set of related concerns, their reifications into models, and the realization of these models (see Figure 4).

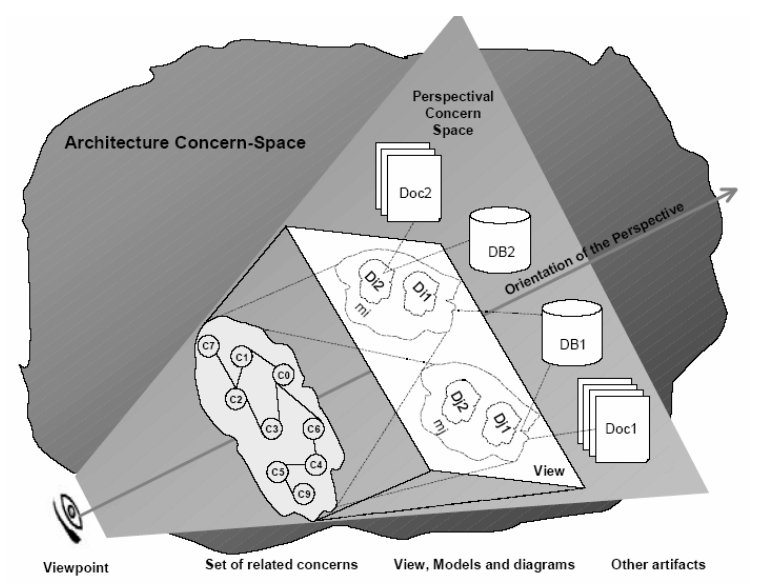

Figure 4. A Perspectival Concern-Space in Overview [Kan03]

The PCS approach uses UML to specify aspect-oriented software architectures, and it extends UML by defining a profile that supports the modelling of aspects and components. The profile simulates aspects by means of architectural connectors based on the idea that aspects act as coordinators among components to intercept their interactions and then replace or add behaviour either before or after them [Kan02b]. As a result, PCS is based on an original ADL without connectors, whose aspect-oriented behaviour is introduced by means of connectors. Components and aspects are modelled by means of UML classes that have been profiled in order to have ports through publishing services. In addition, aspect classes are distinguished by component clasess by means of the $<<$ aspect $>>$ stereotype [Kan02a]. A disadvantage of this 
PRISMA: Aspect-Oriented Software Architectures

combination of aspects and software architectures is the loss of the advantages that connectors provide to ADLS and the opportunity to specify how concerns crosscut the coordination rules of connectors.

The PCS approach is supported by the ConcernBase tool [Kan03]. This tool provides mechanisms for modelling software systems, and it also allows the translation from UML models to the SADL language [Mor97].

Technological independence is a clear advantage that this approach offers. Yet, at the same time, it is a drawback of PCS because it does not provide support to translate its models to a programming language or to trace from models to implementation. As a result, its models cannot be executed on a technological platform.

\subsubsection{CAM/DAOP: Component-Aspect Model/Dynamic Aspect-Oriented Platform}

CAM/DAOP is an approach that supports the separation of concerns from the design to the implementation stages of the software life cycle. It is composed of the CAM model, the DAOP -ADL [Pin03], and the DAOP platform [Pin05].

The CAM model extends UML in order to specify the components, the aspects and the mechanisms that compose components and aspects. Components are the core functionality that is crosscut by non-functional concerns, which are specified as aspects. In CAM, aspects are presented as special components, which are differentiated from the original ones by means of the $<<$ aspect $>>$ stereotype. Specifically, since its component model does not have the notion of connector (see section 2.4.2), CAM introduces aspects as special connectors among components. The coordination of these connectors is performed by intercepting the services that arrive to or depart from components and by adding behaviour before, after or instead of their services. As a result, CAM does not introduce a new concept in software architectures for modelling aspects; it uses a refined version of the connector concept in order to simulate the behaviour of aspects and the composition of aspects and components. One of the advantages of this model is the fact that the weaving process between aspects and components is defined by means of interfaces. As a result, the encapsulation and reusability of components and aspects are preserved. In addition, this allows CAM to define the weaving process using the interfaces 88 
and also to execute it dynamically [Fue05]. However, the model does not provide original connectors to specify the architecture of the system. As a result, the model loses the advantages that connectors provide to ADLS and the opportunity to specify how concerns crosscut the coordination rules of connectors. Finally, it is important to emphasize the local or remote instantiation of components and the four kinds of instantiation that the CAM model provides for aspects: a single instance for each aspect, one aspect instance for each user of the system, one aspect instance for all the components that play the same role, and one aspect instance for each instance of a component.

CAM specifies aspect-oriented software architectures using its DAOP-ADL. This ADL uses XML to describe components, aspects, and their interactions. On one hand, this is an advantage because it is a standard of data exchange between tools, it is widely extended and there are other languages that support query and management mechanisms for XML documents. On the other hand, this is a disadvantage because XML is not a formal language, and it can involve problems of correctness, accuracy, inconsistency, etc. In addition, it introduces limitations such as mechanisms to validate properties, to automatically generate code without ambiguity, etc. Finally, with regard to the DAOP-ADL, it is important to emphasize that it is independent of technology. As a result, their specifications do not introduce expressions or syntaxes of specific programming languages or technologies.

The DAOP platform has been implemented in Java, and it provides a middleware in order to support the execution of aspects, components, and the dynamic weaving between them over the Java technology. The platform and the DAOP-ADL specifications are integrated because the input of the DAOP platform is the XML document that contains the specification of the architectural model in XML. As a result, the middleware can perform the dynamic weaving since it knows all the information about the architectural model and knows the weavings that can be executed by each one of the aspects. The middleware performs the weaving by intercepting service requests and determining which aspect must be executed. In addition, the $\mathrm{XML}$ document contains the information needed to instantiate components and aspects. For this reason, when the document is loaded by the DAOP platform, the instantiation of 
PRISMA: Aspect-Oriented Software Architectures

components and aspects starts taking into account the instantiation information defined in the document.

\subsubsection{Superimposition}

The work of Sihman and Katz proposes the use of superimposition for incorporating aspects into object-oriented programs. The generic operation of superimposition consists of applying a concept on top of another one. In this approach, aspects are superimposed on top of base applications. This approach creates the SuperJ constructor in order to pre-process aspectoriented superimpositions over AspectJ.

A superimposition consists of a set of aspects and new classes that represent the extension of an application. A SuperJ implements an algorithm to apply a superimposition to a base application. Base applications do not reference superimpositions, and superimpositions can also be defined and compiled independently of base applications. However, when a superimposition is connected to a base application using the SuperJ constructor, the code of the superimposition makes reference to the state of the base application and it is not independent (see Figure 5). In fact, the needed advices and pointcuts are defined inside the aspects of a superimposition. As a result, the behaviour of the aspect and its connections to the base program are not defined separately. Despite the fact that the specification of an aspect is done at a high abstraction level, the specification of advices and pointcuts inside aspects reduces the capabilities of aspect reuse of the approach. In addition, superimposition allows the specification of conditions of applicability or the definition of desired results for the process of applying a superimposition to a base application.

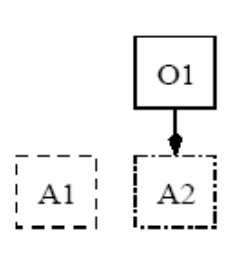

Superimposition

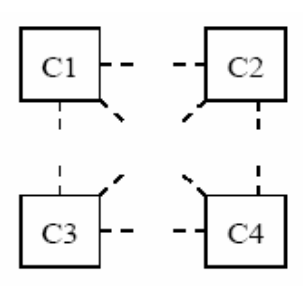

Basic Program

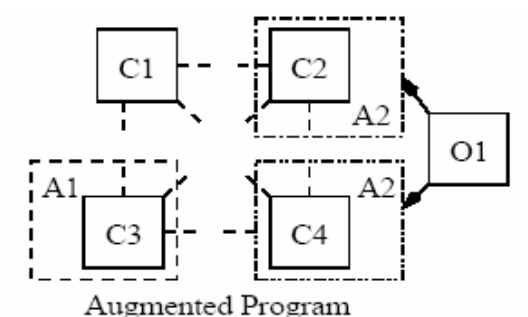

Augmented Program

Figure 5. Superimposition [Sih03] 
This approach allows us to combine superimpositions and to check the constraints that have been defined in a superimposition. It has been implemented using Java, and it uses Aspect $J$ in order to apply this technique over Java base applications. As a result, the Java implementation of the SuperJ makes this model dependent on technology and it is closer to object-oriented programming languages than ADLs.

\subsubsection{TRANSAT}

Transat $[\mathrm{Bar} 04 \mathrm{~b}]$ is an approach for managing the evolution of software architecture specifications using aspect-oriented programming principles. The approach starts from a core architectural model that either needs to be extended during its development process or needs to be evolved during its maintenance process. The mechanisms of extension and evolution are provided using AOP techniques. As a result, this approach incrementally obtains a complete software architecture with business and technical concerns from a business software architecture.

This approach is supported by a framework that allows the evolution of software architectures by integrating new technical concerns. The framework guides the separated definition of technical and business concerns. Business concerns are the core architectural model, and technical concerns are the aspects that extend the basic functionality of the system. The framework provides aspect-oriented mechanisms to weave both.

The core architectural model is defined using its component model, SafArchie [Bar03]. This model is a hierarchical component model that defines software architecture by means of composition relationships. The new technical concerns such as persistence, security, or transaction management are modelled as components. Finally, the weavings between business components and aspect components are defined by means of adapters and weavers. Adapters define the integration rules between technical components and business components, and weavers define the coordination rules between them. In other words, adapters and weavers materialize the integration of the core architecture and their extensions by identifying the join points in the core architecture and by defining the pointcuts at adapters and weavers. 
PRISMA: Aspect-Oriented Software Architectures

The Transat framework consists of a tool called SafArchie Studio [Bar04a]This tool is an extension of ArgoUML, which offers several views of the evolution process depending on the kind of user.

One of the main advantages of this approach is that it is based on ADL for defining the core architectural model. As a result, the formal definition of software architecture and its independence from a technological platform are guaranteed.

Another advantage is the way that evolution is supported. The integration of new requirements does not break the consistency of the original software architecture. In addition, the application of AOP principles to this integration ensures both the separation of concerns in the software architecture extension and better management if new requirements for these concerns arise. Finally, this extension mechanism allows analysts to easily identify where the original software architecture has been modified; they only need to find the adapters and weavers of the complete architecture.

A great limitation of this approach is the constraint of starting the development from a core architectural model without considering concerns from the beginning. Also, the fact that concerns are only technical and not more generic is another drawback. As a result, aspects in this approach are not introduced as a new concept for modelling software architectures. Software architectures are defined using a pure compositional ADL, and aspects only appear as an extension or evolution mechanism of software architectures.

\subsubsection{ASAAM: Aspectual Software Architecture Analysis Method}

ASAAM [Tek04] is the approach that introduces aspect-orientation techniques to the SAAM approach ( see section 2.2), which introduces three perspectives to analyze software architecture specifications: functionality, structure and allocation. As a result, ASAAM is an extension and refinement of SAAM. The steps of ASAAM are the following:

1. Develop a candidate architecture: A candidate architecture is generated taking into account quality attributes and potential aspects.

2. Develop scenarios: The scenarios that define the business rules of the system and possible future changes are created. 
3. Perform scenario evaluations: The scenarios are evaluated and categorized, and potential aspects are identified for each scenario.

4. Assess scenario interaction and classify components: The separation of concerns is assessed for both crosscutting-concerns and non-crosscutting concerns.

5. Refactor the architecture: A refactorization of the architecture is proposed using conventional techniques and aspect-oriented techniques.

This evaluation method of aspect-oriented software architectures has been implemented as an Eclipse add-in called ASAAM-T [Tek05]. The main difference between this approach and the others is the fact that the purpose of ASAAM is to assess an aspect-oriented software architecture instead of specifying and implementing a software architecture. As a result, this approach is a valuable contribution to the field for evaluating if an aspect-oriented software architecture has considered the correct aspects, and if the aspects are factorized in a proper way.

\subsubsection{AVA: Architectural Views of Aspects}

AVA is an approach where aspects are introduced in software architectures as views [Kat03]. The notion of aspect in software architectures is simulated by the architectural view concept. This facilitates the comprehensibility of the model for the software architecture community. However, an aspect is not semantically a view because an aspect has its own behaviour independently of the architectural elements that it affects. Furthermore, this approach constrains the notion of architectural view to an aspect, losing other viewpoints for defining views such as kinds of users, models, level of abstraction, features, etc.

In AVA, an aspect is a module that encapsulates a set of components and their connections that are crosscut by this aspect. As a component can be crosscut by more than one aspect, the dependencies between different aspects must be explicitly specified in order not to lose the consistency of the software architecture. Since it is possible to define several aspects for the same concern in AVA, aspect modules (S,O) can be composed to form a single concern module (C) (see Figure 6). This aspect composition is performed using superimposition, which is an asymmetric operation in which one aspect is applied on top of another one [Kat02]. As a result, the software architecture is completely remodularized in different modules that represent 
PRISMA: Aspect-Oriented Software Architectures

aspects or concerns, depending on the level of abstraction. This modularization distributes components into different modules taking into account the aspects that affect them. The main disadvantage of this remodularization is the loss of the complete software architecture view. However, this concern and remodularization structure of modules allows analysts to easily locate where to introduce new changes, taking into account the concerns that should be modified.

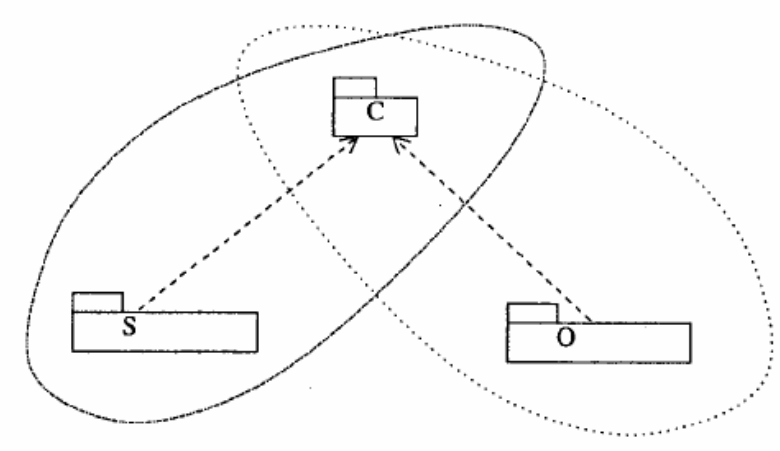

Figure 6. Concern Diagram of AVA [Kat03]

The AVA model has been created by defining a UML profile. As a result, the definition of AVA software architectures is really intuitive because these architectures use the OMG standard. In addition, the use of UML allows analysts to specify software architectures independently of technology and in a graphical way. In the AVA profile, the aspect is a stereotype of the package UML metaclass, and the concern diagram is an extension of the component diagram.

The AVA approach has also been applied to the definition and documentation of pattern systems [Ham05], and the MADE tool has been developed to support it [Ham04].

\subsubsection{AspectLEDA}

AspectLeda is an approach that extends the LEDA ADL [Can00][Can99] with aspect-oriented concepts [Nav05]. This approach consists of two steps: the definition of an initial architectural model and the addition of aspects. The initial architectural model is defined using the LEDA $\mathrm{ADL}$ in order to have the advantages of a formal basis, to validate the software architecture by 
executing a prototype, and to be independent of technology. Once the initial architecture has been defined, the new requirements that emerge during the development and maintenance processes are incorporated in the architecture such as aspects. This is a clear drawback of this approach because it does not give the analyst the chance to introduce aspects at the beginning of the software development process. Aspects are only used at the maintenance stage or at refinement processes of the development stage. In addition, it is important to take into account that not all new requirements of a system are aspects. However, AspectLEDA forces the analyst to introduce new requirements into the model as new aspects without taking into account whether they are aspects or not.

In AspectLEDA, aspects are specified in the way as components because LEDA is an $\mathrm{ADL}$ without connectors. However, aspect components and components of the initial software architecture are defined in different levels. Since AspectLEDA does not have architectural connectors, it cannot specify the concerns that crosscut connectors, and it loses the advantages that connectors provide to ADLs. However, AspectLEDA introduces the notion of coordinator to define the weaving process that synchronizes aspects and components and coordinates both levels. This coordinator preserves the reusability and encapsulation of aspects because the coordination of aspects and components is specified outside aspects.

Finally, it is important to emphasize that this approach is still only a proposal. It does not have a tool to support for its methodology, and it is not able to compile its aspect-oriented software architecture into any technological platform.

\subsubsection{AOCE: Aspect-Oriented Component Engineering}

The Aspect-Oriented Component Engineering approach (AOCE) is based on AOREC (Aspect-Oriented Requierements for Component-Based Systems). AOREC uses the notion of aspect in order to suitably define and categorize the requirements of components in terms of what they provide or require through their services. In AOREC, an aspect is a characteristic of a system for which components provide and/or require services. This approach takes into account some aspects such as user interface, collaboration, persistence, distribution, and configuration. As a result, AOREC uses aspects in order to attain multiple perspectives of the 
PRISMA: Aspect-Oriented Software Architectures

components in order to better understand and reason about the behaviour and semantics of these components.

Since AOCE is the step after AOREC in the development process, AOCE defines aspects in the same way that AOREC does; aspects are specified as components. The definition of an aspect component is done separately from the component specification in order to be independent and reusable. However, an AspectManager must be introduced in order to coordinate aspect components and components. Despite the fact that this approach does not have connectors (because is a component-based approach), it still must introduce a connector called AspectManager to weave aspects and components at run-time.

Apart from not having the notion of connector and not classifying the interfaces of connectors in terms of aspects, the main disadvantage of this approach is the fact that the design language of AOCE is based on a specific component-based platform, the JViews [Grun98] AOP implementation platform, which is not independent of technology.

Finally, it is important to mention that AOREC and AOCE have a tool to support their methodology. They have extended the tool of JViews to support aspects. This tool is called JComposer [Grun98].

\subsubsection{Component Views}

The component views approach [Sto02] is not really an approach to specify aspects of software architectures. The component views approach is an extension of component-based models to define views using concerns as viewpoints. As a result, this approach decomposes the architecture taking into account which components and connections among them are affected by a specific concern. The result of this decomposition is that each view of a concern contains the components and relationships affected by this concern. This approach defines a UML profile to support the definition of these views for software architectures. However, this approach does not introduce new concepts or simulates the notion of aspect because it does not support this notion. It only works with concerns which are only used to analyze componentbased models. Their specification is made using a UML profile. The purpose is not to execute an aspect-oriented component-based model, it is simply to analyze component models. 


\subsubsection{Aspectual Components}

Aspectual Components are proposed as a new kind of component by the work of Lieberherr [Lie99]. They are defined using a generic data model called a participant graph. This graph is then refined to deploy aspects as normal components.

This approach proposes adding a new dimension to aspects over the organization of an object-oriented application. As a result, the first task of the software development process is to decompose software into aspects. The second one is to decompose each aspect into classes following the object-oriented approach. The result of this process is an aspectual component composed of object-oriented classes. However, these aspectual components should be composed with the application base. In other words, the new dimension of aspects must be communicated to the bottom dimension of the application. This communication is achieved by means of connectors that coordinate both dimensions.

Aspectual components can be programmed using Java programming language because this approach does not introduce a new programming constructor; instead, aspectual components are implemented as normal components. However, this approach does not offer a tool to support work with this model.

\subsubsection{Caesar}

Caesar is a model for aspect-oriented programming [Mez03] with its own programming language. This model is characterized by being technology-dependent and by developing a higher-level module to develop aspects independently of the mechanisms for join point interceptions.

Caesar specifies the implementation of aspects and their weaving relationships in a separate way in order to reuse the aspect independently of what the aspect is related to. The main feature that distinguishes Caesar from other aspect-programming models is the concept of Aspect Collaboration Interface (ACI). An aspect is specified by means of an ACI. An ACI decouples the implementation and weavings of aspects. An ACI is composed of two different interrelated modules: an implementation aspect module and a binding aspect module. The former implements the methods that the aspect provides, independently of the context. The latter implements the required methods from a specific context by means of pointcuts and advices. In 
PRISMA: Aspect-Oriented Software Architectures

addition, an ACI can be composed of other ACIs, which provide a complete level of composition in order to define aspects over the base code.

Caesar defines an instantiation mechanism for its ACIs. To instantiate ACIS, the implementation and a specific binding for the aspects must be composed in the same unit; this unit is called weavelet. A weavelet is a class that is composed of the interface that provides and requires. Once, the weavelets are defined; they can be instantiated in a static or dynamic way. This mechanism of instantiation allows several instances of the same weavelet to be defined. It also provides a choice of different weavelets using aspectual polymorphism.

\subsubsection{JASCO}

$\mathrm{JAsCo}$ is originally an aspect-oriented programming language for the Java Beans component model [Suv03]; however, a prototype for .NET platform is currently being developed [Ver03]. As a result, JAsCo is a programming language that is dependent on technology. It introduces three new concepts to extend Java to support aspect-oriented programming, which include aspects, hooks, and connectors.

In JAsCo, aspects are composed of a set of hooks that define how to link an aspect to a specific context. A hook consists of two parts: the pointcut (when the hook is activated) and the advice (what is going to be executed as a result of the activation). Finally, connectors allow the definition of the mappings between a hook and one or more elements of the base code (joinpoint and pointcut correspondence).

The JAsCo execution model is very flexible and provides many advantages. It supports aspectual polymorphism, which is the weaving between aspects and code. This is dynamic because aspects can be added and removed at run-time. However, the referential nature of the dynamic weaving requires an execution platform to intercept the application and insert it into the aspects at execution time. In addition, aspects can be combined to form complex structures by means of inheritance and aggregation relationships.

Finally, it important to mention that there are a pair of tools that support the JAsCo approach. One of them transforms a Java bean into a JAsCo bean, and the other one is the integration of JAsCo into the PacoSuite [Van01], [Wyd01], which allows modelling 
component models at a high abstraction level and also allows generating one or more JAsCo connectors from its models.

\subsubsection{FUSEJ}

FuseJ is a programming language for component-based software architectures onto the Java Beans component model [Suv05b]. This language asserts that there are no aspects and these services can be implemented as a component. It is a platform dependent language that does not make distinctions between normal components and aspect components. It is based on the component architecture presented in Figure 7. Each concern and component is programmed as a component of the Component Layer. The provided and required services of components are sent through the gates of the Gate Layer by preserving the encapsulation of components (black box view). Finally, the coordination among the gates is programmed using connectors of the Connector Layer. However, this connector must be implemented in different ways depending on whether two normal components are being coordinated or a component and an aspect component are being coordinated. In this last case, the coordination is performed using the aspect-oriented primitives to define the pointcuts and advices (to specifying the weaving process).

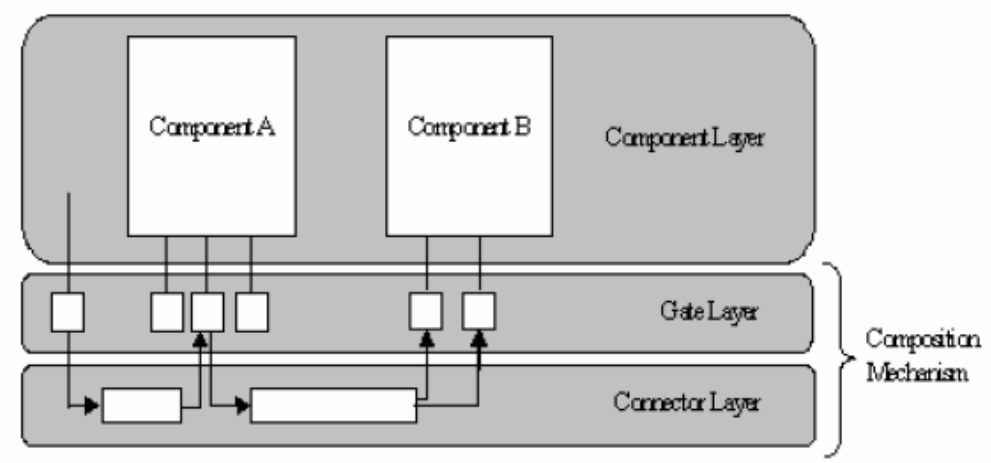

Figure 7. Unified Component Architecture [Suv05b]

With regard to instantiation, aspects are instantiated as regular components; each aspect can have more than one instance. Finally, it is important to emphasize that the tool support for FuseJ is currently being developed. 
PRISMA: Aspect-Oriented Software Architectures

\subsubsection{JAC}

JAC is a framework to develop aspect-oriented distributed applications in Java programming language [Paw04]. The main contribution of the programming model of JAC is the fact that aspects can be distributed. They also have a dynamic nature, which means they can be added and removed at run-time.

JAC provides a set of classes and methods that are extended when a new JAC application is developed. JAC provides two different levels of aspect-oriented programming: the programming level and the configuration level. The former is used when new aspects are programmed from scratch. The latter is used when existing aspects are customized for new requirements.

Since JAC packages normal components inside containers, it also defines aspects as components that are inside containers. The components that define aspects are called aspect components. These containers are remote servers that can represent normal or aspect components. JAC aspect components crosscut normal components that are not necessarily in the same location as the aspect components. As a result, JAC gives support to distributed weaving processes. This need emerges because aspects are treated as components and the weaving process (the pointcuts and advices) are defined inside the aspect. If the weaving process were defined in a different entity of the aspect such as a connector, this distribution need would not arise, because the distributed communication is supported by components and connectors. The main drawback is not the effort needed to support distributed communication in pointcuts, it is the fact that the behaviour of the aspect cannot be reused. JAC loses the reusability of aspects because the relationships for applying the aspect to a specific context are defined inside it.

\subsubsection{JIAZZI}

Jiazzi is an aspect-programming model that extends Java by means of encapsulated code modules called units [McD03]. They are separately compiled and are externally linked code modules that are introduced into a Java program. These units were originally created to obtain higher modularity of code. However, they are currently being used to add aspects to nonaspect-oriented Java programs in a non-invasive way. This is possible because JIAZZI also 100 
provides linking units like connectors to specify the connections of units and a base Java program.

Jiazzi units are composed of Java classes that implement the behaviour of a specific concern. They are compiled independently of linking units and base code; as well as the type checking is also performed internally. In addition, an external compilation is needed to perform the connection between base code and units by means of linking units. In this compilation process, the types of connections must also be checked.

Jiazzi does not extend the syntax of the Java programming language because it introduces aspects as externally linked Java modules. Jiazzi and its interaction with Java are implemented using Java, and it runs perfectly on this technology. Its main drawback is the fact that it is a technology-dependent model and the pre-compilation and encapsulation of its modules before its integration reduces the flexibility to evolve aspects at run-time.

\subsection{COMPARISON OF ASPECT-ORIENTED SOFTWARE ARCHITECTURES}

There are several features that are essential for analyzing and to comparing the different approaches that have been presented in the previous section. The features that have been used as comparison criteria have been selected starting from the premise that an aspect-oriented software architecture approach should completely support the development and maintenance processes of software. It is important to mention that there are important features, such as the instantiation mechanisms and the types checking, that have not been used to compare the different approaches because the proposal does not usually give very much information about these features. The analysis of these features is included in their descriptions above for those approaches that provide information about them.

Next, we detail the features of comparison and the reasons because they have been considered as a classification criteria of aspect-oriented architectural models.

Aspect-oriented model: This feature defines the kind of aspect-oriented model that is integrated with the software architectural model. The four kinds are: asymmetric, symmetric, multidimensional, and composition filters (see section 3.2). This characteristic 
PRISMA: Aspect-Oriented Software Architectures

is important because the integration is completely different depending on the aspectoriented model.

Architectural model: This feature determines whether an architectural model provides connectors for modelling software architectures or not. Those that have connectors provide features that improve the structure and maintenance of software architecture (see section 2.4.2 for details).

D Definition of Aspects: The most distinguishable feature of aspect-oriented architectural models is how they integrate aspects and software architectures. There are two ways of doing this: by simulating the notion of aspect by means of another architectural concept or by defining a new concept in software architecture for aspects. The first way refines the architectural concepts varying their original semantics; and the second one requires understanding a new concept to model software architectures.

Definition of Weavings: This is an important feature of aspect-oriented models, and of aspect-oriented architectural models. The definition of weavings feature specifies where the weaving process between aspects and architectural elements is defined. If the pointcuts and advices are defined inside the aspect, the aspect is dependent on the context that the aspect is connected to. However, if they are defined outside the aspect, the behaviour of the aspect can be reused independently of where they will be connected.

ADL: Another feature that is necessary to take into account when comparing architectural models is whether the ADL is a formal language or not. The formal nature of an ADL is an indispensable property of architectural models if the purpose of the approach is to generate code without ambiguity, to verify properties, to validate behaviour, to trace the different levels of abstraction in a suitable way, to evolve software architectures preserving the consistency of the system and so forth.

Aspect-Oriented Evolution: The evolution of aspect support is an important feature that can improve the evolution and run-time evolution of software architectures. As a result, an approach that provides mechanisms for adding or removing aspects is a great advantage. 
Purpose: The purpose of the approach is an essential feature to be able to compare models. There are aspect-oriented architectural models that give complete support during the development process, others that analyze or evolve models, and still others that fulfil several purposes.

$>$ Technology: This is an important feature that distinguishes the wide variety of aspectoriented architectural models that exists. An aspect-oriented architectural model should be specified in an abstract way by means of an ADL. As a result, the same specification can be applied to different platforms and different programming languages. However, if the model depends on a specific platform and/or programming language, its application and flexibility are considerably reduced.

> Graphical support: The graphical specification of aspect-oriented software architectures is a necessary feature to avoid the complexity of using ADLs. The graphical support is achieved by defining the graphical metaphor of ADLs by means of a new language or by extending a well-known graphical language.

Tool support: A significant feature of the aspect-oriented architectural models is its support by means of a framework that guides the analyst during the development and maintenance processes. A framework can provide a wide variety of facilities such as modelling support, ADL generation, code generation, code execution, validation, verification, evolution, run-time evolution, etc.

A comparison table has been developed from the features and the approaches analyzed in the above section. This table is divided into two separate tables due to the limitation of the page dimensions. Blank cells indicate that no information was available. 


\begin{tabular}{|c|c|c|c|c|c|}
\hline & $\begin{array}{c}\text { Aspect-oriented } \\
\text { model }\end{array}$ & $\begin{array}{c}\text { Architectural } \\
\text { model }\end{array}$ & $\begin{array}{l}\text { Definition of } \\
\text { Aspects }\end{array}$ & $\begin{array}{l}\text { Definition of } \\
\text { Weavings }\end{array}$ & ADL \\
\hline PCS & $\begin{array}{l}\text { Multidimensional } \\
\text { and symmetric }\end{array}$ & $\begin{array}{l}\text { Without } \\
\text { connectors }\end{array}$ & $\begin{array}{l}\text { Aspects like } \\
\text { connectors }\end{array}$ & Inside aspects & $\begin{array}{c}\text { SADL: } \\
\text { Formal } \\
\text { compositional } \\
\text { ADL }\end{array}$ \\
\hline CAM/DAOP & Asymmetric & $\begin{array}{l}\text { Without } \\
\text { connectors }\end{array}$ & $\begin{array}{l}\text { Aspects like } \\
\text { connectors }\end{array}$ & $\begin{array}{c}\text { Outside aspects } \\
\text { using } \\
\text { communication } \\
\text { between } \\
\text { interfaces }\end{array}$ & $\begin{array}{c}\text { DAOP - } \\
\text { ADL: Not } \\
\text { formal, based } \\
\text { on XML }\end{array}$ \\
\hline Superimposition & $\begin{array}{l}\text { Asymmetric: } \\
\text { Two levels: } \\
\text { aspects and } \\
\text { architectures }\end{array}$ & & $\begin{array}{c}\text { Java Classes } \\
\text { inside a } \\
\text { superimposition } \\
\text { layer }\end{array}$ & Inside aspects & \\
\hline TRANSAT & $\begin{array}{l}\text { Asymmetric. } \\
\text { Only technical } \\
\text { aspects }\end{array}$ & $\begin{array}{c}\text { Without } \\
\text { connectors }\end{array}$ & $\begin{array}{l}\text { Aspects like } \\
\text { components. } \\
\text { Aspect } \\
\text { components }\end{array}$ & $\begin{array}{c}\text { Outside } \\
\text { aspects. Using } \\
\text { adapters or } \\
\text { weavers } \cong \\
\text { connectors }\end{array}$ & $\begin{array}{c}\text { SafArchie } \\
\text { component } \\
\text { model }\end{array}$ \\
\hline ASAAM & Asymmetric & Not fixed & Scenarios & $\begin{array}{l}\text { Outside } \\
\text { Aspects }\end{array}$ & Not fixed \\
\hline AVA & Asymmetric & Not fixed & Aspects as views & $\begin{array}{l}\text { Outside } \\
\text { Aspects }\end{array}$ & Not fixed \\
\hline AspectLEDA & $\begin{array}{l}\text { Asymmetric: } \\
\text { Two levels: } \\
\text { Aspects and } \\
\text { architectures }\end{array}$ & $\begin{array}{c}\text { Without } \\
\text { connectors }\end{array}$ & $\begin{array}{c}\text { Aspects as } \\
\text { components }\end{array}$ & $\begin{array}{l}\text { Outside aspects } \\
\text { using } \\
\text { coordinators } \cong \\
\text { connectors }\end{array}$ & $\begin{array}{c}\text { Leda: Formal } \\
\text { Compositional } \\
\text { ADL }\end{array}$ \\
\hline AOCE & Asymmetric & $\begin{array}{c}\text { Without } \\
\text { connectors }\end{array}$ & $\begin{array}{c}\text { Aspects as } \\
\text { components }\end{array}$ & $\begin{array}{c}\text { Outside aspects } \\
\text { using aspect } \\
\text { managers } \cong \\
\text { connectors }\end{array}$ & \\
\hline $\begin{array}{l}\text { Component } \\
\text { Views }\end{array}$ & Asymmetric & & $\begin{array}{l}\text { Not aspects. } \\
\text { Concerns as } \\
\text { viewpoints for } \\
\text { defining } \\
\text { architectural } \\
\text { views } \\
\end{array}$ & & \\
\hline $\begin{array}{c}\text { Aspectual } \\
\text { Components }\end{array}$ & $\begin{array}{l}\text { Asymmetric: } \\
\text { Two levels: } \\
\text { Aspects and } \\
\text { object-oriented } \\
\text { applications }\end{array}$ & & $\begin{array}{c}\text { Aspects as } \\
\text { components: } \\
\text { Aspectual } \\
\text { components }\end{array}$ & $\begin{array}{c}\text { Outside aspects } \\
\text { with } \\
\text { connectors }\end{array}$ & \\
\hline
\end{tabular}




\begin{tabular}{|c|c|c|c|c|c|}
\hline Caesar & Asymmetric & & $\begin{array}{c}\text { Aspect } \\
\text { Collaboration } \\
\text { Interface (ACI) }\end{array}$ & $\begin{array}{c}\text { Separation of } \\
\text { ACI modules } \\
\text { into } \\
\text { implementation } \\
\text { and interaction } \\
\text { of aspects }\end{array}$ & \\
\hline JASCO & Asymmetric & & Aspects & $\begin{array}{c}\text { Hooks and } \\
\text { connectors }\end{array}$ & \\
\hline FUSEJ & Asymmetric & $\begin{array}{c}\text { With } \\
\text { Connectors }\end{array}$ & $\begin{array}{c}\text { Without Aspects: } \\
\text { Components }\end{array}$ & Connectors & \\
\hline JAC & $\begin{array}{c}\text { Aspects as } \\
\text { components: } \\
\text { aspectcomponents }\end{array}$ & Inside aspects & \\
\hline JIAZZI & $\begin{array}{c}\text { Asymmetric: } \\
\text { Two levels: } \\
\text { Aspects and } \\
\text { object-oriented } \\
\text { applications }\end{array}$ & & Units & Linking units & \\
\hline
\end{tabular}

Table 1. First comparison of aspect-oriented software architecture approaches

\begin{tabular}{|c|c|c|c|c|c|}
\hline & $\begin{array}{l}\text { Aspect- } \\
\text { Oriented } \\
\text { Evolution }\end{array}$ & Purpose & Technology & $\begin{array}{c}\text { Graphical } \\
\text { support }\end{array}$ & Tool support \\
\hline PCS & & $\begin{array}{c}\text { Development } \\
\text { of AO } \\
\text { Software } \\
\text { Architecture }\end{array}$ & Independent & $\begin{array}{c}\text { UML } \\
\text { profile: } \\
\text { Aspect is a } \\
\text { stereotype of } \\
\text { a UML class }\end{array}$ & $\begin{array}{l}\text { ConcernBase } \\
\text { tool: modelling } \\
\text { support, ADL } \\
\text { generation from } \\
\text { UML, no code } \\
\text { generation, no } \\
\text { execution }\end{array}$ \\
\hline CAM/DAOP & $\begin{array}{l}\text { Dynamic } \\
\text { weaving but } \\
\text { not adding } \\
\text { and } \\
\text { removing } \\
\text { aspects at } \\
\text { run-time }\end{array}$ & $\begin{array}{l}\text { Development } \\
\text { of AO } \\
\text { Software } \\
\text { Architecture }\end{array}$ & Independent & UML profile & $\begin{array}{c}\text { DAOP } \\
\text { platform: Java } \\
\text { Technology, } \\
\text { modelling } \\
\text { support, DAOP } \\
\text { middleware for } \\
\text { code execution }\end{array}$ \\
\hline Superimposition & & $\begin{array}{c}\text { Programming } \\
\text { aspect-oriented } \\
\text { Java } \\
\text { applications } \\
\text { and verifying } \\
\text { properties of } \\
\text { aspect-oriented } \\
\text { superimposition }\end{array}$ & $\begin{array}{l}\text { Dependent } \\
\text { on Java } \\
\text { technology }\end{array}$ & & \\
\hline
\end{tabular}


PRISMA: Aspect-Oriented Software Architectures

\begin{tabular}{|c|c|c|c|c|c|}
\hline TRANSAT & & $\begin{array}{l}\text { Only evolution } \\
\text { support, the } \\
\text { initial aspect- } \\
\text { oriented } \\
\text { specification is } \\
\text { not supported. }\end{array}$ & Independent & UML profile & $\begin{array}{l}\text { SafArchie } \\
\text { Studio. } \\
\text { Extension of } \\
\text { ArgoUML }\end{array}$ \\
\hline ASAAM & & $\begin{array}{c}\text { Analysis of } \\
\text { Software } \\
\text { Architectures }\end{array}$ & Independent & $\begin{array}{c}\text { UML } \\
\text { profile: } \\
\text { scenarios }\end{array}$ & ASAAM-T \\
\hline AVA & & $\begin{array}{c}\text { Development } \\
\text { of AO } \\
\text { Software } \\
\text { Architecture }\end{array}$ & Independent & $\begin{array}{c}\text { UML } \\
\text { profile: } \\
\text { aspect is an } \\
\text { stereotype of } \\
\text { a UML } \\
\text { package that } \\
\text { contains an } \\
\text { extension of } \\
\text { component } \\
\text { diagram }\end{array}$ & $\begin{array}{l}\text { MADE tool: } \\
\text { modelling } \\
\text { support }\end{array}$ \\
\hline AspectLEDA & & $\begin{array}{l}\text { Development } \\
\text { of AO } \\
\text { Software } \\
\text { Architecture }\end{array}$ & Independent & & \\
\hline AOCE & $\begin{array}{l}\text { Dynamic } \\
\text { weaving }\end{array}$ & $\begin{array}{l}\text { Development } \\
\text { of AO } \\
\text { Software } \\
\text { Architecture }\end{array}$ & $\begin{array}{l}\text { Dependent } \\
\text { on JViews }\end{array}$ & & $\begin{array}{l}\text { JComposer: An } \\
\text { extension of the } \\
\text { JViews tool }\end{array}$ \\
\hline $\begin{array}{c}\text { Component } \\
\text { Views }\end{array}$ & & $\begin{array}{c}\text { Analysis of } \\
\text { software } \\
\text { architectures }\end{array}$ & Independent & UML profile & \\
\hline $\begin{array}{c}\text { Aspectual } \\
\text { Components }\end{array}$ & & $\begin{array}{c}\text { Programming } \\
\text { aspect-oriented } \\
\text { Java } \\
\text { applications }\end{array}$ & $\begin{array}{l}\text { Dependent } \\
\text { on Java } \\
\text { technology }\end{array}$ & & \\
\hline Caesar & & $\begin{array}{l}\text { Programming } \\
\text { aspect-oriented } \\
\text { Caesar } \\
\text { applications }\end{array}$ & $\begin{array}{c}\text { Dependent } \\
\text { on Caesar } \\
\text { programming } \\
\text { language }\end{array}$ & & $\begin{array}{l}\text { Programming } \\
\text { framework }\end{array}$ \\
\hline JASCO & $\begin{array}{c}\text { Dynamic } \\
\text { weaving and } \\
\text { support for } \\
\text { adding and } \\
\text { removing } \\
\text { aspects at } \\
\text { run-time }\end{array}$ & $\begin{array}{l}\text { Programming } \\
\text { aspect-oriented } \\
\text { application }\end{array}$ & $\begin{array}{l}\text { Dependent } \\
\text { on Java or } \\
\text {.Net } \\
\text { technology }\end{array}$ & & $\begin{array}{l}\text { Programming } \\
\text { framework }\end{array}$ \\
\hline
\end{tabular}




\begin{tabular}{|c|c|c|}
\hline FUSEJ & $\begin{array}{l}\text { Programming } \\
\text { aspect-oriented } \\
\text { applications } \\
\text { onto Java } \\
\text { Beans }\end{array}$ & $\begin{array}{c}\text { Dependent } \\
\text { on the Java } \\
\text { Beans } \\
\text { component } \\
\text { model }\end{array}$ \\
\hline JAC & $\begin{array}{c}\text { Programming } \\
\text { aspect-oriented } \\
\text { Java } \\
\text { applications } \\
\end{array}$ & $\begin{array}{l}\text { Dependent } \\
\text { on Java } \\
\text { technology }\end{array}$ \\
\hline JIAZZI & $\begin{array}{c}\text { Programming } \\
\text { aspect-oriented } \\
\text { Java } \\
\text { applications } \\
\end{array}$ & $\begin{array}{l}\text { Dependent } \\
\text { on Java } \\
\text { technology }\end{array}$ \\
\hline
\end{tabular}

Table 2. Second comparison of aspect-oriented software architecture approaches

\subsection{CONCLUSIONS}

After the analysis and comparison of different approaches for aspect-oriented software architecture, it is possible to conclude that these proposals at the architectural level usually extend ADLs without connectors and mainly follow an asymmetric model by considering functionality as architectural components. Despite the fact that there has been a lot of work done, these proposals are only focused on a single specific purpose: the analysis, evolution or development of software architectures. They do not pursue several purposes simultaneously to provide a complete development and maintenance support. Furthermore, they always introduce the notion of aspect by using original architectural concepts, despite the fact that they do not provide the suitable semantics for aspects. As a result, it is necessary to provide an aspectoriented model for symmetric aspect-oriented models and ADLs with connectors. This model should include:

$>$ A suitable semantics for the aspect concept

$>$ A graphical modelling metaphor

$>$ Analysis and evolution capabilities

$>$ Technological support in order to execute the aspect-oriented architectural models that have been defined independently of technology

$>$ A guided support during the development and maintenance processes of software 
PRISMA: Aspect-Oriented Software Architectures

The PRISMA approach has been defined to fulfil these needs. PRISMA integrates an aspect-oriented symmetric model with an ADL that has connectors thereby, creating its own aspect-oriented architectural model that corresponds to an Aspect-Oriented ADL (AOADL). In this thesis, the PRISMA model and framework are presented as an important step forward in the combination of the aspect-oriented paradigm and software architectures. 


\section{PART III}

\section{PRELIMINARIES}

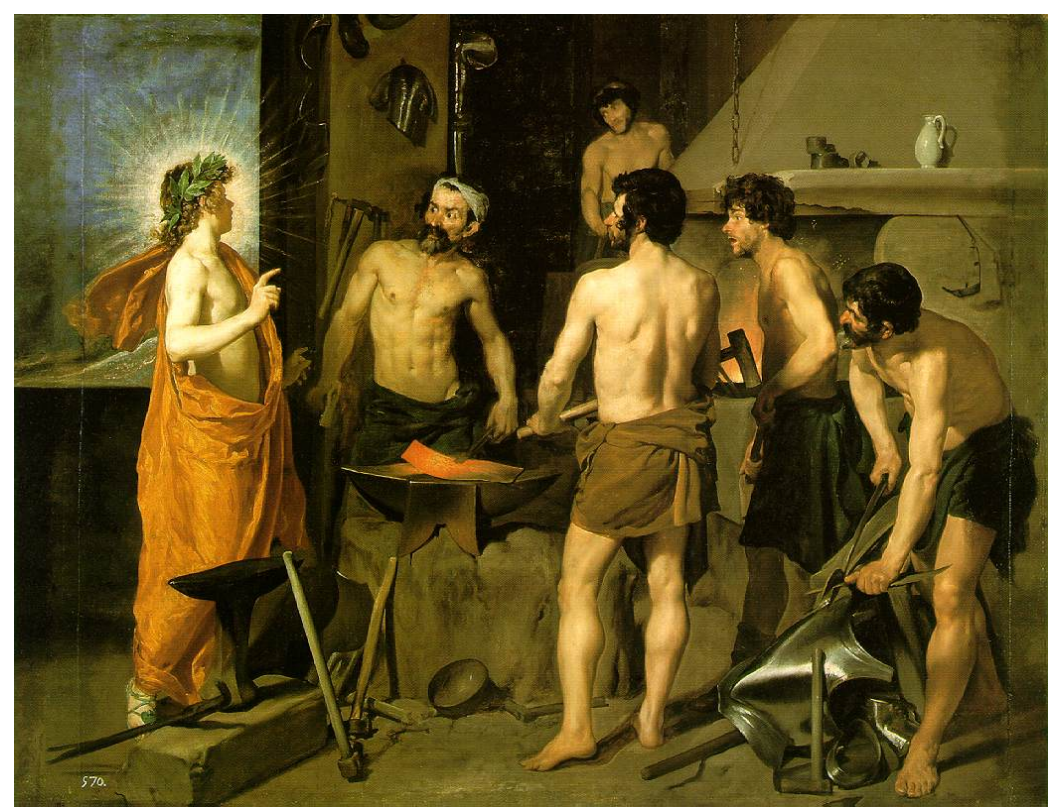

La Fragua de Vulcano, Diego Velázquez, 1630 

$<<$ Sincerity and truth are the basis of every virtue $>>$

Confucius

The main purpose of this chapter is to provide an introduction to the case study that has been chosen to demonstrate the PRISMA approach and to introduce the formalisms that have been used to describe software architectures in PRISMA and to formalize the PRISMA model.

\subsection{TELEOPERATION SYSTEMS: THE TEACHMOVER ROBOT}

There is a wide variety of domains that can take advantage of the PRISMA approach for developing software systems. PRISMA has been put into practice in the tele-operation domain, which unlike academic examples, provides real problems that must be solved in real industrial systems. This domain has been chosen because it offers a framework for applying software engineering techniques.

The main purpose of this section is to provide an introduction to the tele-operation domain as well as to present the suitability of tele-operation systems to apply an aspect-oriented software architecture approach such as PRISMA. In addition, the specific robot that has been completely developed using PRISMA is presented. This robot is used throughout the thesis in order to illustrate the PRISMA approach and its main concepts. 
PRISMA: Aspect-Oriented Software Architectures

\subsubsection{The Tele-operation Domain}

Tele-operation systems are control systems that depend on software to perform their operations. Designing these systems is a difficult task that must integrate mechanical and electrical elements with software components in the same system. They are used for teleoperating mechanisms (robots, vehicles, and tools) that handle inspection and maintenance tasks. This thesis focuses specifically on robotic tele-operated systems. Tele-operated robots are software intensive systems that are used to perform tasks that human operators cannot carry out due to the dangerous nature of the tasks or the hostile nature of the working environment.

The importance of considering the software architecture in robotic tele-operated systems is well known [Cos00]. However, despite the fact that robotic tele-operated systems usually have many common requirements in their definition and many common components in their implementation, it is impossible for a single architecture to be flexible enough to cope with all the variability of the domain. Therefore, a further step is needed to provide a flexible and extensible architectural framework to develop systems with different requirements and commonalities. There have been numerous efforts to provide developers with frameworks such as [Bru02], [Sch01] and [Vol01]. All of them make very valuable contributions that simplify the development of systems. However, the way that the component-oriented approach has been applied may reduce some of its benefits. These frameworks are object-oriented or componentoriented frameworks that rely on object-oriented technologies and that highly depend on a given infrastructure (Linux O.S. and the $\mathrm{C}++$ language). As a result, a technology-independent framework is necessary. This framework should provide mechanisms to define abstract software architectures that can be mapped into specific software architectures as well as mechanisms to dynamically evolve the interaction patterns among components. In addition, tele-operated systems have a wide range of common concerns in their domain. These concerns can be modelled as aspects in order to take advantage of AOSD. Some of these candidate aspects of the tele-operation domain are distribution, safety, mobility, security, coordination, etc.

The European project EFTCoR (Environmental Friendly and Cost-effective Technology for Coating Removal) [EFT02], in which the DSIE (System Division and Electronic 112 
Engineering) Group of the Polytechnic University of Cartagena participates, has developed the design and construction of a robotic teleoperated system. This system is a family of robots called EFTCoR [Fer05]. The EFTCoR is a robotic platform that cleans the hulls of ships in a way that reduces the environmental pollution. This robotic tele-operated system has strong requirements in terms of adaptability to different devices, operator safety, response time, dynamic reconfiguration, etc. As a result, the DSIE group decided to apply PRISMA to these kinds of systems in order to cope with these requirements. Since the EFTCoR is a family of robots that are very large (big dimensions) and very heavy (high tonnage), a complete development of a small-scale robot has been done before developing the software architecture of EFTCoR. This robot is called TeachMover [TEA06]. The TeachMover is simpler than EFTCoR, but it has the same architectural features, which, in a near future, will permit the development of the EFTCoR tele-operated system reusing the implemented components of the TeachMover robot.

\subsubsection{The TeachMover Robot}

The TeachMover robot is a robotic arm that is frequently used to teach the fundamentals of robotics (see Figure 8). This robot was specially designed for the purpose of simulating the behaviour of large and heavy industrial robots.

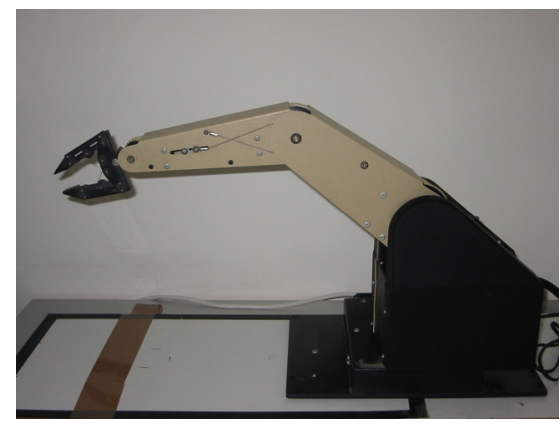

Figure 8. The TeachMover Robot

\subsubsection{The morphology of the TeachMover Robot}

The TeachMover is formed by a set of joints that permit the movement of the robot. These joints are: Base, Shoulder, Elbow and Wrist. In addition, it has a Tool to perform different tasks (see Figure 9). The movements that the robot is able to perform are: the rotation of the robot 
PRISMA: Aspect-Oriented Software Architectures

using the base, the articulation of the elbow and shoulder joints, and the rotation of the wrist. In this case, the Tool is a gripper, whose open and close actions allow the robot to pick up and deposit objects.

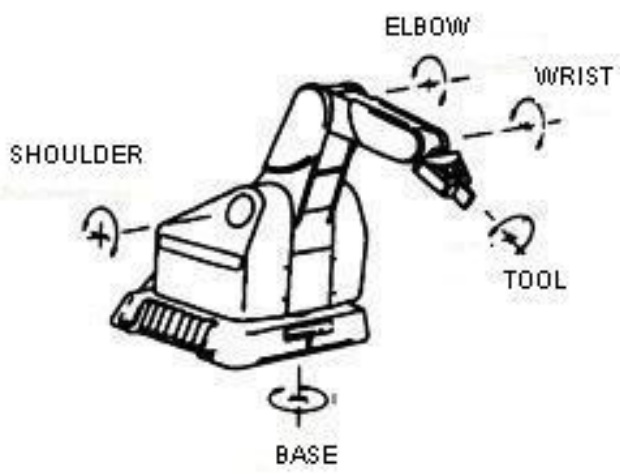

Figure 9. Joints of the TeachMover robot

The robot has six electric step motors for driving the direction of the movements of each joint. These motors perform the movements through gears that are joined by a cable system. The TeachMover can be moved at a specific speed by means of half-steps or inverse cinematics. A half-step movement moves the robot using the number of teeth that a gear of a joint must be moved as a measure. And, an inverse cinematic movement moves the robot using a specific point in the space as a measure. These features, together with the features of the gripper, allow the robot to move objects from an initial position to a final one.

In addition, safety directives of the robot require its movements to be checked to make sure that they are safe for the robot and the environment that surrounds it. The internal safety of the robot is preserved by establishing a set of constraints that forbid certain movements that will break the gears of the robot due to the position of the gears inside the robot. These constraints are defined by establishing minimum and maximum values for the movements of each joint. These values are specified in degrees as follows:

$>$ Base: $\pm 90^{\circ}$

$>$ Shoulder: $+144^{\circ},-35^{\circ}$

$>$ Elbow: $+0^{\circ},-149^{\circ}$ 
Gradient of the Wrist: $\pm 90^{\circ}$

$>$ Rotation of the Wrist: $\pm 180^{\circ}$

$>$ Opening of the gripper: 0 inches, +3 inches $(7,62 \mathrm{~cm}$.)

The robot has a sensor to pick up objects without breaking them. The weight of the objects that the robot is able to carry when its arm is stretched out is 450 grammes. Furthermore, the gripper presses the objects with a maximum pressure of 14 Newtons. Finally, the speed of movements fluctuates between 0 or 7 inches per second $(178 \mathrm{~mm} / \mathrm{s})$ depending on the load that the robot carries when the movement is performed.

It is important to mention that the movements of the robot are commanded by an operator from a computer. This communication between the computer and the robot is possible by means of the serial/RS232C port. In order to stop the robot in situations of emergency, the robot has an interruption mechanism for disconnecting the power of the robot by means of software. This is possible because this interruption mechanism is connected to the parallel port of the computer.

All these features allow the TeachMover robot to simulate the movements of most of the industrial tele-operated robots that are currently in use. This robot allows the testing and verification of new solutions to be applied to more complex robotic systems in the future.

\subsubsection{The Software Architecture of the TeachMover Robot}

The TeachMover architecture has different levels of abstraction for its components, connectors and the interactions among them. The lowest abstraction level of the robot architecture has sensors and actuators as basic components, which are communicated with the hardware joints of the robot. The functionality of the actuators and sensors are the following:

Actuator: An actuator sends commands to a joint of the robot. These commands are performed by the joint or the tool.

Sensor: A sensor reads the results of the commands in order to know whether or not they have been performed successfully. 
PRISMA: Aspect-Oriented Software Architectures

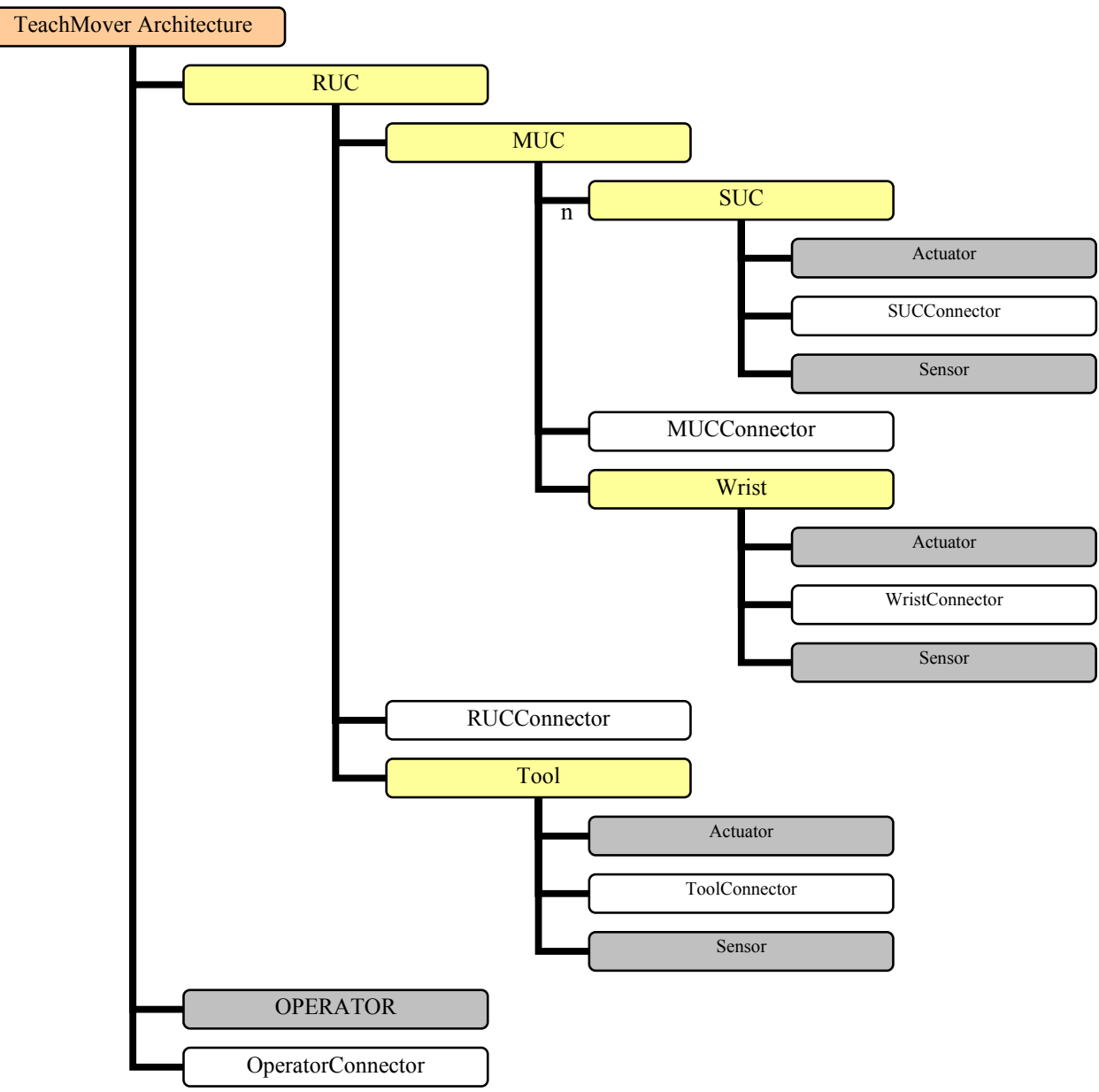

Figure 10. Architectural Elements of the TeachMover Software Architecture

An actuator and a sensor are coordinated by means of a connector. These three architectural elements (actuator, sensor, and SUCconnector) are encapsulated inside a complex component called the Simple Unit Controller (SUC) (see Figure 10). However, two special SUCs have been identified in order to take into account the peculiarities of the wrist joint and the tool. The SUC, the Wrist SUC and the Tool SUC must be composed and coordinated in order to form the complete structure and functionality of a tele-operated robot. This composition generates different levels of granularity (see Figure 10): 
Dechanism Unit Controllers (MUCs): This architectural element type represents the arm of the robot, which is composed of the SUC and Wrist SUC coordinated by means of a connector.

> Robot Unit Controllers (RUCs): This architectural element represents the robot, which is composed of MUCs and Tool SUCs coordinated by means of a connector.

$>$ The Architectural Model: This level represents the interactions between operators and robots through a connector.

\subsection{FORMALISMS}

Since in this thesis architectural elements are observable processes that have state and behaviour, the formalisms that are used to formalize the PRISMA model are a Modal Logic of Actions [Sti92] and $\pi$-calculus algebra [Mil93]. $\pi$-calculus is used to specify and formalize the processes of the PRISMA model, and the Modal Logic of Action is used to formalize how the execution of these processes affects the state of architectural elements.

\subsubsection{Modal Logic of Actions}

A variant of the Modal Logic of Actions presented in [Sti92] is used in this thesis. In addition, this Modal Logic of Actions has been used for implementing obligations, prohibitions, and permissions. As a result, it permits the analysis and formulation of assertions of processes that change the execution environment. A formula of this Modal Logic of Actions is written following the structure $\psi[\mathrm{a}] \varphi . \psi$ and $\varphi$ are well-formed formulae (wff) of the first order of the logic, which characterize the state before or after the execution of the action $a$, respectively. ' [ ]' is the operator of "need", and $a$ represents an action. As a result, the meaning of the formulae that are constructed following the pattern $\psi[\mathrm{a}] \varphi$ is the following: "if $\psi$ is satisfied before the execution of $a, \varphi$ must be satisfied after the execution of $a$ ".

The kinds of formula that are used in this thesis are the following:

$>\psi \rightarrow$ a] false: a cannot be executed in those states in which $\psi$ is satisfied.

$>\psi \rightarrow[\neg]$ false: a must be executed in those states in which $\psi$ is satisfied. 
PRISMA: Aspect-Oriented Software Architectures

$\psi \rightarrow[\mathrm{a}] \varphi$ : a can be executed in those states in which $\psi$ is satisfied. If $\psi$ is satisfied before the execution of $a, \varphi$ must be satisfied after the execution of a.

In this thesis, the Modal Logic of Actions is interpreted using a Kripke structure $\left(\Omega, \omega_{0}, \rho\right)$, where $\Omega$ is a set of possible worlds, $\omega_{0}$ is the initial world, and $\rho$ is the relationship of accessibility between the worlds (see Figure 11).

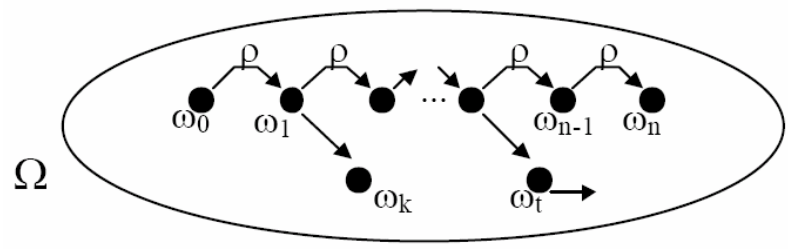

Figure 11. Simple Kripke structure

First-order formulae are valid in one world whereas modal logic of actions formulae are valid in more than one world. This is due to the fact that they are formulae that describe changes of state. As a result, the Modal Logic of Actions interpreted using a Kripke structure describes the effects that the execution of a process produces in the state.

\subsection{2. $\pi$-Calculus}

The formalism that is used to specify and formalize the processes of the PRISMA model is $\pi$ calculus [Mil93]. It has been selected because it is a model to specify concurrent processes. In addition, it is an advance over the Calculus of Communicating Systems (CCS) [Mil80] due to the fact that $\pi$-calculus provides mechanisms for specifying mobility and evolution of concurrent software systems. $\pi$-calculus is presented as the best candidate to describe the processes that are performed in software architectures due to the fact that the computation of architectural elements is concurrently executed, the configurations of software architectures are continuously evolving, and architectural elements must be mobile when software architectures are distributed. For this reason, PRISMA uses $\pi$-calculus to specify the protocols (processes) that architectural elements execute. $\pi$-calculus has also been used to formalize the computational semantics of PRISMA because it provides the necessary primitives to formalize 
PRISMA. As a consequence, it is not necessary to introduce a new formalism to formalize the computational semantics of PRISMA, thus facilitating the understanding of this formalization.

$\pi$-calculus is based on the notion of naming. A name can reference different entities such as an address, an identifier, a pointer, a channel, etc. Unlike object-oriented approaches, the primitive of $\pi$-calculus is the naming of channels, and not the naming of agents. This is a notable difference because it is not possible to directly reference the name of a process from another process that wants to communicate with it. The communication between two processes is only possible if a process references the other process through the name of the channel that permits the access. Another important property is that the name of the channel should be unique because names do not have structure; in other words, it is not possible to use the dot mechanism ${ }^{1}$ as in object-oriented programming approaches to reference names.

The most widely-known variants of the $\pi$-calculus are: monadic $\pi$-calculus and polyadic $\pi$ calculus . The former only permits sending a single value through the channel (name), and the latter permits sending several values through the channel. Polyadic $\pi$-calculus is the formalism that has been chosen to specify the concurrent processes of PRISMA, since the use of polyadic $\pi$-calculus is easier and more flexible than monadic $\pi$-calculus.

\subsubsection{Main Concepts of $\pi$-calculus}

This section presents the main concepts of the original $\pi$-calculus, i.e., the main concepts of monadic $\pi$-calculus.

The primitive concept of $\pi$-calculus is the name. The other concept that exists in $\pi$-calculus is the process. Processes are built from names by the following syntax:

$$
P::=\sum_{i \in I} \pi_{i} \cdot P_{i}|P| Q|! P|(v x) P \mid 0
$$

In this definition of a process $\mathrm{P}, \mathrm{I}$ is a set of indexes and the prefix $\pi$, which appears in the $\pi . P$ expression, represents an atomic action, exactly the first action that is performed by $\pi . P$. The $\pi$ prefix can have the three forms that are presented following:

\footnotetext{
${ }^{1}$ ClassName.servicename, ClassName.attributename
} 
PRISMA: Aspect-Oriented Software Architectures

$>\mathrm{x}(\mathrm{y})$ : this prefix means that "input the name $\mathrm{y}$ along the link or channel named $\mathrm{x}$ "

$>\bar{x}(\mathrm{y})$ : this prefix means that "output the name $\mathrm{y}$ along the link or channel named $\mathrm{x}$ "

$>\tau:$ this prefix means silence. It represents an internal action

$>$ The operation $\mathrm{P} \mid \mathrm{Q}$, which appears in the definition of $\mathrm{P}$, means that the processes $\mathrm{P}$ and $\mathrm{Q}$ are concurrently executed (parallel composition). The operation !P means replication, that is, to automatically create as many copies as are necessary of the process $\mathrm{P}$ (infinite copies). The operation ( $\mathrm{vx}) \mathrm{P}$ declares a new name call $\mathrm{x}$ and its scope is restricted to the process $\mathrm{P}$. And finally, the process 0 means that it is a null process.

\subsubsection{PRISMA dialect of $\pi$-calculus}

There are several versions of the $\pi$-calculus, as well as variations of the syntax. The version of $\pi$-calculus that has been used in this thesis is not the original version of $\pi$-calculus (monadic $\pi$ calculus). In fact, monadic $\pi$-calculus is the least used version of $\pi$-calculus. This thesis uses a new version of $\pi$-calculus based on polyadic $\pi$-calculus and some of its widely used extensions [Liu95], [San01], [Bar95], [Mil99]. In addition, this version extends the standard polyadic $\pi-$ calculus by providing support for priority, which results in the addition of a new action and modifies the meaning of other actions. Table 3 presents the syntactic conventions that have been adopted in this version of $\pi$-calculus with priorities, some derived operators that have been defined in other versions, and the new actions that have been defined.

The syntax that has been adopted varies from the syntax of monadic $\pi$-calculus presented in the previous section. The main reason for this change is to facilitate the description and analysis of the specifications using a standard keyboard. This has been done to avoid symbols above letters, and symbols that can be confused with each other such as the symbol 'l' that represents the parallel composition operation and the non-deterministic selection operation.

Table 3 shows that the input and output prefixes take the form $\mathrm{x}$ ?(y) and $\mathrm{x} !(\mathrm{y})$, respectively. The input prefix $\mathrm{x}$ ?(y) defines the reception of a service request, and the output prefix $\mathrm{x}$ !(y) defines the invocation of a service or the sending of an answer when the is used interanally. Since the sequence of processes has been expressed by $\mathrm{P} \rightarrow \mathrm{Q}$, the continuation of the process 
after an input, output, or silence prefixes have also been specified using the symbol $\rightarrow$, i.e., $\mathrm{x} ?(\mathrm{y}) \rightarrow \mathrm{P}, \mathrm{x} !(\mathrm{y}) \rightarrow \mathrm{P}$ and $\tau \rightarrow \mathrm{P}$, respectively. As a result, the symbol $\rightarrow$ means the sequence of processes and the sequence of prefixes inside a process.

The original $\pi$-calculus does not have a sequence operator due to the fact that $\pi$-calculus does not provide an adequate notion of termination as CSP algebra provides [Hoa85]. However, most of high-level languages present an intuitive notion of sequential composition, and therefore something similar to this kind of construction should be described in terms of the $\pi$-calculus. As a result, it is quite common to simulate this notion of termination in $\pi$-calculus by using a termination channel as well as the notion of composition of $\pi$-calculus. Specifically, this simulation is obtained by creating processes with the convention of sending a signal through this termination channel when they finish their execution, and receiving this signal before they start their execution. This convetion is even syntactically enforced in some languages that are based on $\pi$-calculus by providing a specific operator such as Pict [Pie00]. This convention is also adopted in this thesis by providing an operator of sequential composition. This operator is introduced as a syntactic sugar in the PRISMA dialect in order to avoid the complexity that the explicit specification of this convention would introduce in the language. As a result, this operator of sequential composition is not exactly a derived operator, it is just a syntactic schema that simplifies the specification of processes.

The sequence operator is usually represented by the symbol ';' as in CSP algebra [Hoa85]. But, the PRISMA dialect of $\pi$-calculus uses the symbol ' $\rightarrow$ ' because it represents the intuitive meaning of the compositional sequence better than the symbol ';'. The arrow does not only represent the sequence graphically, but it also depicts its obligatory direction.

As a result, $\mathrm{P} \rightarrow \mathrm{Q}$ is specified using the PRISMA dialect of $\pi$-calculus, it is assumed that there are two processes are defined as follows:

$$
\begin{array}{ll}
\mathrm{P} 1(\mathrm{p})::=(\text { original definition of } P) \cdot \mathrm{p} !() & \text { for } \mathrm{p} \notin \mathbf{f n}(\mathrm{P}) \\
\mathrm{Q} 1(\mathrm{q})::=\mathrm{q} ?() \text {. (original definition of } Q) & \text { for } \mathrm{q} \notin \mathbf{f n}(\mathrm{Q})
\end{array}
$$

Finally, what it is meant by this syntactic schema is the following structure:

$$
(\mathrm{vx})(\mathrm{P} 1(\mathrm{x}) \mid \mathrm{Q} 1(\mathrm{x}))
$$


PRISMA: Aspect-Oriented Software Architectures

It must be taken into account that this is a simplified definition due to the fact that the process $\mathrm{P}$ could have several branches. This just tries to present the way in which the definition works.

\begin{tabular}{|c|c|c|}
\hline \multicolumn{2}{|l|}{$\mathrm{x}, \mathrm{y}, \mathrm{z}$} & Name ( service, process, parameter, identifier) \\
\hline \multicolumn{2}{|c|}{$->->->$} & \\
\hline \multicolumn{2}{|c|}{$x, y, z$} & Array of names where $x, y, z$ are parameters \\
\hline \multicolumn{2}{|l|}{$\mathrm{D}, \mathrm{E}, \mathrm{F}$} & Constant \\
\hline \multicolumn{2}{|l|}{$\mathrm{P}, \mathrm{Q}, \mathrm{R}$} & Process \\
\hline \multirow{3}{*}{ | } & $\mathrm{P}$ & \\
\hline & $(\mathrm{x}) \mathrm{P}$ & Abstraction \\
\hline & $\langle\mathrm{x}\rangle \mathrm{P}$ & Realization \\
\hline \multirow[t]{14}{*}{$P::=$} & 0 & Null process \\
\hline & $\mathrm{x} ?(\mathrm{y}) \rightarrow \mathrm{P}$ & Input prefix \\
\hline & $\mathrm{x} !(\mathrm{y}) \rightarrow \mathrm{P}$ & Output prefix \\
\hline & $\tau \rightarrow \mathrm{P}$ & Silence prefix \\
\hline & $(v \mathrm{x}) . \mathrm{P}$ & Restriction \\
\hline & $\mathrm{P}+\mathrm{Q}$ & Non-deterministic selection (OR) \\
\hline & $\mathrm{P}|| \mathrm{Q}$ & Parallel composition \\
\hline & $\mathrm{D}(\mathrm{x})$ & Constant application \\
\hline & $* \mathrm{P}$ & Replication \\
\hline & $|\mathrm{x}=\mathrm{y}| \mathrm{P}$ & Comparison \\
\hline & $\mathrm{P} \rightarrow \mathrm{Q}$ & Sequential composition \\
\hline & if $b$ then $P$ else $Q$ & Alternative (derived operator) \\
\hline & $\operatorname{case}(\mathrm{b} \triangleright \mathrm{P} \rightarrow \mathrm{Q})$ & Multiple alternative (derived operator) \\
\hline & (y) : $\mathrm{n}^{\mathrm{o}} \rightarrow \mathrm{P}$ & Priority \\
\hline
\end{tabular}

Table 3. Polyadic $\pi$-calculus with priorities

Since this $\pi$-calculus version is based on polyadic $\pi$-calculus, it is possible to define an array or tuple of names $x, y, z$ in the same way as polyadic $\pi$-calculus establishes. As a result, the input and output prefixes take the form $\mathrm{x} ?\left(\mathrm{y}_{1} \ldots \mathrm{y}_{\mathrm{n}}\right)$ and $\mathrm{x} !\left(\mathrm{y}_{1} \ldots \mathrm{y}_{\mathrm{n}}\right)$, respectively. 
The operation P || Q represents the parallel composition, i.e., P and Q are concurrently executed. As in other versions, this operation is represented by the symbol ' $\|$ ' instead of the symbol ' ' to avoid possible confusions with the operation OR of other languages. In contrast, the operation $\mathrm{P}+\mathrm{Q}$ represents the non-deterministic choice, i.e., either $\mathrm{P}$ is executed or $\mathrm{Q}$ is executed. In this operation, the symbol ' + ' is used instead of the symbol '|' to clearly distinguish the differences with the symbol ' $\|$ '.

$\mathrm{D}(\mathrm{x})$ and $* \mathrm{P}$ are interchangeable operators because they define processes with the same capabilities. The operation $\mathrm{D}(\mathrm{x})$ represents the instantiation of the process that represents the constant $\mathrm{D}$. The operation *P permits the definition of infinite copies of a process without executing it recursively, as is necessary with the $\mathrm{D}(\mathrm{x})$ operation. As in other versions, the replication operation is represented by the symbol '*' instead of the symbol '!' to avoid confusions with the output prefix $\mathrm{x}$ !(y).

(x)P represents an abstraction operation. This operation can also be expressed with the more explicit notation $\left(\lambda x_{1} \ldots \lambda x_{n}\right) P$, which is an abstraction of names from a process. This operation is usually used to define a parametric (abstract) definition of a process (agent). The concretion $(\langle\mathrm{x}\rangle \mathrm{P})$ is the operation that uses concrete parameters in abstract processes that have been previously defined using an abstraction operation.

The operation $|\mathrm{x}=\mathrm{y}| \mathrm{P}$ introduces the mechanism to compare two names and only executes a process when the names are equals. The comparison structures with double (if $b$ then $P$ else $\mathrm{Q}$ ) or multiple (case $(\mathrm{b} \triangleright \mathrm{P} \rightarrow \mathrm{Q})$ ) alternatives have been derived from this operation. These structures appear as derived operators of polyadic $\pi$-calculus [Mil93] and other extensions of $\pi$ calculus such as [Liu95], [San01]. These two derived structures usually substitute the original one because they are more expressive.

As mentioned above, this dialect of $\pi$-calculus extends the standard $\pi$-calculus by introducing the notion of priority. This extension consists of introducing a new action to specify priorities. This action emerges from the need to create a mechanism that establishes the priority of the execution of services that participate in a process through a non-deterministic selection. This mechanism has already been defined for process algebras, specifically for CCS [Cle01]. 
PRISMA: Aspect-Oriented Software Architectures

Priority is especially necessary in those coordination processes that must take into account emergency situations and when several invocations of services can arrive at the same time. The priority action provides the mechanism to define a partial order of the execution of services.

In this thesis, this priority operation has been introduced in $\pi$-calculus. This operation is represented by adding the level of priority after the parameters of the name. The maximum priority is 0 and the minimum is no constraint, depending on the system and the levels of priority that the user wants to define. As a result, the way of defining a reception of a service request or invocation is $\mathrm{x} ?(\mathrm{y}): \mathrm{n}^{\mathrm{o}} \rightarrow \mathrm{P}$ and $\mathrm{x} !(\mathrm{y}): \mathrm{n}^{\mathrm{o}} \rightarrow \mathrm{P}$, respectively. In the case of the TeachMover, the service stop, which stops the movements of the robot in emergency situations, has a higher priority than other services of the software system such as the service movejoint. The invocation of these services using the $\pi$-calculus with priorities is specified as follows:

stop! ():0 + moveJoint!(NewSteps, Speed):1

If a priority is not specified, the minimum priority (no constraint) is assumed. Finally, it is important to note that it is only necessary to define this extension in those systems that require different levels of priority in the execution of services, such as emergency systems.

\subsection{CONCLUSIONS}

This chapter has detailed the preliminary notions of this thesis. Robotic tele-operated systems have been chosen as application domain for the PRISMA approach, since these provide real systems that need real solutions for their development and maintenance processes. Specifically, PRISMA has been applied to the TeachMover tele-operated robot, which is used to illustrate the main concepts of PRISMA as well as its methodology.

The formalisms used in this thesis to describe and formalize the PRISMA model have been presented. The need to represent changes of state in an architectural model as a consequence of service executions has led us to use a Modal Logic of Actions to formalize this behaviour. The concurrent nature of processes that are executed in software architecture as well as the evolving and distributed nature of software architectures have led us to choose the polyadic $\pi$-calculus to describe the protocols of PRISMA and to formalize its execution model. Since complex 124 
Preliminaries

software systems sometimes need to prioritize the execution of their services, a dialect of polyadic $\pi$-calculus called polyadic $\pi$-calculus with priorities has been defined and adopted in this thesis. 
PRISMA: Aspect-Oriented Software Architectures 


\section{PART IV}

\section{PRISMA}

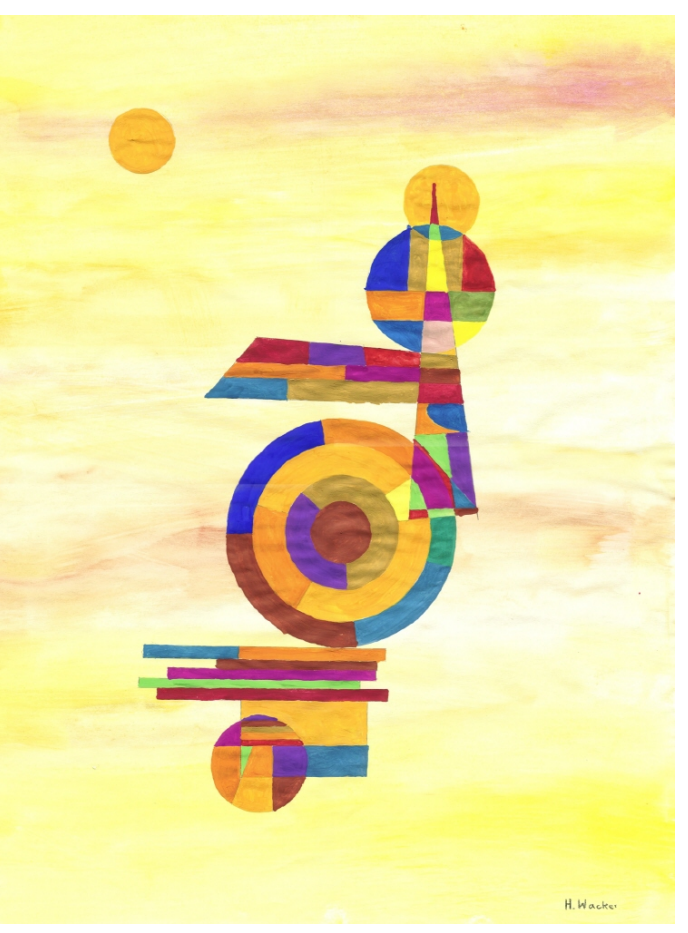

“Computer Software Architecture”, Hermann Wacker [WAC06] 



\section{CHAPTER 6}

\section{THE PRISMA MODEL}

$<<$ Before a diamond shows its brilliancy and prismatic colours

it has to stand a good deal of cutting and smoothing.>>

Anonymous Author

PRISMA provides a model for the definition of complex software systems. Its main contributions are the way in which it integrates elements from aspect-oriented software development and software architecture approaches, as well as the advantages that this integration provides to software development. The PRISMA model introduces the notion of aspect following an architectural model with connectors and a symmetrical aspect-oriented model.

The purpose of this chapter is to define, formalize and exemplify the main concepts of the PRISMA model. First a brief overview of the PRISMA model is presented and then, each one of the concepts is formalized and described in detail.

\subsection{INTRODUCTION TO THE PRISMA MODEL}

PRISMA provides a model for the description of software architectures of complex and large systems. It introduces aspects as first-order citizens of software architectures. This means that, in PRISMA, aspects are not simulated through other architectural concepts such as connectors, views or similar mechanisms as in other approaches. PRISMA creates a new concept for modeling concerns called aspects. As a result, PRISMA specifies different characteristics 
PRISMA: Aspect-Oriented Software Architectures

(distribution, safety, context-awareness, coordination, etc.) of an architectural element (component, connector) using aspects.

From the aspect-oriented point of view, PRISMA is a symmetrical model that does not distinguish a kernel or core entity to encapsulate functionality; functionality is also defined as an aspect. One concern can be specified by several aspects of a software architecture, whereas a PRISMA aspect represents a concern that crosscuts the software architecture. This crosscutting is due to the fact that the same aspect can be imported by more than one architectural element of a software architecture. In this sense, aspects crosscut those elements of the architecture that import their behaviour (see Figure 12).

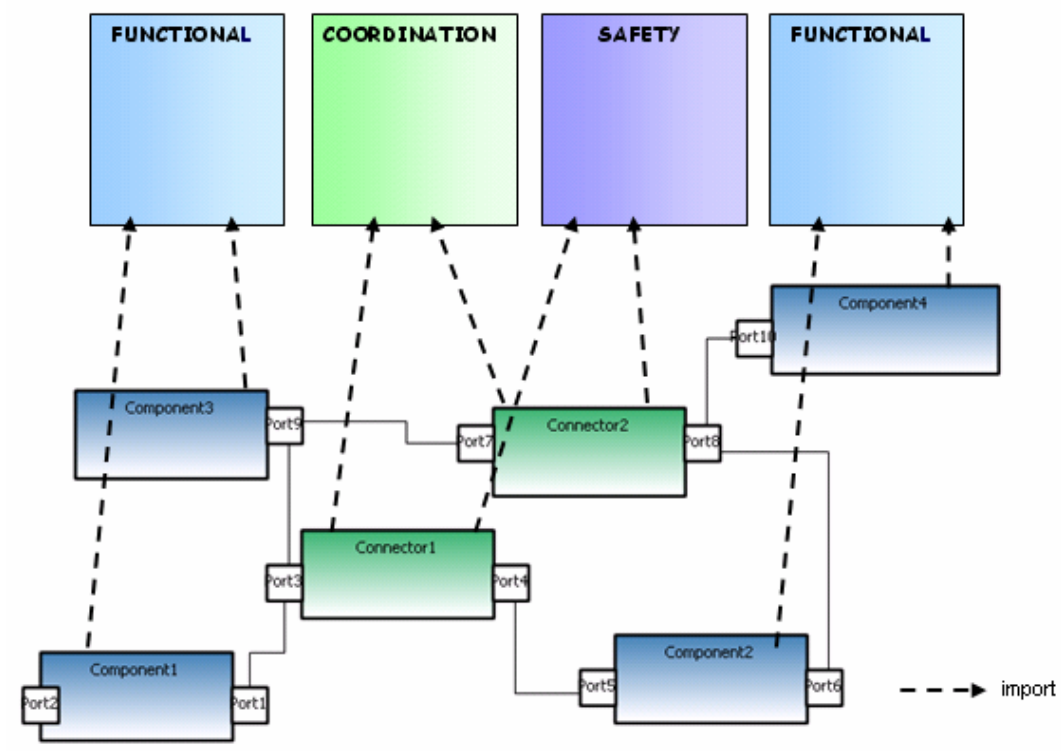

Figure 12. Crosscutting-concerns in PRISMA architectures

The fact that PRISMA is a symmetrical model is an advantage. This facilitates the construction of software architectures since the model does not manage two different concepts (class or component, and aspect) in different ways. In addition, the reusability of functional properties is independent of the architectural element that imports it because the functionality is specified as an aspect. However, if this functionality were implemented as a kernel class of the architectural element, the reuse of the functionality would only be achieved by reusing the full 
architectural element. Consequently, a more uniform model is obtained because of the homogeneity of the concepts that build an architectural element.

A PRISMA architectural element can be seen from two different views: internal and external. In the external view, architectural elements encapsulate their functionality as black boxes and publish a set of services that they offer to other architectural elements (see Figure 13). These services are grouped into interfaces to be published through the ports of architectural elements. Each port has an associated interface that contains the services that are provided and requested through the port. As a result, ports are the interaction points of architectural elements.

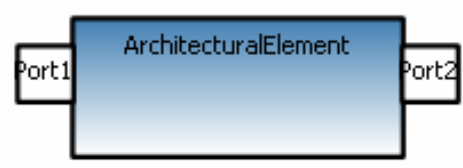

Figure 13. Black box view of an architectural element

The internal view shows an architectural element as a prism (white box view). Each side of the prism is an aspect that the architectural element imports. In this way, architectural elements are represented as a set of aspects (see Figure 14 ) and the weaving relationships among aspects.

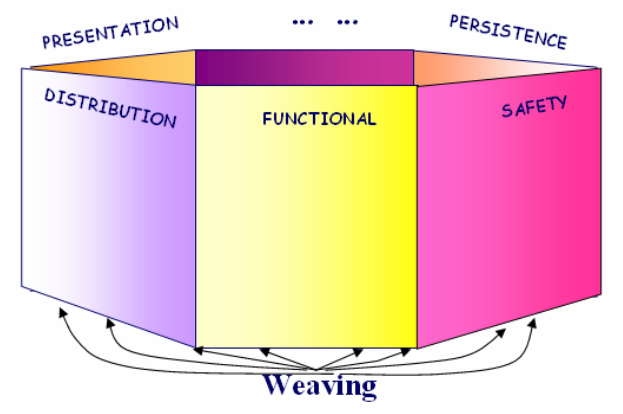

Figure 14. White box view of an architectural element

Since PRISMA is a symmetrical aspect-oriented model that it is applied at the architectural level, the weaving process does not define the pointcuts between the base code and the aspect code and their corresponding advices. In PRISMA, there is no base code; all behaviour of the 
PRISMA: Aspect-Oriented Software Architectures

system is defined as an aspect. As a result, the weaving process is composed of a set of weavings, and a weaving indicates that the execution of an aspect service can trigger the execution of services in other aspects. From the AOP point of view PRISMA weavings can be defined as follows: every service of an aspect is a join point, the services that trigger a weaving are the pointcuts, and the services that are executed as a consequence of weavings are the advices. In PRISMA, in order to preserve the independence of the aspect specification from other aspects and weavings, weavings are specified outside aspects and inside architectural elements. As a result, aspects are reusable and independent of the context of application and weavings weave the different aspects that form an architectural element. This way of specifying weavings achieves not only the reusability of the aspects in different architectural elements, but also the flexibility of specifying different behaviours of an architectural element by importing the same aspects and defining different weavings.

The communications between the white box and black box views is possible by means of interfaces; which are associated to ports and are used by aspects (see Figure 15). Consequently, a request for a service that arrives to a port of an architectural element is processed by an aspect that uses the same interface that is used by this port.

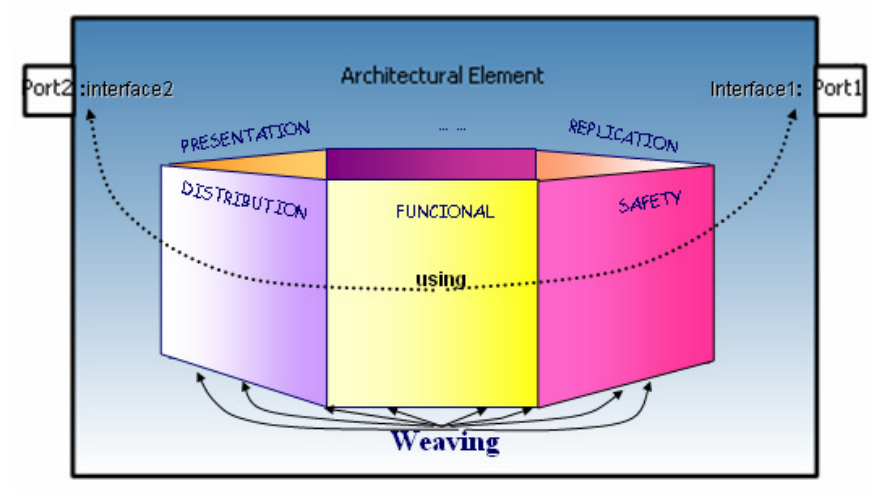

Figure 15. Communication between the white box and the black box views

PRISMA has three kinds of architectural elements: components, connectors, and systems. Components and connectors are simple, but systems are complex components. A component is an architectural element that captures the functionality of software systems and does not act as a 
coordinator among other architectural elements; whereas, a connector is an architectural element that acts as a coordinator among other architectural elements.

Connectors do not have the references of the components that they connect and vice versa. Thus, architectural elements are reusable and unaware of each other. This is possible due to the fact that the channels defined between components and connectors have their references (attachments) instead of architectural elements. Attachments are the channels that enable the communication between components and connectors. Each attachment is defined by attaching a component port with a connector port.

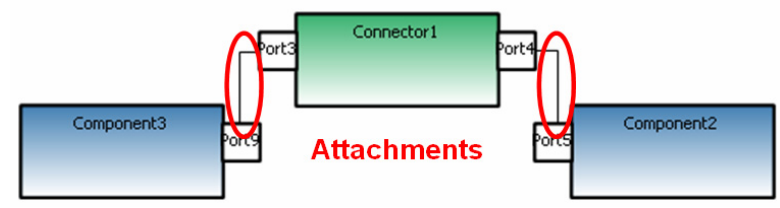

Figure 16. Attachaments

PRISMA components can be simple or complex. The complex ones are called systems. A PRISMA system is a component that includes a set of architectural elements (connectors, components and other systems) that are correctly attached. In addition, a system can have its own aspects and weavings as components and connectors. Since a system is composed by other architectural elements, the composition relationships among them must be defined. These composition relationships are called bindings. Bindings establish the connection among the ports of the complex component (the system) and the ports of the architectural elements that a system contains (see Figure 17).

In PRISMA, the dynamics of aspect-oriented architectures are treated at the meta-level. The meta-level contains the elements that define the PRISMA concepts as data. They can be created, modified and destroyed through the execution of the services of the meta-level. In this way, the execution of services is reflected in the architecture by updating this data (the concept of reflection). As a result, the PRISMA meta-level allows for the creation, destruction and evolution of architectural elements and aspects as well as the dynamic reconfiguration of software architectures. The PRISMA meta-level is represented by means of a metamodel that 
PRISMA: Aspect-Oriented Software Architectures

contains one metaclass for each PRISMA concept. These metaclasses define a set of properties and services for each concept considered in the model (see chapter 7).

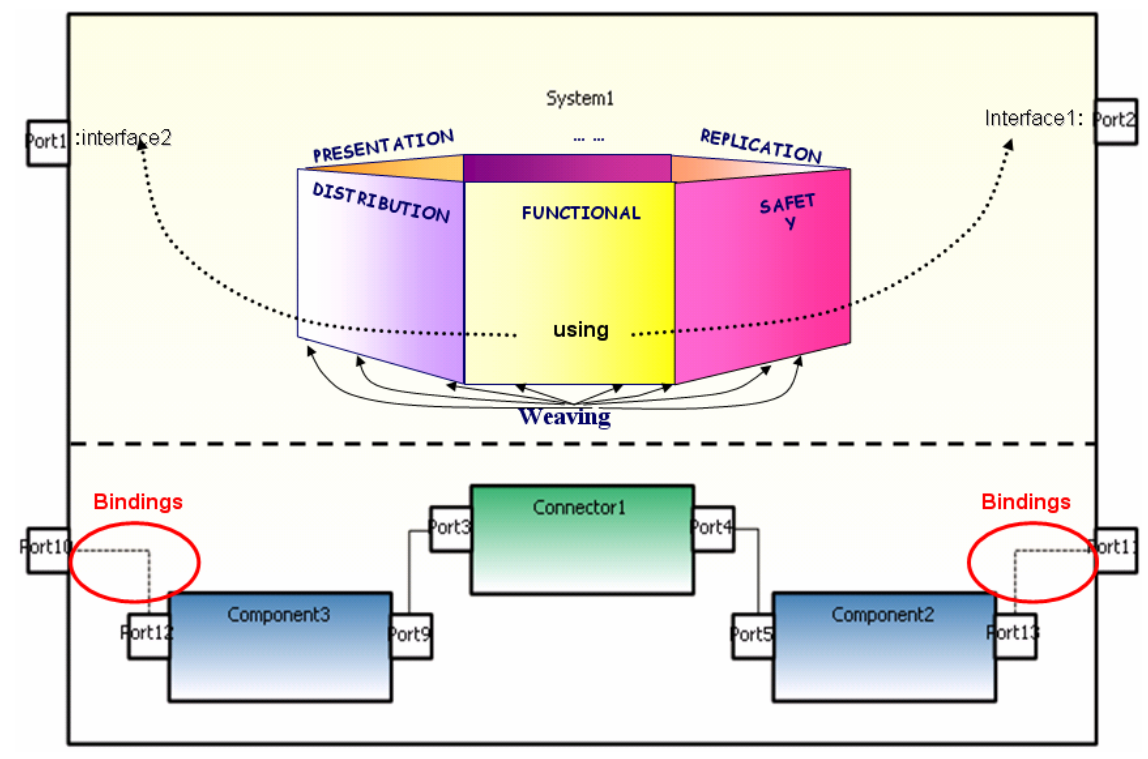

Figure 17. Systems

\subsection{PRISMA FORMALIZATION}

In this section, each one of the concepts is formalized using the Modal Logic of Actions (see section 5.2.1) to formalize the change of state and $\pi$-calculus (see section 5.2.2) to formalize the semantics of the processes and their execution.

\subsubsection{Interface}

An interface publishes a set of services. It describes the signature of the services that can be invoked or requested through that interface. The signature of a service specifies its name and parameters. The data type and the kind (input/output) of parameters are also declared.

An example in the TeachMover robot is the ImotionJoint interface that publishes the services needed to move a joint of the robot (see Figure 18 and Figure 19). This interface publishes two services and specifies their signatures: moveJoint and stop. The moveJoint service allows the movement of a joint indicating the number of half-steps and the speed at 
which the movement must be executed as input parameters. The stop service allows an emergency stop of the joint.

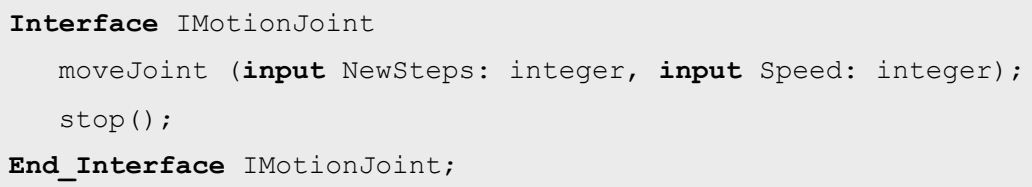

Figure 18. Specification of the interace IMotionJoint

\begin{tabular}{l}
\multicolumn{1}{c}{ IMotionJoint } \\
$\begin{array}{l}\text { move Sorvice } \\
\text { stop }\end{array}$
\end{tabular}

Figure 19. The interface IMotionJoint

\subsubsection{Service}

A service is a process that executes a set of actions to produce a result. Services can be private or public; private services are not published by any interface. In addition, they can be simple or complex. A complex service is composed of a set of services that are executed in a transactional way (all or nothing), these services are called transactions.

\section{Formalization of Services}

Let $\mathrm{S}$ be a service. The semantics of $\mathrm{S}$ is a process in the polyadic $\pi$-calculus, called $\mathrm{P}_{\mathrm{S}}$. This process has a channel $\mathrm{C}_{S}$ through which it can be invoked for execution (see Figure 20). We shall see in section 6.2 .6 that services are not invoked directly by other processes but through weavings that coordinate execution of services within architectural elements.

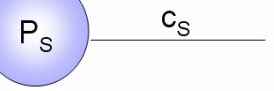

Figure 20. Formalization of a service

Invocation of services 
PRISMA: Aspect-Oriented Software Architectures

Let $\stackrel{\vec{x}}{\vec{x}}=\mathrm{x}_{1} \ldots \mathrm{x}_{\mathrm{n}}$ be the input parameters of $\mathrm{S}$ and $\vec{y}=\mathrm{y}_{1} \ldots \mathrm{y}_{\mathrm{m}}$ be its output parameters. The invocation of $\mathrm{S}$ is formalized by means of an invocation through $\mathrm{C}_{\mathrm{S}}$. In addition, each output parameter $\mathrm{y}_{\mathrm{i}}$ has a return channel $\mathrm{r}_{\mathrm{yi}}$ that is created dynamically at each invocation using the operation restriction of $\pi$-calculus $(v)$. These channels are used to transmit the results of $S$ and to acknowledge when $\mathrm{S}$ has finished executing. As a result, the invocation of a service is captured by the following process:

Service

$$
\left(v \overrightarrow{r_{y}}\right)\left(\mathrm{Cs} !\left(\vec{x}, \overrightarrow{r_{y}}\right) \rightarrow \mathrm{r}_{\mathrm{y} 1} ?(\mathrm{y} 1) \ldots \mathrm{r}_{\mathrm{ym}} ?(\mathrm{ym})\right)
$$

The process $\mathrm{P}_{\mathrm{S}}$ can be divided into the following three actions:

- Reception of requests: The first action of $\mathrm{P}_{\mathrm{S}}$ must be the reception of the messages that arrive at $\mathrm{C}_{\mathrm{S}}$. This reception is specified as follows:

$$
\text { Cs? }(\vec{x}, \vec{r} y)
$$

- Execution: The execution of $\mathrm{P}_{\mathrm{S}}$ consists of processing a set of internal actions. These internal actions create the output parameters $\left(\vec{y}=\mathrm{y}_{1} \ldots \mathrm{y}_{\mathrm{m}}\right)$ and assign them a value. This internal execution is specified as follows:

$$
\left(\begin{array}{ll}
v & y
\end{array}\right)(\tau)
$$

- End: The last action of $\mathrm{P}_{\mathrm{S}}$ consists in sending the output parameters $\left(\vec{y}=\mathrm{y}_{1} \ldots \mathrm{y}_{\mathrm{m}}\right)$ through the return channels $\left(\vec{r}_{y}=\mathrm{r}_{\mathrm{y} 1} \ldots \mathrm{r}_{\mathrm{ym}}\right)$. In this way, the fact that $\mathrm{S}$ has been executed is confirmed. This end of execution is specified as follows:

$$
r_{y} !\left(y_{1}\right) \ldots r_{y m} !\left(y_{m}\right)
$$

As a result, the complete formalization of $\mathrm{P}_{\mathrm{S}}$ is the replicated sequence of these three actions. This replication allows us to execute the service as many times as it is necessary. 
The PRISMA Model

$$
\mathrm{Ps}::={ }^{*}\left(\mathrm{Cs} ?\left(\stackrel{->}{x}, \vec{r}_{y}\right) \rightarrow\left(\begin{array}{ll}
v^{y} & ->
\end{array}\right)\left((\tau) \rightarrow r_{\mathrm{y} 1} !\left(\mathrm{y}_{1}\right) \ldots \mathrm{r}_{\mathrm{ym}} !\left(\mathrm{ym}_{\mathrm{m}}\right)\right)\right)
$$

An example in the Teach Mover robot is the moveJoint service introduced in section 6.2.1.

moveJoint (input NewSteps: integer, input Speed: integer);

As moveJoint does not have output parameters and the system does not need to know when the service has finished, it does not create new return channels in its invocation. As a result, this service can be invoked from any entity of the software architecture as follows:

moveJoint! (NewHalfSteps, Speed)

During the execution of the service, requests are received and a set of actions is performed internally. The latter is captured by the $\tau$ expression (silence action see section 5.2.2).

P movejoint $_{\text {: }}:=$ moveJoint? (NewHalfSteps, Speed) $\rightarrow \tau$

Valuations are used to specify the internal process of a service, that is, the operation $\tau$ (see section 6.2.4).

\subsubsection{Played_Role}

A played_role is a process that defines the behaviour of a specific interface. A played role establishes how and when the services of an interface can be required or provided.

\section{Formalization of Played_Roles}

Let PR be a played_role of an interface I. The semantics of PR is a process called $\mathrm{P}_{\mathrm{PR}}$ that establishes a partial order for the requests and invocations of the services of I. These invocations and requests are formalized as in section 6.2.2.

An example in the Teach Mover robot is the played_role $A C T$ for the interface IMotionJoint (see Figure 21). This played_role defines a non-deterministic selection between the invocation of stop and moveJoint. This means that when this played_role is used in a port (see 6.2.5), it restricts the behaviour of the interface to the invocation of its services. 
PRISMA: Aspect-Oriented Software Architectures

ACT for IMotionJoint = moveJoint ! (NewSteps, Speed) + stop ! ();

Figure 21. Specification of the played_role $A C T$

\subsubsection{Aspect}

An aspect defines the structure and the behaviour of a specific concern of the software system. Examples of concerns are functionality, coordination, safety, distribution, among others.

Structure is defined by a set of attributes, each of which has a value in every state. The state of the aspect at any given moment is determined by the value of its attributes. An aspect can define constraints, which must be satisfied during the execution of the aspect.

An aspect declares a number of interfaces and defines a semantics for the services that these interfaces publish. This semantics captures when the services cannot be executed, how the execution of services changes the state of the aspect, and the order in which they can be executed. This semantics is provided by preconditions, valuations and played_roles (see section 6.2.3), respectively.

An aspect can be private, that is, it does not publish any of its services and it does not define the semantics of any interface. As a result, this kind of aspect does not specify any played_role and all its services are private.

An example of a private aspect is SMotion. This safety aspect describes the behaviour that should be taken into account in order to ensure the safety of the Teach Mover robot, the safety of operators that control it, and the safety the environment that surrounds them. As it can be seen in Figure 22, this aspect does not import any interface because it is private.
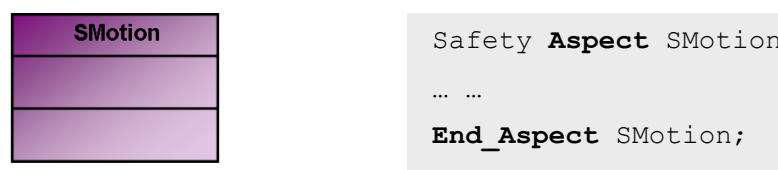

Figure 22. The private aspect SMotion

An example of a public aspect is the coordination aspect CProcessSuc (see Figure 23) that describes the behaviour required to synchronize the actuator and the sensor of a joint in order to 
move the hardware joint. This aspect uses four interfaces to provide this behaviour: IMotionJoint to request that a joint be moved, IRead to know whether the joint has moved or not, IJoint to receive requests for movements and to notify what happened with the request, and IPosition to query and update the position of the joint.
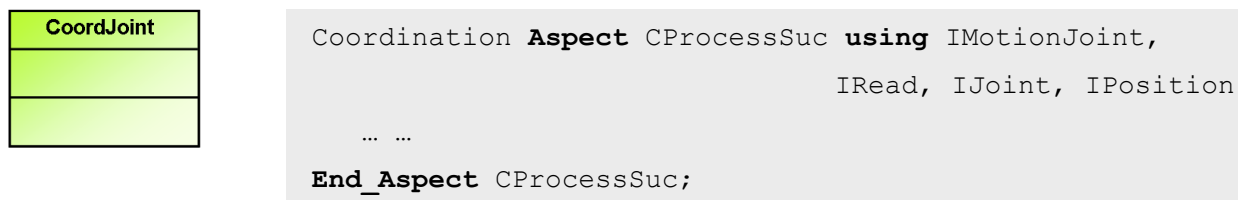

Figure 23. The public aspect CProcessSuc

The behaviour of an aspect is defined by means of a protocol. When an aspect is public, the protocol describes how the different played_roles and private services are coordinated. When an aspect is private, the protocol coordinates the different private services that are specified in the aspect.

The different concerns that have to be taken into account in a software system vary depending on the domain. Those concerns that crosscut the software system are defined as aspects. The domains in which PRISMA has been applied allow the following kinds of aspects to be considered:

$>$ Functional Aspect: A functional aspect captures the semantics of the information system by defining its attributes and behaviour.

> Coordination Aspect: A coordination aspect defines how architectural elements are synchronized while they communicate with each other.

Safety Aspect: A safety aspect specifies the safe values and strategies to apply in order to ensure the accurate and safe execution of the software system.

> Distribution Aspect: A distribution aspect specifies the features and strategies that are related to its distributed communication by means of patterns [Ali03]. A distribution aspect specifies the site where the architectural element is located and indicates when an element needs to be moved [Ali05a] 
PRISMA: Aspect-Oriented Software Architectures

D Contex-Awareness Aspect: A context-awareness aspect provides context-information and supports inspection in order to retrieve the structural properties of the provided information [Jae03].

> Mobility Aspect: A mobility aspect provides mobility services to architectural elements in order to be mobile or to move other architectural elements [Ali06], [Ali05b].

$>$ Integration Aspect: An integration aspect allows the integration of COTS components (Commercial Off-The-Shelf) in PRISMA software architectures in an abstract way.

This thesis is focused on the functional, coordination, safety, and integration aspects. They are the aspects that have been used in the TeachMover.

\section{Formalization of Aspects}

An aspect is defined by the tuple (A, X, $\Phi, \Pi)$ :

A: a set of attributes

X: a set of services (see section 6.2.2)

D: a set of formulae in modal logic of actions

$>$ П: a set of terms in $\pi$-calculus

\section{Formalization of $\Phi$}

$\Phi$ is a set of formulae in modal logic of actions. These formulae define how and when the services of an aspect (X) change the value of the attributes of the aspect (A). They are valuations, preconditions and constraints.

> Valuations: Valuations define the change of the state of an aspect when one of its services is executed. They are formalized by the following formula:

$$
\psi \rightarrow[\mathrm{a}] \varphi
$$

This means that if $\psi$ is satisfied before the execution of a, $\varphi$ must be satisfied after the execution of a. $\psi$ and $\varphi$ are wff that make reference to the states before and after the execution of a, respectively. As a result, valuations define the internal process of a service $(\tau$, see section 6.2.2).

The FJoint aspect defines a service newPosition, its valuation consists in storing the position of the joint (see Figure 24). In this valuation, the $\psi$ wff is empty because it 
is not necessary for defining the state before the execution of moveJoint. The $\varphi$ wff is defined by the expression halfSteps $:=$ halfSteps + NewSteps. $\varphi$ establishes that the attribute halfSteps will have the value of the HalfStepts and NewSteps ${ }^{2}$ addition after the moveJoint execution.

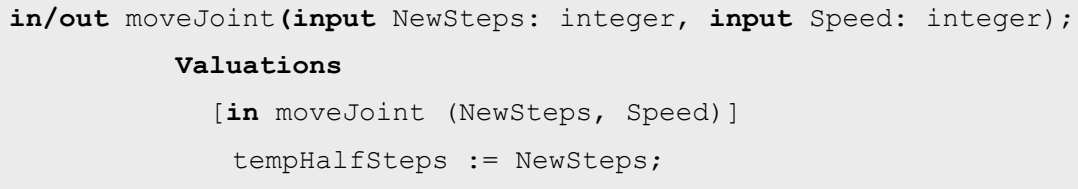

Figure 24. Specificatio of the valuation of the service moveJoint

Preconditions: Preconditions define prohibitions of the execution of actions. They are formalized by the following formula:

$\neg \psi \rightarrow[\mathrm{a}]$ false

This means that if $\psi$ is not satisfied, $a$ cannot be executed; it is prohibited. $\psi$ is a wff that expresses a condition on the state of the aspect.

In the case of the service newPosition, its precondition establishes that the service newPosition can only be executed when the attribute validMovement has the value true (see Figure 25).

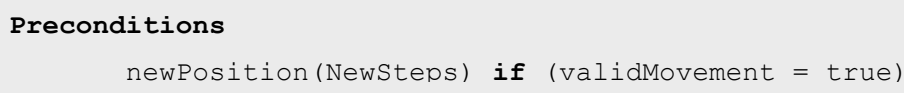

Figure 25. Specification of the precondition of the service newPosition

$>$ Constraints: Constraints are conditions that must be satisfied throughout the entire execution process of the aspect. They can be classified into static or dynamic. Static constraints make reference to one state of the aspect whereas dynamic constraints make reference to several states of the aspect. They use operators of temporal logic: always ${ }^{3}$ for

\footnotetext{
${ }^{2}$ The operator ":=" is used for the $\varphi$ formula instead of using the operator "="because it can be interpreted more intuitively.

${ }^{3}$ If there is a formula without a temporal operator, its assumed that the semantics of the formula is the semantics of the operator always.
} 
PRISMA: Aspect-Oriented Software Architectures

static constraints, and sometimes, since, next or until for dynamic constraints. They are formalized by the following formulae:

$$
\text { always } \psi \text {, sometimes } \psi, \psi \text { since } \psi^{\prime}, \psi \text { next } \psi^{\prime}, \psi \text { until } \psi \text { ' }
$$

\section{Formalization of $\Pi$}

Let $\mathrm{A}$ be a public aspect whose behaviour is specified by played_roles $\mathrm{PR}_{1} \ldots \mathrm{PR}_{\mathrm{n}}$ and the protocol PRT1. Its semantics is the process $\mathrm{P}_{\mathrm{A}}$ defined as follows:

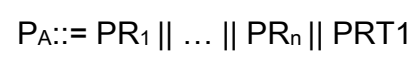

This means that the played_roles and the protocol of a public aspect are processes that are executed concurrently. For this reason, $\mathrm{P}_{\mathrm{A}}$ is defined as their parallel composition. During this concurrent execution, the protocol communicates with the played_roles and the private services of the aspect in order to synchronize them.

Let PRA be a private aspect whose behaviour is specified by the PRT1 protocol. Its semantics is the process $\mathrm{P}_{\mathrm{PRA}}$ defined as follows:

$$
\text { PPRA ::= PRT1 }
$$

This means that the process of a private aspect is its protocol. This is because, as already mentioned, a private aspect does not have played_roles and its protocol only synchronizes the private services of the aspects.

\subsubsection{Port}

Ports are the interaction points of architectural elements (components and connectors). Every port has a type, which is an interface whose behaviour is specified by a played_role for that interface. This played_role (see section 6.2.3) has to be defined in one of the aspects of the corresponding architectural element.

\section{Formalization of Ports}

Let $\mathrm{P}$ be a port of an architectural element whose behaviour is specified by the played_role PR1 (see section 6.2.3) of an aspect corresponding to the architectural element. Its semantics is the process $\mathrm{P}_{\mathrm{P}}$ defined as follows: 


$$
P_{P}::=P R 1
$$

This means that the process of a port is its played_role.

An example is the port PAct (see Figure 26). It belongs to one of the architectural elements of the TeachMover system. This port is typed by the IMotionJoint interface and the ACT played_role, which is defined in the CProcessSuc coordination aspect.

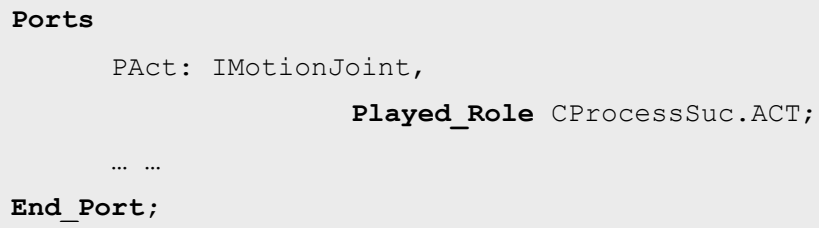

Figure 26. Specification of the port PAct

\subsubsection{Weaving}

A weaving specification defines how the execution of a service of an aspect can trigger the execution of a service of another aspect. The same service can be involved in several weavings.

In order to preserve the independence of the aspect specification from other aspects and weavings, weavings are specified outside aspects and inside architectural elements. As a result, weavings coordinate the different aspects that an architectural element imports.

A weaving is defined by means of operators that describe the order in which services are executed.

A weaving that relates service $\mathrm{s} 1$ of aspect $\mathrm{A} 1$ and service $\mathrm{s} 2$ of aspect $\mathrm{A} 2$ can be specified using the following operators:

$>\mathrm{A} 2 . \mathrm{s} 2$ after A1.s1: A2.s2 is executed after A1.s1

D A2.s2 before A1.s1: A2.s2 is executed before A1.s1

A2.s2 instead A1.s1: A2.s2 is executed in place of A1.s1

$>\mathrm{A} 2 . \mathrm{s} 2$ afterif (Boolean condition) A1.s1: A2.s2 is executed after A1.s1 if the condition is satisfied.

$>\mathrm{A} 2 . \mathrm{s} 2$ beforeif (Boolean condition) A1.s1: if the condition is satisfied, A2.s2 is executed followed by A1.s1; otherwise, only A2.s2 is executed. 
PRISMA: Aspect-Oriented Software Architectures

A2.s2 insteadif (Boolean condition) A1.s1: A2.s2 is executed in place of A1.s1 if the condition is satisfied.

The invocation of A1.s1, the second argument of the weaving, triggers the execution of weaving (pointcut). When a weaving is specified, the operator is chosen from the trigger service point of view; depending on whether the trigger service needs the execution of a service before, after, or instead of it (advice).

\section{Formalization of Weavings}

The semantics of a weaving is a process that intercepts the invocation of a service A1.s1 and either replaces it with, or executes it in relation to, another service A2.s2. A1.s1 and A2.s2 belong to different aspects.

The weaving must be executed each time that A1.s1 is invoked, upon which it executes either A2.s2 instead of A1.s1 or A1.s1 and A2.s2 in the correct order. This means that the invocation of a service does not automatically trigger the execution of its associated process. In terms of our formalization in $\pi$-calculus, and given a service $S$ that the weaving is controlling, the weaving process $P_{W}$ interacts with $P_{S}$ via the channel $C_{S}$ defined in 6.2.2 and provides a channel $\mathrm{CW}_{\mathrm{S}}$ that other processes can use to invoke $\mathrm{S}$ (see Figure 27).

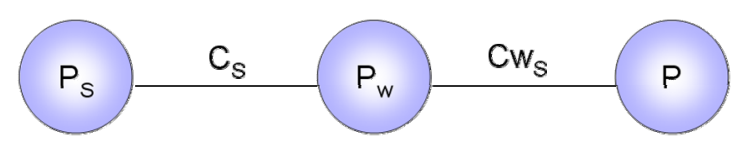

Figure 27. Formalization of a service controlled by a weaving

Taking into account these two channels, the invocation of $\mathrm{S}$ by the weaving process is as follows:

$$
\left(v \overrightarrow{r_{y}}\right)\left(\mathrm{Cs}_{\mathrm{s}} !\left(\vec{x}, \overrightarrow{r_{y}}\right) \rightarrow \mathrm{r}_{\mathrm{y} 1} ?\left(\mathrm{y}_{1}\right) \ldots \mathrm{r}_{\mathrm{ym}} ?\left(\mathrm{y}_{\mathrm{m}}\right)\right)
$$

The invocation of $\mathrm{S}$ by other processes is as follows:

$$
\left(\begin{array}{ll}
v & \vec{r}_{y}
\end{array}\right)\left(\mathrm{CWs} !\left(\vec{x}, \overrightarrow{r_{y}}\right) \rightarrow \mathrm{r}_{\mathrm{y} 1} ?\left(\mathrm{y}_{1}\right) \ldots \mathrm{r}_{\mathrm{ym}} ?\left(\mathrm{y}_{\mathrm{m}}\right)\right)
$$


Each weaving operator defines a process with a specific behaviour. The processes associated with the operators are specified as follows:

\section{A2.s2 after A1.s1}

Let AW be $\mathrm{A} 2 . \mathrm{s} 2$ after $\mathrm{A} 1$.s1. Its semantics is the process $\mathrm{P}_{\mathrm{AW}}$ defined as follows:

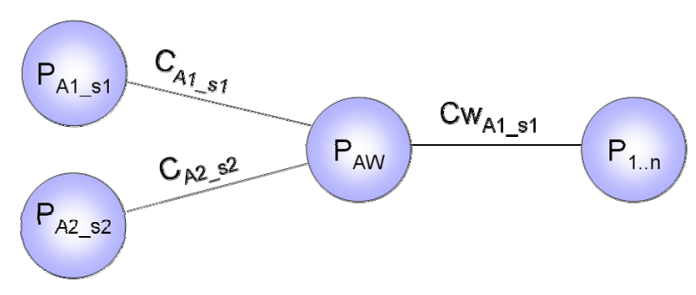

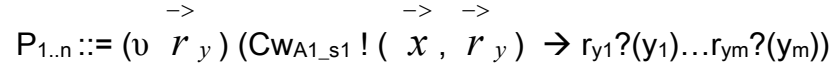

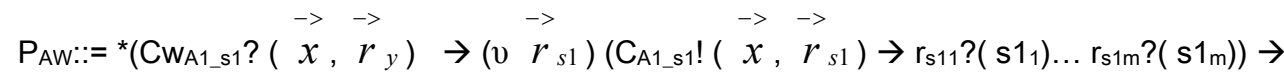

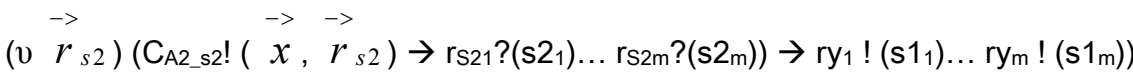

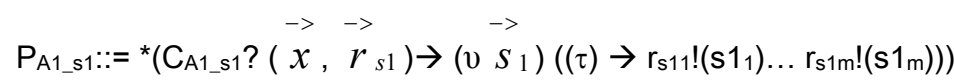

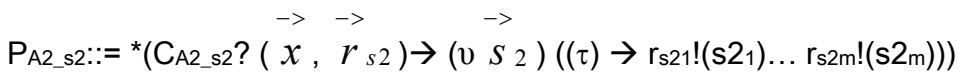

This means that $\mathrm{P}_{\mathrm{AW}}$ receives the invocation of $\mathrm{A} 1 . \mathrm{s} 1$ from another process $\left(\mathrm{P}_{1 . \mathrm{n}}\right)$ through $\mathrm{Cw}_{\mathrm{A} 1 \_\mathrm{s} 1}$. Because $\mathrm{AW}$ is an "after" weaving, $\mathrm{P}_{\mathrm{AW}}$ starts by invoking A1.s1 using $\mathrm{C}_{\mathrm{A} 1 \_s 1}$. Then, $\mathrm{P}_{\mathrm{Al} \_\mathrm{s} 1}$ receives the invocation, executes a set of internal actions, sends the results, and notifies the weaving that execution has finished. Next, the second service of the weaving is executed: $\mathrm{P}_{\mathrm{AW}}$ invokes $\mathrm{A} 2 . \mathrm{s} 2$ using $\mathrm{C}_{\mathrm{A} 2 \_22}$, and $\mathrm{P}_{\mathrm{A} 2 \_2}$ receives the invocation upon which it executes a set of internal actions, sends the results, and notifies the weaving that execution has finished. Finally, $\mathrm{P}_{\mathrm{AW}}$ sends the results to the process that invoked A1.s1. 
PRISMA: Aspect-Oriented Software Architectures

\section{A2.s2 before A1.s1}

Let $\mathrm{BW}$ be $\mathrm{A} 2 . \mathrm{s} 2$ before $\mathrm{A} 1 . \mathrm{s} 1$. Its semantics is the process $\mathrm{P}_{\mathrm{BW}}$ defined as follows:

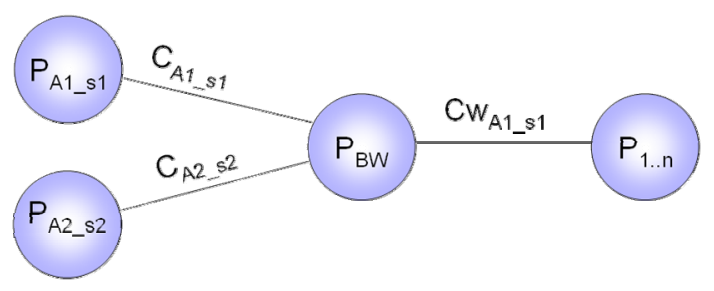

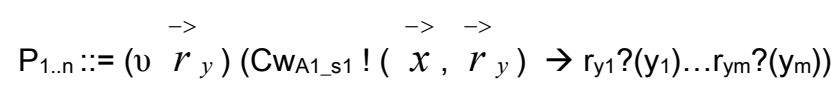

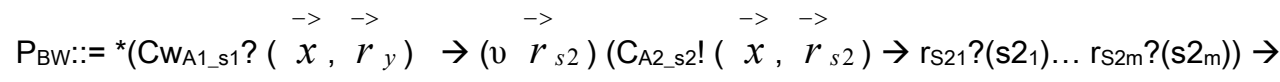

$$
\begin{aligned}
& \left.\left(v \vec{r}_{s 1}\right)\left(\mathrm{C}_{\mathrm{A} 1 \_s 1} !\left(\stackrel{->}{x}, \vec{r}_{s 1}\right) \rightarrow \mathrm{r}_{\mathrm{s} 11} ?\left(\mathrm{~s} 1_{1}\right) \ldots \mathrm{r}_{\mathrm{s} 1 \mathrm{~m}} ?\left(\mathrm{~s} 1_{\mathrm{m}}\right)\right) \rightarrow \mathrm{ry}_{1} !\left(\mathrm{s} 1_{1}\right) \ldots \mathrm{rym} !\left(\mathrm{s} 1_{\mathrm{m}}\right)\right)
\end{aligned}
$$

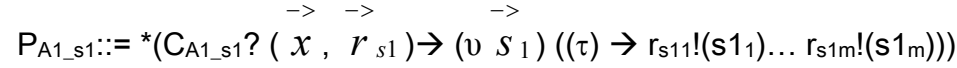

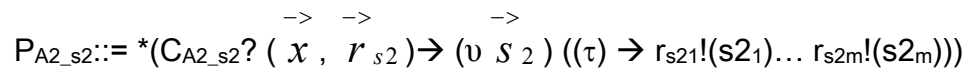

This means that $\mathrm{P}_{\mathrm{BW}}$ receives the invocation of $\mathrm{A} 1 . \mathrm{s} 1$ from another process $\left(\mathrm{P}_{1 . \mathrm{n}}\right)$ through $\mathrm{Cw}_{\mathrm{A} 1 \_\mathrm{s} 1}$. Because $\mathrm{BW}$ is a "before" weaving, $\mathrm{P}_{\mathrm{BW}}$ starts by invoking $\mathrm{A} 2 . \mathrm{s} 2$ using $\mathrm{C}_{\mathrm{A} 2 \_s 2}$. Then, $\mathrm{P}_{\mathrm{A} 2 \_2}$ receives the invocation, executes a set of internal actions, sends the results and notifies the weaving that execution has finished. Next, $\mathrm{P}_{\mathrm{BW}}$ invokes A1.s1 using $\mathrm{C}_{\mathrm{A} 1 \_\mathrm{s}}$, and $\mathrm{P}_{\mathrm{A} 1 \_\mathrm{s}}$ receives the invocation upon which it executes a set of internal actions, sends the results, and notifies the weaving that the execution has finished. Finally, $\mathrm{P}_{\mathrm{BW}}$ sends the results to the process that invoked A1.s1. 


\section{A2.s2 instead A1.s1}

Let IW be A2.s2 instead A1.s1. Its semantics is the process $\mathrm{P}_{\mathrm{IW}}$ defined as follows:

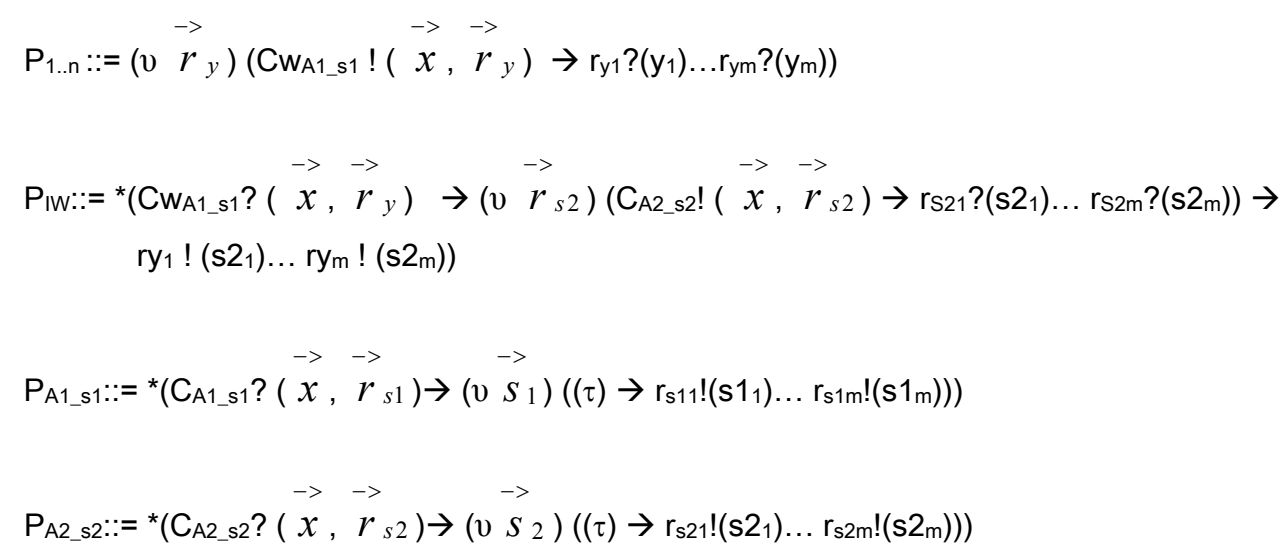

This means that $\mathrm{P}_{\mathrm{IW}}$ receives the invocation of A1.s1 from another process $\left(\mathrm{P}_{1 . \mathrm{n}}\right)$ through $\mathrm{CW}_{\mathrm{A} 1 \_ \text {s1 }}$. Because IW is an "instead" weaving, $\mathrm{P}_{\mathrm{IW}}$ invokes A2.s2 instead of A1.s1, $\mathrm{P}_{\mathrm{IW}}$ invokes $\mathrm{A} 2 . \mathrm{s} 2$ using $\mathrm{C}_{\mathrm{A} 2 \_22}$. Next, $\mathrm{P}_{\mathrm{A} \_\_2}$ receives the invocation, executes a set of internal actions, sends the results, and notifies the weaving that execution has finished. Finally, $\mathrm{P}_{\mathrm{IW}}$ sends the results to the process that invoked A1.s1.

\section{A2.s2 afterif (boolean_condition) A1.s1}

Let AWIF be A2.s2 afterif (Boolean_condition) A1.s1. Its semantics is the process $\mathrm{P}_{\mathrm{AWIF}}$ defined as follows: 
PRISMA: Aspect-Oriented Software Architectures

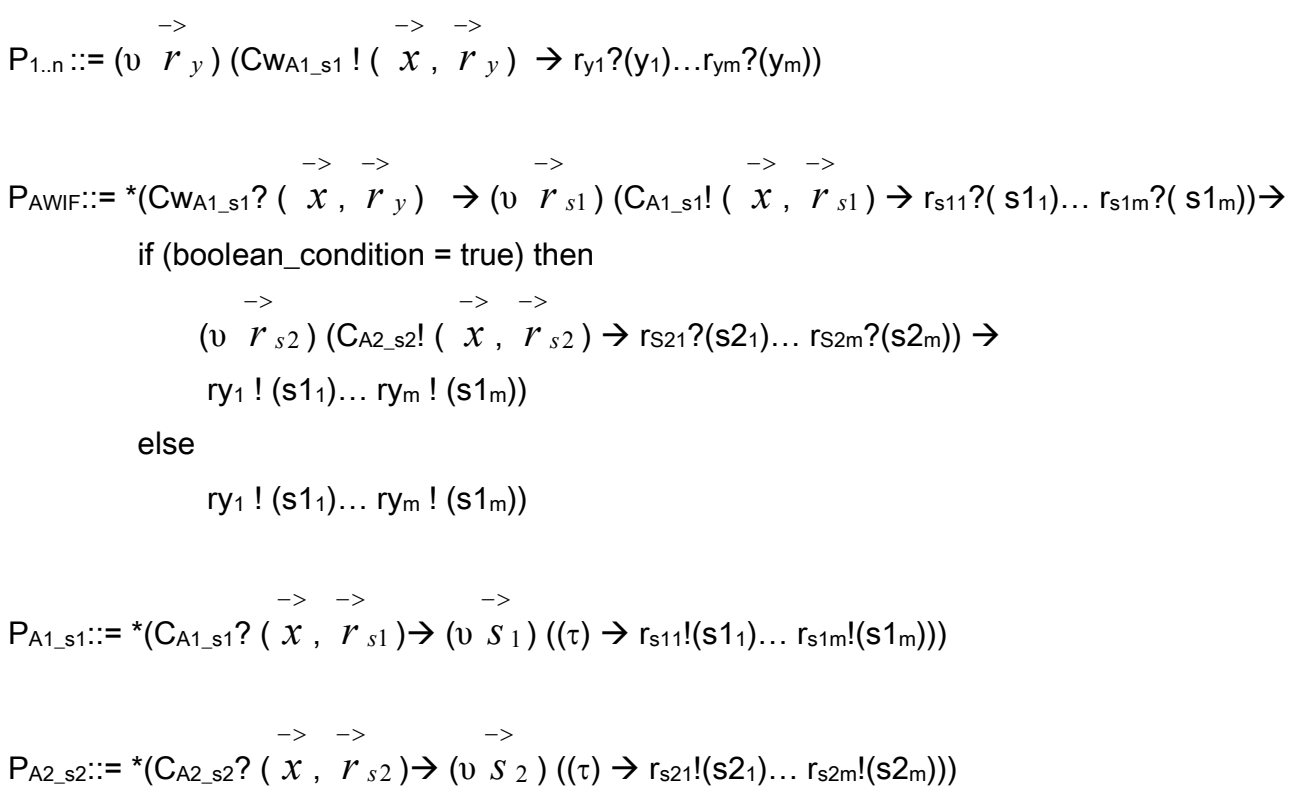

This means that $\mathrm{P}_{\mathrm{AWIF}}$ receives the invocation of $\mathrm{A} 1 . \mathrm{s} 1$ from another process $\left(\mathrm{P}_{1 . n}\right)$ through $\mathrm{CW}_{\mathrm{A} 1 \_\mathrm{s} 1}$. Because $\mathrm{AWIF}$ is an "after" weaving, $\mathrm{P}_{\mathrm{AWIF}}$ starts by invoking $\mathrm{A} 1 . \mathrm{s} 1$ using $\mathrm{C}_{\mathrm{A} 1 \_\mathrm{s} 1}$.

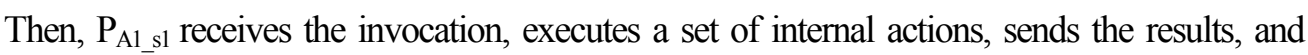
notifies the weaving that execution has finished. Since, the AWIF is a conditional weaving, it has an associated condition. Next, if the boolean condition of the AWIF is true, the second service of the weaving is executed; otherwise $\mathrm{P}_{\mathrm{AWIF}}$ sends the results to the process that invoked A1.s1. In the first case, when the condition is satisfied, $\mathrm{P}_{\mathrm{AWIF}}$ invokes $\mathrm{A} 2 . \mathrm{s} 2$ using $\mathrm{C}_{\mathrm{A} 2 \_\mathrm{s} 2}$ and $\mathrm{P}_{\mathrm{A} 2 \mathrm{~s} 2}$ receives the invocation upon which it executes a set of internal actions, sends the results, 
and notifies the weaving that execution has finished. Finally, $\mathrm{P}_{\mathrm{AWIF}}$ sends the results to the process that invoked A1.s1.

\section{A2.s2 beforeif (boolean_condition) A1.s1}

Let BWIF be A2.s2 beforeif (Boolean_condition) A1.s1. Its semantics is the process $\mathrm{P}_{\mathrm{BWIF}}$ defined as follows:

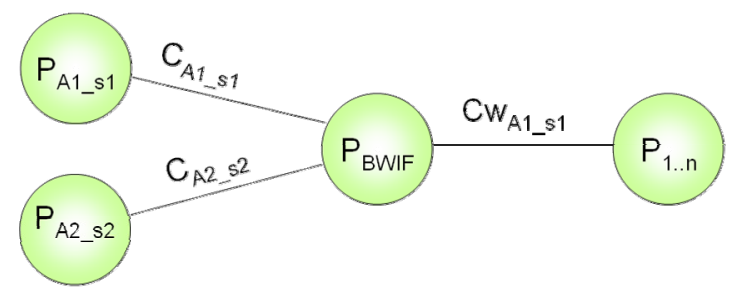

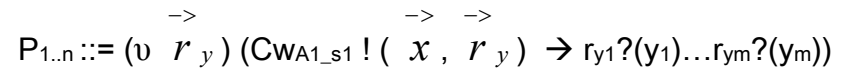

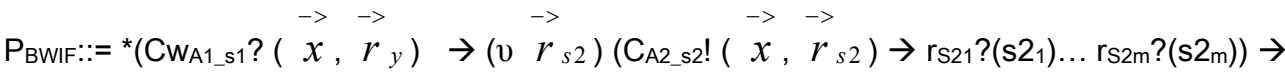

$$
\begin{aligned}
& \text { if (boolean_condition }=\text { true) then } \\
& \left.\left(\begin{array}{ll}
\left(\begin{array}{r}
r_{s 1}
\end{array}\right)\left(\mathrm{C}_{\mathrm{A} 1 \_s 1} !(\vec{x}, \vec{r}\right. \\
s 1
\end{array}\right) \rightarrow \mathrm{r}_{\mathrm{s} 11} ?\left(\mathrm{~s} 1_{1}\right) \ldots \mathrm{r}_{\mathrm{s} 1 \mathrm{~m}} ?\left(\mathrm{~s} 1_{\mathrm{m}}\right)\right) \rightarrow \\
& \mathrm{ry}_{1} !\left(\mathrm{s} 1_{1}\right) \ldots \mathrm{rym} \text { ! (s1m)) } \\
& \text { else } \\
& \left.r y 1 !\left(s 2_{1}\right) \ldots r y m !\left(s 2_{m}\right)\right) \\
& \mathrm{P}_{\mathrm{A} 1 \_s 1}::={ }^{*}\left(\mathrm{C}_{\mathrm{A} 1 \_s 1} ?\left(\vec{x}, \overrightarrow{r_{s 1}}\right) \rightarrow\left(v \overrightarrow{\mathrm{s}_{1}}\right)\left((\tau) \rightarrow \mathrm{r}_{\mathrm{s} 11} !\left(\mathrm{s} 1_{1}\right) \ldots \mathrm{r}_{\mathrm{s} 1 \mathrm{~m}} !\left(\mathrm{s} 1_{\mathrm{m}}\right)\right)\right)
\end{aligned}
$$

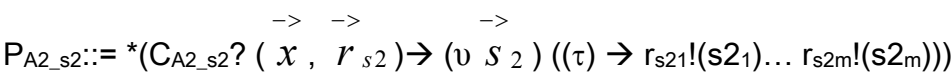

This means that $\mathrm{P}_{\mathrm{BWIF}}$ receives the invocation of $\mathrm{A} 1 . \mathrm{s} 1$ from another process $\left(\mathrm{P}_{1 . \mathrm{n}}\right)$ through $\mathrm{Cw}_{\mathrm{A} 1 \_\mathrm{s} 1}$. Because BWIF is a "before" weaving, $\mathrm{P}_{\mathrm{BWIF}}$ starts by invoking $\mathrm{A} 2 . \mathrm{s} 2$ using $\mathrm{C}_{\mathrm{A} 2 \_s 2}$. Then, $\mathrm{P}_{\mathrm{A} 2 \_2}$ receives the invocation, executes a set of internal actions, sends the results, and notifies the weaving that execution has finished. Next, if the boolean condition of the BWIF is 
PRISMA: Aspect-Oriented Software Architectures

true, the first service of the weaving is executed; otherwise $\mathrm{P}_{\mathrm{BWIF}}$ sends the results of $\mathrm{A} 2 . \mathrm{s} 2$ to the process that invoked A1.s1. In the first case, when the condition is satisfied, $\mathrm{P}_{\mathrm{BWIF}}$ invokes A1.s1 using $\mathrm{C}_{\mathrm{A} 1 \_1}$ and $\mathrm{P}_{\mathrm{A} 1 \_1}$ receives the invocation upon which it executes a set of internal actions, sends the results, and notifies the weaving that the execution has finished. Finally, $\mathrm{P}_{\mathrm{BWIF}}$ sends the results of A1.s1 to the process that invoked A1.s1.

\section{$>$ A2.s2 insteadif (boolean_condition) A1.s1}

Let IWIF be A2.s2 insteadif (Boolean_condition) A1.s1. Its semantics is the process $\mathrm{P}_{\mathrm{IWIF}}$ defined as follows.

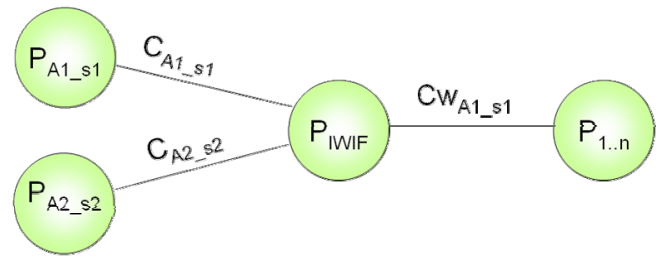

$$
\begin{aligned}
& \mathrm{P}_{1 . . \mathrm{n}}::=\left(\begin{array}{ll}
v & -> \\
r_{y}
\end{array}\right)\left(\mathrm{CW}_{\mathrm{A} 1 \_\mathrm{s} 1} !(\stackrel{->}{x}, \vec{r} y) \rightarrow \mathrm{r}_{\mathrm{y} 1} ?\left(\mathrm{y}_{1}\right) \ldots \mathrm{r}_{\mathrm{ym}} ?\left(\mathrm{y}_{\mathrm{m}}\right)\right) \\
& \text { PIWIF:: }={ }^{*}\left(\mathrm{CW}_{\mathrm{A} 1 \_\mathrm{s} 1} ?\left(\vec{x}, \overrightarrow{r_{y}}\right) \rightarrow\right. \\
& \text { if (boolean_condition }=\text { true) then } \\
& \left(\begin{array}{ll}
v & r_{s 2}
\end{array}\right)\left(\mathrm{C}_{\mathrm{A} 2 \_s 2} !\left(\stackrel{->}{x}, \vec{r}_{s 2}\right) \rightarrow \mathrm{rs} 21 ?(\mathrm{~s} 21) \ldots \mathrm{rs} 2 \mathrm{~m} ?(\mathrm{~s} 2 \mathrm{~m})\right) \rightarrow \\
& \left.r y_{1} !\left(s 2_{1}\right) \ldots r y m !\left(s 2_{m}\right)\right)
\end{aligned}
$$

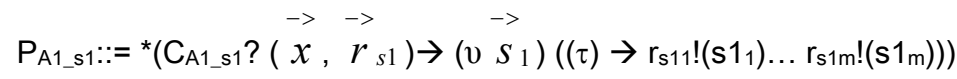

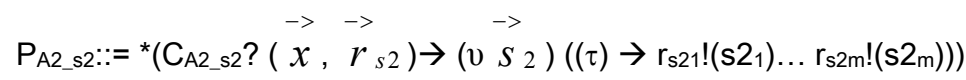


This means that $\mathrm{P}_{\text {IWIF }}$ receives the invocation of $\mathrm{A} 1 . \mathrm{s} 1$ from another process $\left(\mathrm{P}_{1 . \mathrm{n}}\right)$ through $\mathrm{Cw}_{\mathrm{A} 1 \_\mathrm{s} 1}$. Because IWIF is a conditional "instead" weaving, if the boolean condition is true, $\mathrm{P}_{\mathrm{IWIF}}$ invokes A2.s2 instead of A1.s1; otherwise $\mathrm{P}_{\mathrm{IWIF}}$ invokes A1.s1. In the first case, $\mathrm{P}_{\mathrm{IWIF}}$ invokes $\mathrm{A} 2 . \mathrm{s} 2$ using $\mathrm{C}_{\mathrm{A} \_\_2}$. Next, $\mathrm{P}_{\mathrm{A} 2 \_22}$ receives the invocation, executes a set of internal actions, sends the results, and notifies the weaving that execution has finished. Finally, $\mathrm{P}_{\mathrm{AW}}$ sends the results of A2s2 to the process that invoked A1.s1. In the second case, $\mathrm{P}_{\mathrm{IWIF}}$ invokes A1.s1 using $\mathrm{C}_{\mathrm{A} 1 \_\mathrm{s} 1}$. Then, $\mathrm{P}_{\mathrm{A} 1 \_1}$ receives the invocation, executes a set of internal actions, sends the results, and notifies the weaving that execution has finished. Finally, $\mathrm{P}_{\mathrm{IWIF}}$ sends the results of A1_s1 to the process that invoked A1.s1.

The semantics of a set of weavings defined inside an architectural element is the $\mathrm{P}_{\mathrm{W}}$ process:

$$
\begin{aligned}
& P_{W}::=P_{A W 1}\|\ldots\| P_{A W n}\left\|P_{B W 1}\right\| \ldots\left\|P_{B W n}\right\| P_{I W 1}\left\|\ldots .|| P_{I W n}\right\| P_{A W I F 1} \| \ldots .|| P_{\text {AWIFn }}
\end{aligned}
$$

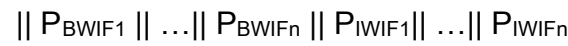

This means that the weavings are executed concurrently, interacting as specified. In addition, the same service can be involved in several weavings of the same architectural element and there is an order for processing the different weavings that a service triggers. This order establishes that weavings are executed from more restrictive to less restrictive. The order is as follows: InsteadIf, Instead, Beforelf, Before, After, Afterlf. The interblocks and infinite loops that could appear using these operators are avoided at the specification time.

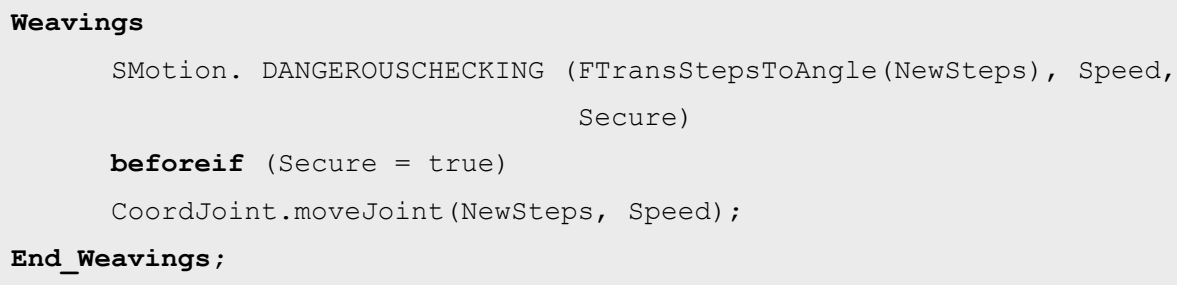

Figure 28. Specification of a weaving between moveJoint and DANGEROUSCHECKING

An example of a weaving appears in the connector that synchronizes the actuator and the sensor of a joint in order to move the hardware joint. This connector imports the SMotion safety 
PRISMA: Aspect-Oriented Software Architectures

aspect and the CProcessSUC coordination aspect presented in 6.2.4. The need for a weaving emerges due to the fact that a joint is moved only after the connector is sure that a movement is safe. The invocation of the moveJoint service (the second argument of the weaving) of CProcessSUC triggers the execution of the weaving (see section 6.2.2). The weaving of the connector implies that the DANGEROUSCHECKING service of SMotion will be executed before the moveJoint service of CProcessSUC. The condition also establishes that the execution of moveJoint must only be performed if the Secure parameter of DANGEROUSCHECKING returns true (see Figure 28).

\subsubsection{Architectural Element}

An architectural element is formed by a set of aspects, their weaving relationships, and one or more ports. These ports represent interaction points among architectural elements. There are two kinds of architectural elements, components and connectors.

\section{Formalization of Architectural Elements}

Let $A R$ be an architectural element, $A_{1} \ldots A_{n}$ the aspects that it imports, $P_{1} \ldots P_{m}$ its ports, and $\mathrm{W}$ its set of weavings.

AR is defined by the tuple $(\mathrm{A}, \mathrm{X}, \Phi, \Pi)$ :

A: the union of the attributes of the aspects $A_{1} \ldots A_{n}$

X: the union of the services of the aspects $A_{1} \ldots A_{n}$

D: the union of the formulae in modal logic of actions of the aspects $A_{1} \ldots A_{n}$

$>\Pi$ : the process $\mathrm{P}_{\mathrm{AR}}$ defined as follows:

$$
\mathrm{P}_{\mathrm{AR}}::=\mathrm{P}_{\mathrm{P} 1}\|\ldots\| \mathrm{P}_{\mathrm{Pm}}\left\|\mathrm{P}_{\mathrm{A} 1}\right\| \ldots\left\|\mathrm{P}_{\mathrm{An}}\right\| \mathrm{P}_{\mathrm{W}}
$$

This means that the processes of the ports, weavings and aspects of the architectural element are executed concurrently. For this reason, $\mathrm{P}_{\mathrm{AR}}$ is defined as their parallel composition.

\subsubsection{Connector}

A connector is an architectural element that acts as a coordinator between other architectural elements. As such, connectors have a coordination aspect. 
An example is the CnctJoint that synchronizes the Actuator and the Sensor of a joint (see Figure 29). This connector imports the SMotion safety aspect and the CProcessSuc coordination aspect as mentioned above and is formed by the follow set of ports and weavings (see Figure 30):

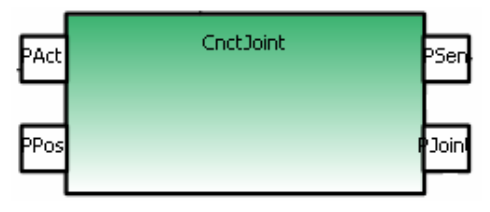

Figure 29. The CnctJoint connector

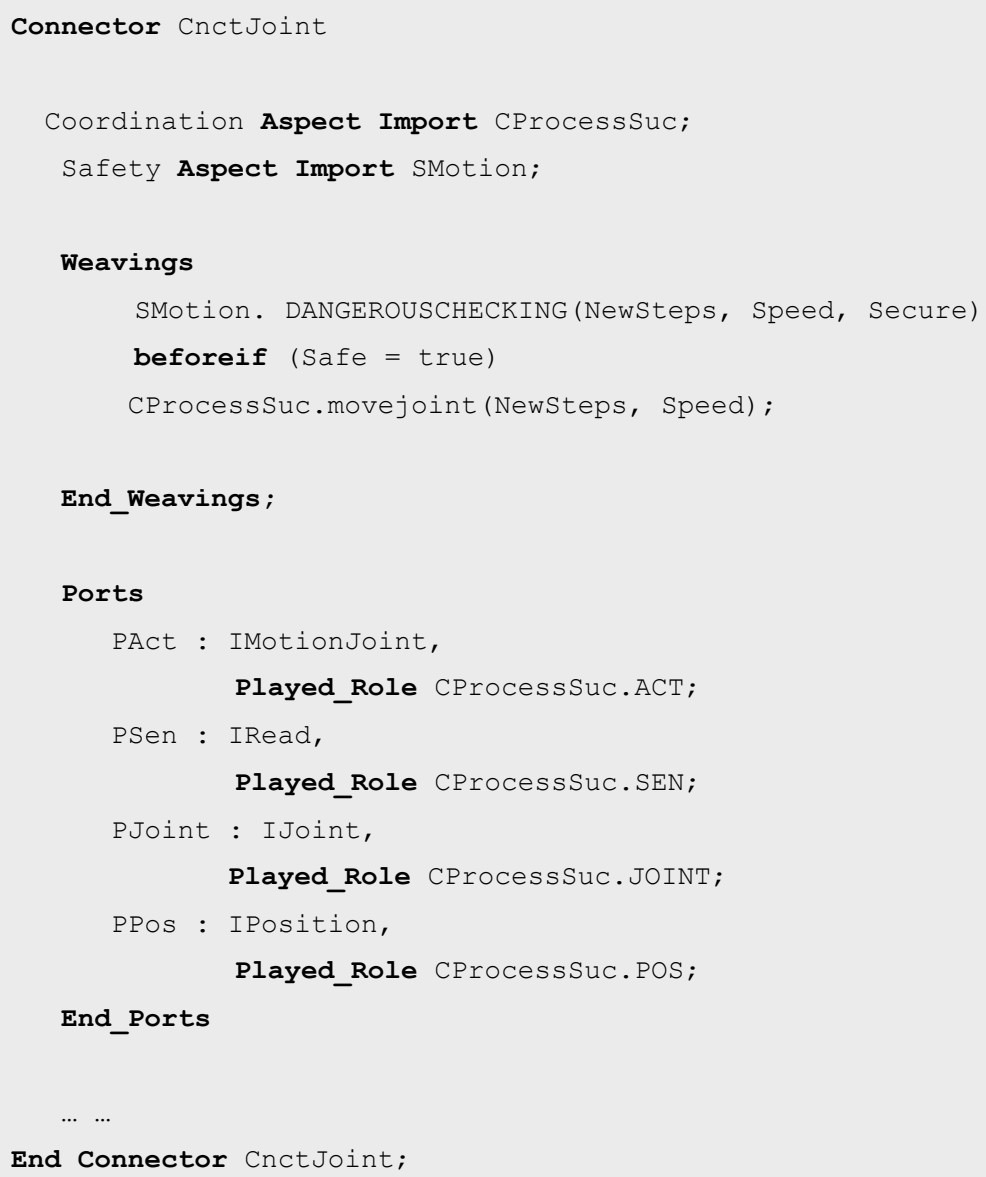

Figure 30. Specification of the connector CnctJoint 
PRISMA: Aspect-Oriented Software Architectures

\subsubsection{Component}

A component is an architectural element that captures a given functionality of a software system. As such, components do not have a coordination aspect.

An example is the Actuator (see Figure 31). It sends commands to a joint of the robot. These commands are performed by the joint or the tool. The Actuator of the TeachMover only imports a functional aspect (see Figure 32).

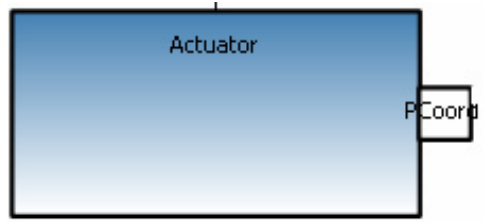

Figure 31. The component Actuator

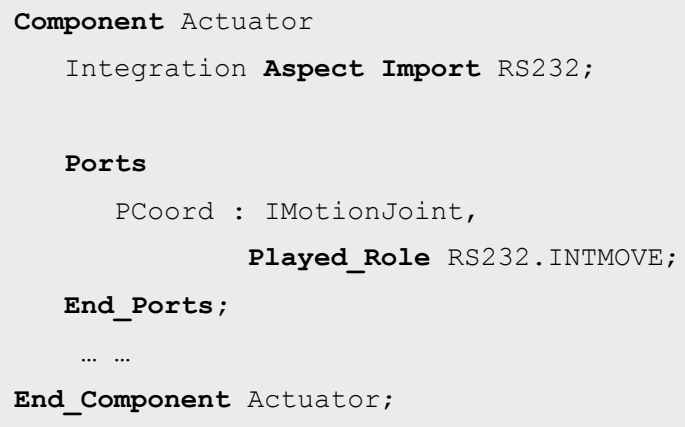

Figure 32. Specification of the component Actuator

\subsubsection{Attachment}

An attachment establishes a connection between two architectural elements, more precisely, between a port of a component and a port of a connector.

An attachment is a channel that transmits the invocations and results of services from one port to another. It solves mismatches of service names and ensures compatibility of played_roles. Played_roles are compatible if:

$>$ They define the semantics of the same interface or one interface is a subset of the other.

$>$ They can communicate with each other, i. e., at least one service requested by one of the played_roles must be provided by the other. 
The PRISMA Model

\section{Formalization of Attachments}

Let ATCH be an attachment that connects ports PC and PCnct. Let PC be the port of a component and PCnct the port of a connector. The semantics of PC and PCnct is defined by two played_roles called $\mathrm{PR}_{\mathrm{PC}}$ and $\mathrm{PR}_{\mathrm{PCnct}}$, respectively (see section 6.2.5). Let $\mathrm{C}_{\mathrm{S} 1} \ldots \mathrm{C}_{\mathrm{Sn}}$ and $\mathrm{Cn}_{\mathrm{S} 1} \ldots \mathrm{Cn}_{\mathrm{Sm}}$ be the services that participate in $\mathrm{PR}_{\mathrm{PC}}$ and $\mathrm{PR}_{\mathrm{PCnct}}$, respectively. The formalization of $\mathrm{PR}_{\mathrm{PC}}$ and $\mathrm{PR}_{\mathrm{PCnct}}$ is defined as in section 6.2.3. As a result, the semantics of $\mathrm{PR}_{\mathrm{PC}}$ is a process that establishes a partial order for the requests and invocations of the services $\mathrm{C}_{\mathrm{S} 1} \ldots \mathrm{C}_{\mathrm{Sn}}$ through the channels $\mathrm{Cc}_{\mathrm{S1}} \ldots \mathrm{Cc}_{\mathrm{Sn}}$. The semantics of $\mathrm{PR}_{\mathrm{PCn} \text { t }}$ is a process that establishes a partial order for the requests and invocations of the services $\mathrm{Cn}_{\mathrm{S1}} \ldots \mathrm{Cn}_{\mathrm{Sm}}$ through the channels $\mathrm{Ccn}_{\mathrm{S} 1} \ldots \mathrm{Cn}_{\mathrm{Sm}}$. The semantics of $\mathrm{ATCH}$ is a process called $\mathrm{P}_{\mathrm{ATCH}}$. $\mathrm{P}_{\mathrm{ATCH}}$ coordinates the services of $\mathrm{PR}_{\mathrm{PC}}$ and $\mathrm{PR}_{\mathrm{PCnct}}$ through the channels that these services provide (see section 6.2.2) (see Figure 33).

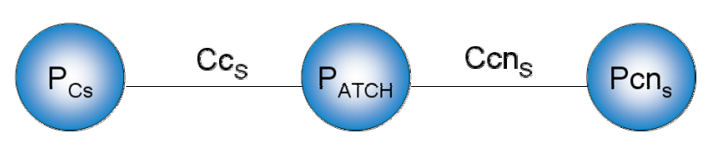

Figure 33. Formalization of Attachments

The formalization of ATCH is presented in two steps as follows:

\section{Invocation from the component}

The process that formalizes the behaviour of Cs when the component invokes a service is defined as follows:

$$
\begin{aligned}
& \mathrm{PC}_{\mathrm{s}}::=\ldots\left(\vec{v} \overrightarrow{r p c_{y}}\right)\left(\mathrm{Ccs}_{1} !\left(\vec{x}, \overrightarrow{r p c_{y}}\right) \rightarrow \operatorname{rpc}_{\mathrm{y} 1} ?\left(\mathrm{y}_{1}\right) \ldots r p \mathrm{rcm}_{\mathrm{y}} ?\left(\mathrm{y}_{\mathrm{m}}\right)\right) \\
& \|\ldots\| \\
& \left({ }^{v r p c_{y}}\right)\left(\mathrm{Ccs}_{n} !\left(\vec{x}, \overrightarrow{r p c_{y}}\right) \rightarrow \operatorname{rpc}_{\mathrm{y} 1} ?\left(\mathrm{y}_{1}\right) \ldots \mathrm{rpc}_{\mathrm{ym}} ?\left(\mathrm{y}_{\mathrm{m}}\right)\right) \ldots
\end{aligned}
$$

The process that formalizes the behaviour of $\mathrm{Cn}_{\mathrm{s}}$ when the component invokes a service is defined as follows:

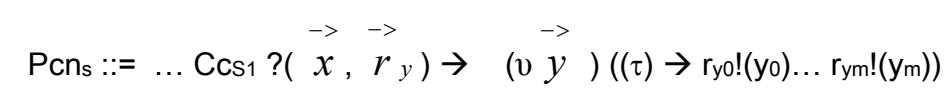


PRISMA: Aspect-Oriented Software Architectures

$$
\begin{aligned}
& \|\ldots\|^{\|} \\
& \operatorname{Ccsn} ?\left(\stackrel{->}{x}, r_{y}\right) \rightarrow\left(\begin{array}{ll}
v & y
\end{array}\right)\left((\tau) \rightarrow r_{\mathrm{y} 0} !\left(\mathrm{y}_{0}\right) \ldots \mathrm{r}_{\mathrm{ym}} !\left(\mathrm{y}_{\mathrm{m}}\right)\right) \ldots
\end{aligned}
$$

The process that formalizes the behaviour of ATCH when the invocation of services is executed from a component is defined as follows:

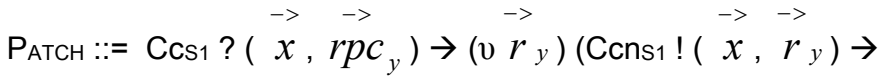

$$
\begin{aligned}
& \left.r_{\mathrm{y} 0} ?\left(\mathrm{y}_{0}\right) \ldots r_{\mathrm{ym}} ?\left(\mathrm{ym}_{\mathrm{m}}\right)\right) \rightarrow \mathrm{rpc}_{\mathrm{y} 0} !\left(\mathrm{y}_{0}\right) \ldots \mathrm{rpc} \mathrm{ym}_{\mathrm{m}} !\left(\mathrm{y}_{\mathrm{m}}\right) \\
& \|\ldots\|
\end{aligned}
$$

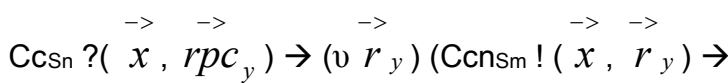

$$
\begin{aligned}
& \left.r_{y 0} ?\left(y_{0}\right) \ldots r_{y m} ?\left(y_{m}\right)\right) \rightarrow \text { rpc }_{y 0} !\left(y_{0}\right) \ldots r p c_{y m} !\left(y_{m}\right) \ldots \ldots
\end{aligned}
$$

This means that the process receives the invocations from $\mathrm{P}_{\mathrm{C}}$ and sends the invocation of the services with the correct name to $\mathrm{P}_{\text {Cnct }}$ in a concurrent way.

\section{Invocation from the connector}

The process that formalizes the behaviour of Cs when the connector invokes a service is defined as follows:

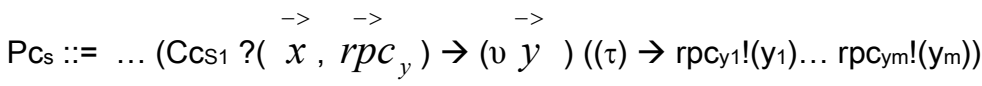

$$
\begin{aligned}
& \|\ldots .\|
\end{aligned}
$$

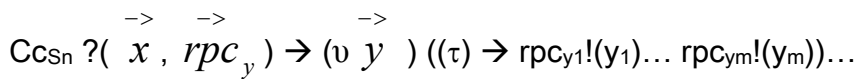

The process that formalizes the behaviour of $\mathrm{Cn}_{\mathrm{s}}$ when the connector invokes a service is defined as follows:

$$
\begin{aligned}
& \mathrm{Pcn}_{\mathrm{s}}::=\ldots\left(\stackrel{->}{r_{y}}\right)\left(\mathrm{Ccs} 1 !\left(\stackrel{->}{x}, \overrightarrow{r_{y}}\right) \rightarrow \mathrm{r}_{\mathrm{y} 0} ?\left(\mathrm{y}_{0}\right) \ldots \mathrm{r}_{\mathrm{ym}} ?(\mathrm{ym})\right) \\
& \|\ldots\| \\
& \rightarrow \rightarrow-> \\
& \left(v r_{y}\right)\left(\operatorname{Ccsn} !\left(x, r_{y}\right) \rightarrow \mathrm{ryo}_{\mathrm{y}} ?\left(\mathrm{y}_{0}\right) \ldots \mathrm{rym}_{\mathrm{ym}} ?\left(\mathrm{ym}_{\mathrm{m}}\right)\right) \ldots
\end{aligned}
$$


The process that formalizes the behaviour of ATCH when the invocation of services is executed from a connector is defined as follows:

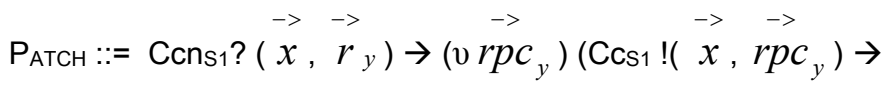

$$
\begin{aligned}
& \left.\mathrm{rpc}_{\mathrm{y} 0} ?\left(\mathrm{y}_{0}\right) \ldots \mathrm{rpc} \mathrm{ym}_{\mathrm{m}} ?\left(\mathrm{ym}_{\mathrm{m}}\right)\right) \rightarrow \mathrm{r}_{\mathrm{y} 1} !\left(\mathrm{y}_{1}\right) \ldots \mathrm{r}_{\mathrm{ym}} !\left(\mathrm{y}_{\mathrm{m}}\right) \\
& \| \text {.... } \|
\end{aligned}
$$

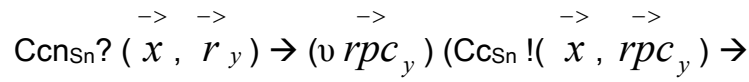

$$
\begin{aligned}
& \left.\mathrm{rpc}_{\mathrm{y} 0} ?\left(\mathrm{y}_{0}\right) \ldots \mathrm{rpc} \mathrm{ym}_{\mathrm{m}} ?\left(\mathrm{y}_{\mathrm{m}}\right)\right) \rightarrow \mathrm{r}_{\mathrm{y} 1} !\left(\mathrm{y}_{1}\right) \ldots \mathrm{rym}_{\mathrm{ym}} !\left(\mathrm{y}_{\mathrm{m}}\right) \ldots \ldots
\end{aligned}
$$

This means that the process receives the invocations from $\mathrm{P}_{\mathrm{Cnct}}$ and sends the invocation of the services with the correct name to $\mathrm{P}_{\mathrm{C}}$ in a concurrent way.

$\mathrm{P}_{\mathrm{ATCH}}$ defines the parallel composition between the processes that define the behaviour of ATCH when the invocation is from a connector and from a component. The process $\mathrm{P}_{\mathrm{ATCH}}$ is defined as follows:

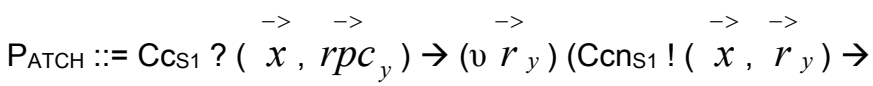

$$
\begin{aligned}
& \left.r_{y 0} ?\left(y_{0}\right) \ldots r_{y m} ?\left(y_{m}\right)\right) \rightarrow r p c_{y 0} !\left(y_{0}\right) \ldots r p c y m !\left(y_{m}\right)\|\ldots \quad\|
\end{aligned}
$$

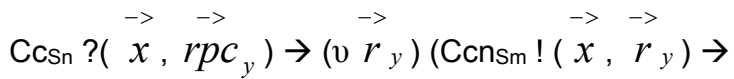

$$
\begin{aligned}
& \left.r_{y 0} ?\left(y_{0}\right) \ldots r_{y m} ?\left(y_{m}\right)\right) \rightarrow r p c_{y 0} !\left(y_{0}\right) \ldots r p c y m !\left(y_{m}\right) \ldots \ldots \\
& \|\ldots\|
\end{aligned}
$$

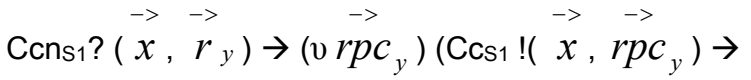

$$
\begin{aligned}
& \left.\mathrm{rpc}_{\mathrm{y} 0} ?\left(\mathrm{y}_{0}\right) \ldots \mathrm{rpc} \mathrm{ym}_{\mathrm{m}} ?\left(\mathrm{y}_{\mathrm{m}}\right)\right) \rightarrow \mathrm{r}_{\mathrm{y} 1} !\left(\mathrm{y}_{1}\right) \ldots \mathrm{r}_{\mathrm{ym}} !\left(\mathrm{y}_{\mathrm{m}}\right)\|\ldots\|
\end{aligned}
$$

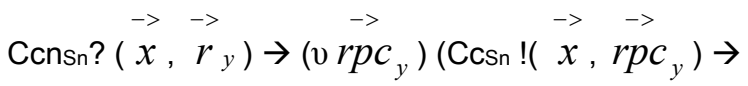

$$
\begin{aligned}
& \left.\mathrm{rpc}_{\mathrm{y} 0} ?\left(\mathrm{y}_{0}\right) \ldots \mathrm{rpc} \mathrm{ym}_{\mathrm{m}} ?\left(\mathrm{y}_{\mathrm{m}}\right)\right) \rightarrow \mathrm{ry}_{\mathrm{y} 1} !\left(\mathrm{y}_{1}\right) \ldots \mathrm{rym}_{\mathrm{ym}} !\left(\mathrm{y}_{\mathrm{m}}\right) \ldots \ldots
\end{aligned}
$$

An example of attachment is the channel that connects the Actuator (see Figure 31) and the CnctJoint (see Figure 29) (see Figure 35 and Figure 34). 
PRISMA: Aspect-Oriented Software Architectures

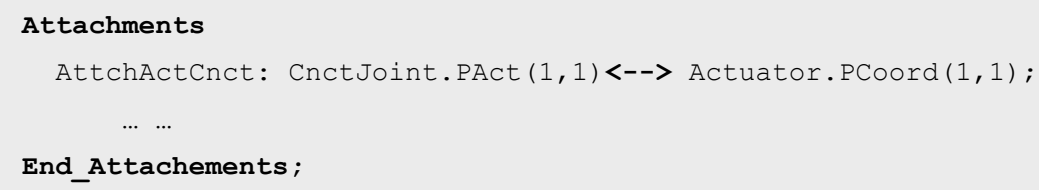

Figure 34. Specification of an attachment between the Actuator and the CnctJoint

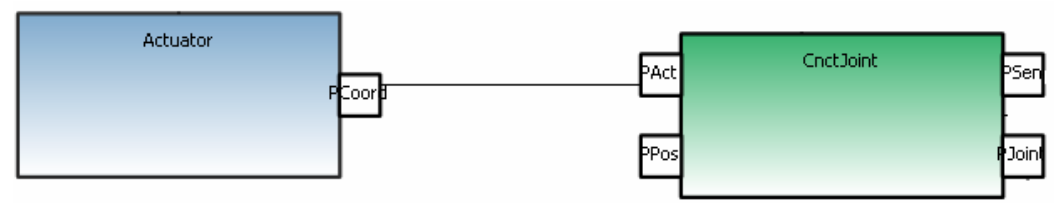

Figure 35. The attachment between the Actuator and the CnctJoint

The channel connects the ports PCoord and PAct, which are defined in the Actuator (see Figure 32) and the CnctJoint (see Figure 30), respectively. Their played_roles INTMOVE and $A C T$ of PCoord and PAct are specified in Figure 36. In addition, the minimum and maximum cardinalities of the attachment are specified. They define the connection pattern by constraining the minimum and maximum instances of the attachment. These cardinalities can be defined as follows:

connector.port $(\min , \max ) \leftarrow \rightarrow$ component.portt(min, $\max )$

$>$ connector.port (min, max) defines how many instances of the attachment can be connected to one instance of the connector through the port

$>$ component.portt(min, max) defines how many instances of the attachment can be connected to one instance of the component through the port.

For example, the semantics of CnctJoint.PAct $(1,1)$ is that an instance of the connector CnctJoint must have one and only one attachment of this type connected to the port CoorAct, and the semantics of Actuator.PCord(1,1) is that an instance of the component Actuator must have one and only one attachment of this type connected to the port RobotAct. 


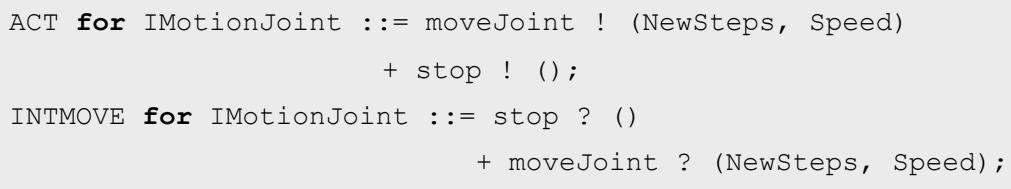

Figure 36. Specification of played_roles $A C T$ and $R O B O T$

The process of the attachment that connects both played_roles is the following:

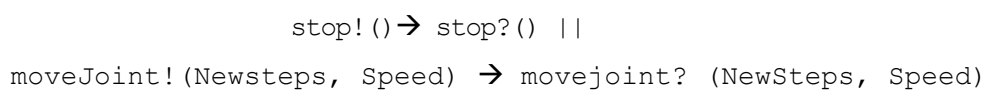

\subsubsection{Binding}

A binding relationship defines the composition between a complex component and one of its architectural elements, specifically, between a port of a complex component and a port of one of its architectural elements. In PRISMA, complex components are called systems (see 6.2.12).

A binding is a relay, a channel that redirects the invocations and results of services from one port to another. It solves mismatches of service names and ensures the compatibility of played_roles. Played_roles are compatible if:

$>$ They define the semantics of the same interface, or one interface is a subset of the other one.

They can communicate with each other, i. e., at least one service requested by one of the played_roles must be requested by the other, or one service provided by one of the played_roles must be provided by the other.

\section{Formalization of Bindings}

Let PAR be the port of an architectural element of a system and let PS be a port of the system. The semantics of PAR and PS are defined by two played_roles called $\mathrm{PR}_{\mathrm{PAR}}$ and $\mathrm{PR}_{\mathrm{PS}}$, respectively (see section 6.2.5). Let $\mathrm{AR}_{\mathrm{S} 1} \ldots \mathrm{AR}_{\mathrm{Sn}}$ and $\mathrm{S}_{\mathrm{S} 1} \ldots \mathrm{S}_{\mathrm{Sm}}$ be the services that participate in $\mathrm{PR}_{\mathrm{PAR}}$ and $\mathrm{PR}_{\mathrm{PS}}$, respectively. The formalization of $\mathrm{PR}_{\mathrm{PAR}}$ and $\mathrm{PR}_{\mathrm{PS}}$ is defined as in section 6.2.3. As a result, the semantics of $P_{\mathrm{PAR}}$ is a process that establishes a partial order for the requests and invocations of the services of $A_{\mathrm{S} 1} \ldots \mathrm{AR}_{\mathrm{Sn}}$ through the channels $\mathrm{C}_{\mathrm{ARs}} \ldots \mathrm{C}_{\mathrm{ARsn}}$. 
PRISMA: Aspect-Oriented Software Architectures

The semantics of $\mathrm{PR}_{\mathrm{PS}}$ is a process that establishes a partial order for the requests and invocations of the services of $\mathrm{S}_{\mathrm{S} 1} \ldots \mathrm{S}_{\mathrm{Sm}}$ through the channels $\mathrm{CS}_{\mathrm{S} 1} \ldots \mathrm{Cs}_{\mathrm{Sm}}$.

Let $\mathrm{BD}$ be a binding that connects the ports PAR and PS. The semantics of BD is a process, called $\mathrm{P}_{\mathrm{BD}}$, that coordinates the services of $\mathrm{PR}_{\mathrm{PAR}}$ and $\mathrm{PR}_{\mathrm{PS}}$ through the channels that they provide for their invocation (see section 6.2.2). When $\mathrm{PR}_{\mathrm{PAR}}$ and $\mathrm{PR}_{\mathrm{PS}}$ are linked by a binding, $\mathrm{PR}_{\mathrm{PS}}$ invokes the services provided by the binding $\mathrm{B}_{\mathrm{S} 1} \ldots \mathrm{B}_{\mathrm{Sp}}$ using the channels $\mathrm{C}_{\mathrm{Bs}} \ldots \mathrm{C}_{\mathrm{Bsp}}$ and receives the service invocations from these channels in order to redirect the invocations from PS to PAR.

The semantics of a $\mathrm{BD}$ is a process, called $\mathrm{P}_{\mathrm{BD}} . \mathrm{P}_{\mathrm{BD}}$ redirects the invocations of the services of $\mathrm{PR}_{\mathrm{PC}}$ and $\mathrm{PR}_{\mathrm{PCnct}}$ through the channels that these services provide and the channels of the services of the binding (see section 6.2.2) (see Figure 37).

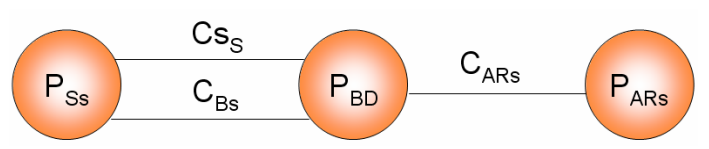

Figure 37. Formalization of Bindings

\section{An invocation arrives to the system}

$P_{S}$ receives invocations of services that arrive to PS and redirects invocations to $P_{B D}$ :

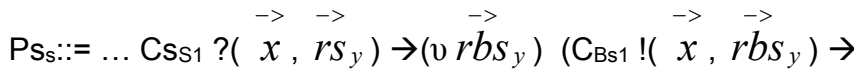

$$
\begin{aligned}
& \text { rbsyo } \left.?\left(\mathrm{y}_{0}\right) \ldots \mathrm{rbs} y m ?\left(\mathrm{y}_{\mathrm{m}}\right)\right) \rightarrow \mathrm{rs} \mathrm{y}_{\mathrm{o}} !\left(\mathrm{y}_{0}\right) \ldots \mathrm{rs} \mathrm{s}_{\mathrm{y}} !\left(\mathrm{y}_{\mathrm{m}}\right) \\
& \|\ldots\| \\
& \text { Cssm ? }\left(\vec{x}, \overrightarrow{r s} s_{y}\right) \rightarrow\left(\begin{array}{c}
\overrightarrow{r b} s_{y}
\end{array}\right)\left(\mathrm{C}_{\mathrm{Bsp}} !\left(\vec{x}, \overrightarrow{r b} s_{y}\right) \rightarrow\right.
\end{aligned}
$$

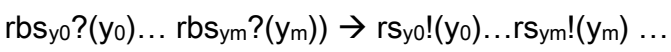

$\mathrm{P}_{\mathrm{ARs}}$ receives invocations from $\mathrm{P}_{\mathrm{BD}}$ and sends them through $\mathrm{PAR}$ to be processed by the architectural element of the system.

$$
\begin{aligned}
& \mathrm{P}_{\mathrm{ARs}}::=\ldots \mathrm{C}_{\mathrm{ARs} 1} ?\left(\overrightarrow{\vec{x}}, \overrightarrow{r_{A R s y}}\right) \rightarrow\left(\begin{array}{ll}
v & \vec{y}
\end{array}\right)\left((\tau) \rightarrow \mathrm{r}_{\mathrm{ARs}} \mathrm{y} 0(\mathrm{y} 0) \ldots \mathrm{r}_{\mathrm{ARs}} \mathrm{ym} !\left(\mathrm{y}_{\mathrm{m}}\right)\right. \\
& \text { || ... }|| \\
& \mathrm{C}_{\text {ARsn }} ?(\stackrel{\vec{x}, \vec{r}}{A R s y}) \rightarrow\left(\begin{array}{ll}
\mathrm{v} & \vec{y}
\end{array}\right)\left((\tau) \rightarrow \mathrm{r}_{\mathrm{ARs}} \mathrm{y} 0 \text { !(yo)... } \mathrm{r}_{\mathrm{ARs}} \mathrm{ym} !(\mathrm{ym}) \ldots\right.
\end{aligned}
$$


The process that formalizes the behaviour of $\mathrm{BD}$ when invocations of services arrive to PS is as follows:

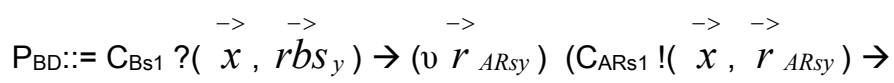

$$
\begin{aligned}
& \left.\mathrm{r}_{\mathrm{ARs}} \mathrm{y} 0 ?\left(\mathrm{y}_{0}\right) \ldots \mathrm{r}_{\mathrm{ARs}} \mathrm{ym} ?\left(\mathrm{ym}_{\mathrm{m}}\right)\right) \rightarrow \mathrm{rbs}_{\mathrm{y} 0} !\left(\mathrm{y}_{0}\right) \ldots \mathrm{rbs}_{\mathrm{ym}} !\left(\mathrm{y}_{\mathrm{m}}\right) \\
& \|\ldots\|
\end{aligned}
$$

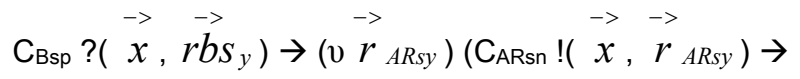

$$
\begin{aligned}
& \text { rARs } \left.\mathrm{y} 0 ?(\mathrm{y} 0) \ldots \text { rARs } y m ?\left(\mathrm{ym}_{\mathrm{m}}\right)\right) \rightarrow \text { rbsyo!(yo)... rbsym!(ym) ... }
\end{aligned}
$$

This means that $\mathrm{P}_{\mathrm{BD}}$ receives the invocations from $\mathrm{PSS}_{\mathrm{S}}$ and sends the invocation of the services with the correct name to $\mathrm{P}_{\mathrm{ARs}}$ in a concurrent way.

2.An invocation from the architectural element of the system

$\mathrm{P}_{\mathrm{ARs}}$ invokes services through PAR:

$$
\begin{aligned}
& \mathrm{P}_{\mathrm{ARs}}::=\ldots\left(\mathrm{v} \stackrel{->}{r_{\text {ARsy }}}\right)\left(\mathrm{C}_{\mathrm{ARs} 1} !\left(\stackrel{->}{x}, \vec{r}_{\text {ARsy }}\right) \rightarrow \mathrm{r}_{\mathrm{ARs} \mathrm{y} 0} ?\left(\mathrm{y}_{0}\right) \ldots \mathrm{r}_{\mathrm{ARs}} \mathrm{ym} ?\left(\mathrm{ym}_{\mathrm{m}}\right)\right) \\
& \|\ldots\| \\
& \rightarrow \quad \rightarrow-> \\
& \left(\text { (v } r_{\text {ARsy }}\right)\left(\mathrm{C}_{\mathrm{ARsn}} !\left(x, r_{\text {ARsy }}\right) \rightarrow \mathrm{rARs}_{\mathrm{y} 0} ?(\mathrm{y} 0) \ldots \mathrm{r}_{\mathrm{ARs}} \mathrm{ym} ?(\mathrm{ym})\right) \ldots
\end{aligned}
$$

$\mathrm{PS}_{\mathrm{S}}$ receives invocations from $\mathrm{P}_{\mathrm{BD}}$ and sends them through PS in order to request them from an external architectural element of the system:

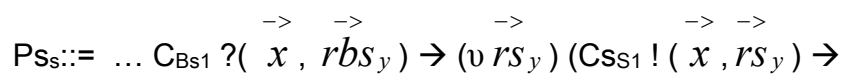

$$
\begin{aligned}
& \left.\mathrm{rs}_{\mathrm{y} 0} ?\left(\mathrm{y}_{0}\right) \ldots \mathrm{rs} y \mathrm{~m} ?\left(\mathrm{ym}_{\mathrm{m}}\right)\right) \rightarrow \mathrm{rbs}_{\mathrm{y} 0} !\left(\mathrm{y}_{0}\right) \ldots \mathrm{rbs}_{\mathrm{ym}} !\left(\mathrm{ym}_{\mathrm{m}}\right) \\
& \|\ldots\|
\end{aligned}
$$

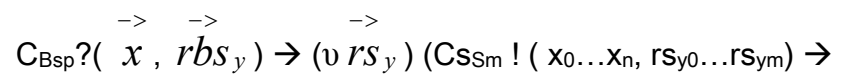

$$
\begin{aligned}
& \left.\mathrm{rS}_{\mathrm{y} 0} ?\left(\mathrm{y}_{0}\right) \ldots \mathrm{rs} \mathrm{sm}_{\mathrm{m}} ?\left(\mathrm{y}_{\mathrm{m}}\right)\right) \rightarrow \mathrm{rbs}_{\mathrm{y} 0} !\left(\mathrm{y}_{0}\right) \ldots \mathrm{rbS}_{\mathrm{ym}} !\left(\mathrm{y}_{\mathrm{m}}\right) \ldots
\end{aligned}
$$

The process that formalizes the behaviour of $\mathrm{BD}$ when invocations of services arrive to PAR is as follows:

$$
\begin{aligned}
\mathrm{P}_{\mathrm{BD}:::=} & \mathrm{C}_{\mathrm{ARs} 1} ?\left(\vec{x}, \overrightarrow{r_{A R s y}}\right) \rightarrow\left(\vec{v} \overrightarrow{b b} s_{y}\right)\left(\mathrm{C}_{\mathrm{Bs} 1} !\left(\vec{x}, \overrightarrow{r b} s_{y}\right) \rightarrow\right. \\
& \left.\operatorname{rbs}_{\mathrm{y} 0} ?(\mathrm{y} 0) \ldots \mathrm{rbs}_{\mathrm{ym}} ?\left(\mathrm{y}_{\mathrm{m}}\right)\right) \rightarrow \mathrm{r}_{\mathrm{ARs} \mathrm{y} 0} !\left(\mathrm{y}_{0}\right) \ldots \mathrm{r}_{\mathrm{ARs}} \mathrm{ym} ?\left(\mathrm{y}_{\mathrm{m}}\right)
\end{aligned}
$$


PRISMA: Aspect-Oriented Software Architectures

$$
\begin{aligned}
& \text { || } \ldots \text {. }
\end{aligned}
$$

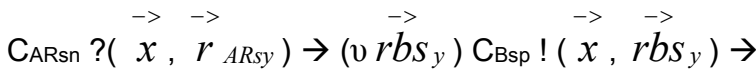

$$
\begin{aligned}
& \left.\mathrm{rbs}_{\mathrm{y} 0} ?\left(\mathrm{y}_{0}\right) \ldots \mathrm{rbs}_{\mathrm{ym}} ?\left(\mathrm{y}_{\mathrm{m}}\right)\right) \rightarrow \mathrm{r}_{\mathrm{ARs}} \mathrm{y} 0 !\left(\mathrm{y}_{0}\right) \ldots \mathrm{r}_{\mathrm{ARs}} \mathrm{ym} ?\left(\mathrm{y}_{\mathrm{m}}\right)
\end{aligned}
$$

This means that $\mathrm{P}_{\mathrm{BD}}$ receives the invocations from $\mathrm{P}_{\mathrm{ARs}}$ and sends the invocation of the services with the correct name to $\mathrm{Ps}_{\mathrm{S}}$ in a concurrent way.

$\mathrm{P}_{\mathrm{BD}}$ defines the parallel composition between the processes that define the behaviour of $\mathrm{BD}$ when the invocation is from the system and from a architectural element. The process $\mathrm{P}_{\text {ATCH }}$ is defined as follows:

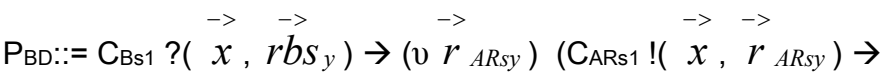

$$
\begin{aligned}
& \left.\mathrm{r}_{\text {ARs yo }} \text { ? }\left(\mathrm{y}_{0}\right) \ldots \mathrm{r}_{\text {ARs } y m} ?\left(\mathrm{y}_{\mathrm{m}}\right)\right) \rightarrow \mathrm{rbs}_{\mathrm{y} 0} !\left(\mathrm{y}_{0}\right) \ldots \mathrm{rbs}_{\mathrm{ym}} !\left(\mathrm{ym}_{\mathrm{m}}\right)\|\ldots\|
\end{aligned}
$$

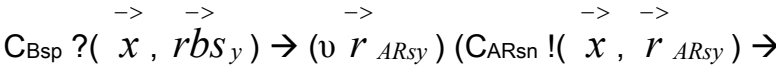

$$
\begin{aligned}
& \left.\mathrm{r}_{\mathrm{ARs}} \mathrm{y} 0 \text { ? }\left(\mathrm{y}_{0}\right) \ldots \mathrm{r}_{\mathrm{ARs}} \mathrm{ym} ?\left(\mathrm{y}_{\mathrm{m}}\right)\right) \rightarrow \mathrm{rbs}_{\mathrm{y} 0} !\left(\mathrm{y}_{0}\right) \ldots \mathrm{rbs}_{\mathrm{ym}} !\left(\mathrm{y}_{\mathrm{m}}\right) \\
& \|\ldots .\| \\
& \mathrm{C}_{\mathrm{ARs} 1} ?\left(\stackrel{->}{x}, \vec{r}_{A R s y}\right) \rightarrow(v \overrightarrow{v b s} y)\left(\mathrm{C}_{\mathrm{Bs} 1} !\left(\vec{x}, \overrightarrow{r b} s_{y}\right) \rightarrow\right. \\
& \left.\mathrm{rbs}_{\mathrm{y} 0} ?\left(\mathrm{y}_{0}\right) \ldots \mathrm{rbs}_{\mathrm{ym}} ?\left(\mathrm{y}_{\mathrm{m}}\right)\right) \rightarrow \mathrm{r}_{\mathrm{ARs}} \mathrm{y} 0 \text { ! }\left(\mathrm{y}_{0}\right) \ldots \mathrm{r}_{\mathrm{ARs}} \mathrm{ym} ?\left(\mathrm{y}_{\mathrm{m}}\right)\|\ldots\|
\end{aligned}
$$

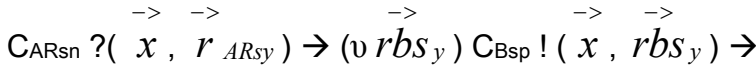

$$
\begin{aligned}
& \left.\mathrm{rbs}_{\mathrm{y} 0} ?\left(\mathrm{y}_{0}\right) \ldots \mathrm{rbS}_{\mathrm{ym}} ?\left(\mathrm{y}_{\mathrm{m}}\right)\right) \rightarrow \mathrm{r}_{\mathrm{ARs}} \mathrm{y} 0 !\left(\mathrm{y}_{0}\right) \ldots \mathrm{r}_{\mathrm{ARs}} \mathrm{ym} ?\left(\mathrm{y}_{\mathrm{m}}\right)
\end{aligned}
$$

An example of binding is the channel that communicates the CnctJoint connector (see Figure 29) and the Joint system (see Figure 38 and Figure 39).

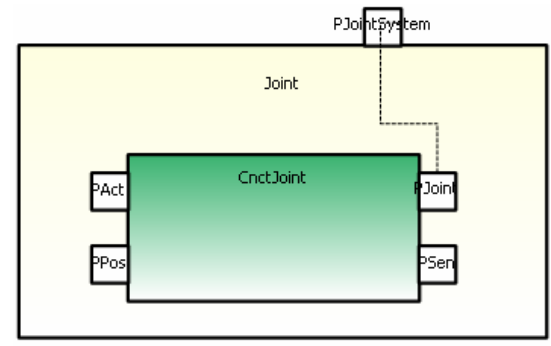

Figure 38. The binding between the CnctJoint and the Joint of the TeachMover 


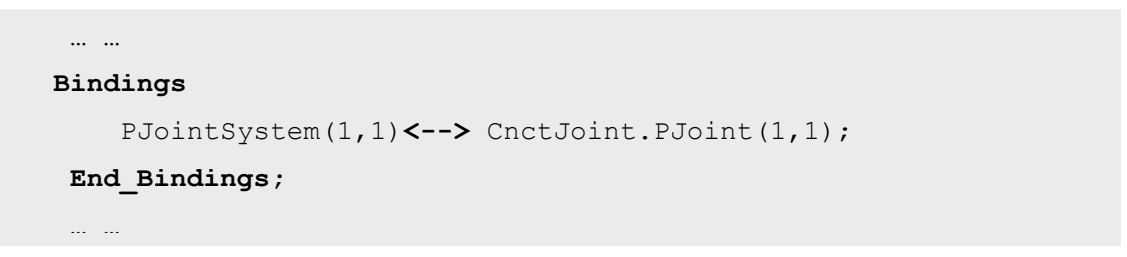

Figure 39. Specification of a binding between the Joint and the CnctJoint

The channel connects the ports PJointSystem and PJoint, which are defined in the Joint (see Figure 38) and the CnctJoint (see Figure 30), respectively. They have the same played_role, JOINT of the CnctJoint (see Figure 40). In addition, the minimum and maximum cardinalities of the binding are specified as in the definition of attachments (see section 6.2.10).

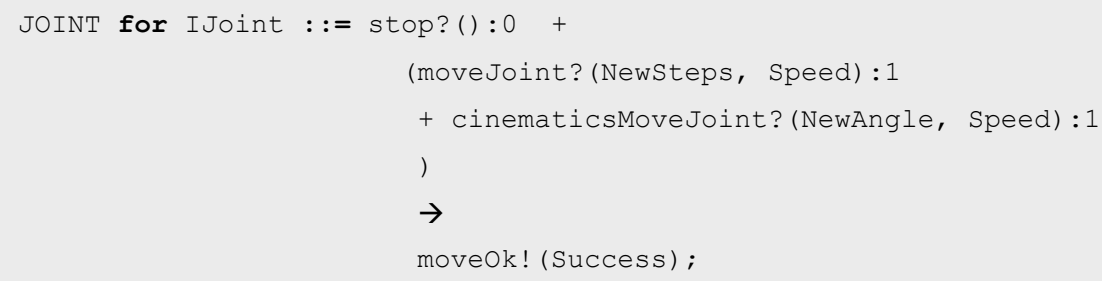

Figure 40. Specification of the played_role JOINT

The played_role JOINT is adapted to the PJointSystem in order to be able to communicate with the binding channel. This adaptation is performed automatically by the system and the user does not have to explicitly specify it. This communication is established as follows:

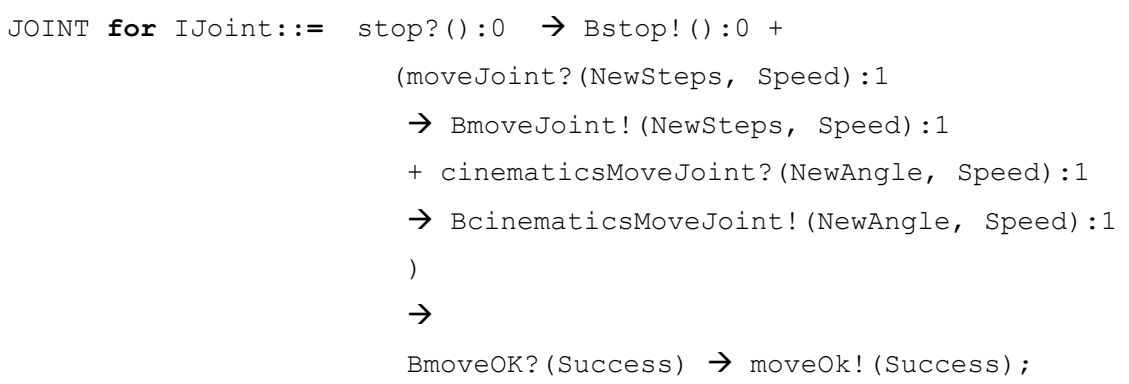

The binding process that connects the two played_roles is the following: 
PRISMA: Aspect-Oriented Software Architectures

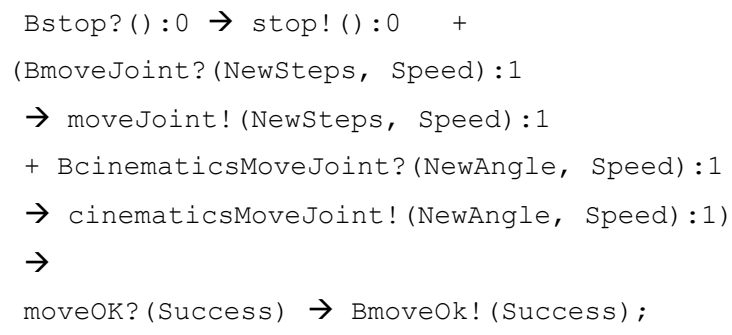

\subsubsection{System}

PRISMA components can be simple or complex. Complex components are called systems. A PRISMA system is a component that includes a set of connectors, simple components, and other systems that are correctly attached to one another.

The difference between a system and a simple component is that a system is composed of a set of architectural elements (simple components, systems, and connectors) and the attachments and bindings that connect them.

As a component, a system captures the functionality of a software system and does not act as a coordinator. The composition of architectural elements can lead to new aspects. These aspects and their new weavings belong to the system. Since systems address the functionality of software architectures in the same way as simple components, they do not have any coordination aspects.

It is important to take into account that interfaces and played_roles that type the ports of a system must be specified by one of the system aspects, or they must be specified by one of the ports of the system architectural elements.

A system is specified as a pattern, so that, it can be reused in any software architecture where necessary. This permits not only defining how its architectural elements are connected, but also constrains the number of instances that can be created for each one of its architectural elements. These constraints concern the minimum and maximum cardinalities, whose default values are one and infinite, respectively.

Sometimes, the aspects of a system require services from the architectural elements of the system and vice versa. For this reason, systems must provide a mechanism for their aspects to communicate with their architectural elements. However, architectural elements can only 
communicate with other architectural elements by means of their ports and the attachments or bindings that link them; similarly, aspects can only communicate with other aspects by means of weavings. A global access between the different aspects and architectural elements of a system is a simple solution to this problem. However, it is also important to keep in mind that a system and its architectural elements can be distributed. Therefore, in order to prepare PRISMA to develop distributed software architectures in the future, the global access solution may not be possible because the system architectural elements and the system could be located in different sites of the network. For this reason, a solution must be found for new aspects that result from composition in order to communicate with the architectural elements of the system without using global access. Moreover, this solution should not violate the aspect-oriented and software architecture approaches, i.e, aspects can only communicate with other aspects by means of weavings, and architectural elements can only communicate with other architectural elements by means of their ports and connection relationships (attachments and bindings). In order to enforce these rules, the new aspects of a system that result from the composition are wrapped inside a component. This way, the aspects of the system can communicate with the architectural elements by means of the ports of the wrapper component. As a result, the main properties of aspects and architectural elements are preserved as well as their reuse and independence.

Composition of systems and their architectural elements can be inclusive or weak. An inclusive composition means that the only way to interact with the architectural elements is through the ports of the system. A weak composition means that it is possible to communicate directly with the architectural elements of the system.

\section{Formalization of Systems}

Let $\mathrm{SC}$ be the component that wraps the new aspects of a system that result from the composition; let $\mathrm{P}_{1} \ldots \mathrm{P}_{\mathrm{m}}$ be its ports; let $\mathrm{A}_{1} . . \mathrm{A}_{\mathrm{n}}$ be the aspects of the system; and let $\mathrm{W}$ be the set of weavings among them. 
PRISMA: Aspect-Oriented Software Architectures

$\mathrm{SC}$ is defined by the tuple $(\mathrm{A}, \mathrm{X}, \Phi, \Pi)$ :

$>A$ : the union of the attributes of the aspects $A_{1} \ldots A_{n}$

$>\mathrm{X}$ : the union of the services of the aspects $\mathrm{A}_{1} \ldots \mathrm{A}_{\mathrm{n}}$

$>\Phi$ : the union of the formulae in modal logic of actions of the aspects $A_{1} \ldots A_{n}$

$>\Pi$ : the process $\mathrm{P}_{\mathrm{SC}}$ defined as follows:

$$
P_{s c}::=P_{P 1}\|\ldots\| P_{P m}\left\|P_{A 1}\right\| \ldots\left\|P_{A n}\right\| P_{W}
$$

Let $\mathrm{S}$ be a system, $\mathrm{SC}$ the component that wraps its aspects and weavings, $\mathrm{P}_{1} . . \mathrm{P}_{\mathrm{n}}$ its ports, $\mathrm{AR}_{1} . . \mathrm{AR}_{\mathrm{m}}$ the architectural elements that it contains, and $\mathrm{ATCH}_{1} . . \mathrm{ATCH}_{\mathrm{n}}$ and $\mathrm{BD}_{1} . . \mathrm{BD}_{\mathrm{p}}$ the attachments and bindings that interconnect them, respectively. The semantics $\mathrm{S}$ is defined by the process $\mathrm{P}_{\mathrm{S}}$ :

$$
P_{S}::=P_{S C}\left\|P_{A R 1}\right\| \ldots\left\|P_{A R m}\right\| P_{A T C H 1}\|\ldots\| P_{A T C H n}
$$

It important to keep in mind that the set of attachments $\mathrm{ATCH}_{1} \ldots \mathrm{ATCH}_{n}$ not only contains the attachments among the architectural elements that the system contains, but also the attachments between the SC and these architectural elements. In the same way, the set of bindings $\mathrm{BD}_{1} \ldots \mathrm{BD}_{\mathrm{p}}$ not only contains the bindings between the system and its architectural elements, but also the bindings between the system and the component $\mathrm{SC}$ to publish the system behaviour.

An example of system is the Joint system of the TeachMover robot (see Figure 41). This system defines a pattern to specify architectures for joints of robots such as a base, elbow, shoulder, etc. The system Joint imports a functional aspect to manage the position of the joint. It defines a port to communicate with the rest of the robot parts. The system also imports the types that are necessary to define the architectural pattern so that joints can be defined and the number of instances can be constrained. In this case, the types are the Actuator and Sensor components and the connector, and the default cardinality of instantiation has been applied, i.e., one instance as minimum and one instance as maximum. Finally, the attachments between the imported elements and the binding are defined. 


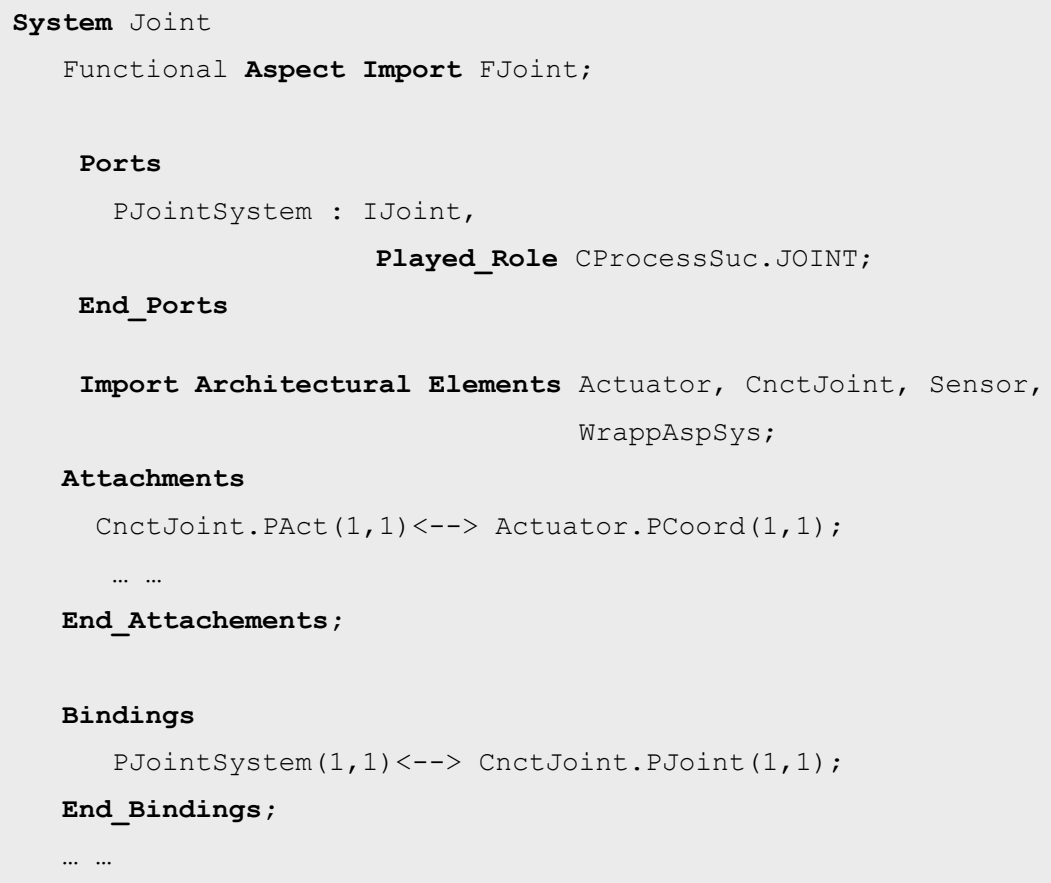

Figure 41. The Joint system

\subsection{CONCLUSIONS}

The PRISMA model has been presented in this chapter. This model allows us to describe software architectures of complex and large systems and to improve their reusability and maintainability. This is possible because the application of aspect-oriented software development to software architectures provides different levels of reusability and maintenance: the concern level (aspects) and the functional level (architectural elements).

> The concern level places the properties of a concern inside an aspect. As a result, the modification of a concern is easily found in the aspects of this concern, and the aspects of a concern can be reused by any architectural element that needs their properties.

$>$ The functional level places functional or coordination processes of the business rules of the software system in components and connectors, respectively. These can be easily found in order to be reused or modified. 
PRISMA: Aspect-Oriented Software Architectures

Another important property of this model is the fact that the weavings between aspects and the relationships among architectural elements are defined outside aspects, which improves their reusability and maintenance.

Instead of using a kemel or core entity to encapsulate functionality, aspects to define nonfunctional requirements and their weavings, this model only uses aspects and weavings to define architectural elements. The symmetrical way in which aspects are introduced in PRISMA software architectures provides homogeneity to the model and a clean and novel way of modelling software architectures. In PRISMA, architectural elements and aspects are used as it they were pieces of a puzzle that fit together to form a software architecture. This way of specifying PRISMA software architectures is presented in detail in chapter.

This chapter also has presented the formalization of the model combining the Modal Logic of Actions and $\pi$-calculus to cope with both the structure and the behaviour of PRISMA software architecture. This formalization defines the properties of the model and provides a formal language that is independent of technology (see chapter 8) to specify PRISMA software architectures and to automatically generate code from its specifications (see section 9.2).

The work related to the PRISMA model has produced a set of results that are published in the following publications:

> Jennifer Pérez, Manuel Llavador, Jose A. Carsí, Jose H. Canós, Isidro Ramos, Coordination in Software Architectures: an Aspect-Oriented Approach, fifth Working IEEE/IFIP Conference on Software Architecture (WICSA), IEEE Computer Society Press, pp. 219-220, ISBN: 0-7695-2548-2, Pittsburgh, Pennsylvania, USA, 6-9 November, 2005 (position paper)

> Nour H. Ali, Jennifer Pérez, Isidro Ramos, Jose A. Carsi, Aspect Reusability in Software Architectures. The 8th International conference of Software Reuse (ICSR), July, 2004. (poster)

$>$ Jennifer Pérez, Isidro Ramos, Javier Jaén, Patricio Letelier, Elena Navarro, PRISMA: Towards Quality, Aspect Oriented and Dynamic Software Architectures, 3rd IEEE International Conference on Quality Software (QSIC 2003), IEEE Computer Society Press, pp.59-66, ISBN: 0-7695-2015-4, Dallas, Texas, USA, November 6 - 7, 2003. 168 
> Jennifer Pérez, Isidro Ramos, Javier Jaén, Patricio Letelier, PRISMA: Development of Software Architectures with an Aspect Oriented, Reflexive and Dynamic Approach, Dagstuhl Seminar No 03081, Report N 36 "Objects, Agents and Features",Copyright (c) IBFI gem. GmbH, Schloss Dagstuhl, D-66687 Wadern, Germany . Eds.H.-D. Ehrich (Univ. Braunschweig, D), J.-J. Meyer (Utrecht, NL), M. Ryan (Univ. of Birmingham, GB), pp. 16, Germany, January, 2003.

> Jennifer Pérez,Nour H. Ali, Isidro Ramos, Jose A. Carsí, PRISMA: Aspect-Oriented and Component-Based Software Architectures, Workshop on Aspect-Oriented Software Development (DSOA), Conference on Software Engineering and Databases (JISBD), Technical Report TR-20/2003 of the Polytechic School of the University of Extremadura, pp. 27-36, Alicante, November, 2003. (In Spanish)

> Jennifer Pérez, Isidro Ramos, OASIS as a Formal Support for the Dynamic, Distributed and Evolutive Hypermedia Models, Technical Report DSIC-II/22/03, pp. 144, Polytechnic University of Valencia, October 2003. (In Spanish)

> Jorge Ferrer, Ángeles Lorenzo, Isidro Ramos, José Ángel Carsí, Jennifer Pérez, Modeling Dynamic Aspects in Architectures and Multiagent Systems, Logic Programming and Software Engineering (CLPSE), pp. 1-13, Copenhagen, Denmark, affiliated with ICLP, july 2002. 
PRISMA: Aspect-Oriented Software Architectures 


\title{
CHAPTER 7
}

\section{THE PRISMA METAMODEL}

\author{
$<<$ There are two distinct classes of what are called thoughts: \\ those that we produce in ourselves by reflection and the act of thinking, \\ and those that bolt into the mind of their own accord. $>>$
}

Thomas Paine

Metamodels define models and establish their properties in a precise way. In addition, metamodels facilitate the automation and maintenance of software development thanks to the support that modeling tools currently offer for these tasks. In order to take advantage of these properties, the PRISMA meta-level is represented by means of a metamodel that contains a set of metaclasses that are related to each other. These metaclasses define a set of properties and services for each concept considered in the model. The metaclasses and their relationships define the structure and the information that is necessary to describe PRISMA architectural models. In addition, the PRISMA metamodel defines the constraints that must be satisfied by a PRISMA architectural model. These constraints guide the methodology for modelling PRISMA architectural models. At the end of the modelling process, all of them must be satisfied in order to ensure that an architectural model is correct.

The PRISMA metamodel has been specified using the class diagram of the Unified Modelling Language (UML) 1.5. and the Object Constraint Language (OCL) 2.0 [UML06]. UML has been used to model the metaclasses and their relationships, attributes and services. OCL has been used to specify the constraints of the metamodel. The choice of these languages 
PRISMA: Aspect-Oriented Software Architectures

over others is based on the fact that they are standards and are widely extended languages. As a result, they facilitate the comprehension of the model by new users.

In this chapter, each one of the packages of the PRISMA metamodel is presented in detail. In addition to the metaclasses, relationships and constraints that packages consist of, the attributes and services of the metaclasses are also explained. The services that are presented are those that allow the construction of PRISMA architectural models.

\subsection{THE PRISMA METAMODEL}

The PRISMA metamodel is composed of three main packages: Types, Architecture Specification, and Common (see Figure 42 ).
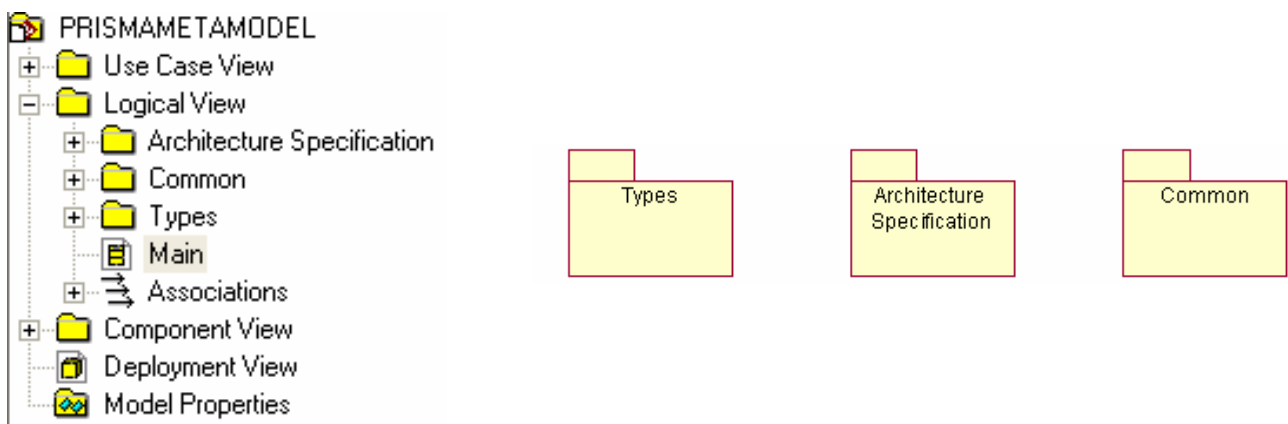

Figure 42. Main packages of the PRISMA metamodel
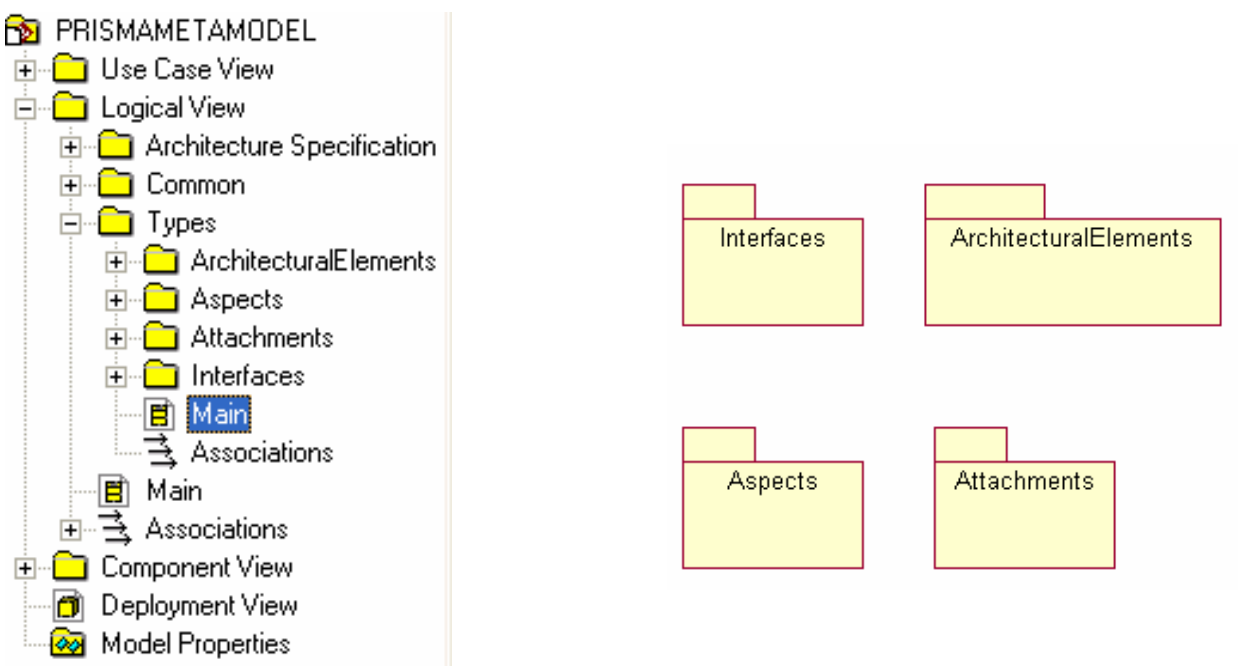

Figure 43. The package Types of the PRISMA metamodel 
The package Types contains the packages Interfaces, Aspects, Architectural Elements and Attachments of the PRISMA model (see Figure 43). These packages define the properties of PRISMA types.

The package Architecture Specification defines the elements that form an architectural model using the types that are defined in the package Types. This package provides the mechanisms to build an architectural model.

The package Common defines the utilities that are necessary to develop any kind of model. These utilities are structured in five sub-packages: DataTypes, Parameters, Constants, Formulae and Process. These sub-packages provide mechanisms to define data types, parameters, constant values, formulae of different kinds and complex process, respectively (see Figure 44).
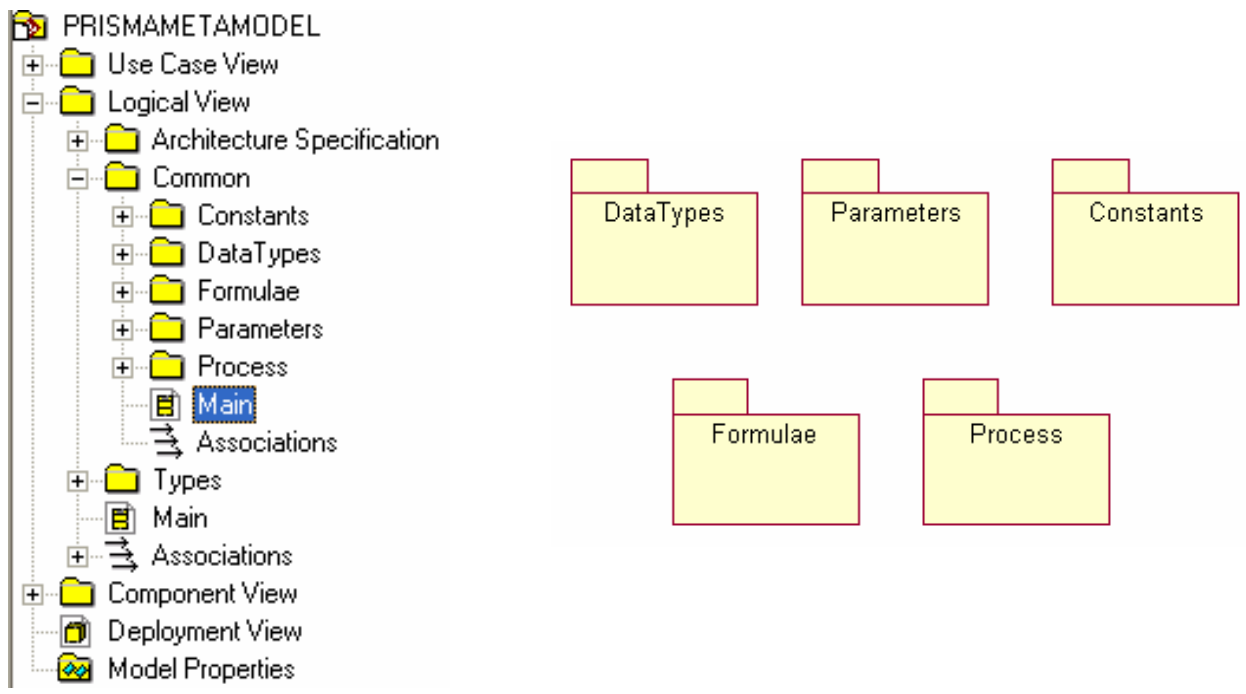

Figure 44. The package Common of the PRISMA metamodel

\subsection{THE PACKAGE “TYPES”}

\subsubsection{The Package "Interfaces"}

An interface publishes a set of services. This set of services is composed of at least one service, and there is no limit to the number of services that can be specified (see Figure 45). The services that make up an interface are called InterfaceServices. These services are defined in an 
PRISMA: Aspect-Oriented Software Architectures

abstract way, without specifying whether they are going to be provided (in), requested (out), or provided and requested (in/out) by architectural elements. An InterfaceService only specifies its signature.

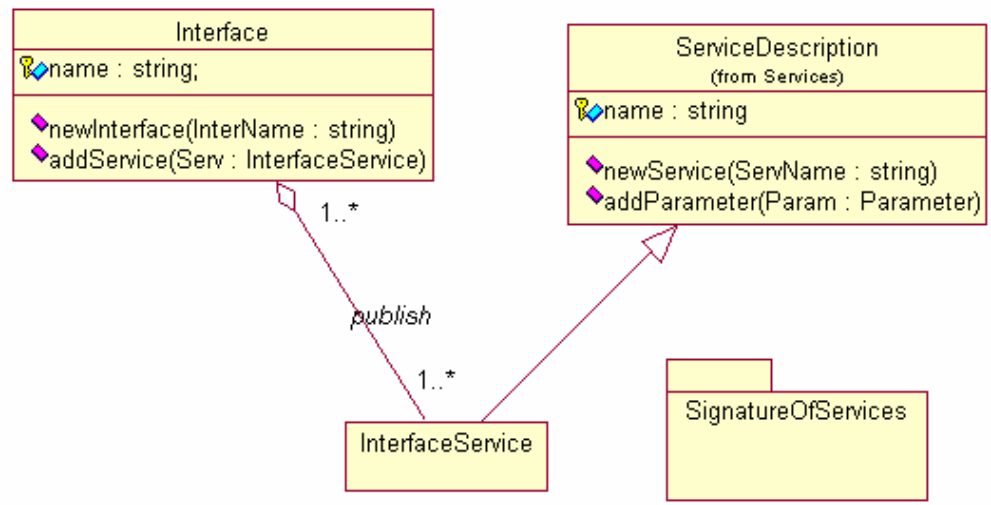

Figure 45. The package Interfaces of the PRISMA metamodel

The signature of a service specifies its name and parameters. The parameters are defined in a specific order. The data type and kind (input/output) of parameters are also defined (see Figure 46).

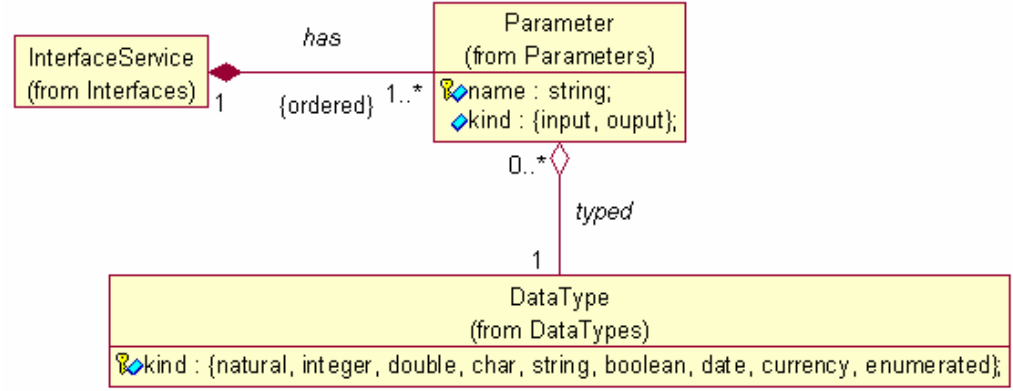

Figure 46. The package SignatureOfService of the PRISMA metamodel

The metaclass InterfaceService inherits its properties from the metaclass ServiceDescription. This class allows the creation of services using the service newService, whose parameter defines the name of the service that is created. The attribute name stores the value of the ServName parameter of newService. The service addParameter adds parameters to services (see the Parameter metaclass in Figure 76). 
The metaclass Interface creates interfaces. The service newInterface creates a new interface, whose parameter defines the name of the interface that is created. The service addService adds a service to the interface, whose parameter provides the InterfaceService that is added to the interface.

\subsubsection{The package "Aspects"}

An aspect defines structure and behaviour of a specific concern of the software system. The Aspects package includes all the metaclasses that are necessary to specify an aspect. The structure and the behaviour of aspects are defined by attributes, services, preconditions, valuations, constraints, played roles and protocols. These concepts are sub-packages of the Aspects package (see Figure 47).
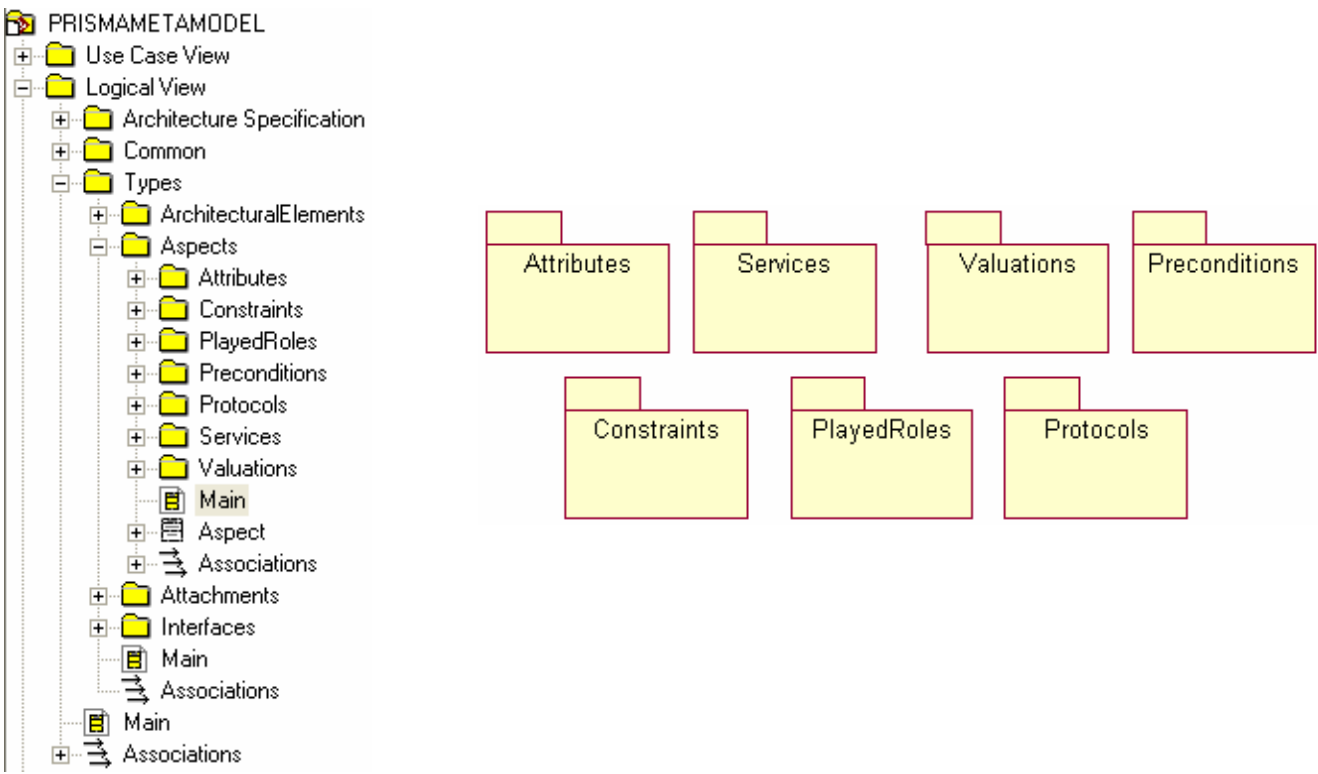

Figure 47. The sub-packages of the package Aspects of the PRISMA metamodel

The metaclass Aspect (see Figure 48) has two attributes, name and concern. These attributes store the name of the aspect and the concern that the aspect belongs to, respectively. The service newAspect creates a new aspect, whose parameters define the name, the concern and the protocol of the aspect. 


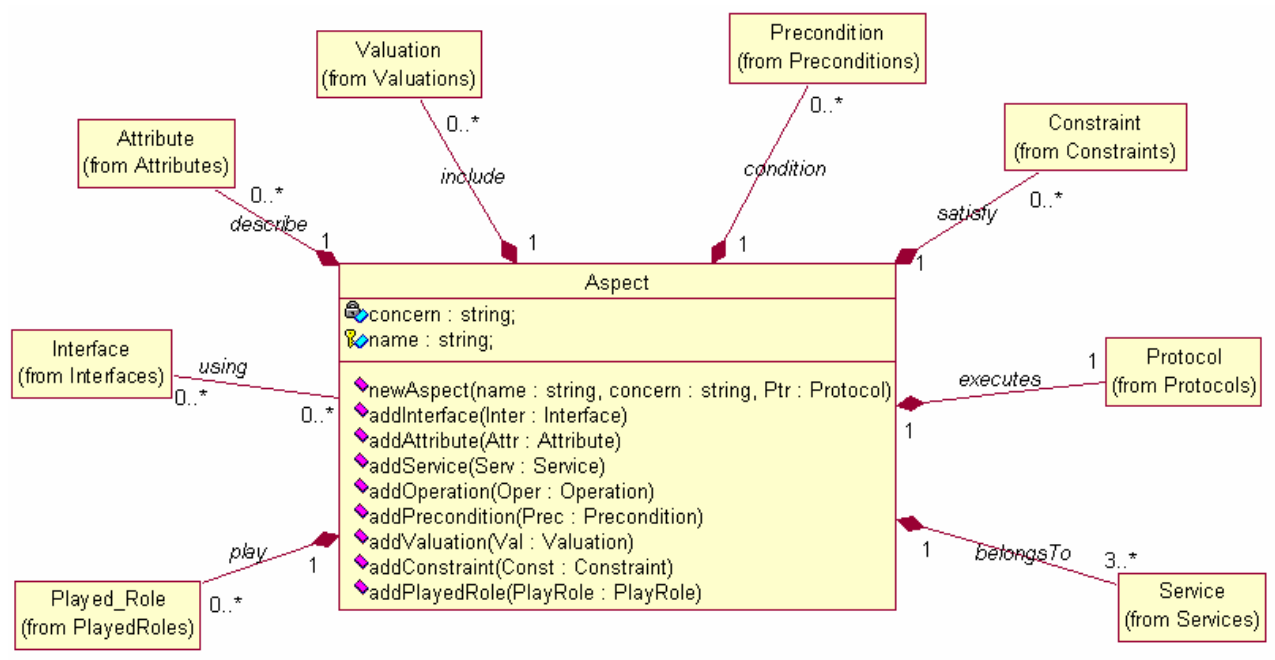

Figure 48. The metaclass Aspect of the package Aspects of the PRISMA metamodel

Aspects may need to store information to successfully perform their computation. For this reason, the Aspect metaclass has an aggregation relationship with the Attribute metaclass. This relationship aggregates the attributes that an aspect is composed of (see the describe aggregation relationship in Figure 48). This aggregation is established by invoking the addAttribute service. This service adds attributes to an aspect through its Attr parameter by providing the attribute that is added to the aspect. Aspects may need to constrain the value of attributes. For this reason, an aspect can be composed of constraints that determine the value of aspect attributes (see the satisfy aggregation relationship in Figure 48).

Aspects must be composed of three or more services. The three services that are required are the following: The services begin and end to start and finish the execution of the aspect, and at least one service to perform the necessary computations of an aspect (see the belongsTo aggregation relationship in Figure 48). Aspect services can be private or public. Public services of an aspect are those that are published by an interface whose semantics is defined by the aspect. As a result, aspects import the interfaces whose semantics they define (see the using association relationship in Figure 47). In order to associate interfaces and services to aspects, 
the aspect metaclass provides the addInterface and addService services, whose Inter and Serv parameters provide the interface and the service that are added to aspects.

In order to define the semantics of aspect services, aspects are composed of preconditions, valuations, played_roles and a protocol (see the aggregation relationships condition, include, play and executes in Figure 48). For this reason, the Aspect metaclass has three services to associate preconditions, valuations, and played_roles to aspects. These services are addPrecondition, addValuation, and addPlayedRole, respectively.

In addition to these attributes, services, and relationships, the metaclass Aspect has an associated set of constraints to completely model its properties (see Figure 49).

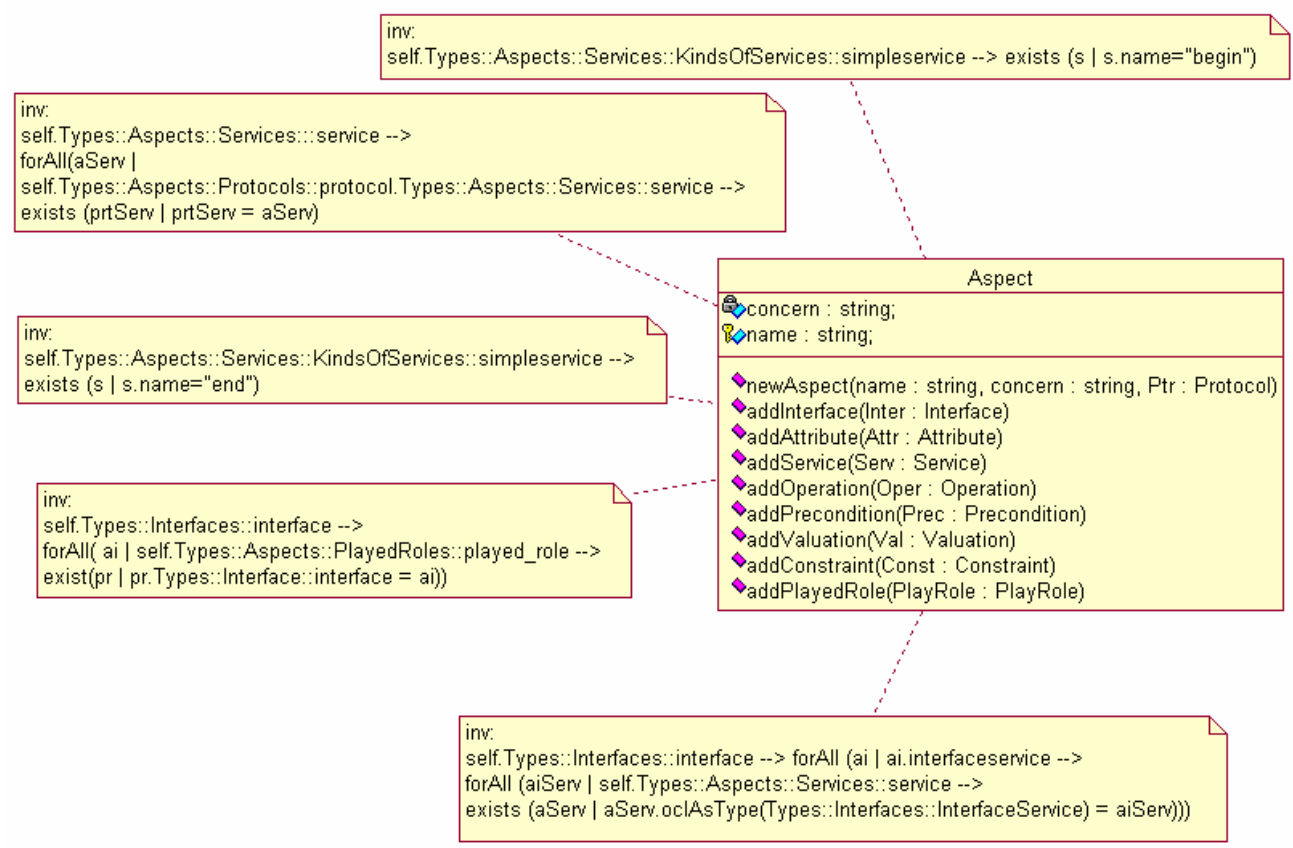

Figure 49. Constraints of the metaclass Aspect

These constraints correspond to the OCL rules shown in Figure 49. They specify the following:

$<<$ Every aspect has a "begin" service $>>$

$<<$ Every aspect service must participate in the protocol of the aspect $>>$

$<<$ Every aspect has an "end" service $>>$ 
PRISMA: Aspect-Oriented Software Architectures

$<<$ For each interface that an aspect imports, the aspect must define at least a played_role associated to this interface $>>$

$<<$ Every service of an interface that is imported by an aspect must be a service of the aspect $>>$

\subsubsection{The package "Attributes"}

Attributes store a value of a specific data type. Therefore, each aspect attribute must be associated to a data type (see Figure 50).

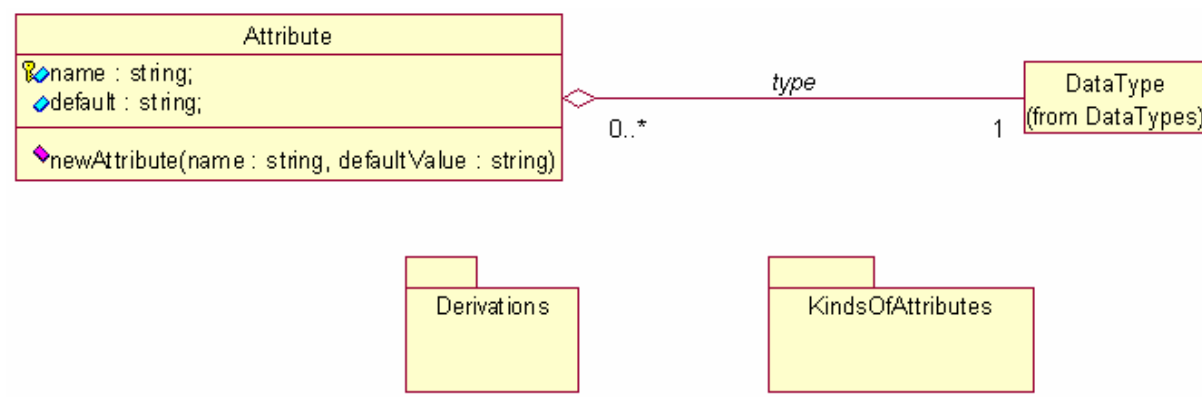

Figure 50. The package Attributes of the PRISMA metamodel

The metaclass Attribute has two attributes, name and default. The attribute name stores the name of the aspect and the sttribute default stores the default value of the attribute when necessary. The service newAttribute creates a new attribute, whose parameters define the name and the default value of the attribute.

In PRISMA, it is possible to define different kinds of attributes. The semantics of each kind is defined in the kindsofAttributes sub-package of the Attributes package. Attributes can be classified into derived and non-derived attributes (see Figure 51). Derived attributes calculate their values on demand by applying a derivation rule. The derivation rule is associated to the derived attribute (see Figure 52). Non-derived attributes store their values, and it is possible to constrain the fact that they must contain a value by means of the notNull attribute (see the NonDerivedAttribute metaclass in Figure 51). If notNull is true, the attributes must contain a value; if notNull is false, no value is required. In order to correctly define the semantics of non- 
derived attributes, there are two constraints associated to the NonDerivedAttribute metaclass. These constraints correspond to the OCL rules shown in Figure 51. They specify the following: $<<$ For each non-derived attribute that cannot contain a null value, there is a postcondition of the begin service valuation that must provide a value to the attribute $>>$

$<<$ The"notNull" attribute of a constant attribute is always true $>>$

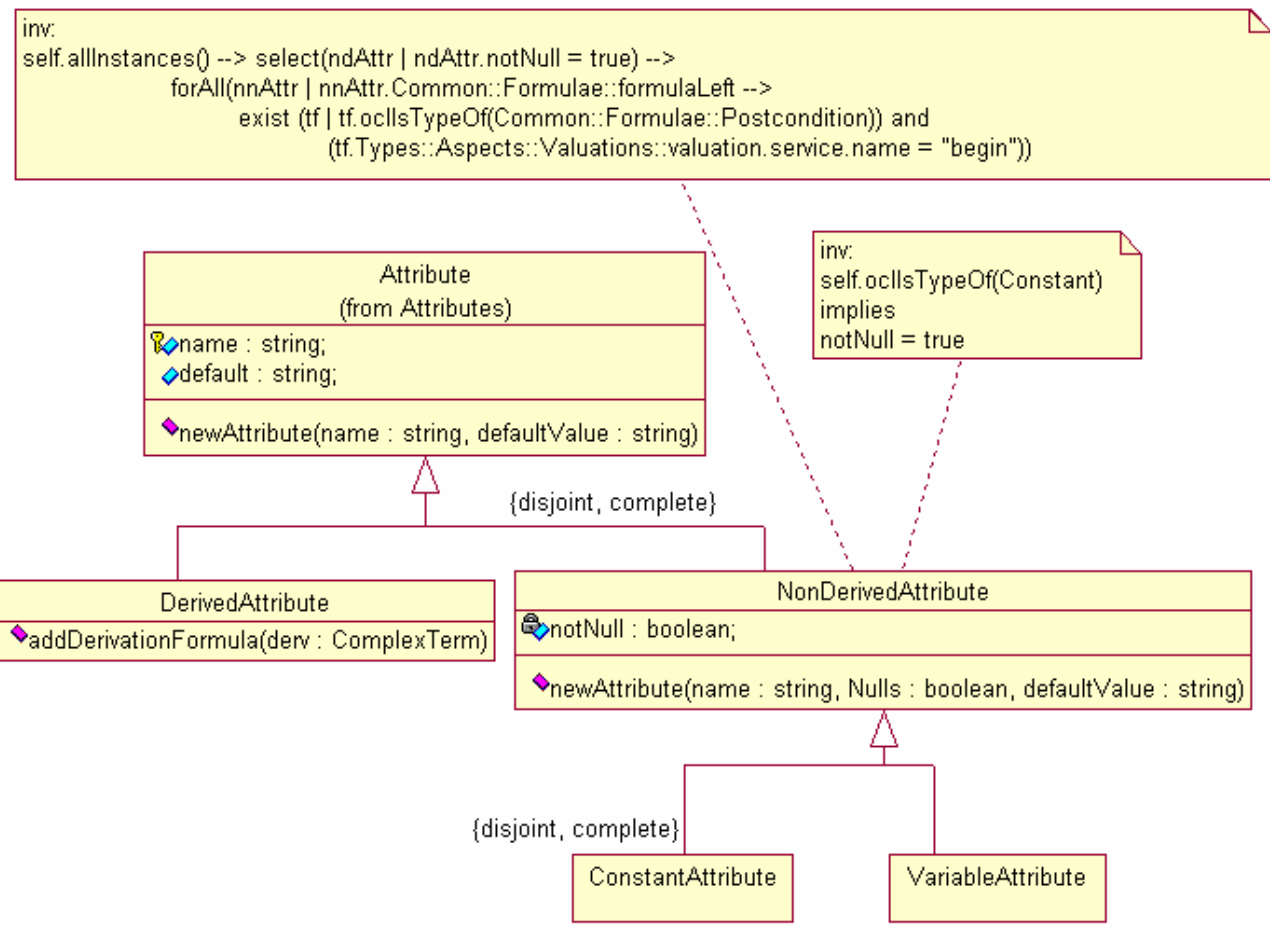

Figure 51. The package KindsOfAttributes of the PRISMA metamodel

Non-derived attributes can be constant or variable. Constant attributes store values that cannot change; i.e., they cannot be modified during the execution of the aspect. Also, variable attributes store values that can be modified during the execution of the aspect.

\begin{tabular}{|c|c|c|c|}
\hline DerivedAttribute & & calculate & ComplexTerm \\
\hline
\end{tabular}

Figure 52. The package Derivations of the PRISMA metamodel 
PRISMA: Aspect-Oriented Software Architectures

\subsubsection{The package "Services"}

The metaclass Service is a specialization of the metaclass InterfaceService (see Figure 53). As a result, it inherits all its properties and services. The metaclass Service defines that every service of an aspect must be characterized by the behaviour that it offers in the context of the aspect. This means that the service can either be provided, requested or both by the aspect. This characteristic is specified by the type attribute of the metaclass. The type values are in, out and in/out to define the behaviour of a server (provide), a client (request), or both a server and aclient (provide and request), respectively. A service of an aspect can also have an alias. An alias permits changing the name of an InterfaceService inside the aspect. This metaclass stores the alias name and provides the newAlias service to change the name. The parameters of newAlias are the service whose name is going to be changed and the new alias.

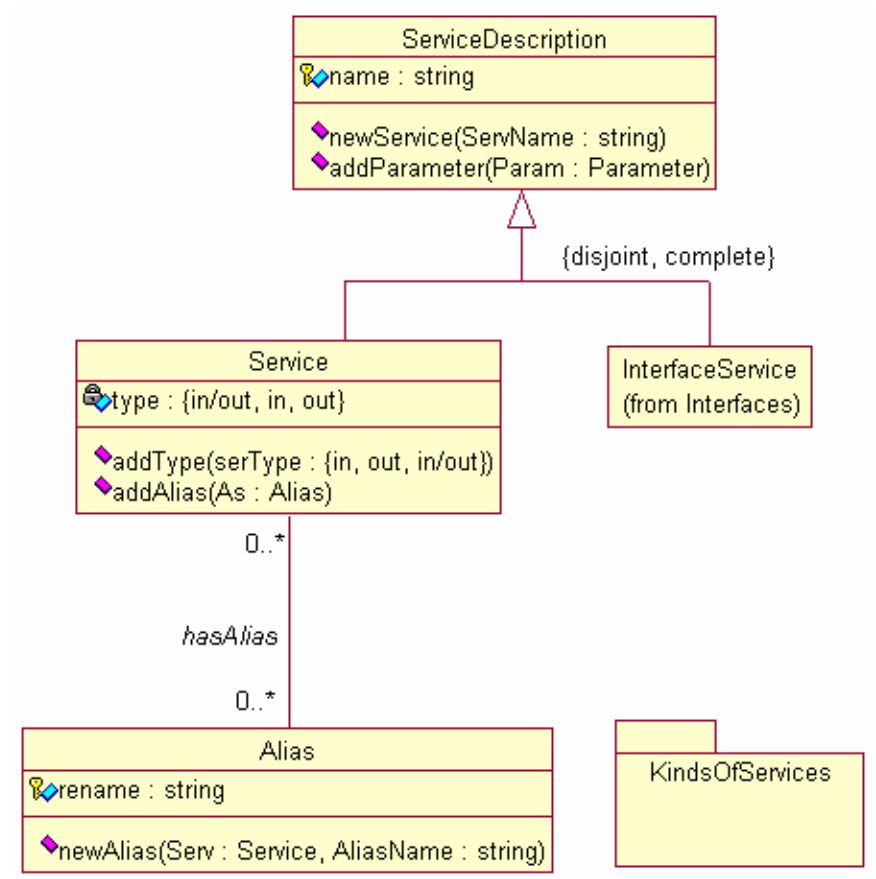

Figure 53. The package Services of the PRISMA metamodel

There are two kinds of services: simple services and transactions (see Figure 54). A transaction is a complex service that it is composed of more than one service and is executed in a transactional way (all or nothing) (see the composedService aggregation in Figure 54). A 
transaction describes a process, which models how and when the different services that compose the transaction are executed. As a result, a transaction is a specialization of the Process metaclass (see Figure 80).

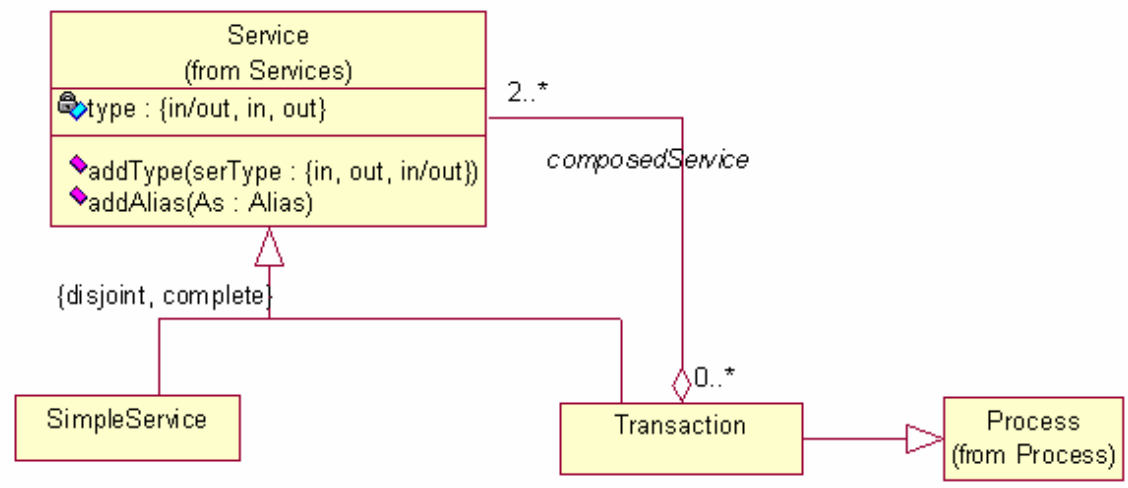

Figure 54. The package KindsOfServices of the PRISMA metamodel

\subsubsection{The package "Constraints"}

Constraints are formulae that establish conditions on the state of the aspect that they belong to.

As a result, each time that a service execution is finished, the value of each attribute must satisfy the aspect constraints. As explained in (see section 6.2.4), there are two kinds of constraints: static and dynamic (see Figure 55).

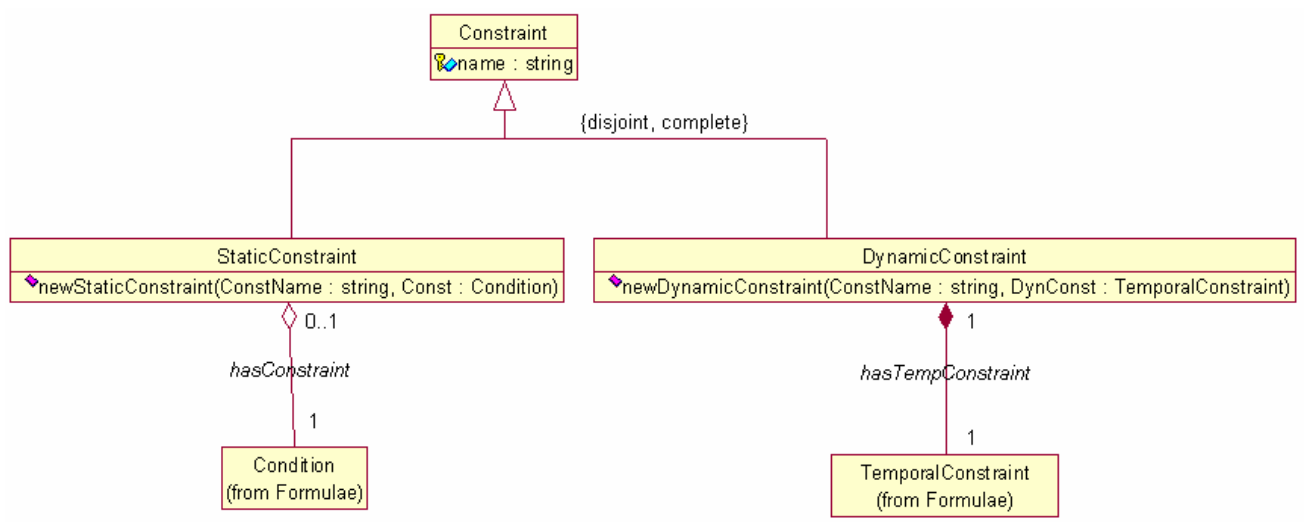

Figure 55. The package Constraints of the PRISMA metamodel

The metaclass Constraint is an abstract class that has only one attribute, the name of the constraint. This metaclass is specialized into two metaclasses: StaticConstraint and 
PRISMA: Aspect-Oriented Software Architectures

DynamicConstraint. Each one of them provides a constructor service to create instances of static and dynamic constraints, respectively. The service newStaticConstraint creates a new static constraint giving the name and the condition of the constraint as parameters. The service newDynamicConstraint creates a new dynamic constraint, whose parameters define the name of the constraint and a condition that uses a temporal operator (see the Formulae package in Figure 78).

\subsubsection{The package "Preconditions"}

Preconditions establish the condition that must be satisfied to execute an aspect service. Therefore, the metaclass Precondition has the aggregation relationship establishCondition and aggregation relationship constrains with the metaclasses Condition and Service, respectively (see Figure 56). The first aggregation establishes that a precondition must define the service that it affects. The second aggregation establishes the condition that must be satisfied to execute the service.

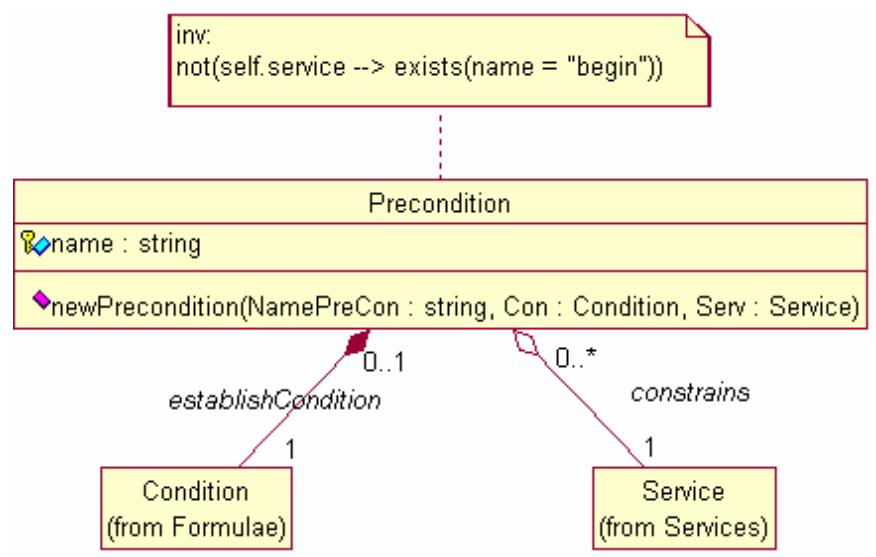

Figure 56. The package Preconditions of the PRISMA metamodel

The metaclass Precondition only has one attribute, the name of the precondition. The service newPrecondition creates a new precondition, whose parameters define the name, the condition that must be satisfied, and the service that will only be executed if the condition is satisfied.In addition, the metaclass Precondition has an associated constraint in order to ensure that the aspect execution is not conditioned by a precondition. This constraint corresponds to the OCL rule shown in Figure 56. It specifies the following: 
The PRISMA Metamodel

$<<A$ service begin does not have preconditions associated to it since the start of the aspect execution cannot be conditioned by the aspect itself $>>$

This constraint is necessary because preconditions are used to define the business logic of the software system and not to define the mechanisms of creating, destroying or executing instances.

\subsubsection{The package "Valuations"}

Valuations establish how the service executions affect the aspect state. This semantics is specified by means of two conditions: one that must be satisfied before the service execution and another that must be satisfied after the service execution. For this reason, the metaclass Valuation has three aggregation relationships with the metaclasses Condition, Service and Postcondition (see Figure 57).

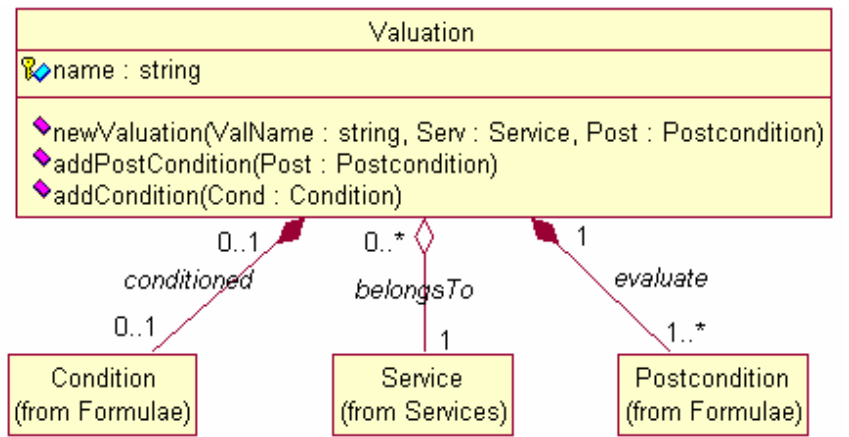

Figure 57. The package Valuations of the PRISMA metamodel

The metaclass Condition defines the condition that specifies the state of the aspect before the service execution. This condition is optional, this means it does not have to be specified when the state before the execution is not relevant to the state change (see the conditioned aggregation in Figure 57). However, the specification of the condition after the service execution is mandatory. The postcondition must be satisfied after the service execution. Since the metaclass Postcondition defines the change in one attribute or parameter and a valuation can affect several attributes or parameters, a valuation can have more than one postcondition associated to it in order to model the service changes in several attributes and/or parameters (see the evaluate aggregation in Figure 57). 
PRISMA: Aspect-Oriented Software Architectures

The metaclass Valuation has only one attribute, the name of the valuation. The service newValuation creates a new valuation, whose parameters define the name, the service that produces the change of state, and the condition that must be satisfied after the service execution. Moreover, it has two services addCondition and addPostCondition. The addCondition adds a condition to the valuation. This condition must be satisfied before the service execution. The addPostCondition adds more that one postcondition when the valuation affects several attributes or parameters.

\subsubsection{The package "PlayedRoles"}

PlayedRoles establish how the services of an interface can be executed. As a result, a played_role defines a process that orchestrates the service execution of a specific interface. Since the metaclass Played_Role defines a process, it inherits the properties of the metaclass Process (see the Processes package in Figure 80).

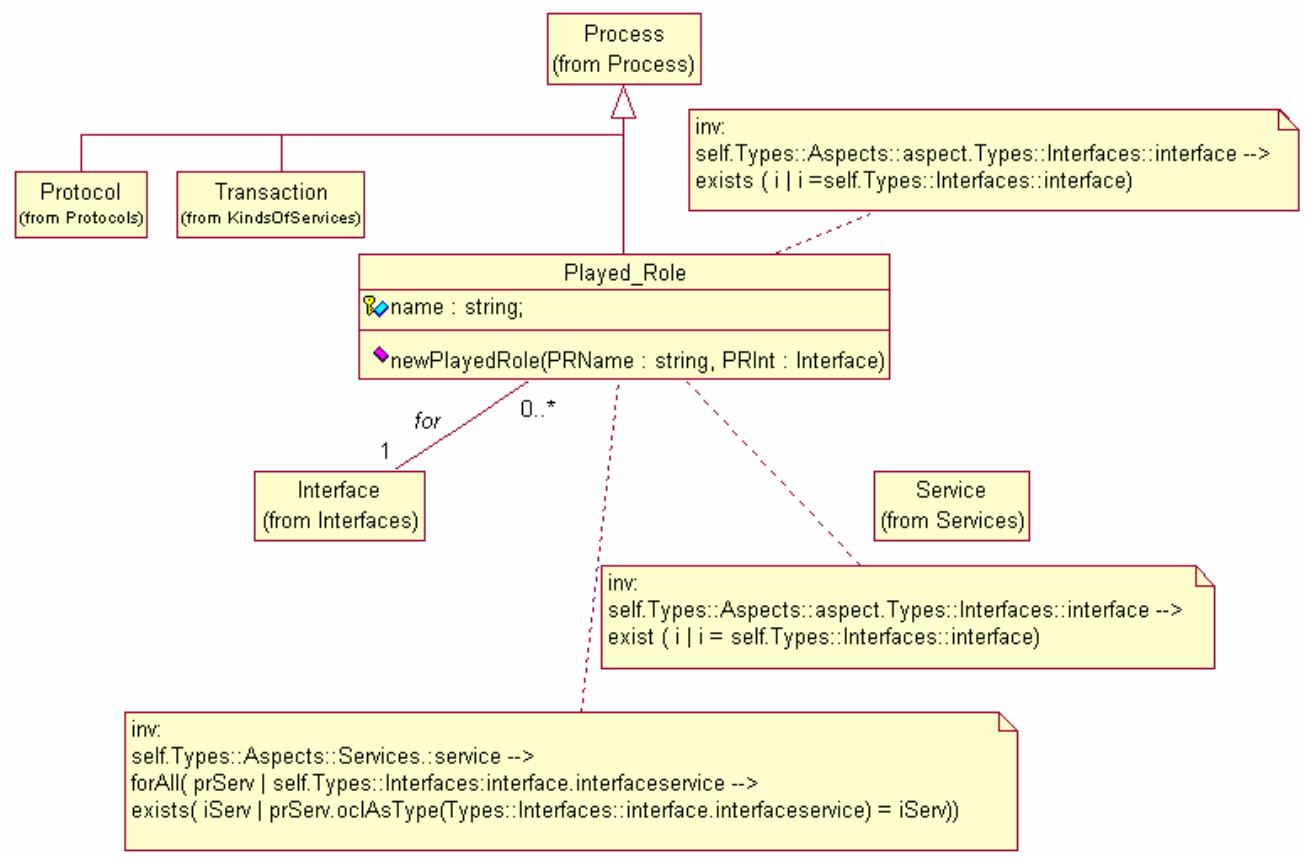

Figure 58. The package PlayedRoles of the PRISMA metamodel

The metaclass Played_Role has two association relationships with the metaclasses Interface and Service (see Figure 58). A played_role defines the behaviour of only one interface (see the 
the for association in Figure 58) and describes the execution process of more than one service (see the order association in Figure 58). However, the played_role cannot be related to any interface or service of the software system. As a result, these relationships are constrained by three constraints. These constraints correspond to the OCL rules shown in Figure 58. They specify the following:

$<<$ Every interface that an aspect imports must have associated a played_role $>>$

$<<$ The interface of a played role is one of the interfaces that imports the aspect that the played_role belongs to $>>$

$<<$ Every service that participate in a played_role must be a service of the played_role interface $>>$

The metaclass Played_Role has one attribute, the name of the played_role. The service newPlayedRole creates a new played_role, whose parameters define the name and the interface. The behaviour of this interface is defined by the played role.

\subsubsection{The package "Protocols"}

A protocol establishes how the services of an aspect can be executed. As a result, a protocol defines a process that coordinates the private and public services of an aspect. Since the metaclass Protocol defines a process, it inherits the properties of the metaclass Process (see the Processes package in Figure 80).

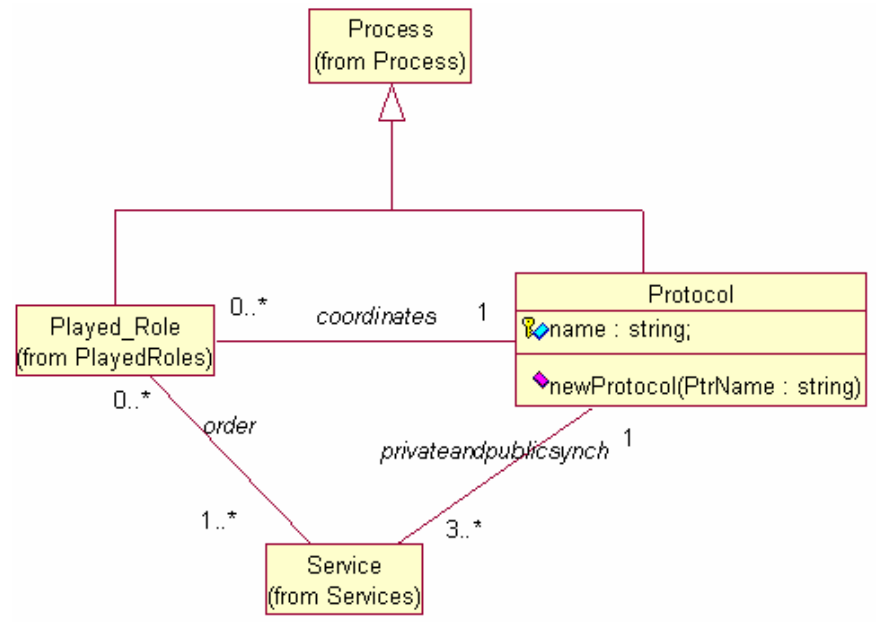

Figure 59. The package Protocols of the PRISMA metamodel 
PRISMA: Aspect-Oriented Software Architectures

A service can be private or public and only belongs to one aspect. For this reason, a service can only participate in one protocol: the protocol of the aspect that it belongs to (see the privateandpublicsynch association Figure 59). In addition, each service of the aspect must participate in its protocol (see the constraint in the Aspect package in Figure 49). These services can be either private or public services, but there must be at least three: the begin and end services of an aspect, and one service to perform the computation of the aspect (see the privateandpublicsynch association in Figure 59).

A protocol is the glue of the played_roles and the private services of the aspect. As a result, the protocol coordinates the many different played_roles that have been defined in the aspect that it belongs to. However, a played_role is only coordinated one protocol, its aspect protocol (see the coordinates association in Figure 59). Played_roles are specified using the public services of an aspect. Since aspect services can be private or public, those that are private are not related to played_roles (see the order association in Figure 59).

The metaclass Protocol has one attribute, the name of the protocol. The service newProtocol creates a new protocol by providing the name of the protocol as a parameter.

\subsubsection{The package "ArchitecturalElements"}

In PRISMA, there are three kinds of architectural elements: components, connectors, and systems (see Figure 60). The package ArchitecturalElements defines the metaclass ArchitecturalElement. It is an abstract metaclass that specifies the commonalities of the three kinds of PRISMA architectural elements. In addition, it includes all subpackages that define the concepts required to specify PRISMA architectural elements.

The metaclass ArchitecturalElement has two aggregation relationships with the metaclassess Port and Weaving, and one association relationship with the metaclass Aspect (see Figure 61). An architectural element has at least one port; the port is part of the architectural element and does not have its own entity without the architectural element. In other words, the aggregation between the port and the architectural element is inclusive (see the has aggregation in Figure 61). An architectural element imports at least one aspect and an aspect can be imported by one or more architectural elements of the software system (see the imports 
association in Figure 61). In addition, an architectural element can include a set of weavings to synchronize its aspects. These Weavings are related to the architectural element by means of an inclusive aggregation (see the weaves aggregation in Figure 61).
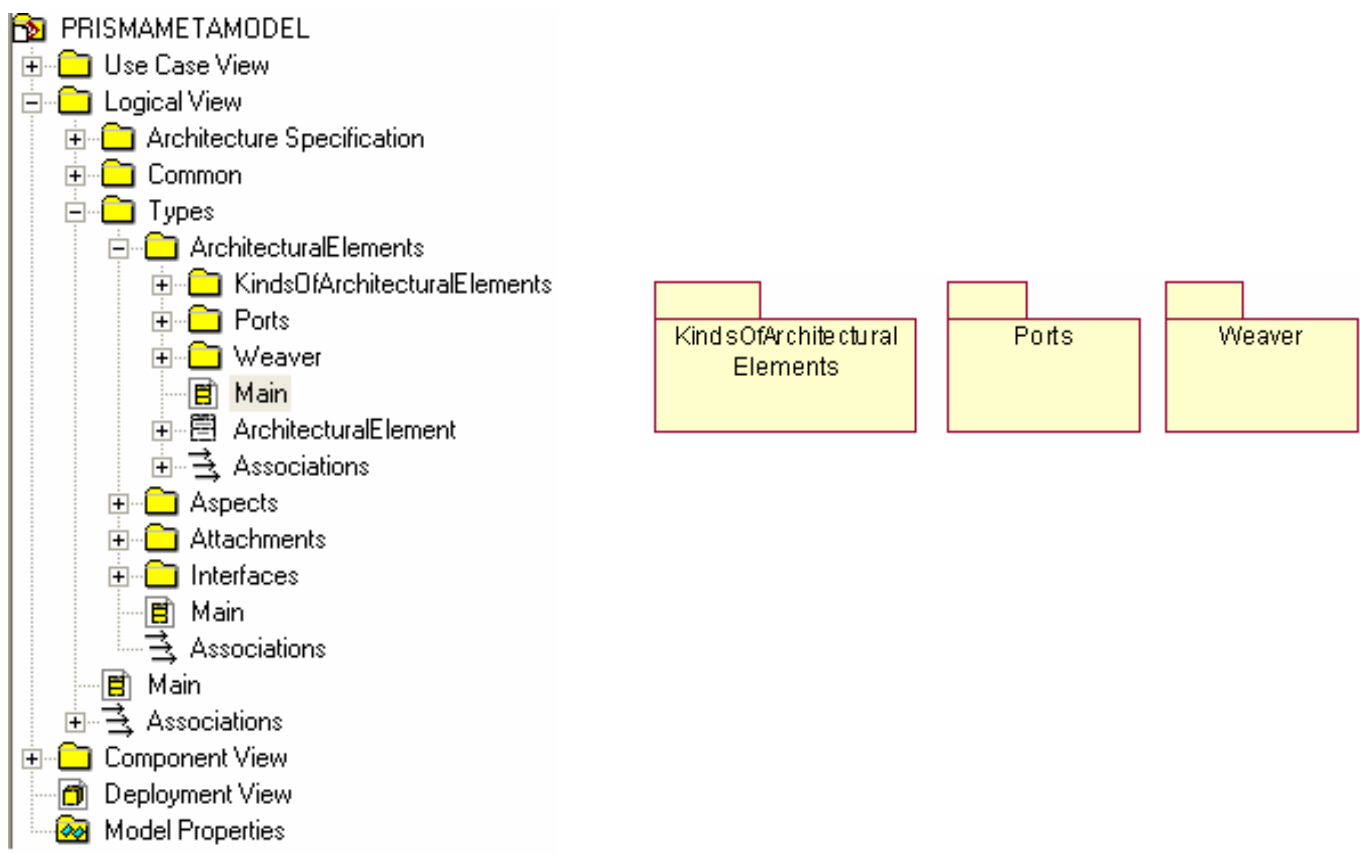

Figure 60. The subpackages of the package ArchitecturalElements of the PRISMA metamodel

The metaclass ArchitecturalElement has one attribute, the name of the architectural element. In addition, it has three services addAspect, addPort, addWeaving to associate aspects, ports, and weavings to the architectural element, respectively (see Figure 61). It is important to emphasize that this metaclass does not have a constructor (new service) because it is an abstract class that cannot be instantiated.

The metaclass ArchitecturalElement has two constraints associated to it in order to completely define its properties. These constraints correspond to the OCL rules shown in Figure 61. They specify the following:

$<<$ There are no two ports of an architectural model that have the same interface and the same played_role associated $>>$

$<<$ An architectural element cannot import more than one aspect of the same concern $>>$ 
PRISMA: Aspect-Oriented Software Architectures

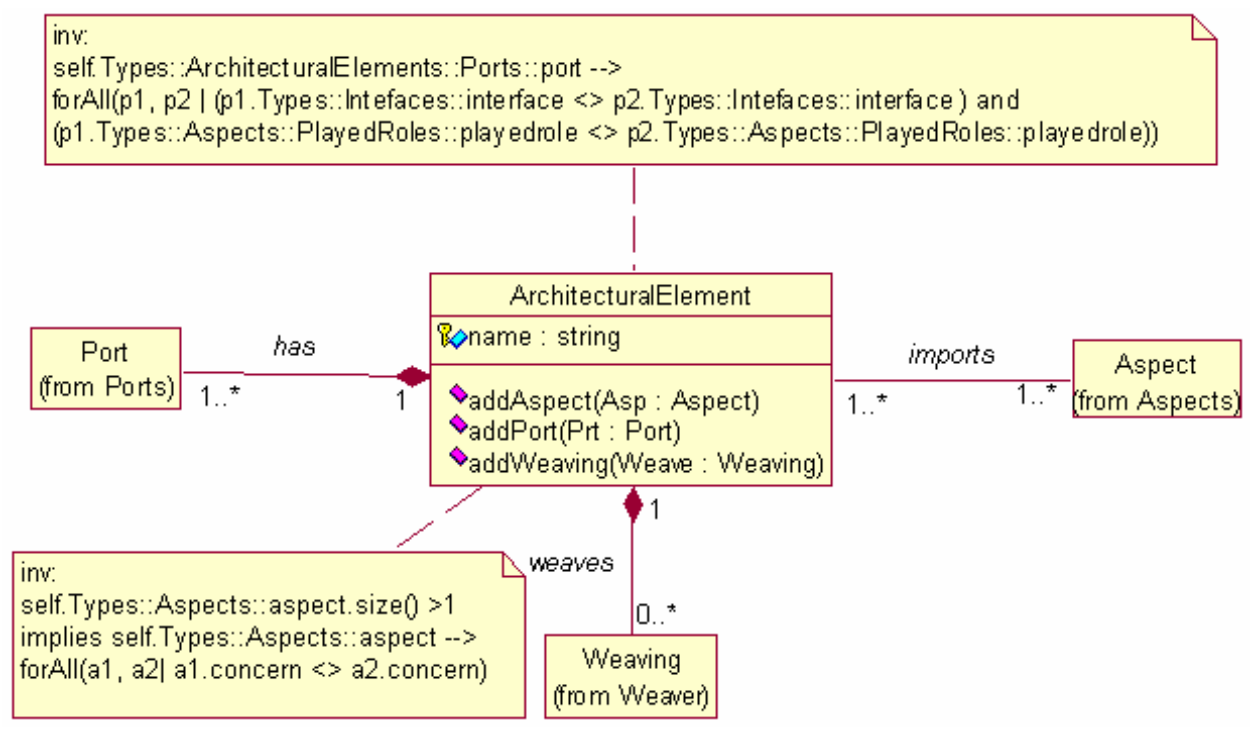

Figure 61. The package ArchitecturalElements of the PRISMA metamodel

The package KindsOfArchitecturalElements is a subpackage of the package ArchitecturalElements, and it classifies architectural elements into components and connectors. As a result, this package specifies that components and connectors inherit the properties of the ArchitecturalElement metaclass, and it also contains the packages that define components and connectors.

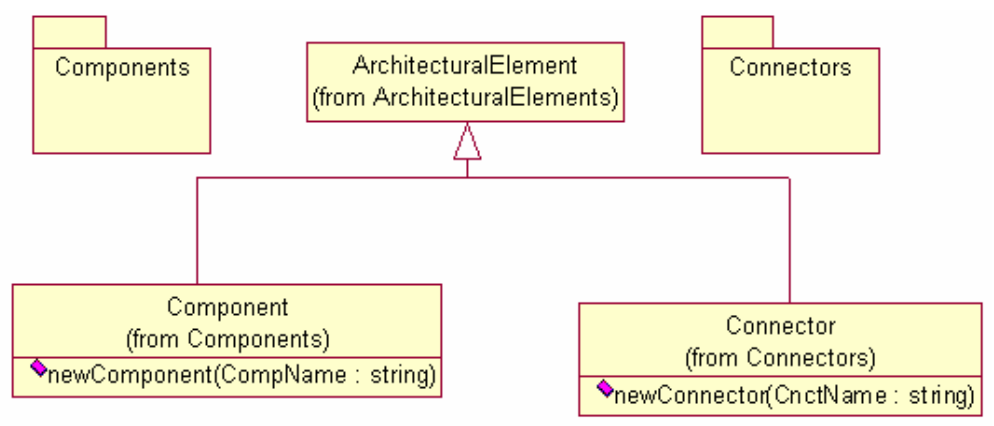

Figure 62. The package KindsOfArchitecturalElements of the PRISMA metamodel 
The PRISMA Metamodel

\subsubsection{The package "Weaver"}

The package Weaver defines the weavings of architectural elements. It contains the metaclass weaving which is formed by two aspect services. One of the services, the pointcut service, triggers the execution of the weaving; the other service, the advice service, is executed as a consequence of the weaving. The relationships between the weaving and these two services are modelled in the metamodel by means of two aggregations (see Figure 63). In addition, if the weaving is conditional, it has a condition associated to it.

There are certain constraints that must be satisfied in order to associate the appropiate services to a weaving definition. As a result, the metaclass Weaving has constraints associated to it. These constraints correspond to the OCL rules shown in Figure 64. They specify the following:

$<<$ The services that participate in the weaving must belong to aspects that are imported by the architectural element in which the weaving is defined $>>$

$<<$ If the weaving uses a conditional operator, it must have a condition associated to it. However, if the weaving does not use a conditional operator, it cannot have a condition associated to it $>>$

$<<$ The services that participate in a weaving must belong to different aspects $>>$

$<<$ The aspects of the services that participate in a weaving must define different concerns $>>$

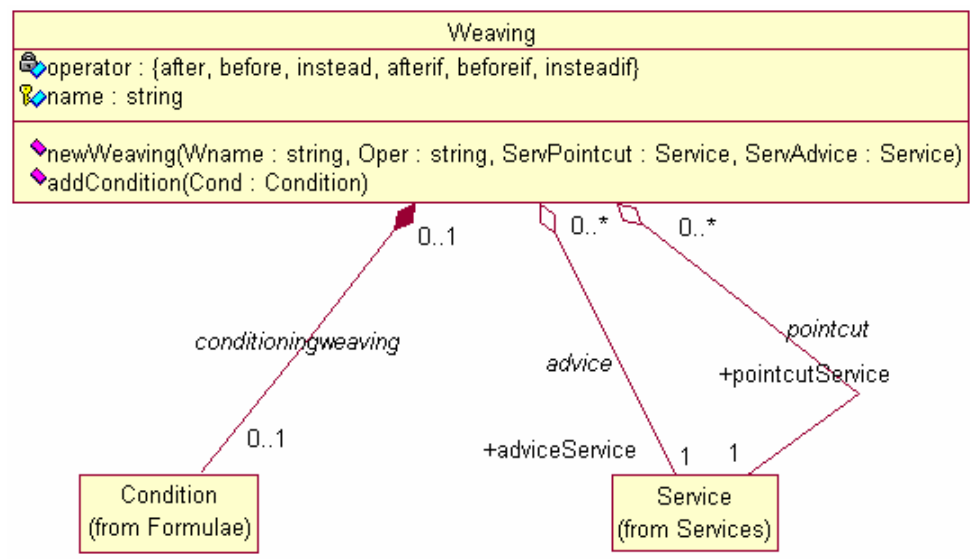

Figure 63. The package Weaver of the PRISMA metamodel 
PRISMA: Aspect-Oriented Software Architectures

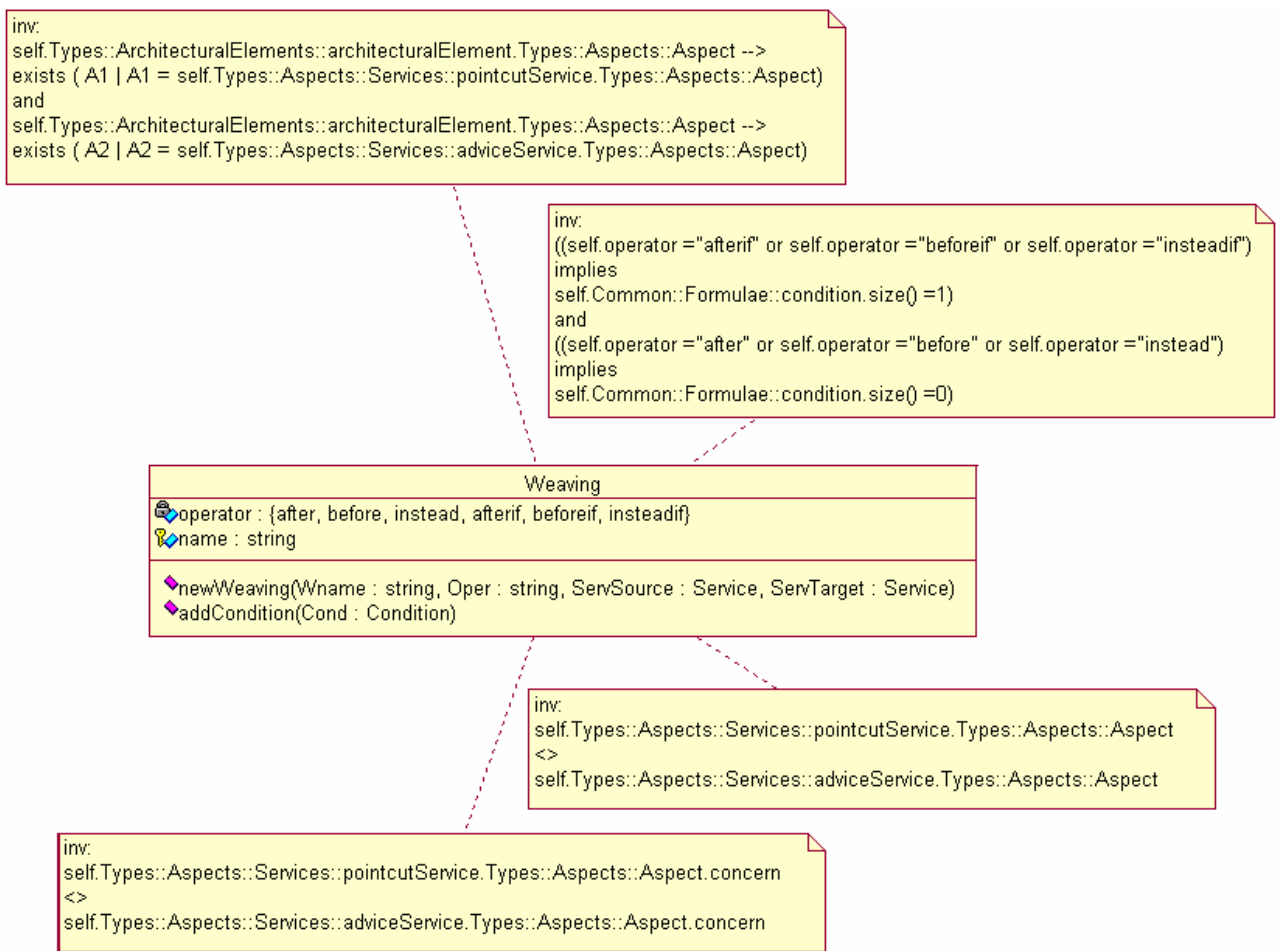

Figure 64. Constraints of the metaclass Weaving

The metaclass Weaving has two attributes, name and operator (see Figure 63). These store the name of the aspect and the operator that the weaving applies to the service execution, respectively. The service newWeaving creates a new aspect; whose parameters define the name, the operator of the weaving, and the two services that participate in the weaving. In addition, the metaclass provides a service for adding a condition to a weaving when it uses a conditional operator. This service is called addCondition.

\subsubsection{The package "Components"}

The package Components defines simple and complex components (see Figure 65). Since components cannot be coordinators of the software system, there is a constraint that specifies that a component cannot import an aspect whose concern is coordination. 
The metaclass Component provides a service to create components. This service is called newComponent, and its parameter is the name of the component that is created as a result of the service execution.

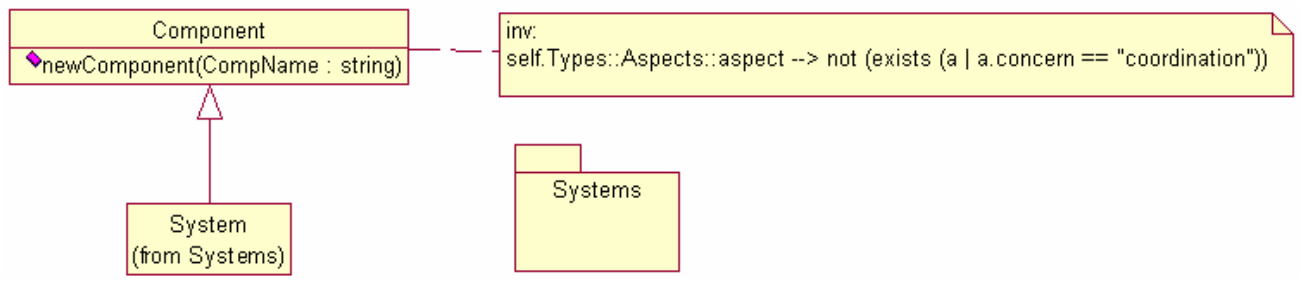

Figure 65. The package Components of the PRISMA metamodel

Since systems are complex components, they inherit all the properties of components. For this reason, the package that defines a system is a subpackage of the Component metaclass.

\subsubsection{The package "Connectors"}

The package Connectors defines the connector architectural element (see Figure 66). Since connectors act as coordinators of components, the metaclass Connector has an associated constraint that specifies that a connector must import an aspect whose concern is coordination (see the first constraint that appears in Figure 66).

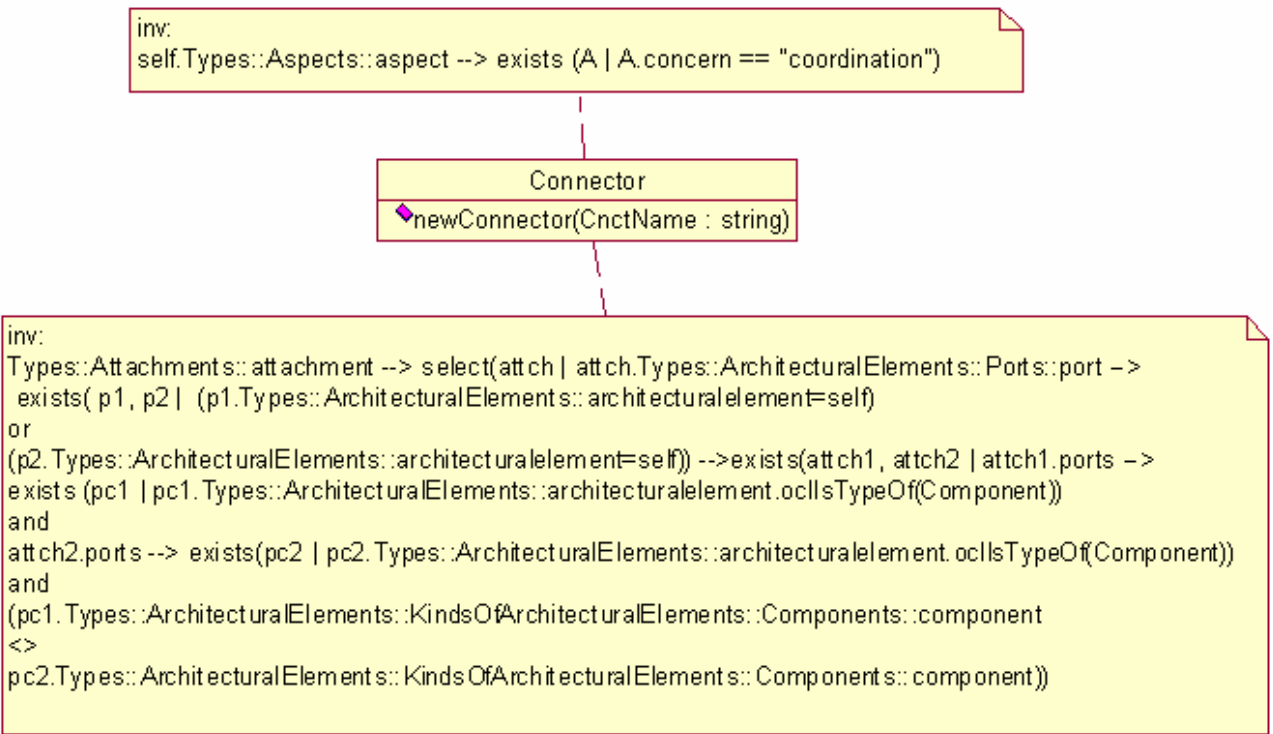

Figure 66. The package Connectors of the PRISMA metamodel 
PRISMA: Aspect-Oriented Software Architectures

Morevover, the metaclass Connector has another constraint associated to it that specifies the following:

$<<$ A connector must have at least two attachments associated to it, and each attachment must connect the connector to two different components $>>$

The metaclass Connector provides a service to create connectors. This service is called newConnector, and its parameter is the name of the connector that is created as a result of the service execution

\subsubsection{The package "Attachments"}

Attachments define types of communication channels between the ports of a component and the port of a connector. As a result, the metaclass Attachment is related to the metaclass Port by means of an association relationship (see Figure 73). This relationship establishes that the attachment must be related to two ports. However, it is necessary to constrain this association with a constraint in order to establish that one of the ports must belong to a component and that the other one must belong to a connector.

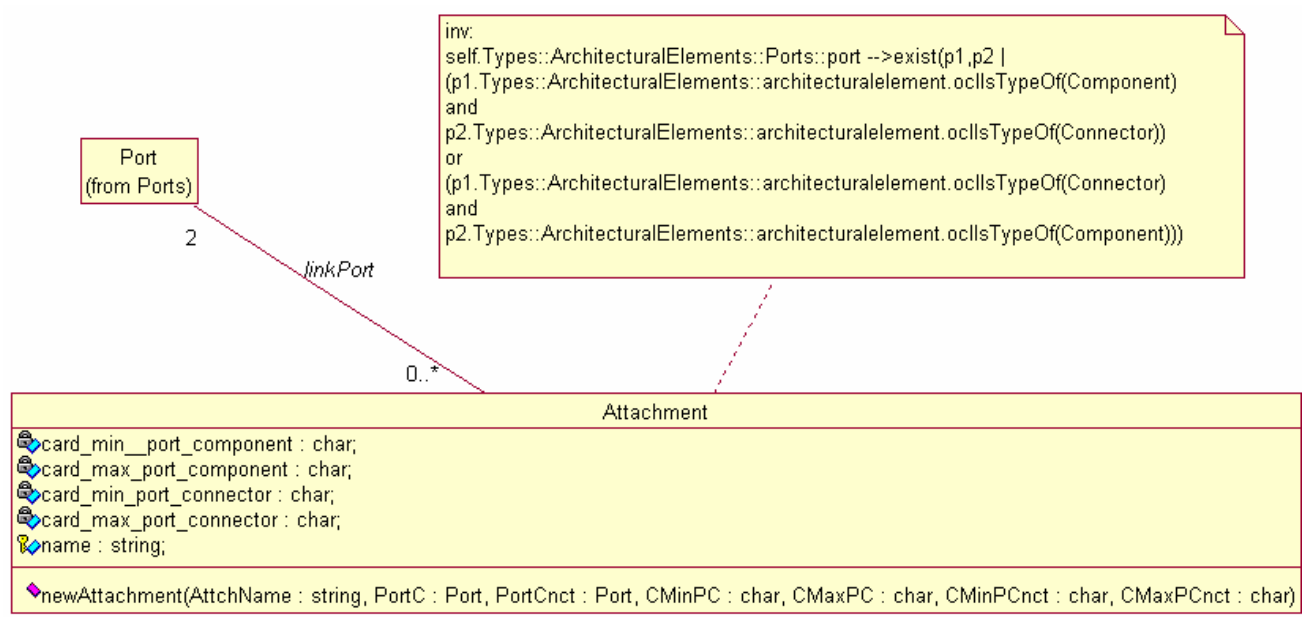

Figure 67. The package Attachments of the PRISMA metamodel

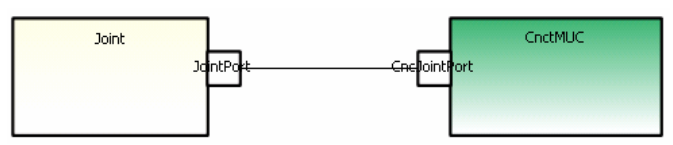

Figure 68. The attachment between the Joint and the CnctMUC of the TeachMover 


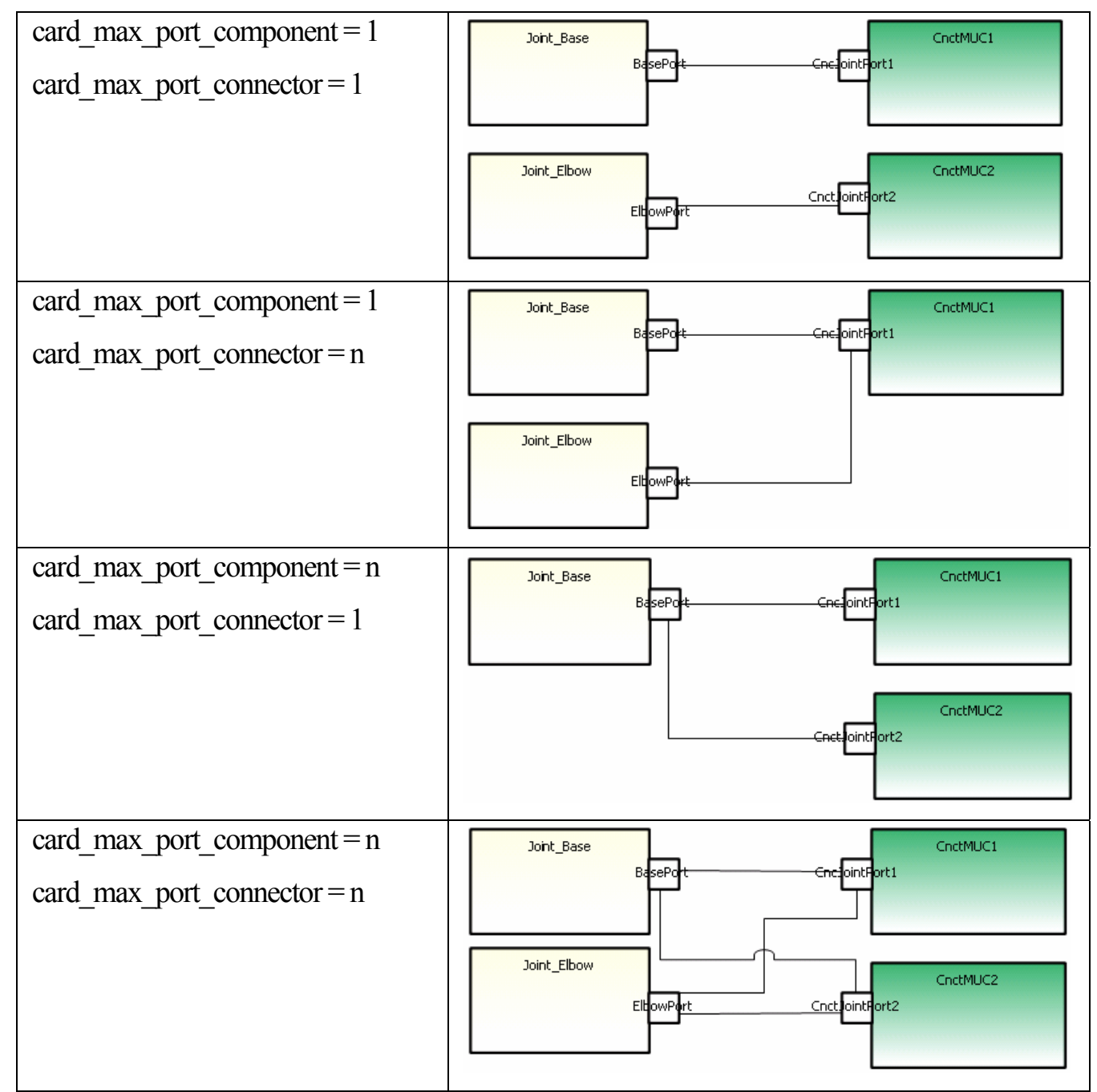

Figure 69. Different configuration of an architecture depending on the maximum cardinality of an attachment

In addition to the attachment name for storing the name of the attachment, the metaclass Attachment has four more attributes to specify the attachment communication pattern, i.e., the instantiation pattern of the attachment. It is necessary to constrain how many instances of the attachment can be attached to the port of the component instance and the port of the connector instance. The attribute card_min_port_component specifies the minimum number of attachment instances that must be connected to one instance of this component through the 
PRISMA: Aspect-Oriented Software Architectures

port. The attribute card_max_port_component specifies the maximum number of attachment instances that must be connected to one instance of this component through the port. The attribute card_min_port_connector specifies the minimum number of attachment instances that must be connected to one instance of this connector through the port. The attribute card_max_port_connector specifies the maximum number of attachment instances that must be connected to one instance of this connector through the port. Figure 69 shows an example of how the maximum cardinality of the attachment can vary the configuration of the architecture at the instance level. The attachment defined between the Joint system and the CnctMUC connector of the TeachMover architecture is used to exemply this variation (see Figure 35).

Moreover, the metaclass Attachment has the service newAttachment to create a new attachment. Its parameters are the name of the attachment that is created as a result of the service execution, the component port and the connector port that it connects, and the minimum and maximum cardinalities for each one of the ports.

\subsubsection{The package "Systems"}

The package Systems defines complex components (see Figure 70). Systems are complex components that are composed of a set of architectural elements and their attachments. For this reason, the metaclass System has an aggregation with each one of the metaclasses Component,

\section{Connector and Attachment.}

The architectural elements that compose a system can be directly related to other elements or their access can only be possible through the system. These two kinds of composition are referential and inclusive, respectively. The analyst can model any of these compositions for the architectural elements of the system, depending on the requirements of the system. However, the definition of an inclusive composition between a system and an architectural element requires the definition of a channel between a system port and a port of the architectural element. This channel is required to resend the provided and requested services of the architectural element through the system port. These channels are called bindings. Therefore, 
the metaclass System has an aggregation relationship with the metaclass Binding. Bindings are defined in the Bindings subpackage of the System package.

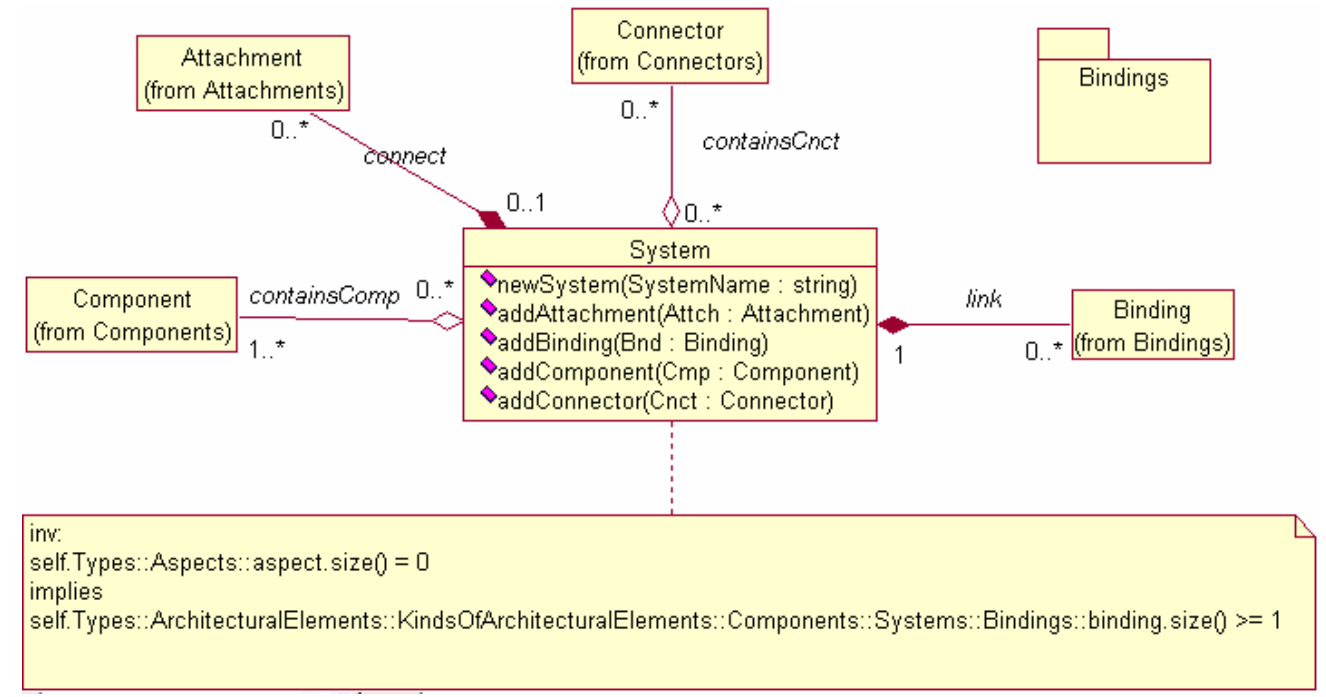

Figure 70. The package Systems of the PRISMA metamodel

The metaclass System must be related to at least one component in order to be complex (see the containsComp aggregation in Figure 70). In addition, it has an associated constraint that ensures a correct composition. It specifies the following:

$<<$ If a system does not import any aspect, the system must have at least one binding associated to it $>>$

The metaclass System has four services. The service newSystem creates a new system by providing the name of the system as a parameter. In addition, the services addComponent, addConnector, addAttachment and addBinding add components, connectors, attachments and bindings, respectively, to the system.

\subsubsection{The package "Bindings"}

The metaclass Binding is related to the metaclass Port by means of two association relationships (see Figure 73). These associations establish that the binding must be related to a system port and an architectural element port. However, the architectural element that the port belongs to must be one of the architectural elements of the system. This constraint is applied 
PRISMA: Aspect-Oriented Software Architectures

not only at the types level, but also at the configuration level (instances). For this reason, the metaclass Binding has an associated constraint that specifies this requirement.

In addition to the attribute name for storing the name of the binding, the metaclass Binding has four more attributes to specify the communication pattern of the binding. As a result, the attribute card_min_port_AR specifies the minimum number of binding instances that must be connected to one instance of this architectural element through the port. The attribute card_max_port_AR specifies the maximum number of binding instances that must be connected to one instance of this architectural element through the port. The attribute card_min_port_Sys specifies the minimum number of binding instances that must be connected to one instance of this system through the port. The attribute card_max_port_Syst specifies the maximum number of binding instances that must be connected to one instance of this system through the port.

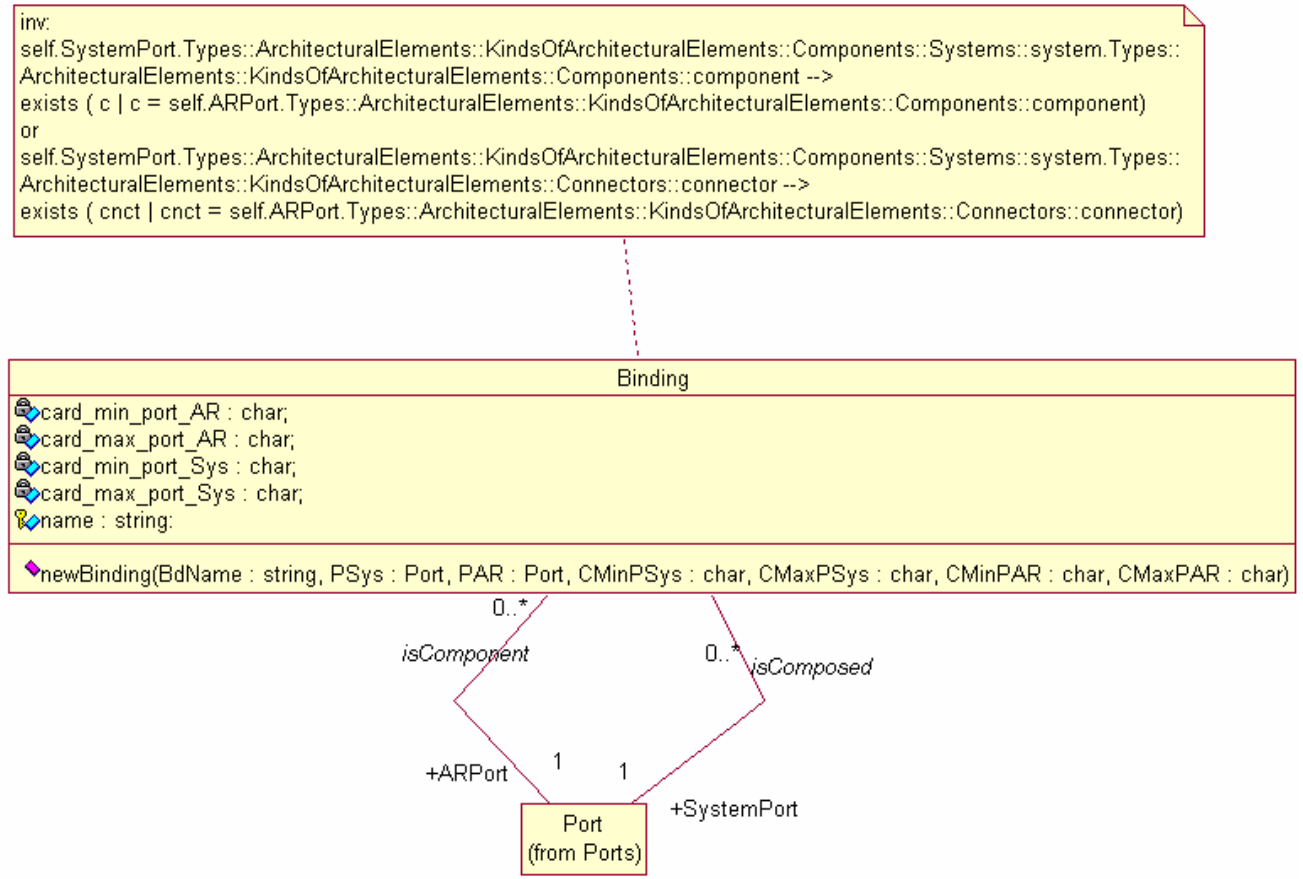

Figure 71. The package Bindings of the PRISMA metamodel 
Moreover, the metaclass Binding has the service newBinding to create a new binding. Its parameters are the name of the binding that is created as a result of the service execution, the system port and architectural element port that it connects, and the minimum and maximum cardinalities for each one of the ports.

\subsubsection{The package "Ports"}

Ports publish the services of an interface and constrain how these services can be provided or requested by means of a played_role. For this reason, the metaclass Port has two aggregation relationships with the metaclasses Interface and Played_Role (see Figure 72).

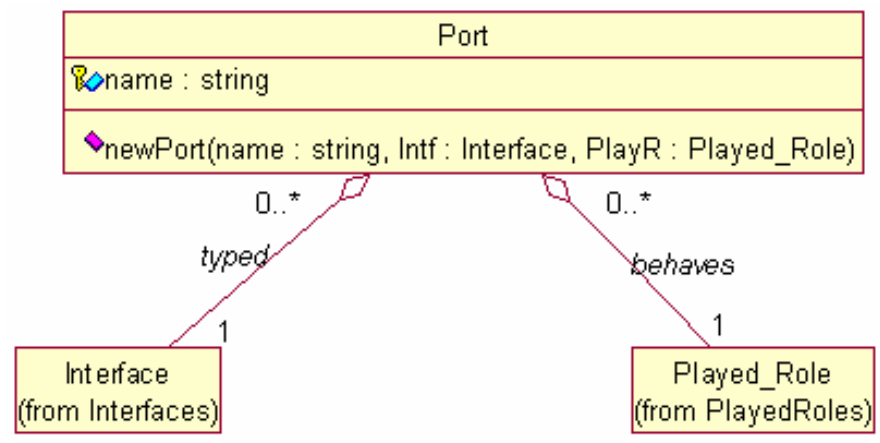

Figure 72. The package Ports of the PRISMA metamodel

However, ports cannot be related to any interface or played_role of the software system. As a result, these relationships are constrained by the following two constraints that correspond to the two that appear in Figure 73:

$<<$ If the architectural element that the port belongs to is not a system, the played_role of the port must be defined for one of the aspects that the architectural element imports. In addition, the interface of the played_role must be the same one as the interface of the port $>>$

$<<$ If the architectural element that the port belongs to is a system, there are two possible options: 1) either the played_role of the port is defined for one of the aspects that the system imports, and the interface of the played_role is the same as the interface of the port; 2) or the port has the same interface and played_role as one port of the architectural element of the system $>>$ 
PRISMA: Aspect-Oriented Software Architectures

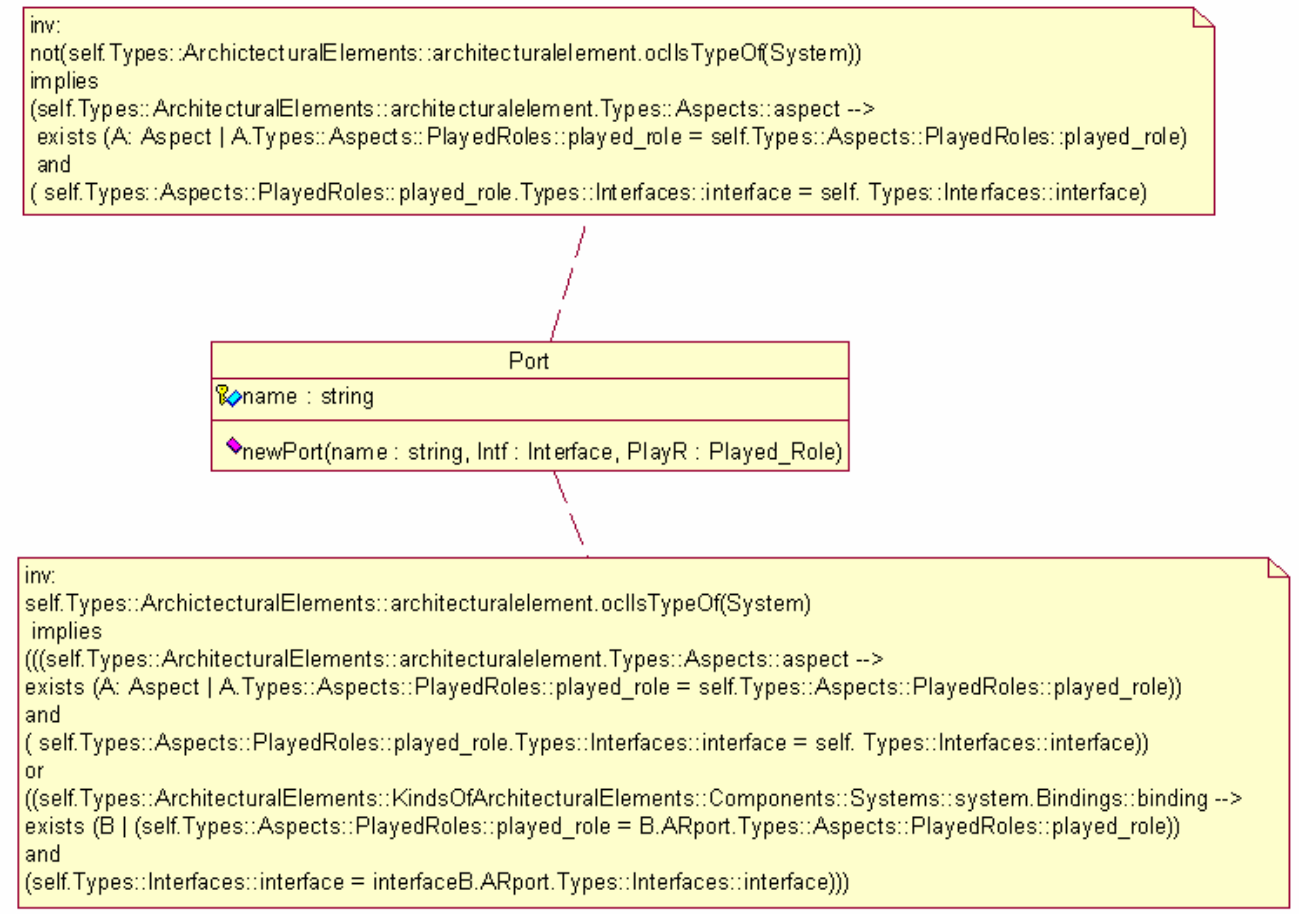

Figure 73. Constraints of the metaclass Port

The metaclass Port has one attribute, the name of the port. The service newPort creates a new port by providing the name of the port, the interface and the played_role as parameters.

\subsection{THE PACKAGE “ARCHITECTURE SPECIFICATION”}

The package Architecture Specification defines how a PRISMA architecture can be defined using the types defined in the package Types. The metaclass PRISMAArchitecture has five aggregation relationships with each one of the first-order citizens of the PRISMA model. They are components, connectors, aspects, interfaces, and attachments. Since components, connectors, interfaces, and aspects are reusable, they can be used by more than one architectural element (see Figure 74). 


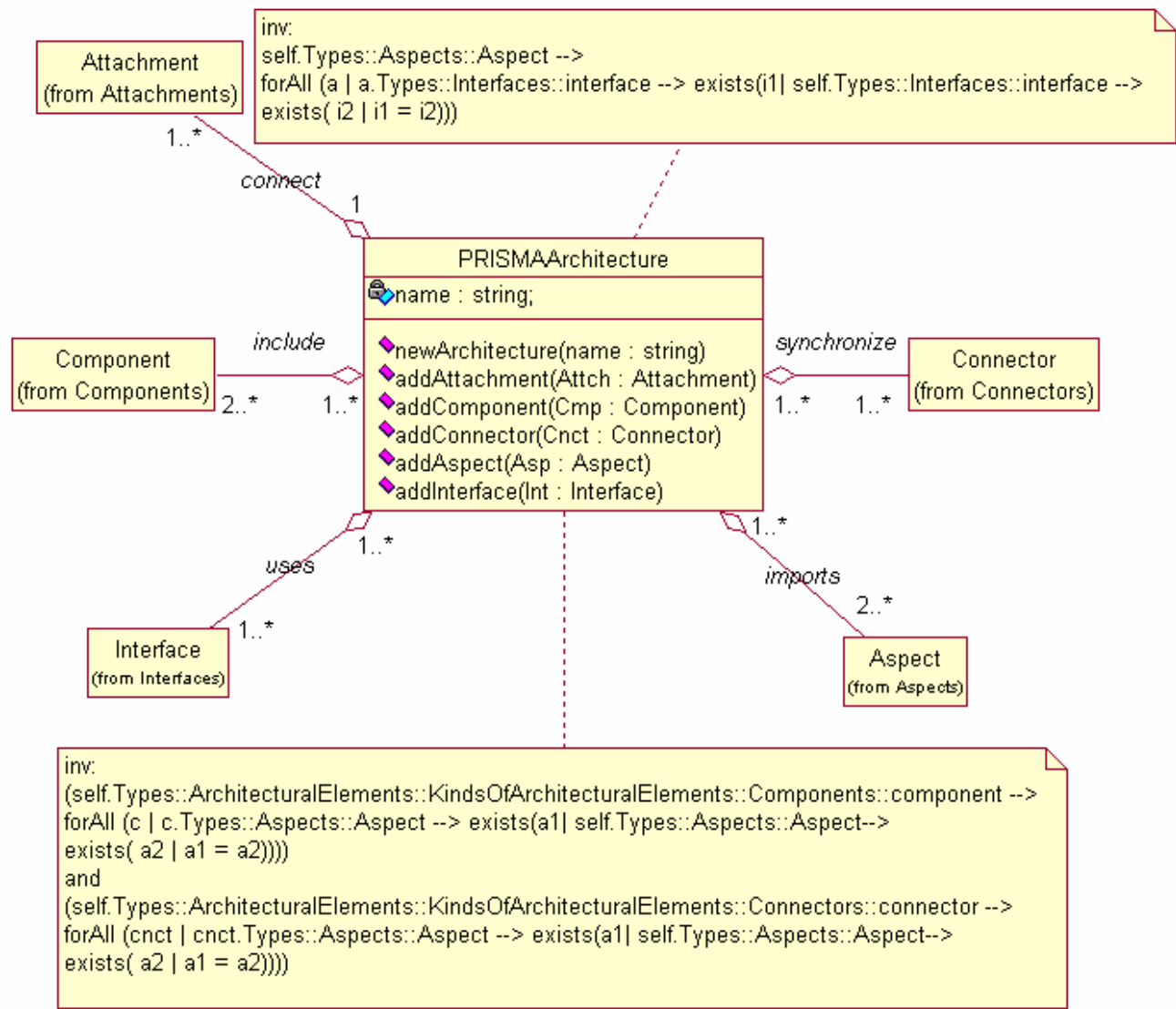

Figure 74. The package Architecture Specification of the PRISMA metamodel

The metaclass PRISMAArchitecture has one attribute, the name of the architectural model. The service newArchitecture creates a new architectural model by providing its name as a parameter. In addition, the metaclass provides five services to add attachments, components, connectors, interfaces and aspects to the architectural model. In order to ensure that a model is correctly defined, the metaclass PRISMAArchitecture has a set of constraints associated to it (see Figure 74). Their meaning is the following:

$<<$ An architectural model must include every aspect that is imported by its components and/or connectors $>>$

$<<$ An architectural model must include every interface that is used by its aspects $>>$ 
PRISMA: Aspect-Oriented Software Architectures

\subsection{THE PACKAGE “COMMON”}

The package Common defines the utilities that are necessary to develop any kind of model. These utilities consist of providing mechanisms to define data types, parameters, constant values, different kinds of formulae and complex processes. Since these are utilities that are common to all models, they are not going to be explained in detail in this thesis. However, the different packages that the Common package is composed of are shown in the figures that are presented in this section in order to provide the complete specification of the PRISMA metamodel.

\section{DataType}

Qkind : $\{$ natural, integer, double, char, string, boolean, date, currency, enumerated $\}$;

Figure 75. The package DataTypes of the PRISMA metamodel

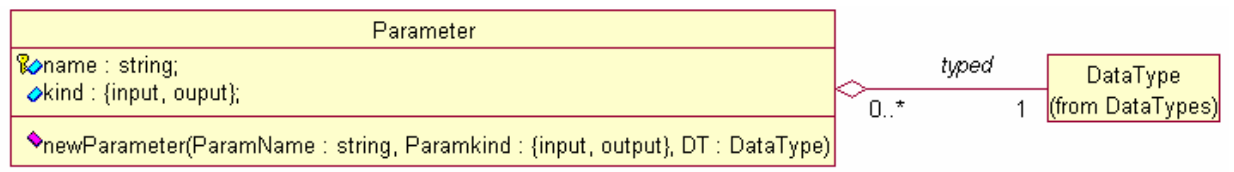

Figure 76. The package Parameters of the PRISMA metamodel

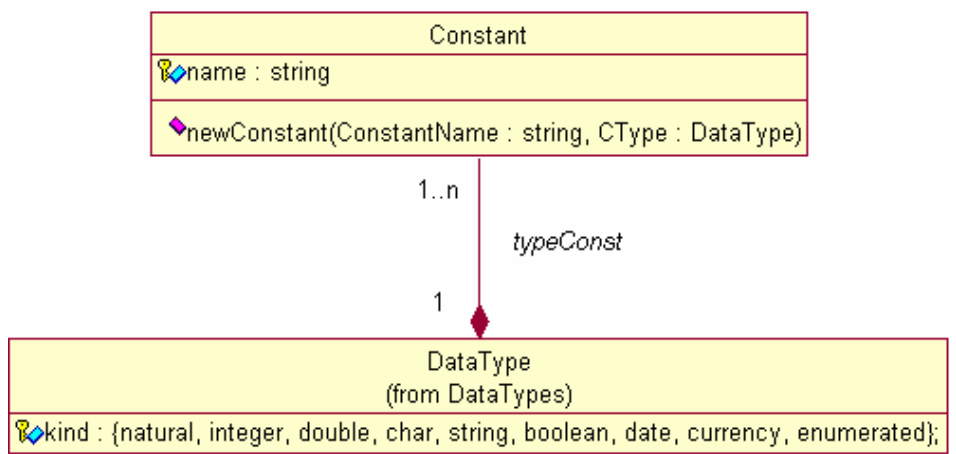

Figure 77. The package Constants of the PRISMA metamodel 
The PRISMA Metamodel

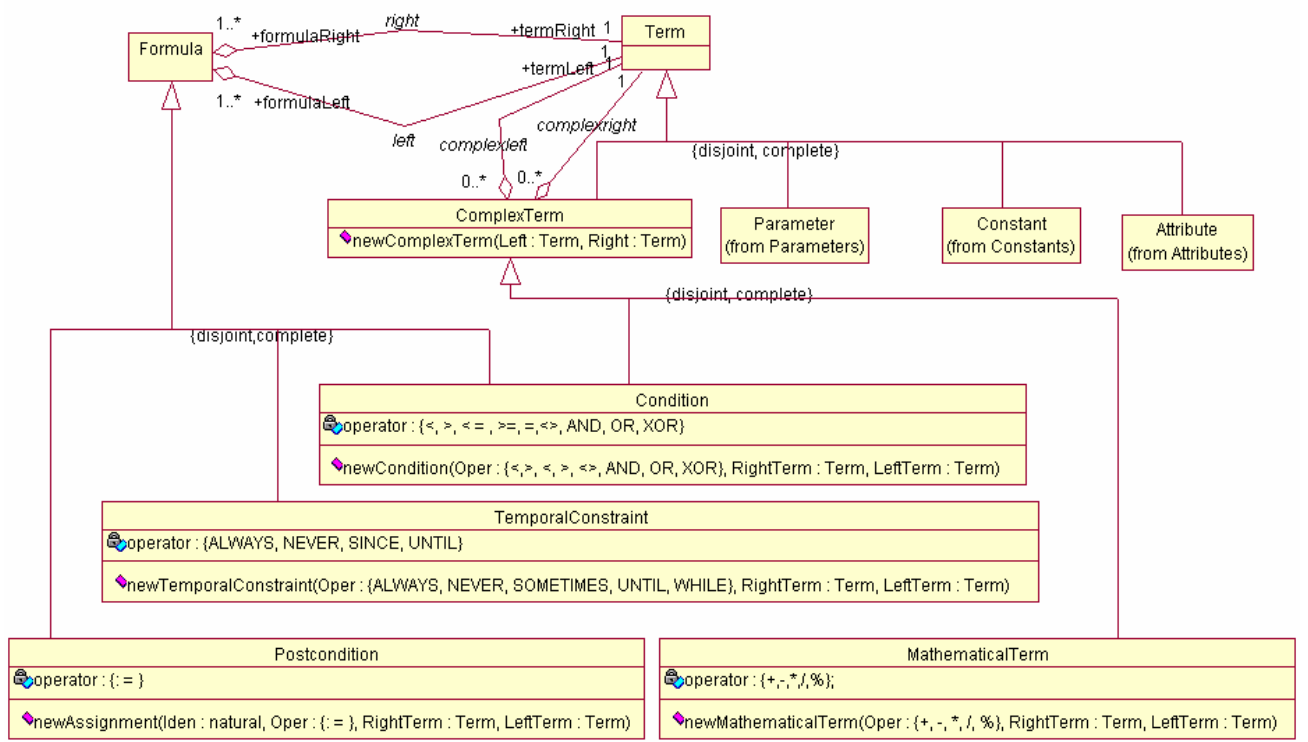

Figure 78. The package Formulae of the PRISMA metamodel

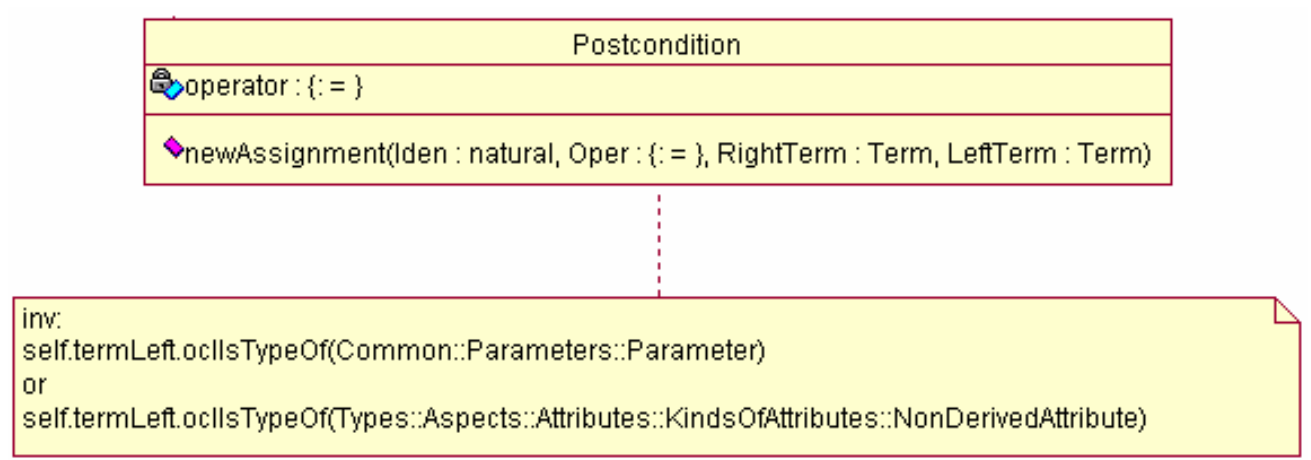

Figure 79. The constraint of the metaclass Postcondition of the Package Formulae 
PRISMA: Aspect-Oriented Software Architectures

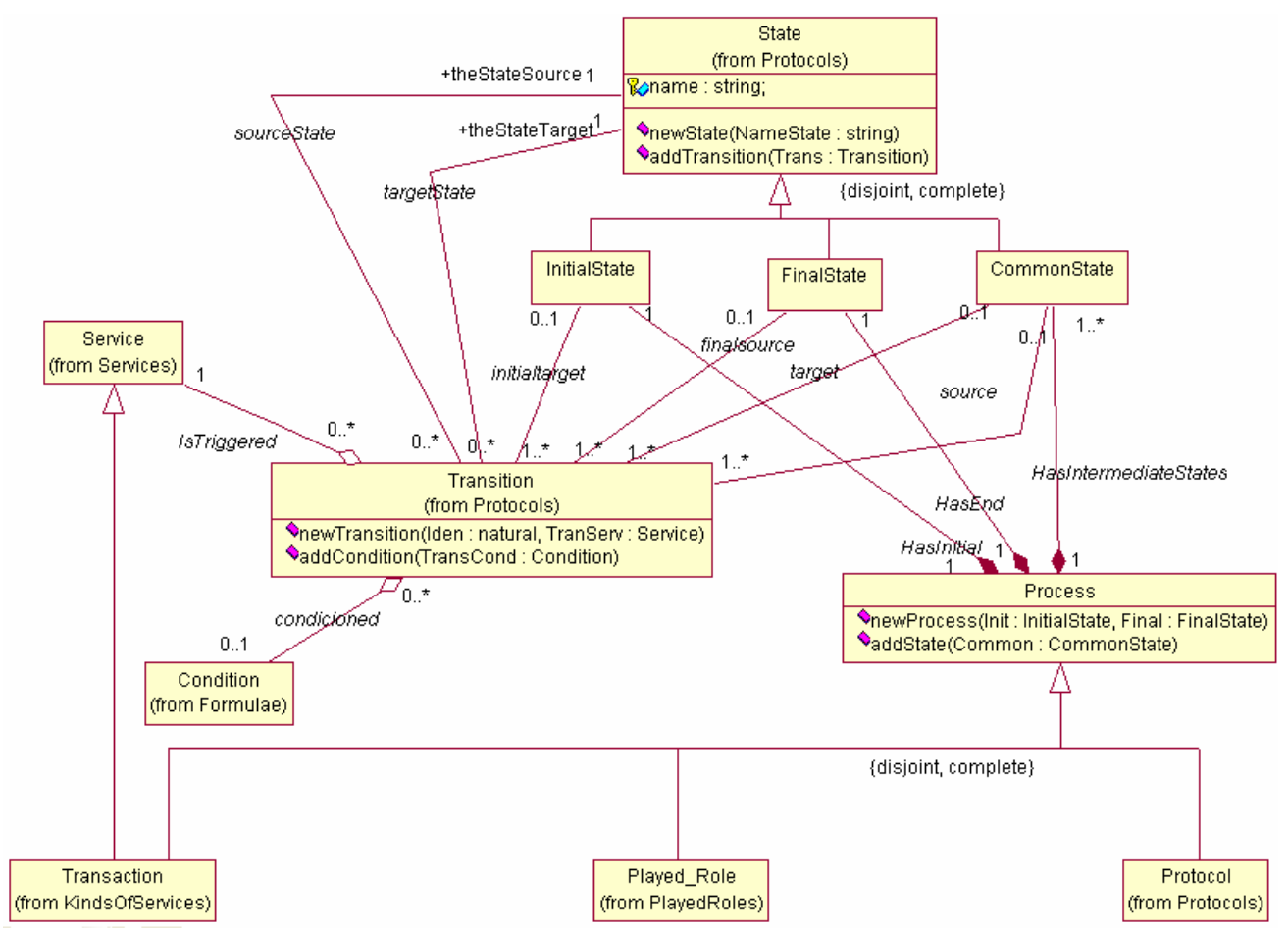

Figure 80. The package Processes of the PRISMA metamodel

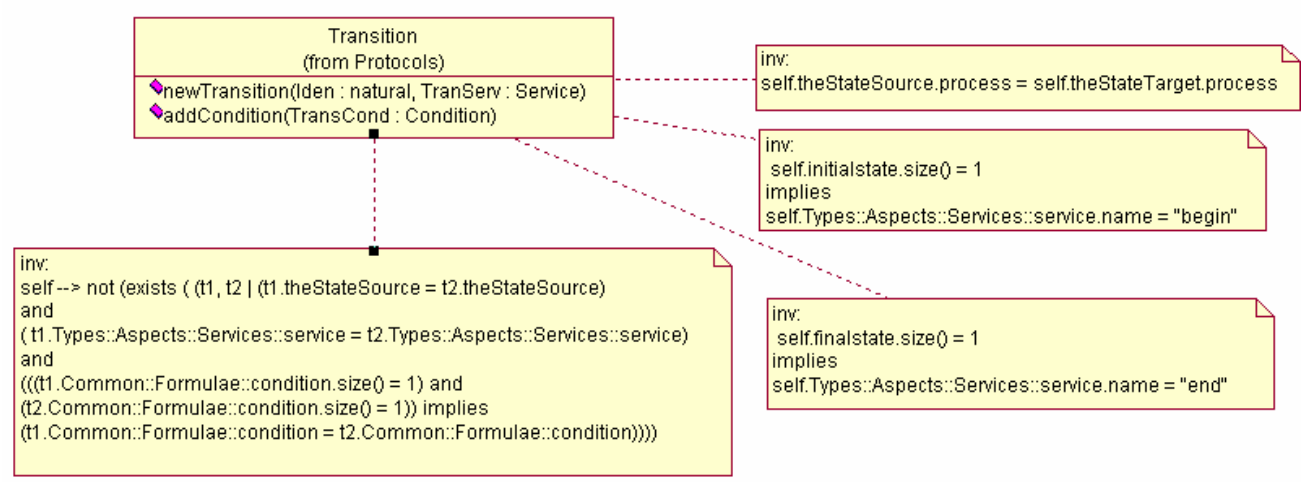

Figure 81. Constraints of the metaclass Transition of the package Processes 
The PRISMA Metamodel

\subsection{CONCLUSIONS}

The PRISMA metamodel has been presented in this chapter. This metamodel permits the creation of PRISMA architectural models in a correct way and the accurate definition of their properties. In addtition, it facilitates the integration of the PRISMA model into modelling tools that support the incorporation of new metamodels.

The PRISMA metamodel defines the required metaclasses, their properties and services, and their relationships with each other. In addition, the metamodel specifies the constraints to ensure that the definition of an architectural model is correct.

In the thesis, only the services for creating architectural models are presented. Each metaclass provides a set of services to create the concept that it defines, as well as a set of services to relate the concept to other concepts. It is assumed that each one of the metaclasses has the necessary services to destroy and delete architectural models in a consistent way.

The metamodel is the repository structure that stores PRISMA architectural models, preserving the reusability of interfaces, architectural elements and aspects. In addition, the metamodel introduces a methodology to follow when a PRISMA architectural model is specified by means of constraints.

The PRISMA metamodel has been defined to be able to support evolution at run-time in the future. This evolution could be supported at different levels of granularity by adding the evolution services to the different metaclasses of the metamodel. This would consist of adding or removing architectural elements of the model, or adding or removing properties of an aspect (attributes, services, etc). In addition to adding evolution services to the metamodel, a mechanism to invoke these services at run-time should be provided to support run-time evolution.

The PRISMA metamodel with its properties of creation, destruction and evolution has been published in the following publications:

> Jennifer Pérez, Nour Ali, Jose A. Carsí, Isidro Ramos, Dynamic Evolution in AspectOriented Architectural Models, Second European Workshop on Software Architecture, Springer LNCS 3527, pp.59-16, ISSN: 0302-9743, ISBN: 3-540-26275-X , Pisa, Italy, June 2005. 
PRISMA: Aspect-Oriented Software Architectures

Jennifer Pérez, Isidro Ramos, Jose A. Carsí, A Compiler to Automatically Generate the Metalevel of Specifications using Properties of the Base Level, Technical Report, DSICII/23/03, pp. 107, Polytechnic University of Valencia, October, 2003.(In Spanish) 


\section{CHAPTER 8}

\section{THE PRISMA ASPECT-ORIENTED ARCHITECTURE DESCRIPTION LANGUAGE}

$<<$ Language is the dress of thought $>>$

Samuel Johnson

The PRISMA approach provides an Architecture Description Language (ADL) to specify software architectures following the PRISMA model. Since the PRISMA model combines AOSD with software architectures, its language is an Aspect-Oriented Architecture Description Language (AOADL).

The PRISMA AOADL provides the syntactical constructions that allow the description of PRISMA architectural models. It is based on a Modal Logic of Actions [Sti92] and the dialect of polyadic $\pi$-calculus [Mil93] that is presented in section 5.2. In addition, some of the syntactical constructions of the language and the Modal Logic of Actions that is used come from the OASIS language [Let98]. OASIS is a formal language that defines conceptual models of information systems. As a result, the PRISMA AOADL defines the semantics of the architectural models in a formal way.

The PRISMA AOADL defines the architectural elements at different levels of abstraction: the type definition level and the configuration level. The type definition level defines architectural types with a high abstraction level in order to be reused by other types or specific architectures. 
PRISMA: Aspect-Oriented Software Architectures

The configuration level designs the architecture of software systems by creating and interconnecting instances of the defined architectural elements in the type definition level. In other words, it specifies the topology of a specific architectural model.

The purpose of this chapter is to present the structure and syntax of the PRISMA AOADL in detail. In this chapter, only the most important structures are presented. The complete BNF of the PRISMA AOADL and its notation is presented in detail in appendix A

\subsection{THE TYPE DEFINITION LEVEL}

The type definition level of PRISMA defines architectural patterns (complex components) and the first-class citizens of the language: interfaces, aspects, components, and connectors.

\subsubsection{Interface}

An interface describes the signature of the services that can be provided or requested through that interface. The specification of the interface consists of specifying its name and the list of services that it publishes between the reserved words Interface and End_Interface. Services are specified by defining their signature. The signature of a service specifies its name and parameters. The data type and the kind (input/output) of parameters are also declared. The template for specifying an interface is presented in Figure 82.

With regard to the nomenclature of concepts, it is recommended to follow an agreement during the specification. PRISMA proposes that the names of interfaces start with the letter I in uppercase. It is also recommended that the first letter of parameters be written with a capital letter and the first letter of services be written with a small letter.

An example of an interface in the TeachMover robot is the IMotionJoint interface, which publishes the moveJoint and stop services (see Figure 83). The moveJoint service has two input parameters whose names are NewSteps and Speed. Both parameters store values of the integer data type and are input parameters. Input parameters are those that provide information for the service execution; whereas output parameters are those that provide the result of the execution of the service. In addition, it is possible to declare services without parameters; an example is the stop service that the ImotionJoint also publishes. 


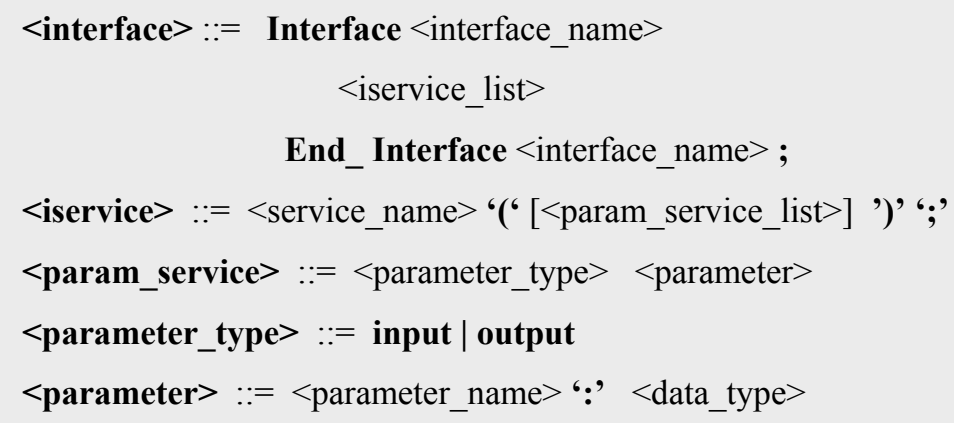

Figure 82. Specification template of interfaces

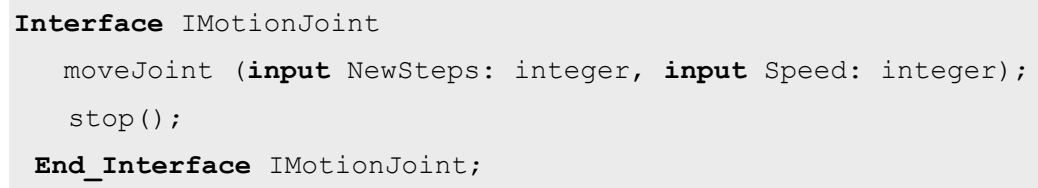

Figure 83. Specification of the interface IMotionJoint

\subsubsection{Aspects}

An aspect defines the structure and the behaviour of a specific concern of the software system. A common syntax of aspects has been defined independently of the concern that they define. The template for specifying an aspect is composed of several sections (see Figure 84).

The aspect specification is delimited by the reserved words Aspect and End_Aspect. The head of an aspect specifies its name and the kind of concern it defines: functional, coordination, safety, etc. Moreover, interfaces whose semantics are defined by aspects are detailed next to the reserved word using.

Figure 85 shows the head of the aspect CProcessSuc of the TeachMover software architecture. Since the aspect specifies coordination rules, the concern of the aspect is Coordination. This aspect uses four interfaces: IMotionJoint, IRead, IJoint, and IPosition. As a result, they are specified in the head of the aspect definition. 
PRISMA: Aspect-Oriented Software Architectures

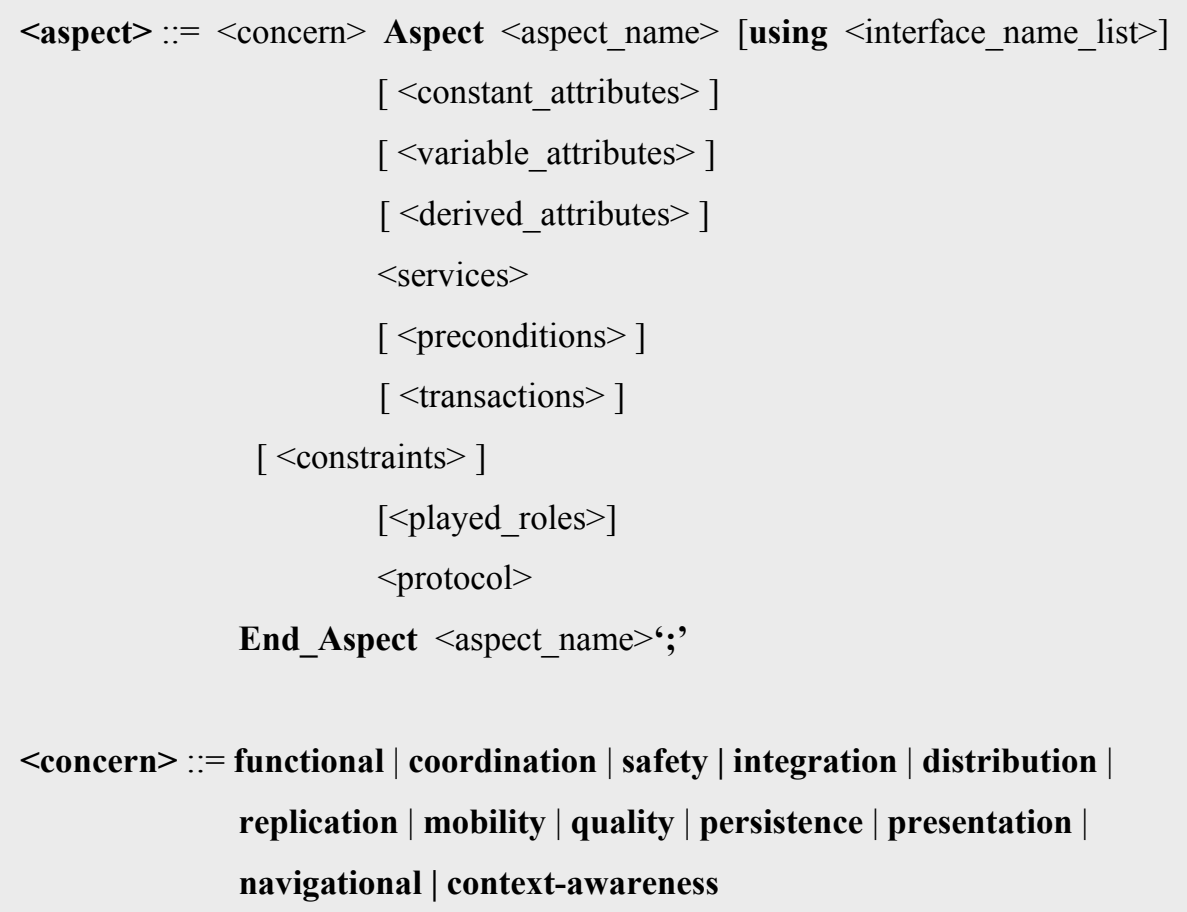

Figure 84. Specification template of aspects

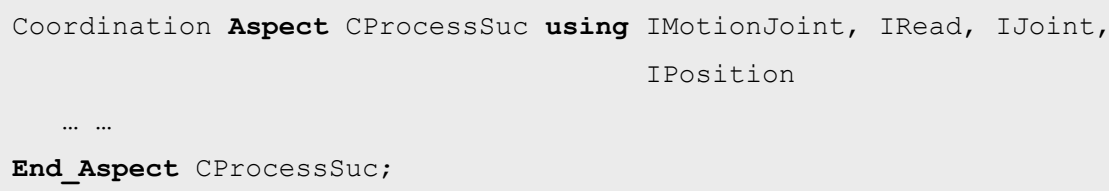

Figure 85. Specification of an aspect head with interfaces (CProcessSuc)

However, it is also possible to define an aspect without using any interface. This is the case of the SMotion aspect of the TeachMover software system (see Figure 86).

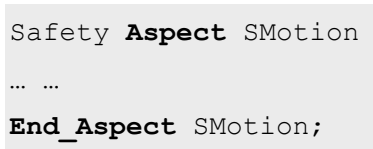

Figure 86. Specification of an aspect head without interfaces (SMotion)

The sections that the aspect is composed of are explained in the following subsections. 


\subsubsection{Attributes}

Attributes are specified inside an aspect. They are necessary to store information about the characteristics of the aspect. Attributes are preceded by the reserved word Attributes and they have a name and a data type. The data type defines the kind of values that the attribute can store. There are three kinds of attributes:

Constant: The stored values cannot change

$>$ Variable: The stored values can be changed

$>$ Derived: The value is calculated on demand applying its derivation rule.

The template for specifying these three kinds of attributes is presented in Figure 87. In the aspect specification, the different kinds of attributes are denoted by the reserved words Constant, Variable, or Derived.

Variable and constant attributes can store a value by default, which can only be modified when the attribute is variable. In addition, variable attributes can specify that they must always store a value by specifying the reserved word NOT NULL after their data type specification.

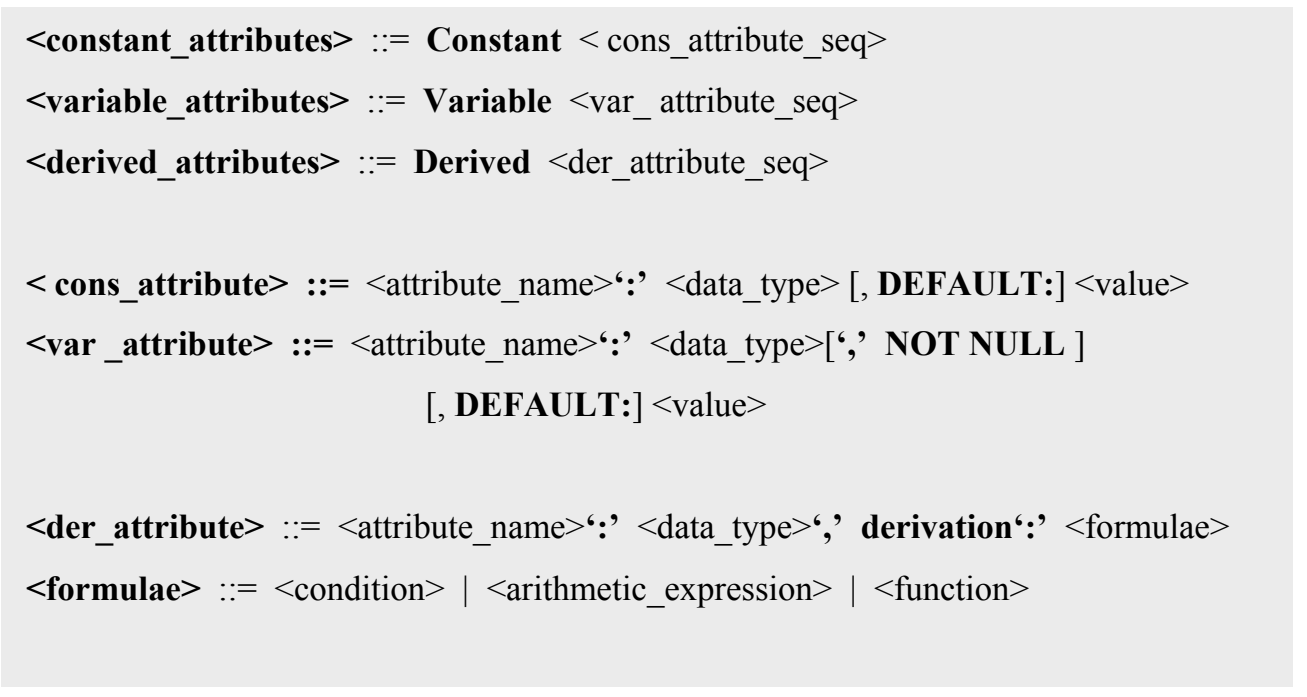

Figure 87. Specification template of attributes

Derived attributes must specify the derivation formula that describes how to calculate their value. This formula can be a condition, an arithmetic expression, or a function that has been defined by the user. 
PRISMA: Aspect-Oriented Software Architectures

With regard to the nomenclature of concepts, PRISMA proposes that the first letter of an attribute be written in small letters to clearly distinguish attributes from parameters, which start whith capital letters.

An example of the specification of constant attributes is the specification of the attributes of the SMotion aspect (see Figure 88). This aspect specifies the minimum, maximum attributes, whose data type is integer and they must store a value.

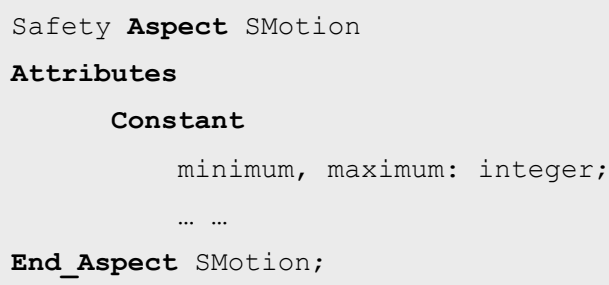

Figure 88. Specification of the constant attributes of the aspect SMotion

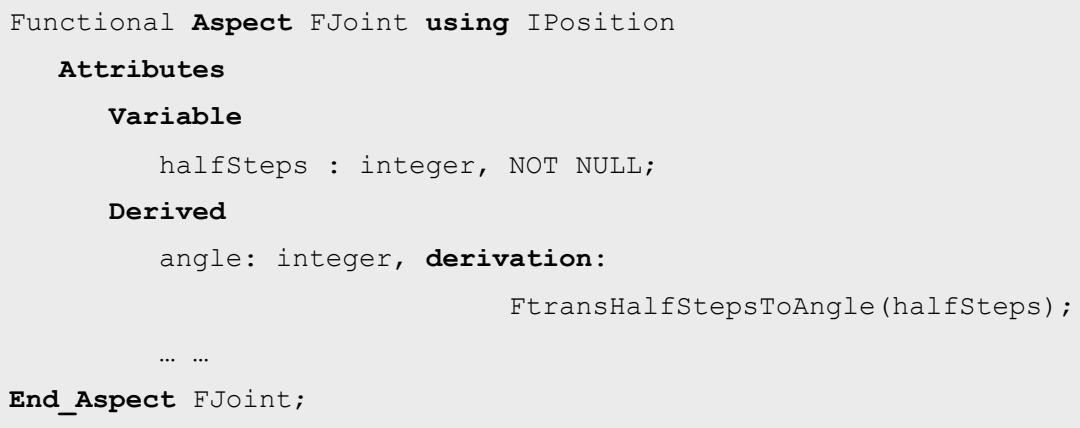

Figure 89. Specification of the variable and derived attributes of the aspect FJoint

Another example is the specification of the attributes of the FJoint aspect, which shows how to specify variable and derived attributes (see Figure 89). It specifies one variable attribute, halfSteps. Its data type is integer and is constrained by the NOT NULL property. Therefore, it cannot be empty.

In addition, the aspect specifies the derived attribute angle. In this case, the attribute is calculated using a function defined by the user. The function FtransHalfStepsToAngle 
transforms a datum from a half-steps measure to a degree measure in order to know angle corresponds to a specific number of half-steps.

\subsubsection{Services}

An aspect defines the semantics of services. The specification of services is preceded by the Services reserved word. The set of services specified in an aspect must contain the begin service, the end service, and the interface services that the aspect defines. begin and end services do not mean that it is possible to instantiate an aspect by itself. These services can only be requested from the creation and destruction services of the architectural elements that import the aspects that begin and end services belong to (see the creation and destruction services of architectural elements in Figure 105).

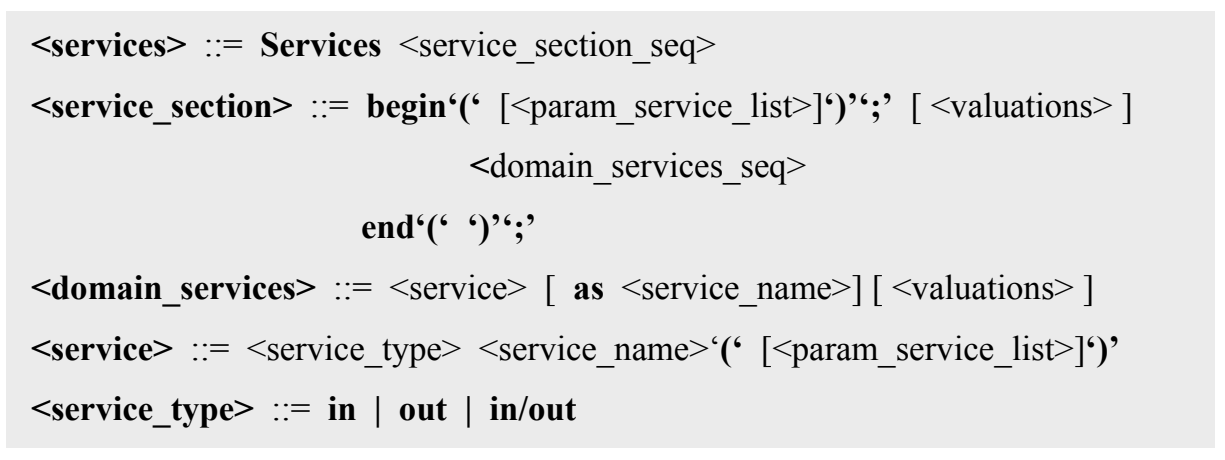

Figure 90. Specification template of services

The specification of a service consists of defining its name and parameters. Sometimes it is necessary for the service name of the aspect to be different from the service name of the interface that it belongs to. The language provides a mechanism to rename the service without losing the traceability between the aspect service and the interface service. The aspect defines an alias name for the service using the reserved word $a$ s. The $a$ indicates that the service name of the aspect is an alias and that it is the same service as the interface service, whose name is specified after the as. A hypothetical alias for the service moveJoint of the interface IMotionJoint is defined in the example shown in Figure 91. 
PRISMA: Aspect-Oriented Software Architectures

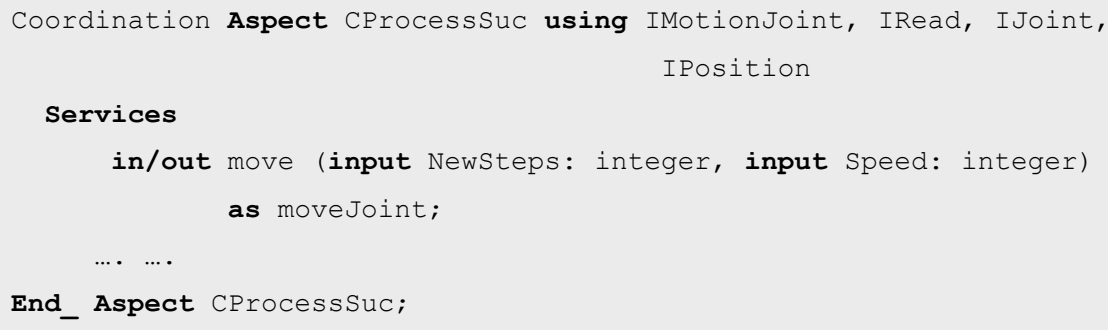

Figure 91. Specification of aliases

In addition, it is important to take into account that the same service can have four different behaviours. The first behaviour is when a service is requested, the second behaviour is when a request service is received and executed, the third behaviour is when the service sends its execution result, and the fourth behaviour is when an aspect receives the results of a service execution. The possibility of having these four behaviours depends on whether or not the service returns a result. In other words, a service has these four behaviours if it has output parameters; otherwise the service only has the first and second behaviours. These behaviours are distinguished taking into account the kinds of parameters of the service as well as the reserved words in, out, in/out. The patterns of specification to define a specific behaviour are the following:

A service without output parameters

- in: The service is provided and executed by the aspect. This is the case of the moveJoint service of the $R S 232$ aspect.

in moveJoint (input NewSteps:integer, input Speed:integer)

- out: The service is requested by the aspect. This is the case of the moveJoint or stop services of the functional aspect that models the behaviour of the operator (the aspect. FOperator).

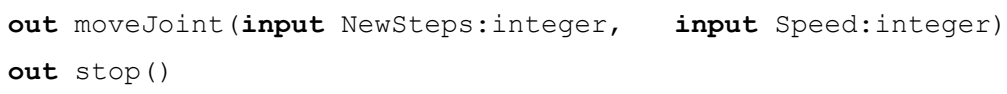


- in/out: This is the way of specifying that the service has both the in and out behaviours without repeating the specification of the service, which means that:

- The service is provided and executed by the aspect, and the service is also requested by the aspect. This is the case of the moveJoint service of the CProcessSuc aspect:

in/out moveJoint (input NewSteps:integer, input speed:integer)

A service with output parameters

- in/out: It defines two possible behaviours

- The service is provided and executed by the aspect (in), and the aspect sends the results of the service execution $(o u t)$. This is the case of the check service of the SMotion aspect.

in/out check (input Degrees: integer,

output MovSecure: boolean)

- The service is requested by the aspect (out) and the aspect receives the result of the service (in). This is the case of the moveOk service of the CProcessSuc aspect.

in/out moveok (output Success: boolean);

As can be seen from these patterns, when the service does not have any output parameter, the properties in, out and in/out define the behaviour of the services without ambiguities. However, when the service has output parameters, the property in/out has two possible meanings. This ambiguity disappears thanks to the definition of the service valuations presented below. 
PRISMA: Aspect-Oriented Software Architectures

\subsubsection{Valuations}

A service can have one or more valuations associated to it (see Figure 90). Valuations specify the changes in the value of attributes and parameters by the execution of services. The template for specifying the service valuations is presented in Figure 92.

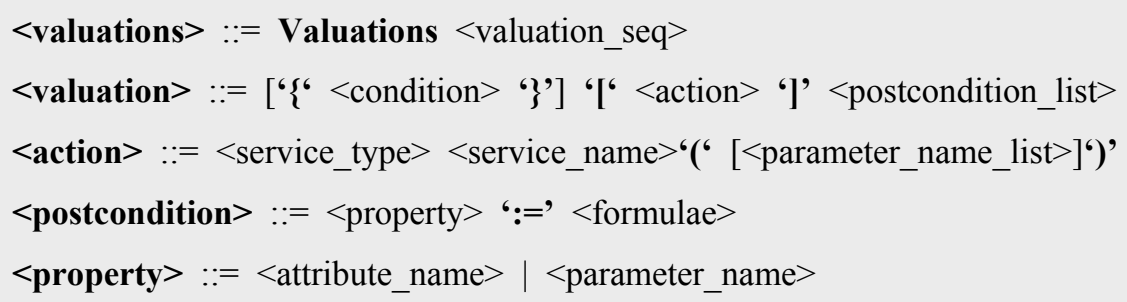

Figure 92. Specification template of valuations

The valuations of a service are preceded by the reserved word Valuations. The specification of a valuation consists of three sections: condition, action and postcondition. A condition is validated in the aspect state before its execution, and its specification is optional. The action specifies the service that executes the action by specifying its name and the name of its parameters. In addition, the action specifies the property in or out of the service in which the valuation must be performed. The property that can be used in the valuation definitions depends on the service specification, i.e., if the service has an in/out property it is possible to define out valuations and in valuations; whereas if the service only has an out property or the service only has an in property it is only possible to define out valuations or in valuations, respectively.

The semantics of the valuation depends on the in and out properties and the use of the parameters inside the valuation. The meaning of the postcondition is presented following the same classification used above for the services properties and parameters.

A service without output parameters

- in: The service is provided and executed by the aspect.

- Valuation in: The postcondition must be satisfied after the service execution.

- out: The service is requested by the aspect. 
- Valuation out: The postcondition must be satisfied after the service request

- in/out: The service is provided and executed by the aspect, and the service is also requested by the aspect. In this case, it is possible to define the two kinds of valuations:

○ Valuation in: The postcondition must be satisfied after the service execution

- Valuation out: The postcondition must be satisfied after the service invocation

$>$ A service with output parameters

A service with output parameters always has an in/out behaviour. Its semantics varies depending on the service is provided or requested.

- in/out: It defines two possible behaviours. To specify one behaviour or the other, the valuation must be specified as is presented bellow:

- The service is provided and executed by the aspect, and the aspect sends the results of the service execution.

- Valuation in: The postcondition must be satisfied after the service execution. The output parameters of the service cannot be used as a right term of the valuation in any case, neither in the condition nor in the postcondition, due to the fact that the service has not been executed and its output parameters do not have value. In fact, output parameters are usually used as a left term of the postcondition in order to satisfy the condition that requires output parameters to have a value after the service execution.

An example of this case is the check service of the SMotion aspect that has two associated in valuations that define the state of the aspect after the service execution. This is due to the fact 
PRISMA: Aspect-Oriented Software Architectures

that the output parameter is not used as a right term of conditions. In addition, the postcondition establishes the condition that must be satisfied (left term) as a result of the service execution (see Figure 93). In this case, the value of the output parameter value must be equal to a specific value, eithertrue or false.

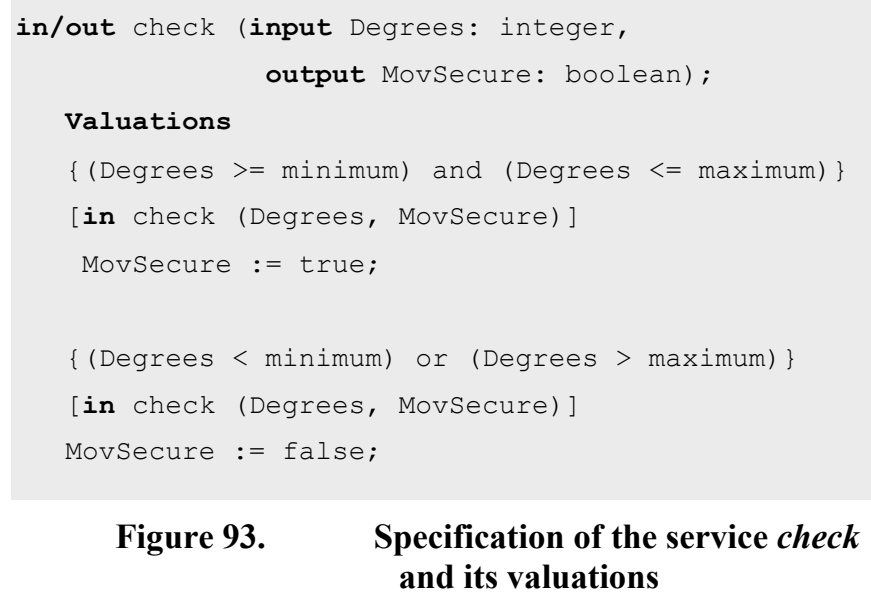

Figure 93. Specification of the service check and its valuations

- Valuation out: The postcondition must be satisfied after sending the result. The semantics of the out valuation is conditioned by the semantics of the in valuation. If the in obtains a result, the out is related to sending the result. Since the service has already been executed and the output parameters have value, they can be used in the condition and in the right terms of postcondition.

- The service is requested by the aspect and the aspect receives the result of the service.

- Valuation in: The postcondition must be satisfied after the reception of the service result. Since the service has already been executed and the output parameters have value, they can be used in the condition and in the right terms of postcondition. 
The PRISMA Aspect-Oriented Architecture Description Language

An example of this case is the moveOk service of the CProcessSuc aspect that has two associated in valuations that define the state of the aspect after the reception of the service result. This is due to the fact that its output parameter has a value that is used in the condition to establish a different action depending on its value (see Figure 94).

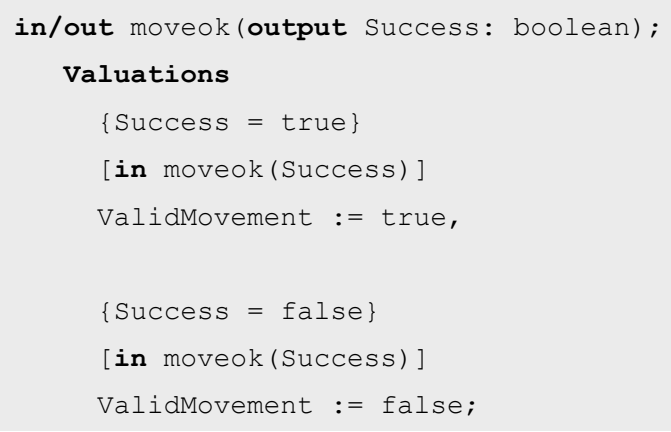

Figure 94. Specification of the service moveOk and its valuations

- Valuation out: The postcondition defines the state of the aspect after the service request. The output parameters of the service cannot be used as a right term of the valuation in any case, neither in the condition nor in the postcondition due to the fact that the service has not been executed and its output parameters do not have value.

\subsubsection{Preconditions}

An aspect can define preconditions to establish conditions that must be satisfied to execute aspect services. The specification of preconditions is preceded by the reserved word Preconditions. The precondition specification consists of defining a condition and the service that the condition affects. The service is specified by describing its name and the name of its parameters in brackets. The condition is preceded by the reserved word if and is specified in curly brackets (see Figure 95). 
PRISMA: Aspect-Oriented Software Architectures

An example of a precondition is the precondition of the CProcessSuc aspect shown in Figure 96. In this case, the precondition establishes that the newposition service can only be executed if the validMovement attribute has the value true. This precondition defines that the position of a joint is only modified when the movement has been successfully performed.

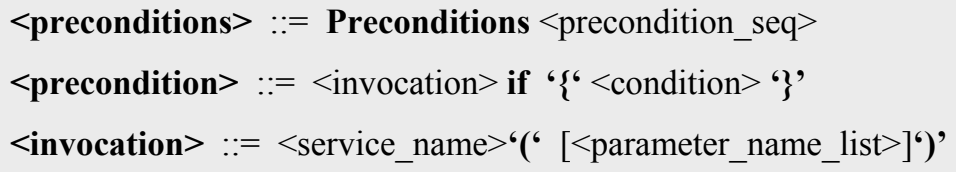

Figure 95. Specification template of preconditions

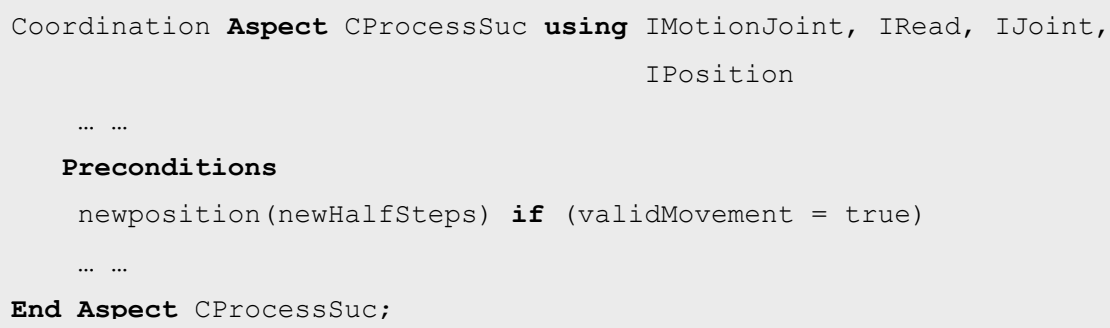

Figure 96. Specification of preconditions

\subsubsection{Constraints}

An aspect can define constraints to condition the value of its attributes. The specification of constraints is preceded by the reserved word Constraints. The constraint specification consists of defining a static or dynamic condition (see Figure 97). If the condition is dynamic, it uses ones of the temporal operators offered by the PRISMA AOADL: always, never, since, until, and their possible combinations.

In the TeachMover, none of its aspects have the specification of a constraint. However, it is possible to imagine a hypothetical constraint for the halfSteps attribute of the FJoint aspect, in which it was specified that the halfSteps attribute must always contain a value higher than 10 (see Figure 98). 


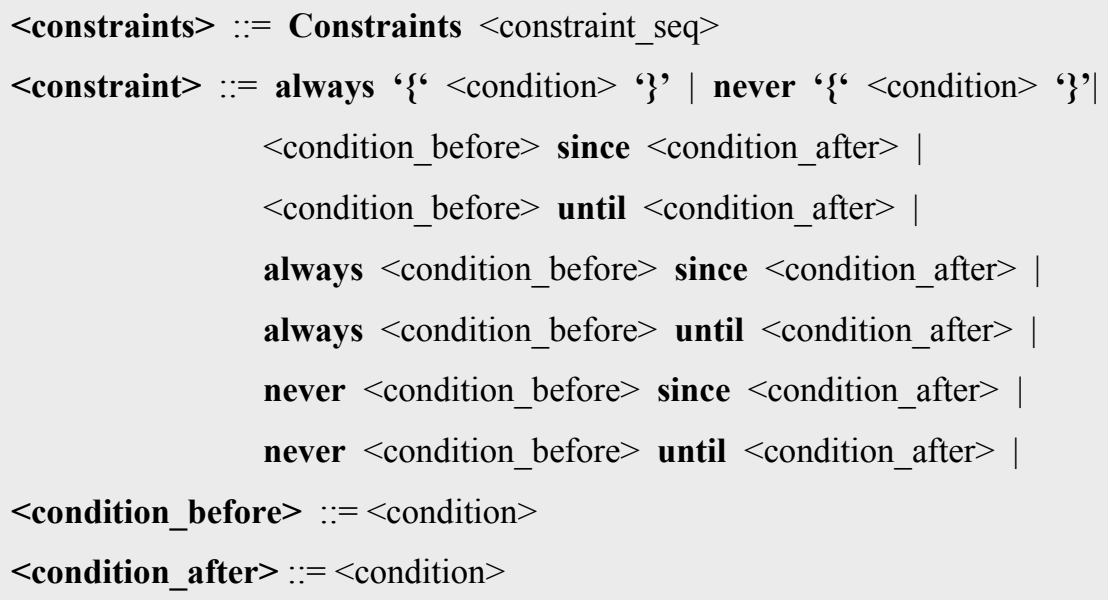

Figure 97. Specification template of constraints

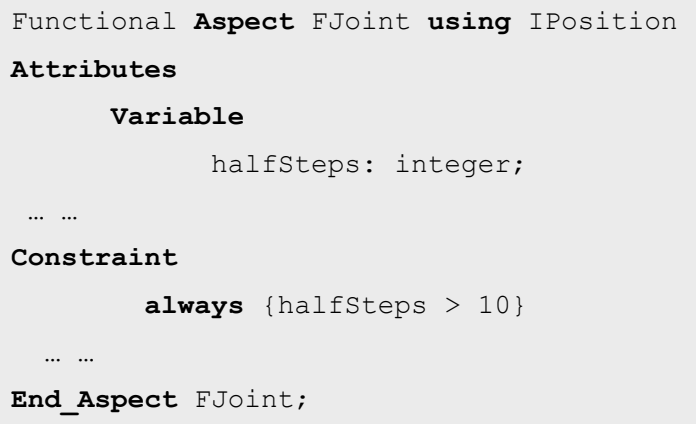

Figure 98. Specification of constraints

\subsubsection{Transactions}

An aspect can not only define simple services, it can also define complex services composed of simple services and/or other complex services. These complex services are transactions due to the fact that they are executed in a transactional way. In other words, the services that compose a transaction are atomically executed (all or none). As a result, if the execution of one of the transaction services fails, the execution of the services that have already been executed is undone.

The specification of transactions is preceded by the reserved word Transactions. In the first instance, the specification of a transaction consists of defining its name in capital letters and the 
PRISMA: Aspect-Oriented Software Architectures

list of parameters that are necessary to execute the transaction. This list of parameters is specified in brackets. In the second instance, the process that describes the transaction is specified after a colon (see Figure 99). The process specification consists of a set of processes that coordinate a set of services. These processes are specified and synchronized with each other using the $\pi$-calculus with priorities (see section 5.2.2). Each one of these processes has a name (channel) that identifies it and allows the rest of the states to call it. In the case of the first transaction process, its name is always the transaction name.

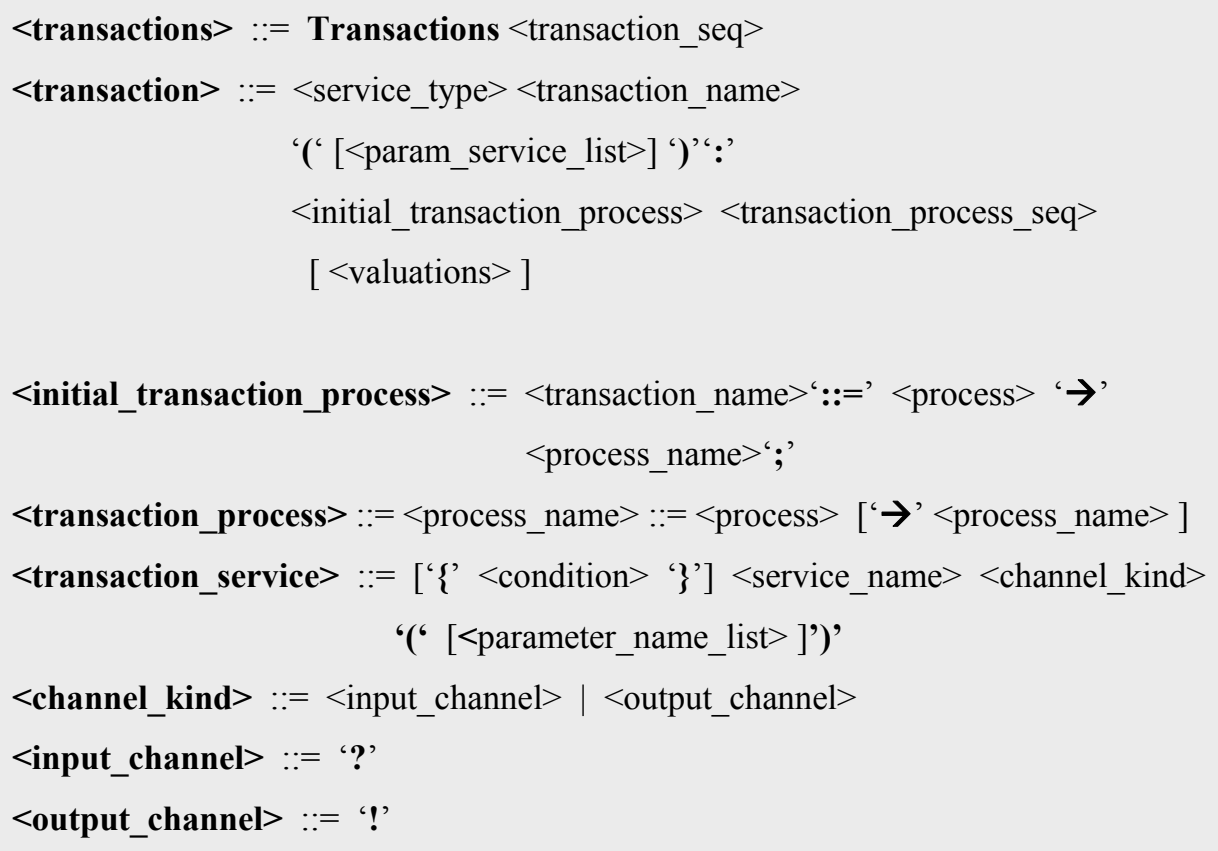

Figure 99. Specification template of transactions

An example of a transaction is the transaction DANGEROUSCHECKING of the aspect SMotion (see Figure 100). This transaction receives the needed information about the movement as input parameters. It provides the information about whether or not the movement is safe as a result of its execution. This transaction is composed of two checking services: controlSpeed and check. First, controlSpeed is executed to check if the speed is suitable for the movement; and second, check is executed to ensure that the movement is safe for the robot, the operator, and the environment that surrounds them. The execution of these two services 220 
The PRISMA Aspect-Oriented Architecture Description Language

together determines whether or not the movement is safe. The result is specified by means of a valuation that establishes the value of the Secure output parameter, and is based on the results obtained by the simple services that compose the transaction.

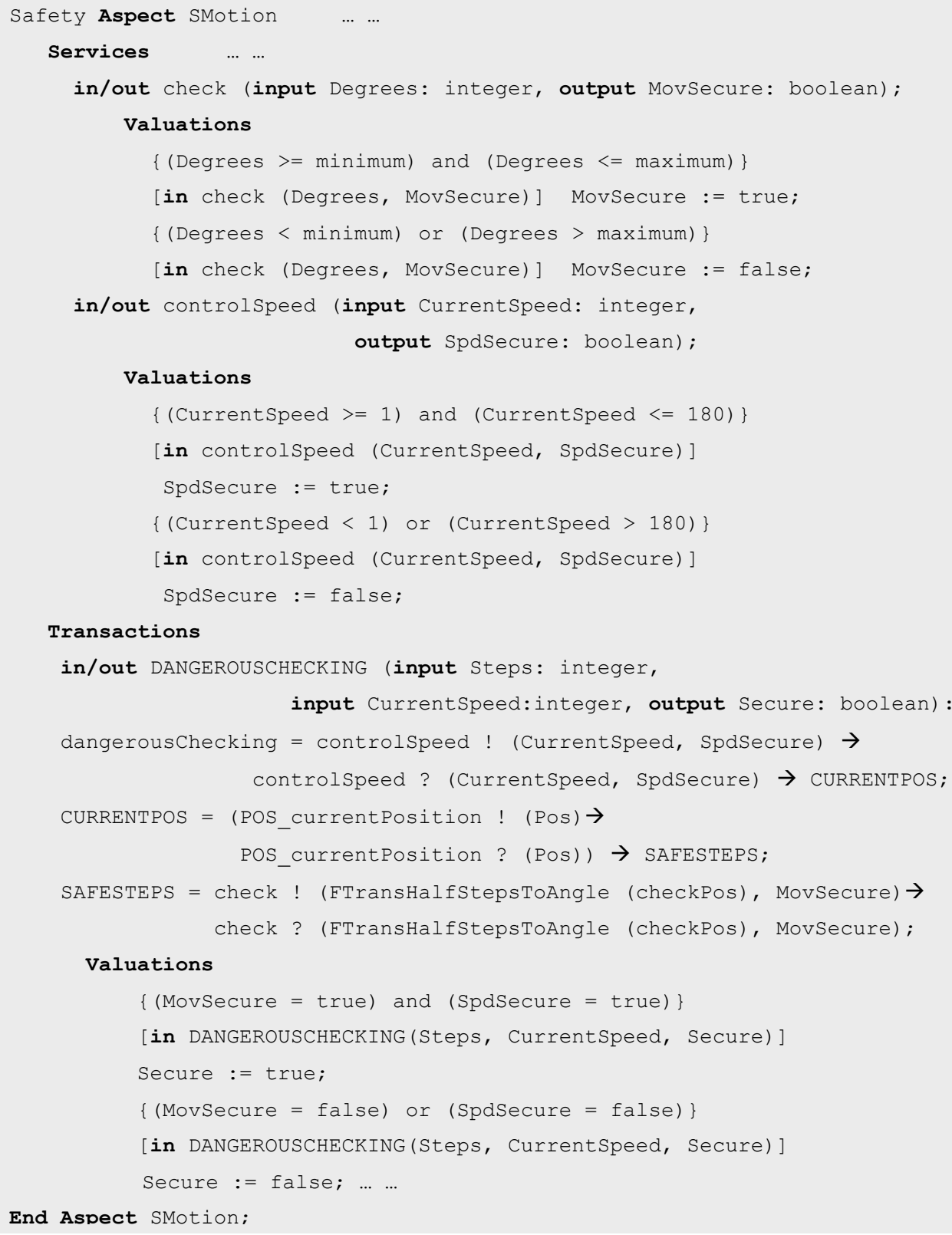

Figure 100. Specification of transactions 
PRISMA: Aspect-Oriented Software Architectures

\subsubsection{Played_Roles}

An aspect also defines the set of roles that can be played taking into account the semantics of services. They are called played_roles and their definition is preceded by the reserved word Played_Roles. A played_role specification consists of its name in capital letters and a process. The process is specified using the dialect of $\pi$-calculus with priorities. Every service that composes a played_role process belongs to the same interface. This interface is specified after the played_role name with the reserved word for and the name of the interface (see Figure $101)$.

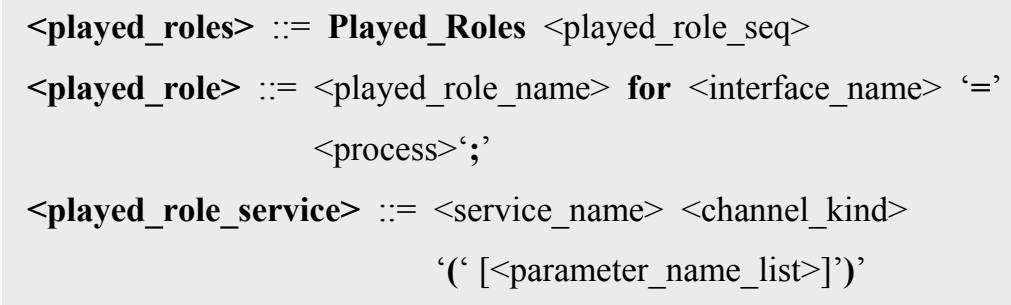

Figure 101. Specification template of played_roles

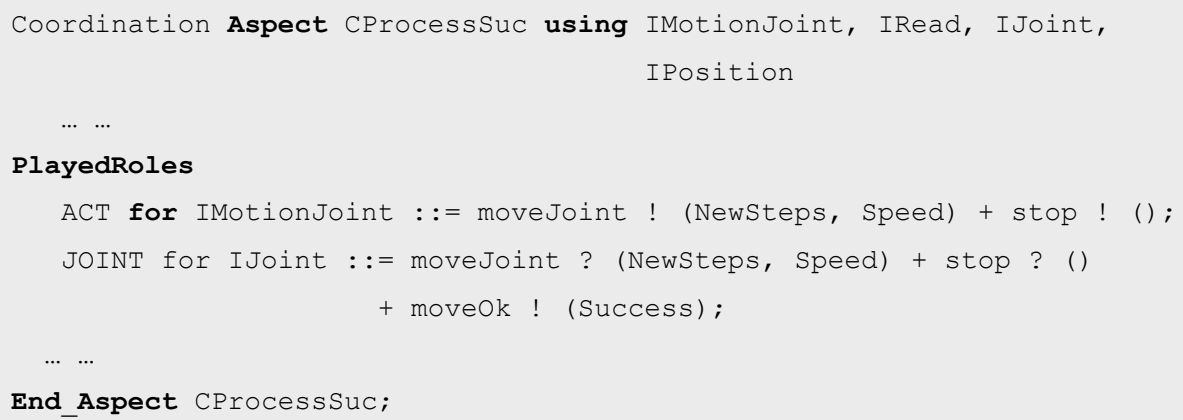

Figure 102. Specification of played_roles of the aspect CProcessSuc

For example, the CProcessSuc aspect defines some played_roles. Two of them are the $A C T$ and JOINT played_roles. ACT defines the client behaviour for the movejoint and stop services, i.e., it requests the services. Whereas, JOINT defines the server behaviour for these services, and it also defines the client behaviour of the moveO $k$ service (see Figure 102). 


\subsubsection{Protocols}

The language that has been used to specify protocols is a high-level language that is based on the dialect of $\pi$ - calculus with priorities. This high-level Language for PRISMA Protocols (LPP) has been defined due to the fact that the PRISMA services are context-aware in protocols. As a result, protocols must be specified taking into account the context of the service.

LPP introduces the context of a prefix using the $\pi$-calculus with priorities as a base language. This prefix is an action that has been created because in PRISMA, it is sometimes necessary to know the different contexts where a name is used (see Table 4).

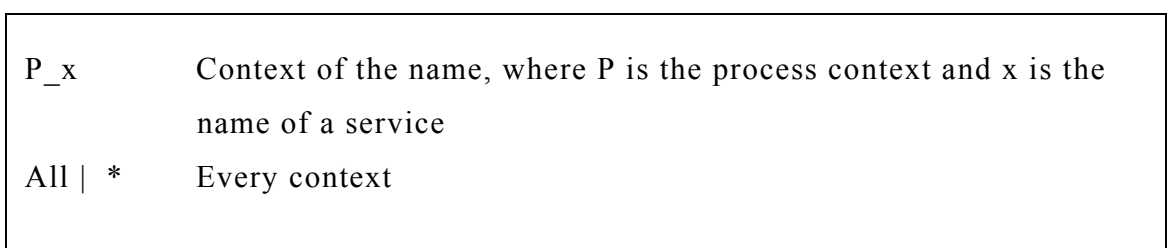

\section{Table 4. Prefixes with Context of LPP}

The context information is an important advantage to constrain the invocation of a name in a specific context. The context of the name is added as a prefix of the name as $\mathrm{P}_{-} \mathrm{x}$, with $\mathrm{P}$ being the process context and $\mathrm{x}$ the name of the service. This expression might be confused with a hierarchy; however, it is not a hierarchy. In fact, the name is not a hierarchical composition of names as when the dot naming uses dots for naming (P.x). $\mathrm{P}_{-} \mathrm{x}$ is the complete name and cannot be divided. This is syntactic sugar, which means that a name $\mathrm{x}$ is used in the process $\mathrm{P}$. In the case that this same name $x$ is used by another process $\mathrm{Q}$, it can be distinguished by the expression Q $\mathrm{x}$. However, it is important not to lose sight of the fact that $\mathrm{x}$, P_x, and Q_ $\mathrm{x}$ are the same names. As a result, this equivalence must be explicitly specified.

An example in which this action could be useful is the following:

$<<$ In the business rules of a bank system, there is a process $\mathrm{W}$ that executes a set of internal actions that allow the withdrawal of money from a bank account. This process can be invoked with the withdrawal name. In addition, there are two different processes, VIPC and C. VIPC describes the actions that a VIP customer of the bank can execute, and $\mathrm{C}$ describes the actions that a normal customer of the bank can execute. Finally, the process B specifies the actions that the bank can execute as well as the coordination rules of VIPC and C. The three processes 
PRISMA: Aspect-Oriented Software Architectures

VIPC, $\mathrm{C}$, and $\mathrm{B}$ can execute the $\mathrm{W}$ process using the prefix withdrawal!). However, B must establish when the withdrawal name can be requested by a VIP customer, a normal customer, or the bank, depending on its state. Note that in the specification of the process B, there is no way of distinguishing who is invoking the withdrawal from the process B:

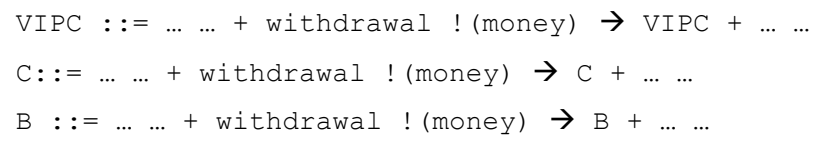

As a result, in B the context action is necessary to establish when the withdrawal can be executed and by whom. The way of defining the different execution contexts of the $\mathrm{W}$ process is by using the context action specified as VIPC_withdrawal!(), C_ withdrawal!), withdrawal!(). An example in which process B constrains the invocation of the withdrawal name from the process VIPC is presented below. This constraint is defined using a variable that provides the bank the information about whether or not there are VIP customers in the bank.

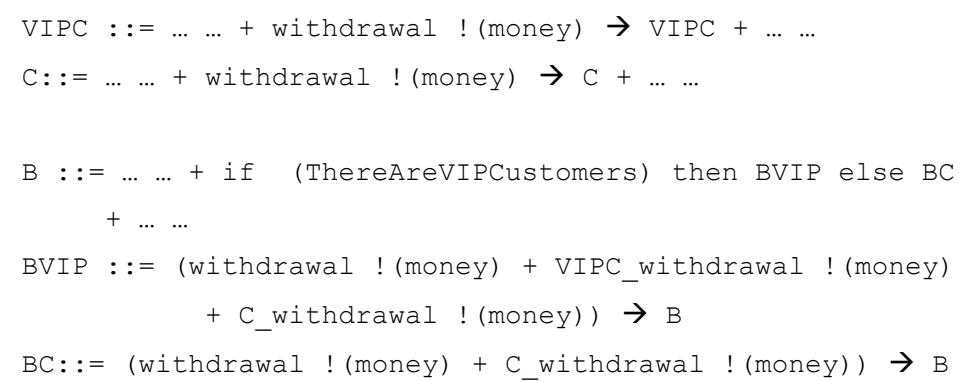

Being:

withdrawal !(money) = VIPC_withdrawal !(money) = C_withdrawal !(money)

$>>$

In PRISMA, this action is necessary to specify protocols, which are the glue of the set of services of the aspect and the different played_roles (processes) that have been defined inside the aspect. To facilitate the definition of protocols, it is assumed that the context specifications whose names are the same, without considering the context prefix, refer to the same name. As a result, PRISMA performs this equivalence $\left(\mathrm{P}_{\mathrm{X}} \mathrm{x}=\mathrm{Q} \mathrm{x}=\mathrm{x}\right)$ automatically in order to suitably process the behaviour specified. 
An aspect must define its protocol. The protocol specification is preceded by the reserved word Protocol (see Figure 103). Since protocols are the glue of the private services and played_roles, the specification of protocols consists of a set of processes that coordinates a set of private services and/or services of the played_roles. Each process that forms a protocol represents a state of the aspect where the services of this process can be executed. Some of these services can produce changes in the aspect state that are relevant for the business logic of the software system. As a result, these changes can vary the set of services that can be executed. The new state of the aspect is modelled by another process that only allows the execution of the permitted services. Thus, the services that change the aspect state provoke a transition between the current process (state) and the process that models the new state of the aspect.

Furthermore, the different processes that make up an aspect can be executed in a concurrent way. The specification of each protocol process consists of specifying its name and the processes that coordinate the services that the protocol is composed of. The name is specified before the symbol '::=', and the process is specified after it. The name of the first process of the protocol specifies the name of the aspect in small letters, whereas it is recommended that the rest of process names be specified in capital letters.

The protocol services are specified using the LPP language. As a result, those services that participate in played_roles must specify their context in order to clearly identify the played_role that they belong to. This is true especially for those cases where one service participates in more than one played_role. In addition, in those software systems in which the priority is required, each service must specify its priority. As a result, when a process has a non-deterministic selection, it can determine the order of service executions.

An example of a protocol is the protocol that glues the $A C T$ and JOINT played roles of the CProcessSuc aspect presented in Figure 102. The partial view of the protocol that it is presented in Figure 104 specifies a non-deterministic choice among the execution request of the services end, moveJoint and stop. This means that the aspect can receive execution requests of the services end, moveJoint or stop. However, if several execution requests arrive at the same time and one of them makes reference to the stop service, the stop service is executed before the others due to the fact that it has the maximum priority (0). The stop service is modelled in 
PRISMA: Aspect-Oriented Software Architectures

the example TeachMover with priorities since this service is used for emergency situations in which the robot must be urgently stopped.

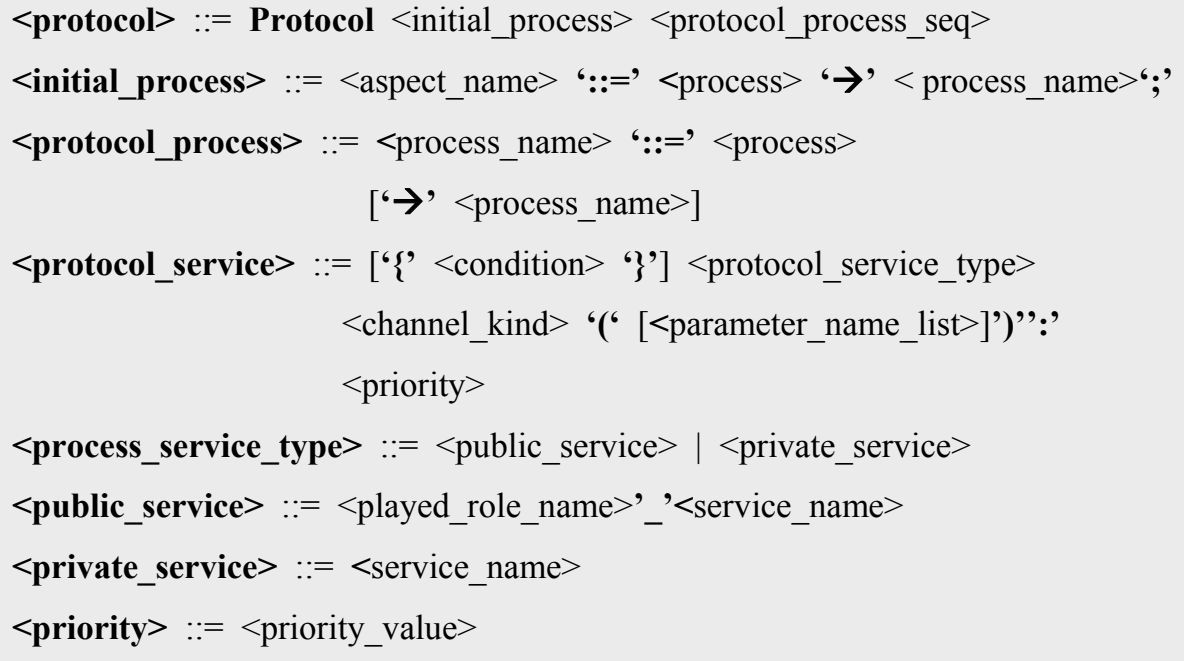

Figure 103. Specification template of protocols

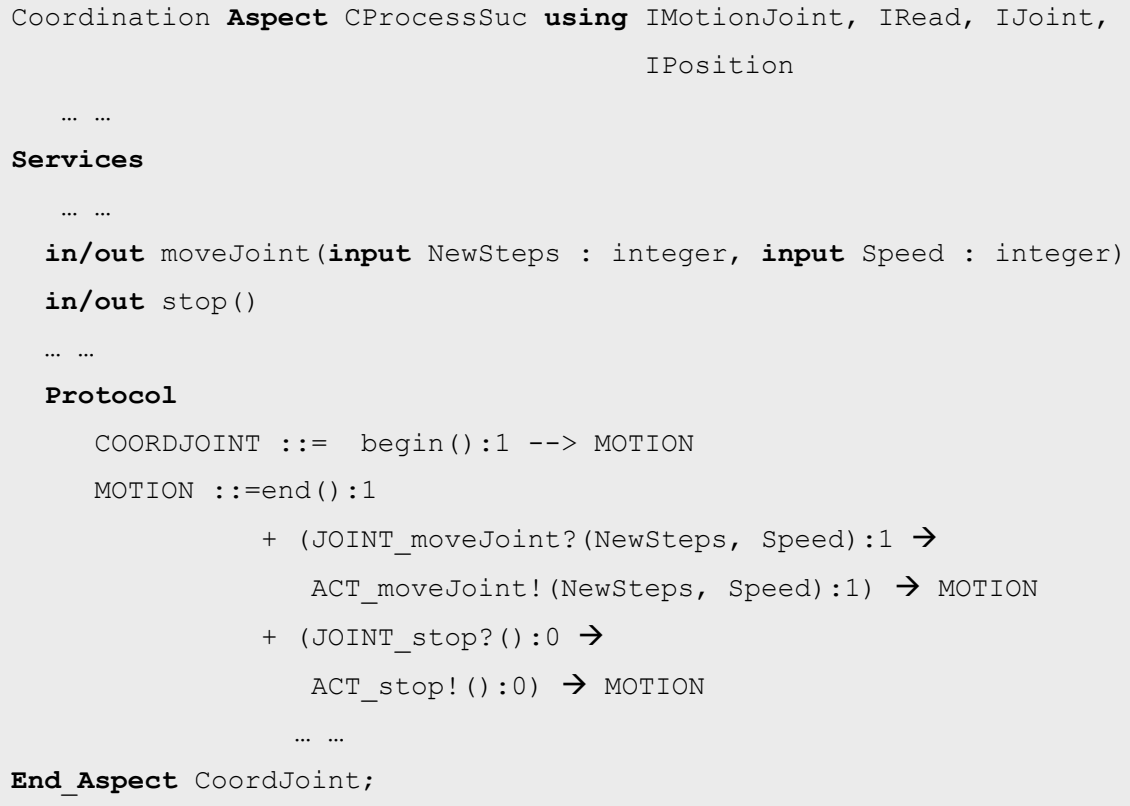

Figure 104. Specification of the protocol of the aspect CProcessSuc 
In addition to the non-deterministic choice, the protocol establishes that after the processing of the execution request of the JOINT played_role, the same service must be requested using the $A C T$ played_role in order to be performed by the robot. In this case, the parameter values are the same because their values have not been changed by their valuations. In this way, the processes of both played_roles are coordinated by the protocol.

\subsubsection{Simple Architectural Elements: Components and Connectors}

A simple architectural element is specified with the set of ports, the aspects it is formed of, and the aspect weavings.

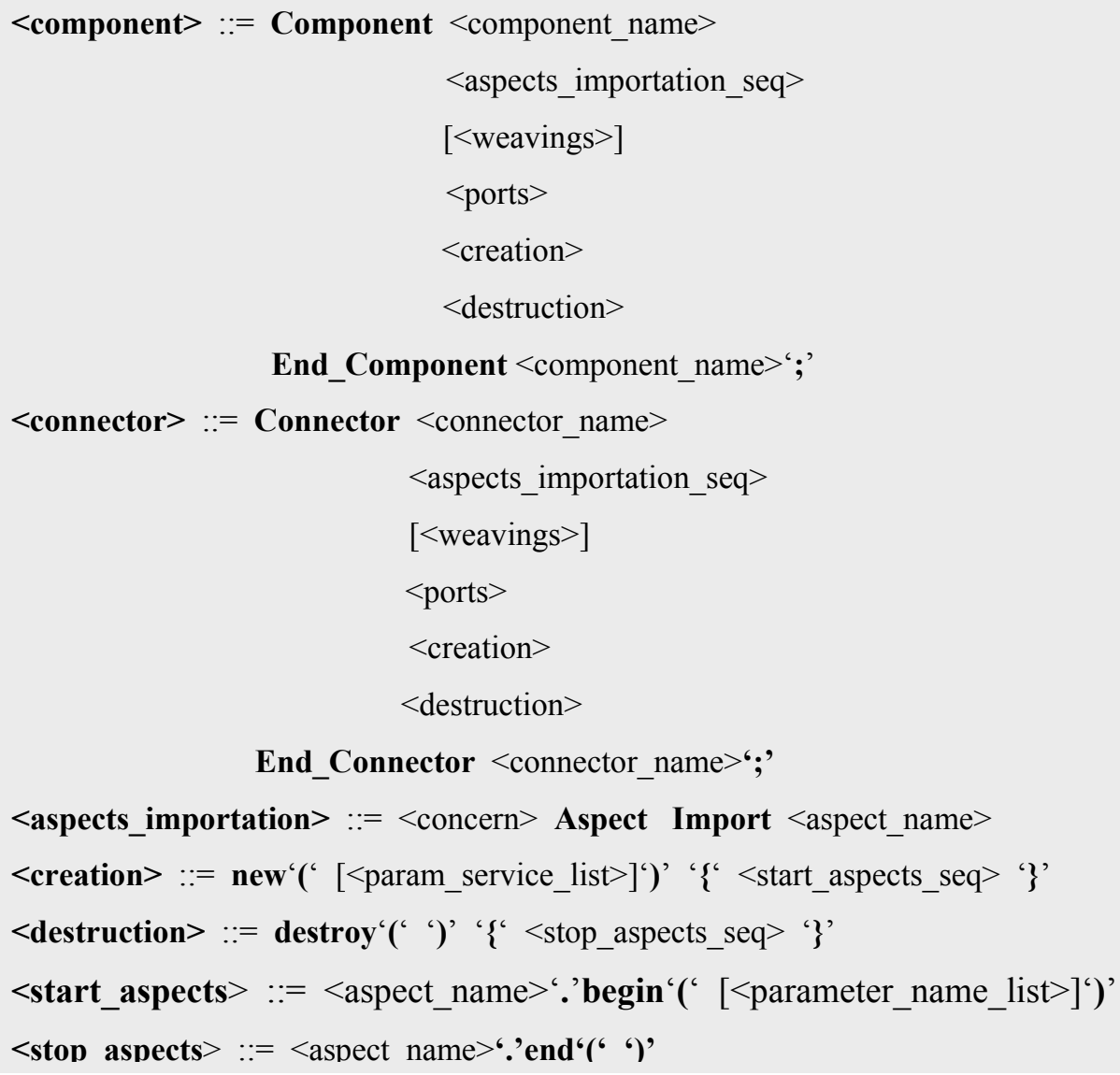

Figure 105. Specification template of simple architectural elements 
PRISMA: Aspect-Oriented Software Architectures

The aspect specification in section 8.1.2 shows that an aspect definition does not include the points where an aspect needs to coordinate with the other aspects (aspect weavings). This independence of the aspect specification from other aspects and weavings makes aspects reusable. Furthermore, the fact that the specification of weavings is inside architectural elements provides the flexibility of specifying different behaviours of an architectural element by importing the same aspects and defining different weavings. Therefore, when architectural elements are defined, they import the aspect and define their weavings. An aspect weaving is specified by determining the aspects that participate in the weaving, the services of the aspects where they are weaved, and the weaving operators.

Components and connectors are simple architectural elements in PRISMA. Their specification is performed in the same way; the only difference is the reserved words that precede and end their specifications. These reserved words are Component...End_Component and Connector...End_Connector for the specification of components and connectors, respectively. The specification of simple architectural elements consists of a name, the importation of needed aspects, the weavings among the aspects, a set of ports, and the two sections that allow the creation and destruction of the architectural element (see Figure 105).

The importation of an aspect is specified by defining the concern and the name of the aspect. Between the concern and the name, the reserved words Aspect Import must be specified (see Figure 105).

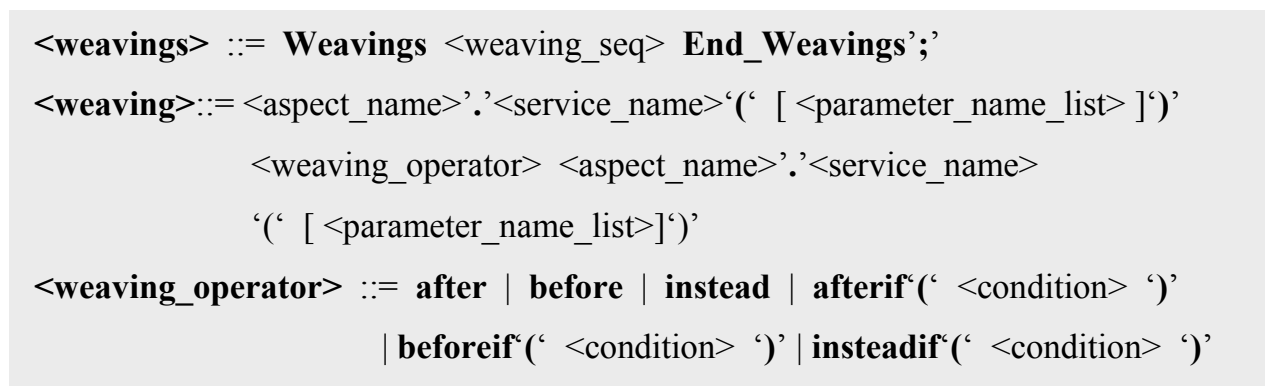

Figure 106. Specification template of weavings

The weavings section is optional because an architectural element can import aspects without having to synchronize them. The weaving section is preceded by the reserved word 
Weavings and is ended by the reserved word End_Weavings. The specification of a weaving consists of the following: specifying the service that is executed as a consequence of the weaving (advice) and the aspect that it belongs to; the weaving operator; and the service that triggers the weaving (pointcut) and the aspect that it belongs to. If the weaving operator is a conditional operator, the condition must be specified (see Figure 106).

The port section specifies the set of ports of the architectural element. This section is preceded and ended by the reserved words Ports and End_Ports, respectively. Each port is specified by defining its name, the interface that it publishes, and the played_role that it associates to the interface. The played_role specification is preceded by the reserved word Played_Role (see Figure 107).

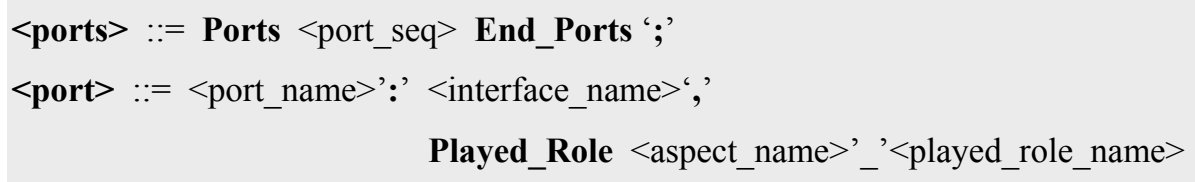

Figure 107. Specification template of ports

Finally, the creation and destruction sections must be specified (see Figure 105). On the one hand, the creation section is preceded by the reserved word new and the list of parameters. Next, the invocations of the begin services of the imported aspects are specified in curly brackets. The destruction section is preceded by the reserved word destroy. Next, the invocations of the end services of the imported aspects are specified in curly brackets.

An example is the CnctJoint connector that synchronizes the Actuator and the Sensor of a joint. This connector imports the SMotion safety aspect and the CProcessSuc coordination aspect. The two aspects are coordinated by means of a weaving that ensures that movements of the robot are safe for the robot. In addition, the connector has four ports: PAct, PSen, PJoint and PPos. All of them export the services of different interfaces and/or have a different played_role defined for the interface. In this case, all the played_roles are defined by the CProcessSuc aspect. After the port definitions, the creation section is specified by invoking the begin services of the SMotion and CProcessSuc aspects. Finally, the destroy service of the connector is specified by invoking the end services of both aspects. 
PRISMA: Aspect-Oriented Software Architectures

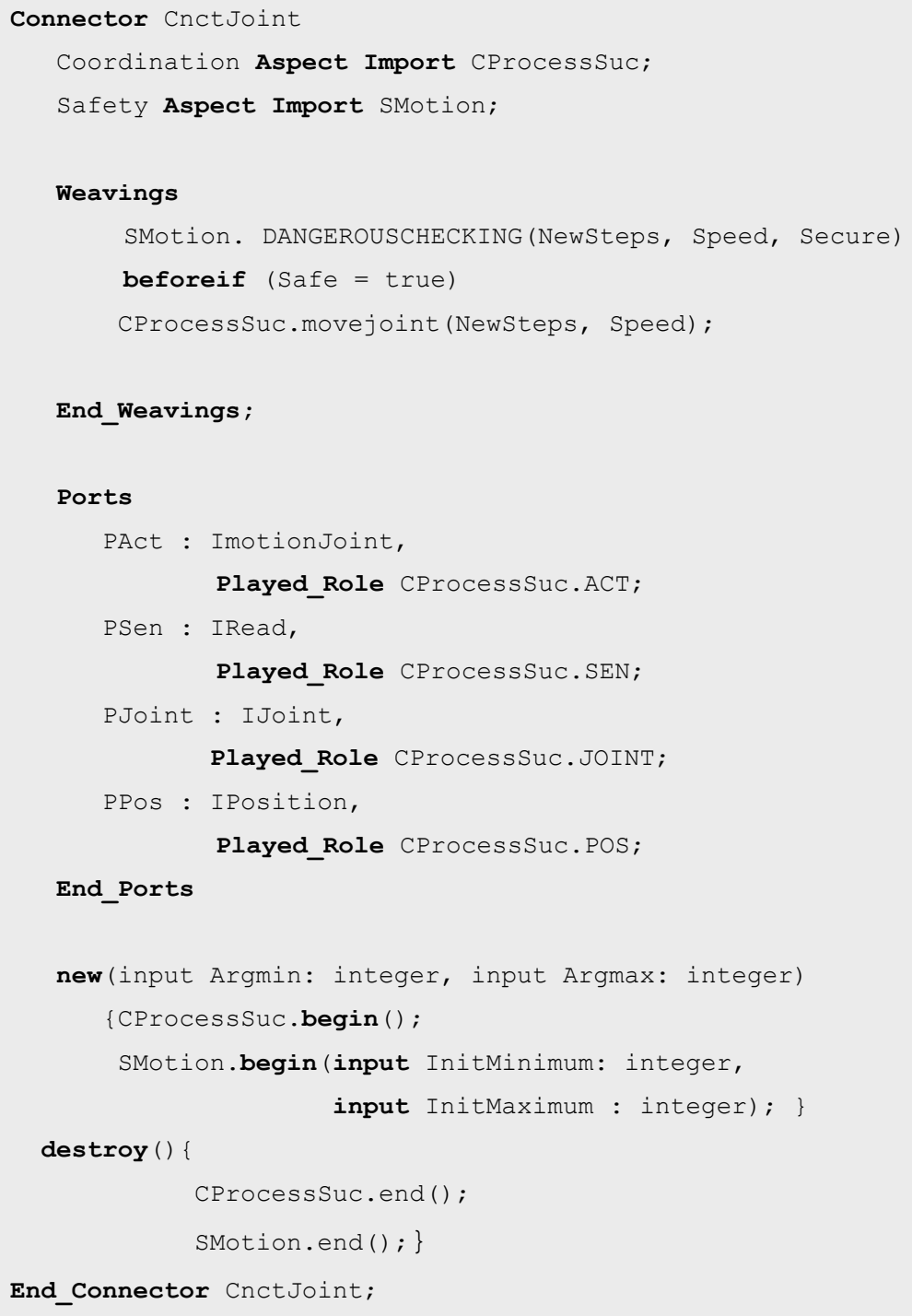

Figure 108. Specification of the connector CnctJoint

\subsubsection{Attachments}

Attachment specifications define the connection among ports of components and connectors. They can be specified inside systems (complex components) to connect the components and connectors that compose systems or outside systems as part of the software architecture. They are specified in the same way in both cases. 


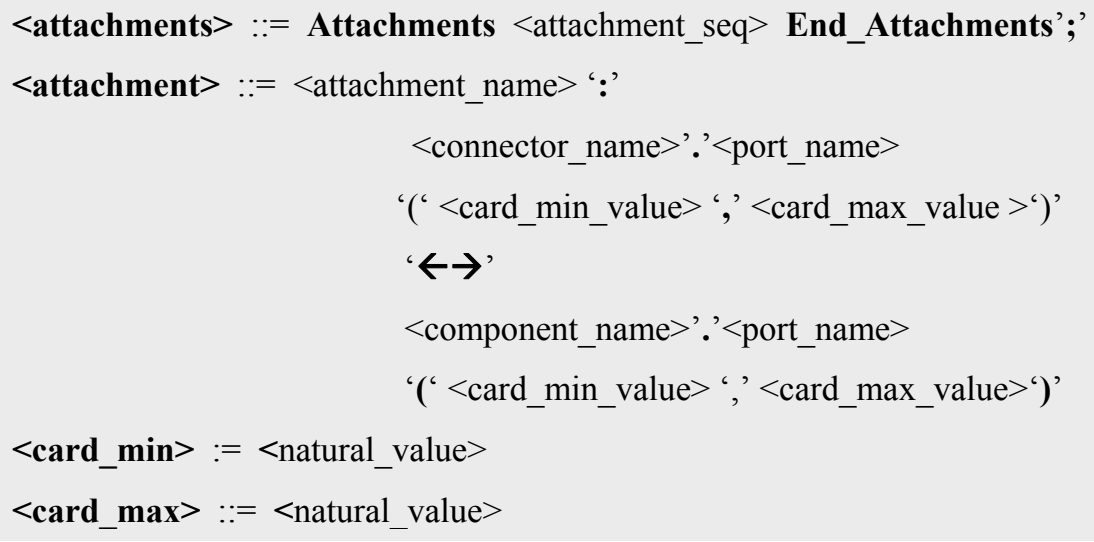

Figure 109. Specification template of attachments

The specification of attachments is preceded and ended by the reserved words Attachments and End_Attachments, respectively. Each attachment is specified by defining its name followed by the specification of the connector port and the component port separated by the symbol ' $\leftarrow$ $\rightarrow$ '. The connector port specification consists of specifying the connector name, the port name and the minimum and maximum cardinalities. The component port specification consists of specifying the component name, the port name and the minimum and maximum cardinalities (see Figure 109).

An example of an attachment is the attachment AttchActCnct that connects the CnctJoint and the Actuator through their respective ports PAct and PCoord. In this case, their cardinalities establish than only one attachment instance can be defined between the ports of the same instances of CnctJoint and Actuator.

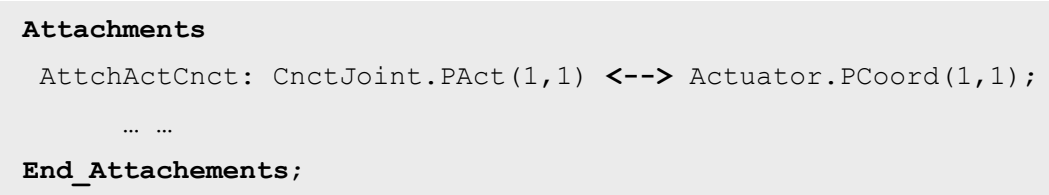

Figure 110. Specification of an attachment between the Actuator and the CnctJoint

\subsubsection{Systems}

The specification of systems permits the definition of architectural patterns that can be reused to define the configuration of one or several software architectures. A system specification is 
PRISMA: Aspect-Oriented Software Architectures

preceded and ended by the reserved words System and End_System, respectively. The specification of a system consists of a name, the importation of needed aspects, the weavings among aspects, a set of ports, the importation of architectural elements, the attachments among architectural elements, the bindings between the system and the architectural elements, and the two sections create and destroy systems (see Figure 111). It is important to keep in mind that a system can be defined without aspects and weavings, attachments or bindings. As a result, these sections of the system specification are optional.

Systems import aspects and define the weavings among these aspects in the same way as simple architectural elements (see Figure 105 and Figure 106). The ports of the system are specified in the same way that it is presented in Figure 107. In addition, the set of architectural elements, that are necessary to define the system are imported, and the number of instances that can be specified at configuration time are constrained. These architectural elements can be components, connectors and other systems, and their specification is defined by specifying the name and the minimum and maximum number of instances that can be created for each architectural element at configuration time.

Moreover, the connections among the different types of architectural elements are specified in order to define the architectural pattern of the system. The attachments inside a system are defined in the same way that is presented in section 8.1.4. The bindings among the system ports and the connector or/and component ports that the system is composed of are specified.

The specification of bindings is preceded and ended by the reserved words Bindings and End_Bindings, respectively. Each binding is specified by defining its name followed by the specification of the system port and the architectural element port separated by the symbol ' $\leftarrow \rightarrow$ '. The system port specification consists of specifying the port name and the minimum and maximum cardinalities, whereas the architectural element port specification consists of specifying the architectural element name, the port name, and the minimum and maximum cardinalities (see Figure 112). 


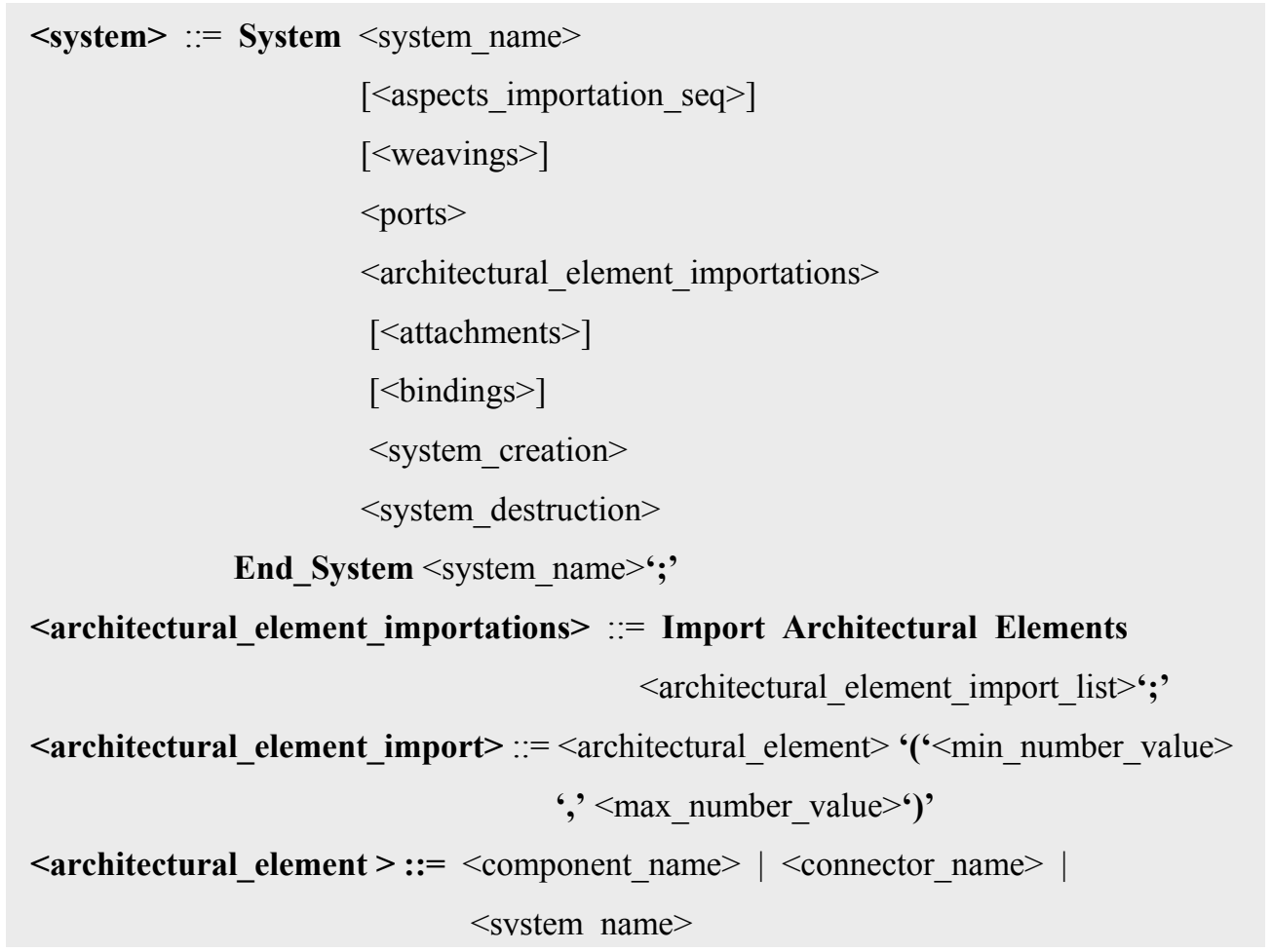

Figure 111. Specification template of systems

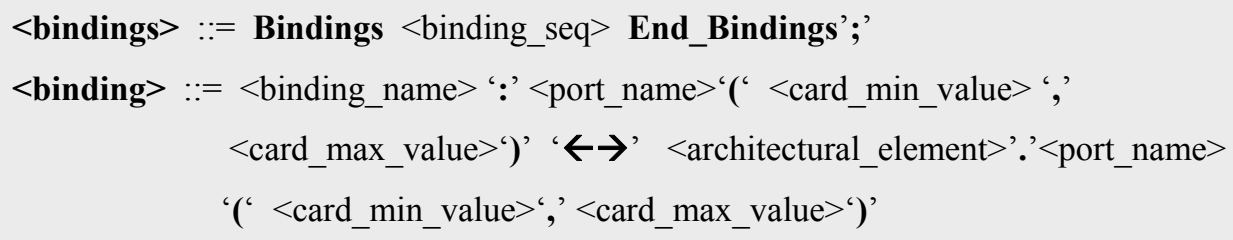

Figure 112. Specification template of bindings

Finally, the creation and destruction sections must be specified (see Figure 113). These sections specify the signature of the services for creating the system at configuration time. These sections establish the parameters and requests required to correctly create the elements that compose the system. Then, the parameters and requests are instantiated at configuration time. The creation section is preceded by the reserved word new, the list of parameters when the system imports aspects, and the list of the number of instances of each architectural element or channel of the system. This number establishes the exact number of instances that must be 
PRISMA: Aspect-Oriented Software Architectures

created for each architectural element, attachment and binding at configuration time. Then, the requests of the begin services of the imported aspects and the requests of the new services of architectural elements, attachments and bindings are specified in curly brackets. The begin service of aspects requires the parameters that are necessary in order to start the execution at configuration time. The new service of architectural elements also requires the value of the parameters that are necessary in order to create the architectural element. The new services of binding and attachments relationships need to know the instance name of the instances that they connect at configuration time.

The destruction section is preceded by the reserved word destroy(). Then, the requests of the end services of the imported aspects and the destroy services of the architectural elements, attachments and bindings are specified in curly brackets. With regard to the attachment and binding creation, the template fixes the parameters. This is due to the fact they always have the same number of parameters, and the only thing that varies is the name of the instances that they connect.

An example is the Joint system, which imports a functional aspect that allows the system to manage its position (see Figure 41). It has a port (PJoinSystem) to communicate with external architectural elements (see section Ports, Figure 41). The Joint is composed of an Actuator, a Sensor, and a CnctJoint connector that synchronizes them (see section Import Architectural Elements, Figure 41). In addition, since it has a functional aspect that needs to communicate with the CnctJoint in order to suitably update the joint position, a component that acts as a wrapper of the functional aspect has been specified. This component wrapper is called WrappAspSys and permits the communication between the aspect of the system and the CnctJoint through an attachment (see section 6.2.12). This attachment (AttchPos) and two more attachments have been defined to communicate the Actuator with the CnctJoint (AttActCnct) and the Sensor with the CnctJoint (AttSenCnct) (see the Attachments section, Figure 41).Also, a binding (BndJCnct) has been defined to publish the services of the CnctJoint through the PJoint port to the exterior of the Joint (see the Bindings section, Figure 41). 


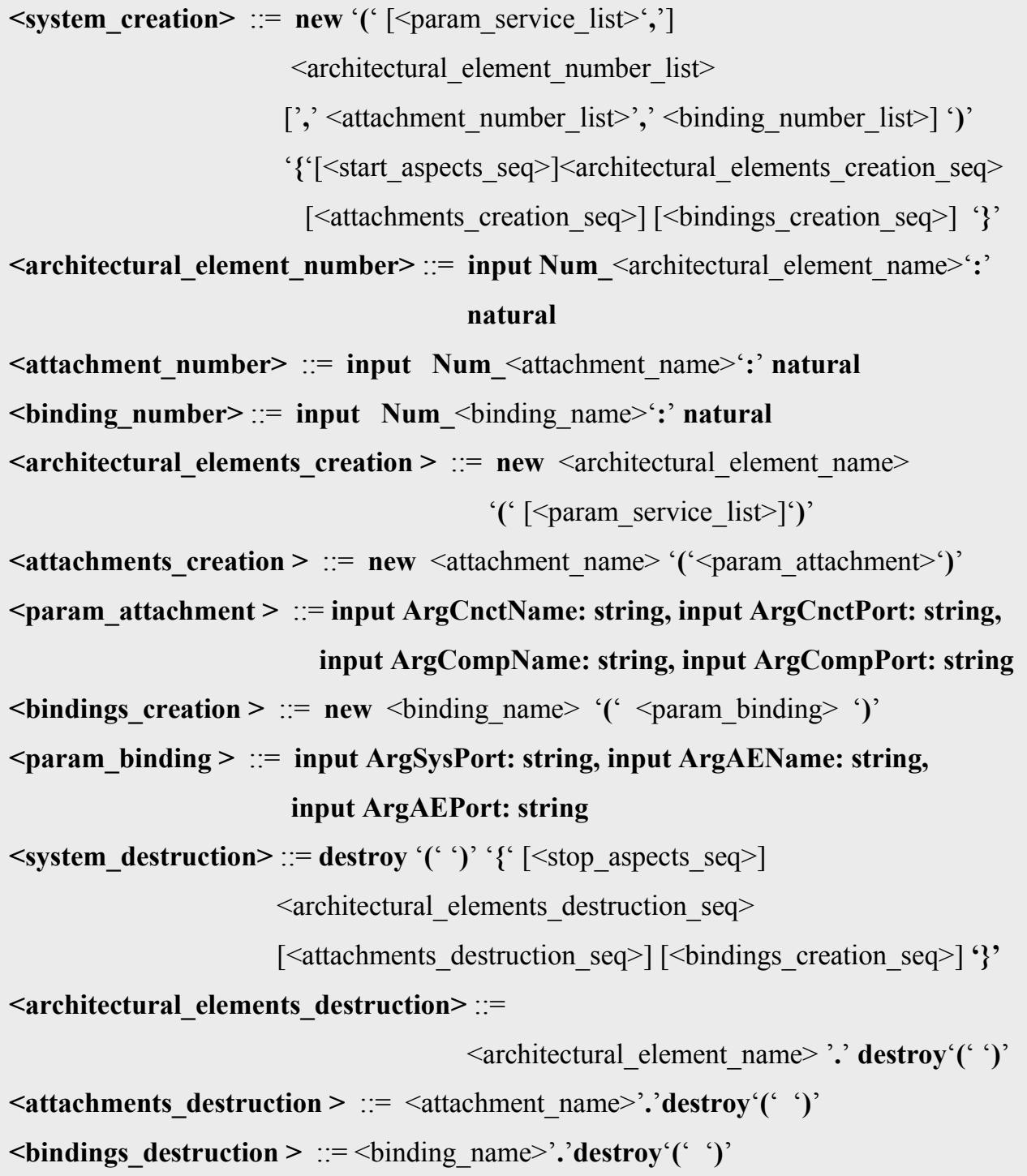

Figure 113. Specification template of the creation and destruction of systems 
PRISMA: Aspect-Oriented Software Architectures

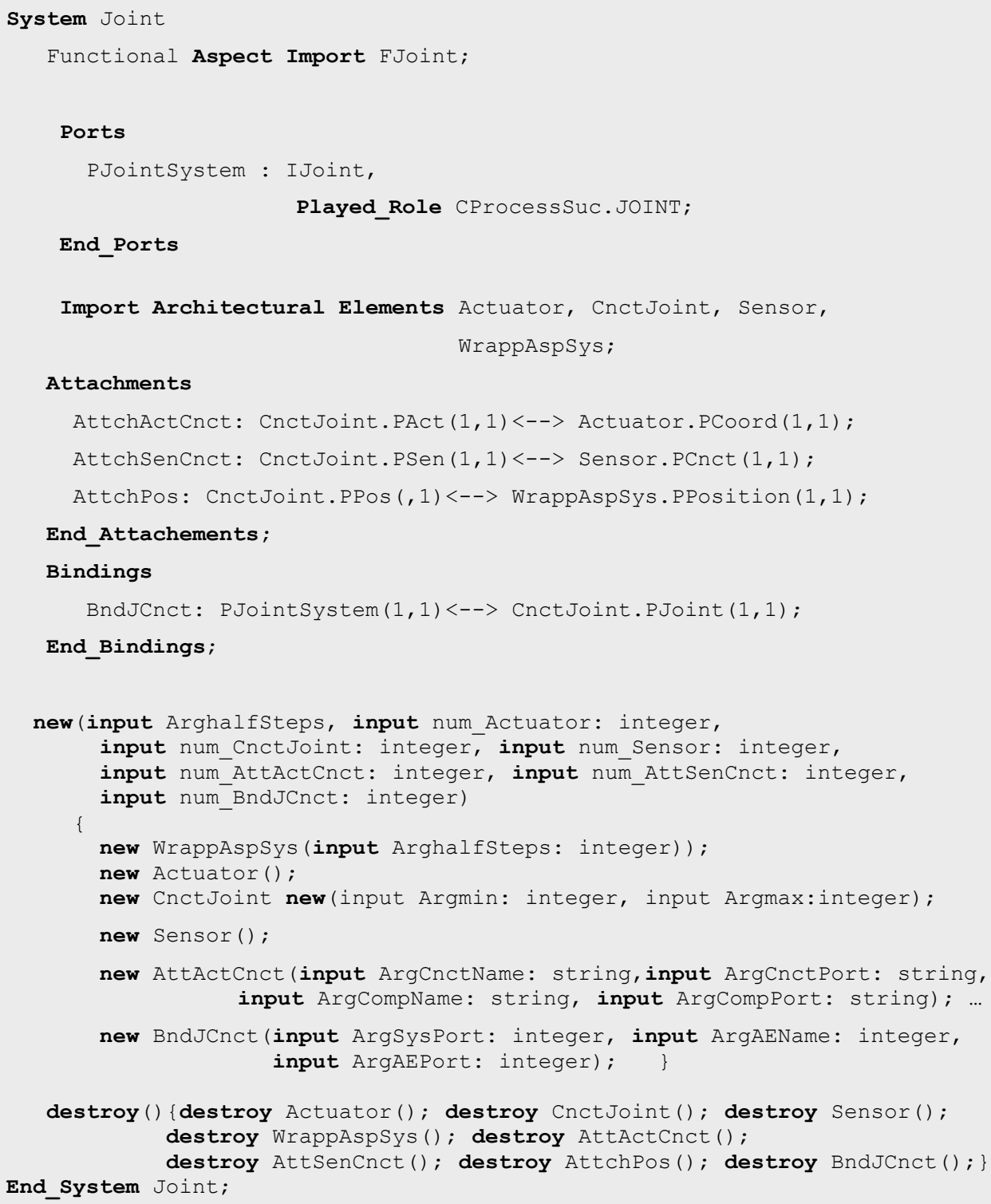

Figure 114. Specification of the system Joint

After these definitions, the new service is specified (see the new section, Figure 41). The list of parameters of the new service consists of the following parameters: a parameter that provides the initial position of the joint (ArghalfSteps), which is a value that is required by the FJoint 
aspect, and the parameters that provide the cardinalities of each architectural element, binding, and attachment of the system. These cardinalities allow the Joint to know how many instances of each type must be created at configuration time.

Note that there are no parameters to provide the cardinalities for the WrappAspSys and the AttchPos. This is due to the fact that they are not architectural elements and attachments of the software system. They are mechanisms that are created on purpose in order to permit the communication between the system aspects and the architectural elements of the system. They are only instantiated once.

The Joint new service specifies the request of the begin service of its imported aspects and the new services of architectural elements, attachments and bindings that the joint includes (see the new section, Figure 41). Since a wrapper is used for the system aspects, the begin service of the FJoint aspect is invoked through the new service of the WrappAspSys. In addition, the request of the new services for the CnctJoint, Sensor, Actuator, AttActCnct, BndJCnct, etc, are specified. Those new services that need parameters to be properly instantiated must also be specified. A clear example is the CnctJoint, which need a set of parameters to provide a value to some of the attributes of the SMotion aspect (see Figure 88).

Finally, the destroy service of the Joint is specified by invoking the destroy services of the architectural elements that it imports.

\subsection{THE CONFIGURATION LEVEL}

The configuration level is used to define a specific architectural model for a software system. In order to do this, all required connector, component and system types should be instantiated. Then, all attachment and binding instances should be connected among these type instances. At this point, the new services are instantiated and the constraints that have been defined for systems are validated in order to ensure pattern satisfaction. 
PRISMA: Aspect-Oriented Software Architectures

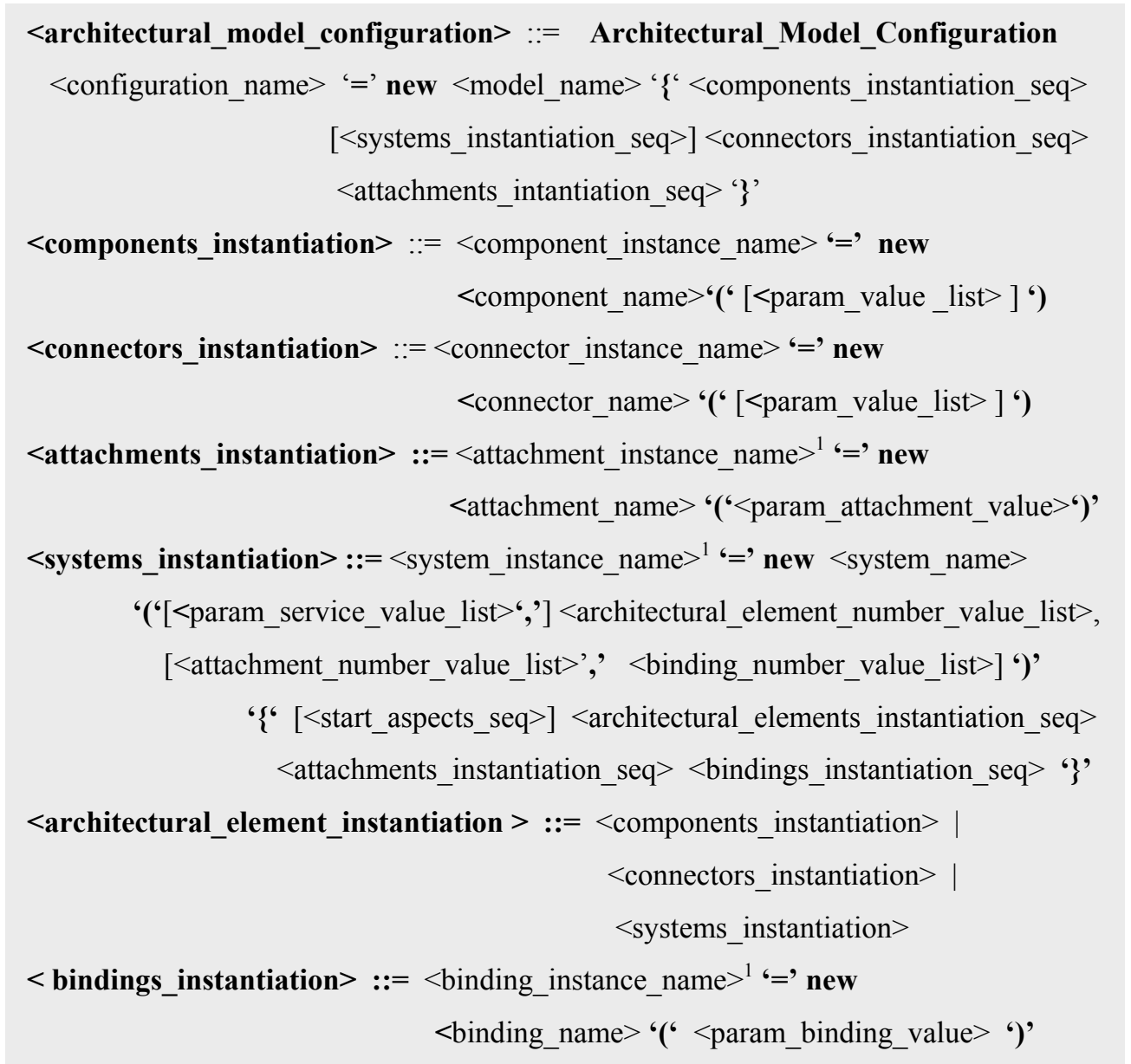

Figure 115. Specification template of configurations of architectural models

The architectural model specification is preceded by the reserved word Architectural_ModelConfiguration and the name of the architectural model configuration. The specification of the new service is defined in curly brackets and is preceded by the reserved word new and the architectural model name. The specification of the new service consists of the request of the new services of the architectural elements, attachments and bindings that compose the configuration in order to instantiate them. 


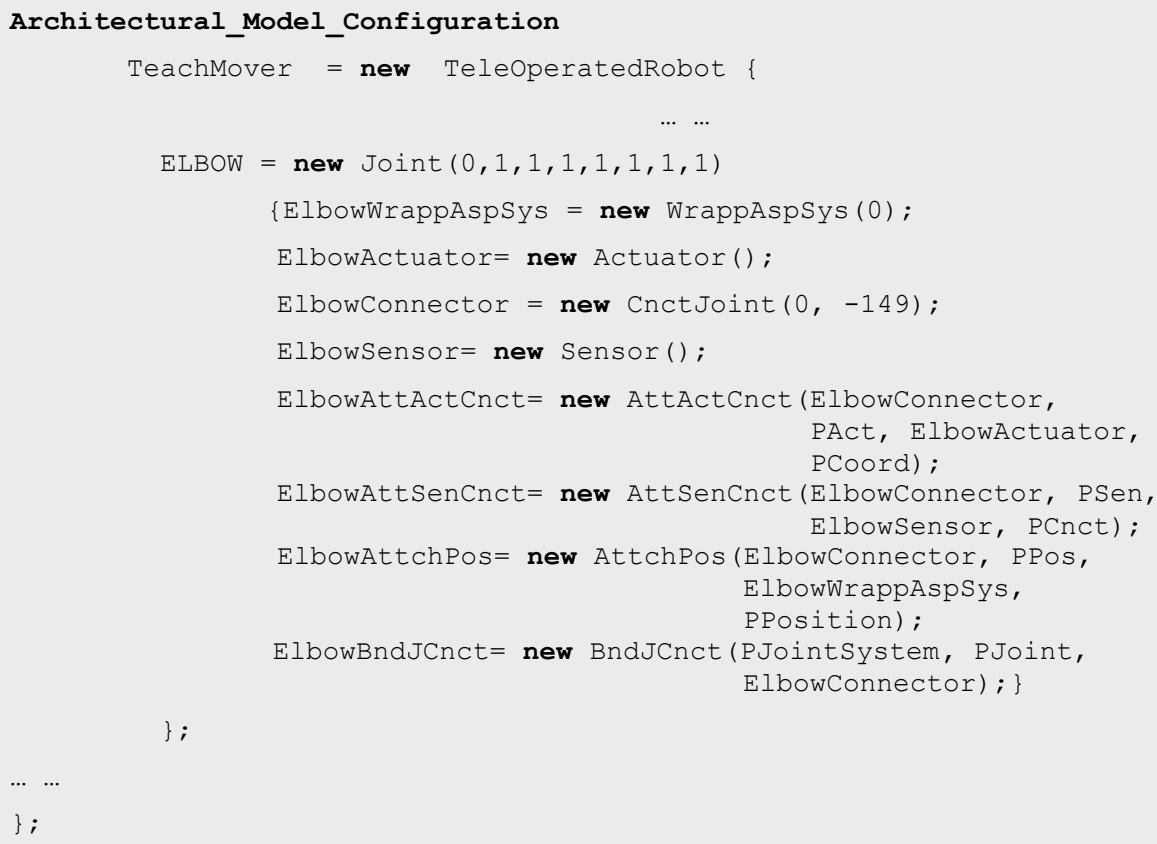

Figure 116. The TeachMover architectural model (The Base Configuration)

An example is the architectural model of the TeachMover. The specific configuration of the Elbow joint is presented in Figure 116. This configuration consists of instantiating the architectural elements of a Joint by providing the needed values to obtain the Base. In addition, the attachments and binding relationships are also instantiated by connecting the instances instead of the architectural element types.

\subsection{CONCLUSIONS}

The PRISMA AOADL has been presented in this chapter. This language allows us to define PRISMA architectural models. The structure, design and maintainability of architectures specified in the PRISMA AOADL are improved by reusing entities at different levels of granularity (interfaces, aspects, components, connectors and systems). This reusability is achieved thanks to the independence of the concept specifications. In other words, interfaces are specified without referencing aspects and ports, aspects are specified without referencing 
PRISMA: Aspect-Oriented Software Architectures

other aspects or architectural elements, and architectural elements are specified without referencing other architectural elements. The references among aspects are specified by means of weavings, and the references among architectural elements are specified using attachments and bindings. Therefore, an interface can be reused by several aspects and ports; an aspect can be reused by several architectural elements and can be synchronized in different ways and with different aspects inside these architectural elements; and an architectural element can be attached and bound to several architectural elements.

This reusability is also improved by means of the division of the language into two levels of abstraction: types and configuration. The types defined at the type definition level can be reused by the configuration level to specify different software architectures such as the TeachMover, EFTCoR, etc. At the same time, the division of the language into two levels of abstraction provides two more important advantages. One of the advantages is the fact that it is possible to independently manage types and the specific configuration of an architectural model, which improves the reusability of types and the maintenance of the system. The other advantage is that it is possible to easily differentiate between a change in a type and a change in the configuration of the architecture. As a result, this provides a suitable framework to introduce evolution mechanisms at two different levels of abstraction: types and configuration.

In addition, the fact that the PRISMA AOADL integrates AOSD in software architectures provides better maintenance. This is due to the fact software architectures are well modularized by a functional decomposition; however, in PRISMA this modularization has been improved by the specification of the crosscutting-concerns inside aspects. Thus, if a change in the features of a specific concern is required, it would only be necessary to modify or change the aspect that defines the concern, and every architectural element that imports it will automatically be updated. As a result, the changes of the functionality are well located thanks to the architectural elements, and the changes of crosscutting-concerns are well located thanks to aspects.

It is important to take into account that most ADLs only permit the specification of the skeleton of architectures and the services that are interchanged among their different architectural elements. The PRISMA AOADL has greater expressive power and can specify 
more features and requirements using aspects. This complete specification of the system requirements facilitates code generation.

Finally, it is important to emphasize that the PRISMA AOADL is a formal language that allows the validation and verification of PRISMA architectural models and facilitates the task of automatically generating code from its specifications. Since PRISMA AOADL is a language independent of the technology, the same PRISMA architectural model can also be compiled into different programming languages and technologies, thereby reducing development time and preserving the traceability between an architectural model and its application code.

The work related to the PRISMA ADL has produced a set of results that have been materialized in the following publications:

> Jennifer Pérez, Nour Ali, Jose Ángel Carsí, Isidro Ramos, Designing Software Architectures with an Aspect-Oriented Architecture Description Language, 9th Symposium on the Component Based Software Engineering (CBSE), Springer Verlang LNCS 4063 ,pp. 123-138, ISSN: 0302-9743, ISBN: 3-540-35628-2, Vasteras, Suecia, June 29th-July 1st, 2006.

> Jennifer Pérez, Isidro Ramos, OASIS as a Formal Support for the Dynamic, Distributed and Evolutive Hypermedia Models, Technical Report DSIC-II/22/03, pp. 144, Polytechnic University of Valencia, October 2003. (In Spanish) 
PRISMA: Aspect-Oriented Software Architectures 


\section{PART V}

\section{THE PRISMA FRAMEWORK}

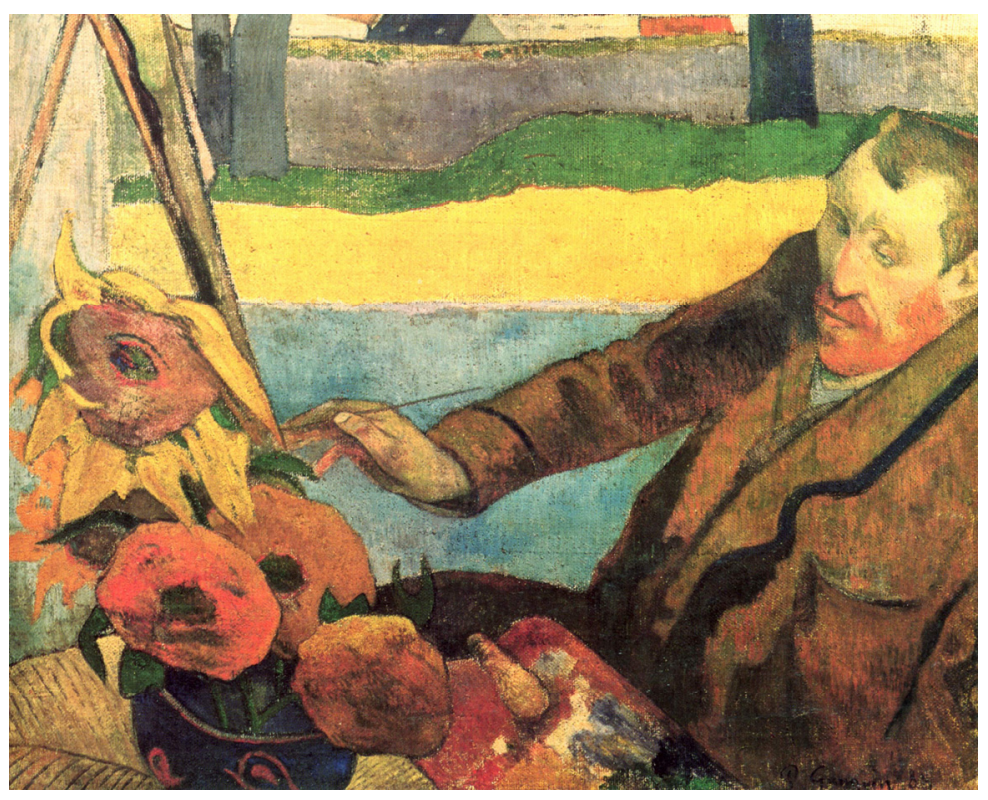

“Van Gogh Painting Sunflowers”, Paul Gauguin, 1888 



\section{CHAPTER 9}

\section{THE PRISMA CASE}

$<<$ Knowledge is a treasure, but practice is the key to it. $>>$

Thomas Fuller

Some new approaches have recently emerged in order to improve software development. Their common characteristic is that they try to improve the early stages of the software life cycle by automating their activities as much as possible by following Model-Driven Development (MDD). MDD is a software development paradigm that is based on models that use automatic generation techniques in order to obtain the software product. MDD is included within ModelDriven Engineering (MDE), which increases the variety of software artefacts that can be represented as models (ontologies, UML models, relational schemas, XML schemas, etc). The use of models to develop software provides solutions that are independent of technology, whose source code can be obtained by means of automatic code generation techniques for different technologies and programming languages. The high level of abstraction that models provide permits working with metamodels in the same way as with specific models or domainspecific models.

Since the PRISMA model is a technology-independent model, the PRISMA approach follows the MDD paradigm to obtain its advantages during the development and maintenance processes of PRISMA architectures. The main goal of the PRISMA approach is to give support to the development of technology-independent aspect-oriented software architectures, which could be compiled for different technological platforms and languages using automatic code generation techniques. A PRISMA CASE has been developed to give support to the 
PRISMA: Aspect-Oriented Software Architectures

PRISMA approach following the MDD paradigm. PRISMA CASE currently supports the generation of aspect-oriented C\# code that is executable on .NET technology. The PRISMA CASE is composed of the PRISMA metamodel, a graphical modelling tool, a model compiler, and a middleware (see Figure 117).

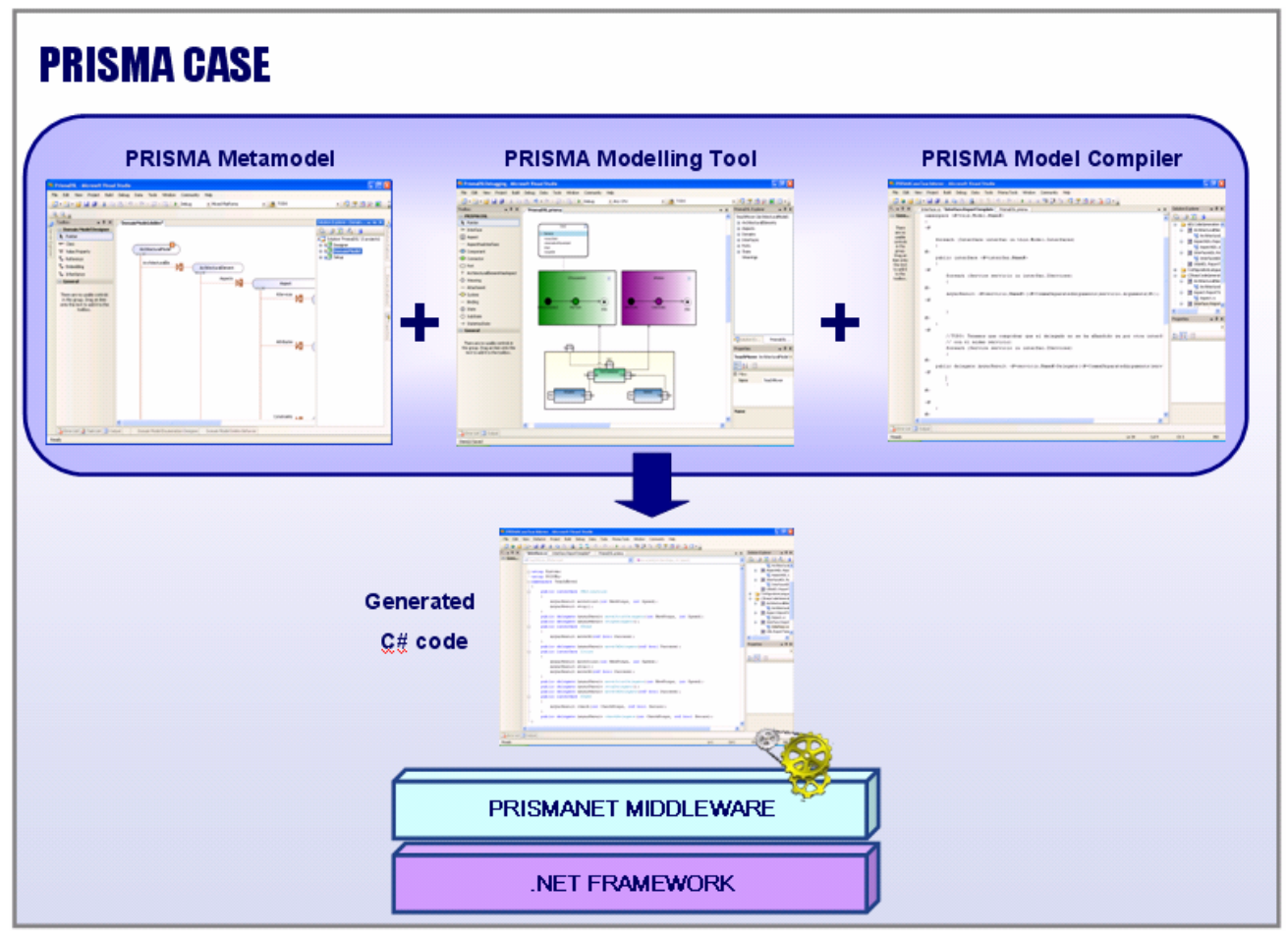

Figure 117. PRISMA CASE

The PRISMA metamodel is part of the PRISMA CASE since the metaclassess that allow the creation of PRISMA aspect-oriented software architectures, as well the OCL rules of the PRISMA metamodel, must be available in the CASE tool. They are necessary to be able to model PRISMA architectural models and to make sure that they satisfy the PRISMA model.

The PRISMA AOADL is a formal language. Even though the use of a formal language clearly provides advantageous characteristics, the use of a formal language is really difficult. For this reason, PRISMA CASE provides a graphical language and a graphical modelling tool to model PRISMA software architectures using an intuitive and friendly graphical AOADL 
Since PRISMA CASE must generate executable C\# code in .NET technology and the .NET framework does not provide support for the Aspect-Oriented approach, this thesis presents a .NET middleware that has been developed to provide a solution. This middleware is called PRISMANET. PRISMANET extends the .NET technology through the execution of aspects on the .NET platform in accordance with the PRISMA model.

Finally, the PRISMA model compiler has been developed to automatically generate C\# code from the PRISMA architectural models that are defined using PRISMA CASE.

This chapter presents the PRISMA CASE in detail. First, it is explained how the PRISMA CASE supports the metamodel and how the modelling tool gives support to the graphical PRISMA AOADL. Second, the PRISMA model compiler and its code generation patterns are introduced. Then, the chapter explains how the configuration of software architectures is integrated in PRISMA CASE and how PRISMA configurations can be executed. Finally, the PRISMANET middleware is presented to show how the execution of PRISMA software architectures is supported by the .NET platform.

\subsection{GRAPHICAL MODELLING TOOL}

The PRISMA CASE provides a graphical language and a modelling tool to support more intuitive and friendly aspect-oriented software architecture modelling. However, a formal specification is more suitable for some details of PRISMA specifications; for instance, the specification of formulae such as valuations or preconditions. Thus, only the main concepts and their relationships are graphically specified; the rest of the concepts are represented using the AOADL (see chapter 8 and appendix A), and their specifications are included in the definition of the corresponding graphical shapes.

\subsubsection{PRISMA UML Profile}

UML is a standard of the OMG and a widely extended notation [UML06]. These characteristics make graphical notations based on UML more understandable to users. There are a wide variety of tools based on UML that interchange UML models using XMI documents and provide extension mechanisms. This means there is no need to develop a tool 
PRISMA: Aspect-Oriented Software Architectures

from scratch in order to create a customized tool. Therefore, UML was initially chosen as a graphical notation for PRISMA.

Although UML is intended for object-oriented modelling, thanks to its extension mechanisms that are associated to the definition of a UML profile, it can be customized to the particular needs of new models. These extension mechanisms are stereotypes, tagged values, and constraints, which are all used to define new derived concepts (metaclasses) from the standard UML metaclasses. Thus, a set of specific extensions is called a UML profile.

A PRISMA profile was developed not only to have a graphical notation that follows a standard, but also to achieve the PRISMA support by any modelling tool that supports UML or XMI documents. This profile includes all the necessary extensions for using UML as a graphical notation for PRISMA specifications. It has been implemented by extending the metaclasses of the UML metamodel version 1.5. and by preserving the satisfaction of the requirements that Aldawud stated for defining a UML profile for AOSD. This extension is necessary because, despite the fact that UML 1.5 includes the following concepts: component, connector and interface (required or provided), the provided expressivity is too basic in comparison with PRISMA. Furthermore, UML 1.5. does not include the aspect, port, weaving, attachment and binding concepts. A simplified version of the PRISMA UML profile is presented in appendix B.

One of the advantages of defining a UML profile is that modelling tool support can be easily obtained by means of available tools based on UML, such as Rational Rose [RAT06], Argo UML [ARG06], Visio [VIS06], etc. Most of them provide mechanisms to extend their functionality. This means that there is no need to develop a tool from scratch but that any of the existing ones can be extended. Several of these tools were considered and reviewed as support for PRISMA modelling, but Visio was finally selected for the following reasons:

1. Visio allows straightforward management, both for using and modifying shapes. This characteristic is highly relevant for PRISMA purposes because all the kinds of concepts that are included in the PRISMA profile can easily have different shapes related to their functionality. 
2. Visio provides an add- to translate a UML model to XMI. This means that other tools such as those listed in [OCL06] can be used for semantic analysis. This provides for simple consistency checks and type checking in terms of defined OCL constraints of the PRISMA profile.

A lightweight extension of Visio called EGV-PRISMA was developed by means of a template to support the above mentioned needs of the PRISMA profile [Per06b], [Per06a]. However, Visio does not provides a repository where the PRISMA metamodel can be introduced and where its constraints can be verified during the modelling process. The models can only be semantically verified when the model is finished, using other tools such as the ones that appear in [OCL06]. Furthermore, Visio does not provide mechanisms to develop a model compiler that automatically generates the source code from the graphical models.

Since Visio does not provide a framework that could support all the requirements that a PRISMA analyst has, the selection of another tool that would support these needs was mandatory. As a result, DSL tools were selected to develop the PRISMA framework because they provide specific mechanisms to define models, graphical support and code generation templates. However, DSL tools are not based on a standard such as UML, an ad-hoc graphical AOADL has been defined for the PRISMA CASE.

\subsubsection{Domain-Specific Language Tools (DSL Tools)}

The PRISMA approach follows the MDD paradigm. There are two main approaches that apply this paradigm. They are the Model-Driven Architecture (MDA) approach proposed by the OMG [MDA06], and the Software Factories approach proposed by Microsoft [Gre04]. MDA deals with the lack of software system adaptation to different technologies and programming languages. Software Factories leads to the reuse of architectures, software components, techniques and tools to improve software development. The set of tools and techniques that give support this approach are integrated into DSL Tools (Domain Specific Languages Tools) [DSL06].

DSL Tools is the framework that has been selected to develop and support the development of PRISMA aspect-oriented software architectures. It is a set of tools for creating, editing, 
PRISMA: Aspect-Oriented Software Architectures

visualizing, and using domain-specific models to automate and improve the software development process (see Figure 118). This set of tools is integrated into the Visual Studio 2005 framework to define domain models with their customized graphical representations. The tools and mechanisms that compose this framework are the following:

> Project wizard: The framework allows you to define your own customized solution where the specific model, its graphical representation and its code generators are defined. Its integration inside the Visual Studio 2005 permits the validation of the generated code by executing it in the framework. The wizard requires the choice of one of the templates that it offers on which to base the definition of a domain model. There are currently four templates available:

- Minimal Language: This is the generic template for the definition of any domain model.

- Activity Diagrams: This is a template for the activity diagrams of UML

- Class Diagrams: This is a template for the class diagrams of UML

- Use Case Diagrams: This is a template for the use cases diagrams of UML

Model designer: The Model Designer tool creates a domain model project and permits the definition of a domain model associated to this project. In the domain model, concepts are represented in a graphical way by classes and relationships (see Figure 118). Classes have value properties and participate in relationships. A domain model defined in the model designer is translated to .NET Framework classes. These C\# classes are provided with an interface to access and update value properties, to navigate across relationships, and to enable an object to participate in a relationship. In addition, the classes can be extended to introduce verification and validation rules depending on whether the domain model is a meta-model or a model, respectively. 


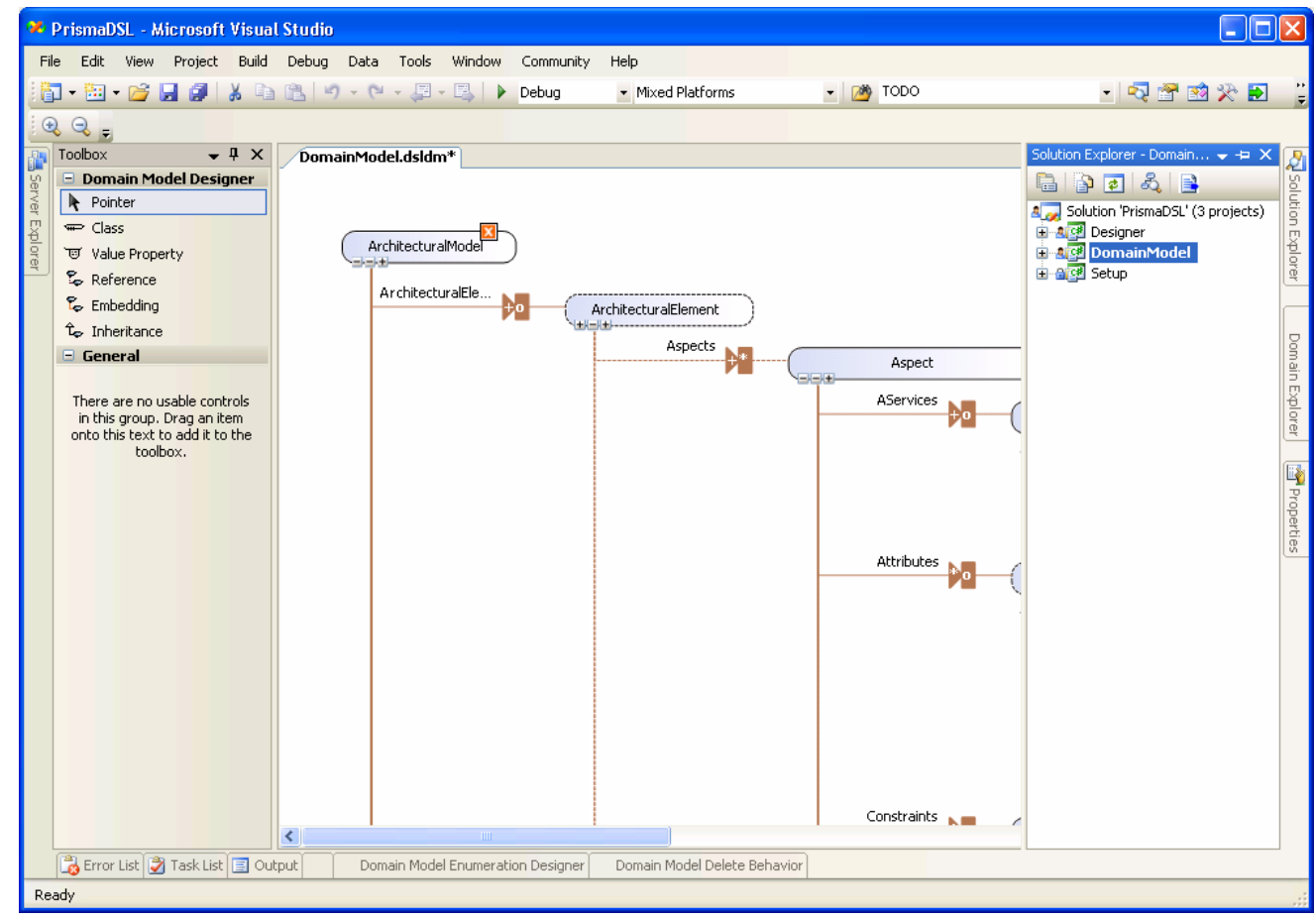

Figure 118. DSLTools Framework: Domain Model of PRISMA

Graphical designer: The Model Designer tool stores the definition of the domain model in an XML document (see Solution Explorer in Figure 118). In this XML document, the design decisions and the graphical representation of each one of the concepts of the model can be defined without any manual coding. These definitions have an effect on the generated solution, where it is possible to define specific models following the domain model defined in the domain model project. The Graphical Designer tool creates the designer project, i.e., the diagram types that are associated to a domain model, the concepts that can be drawn in each diagram as well as the shapes that are associated to each concept and the toolboxes.

$>$ Other projects: In addition to the two predefined Domain Model and Designer projects, DSL can add as many projects as necessary to develop a customized modelling tool.

Onces the DSL solution has been completely defined, it can be compiled and executed. As a result, a modelling tool for the domain specific model that has been defined is generated. This 
PRISMA: Aspect-Oriented Software Architectures

tool is called Debugging Solution. This generated tool provides a customized toolbox, a tree that is organized according to the metamodel in which concepts can be queried and updated, mechanisms for browsing through the tree, and mechanisms to update and query the properties of the defined concepts. This solution not only provides a customized modelling tool, it also provides a new project in its solution explorer called Code Generation Designer, which provides a set of templates in order to automatically generate the code using a set of code generators. These templates help users to define a model compiler in an easy way by browsing through the concepts that have been modelled and stored in the metamodel (DSL Domain Model). The DSL code generators take the templates, the domain model definition and its XML document as inputs of the code generation process. The output of this process is generated by the code generators following the defined templates and substituting the parameters for the concepts stored in the metamodel.

\subsubsection{PRISMA as a Domain-Specific Language}

DSL tools have been created to model specific models such as the model of a web page, a banking system, a tele-operated system, etc. The debugging solution of this model is then used ftoor define specific web pages, banks systems, tele-operated systems with domain-specific tool boxes and concepts that have been defined in the Domain Model and the Designer projects. However, the PRISMA model is a metamodel that permits the definition of PRISMA models whose instantiation defines specific systems. In this sense, PRISMA has taken a step forward. In PRISMA, DSL tools are used to define a metamodel as a domain-specific language, i.e., aspect-oriented software architectures are defined as a domain-specific model.

In order to develop PRISMA CASE, a DSL project has been generated using the Minimal Language template (see section 9.1.2).This is the template that provides mechanisms for introducing the PRISMA metamodel in DSL Tools. The predefined projects DomainModel and Designer are generated as a result of this template selection.

The project DomainModel provides a toolbox to define the domain-specific model in DSL (see Figure 119). In PRISMA, every metaclass and relationship of the PRISMA metamodel is 
been introduced in the domain model. For example, Figure 120 shows the definition of the architectural element and aspect concepts in the DomainModel.

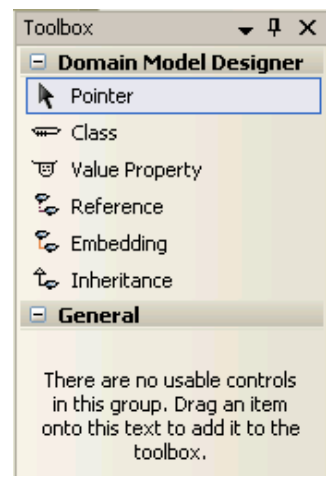

Figure 119. Toolbox of the Domain Model

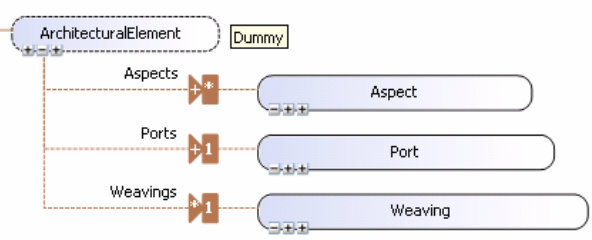

(a) Architectural Element

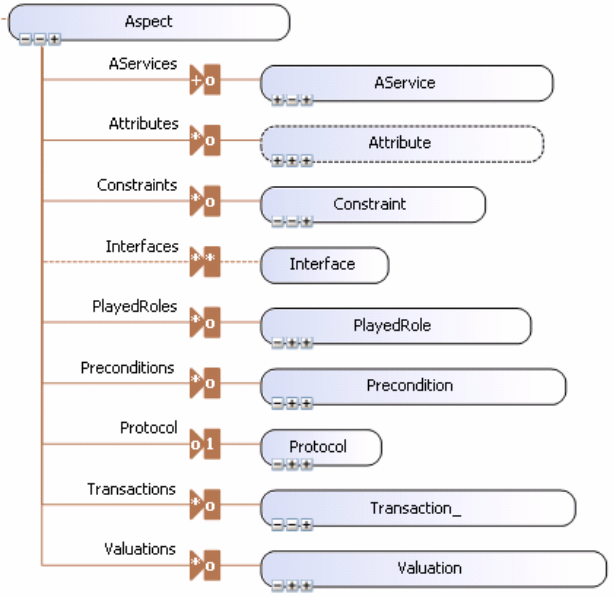

(b) Aspect

Figure 120. Definition of Architectural Elements and Aspects in the DomainModel of DSL

All the classes of the PRISMA domain model are translated to partial C\# classes in order to access and update value properties, to navigate across relationships, and to enable an object to participate in a relationship. These partial classes can be implemented by parts and/or by different files. Therefore, a folder Verification has been created in the Domain Model, which includes a set of DSL files that allow the implementation of verification rules (see Figure 121). 
PRISMA: Aspect-Oriented Software Architectures

This folder also includes the files that extend the partial classes of PRISMA types that have associated constraints which must be verified during the modelling process. These constraints are presented in chapter 7 using OCL language.

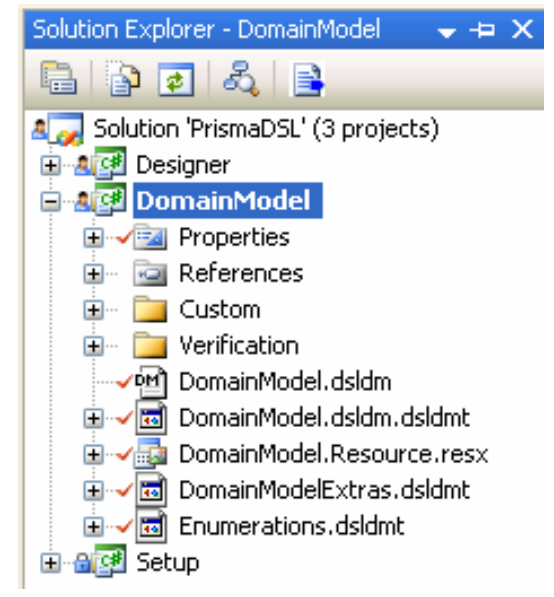

Figure 121. PRISMA Domain Model of DSL

DSL tools distinguish between two kinds of verification: verification rules that must always be satisfied (hardconstraints), and verification rules that must be satisfied once the model has been completely finished (verification rules). The Verification folder of the Domain Model contains the Verification Rules. Verification rules are not verified while the user is modelling, they are verified when it is explicitly requested by the user or when the model is saved. These Verification Rules are PRISMA constraints that act as warnings during the modelling process. These warnings must be rectified before the model is finished so that, it is compliant with the PRISMA metamodel. For example, an architectural element must always have at least one aspect associated to it. However, the structure of the architectural model can be modelled without associating the behaviour and then, the architectural model relationships with aspects can be modelled.

The Designer project of PRISMA associates a graphical metaphor to each PRISMA class of the domain model that requires it. This project also stores the graphical representations selected for the PRISMA concepts and implements the PRISMA hardconstraints (see Figure 122). 


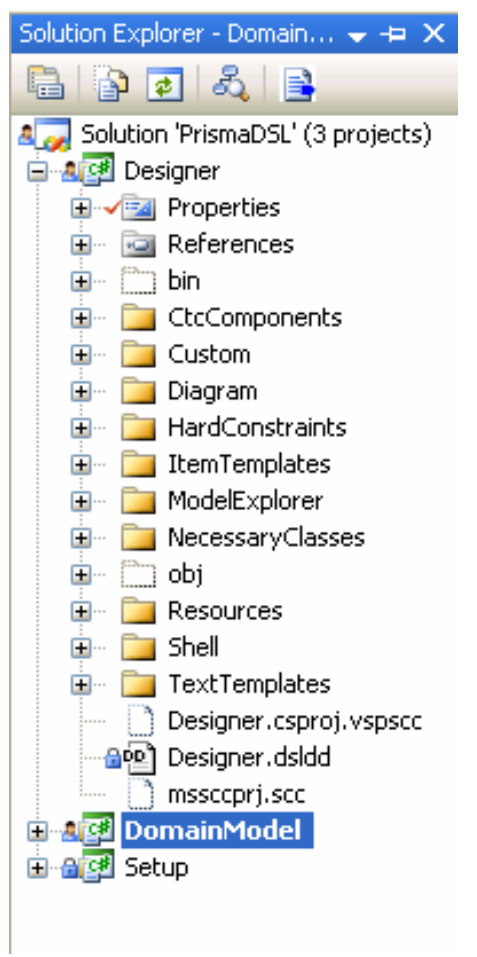

Figure 122. PRISMA Designer of DSL

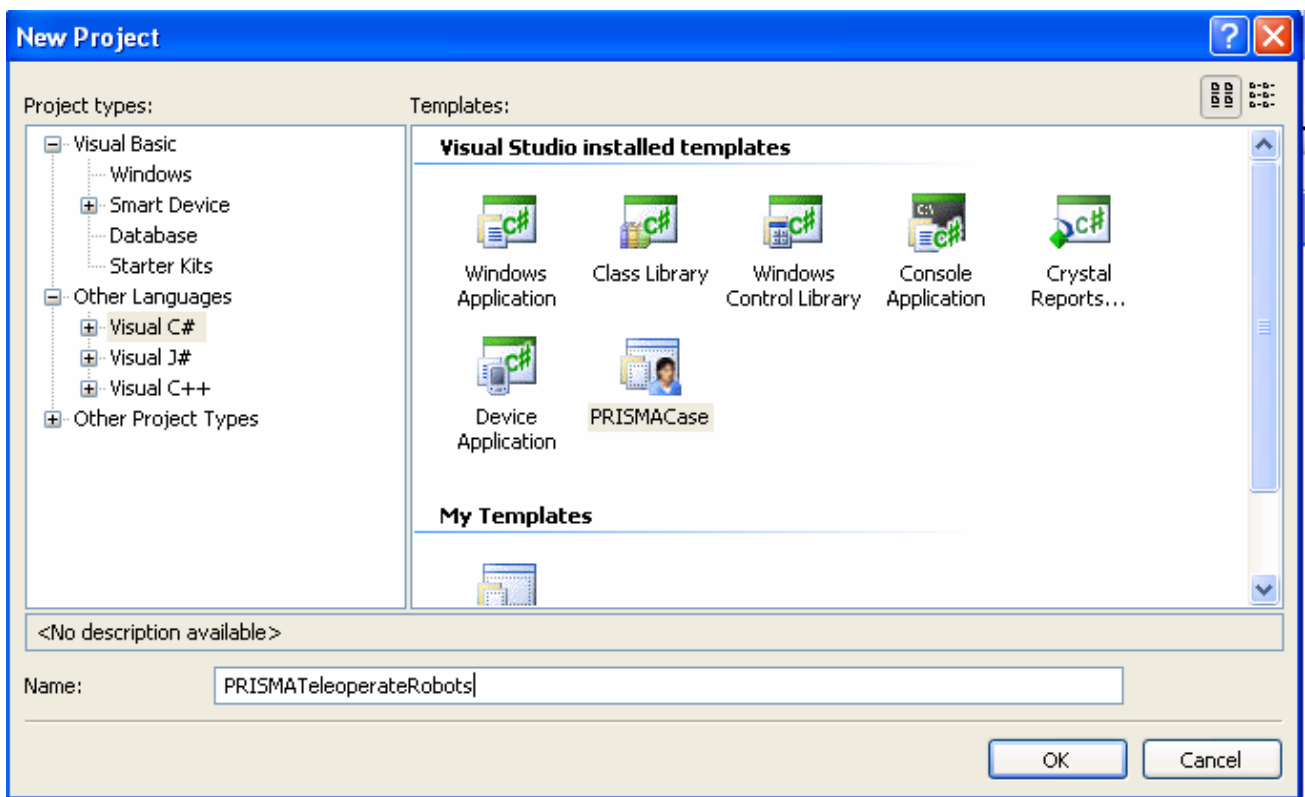

Figure 123. The Visual Studio Project of PRISMA 
PRISMA: Aspect-Oriented Software Architectures

Hardconstraints are verified while the user is modelling. They are very close to the graphical metaphor and they do not permit links between certain graphical entities, compositions of certain entities, changes of name, etc. For example, two aspects cannot be linked using an attachment.

A new project has been added to the PRISMA domain-specific model in order to generate a setup for the PRISMA CASE and to facilitate its installation for users (see Figure 122). This setup creates a new kind of project for Visual Studio 2005 called PRISMACase (see Figure 123). The creation of a PRISMA CASE project consists of launching PRISMA CASE and starting the development process.

\subsubsection{The PRISMA Modelling Tool}

The PRISMA Modelling tool is generated from the projects defined in the above section. The modelling tool is composed of a toolbox, a drawing sheet, a model explorer, a window of properties and a PRISMA menu (see Figure 124).

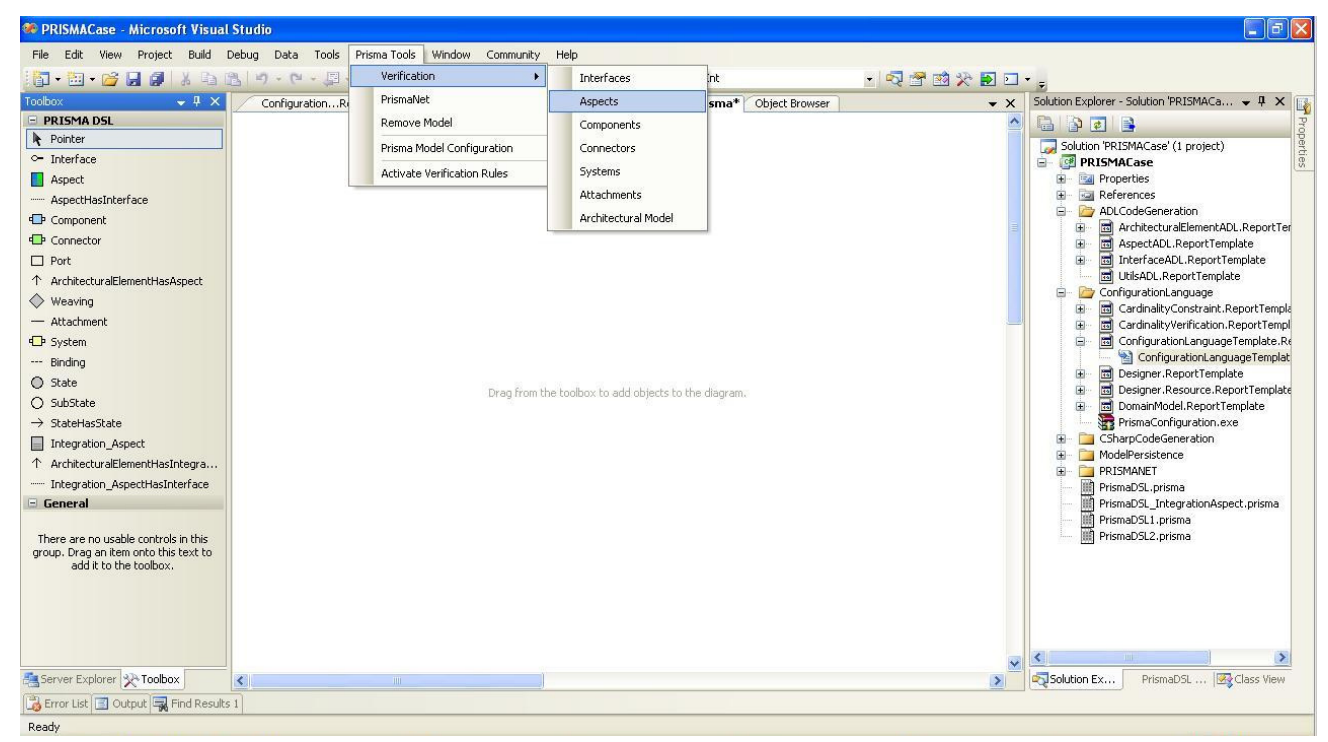

Figure 124. PRISMA Modelling Tool

\subsubsection{The Graphical AOADL of PRISMA}

The toolbox of the PRISMA modeling tool provides a gallery of shapes that permits the graphical modelling of PRISMA models by dragging and dropping the shapes to the drawing 
sheet (see Figure 125). In PRISMA, only the main concepts and their relationships are graphically represented; the rest of the concepts are specified using the AOADL and are included in the definition of the corresponding shapes.

The graphical AOADL is shown below detailing how to complete the specification of the graphical concepts using the model explorer and the window of properties that the PRISMA modelling tool offers.

\begin{tabular}{|c|c|}
\hline \multirow{2}{*}{\multicolumn{2}{|c|}{$\begin{array}{l}\text { Toolbox } \\
\text { — PRISMA DSL }\end{array}$}} \\
\hline & \\
\hline \multicolumn{2}{|c|}{ * Pointer } \\
\hline \multirow{3}{*}{\multicolumn{2}{|c|}{$\begin{array}{l}\text { \% Interface } \\
\square \text { Aspect } \\
\text {........ AspectHasInterface }\end{array}$}} \\
\hline & \\
\hline & \\
\hline \multicolumn{2}{|c|}{ 口? Component } \\
\hline \multicolumn{2}{|c|}{ घ Connector } \\
\hline \multicolumn{2}{|c|}{$\square$ Port } \\
\hline \multicolumn{2}{|c|}{$\uparrow$ ArchitecturalElementHasAspect } \\
\hline \multicolumn{2}{|c|}{$\diamond$ Weaving } \\
\hline \multicolumn{2}{|c|}{ - Attachment } \\
\hline \multicolumn{2}{|c|}{ ロ System } \\
\hline \multicolumn{2}{|c|}{--- Binding } \\
\hline \multicolumn{2}{|c|}{ State } \\
\hline \multicolumn{2}{|c|}{ Substate } \\
\hline \multicolumn{2}{|c|}{$\rightarrow$ StateHasState } \\
\hline \multicolumn{2}{|c|}{ 目 Integration_Aspect } \\
\hline \multicolumn{2}{|r|}{$\uparrow$ ArchitecturalElementHasIntegration_Aspect } \\
\hline & - Integration_AspectHasInterface \\
\hline
\end{tabular}

Figure 125. PRISMA Tool Box

\section{$>$ Interfaces}

Interfaces are represented by a rectangle with its corners rounded off (see Figure 126). The interface includes the definition of the services that it publishes. The services can be added using the context-menu that is associated to interfaces (see step 1, Figure 127). Then, their arguments and argument properties can be defined using the model explorer and the window of properties, respectively (see steps 2 and 3, Figure 127). 
PRISMA: Aspect-Oriented Software Architectures

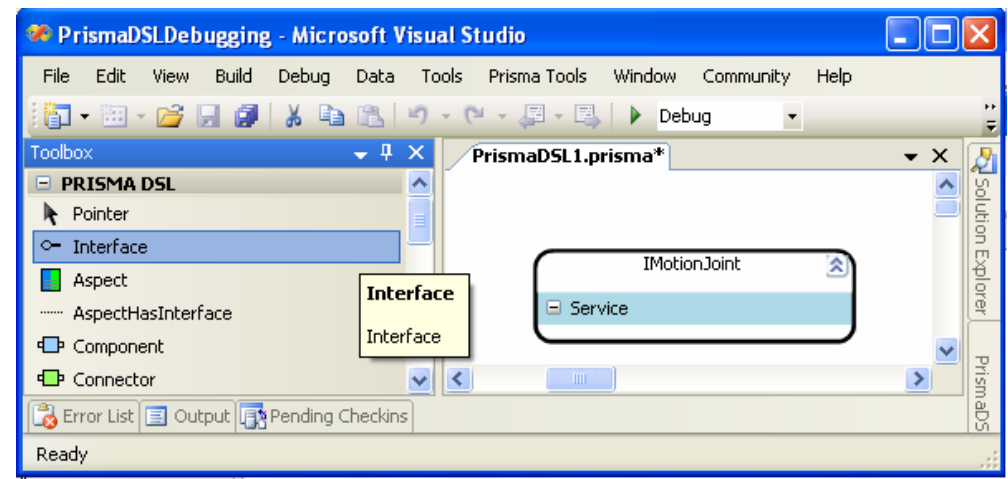

Figure 126. Interface Shape

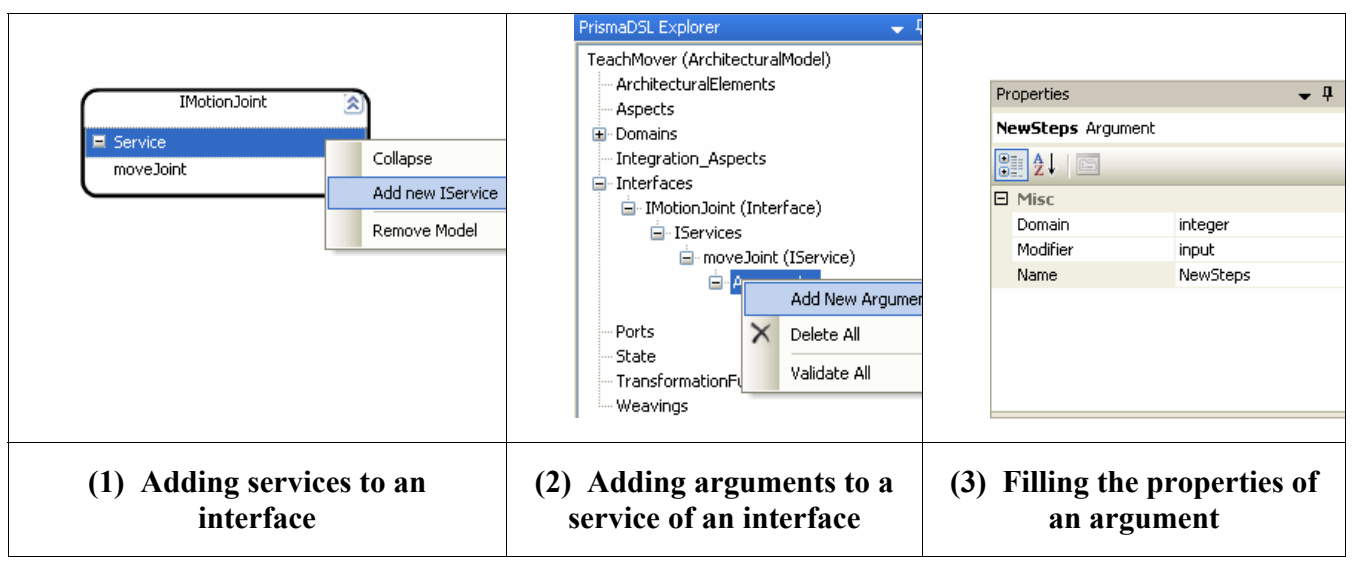

Figure 127. Modelling Services of Interfaces

\section{Aspects}

Aspects are represented by a rectangle (see Figure 128). The aspect includes the definition of attributes, services, valuations, preconditions, constraints, played_roles and transactions. They can be added using the context-menu that is associated to each concept as in step 1 of Figure 127. Ten, their properties can be filled using the model explorer and the window of properties as presented in steps 2 and 3 of Figure 127. Each aspect defines properties of a specific concern. Each concern has a colour associated to it and, depending on the value of the aspect concern, the aspect is painted in one colour or another. For example, the concern coordination has the green colour associated to it (see Figure 128). 
The importation of interfaces by an aspect is specified using the link AspectHasInterface, which is provided by the tool box. This link automatically includes the interface services as services of the aspect that is linked to the interface (see Figure 129).

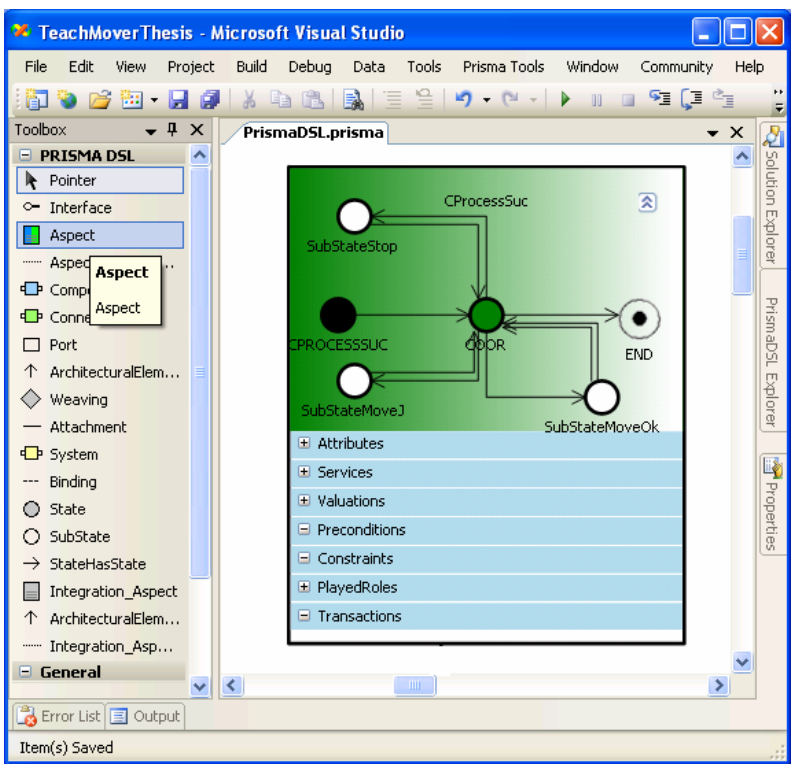

Figure 128. Aspect Shape

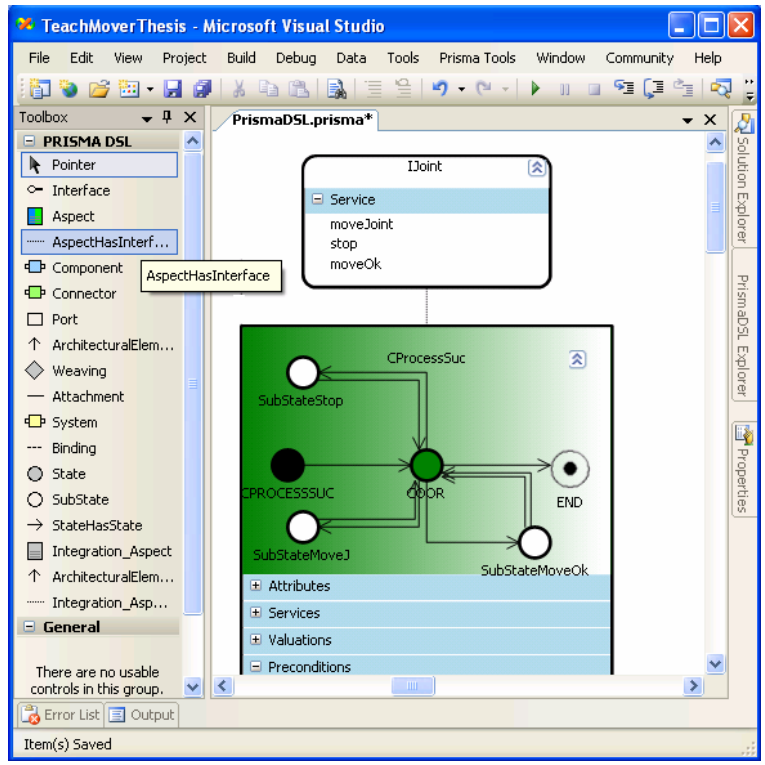

Figure 129. Link Shape between Aspects and Interfaces 
PRISMA: Aspect-Oriented Software Architectures

The aspect also includes the specification of its protocol. The protocol is modelled in a graphical way. Protocols are specified by modelling a State Transition Diagram (STD) (see Figure 130). The tool box provides a set of shapes to model the protocol of an aspect. These shapes are states, substates, and transitions between these states (see Figure 125).

States define relevant states of the software system business logic. A state establishes the services that can be executed when the aspect execution is placed in it. These states are graphically characterized because they are painted in the same colour as the aspect (see Figure 130).

Substates help to define sequences of services that may or may not be transactional. They are not states of the business logic of the software system and are characterized by always being painted white (see Figure 130).

Transitions define the jump from one source state to another target state. Both the source state and the target state can be states or substates (see Figure 130). The transition between states has a service associated to it and can also have a condition, a played_roled and a transaction associated to it. These properties of the transition are filled using the window of properties.

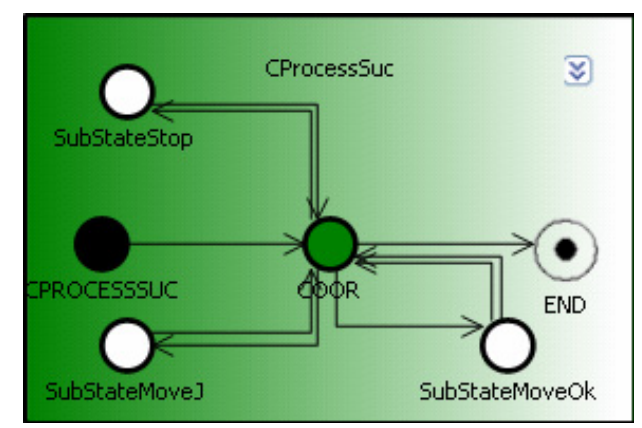

Figure 130. STD for modelling protocols

In addition to these generic shapes for modelling aspects and the concepts related to them, two specialized shapes have been defined in the toolbox to define an aspect that has properties that are different to the rest. The first one is the integration aspect, which is the mechanism for integrating COTS in PRISMA software architectures (see section 10.2), it is graphically 260 
represented by a rectangle. Integration aspects are painted grey to denote their wrapper semantics viewed as a black box (see Figure 125). The second one is the link to connect this kind of aspects to interfaces (Integration_AspectHasInterface), it is graphically represented by a line.

\section{$>$ Components, Connectors and Systems}

Components, connectors and systems are represented by rectangles that have one pin for each one of the ports that are associated to them (see Figure 128). The components, connectors and systems are distinguished by the colour. Components are blue, connectors are green, and systems are yellow.

\begin{tabular}{|l|l|l|}
\hline Cort1 & Component1 & Connector1 \\
\hline (1) Component & (2) Connector & (3) System \\
\hline
\end{tabular}

Figure 131. Shapes of Architectural Elements

Since the number of ports is not fixed, there is a rectangular white shape that corresponds to the port so that as many ports as are necessary can be added to each architectural element. When the port shape is drawnand droppedon top of an architectural element, the port is automatically associated to this architectural element. The use of the window of properties makes it possible to define the interface and played_role that types the port.

The importation of aspects by architectural elements is specified using the link ArchitectecturalElementHasAspect, is provided by the tool box. In addition, there is another specialized link for importing the integration aspect called ArchitectecturalElementHasIntegrationAspect.

Since systems are composed of other architectural elements, the architectural elements that are dropped on top of systems are automatically included in the systems. The association of architectural elements to systems can also be done by hand using the system window of properties. 
PRISMA: Aspect-Oriented Software Architectures

$>$ Weavings

Weavings are represented by a diamond that joins the aspects that participate in the weaving using incoming and outgoing arrows (see Figure 132). The arrows determine the direction in which the weaving service is executed, i.e., the aspect service with the outgoing arrow is the first to be executed, and the aspect service with the incoming arrow is the second to be executed. The services that participate in the weaving as well as the operator are specified using the window of properties. After specifying the operator, the operator is displayed in the center of the diamond.

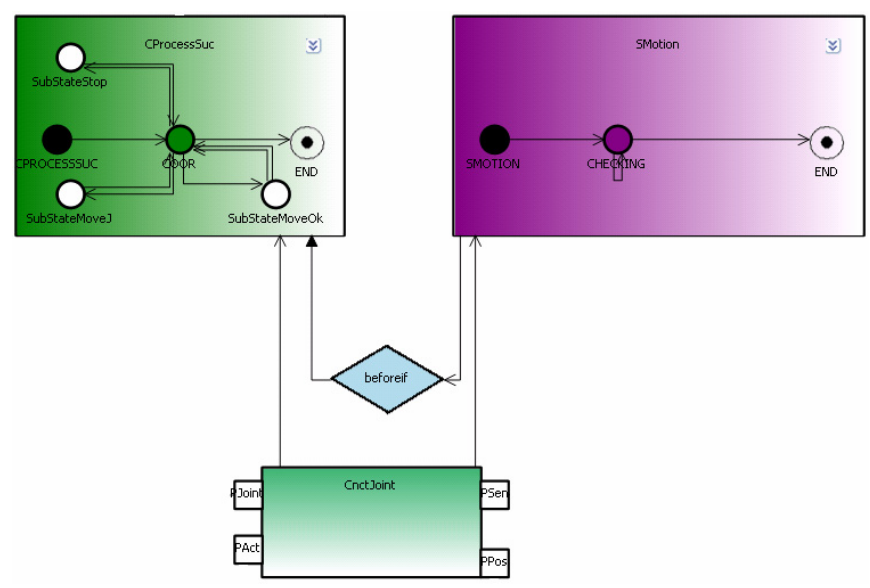

Figure 132. Weaving Shape

Attachments

Attachments are represented by a line that links a component port to a connector port (see Figure 133). The properties of the attachment are specified using the window of properties.

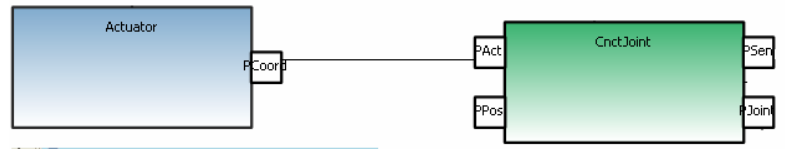

Figure 133. Attachment Shape

Bindings

Bindings are represented by a dashed line that links an architectural element port to a system port (see Figure 134). The properties of the binding are specified using the window of properties. 


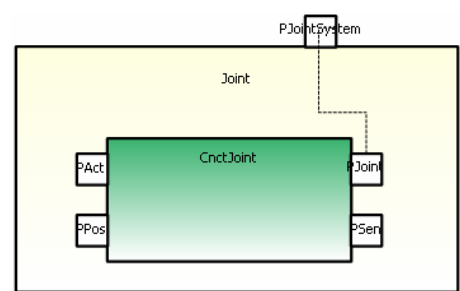

Figure 134. Binding Shape

\subsubsection{Verification of PRISMA Models}

The hardconstraints that have been defined in DSL tools are checked throughout the entire modeling process. As a result, when the user tries to model something that violates a hardcontstraint, the PRISMA modeling tool shows a prohibition sign.

The verification rules defined in DSL tools are checked each time that the model is saved. The list of errors is displayed in the Error List window (see Figure 135).

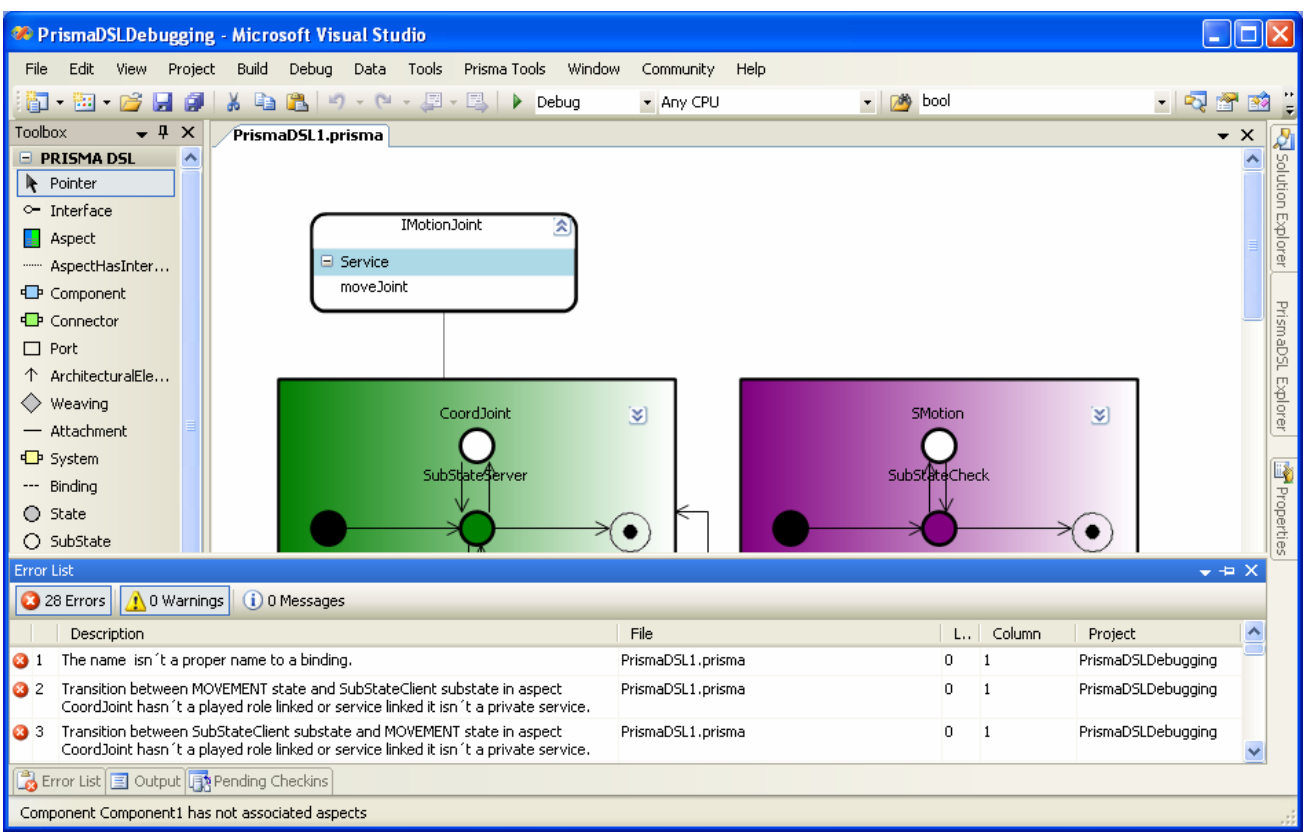

Figure 135. Error List

Verification rules can also be checked whenever the user requires it. The PRISMA menu offers the option of checking these rules in a complete or partial way (see Figure 136). The 
PRISMA: Aspect-Oriented Software Architectures

complete way checks all the verification rules, while the partial way allows you to only check one kind of PRISMA type. The options that are provided to check an architectural model in a partial way are the following: interfaces, aspects, components, connectors, systems and attachments. For example, if the user requests the Interface Verification, only the rules associated to interfaces are checked. The advantage of this partial verification is that the user can incrementally check the models and focus on the problems of a specific type of the model.

\begin{tabular}{l|l|}
\hline \multicolumn{1}{|c|}{ Prisma Tools Window Community } & \multicolumn{1}{l|}{ Help } \\
\hline \multicolumn{1}{|c|}{ Verification } & Interfaces \\
\hline PrismaNet & Aspects \\
Remove Model & Components \\
\hline Prisma Model Configuration & Connectors \\
Activate Verification Rules & Systems \\
\hline & Attachments \\
& Architectural Model \\
\hline
\end{tabular}

Figure 136. Verification Menu

In addition, the graphical modelling tool offers the mechanism of checking only one element of the architectural model. This is possible by executing the option that appears in the contextual menu that is associated to the element that the user wants to check. For example, Figure 137 shows the contextual menu that only verifies the interface IMotionJoint.

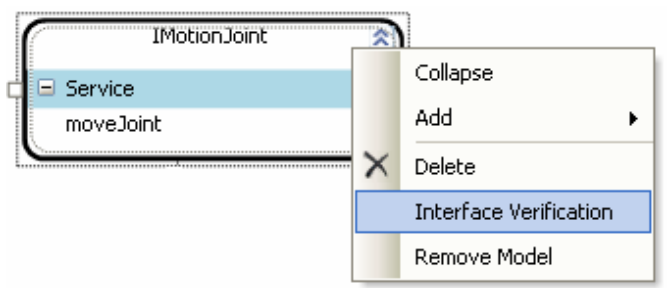

Figure 137. Contextual Menu of an Element

\subsection{MODEL COMPILER}

The PRISMA modelling tool also provides a set of templates to automatically generate the code from the models that have been graphically modelled. The templates for generating the AOADL specification and the C\# code are already available. They can be extended to generate 
the code for other languages. The templates and the command to execute the generators that produce the result are provided by the window Solution Explorer of PRISMA CASE. This command is called Transform All Templates. Its execution calls the code generators that execute the code generation templates of PRISMA by substituting the parameters for the elements that have been modeled. The PRISMA templates are stored in two different folders: ADLCodeGeneration and CSharpCodeGeneration. These folders contain the templates to generate each PRISMA type and the file that contains the result of the last Transform All Templates execution (see Figure 138).

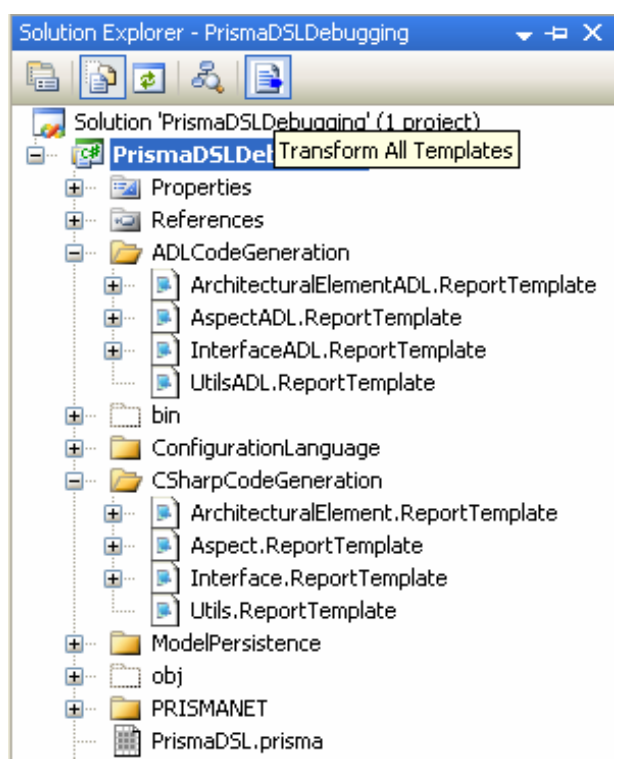

Figure 138. PRISMA Code Generation Templates

The ADLCodeGeneration folder contains the formal specification of the software architecture that has been modelled following the PRISMA AOADL. Despite the fact that the specifications are introduced in the graphical shapes using the PRISMA AOADL, the AOADL generation permits the user to see the complete textual specification of the model.

The CSharpCodeGeneration folder contains the C\# code generation, which allows the execution of the specified software architecture on the PRISMANET. The implementation of a specific PRISMA software architecture is performed by extending the classes provided by 
PRISMA: Aspect-Oriented Software Architectures

PRISMANET (see section 9.4.2). In order to develop these code generation templates, a set of patterns has been identified and defined to generate the $\mathrm{C} \#$ code for each one the PRISMA concepts to be executed over PRISMANET. Next, the mappings of components and aspects are presented in a simplified way by using the Actuator component and the FunctionActuator aspect as an example. The complete list of patterns is presented in detail in [Cab05].

\subsubsection{Components}

A component is implemented as a serializable C\# class. This class is serializable in order to enable mobility in future versions of PRISMA CASE. This class inherits from the ComponentBase class of PRISMANET, which implements the component of the PRISMA model. The component name is the same as the one in the PRISMA specification. The set of ports and aspects that make up a component are included by invoking the constructors of the port and aspect PRISMANET classes. Both classes implement the port and aspect elements of the PRISMA model. For example, Figure 139 shows an initial specification of the component Actuator and the C\# code that the component pattern of the PRISMA CASE has automatically generated from the specification.

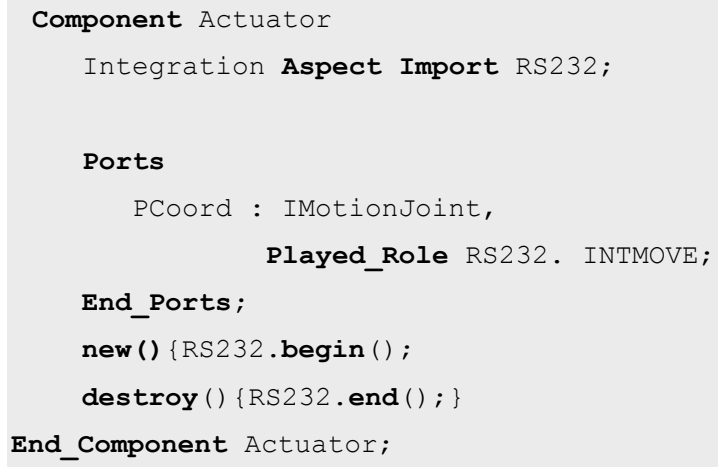




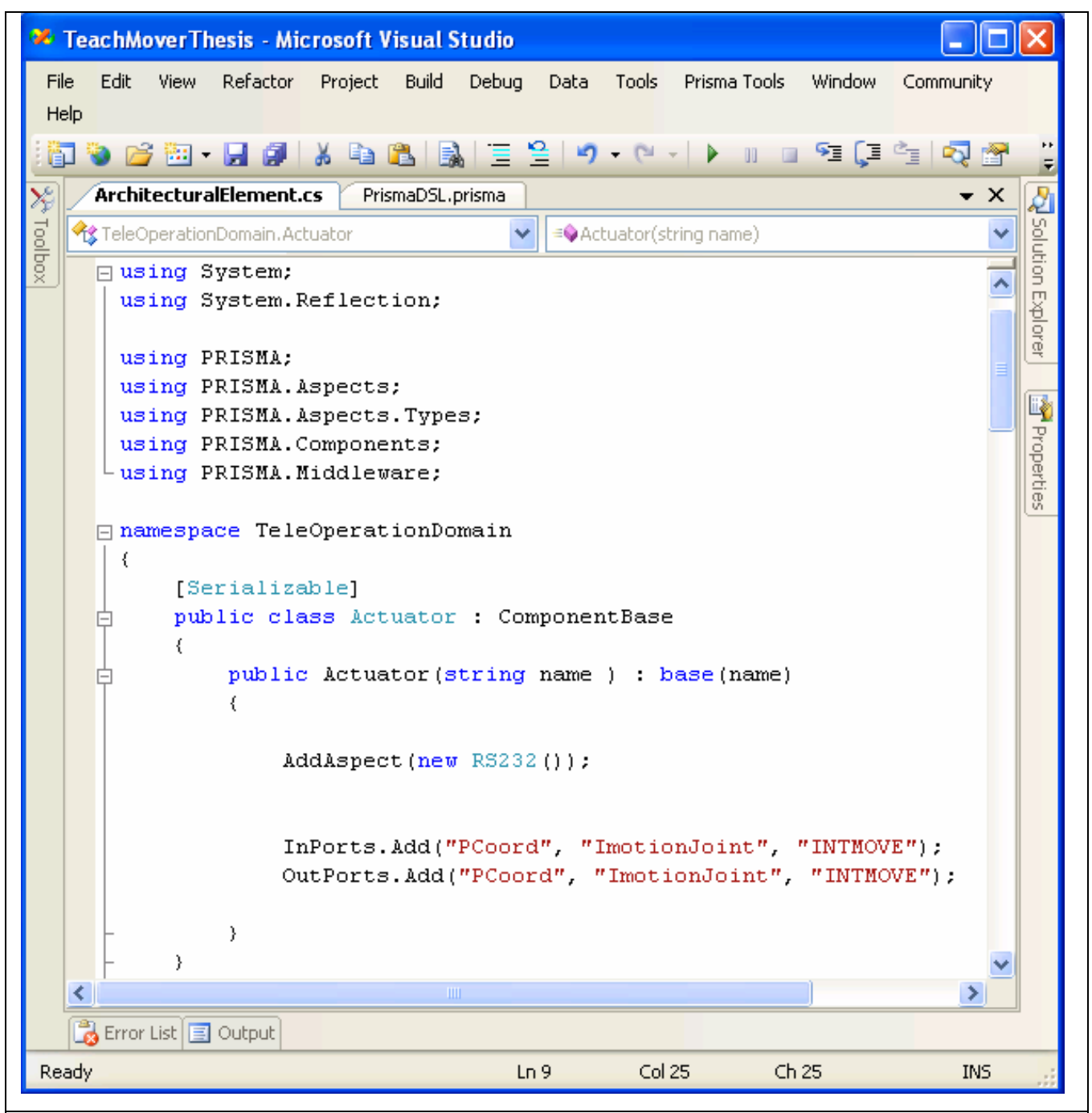

Figure 139. The generated C\# code of the component Actuator

\subsubsection{Aspects}

An aspect is implemented as a serializable C\# class to enable mobility in the network. This class inherits from the AspectBase class of PRISMANET, which implements the aspect element of PRISMA model. The aspect name is the same as the name in the PRISMA specification as well as the interface that it implements. Moreover, the kind of aspect is specified in the implementation as defined in the specification. 
PRISMA: Aspect-Oriented Software Architectures

The attributes of an aspect are implemented as variables of the C\# class. The services specified by a PRISMA aspect are implemented as methods of the aspect class. The played_roles that make up an aspect are included by invoking the constructors of the played_role PRISMANET class. The different states of the protocol are implemented as internal variables that are checked inside of the service methods in which the priority of each method is taken into account. The implementation of these methods follows a pattern that implements the set of steps that must be executed in order to properly execute a PRISMA service. These steps are the following:

1. Verification of Protocol: It must be verified that there is a valid transition for the required service. This transition must depart from the state that the protocol is executing at that moment.

2. Verification of Preconditions: It must be verified that every precondition associated to the service is satisfied.

3. Selection of Valuations: The valuations that must be executed must be selected depending whether or not their conditions are satisfied or not.

4. Execution of Valuations: The variables that represent the attributes or parameters that must be updated due to the service method execution are modified.

5. Verification of Constraints: It must be verified that the constraints of the aspect are satisfied by the new state of the aspect that has been reached due to the service method execution. This verification is performed using exceptions as the verifications of protocols and preconditions.

6. Execution of Service Sequences: There are services whose execution implies the automatic execution of other services. In this case, these services are invoked.

7. Change of the protocol: If the method execution implies a change in the protocol state, this change is performed by updating the state variable. 
For example, Figure 140 shows the partial specification of the CProcessSuc coordination Aspect (see the complete specification in C.3) and the C\# code that the aspect pattern of the PRISMA CASE has automatically generated from the specification.

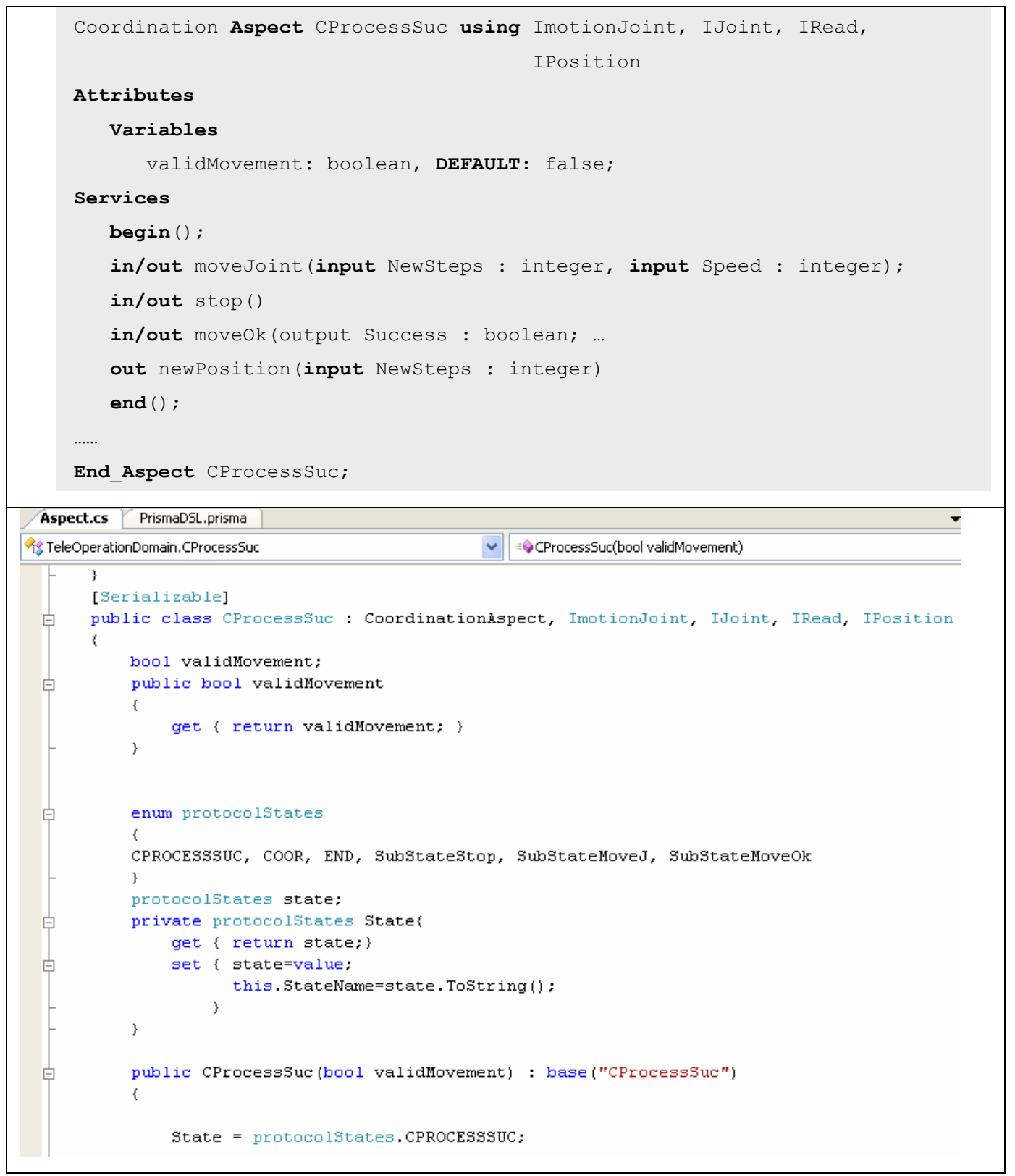


PRISMA: Aspect-Oriented Software Architectures

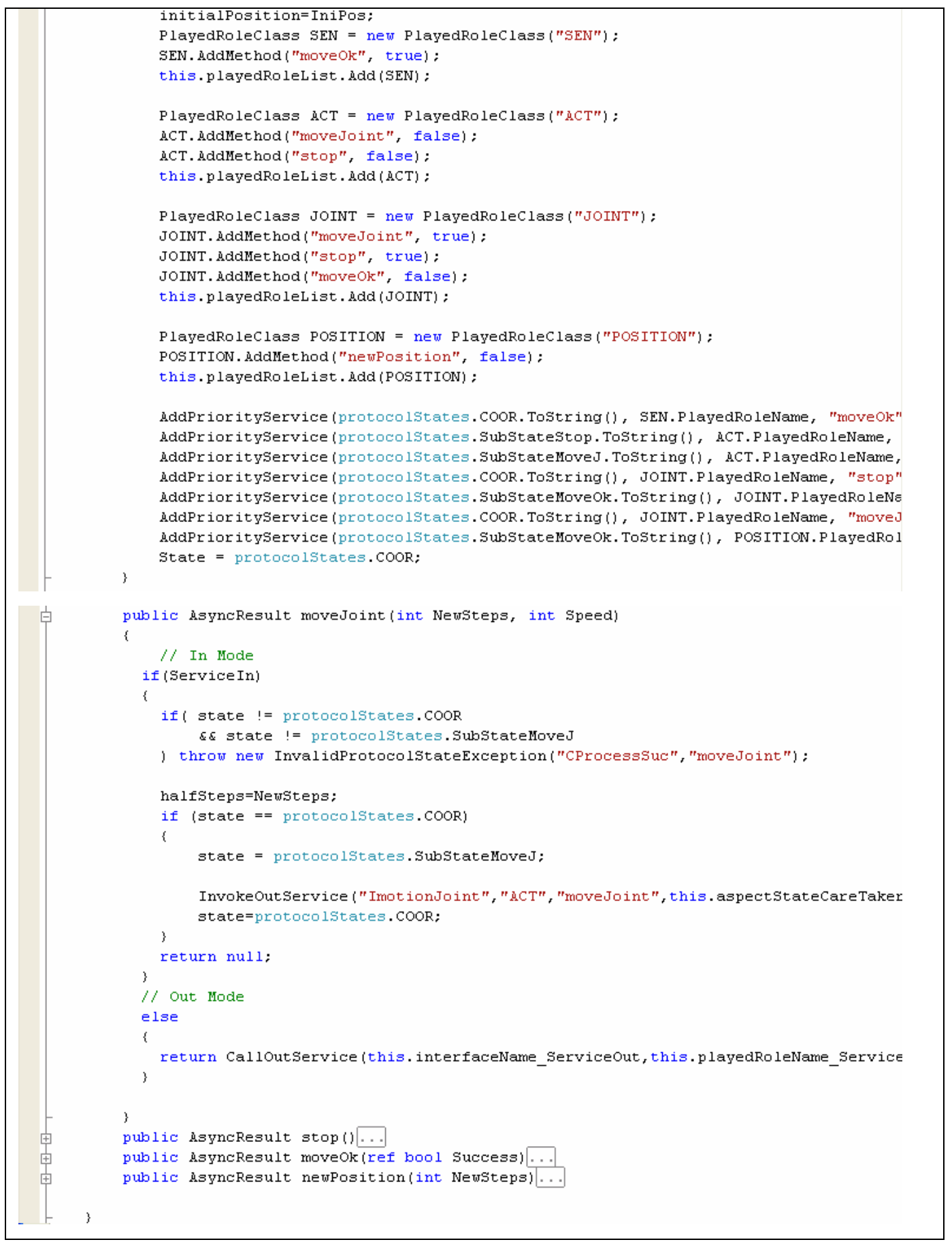

Figure 140. The generated C\# code of the aspect FunctionActuator 


\subsection{CONFIGURATION MODEL}

The PRISMA model that has been introduced in the Domain Model of DSL tools is a metamodel, and the modelling tool (debugging solution) that has been developed from this model provide us mechanisms to specify PRISMA aspect-oriented software architectures. However, it is necessary to instantiate and to configure these architectures into specific ones and provide the user mechanisms to do so. In order to cope with these needs, PRISMA CASE automatically generates a domain specific graphical modelling tool to configure the software architectures that have been defined using PRISMA CASE. As a result, the PRISMA modelling tool is composed of two different tools: the modelling type tool and the modelling configuration tool. The modelling configuration tool is generated from the models that have been specified using the modelling type tool. As a result, the PRISMA graphical modelling is compliant with the PRISMA AOADL, which is also divided into types and configuration (see Figure 141).

A configuration modelling tool is generated for each PRISMA software architecture that is modelled using the PRISMA modelling type tool. The configuration modelling tool is used to develop specific software architectures using the PRISMA types defined in PRISMA type modelling tools as modelling primitives.

The capacity to store all the information needed to automatically generate a domain-specific tool permits the use of PRISMA architecture as a domain specific language in other model specifications. It also permits the generation of the specification of the configuration language. This information is generated using the code generators of DSL and is stored in the persistence and configuration language folders of PRISMA type modelling tool (see Figure 142). The information of these folders is the input for creating the new project for the domain-specific PRISMA software architecture that has been defined. 
PRISMA: Aspect-Oriented Software Architectures

(1) PRISMA Type Modelling Tool

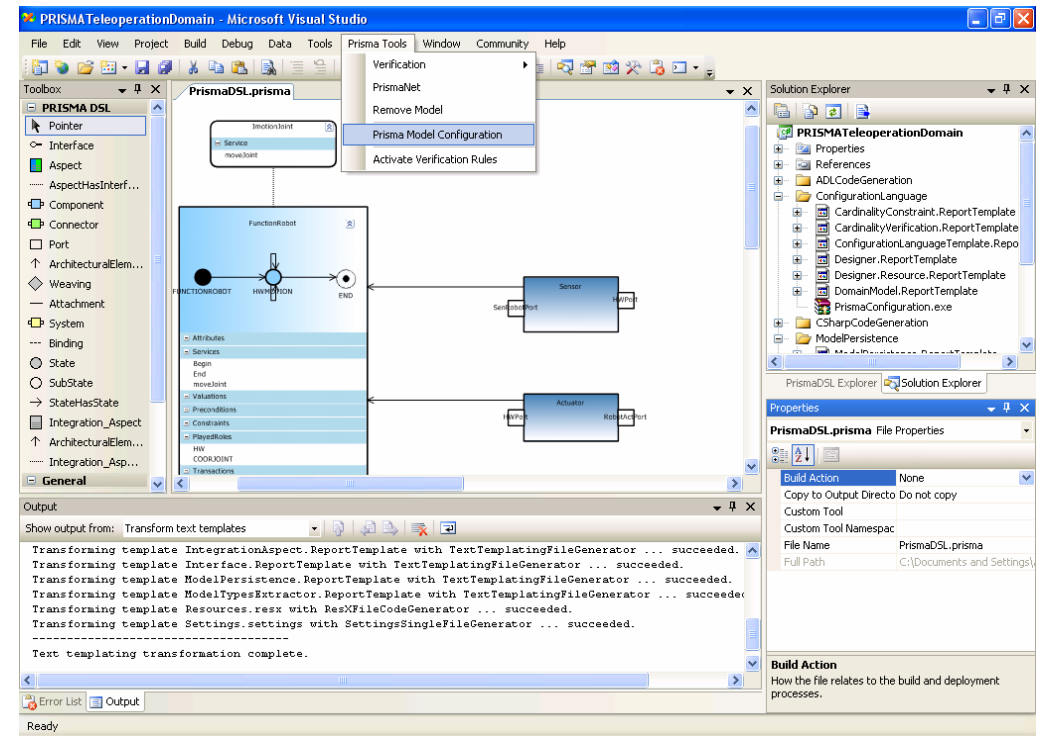

(2) PRISMA Configuration Modelling Tool

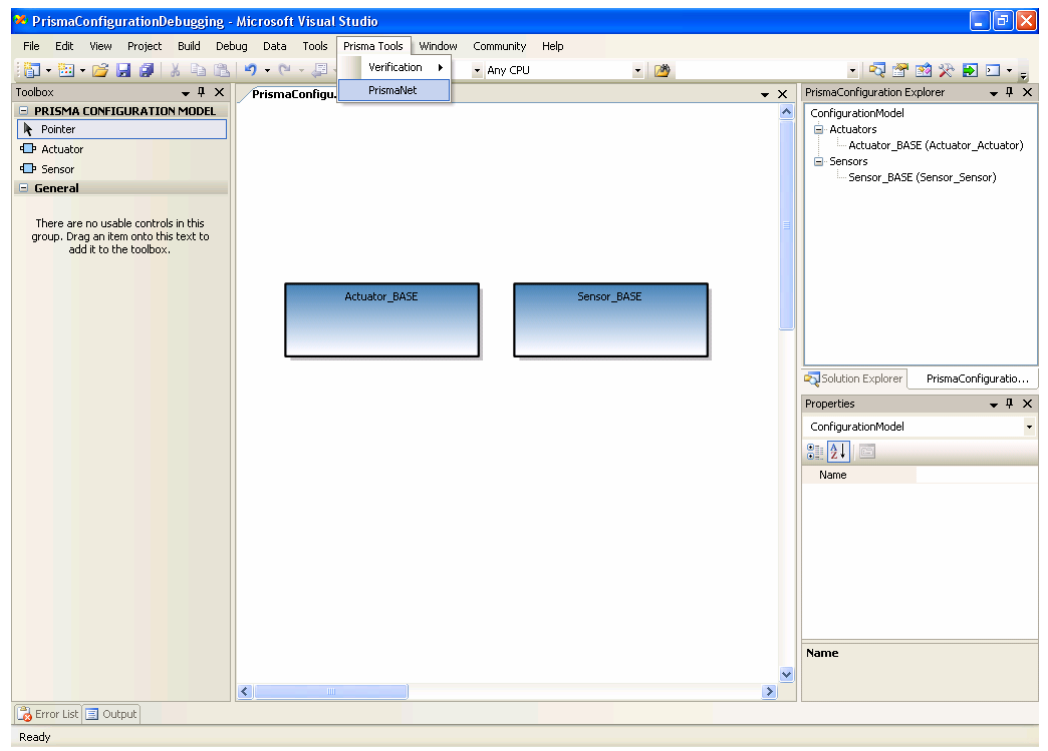

Figure 141. Generation and Execution of the PRISMA Modelling Configuration Tool 


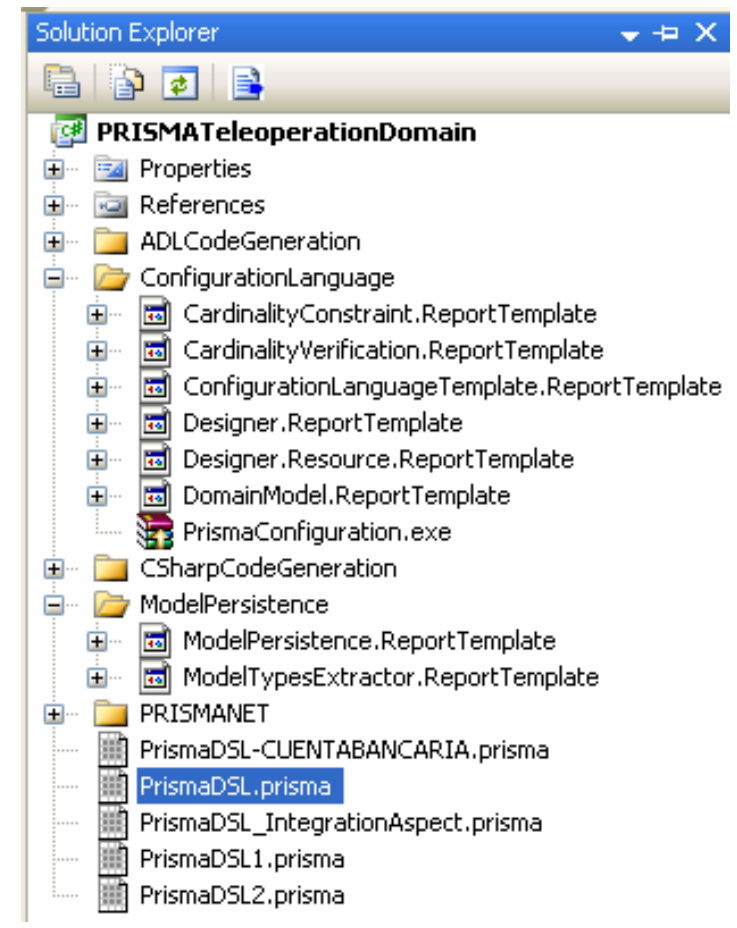

Figure 142. Model Persistence and Configuration Language Information

The automatic generation of a tool for modelling configurations of a PRISMA software architectures is performed by executing the PRISMA Model Configuration option of the menu PRISMA after the transformation of all templates has been done (see step 1 in Figure 141). Next, a new project is automatically created and can be used as a configuration modelling tool. Step 2 of Figure 141 shows how the Actuator and Sensor types defined in step 1, Figure 141 appear in the tool box of the Configuration Modelling Tool as shapes for modelling. It shows how these types have been dragged and dropped on the drawing sheet generating two instances. As a result, the base joint is modelled by defining its actuator and sensor.

In addition, the configuration modelling tool provides a command to transform its templates to obtain the AOADL specification that corresponds to the configuration that has been defined using the tool. Finally, this tool permits the execution of the generated code. In order to do this, the PRISMA menu offers the option PRISMANET, which executes the middleware PRISMANET and instantiates the defined configuration. As a result of this execution, a 
PRISMA: Aspect-Oriented Software Architectures

generic GUI is launched to interact with the architecture by invoking its services and checking the value of its attributes (see Figure 143). The main purpose of the generic GUI is to assist the user in checking the behaviour of the architecture without having to worry about aesthetic details and without forcing the user to define a GUI in order to obtain a result.

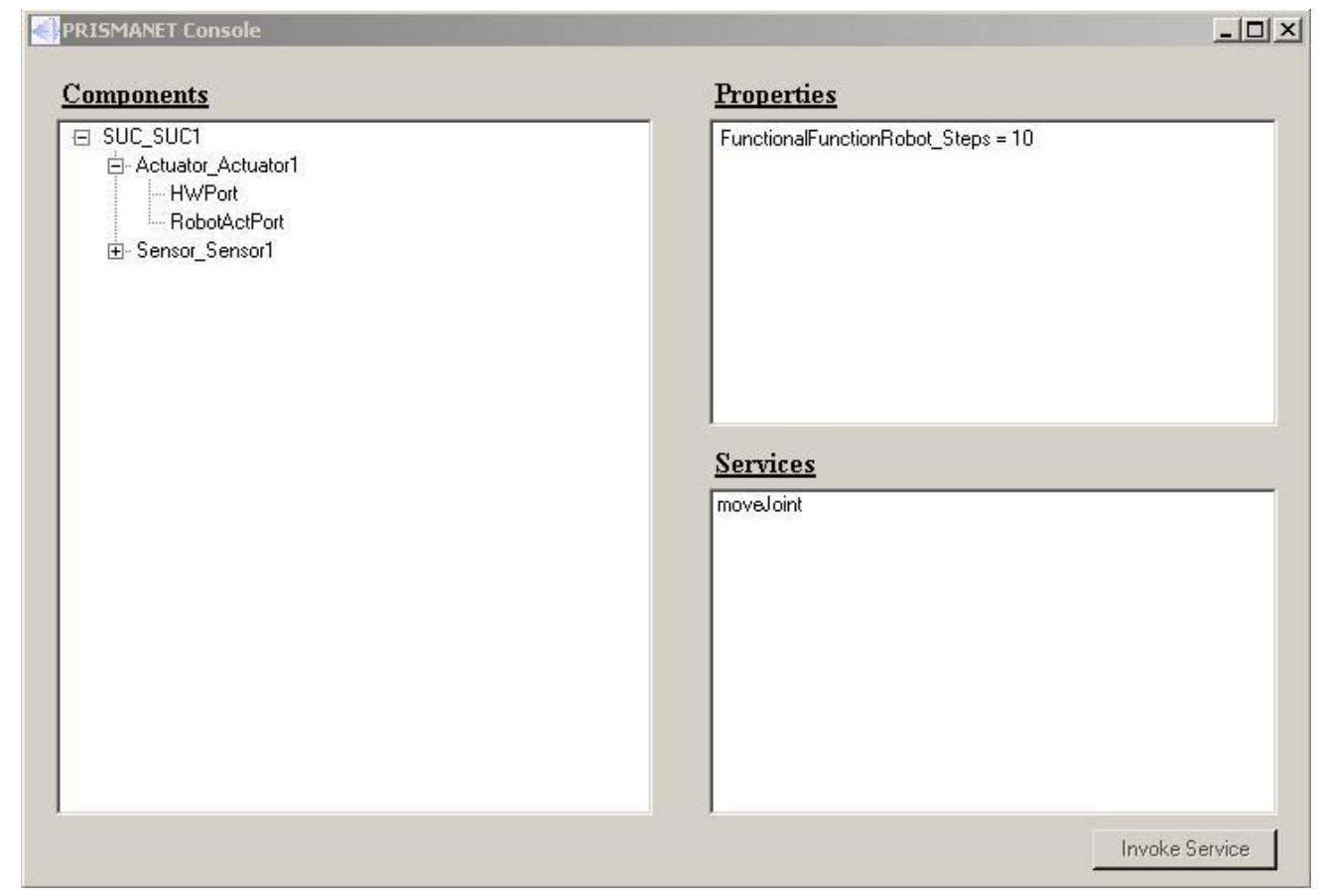

Figure 143. Generic GUI of PRISMA Applications

However, it is important to mention that the use of this interface is not mandatory. In other words, if the users prefer to define their own specialized forms, they can do so. They only have to integrate them with the architecture by using the presentation aspect that the PRISMA modelling tool offers. Users must define the services to interact with the defined forms inside the presentation aspect and to synchronize them with the rest of aspects that belong to the same architectural element by means of weavings. The forms are included in the project folder so that the GUI of the user is executed instead of the generic interface that PRISMA CASE provides by default. 
The PRISMA CASE

\subsection{PRISMANET}

PRISMANET implements the PRISMA model by extending the .NET technology with the incorporation of aspects. PRISMANET is the platform-dependent model of PRISMA, which permits PRISMA software architectures to be developed and executed on the .NET platform.

PRISMANET offers extra functionalities and characteristics that .NET does not directly provide. It allows for the execution of aspects, the concurrent execution of aspects and architectural elements, the loading of components, the creation of execution threads, the management of the local components, etc.

Even though PRISMANET has only been used for software architectures that are locally executed, due to the distributed nature of software, the distributed execution of software architectures will be supported by PRISMANET in the near future. To completely reuse PRISMANET when it will be extended to support distribution and mobility capabilities, aspects and components have been developed so that they can be executed and can communicate with each other in both a local and distributed way.

This section presents how the PRISMA model, its execution model, and its aspect-oriented properties have been implemented in the .NET technology.

\subsubsection{PRISMANET Architecture}

The .NET framework consists of several layers at different levels of abstraction. Not all the technologies that support AOP in the .NET platform are applied to the same layer of the .NET framework. Figure 144 shows the different layers that .NET framework consists of and also classifies the AOP .NET technologies according to the layers they have been applied to. These technologies are analyzed in section 3.3.3. 


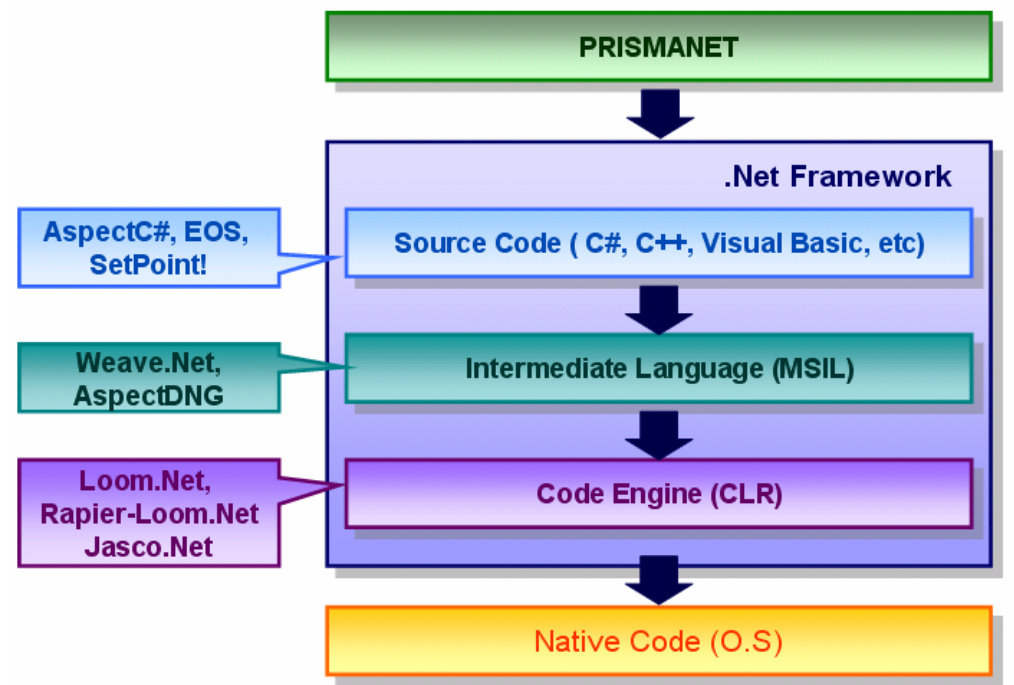

Figure 144. Layer classification of technologies that support AOP in .NET framework

The AOP .NET technologies can be classified into three kinds. These kinds of AOP .NET technologies are defined by the way in which the base code and the aspect code are weaved and by the layer in which this weaving process is performed [Jac04]. These three kinds of AOP .NET technologies are the following:

AOP .NET technologies applied to the Common Language Runtime (CLR) layer:

This kind of AOP .NET technology extends the CLR by adding new mechanisms to perform weavings (see Figure 144). The advantage of this kind of AOP .NET technology is that its approach is independent of the programming language. However, one of its inconveniences is that the pointcuts are limited by the mechanisms used for weaving the aspect code and the base code at the CLR level. Moreover, since the CLR is modified, the standard debugging tools cannot be used.

$>$ AOP .NET technologies applied to the MicroSoft Intermediate Language (MSIL) layer: This kind of AOP .NET technology modifies the MSIL to weave the base code and the aspect code (see Figure 144). Most of these technologies modify the MSIL of the assemblies to intercept the methods at runtime, thereby executing aspects. The advantage of this kind of AOP .NET technology is that its approach is independent of the 
programming language. However, the standard debugging tools cannot be used as in the previous kind, and it does not allow for the dynamic definition of weavings.

$>$ AOP .NET technologies applied to the Common Language System/Common Type System (CLS/CTS) layer: This kind of AOP .NET technology extends the CLS/CTS to weave the base and aspect code at compilation time (see Figure 144). One of the main inconveniences of these technologies is their dependence on the programming language. Another inconvenience is the need to adapt compilers of the programming languages that this kind of technology extends in order to adjust them to the evolution of these programming languages.

These three kinds of AOP Technologies share an additional inconvenience. They depend on the .NET platform. As a result, they must be modified in order to be compatible with future versions of the .NET platform. In order to avoid these inconveniences, PRISMANET has been applied to a higher layer of abstraction without extending the development platform. PRISMANET implementation is localized in a new layer that sits over the .NET framework (see Figure 144). This can be done because PRISMA is a technology-independent model. PRISMANET implementation has been carried out in C\# language using the standard techniques and mechanisms that the .NET framework provides, that is, without extending the development platform. As a result, PRISMANET does not have to be updated to the new versions of the CLR, MSIL and so forth, and it can be executed in the .NET platform without having to do anything else other than starting the execution of the middleware. The most important .NET technology mechanisms [Rob03] that have been used are: delegates, reflection, serialization and .NET Remoting [MIC05].

The PRISMANET architecture is constituted by four main modules (see Figure 145):

D The PRISMA Execution Model: This module implements the basic functionality of the PRISMA types. This implementation is divided into two modules that contain the classes that implement this functionality: the Types and Communications modules. The Types module implements aspects and architectural elements, and the Communications module implements attachments and bindings. As a result, the implementation of the PRISMA application is achieved by extending these classes. 


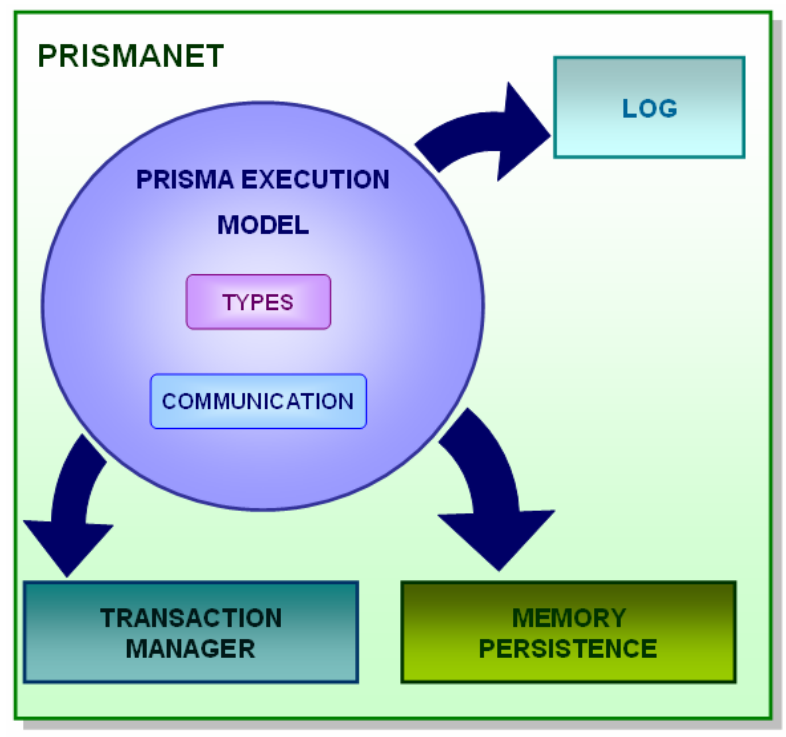

Figure 145. PRISMANET Middleware

> Memory Persistence: This module provides services to manage and maintain the instances of architectural elements that are stored in the main memory during their execution. The middleware manages the instances that are locally executed. Some of these services are the loading of architectural elements instances, the creation of execution threads, and the management of architectural element lists.

$>$ Transaction Manager: This module provides services to suitably execute transactions.

Log: This module logs every operation that it is performed by the middleware in order to register the execution history of software architectures.

PRISMANET has to be running on all PCs where a PRISMA application is being executed. The middleware manages the architectural elements instances that are being executed in the PC, providing the necessary services to its instances. As a result, there are two kinds of communications concerning PRISMANET and the applications that run on it:

Communication among PRISMANET and the architectural element instances to ask for maintenance services.

> Communication among different PRISMA components as a result of the execution of the application. 


\subsubsection{PRISMA model implementation}

Each concept defined in the PRISMA model has been implemented in the module PRISMA Execution Model of PRISMANET. The implementation has been carried out preserving the following features:

$>$ The implementation has to be as close as possible to the PRISMA model in order to facilitate automatic code generation. Therefore, PRISMANET is the PRISMA model that is dependent on the .NET technology, i.e., it is the platform-dependent model of PRISMA.

$>$ The execution of attachments, connectors and components must be concurrent in order to be compliant with the PRISMA Model Formalization (see section 6.2). In addition, the concurrency among the different aspects that make up a component must be preserved.

The Types and Communication modules that compose the module PRISMA Execution Model have been grouped by namespaces of .NET in order to clearly identify the implementation of each one of the PRISMA types (see Figure 146).

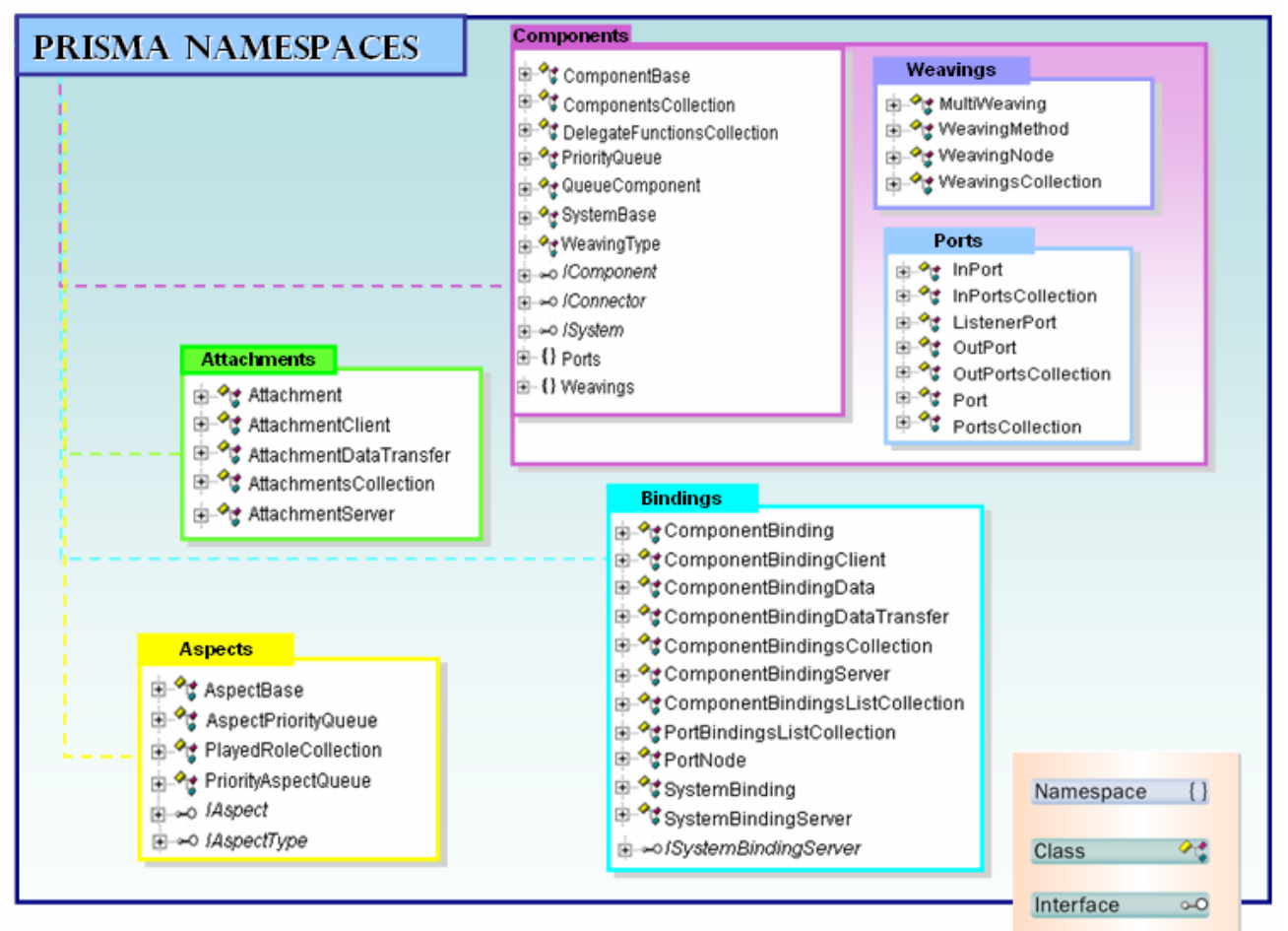

Figure 146. Namespaces of the module PRISMA Execution Model 
PRISMA: Aspect-Oriented Software Architectures

\subsubsection{Asynchronous executions}

There are mechanisms that the .NET platform offers to support asynchronous invocations of services such as the classes Delegate and AsyncResult. However, these mechanisms are managed by the .NET platform and they cannot be customized for the specific needs of a new execution model such as the PRISMA model. Therefore, a new class AsyncResult has been created to implement the asynchronous invocations of the PRISMA model by hiding the specific details of the .NET platform, and by dealing with the PRISMA peculiarities such as the management of requests, the service execution based on priorities, the request resendings to other architectural elements, etc.

The class AsyncResult is the structure that allows the client of a service to verify whether or not its request has been successfully executed once the service execution has finished. If it has been successfully executed, the results can be obtained. For this reason, in PRISMA, every service provided by a server that can be asynchronously executed must return an AsyncResult structure.

\section{Execution of an asynchronous invocation}

PRISMA servers have a mechanism that stores the requests that they receive while they are executing another request. This mechanism consists of creating a delegate and an AsyncResult structure for each request that the server receives and storing them to be processed afterwards. Then, the server sends the reference of the AsyncResult structure that the server has created to the client. Thus, the client does not have to wait for the result and can continue its execution.

When the server processes the client request, it executes the delegate associated to this request, i.e., it executes the request service and stores the results in the AsyncResult structure associated to the request. Since the client has the reference of the AsyncResult structure, this structure can be queried by the client whenever necessary. The client checks the request execution as well as the results that have been obtained from it.

This execution model together with the AsyncResult structure allows for the asynchronous execution of the requests of PRISMA services. 


\subsubsection{Aspects}

The aspect type has been implemented as a C\# class called AspectBase of the PRISMANET (see Figure 147). This class stores the name of the aspect and its thread reference as well as other properties. The AspectBase class has the references of the component and the middleware that it belongs to in order to request services from them. This class offers services to start the execution of the aspect thread, to stop the execution of the aspect thread, and to abort the execution of the aspect thread.

A played_role has been implemented as a C\# class called PlayedRoleClass (see Figure 147), which stores information about the services of the played_role and their priorities. The played roles of an aspect are stored in the class PlayedRoleCollection that the aspect is related to (see Figure 147).

The class AspectBase also contains a priority queue to deal with the service requests that arrive to the aspect in the order specified by the protocol (see Figure 147).

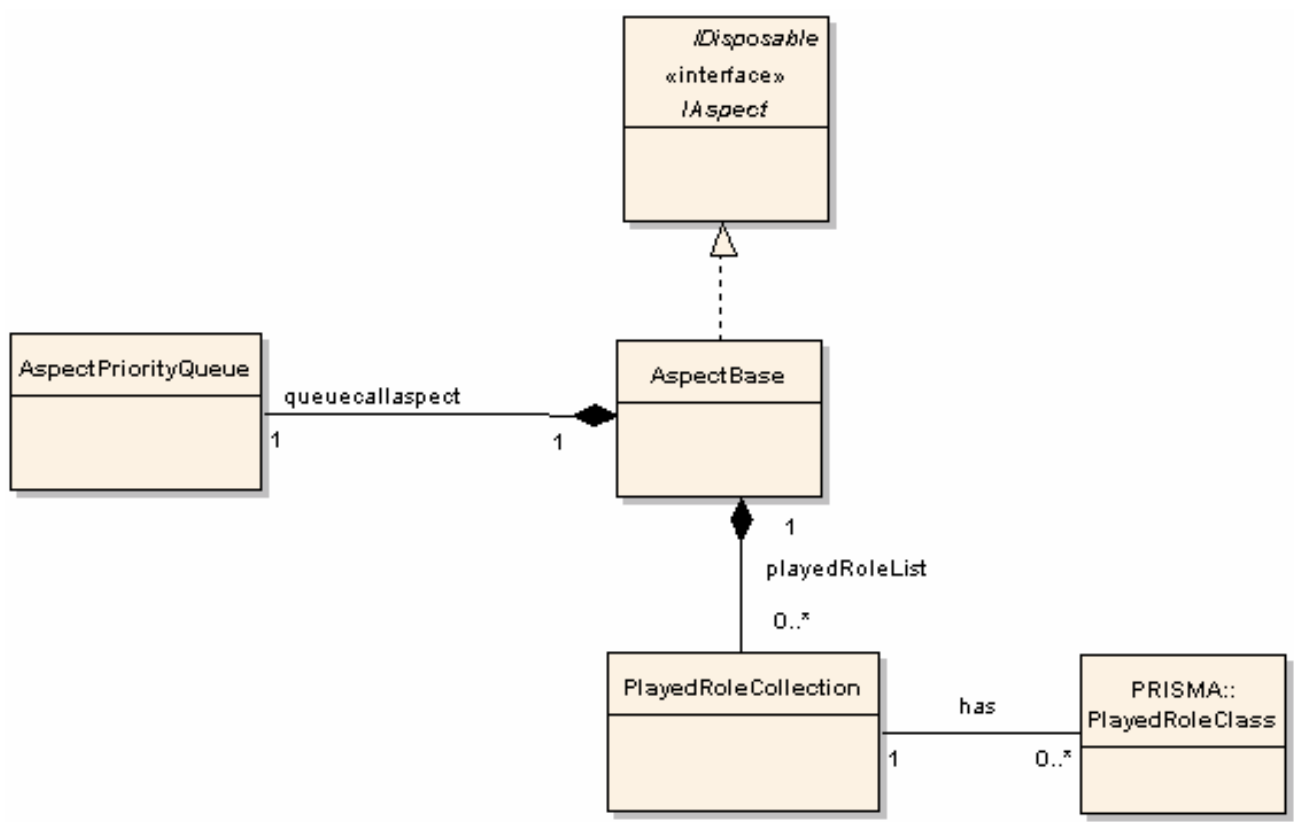

Figure 147. Main classes of the namespace Aspects of PRISMANET ${ }^{4}$

\footnotetext{
${ }^{4}$ The set of classes that appear in the figure have been automatically generated from the source code of PRISMANET using the Sparx tool http://www.sparxsystems.com/
} 
PRISMA: Aspect-Oriented Software Architectures

The kinds of aspects that can be defined in the PRISMA model are unlimited. However, each one has the functionality described above. Therefore, the kinds of aspects are subclasses of the class AspectBase and inherit this functionality (see Figure 148).

PRISMANET allows the implementation of specific aspects by creating C\# classes that inherit from one of the classes that represents one kind of aspect. These specific aspects must be serializable to enable the mobility of aspects in future PRISMA distributed architectures. These aspect classes are packaged in an assembly to facilitate their integration in a repository and their future distribution over the network.

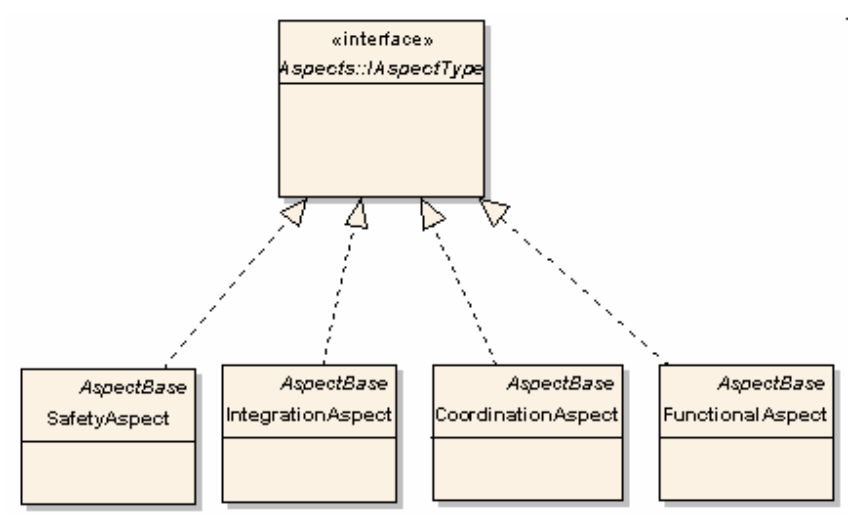

Figure 148. Classes of some of the kinds of aspects ${ }^{4}$

\section{Dxecution Model of Aspects}

Due to the fact that the middleware must guarantee the execution of services without blocking the requesters, when an aspect service requires its execution at the same time that another service is being processed, the aspect stores the service that cannot be immediately attended in a priority queue.

An aspect is divided into two parts for the management of services: a public part and a private part. The private part specifies the functionality that must be executed by each service of the aspect, whereas the public part receives the requests of the services and adds them to the aspect queue (see public and private methods in Figure 149). 


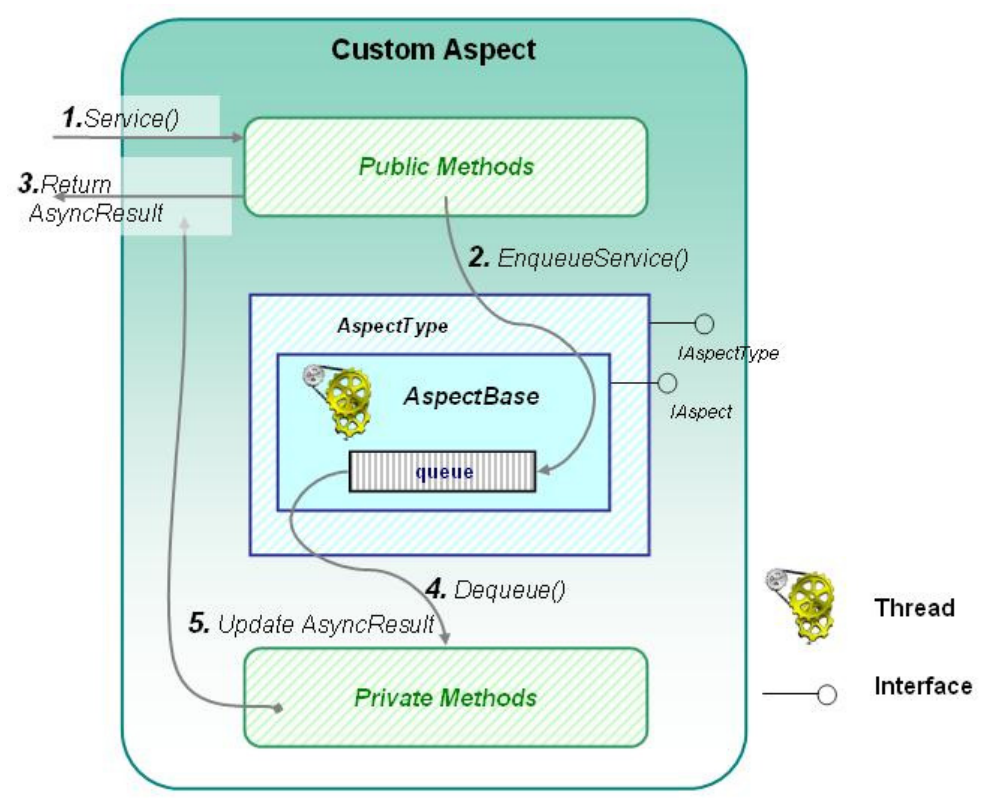

Figure 149. Execution Model of an Aspect

The execution of an aspect service is performed as described below:

$<<$ When the execution of a service is requested from an aspect, the request comes from the public method of the service (see step 1, Figure 149). The request is added to the queue of the aspect with all the needed information to execute it (see step 2, Figure 149). To prevent the requester that is waiting for the result from being blocked, the reference of the AsyncResult structure is sent to the requester as explained in section 9.4.2.1 (see step 3, Figure 149). Requests are then extracted from the queue in order to be executed taking into account the priorities of the services and whether they can be executed in the state that the aspect is in that moment. The requests are extracted from the queue following a FIFO policy ordered by the service priority. As a result, the requests with higher priority are executed first and the requests with the same priority are ordered using the standard FIFO policy (see step 4, Figure 149). It must also be taken into account that sometimes the first request of the queue cannot be executed because its execution is not allowed by the state of the protocol. In this case, the request is not extracted from the queue, and its number of execution attempts is increased and 
PRISMA: Aspect-Oriented Software Architectures

the next request in the queue is executed. After its execution, the execution of the first request of the queue is tried again because the state of the protocol could have been changed by the previous execution. In this way, the aspect thread gives the previously failed services the chance of being executed. However, if a service request fails the maximum of number of execution attempts that has been determined by PRISMANET, the request is extracted from the queue and generates an exception that is sent to the requester. If the first request of the queue can be processed, the functionality of its corresponding private method is executed and the result of the execution is stored in its AsyncResult structure (see step 5, Figure 149). Finally, this structure will be queried by the requester to obtain the results of the service execution. $>>$

It is important to emphasize that while the aspect thread is executing a private method the public method can concurrently receive new requests and can add them to the queue. It must also be emphasized that to stop the execution of an aspect appropiately, three steps must be performed: 1. reject new requests from the public part; 2 . execute the requests that are already stored in the queue until it is empty, and 3. stop the aspect thread.

\subsubsection{Simple Architectural Elements: Components and Connectors}

The architectural element type has been implemented as a C\# class of PRISMANET called ComponentBase. This class stores the name of the architectural element, its own thread and its middleware references, the dynamic list of aspects, the dynamic list of weavings, and the references to the ports to be able to receive and request services. In addition, the ComponentBase class offers services to initiate the architectural element thread execution, to stop the architectural thread execution, to abort the architectural element thread execution, to query if an aspect of the architectural element is weaved with another aspect, and to add aspects and weavings from an architectural element. Finally, it is important to emphasize that the architectural element contains a queue that guarantees the execution of services without blocking the requesters by storing the service requests that cannot be immediately attended to. 


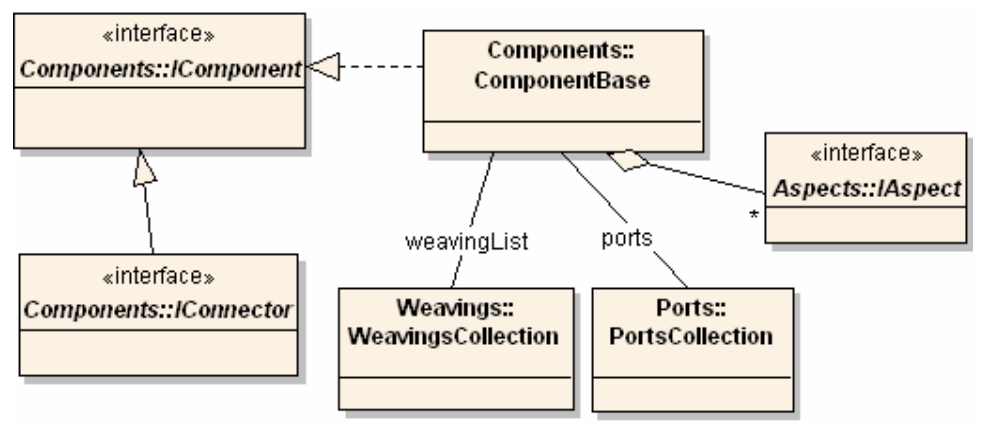

Figure 150. Main classes of the namespace Components of PRISMANET ${ }^{4}$

Connectors have been implemented as components because the functionality that they must provide in the middleware is the same. The difference between components and connectors is that connectors implement the interface IConnector and components implement the interface IComponent. However, the interface IConnector inherits the properties of IComponent, but it does not extend its properties. In fact, this interface is only used as an identification mechanism between components and connectors.

As a result, PRISMANET allows the implementation of a specific architectural element by creating a C\# class that inherits from the ComponentBase class. It is important to keep in mind that architectural elements must be serializable in order to enable their mobility in future PRISMA distributed architectures.

\section{Ports}

The port type has been implemented as a C\# class called Port. The ports of an architectural element are classified into input (inPorts) and output (outPorts) ports. As a result, inPorts and outPorts inherit their properties from the class Port. This class stores the name of the port, the references to the interface and the played_role that is associated to it, and the list of attachments that are connected to it (see Figure 151). 
PRISMA: Aspect-Oriented Software Architectures

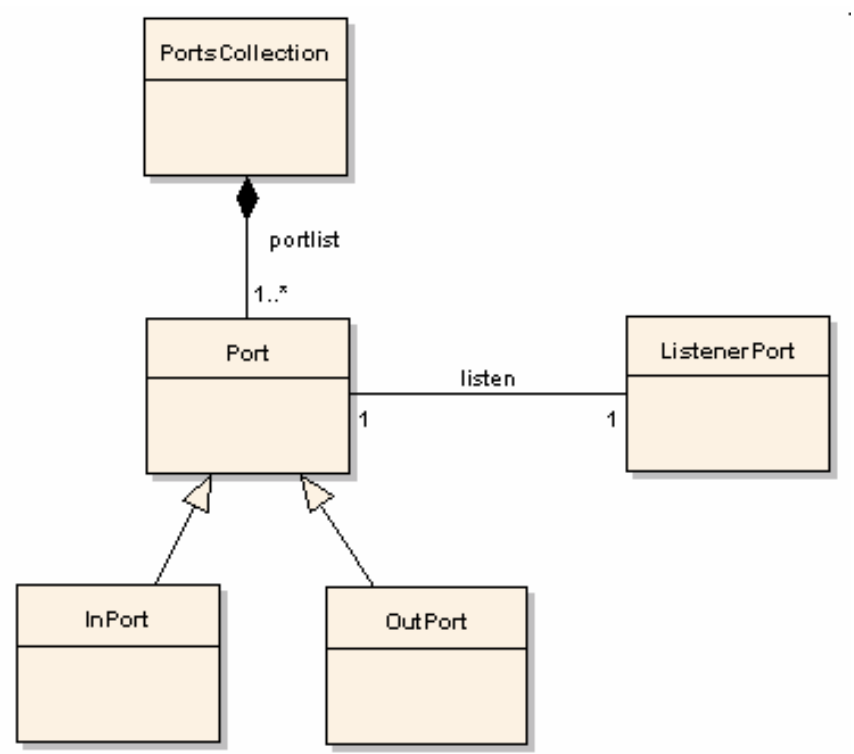

Figure 151. Main classes of the namespace Ports of PRISMANET ${ }^{4}$

InPorts publish the services that architectural elements provide. An InPort publishes an interface that is implemented by one of the architectural element aspects. In addition, an InPort is in charge of adding the received service requests to the architectural element queue.

OutPorts are in charge of providing mechanisms to send service requests to other architectural elements. An OutPort only permits the sending of those service requests that belong to the OutPort interface.

Ports offer reflection mechanisms to be dynamically queried, and thereby to dynamically get the list of interfaces that are associated to the architectural element ports making dynamic communications feasible. In addition, the implementation of ports also allows the isolation of aspect services and the services that architectural elements publish through their ports. Since the aspect that implements the port interface is known, ports have a set of delegates that point to each of the aspect services that must be executed when an interface service is requested. As a result, when a service interface is requested, its corresponding delegate is stored in the architectural element queue. 


\section{Weavings}

An architectural element contains a weaving manager that checks whether or not a service has associated weavings. If so, the weaving manager executes them synchronously, and, if not, the service is resent to the corresponding aspect.

There are no previous .NET works that implement weavings at the source code level and that could dynamically perform weavings (see Figure 144). From the different strategies of implementing weavings, the static code injection strategy provides the best performance, and the code interception strategy is the most flexible. Therefore, in order to balance the advantages of performance and flexibility, weavings have been implemented as a dynamic linked list with three levels of depth. This list is part of the weaving manager of the architectural element and contains the weavings that belong to the architectural element. Thus, this weaving implementation facilitates the management of the weavings.

The dynamic list is implemented by the C\# class WeavingsCollection (see Figure 152). Each element of this dynamic list is an instance of the C\# class AspectTypeNode, which contains the aspect type and another dynamic list called weavingAspectList. Each element of the WeavingAspectList is an instance of the C\# class WeavingNode. This class stores the service name, which triggers the weaving execution as well as a delegate of this service for its dynamic invocation. It also stores three more lists, each of which belongs to a weaving operator (after, before, instead) and contains instances of the C\# class WeavingMethod. This class stores the delegate, which points to the method that must be executed as a result of the weaving (advice service). It also stores the method that has triggered the weaving execution (pointcut service), and the weaving operator (after, before, instead, afterif, beforeif, insteadif) (see Figure $152)$.

The weaving manager executes weavings. This weaving execution consists of searching the methods that are involved in the weaving by going through the lists beforeList, afterList and insteadList. The execution of the corresponding delegates must be performed in the correct order. To preserve this order, the first services in being executed are the advice services of the weaving that are stored in WeavingNode.beforeList, and then their pointcut services are executed. The second services in being executed are the advice services of the weaving that are 
PRISMA: Aspect-Oriented Software Architectures

stored in WeavingNode.insteadList. Finally, the third services in being executed are the pointcut services of the weaving that are stored in the WeavingNode.afterList., and then their advice services are executed.

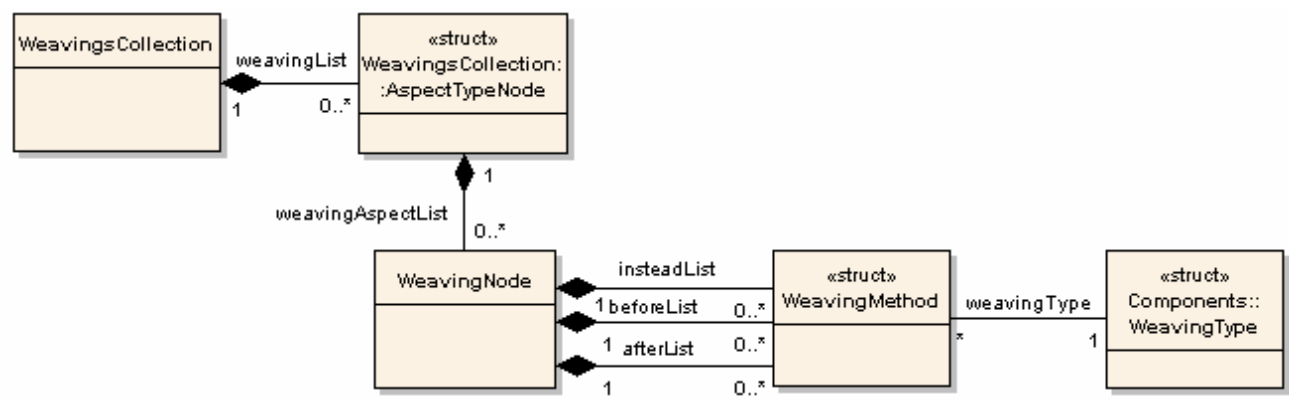

Figure 152. Dynamic List of Weavings ${ }^{4}$

Execution Model of Architectural Elements

An architectural element is composed of input (InPorts) and output (OutPorts) ports, a queue that temporally stores the services that will be processed by the thread of the architectural element, a weaving manager, and a set of aspects that are concurrently executed.

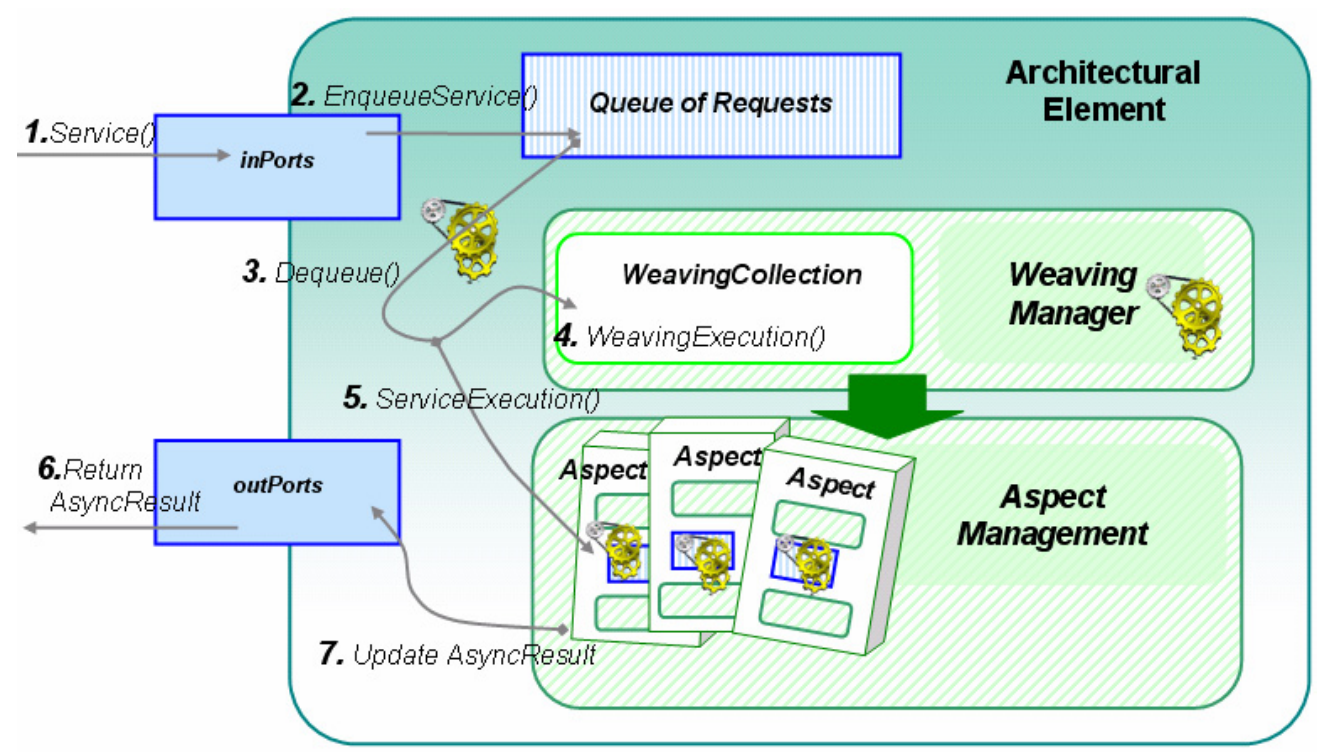

Figure 153. The execution model of an architectural element 
Taking into account the properties of the elements that architectural elements are composed of, the execution of a service that is requested from an architectural element performs the following process:

$<<$ When the execution of a service is requested from an architectural element, the request comes from the port that publishes the service (see step 1, Figure 153). The port sends the request to the queue of the architectural element (see step 2, Figure 153). Due to the fact that several ports can try to add requests to the queue at the same time, the queue is protected from concurrent access using monitors. As a result, only one port can add a request to the queue at the same time.

Once the architectural element thread extracts the requested service from the queue, the architectural element checks if the requested service has weavings associated to it (see step 3, Figure 153). If the service does not have any weavings, its delegate is asynchronously executed so that the architectural element can process another request from the queue. The delegate execution consists of adding the service to the queue of the corresponding aspect. Then, the aspect thread executes the service (see step 5, Figure 153). However, if the service has weavings associated to it, before executing step 5 , the service is sent to the weaving manager (see step 4, Figure 153). The manager processes weavings by creating its own thread and freeing the component from this task. $>>$

With regard to starting or stopping an architectural element, when the middleware calls the start service of an architectural element, the architectural element calls the startAspect service of each one of its aspects. When the middleware calls the stop or abort services of an architectural element, the threads of its aspects must also be stopped or aborted. To stop an architectural element in a secure way, a set of operations must be performed to achieve a secure state that will permit the start of the architectural element execution in the future. An architectural element is in a secure state when it does not have requests in its aspect queues and there are no executing services. These operations consist of rejecting new services in their queues and processing all the services that were already stored in the queue before the stop execution. 
PRISMA: Aspect-Oriented Software Architectures

\subsubsection{Communication: Attachments and Bindings}

PRISMANET supports the communication of the architectural elements without making architectural elements aware of each other. To make architectural elements as reusable as possible, they do not have references to the other architectural elements that they communicate with. The communication among components is the responsibility of attachments and bindings. Thus, an attachment has the references of the communicating components and connectors, and a binding has the references of the communicating systems and architectural elements that systems are composed of.

Attachments

To support attachments, PRISMANET contains three classes: the class Attachment, the class AttachmentServerBase and the class AttachmentClientBase (see Figure 154). For each component port, there is at least one instance of an Attachment class. When a component instance is created, PRISMANET creates the instances of the attachments associated to each port. Each PRISMA port has been implemented in two parts, a client port (outPort) and a server port (inPort) (see Figure 151). At the same time, attachments have also been implemented in two parts, a Server Attachment (AttachmentServerBase) and a Client Attachment (AttachmentClientBase). An instance of the Attachment class automatically instantiates an AttachmentClientBase and an AttachmentServerBase class.

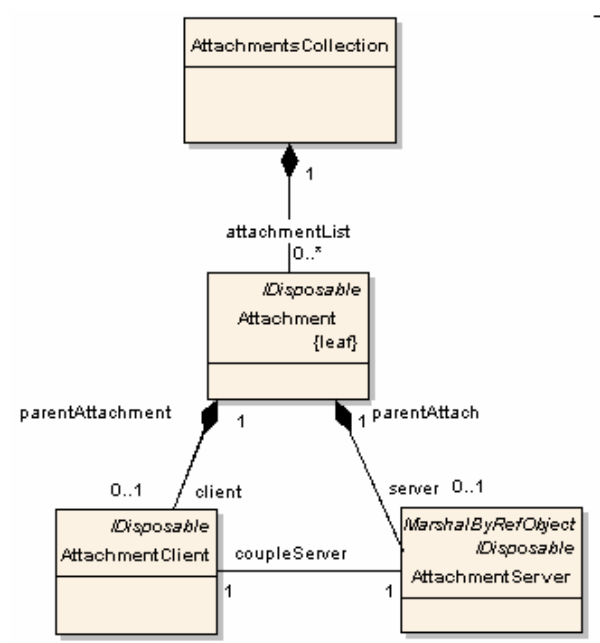

Figure 154. Main classes of the namespace Attachments of PRISMANET ${ }^{4}$ 
Figure 155 shows how a component and a connector are connected to each other through an attachment. The ports of both the component and the connector have an attachment associated to them. Specifically, the component port has the attachment $\mathrm{X}$ associated to it and the connector port has the attachment $\mathrm{Y}$ associated to it. The AttachmentClientBase of the attachment $\mathrm{X}$ listens to the component outPort (see step 1, Figure 155 ) and resends services to the AttachmentServerBase of the connector attachment Y (see step 2, Figure 155 ). Then, AttachmentServerBase of the attachment $\mathrm{Y}$ resends services to the connector inPort in order to be executed by it (see step 3, Figure 155 ). The communication in the inverse direction is performed in the same way. The AttachmentClientBase of the attachment $\mathrm{Y}$ listens to the connector outPort (see step 4, Figure 155) and resends the services to the AttachmentServerBase of the component attachment X (see step 5, Figure 155 ). Finally, the AttachmentServerBase of the attachment $\mathrm{X}$ resends the services to the component import in order to be executed (see step 6, Figure 155 ).

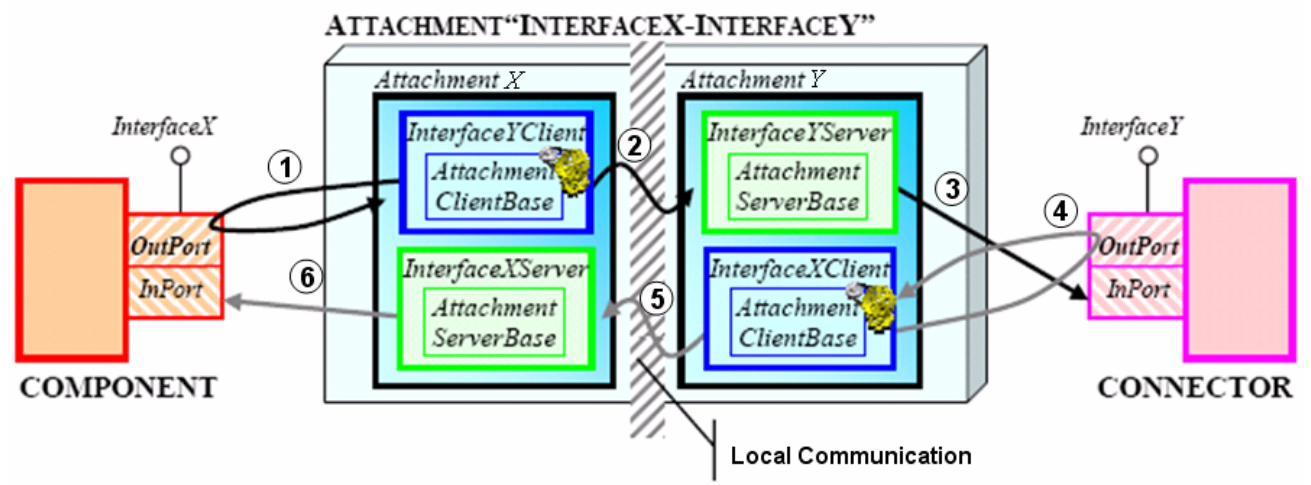

Figure 155. The execution model of an attachment

An AttachmentClientBase instance has a thread that listens to a specific outPort of an architectural element instance. When the AttachmentClientBase instance detects that there is a request in the outPort, the request is resent to the instance of an AttachmentServerBase. Thus, the AttachmentClientBase instance has a reference or a proxy of the AttachmentServerBase. The AttachmentServerBase is a MarshalByRefObject class of the .NET Remoting framework. This is necessary to create a proxy of the instance to allow the AttachmentClientBase instance to access to it remotely in a future version of PRISMANET. 
PRISMA: Aspect-Oriented Software Architectures

All elements that are involved in an attachment are related to each other in one way or another. As a result, it is possible to define an invocation cycle that involves all the elements that participate in the attachment. When an element that participates in this cycle receives a request, it must process the established actions and propagate the invocation to the next element in the cycle. The execution order of the cycle, taking into account the attachment presented in Figure 155, is the following:

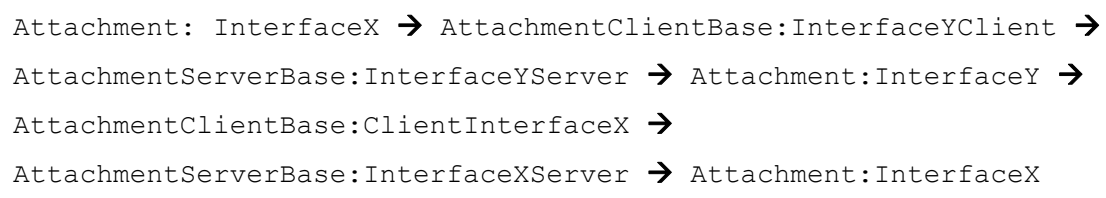

This cycle determines the execution model of an attachment. The invocation cycle starts and stops at the same point to detect when the communication has been finished.

The attachments are solely responsible for the communication of the components and connectors. PRISMANET only participates in the creation of attachment instances between its component and connectors instances. To store the list of attachments in its site, the middleware has an AttachmentCollection class (see Figure 154). Since middleware does not manage the communication process, its implementation is simplified and the independence and maintainability of attachments are increased.

\section{Bindings}

Bindings connect system ports and ports of the architectural elements that the system is composed of. Bindings have been designed in a way similar to attachments. Bindings consist of two parts: the part of the system (SystemBinding) and the part of the architectural element that the system is composed of (ComponentBinding) (see Figure 156). The difference between attachments and bindings is introduced by a binding manager, which is called SystemBinding. There is only one SystemBinding for each system, i.e., there is not a SystemBinding for each system port as occurs in attachments. As a result, SystemBinding contains the references of every binding connected to the system ports. In addition, the SystemBinding does not have a server part and a client part as attachments have; it only has a server part., which is called 292 
SystemBindingServer (see Figure 156). The SystemBindingServer is in charge of localizing the system's architectural elements that are connected to bindings and resending them the requests that arrive to the bindings. However, the part of the binding related to the system's architectural element (ComponentBinding) is implemented in the same way as attachments. The ComponentBinding has a ComponentBindingClient that listens to its outPort and resends services to the SystemBindingServer of the system as well as a ComponentBindingServer that receives the request through its inPort (see Figure 156).

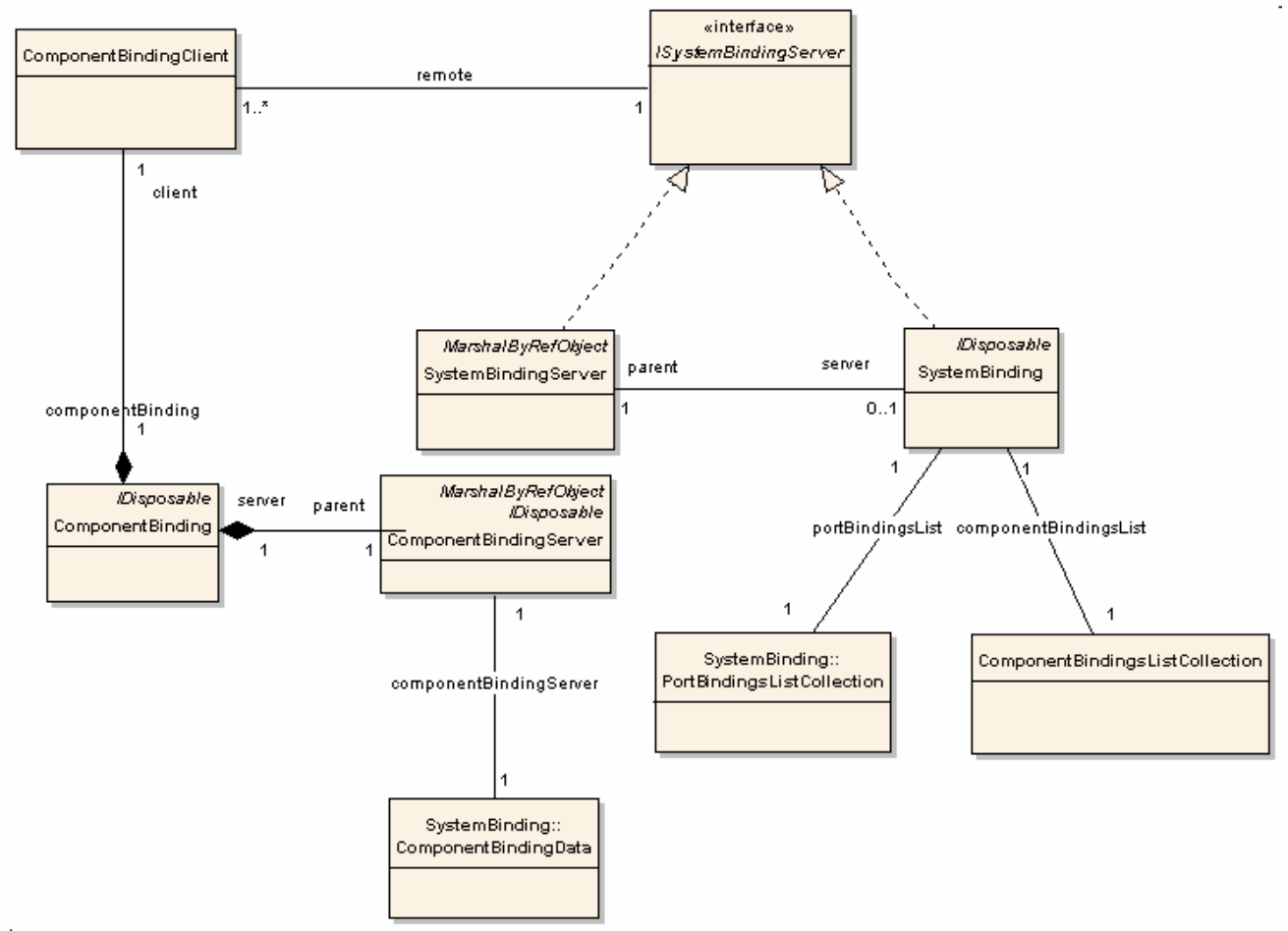

Figure 156. Main classes of the namespace Bindings of PRISMANET ${ }^{4}$

When the SystemBindingServer detects that there is a request in a system inPort, the request is added to the system queue (see step 1, Figure 157). Then, when the request is extracted from the queue by the SystemBinding (see step 2, Figure 157), and it resends the request to the correspondent ComponentBidingServer. When the ComponentBindingServer receives the request (see step 4, Figure 157), it is sent to the InPort (see step 4, Figure 157), and it is 
PRISMA: Aspect-Oriented Software Architectures

processed by the architectural element. On the other hand, when an internal architectural element sends a request through its Outport (see step 5, Figure 157), since its componentBindingClient is listening to the OutPort all the time, it realizes that there is a request and it resends the request to the SystemBindingServer (see step 6, Figure 157). Finally, the SystemBindingServer sends the request through the corresponding outPort of the system (see step 7, Figure 157).

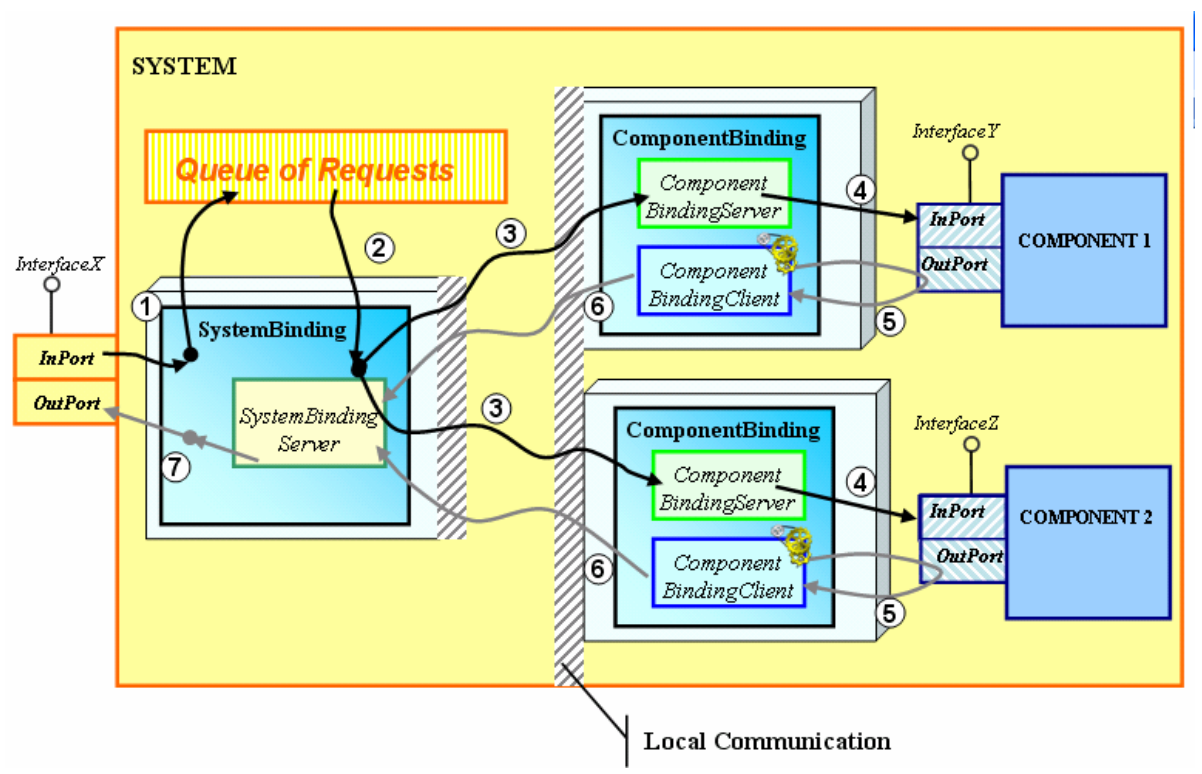

Figure 157. The execution model of bindings

\subsubsection{Complex Architectural Elements: Systems}

Systems are been implemented as a specialized class of ComponentBase called SystemBase.

This class inherits the properties of components and defines a set of services that characterize the system. These services allow components, connectors, attachments and bindings to be added to the system. They are grouped by the interface ISystem, which is implemented by the class SystemBase (see Figure 158).

The class SystemBase is in charge of maintaining the list of architectural elements, attachments and bindings that the system is composed of. In addition, SystemBase invokes the services needed by the middleware to start the execution of those elements that the system is composed of. 
Due to the fact that a system is composed of a set of elements, it overwrites the start and stop services of the ComponentBase class by extending their behaviour to start the execution of these elements.

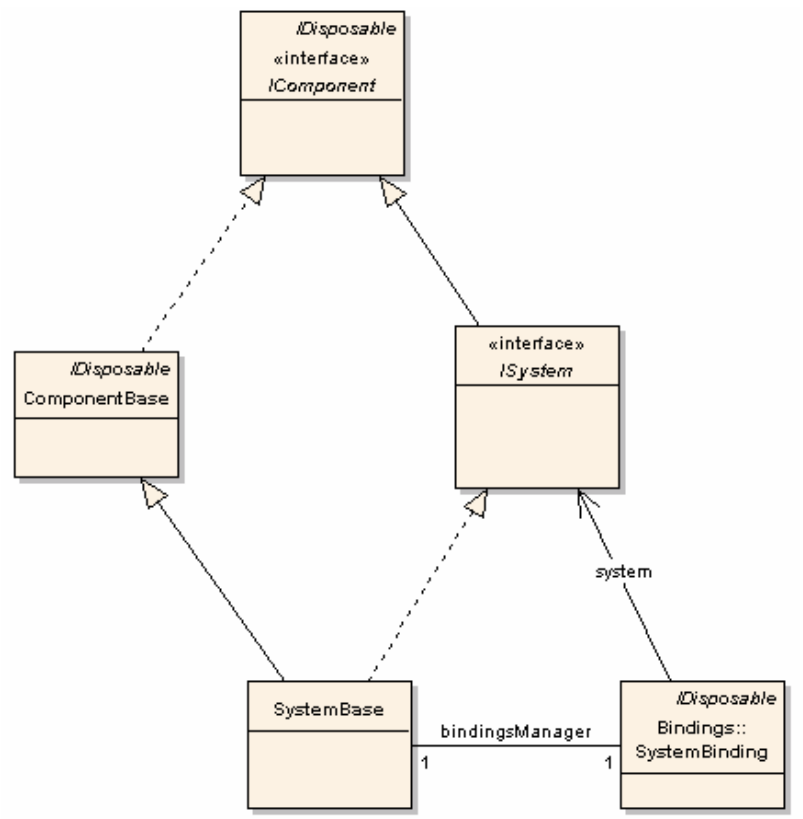

Figure 158. Main classes of the namespace Systems of PRISMANET ${ }^{4}$

\subsubsection{Memory Persistence}

The class MiddlewareSystem implements the functionality that is dependent on the .NET technology (see Figure 159). It provides a wide variety of services to the PRISMA architectural elements so that they are properly maintained. This maintenance is managed by MiddlewareSystem and its different functionalities have been structured in different related classes. These classes are the class ElementManagementServices, which manages the architectural elements and connections, and the class TypeManagementService, which manages types (see Figure 159). 
PRISMA: Aspect-Oriented Software Architectures

The class ElementManagementServices manages the creation and destruction of instances of components, connectors, systems, attachments and bindings. It also starts and stops the threads of the instances that it creates.

The class TypeManagementServices searches the different types that are required by architectural models and loads their assemblies. Its services are used to dynamically create ports, attachments, and bindings.

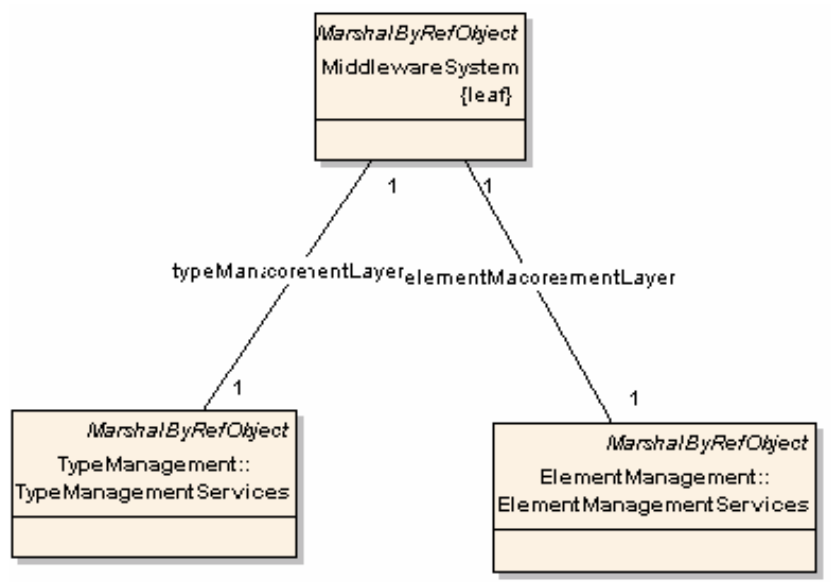

Figure 159. Main classes of the namespace Middleware of PRISMANET ${ }^{4}$

\subsubsection{Transaction Manager}

The Transaction Manager is a module of the middleware that provides services to suitably execute transactions (see Figure 145). The execution of a PRISMA transaction can involve more than one aspect since that it can invoke services that belong to different aspects of the transaction aspect. These aspects can also be imported by either the same architectural element that imports the transaction aspect or other different architectural elements. As a result, to correctly perform a transaction and to guarantee its atomic execution, the aspects involved in the transaction must be aware of when they are executing a transaction. In order to cope with this need, transactional contexts have been implemented in PRISMA.. 
A transactional context defines the boundaries of a transaction. In PRISMA, transactional contexts notify aspects if they are executing a service that is involved in a transaction. Therefore, the aspect is blocked by the transactional context and it only executes requests that come from this transactional context until the transaction finishes. This notification is performed by adding the transactional context name to the service request that is temporarily stored in the queue of the aspect.

The class TransactionManager is responsible for the transactional context management. When the execution of a transaction is started, the middleware is notified and a new entry to the TransactionalContext list of the Transaction Manager is added (see Figure 160). TransactionManager stores an incremental sequence number, a reference to the MiddlewareSystem, the TransactionalContext list and the reference of the TransactionLog file.

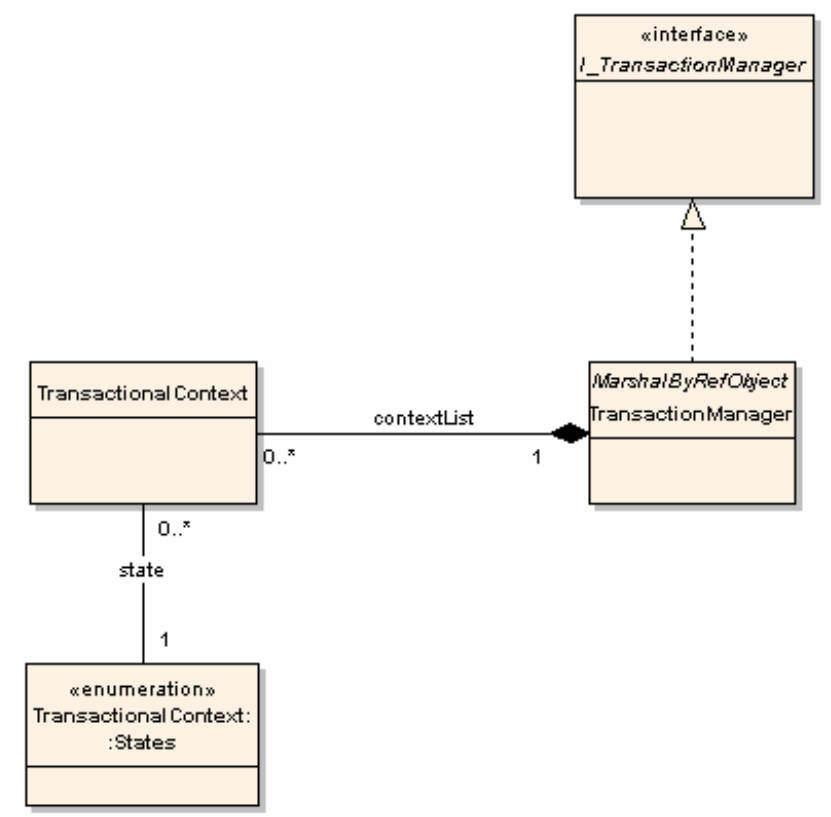

Figure 160. Main classes of the namespace TransactioManager of PRISMANET ${ }^{4}$

The class TransactionalContext has a context identifier and maintains the information related to the transaction failures. It stores which is the state of the transaction and when it finishes. 
PRISMA: Aspect-Oriented Software Architectures

It is important to take into account that there can be transactions that have other nested transactions [Mos87]. A transactional hierarchy is created, which can be represented as a tree whose root is the initial transaction. Thus, each transaction that is involved in a nested transaction has an associated nesting level number, where 0 is the nesting level number of the root, and where the highest nesting level number is the most nested transaction. The class TransactionalContext is in charge of storing the nesting level number of each transaction.

When a transaction has nested transactions and one of them fails, every action performed by the nested transaction will be undone unless the transaction can treat the failure. The commit of a nested transaction is considered to be partial commit until it has beenconfirmed that every transaction that includes the nested transaction does not fail. In fact, the only commit that is not partial in a hierarchical transaction is the commit of the root transaction. As a result, a transactional context has the following possible states (see the relationship state in Figure 160):

$>$ ACTIVE : The transaction is being executed

$>$ PRECOMMITTED : The transaction has finished. However, since it belongs to a transactional hierarchy, it must wait for the end of all the transactions of the hierarchy in order to confirm the commit or execute a rollback..

> PREROLLBACK : The transaction has finished. However, since it belongs to a transactional hierarchy, it must wait for the end of the root transaction because it establishes the execution order of the transitions that it nests.

> COMMITTED : The transaction has finished successfully.

$>$ ROLLBACKED : The transaction has finished due to a failure.

There is a TransactionLog file for each transactional context, in which all the actions of the transactional process are recorded. Each entry of the TransactionLog has a sequence number, a reference to the previous sequence number, and the inverse action of the action that has been performed. As a result, each transactional context has a TransactionLog file that is executed by the middleware when a transaction fails. This file execution reestablishes the state of the architecture that there was after the transaction execution. 
The PRISMA CASE
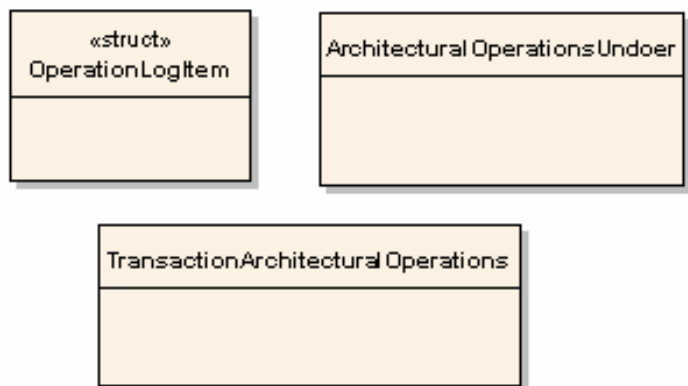

Figure 161. Main classes of the TransactioLog of PRISMANET

\subsection{5. $\log$}

The Log is a module of PRISMANET that logs every operation that is performed by the middleware in order to register the execution history of software architectures (see Figure 145). It is important to emphasize that this log is different from the TransactionLog; its functionality consists of recording the actions that are executed by the middleware like as if it were a blackboard. The only purpose of this $\log$ is to provide information about the software architecture execution. This log is shown by PRISMANET during its execution and provides the updated information at runtime (see Figure 162 ).

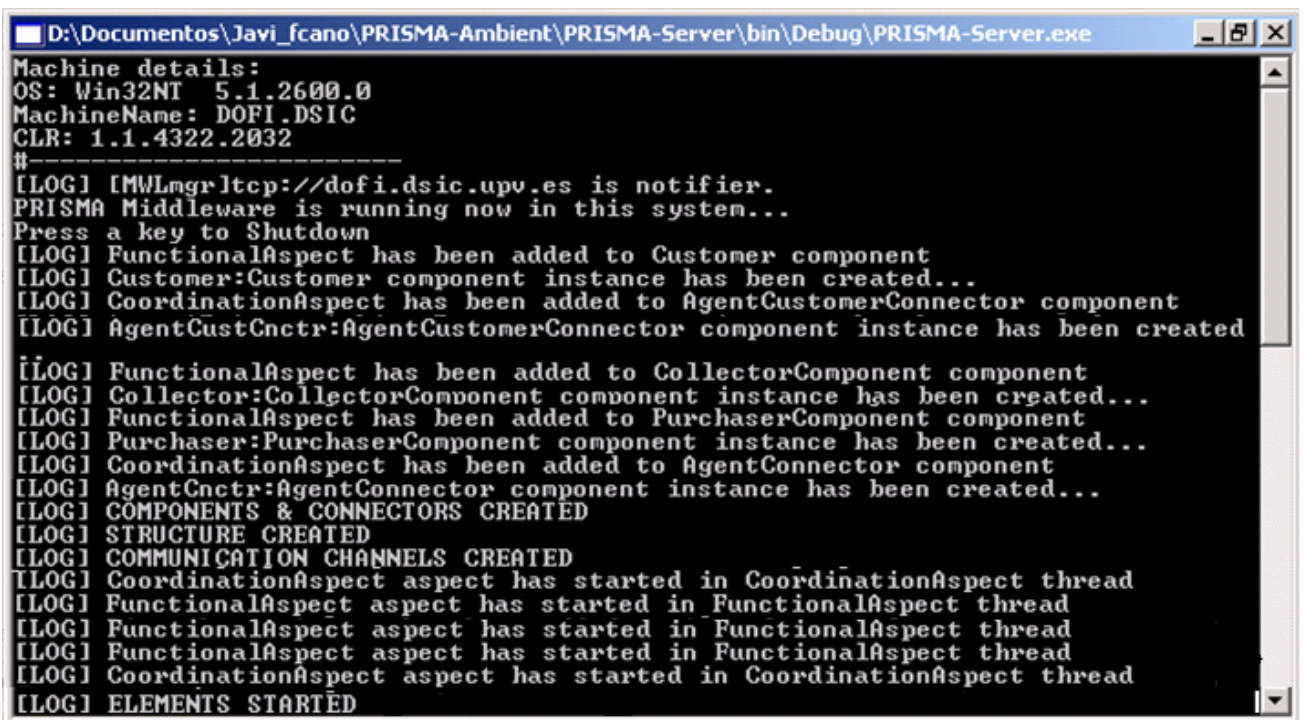

Figure 162. Execution of a PRISMANET Log 
PRISMA: Aspect-Oriented Software Architectures

\subsection{CONCLUSIONS}

PRISMA CASE has been presented in this chapter. PRISMA CASE is a framework that provides complete support for developing software systems following the PRISMA approach. It is composed of a set of tools that is suitably integrated to provide a unique framework that gives support to the user throughout the software life cycle. This integration also provides traceability during the different stages of the software life cycle and facilitates the maintenance of the developed software products.

This set of tools includes the PRISMA Types Graphical Modelling Tool with its code generation patterns, the PRISMA Configuration Graphical Modelling Tool with its code generation patterns, the generic Graphical User Interface for PRISMA applications, and the middleware PRISMANET.

The PRISMA Types and Configuration Graphical Modelling Tools give support to the developmento of PRISMA software architectures following the MDD approach and using the PRISMA AOADL in a graphical way. As a result, PRISMA offers mechanisms to develop software architectures in a more intuitive and friendly way and mechanisms to verify their models. In addition, the code generation patterns that PRISMA modelling tools offer allow automatically generate executable C\# code on PRISMANET from the specified graphical models. Thus, PRISMA CASE deals with the traceability between software architectures and implementation and reduces the time and cost invested in the development and maintenance processes.

PRISMA CASE provides a generic Graphical User Interface to execute software architectures. This is an important advantage because it is a simple way of validating that software architectures provide the behaviour expected by the user without having to develop a customized graphical user interface.

RISMANET is an innovative middleware based on the PRISMA model, which allows the implementation of complex, aspect-oriented and component-based software systems using C\# language. PRISMANET has been developed with $\mathrm{C \#}$ language using the standard techniques that the framework provides, that is, without extending the development platform. As a result, PRISMANET can be executed on every computer that has the .NET framework installed. 300 
PRISMANET offers extra functionalities for the .NET platform. It allows the execution of aspects, the configuration of software architectures (local and distributed), the communication among different components that are unaware of each other, etc. PRISMANET has also been tested in case studies such as the TeachMover tele-operated robot, banking systems, electronic auctions, etc.

This chapter demonstrates that all the tools and mechanisms that PRISMA CASE provides make PRISMA a mature and well-supported approach for developing large and complex software systems.

The work related to the PRISMA model has produced a set of results that is published in the following publications:

Jennifer Pérez, Elena Navarro, Patricio Letelier, Isidro Ramos, A Modelling Proposal for Aspect-Oriented Software Architectures, 13th Annual IEEE International Conference and Workshop on the Engineering of Computer Based Systems (ECBS), IEEE Computer Society , pp.32-41, ISBN: 0-7695-2546-6, Potsdam, Germany (Berlin metropolitan area), March 27th-30th, 2006.

> Jennifer Pérez, Elena Navarro, Patricio Letelier, Isidro Ramos, Graphical Modelling for Aspect Oriented SA, 21st Annual ACM Symposium on Applied Computing, ACM, pp. 1597-1598, ISBN: 1-59593-108-2, Dijon, France, April 23 -27, 2006. (short paper)

> Jennifer Pérez, Nour Ali, Cristobal Costa, José Á. Carsí, Isidro Ramos, Executing AspectOriented Component-Based Software Architectures on .NET Technology, 3rd International Conference on .NET Technologies, pp. 97-108, Pilsen, Czech Republic, May-June 2005.

> Cristóbal Costa, Jennifer Pérez, Nour Ali, Jose Angel Carsi, Isidro Ramos, PRISMANET middleware: Support to the Dynamic Evolution of Aspect-Oriented Software Architectures, X Conference on Software Engineering and Databases (JISBD), pp. 27-34, ISBN: 84-9732-434-X, Granada, September, 2005. (In Spanish) 
PRISMA: Aspect-Oriented Software Architectures

> Rafael Cabedo, Jennifer Pérez, Nour Ali, Isidro Ramos, Jose A. Carsí, Aspect-Oriented C\# Implementation of a Tele-Operated Robotic System, III Workshop on Aspect-Oriented Software Development (DSOA), X Conference on Software Engineering and Databases (JISBD), pp. 53-59, ISBN: 84-7723-670-4, Granada, September, 2005. (In Spanish)

> Rafael Cabedo, Jennifer Pérez, Jose A. Carsí, Isidro Ramos, Generation and Modelling of PRISMA Architecture using DSL Tools, IV Workshop DYNAMICA - DYNamic and Aspect-Oriented Modeling for Integrated Component-based Architectures, pp.79-86, Archena, Murcia, November, 2005. (In Spanish)

> Cristobal Costa, Jennifer Pérez, Jose Ángel Carsí, Study and Implementation of an Aspect-Oriented Component-Based Model in .NET technology, Technical Report, DSICII/12/05, pp. 198, Polytechnic University of Valencia, September, 2005. (In Spanish)

> Nour Ali Jennifer Pérez, Cristobal Costa, Jose A. Carsí, Isidro Ramos, Implementation of the PRISMA Model in the .Net Platform,II workshop DYNAMICA - DYNamic and Aspect-Oriented Modeling for Integrated Component-based Architectures, Conference on Software Engineering and Databases (JISBD), pp. 119-127, Málaga, November, 2004. 


\section{CHAPTER 10}

\section{THE PRISMA METHODOLOGY}

$<<$ Art and science have their meeting point in method. $>>$

Edward Bulwer-Lytton

Every development approach has an associated methodology that must be followed in order to obtain a quality software and to reduce the cost and the time invested in its development process. The PRISMA approach defines its own methodology for developing software systems.

This methodology follows the MDD and is divided into three stages: detection of architectural elements and aspects, software architecture modelling, and code generation and execution. These three stages are applied by the analyst of the software system in an iterative and an incremental way depending on his/her needs.

In this chapter, the PRISMA methodology is presented in detail using the TeachMover robot case study as an example. The different stages of the methodology are described considering that the development process is started from scratch. However, the development of a software system can be done starting from other software architectures that have been previously developed in a partial or complete way. In this sense, the methodology also considers the integration of COTS in PRISMA software architecture in order to reuse software and to reduce the development time. 
PRISMA: Aspect-Oriented Software Architectures

\subsection{STAGES OF THE PRISMA METHODOLOGY}

\subsubsection{First Stage: Detection of Architectural Elements and Aspects}

The first tasks for developing software architectures are to identify which architectural elements make up the software architecture and to detect the aspects that crosscut this software architecture. Several works have been proposed for the identification of components, they are usually based on a widely-extended criterion that consists of the functional decomposition of software. One of these works applies this criterion to multi-agent systems [Nor98] and another work applies this criterion to software architectures (the Architecture Based Design Method $(\mathrm{ABD}))$ [Bac00]. A detailed survey of criteria to identify software components can be found in [Ira00].

Other works have been developed for the identification of aspects in requirements specifications such as [Ban04], [Mor05a], [Nav03], [Sou03], [Sut02], and [YuY04]. An extended survey of these can be found in [Chi05] (see section 3.3.1). The identification of aspects is one of the new challenges that have emerged as a consequence of introducing aspects in software architectures. However, there is no defined, consolidated, guided process to identify architectural elements and the aspects that crosscut them. Until now, the detection of architectural elements and aspects has usually been performed from the requirements document in an intuitive way.

In PRISMA, the computational units (components) and the coordination units (connectors) are identified from the requirements specification of software systems, and the concerns that crosscut the software architecture can be detected.

\subsubsection{Identification of Architectural Elements}

The identification of architectural elements in the TeachMover robot has been done using the manual of the robot. The manual shows that the TeachMover is composed of a set of components that represent a set of joints, a set of connectors that coordinate the joints, and a set of systems that allows the composition of joints to form a robot. The result of this identification is the set of components and connectors that is presented in detail in section 5.1.2.2. 
Both architectural elements and the services that must be interchanged among them can be identified during this stage. These services can be requested and/or provided by the architectural elements. Since a PRISMA interface is a set of services that is provided and/or requested by means of the ports of architectural elements, the identification of these services implies the identification of the interfaces. The ports of architectural elements and the interconnections among these ports can also be detected taking into account the identified interfaces.

\subsubsection{Identification of Crosscutting Concerns}

The concerns that crosscut the software system must be identified from the requirements specification in order to modularize them into reusable entities called aspects. In the case of the TeachMover, the crosscutting concerns that we have identified are the following:

Functional: The purpose of the TeachMover software system is to move the robot. The robot has a motor to accurately perform movements by half-steps. A half-step is an angular advance that is produced by a stimulating impulse. In the case of the TeachMover, the movements can be requested using half-steps or inverse cinematics (moving to a specific point in space). This functionality, together with the gripper functionalities, allows the robot to move objects from an initial position to a final one. The movements of the robot are ordered by an operator from a computer.

$>$ Safety: Safety directives are necessary for monitoring the TeachMover movements in order to make sure that the movements are safe for the robot, the operator, and the environment that surrounds them.

$>$ Coordination: The inner behaviour of joints (SUCs) and the movements in which more than one joint has to be moved must be coordinated. In addition, the requests of the operator and the performance of the movements must be synchronized.

\subsubsection{Second Stage: Software Architecture Modelling}

Once the interfaces, aspects, architectural elements and their ports have been identified, the skeleton of the architectural elements and the aspects can be defined. The analyst is then ready to start the modelling process of the software architecture using the PRISMA CASE. This stage 
PRISMA: Aspect-Oriented Software Architectures

can be divided into five modelling steps: Interfaces, Aspects, Simple Architectural Elements, Systems, and Configuration (see Figure 163).

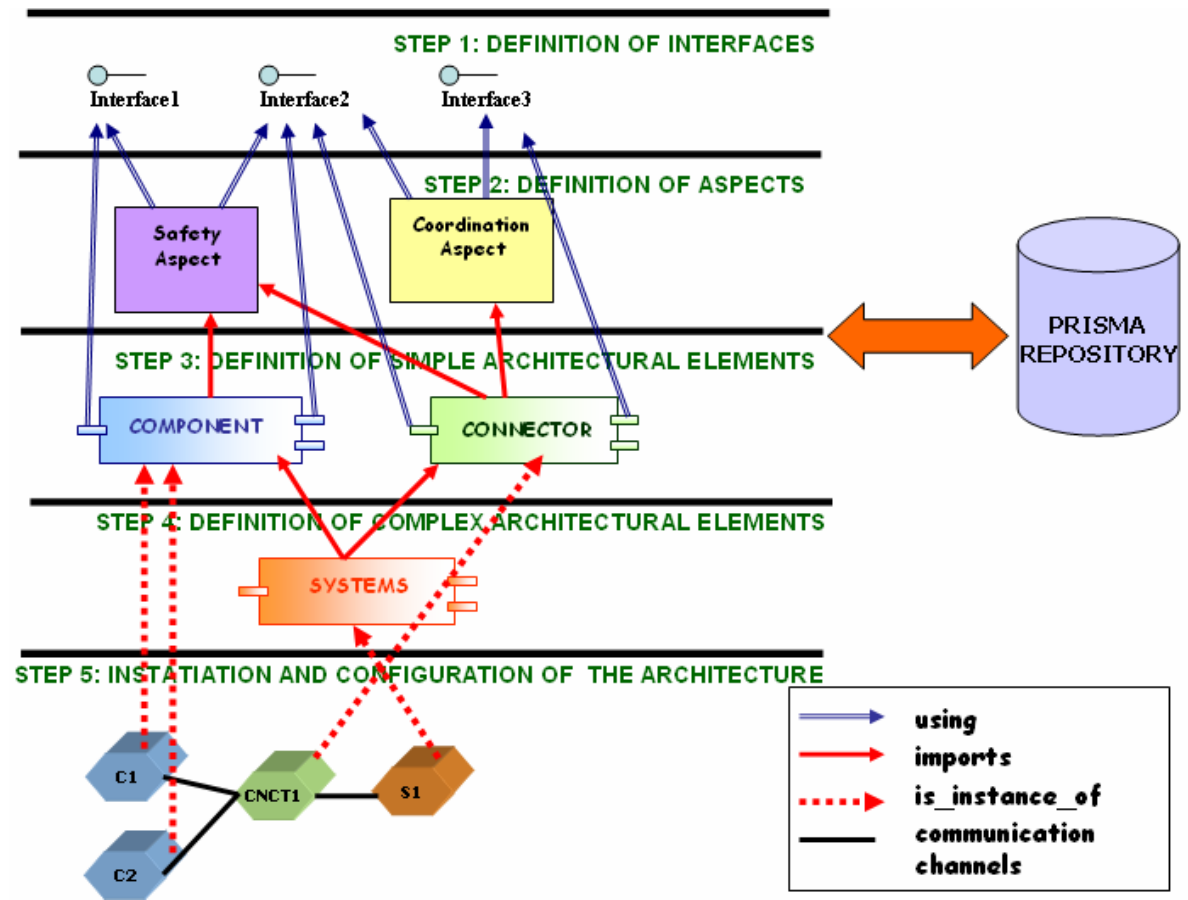

Figure 163. The methodology of the PRISMA approach

It is important to keep in mind that the enumeration of these steps is not in a restrictive order. The enumeration simply indicates the dependencies between the different concepts that arise when the architectural model is being modelled.

$>$ To configure an architectural model, the concepts that are instantiated during the configuration process must have been previously defined.

> To completely define a complex architectural element, the architectural elements that it consists of must have been previously defined.

$>$ To completely define an architectural element, the aspects that it imports and the interfaces that their ports use must have been previously defined.

$>$ To completely define an aspect that uses interfaces, the interfaces must have been previously defined. 
Even though the order of these can be different, it should be followed to completely define an architectural model. In other words, it does not mean that partial descriptions of the architectural elements, aspects or architectural models cannot be performed during the development process. The analyst can start the modelling process from either the steps 1,2,3 or 4 , obtaining partial solutions of the model, and can go backward or forward depending on his/her needs.

\subsubsection{Interfaces}

Interfaces are specified in step 1 of the PRISMA architecture modelling stage,. This is due to the fact that it is not necessary to previously define other elements of the model. Interfaces are stored in a PRISMA repository for reuse (see step 1, Figure 163).

The specification of the interfaces identified in the stage presented in section 10.1.1.1 consists of modelling the interface services and their signatures. This task is performed using the graphical constructors provided by the Modelling Type Tool of PRISMA CASE. The specifications of the interfaces of the joint of the TeachMover are presented in detail in appendix C.

\subsubsection{Aspects}

Aspects are modelled in step 2 of the PRISMA architecture modelling stage using the graphical shapes provided by the PRISMA Modelling Type Tool and the Modal Logic of Actions.

The aspects that specify the semantics of the services of an interface must be defined after the interface has been defined. This is why interfaces are defined in step 1 and aspects are defined in step 2 of the methodology. However, this does not constrain the specification order. Either the interface is defined before the aspect, or the services are initially defined as private services of the aspect and are then changed to publish services by means of an interface. Furthermore, when the needed interfaces are reused from the PRISMA CASE repository, step 1 is not necessary.

The aspects are defined by taking into account the crosscutting concerns identified in stage 3.1.1. The number of aspects for the same concern is decided by the analyst, taking into account the software system and criteria such as reusability and/or understanding. Depending 
PRISMA: Aspect-Oriented Software Architectures

on the analyst's criteria, he/she will define one aspect for a concern or several aspects for the same concern. Aspects are reusable entities that define a specific behaviour of a crosscutting concern and are, therefore, stored in a PRISMA repository (see step 2, Figure 163). As a result, not only can aspects be used more than once in a software architecture description, they can also be reused in different software architectures. The specifications of the aspects of the joint of the TeachMover are presented in the appendix C.

\subsubsection{Simple Architectural Elements}

The definition of simple architectural elements is performed in step 3 of the PRISMA architecture modelling stage. The aspects that are defined in step 2 are used to completely define these architectural elements. An architectural element imports the aspects that define the concerns that it requires. For this reason, aspects must be defined before architectural elements.

The same aspect is imported by each architectural element that needs to take into account the same behaviour of this concern (crosscutting concerns). As a result, an aspect can be imported by one or more architectural elements (see steps 2 and 3 of Figure 163). It is important to note that the changes performed in an aspect also affect every architectural element that imports this aspect.

The architectural elements that are defined in this step are types that are reusable by different software architectures because they are stored by PRISMA CASE. The storage of PRISMA architectural elements implies the storage of the aspects that they import. In addition, the reusability of the aspect can be due to the fact that it is reused in many architectural elements and also when the architectural element that imports the aspect is reused.

The simplest components that are found in the joint system of the TeachMover are specified in the appendix $\mathrm{C}$.

\subsubsection{Systems}

Systems are defined for the definition of PRISMA software architectures in step 4 of the PRISMA architecture modelling stage. To completely specify a system, the architectural elements that the system is composed of should be previously defined. In addition, the communication channels that permit the communication among them are defined. It is 
important to emphasize that the attachments are only defined if the system includes components and connectors that must be coordinated.

Besides having reusable aspects, components, and connectors, systems are also reusable. They are defined as patterns or architectural styles that can be reused in any software architecture whenever they are needed. For this reason, they are stored by PRISMA CASE.

It is important to note that any changes that occur in aspects affect the architectural elements that import them and, consequently, affect the systems that import these architectural elements. Moreover, the changes that occur in architectural elements that are imported by a system also affect the system (see step 3, Figure 163).

In the case of the TeachMover, there are several systems, at different levels of granularity. These systems are guided by the skeleton identified in the stage 10.1.1 (see Figure 10 in section 5.1.2.1)

The highest layer of composition provides the most abstract view of the software architecture, which is called the Architectural Model. It is important to emphasize that since the architectural model does not define a system that encapsulates it, bindings do not need to be defined.

\subsubsection{Configuration of Software Architectures}

Finally, the architectural elements that have been defined in the previous steps and have been stored by the PRISMA CASE are instantiated in step 5. In order to understand the step trace of the approach, it is important to take into account that instances have all the properties and behaviours of their architectural elements, and as a consequence, instances have the properties and behaviours of the aspects that their architectural elements import.

A specific software architecture is defined by connecting a set of components, systems, and connector instances with each other (see Figure 163). This step of the modelling stage is performed using the PRISMA Modelling Configuration Tool for modelling tele-operated systems (see section 9.3). This tool has been generated from types defined in previous steps of this stage using the PRISMA Modelling Type Tool.. The instantiation of the architectural elements of a model and the definition of attachment and binding relationships among 
PRISMA: Aspect-Oriented Software Architectures

instances is necessary to obtain an executable architectural model. This configuration is the input of the third stage of the PRISMA methodology

\subsubsection{Third Stage: Code Generation and Execution}

Once a specific configuration is defined, the automatic C\# code generation can be performed. This generation is possible thanks to the code generation templates of the PRISMA CASE, which isolate the specification from the source code preserving its independence.

The PRISMANET and the generated application can then be executed. The user can interact with the application using the generic console that the PRISMA CASE provides.

In the Teach Mover case, an application has been generated to move the robot using an aspect-oriented $\mathrm{CH}$ code that has been automatically generated from a formal aspect-oriented software architecture specification.

\subsection{INTEGRATION OF COTS IN PRISMA}

The use of Commercial Off-The-Shelf(COTS) during the development process has increased in the last few years due to the market competitiveness [Obe97],[Car00]. This increase has led developers to try to reduce the time and cost required to develop a software product. As a result, developers are using the software components of other companies more and more. These software components provide the functionality that developers need and have been tested and have a quality guarantee. The use of COTS is supported by the PRISMA approach to avoid having to remodel already existing software components, and to improve the development time. PRISMA provides mechanisms to integrate COTS in its software architectures without violating the principles of the PRISMA model.

COTS integration is usually presented as a handicap for developers because there are a lot of incompatibilities with programming languages, frameworks, platforms, communications, etc. Therefore, COTS must be adapted so that can be reused in a software system. There are three well-known techniques for integrating COTS in software systems: wrappers, gluewares, and proxies. Wrappers wrap COTS in a kind of software artefact of the software system; gluewares are intermediaries between the COTS and the software components of the software system; and proxies are adapters that hide the incompatibilities between COTS and the 310 
components of the software system. PRISMA uses the wrapper technique to integrate COTS in its aspect-oriented software architectures. Aspects are the PRISMA type that has been selected to wrapp COTS. A a new kind of aspect has been defined to do this, it is called Integration Aspect (see section 9.1.4.1).

An integration aspect contains a reference to the COTS that it is related to and has an associated PRISMA interface that defines all the services that the COTS provides. In order for the COTS integration to be compliant with the PRISMA model, the aspect must be imported by an architectural element of the model so that it can publish its services through its ports and communicate with other architectural elements through attachments or bindings.

The code generation template of integration aspects is different from other kinds of aspects because they only have references to COTS services. Moreover, to properly execute COTS services, the assemblies of COTS must be stored in the same folder as the PRISMA project. This is so that the assemblies of COTS can be added to the compilation process and to start their executions in the same way as other PRISMA software architectural elements.

\subsection{CONCLUSIONS}

This chapter has presented the PRISMA methodology for developing PRISMA aspectoriented software architectures. This methodology allows the development of aspect-oriented software architectures as if aspects and architectural elements were building blocks. Thus, it is possible to work with them in different ways to obtain different results. This flexibility and facility for working is achieved thanks to the modelling mechanisms provided by PRISMA CASE and the fact that aspects and architectural elements are independent entities that can be imported by different entities of the same software architectures.

The PRISMA CASE also promotes the reuse of architectural elements and aspects in different software architectures because their specifications are stored for reuse. For example, most of the architectural elements of the TeachMover can be reused in the other software architectures of tele-operated robots, such as the EFTCoR. The reused components and/or aspects can be reused as they were defined, or they can be updated and/or extended without having to start from scratch. However, it should be noted that even though reuse is limited by 
PRISMA: Aspect-Oriented Software Architectures

the system domain, a repository of PRISMA aspects and architectural elements can be a great contribution for reuse in product families such as the tele-operation family. Furthermore, a large repository with good searching mechanisms can provide excellent support for the development and maintenance of software.

It is also important to take into account that the step order of the methodology is not a restrictive order. The analyst is not forced to define all the interfaces of the system to specify the aspects nor is the analyst forced to define all the aspects to start the definition of simple architectural elements, etc. The analyst can completely define some interfaces, aspects, and architectural elements of the software system to obtain a part of a software architecture. Then, he/she can go back and define another piece of the software architecture starting from steps 1 , 2, 3, or 4 depending on his/her needs, and so on. The steps that have been presented only define the elements that must be defined before the specification of other elements. These steps can be applied in an incremental and iterative way by the analyst.

Finally, this chapter has introduced the integration of COTS in PRISMA models as part of the PRISMA methodology. This is an important characteristic due to the fact that the use of COTS is starting to be widely used, and any development approach that needs to reduce the development time must provide it. PRISMA uses aspects as wrappers of COTS. These aspects must be imported by an architectural element in order to publish their services through ports that enable their communication with other architectural elements. This is a novel and advantageous way of introducing COTS in software architectures because the COTS services can be requested and received and also can be extended by weaving them with other aspects that architectural elements import. As a consequence, COTS can be easily extended using the aspect-oriented mechanisms that PRISMA offers.

The work related to the PRISMA model has produced a set of results that are published in the following publications:

> Rafael Cabedo, Jennifer Pérez, Isidro Ramos, The application of the PRISMA Architecture Description Language to an Industrial Robotic System, Technical Report, DSIC-II/11/05, pp.180, Polytechnic University of Valencia, September 2005. (In Spanish) 
$>\mathrm{M}^{\mathrm{a}}$ Eugenia, Nour Ali, Jennifer Pérez, Isidro Ramos, Jose A. Carsí,DIAGMED: An Architectural model for a Medical Diagnosis, IV workshop DYNAMICA - DYNamic and Aspect-Oriented Modeling for Integrated Component-based Architectures, pp. 1-7, Archena, Murcia, November, 2005. (In Spanish)

> Jennifer Pérez, Rafael Cabedo, Pedro Sánchez, Jose A. Carsí, Juan A. Pastor, Isidro Ramos, Bárbara Álvarez, PRISMA Architecture of the Case Study: an Arm Robot, II workshop DYNAMICA - DYNamic and Aspect-Oriented Modeling for Integrated Component-based Architectures, Conference on Software Engineering and Databases (JISBD), pp. 119-127, Málaga, November 2004. (In Spanish)

> Jennifer Pérez, Nour Ali, Jose A. Carsí, Isidro Ramos, PRISMA Architecture of the Robot 4U4 Case Study, Technical Report DSIC-II/13/04, pp. 72, Polytechnic University of Valencia, 2004. (In Spanish)

> Jennifer Pérez, Nour H. Ali, Isidro Ramos, Juan A. Pastor, Pedro Sánchez, Bárbara Álvarez, Development of a Tele-Operation System using the PRISMA Approach, VIII Conference on Software Engineering and Databases (JISBD), pp. 411-420, ISBN: 84688-3836-5, Alicante, November, 2003. (In Spanish) 



\section{PART VI}

\section{CONCLUSIONS AND FURTHER}

\section{RESEARCH}

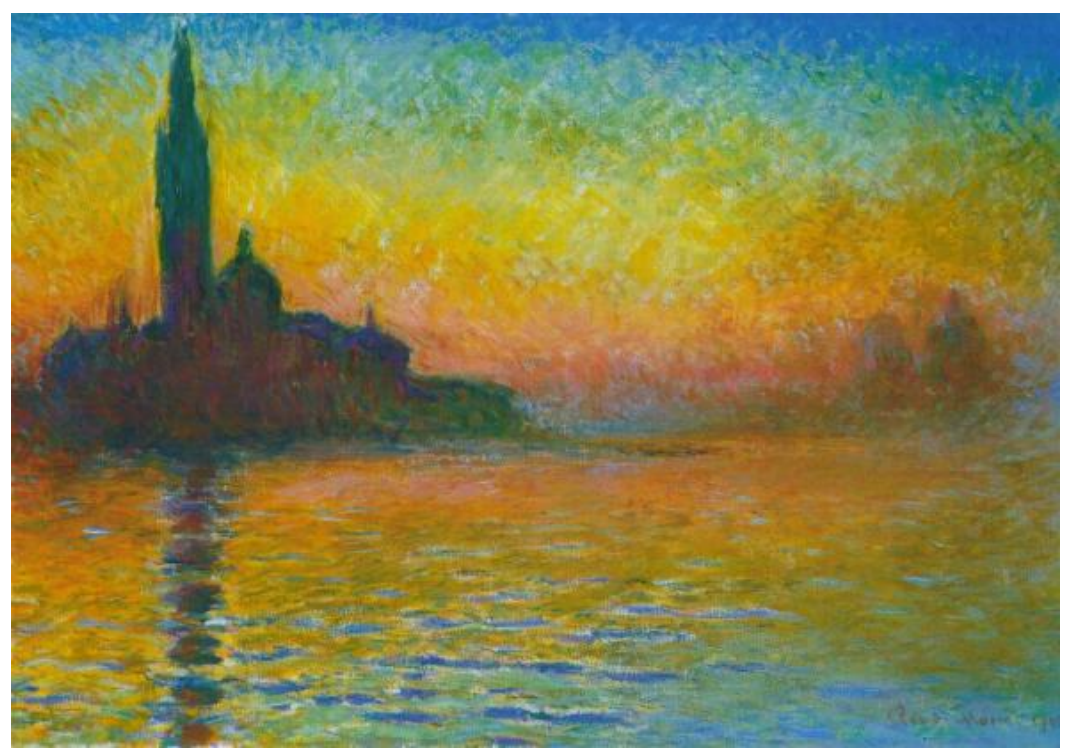

“San Giorgio Maggiore at Dusk”, Claude Monet, 1908 



\section{CHAPTER 11}

\section{CONCLUSIONS AND FURTHER RESEARCH}

$<<$ But all endings are also beginnings. We just don't know it at the time. $>>$ Mitch Albom

This chapter presents and analyzes the main contributions of the thesis. It also presents future work that can be done to continue this research and to extend the results that have already been obtained.

\subsection{CONCLUSIONS}

The complexity of current software systems and the fact that their non-functional requirements have become very relevant to the user are challenges to be faced in all software development. Software Architectures and AOSD have emerged to overcome these needs. Software Architectures reduce the complexity of software development and improve its maintenance by increasing software reuse. AOSD improves the quality of software and accurately satisfies the needs of the user giving equal importance to functional and non-functional requirements from the early stages of the software life-cycle. AOSD also reduces the complexity of software development and improves its maintenance by increasing the reusability of software.

In order to take advantage of Software architectures and AOSD, several approaches have emerged to combine both approaches providing all their advantages together. However, these approaches usually extend architectural models without connectors and mainly follow an asymmetric model. They are only focused on a single specific purpose: analysis, evolution or 
PRISMA: Aspect-Oriented Software Architectures

development of software architectures without attempting to provide complete development and maintenance support. Furthermore, these approaches always introduce the notion of aspect by using original architectural concepts, despite the fact that they do not provide the suitable semantics for aspects. This thesis presents a new software development approach that integrates Software Architectures and AOSD to fulfil these needs.

In this thesis, the PRISMA approach is presented as an important advance in the combination of the aspect-oriented paradigm and software architectures. The PRISMA approach integrates an aspect-oriented symmetric model with an architectural model that has the notion of connector. The PRISMA approach is also based on the Model-Driven Development (MDD) to provide complete support during the development and maintenance processes of software.

In PRISMA, aspects and software architectures are smoothly integrated with a clear semantics, which has been formalized using a Modal Logic of Actions and $\pi$-calculus. In addition, a PRISMA metamodel has been defined to define the PRISMA model and to establish its properties in a precise way. This metamodel has facilitated the automation and maintenance tasks of PRISMA software architectures since modelling tools are based on metamodels to support these tasks. In this case, the metamodel has been introduced in DSL Tools to develop the PRISMA framework.

Another important contribution of this thesis is the Aspect-Oriented Architecture Description Language (AOADL), which supports the PRISMA model. This AOADL allows the definition of PRISMA aspect-oriented software architectures, not only providing components and connectors as first-order citizens of the language, but also provides aspects and interfaces. The structure, design and maintainability of architectures specified in the PRISMA AOADL are improved by defining and reusing entities at different levels of granularity (interfaces, aspects, components, connectors and systems). This improvement is possible since (1) AOADL provides interfaces and aspects, (2) weavings are defined outside aspects, (3) aspects are defined independently of architectural elements, and (4) architectural elements are defined without being aware of the architectural elements that are connected to them. As a result, an interface can be used by several aspects, an aspect can be used by several architectural 318 
elements, and an architectural element can be used by several software architectures. In addition, the precise semantics of the PRISMA AOADL and its independence of technology provide the opportunity to validate PRISMA software architecture properties and to compile models for different programming languages and platforms. The language division into two levels of abstraction (types and configuration) permits the stored types defined at the type definition level to be reused by the configuration level to define a specific software architecture as many times as necessary. Since the use of a formal language is a hindrance for many users, a graphical AOADL has been designed to describe formal software architectures using a friendly graphical notation. This graphical modelling language is supported by the modelling tool that PRISMA CASE offers.

PRISMA AOADL has been created to specify industrial projects where the software systems are complex, open, and active such as the TeachMover robot. It is important to keep in mind that most ADLs only allow us to specify the skeleton of architectures and the services that are interchanged among their different architectural elements. However, the PRISMA AOADL has great expressive power to specify more features and requirements related to the software system by means of aspects. Therefore, PRISMA AOADL is not only able to specify simple architectural systems for academic projects such as pipelines, filters, blackboards, etc, but it is also able to completely specify complex software systems.

PRISMA CASE is the framework that supports the complete PRISMA approach by integrating the PRISMA metamodel, AOADL (graphical and textual), model compiler, middleware and methodology. Therefore, PRISMA is presented as a well supported approach for developing software systems.

Another important contribution of this thesis is the PRISMA model compiler, which has been developed using the templates and code generators provided by DSL tools. It permits the compilation of PRISMA architectural models in different programming languages and technologies, thereby reducing the development time and preserving the traceability between architectural models and their application code. The PRISMA model compiler currently generates C\# code and PRISMA textual specifications from graphical PRISMA architectural models. Despite the fact that the .NET framework does not provide support for the Aspect- 
PRISMA: Aspect-Oriented Software Architectures

Oriented approach, PRISMA is able to execute the generated aspect-oriented C\# code on the .NET platform. This is possible thanks to the PRISMANET middleware that has been developed. PRISMANET extends the .NET technology by the execution of aspects on the .NET platform, the concurrent execution of aspects and architectural elements, the loading of components, the creation of execution threads, the management of the local components, etc. Therefore, PRISMANET is an important contribution for the .NET community since it provides mechanisms to support AOP mechanisms.

Just as important is the methodology that has been defined to guide the user during the development process of PRISMA software architectures. This methodology is supported by the PRISMA CASE and consists of three stages that define how to define software architectures from scratch or how to reuse software by importing PRISMA architectural elements and aspects and COTS.

All the contributions of this thesis have been demonstrated using the the TeachMover robot case study. The reuse capabilities of the PRISMA model have been presented by means of the TeachMover case study. The TeachMover architecture has also helped to present the capabilities of the PRISMA modelling tool. The case study has been totally specified and its code has been generated and executed by PRISMACASE.

Software architectures are very important in robotic tele-operated systems [Cos00]. Robotic tele-operated systems must be developed using flexible and extensible architectural frameworks instead of being developed just using programming languages such as ADA95 or programming languages for automatons (for example STEP 7 of Siemens). There have been numerous efforts to provide developers with frameworks such as OROCOS [Bru02], MCA [Sch01] and CLARAty [Vol01]. All of them make very valuable contributions that simplify the development of systems. However, the way that the component-oriented approach has been applied may reduce some of its benefits. These frameworks are object-oriented or componentoriented frameworks that rely on object-oriented technologies and that highly depend on a given infrastructure (Linux O.S. and the $\mathrm{C}++$ language). As a result, a technologyindependence is not provided. Another framework for developing robotic tele-operated systems is ACROSET, which is technology-independent but it does not provides a real 320 
component oriented language for developing their software architectures, they use interface definition languages (such as CORBA-IDL), which are not enough to completely define components. In this thesis, a software engineering approach has been applied to the development of a robotic system. This development takes advantage of the good properties provided by this software engineering approach, especially the reuse of components, maintainability, traceability, technology-independence, code generation techniques, etc.

All these contribution have been published in thirteen international conferences, three international workshops, four national conferences, nine national workshops, and five technical reports. These contributions are the following:

\section{INTERNATIONAL CONFERENCES}

Jennifer Pérez, Nour Ali, Jose Ángel Carsí, Isidro Ramos, Designing Software Architectures with an Aspect-Oriented Architecture Description Language, 9th Symposium on the Component Based Software Engineering (CBSE), Springer Verlang LNCS 4063 ,pp. 123-138, ISSN: 0302-9743, ISBN: 3-540-35628-2, Vasteras, Suecia, June 29th-July 1st, 2006.

> Nour Ali, Jennifer Pérez, Cristóbal Costa, Isidro Ramos, José Ángel Carsí, Mobile Ambients in Aspect-Oriented Software Architectures, IFIP Working Conference on Software Engineering Techniques: Design for Quality- SET 2006, Springer, Volume 227 pp. 37-48, ISSN: 1571-5736, ISBN: 0-387-39387-0, Warsaw, Poland, October, 17-20, 2006.

> Jennifer Pérez, Elena Navarro, Patricio Letelier, Isidro Ramos, A Modelling Proposal for Aspect-Oriented Software Architectures, 13th Annual IEEE International Conference and Workshop on the Engineering of Computer Based Systems (ECBS), IEEE Computer Society , pp.32-41, ISBN: 0-7695-2546-6, Potsdam, Germany (Berlin metropolitan area), March 27th-30th, 2006. 
PRISMA: Aspect-Oriented Software Architectures

> Jennifer Pérez, Elena Navarro, Patricio Letelier, Isidro Ramos, Graphical Modelling for Aspect Oriented SA, 21st Annual ACM Symposium on Applied Computing, ACM, pp. 1597-1598, ISBN: 1-59593-108-2, Dijon, France, April 23 -27, 2006. (short paper)

> Jennifer Pérez, Manuel Llavador, Jose A. Carsí, Jose H. Canós, Isidro Ramos, Coordination in Software Architectures: an Aspect-Oriented Approach, Fifth Working IEEE/IFIP Conference on Software Architecture (WICSA), IEEE Computer Society Press, pp. 219-220, ISBN: 0-7695-2548-2, Pittsburgh, Pennsylvania, USA, 6-9 November, 2005 (position paper)

> Nour Ali, Jennifer Pérez, Isidro Ramos, Jose A. Carsí, Integrating Ambient Calculus in Mobile Aspect-Oriented Software Architectures, Fifth Working IEEE/IFIP Conference on Software Architecture (WICSA), IEEE Computer Society Press, pp. 233-234, ISBN: 07695-2548-2, Pittsburgh, Pennsylvania, USA, 6-9 November, 2005 (position paper)

> Jennifer Pérez, Nour Ali, Cristobal Costa, José Á. Carsí, Isidro Ramos, Executing AspectOriented Component-Based Software Architectures on .NET Technology, 3rd International Conference on .NET Technologies, pp. 97-108, Pilsen, Czech Republic, May-June 2005.

> Nour Ali, Jennifer Pérez, Isidro Ramos, Aspect High Level Specification of Distributed and Mobile Information Systems, Second International Symposium on Innovation in Information \& Communication Technology ISSICT, pp. 14, Amman, Jordania,21-22, April, 2004.

> Nour Ali, Jennifer Pérez Isidro Ramos, Jose A. Carsí , Aspect Reusability in Software Architectures, 8th International conference of Software Reuse (ICSR), July, 2004 (poster)

$>$ Elena Navarro, Isidro Ramos, Jennifer Pérez, Goals Model-Driving Software Architecture, 2nd International Conference on Software Engineering Research, Management and Applications (SERA), pp. 205-212, ISBN:0-9700776-9-6, May 5-8, 2004, Los Angeles, CA, USA. 
> Nour Hussein, Josep Silva, Javier Jaen, Isidro Ramos, Jose Ángel Carsí ,Jennifer Pérez, Mobility and Replicability Patterns in Aspect-Oriented Component- Based Software Architectures, 15th IASTED International Conference, Parallel and Distributed Computing and Systems (PDCS), ACTA Press, ISBN: 0-88986-392-X, ISSN: 10272658, pp. 820-826, Marina del Rey, California, USA, 3-5, November 2003,

> Jennifer Pérez, Isidro Ramos, Javier Jaén, Patricio Letelier, Elena Navarro, PRISMA: Towards Quality, Aspect Oriented and Dynamic Software Architectures, 3rd IEEE International Conference on Quality Software (QSIC 2003), IEEE Computer Society Press, pp.59-66, ISBN: 0-7695-2015-4, Dallas, Texas, USA, November 6 - 7, 2003.

$>$ Elena Navarro, Isidro Ramos, Jennifer Pérez, Software Requirements for Architectured Systems, 11t h IEEE International Requirements Engineering Conference (RE'03), IEEE Computer Society Press, pp. 365-366, ISSN: 1090-705X, ISBN: 0-7695-1980-6, Monterey, California, 8-12 September 2003 (Poster)

\section{INTERNATIONAL WORKSHOPS}

> Jennifer Pérez, Nour Ali, Jose A. Carsí, Isidro Ramos, Dynamic Evolution in AspectOriented Architectural Models, Second European Workshop on Software Architecture, Springer LNCS 3527, pp.59-16, ISSN: 0302-9743, ISBN: 3-540-26275-X , Pisa, Italy, June 2005.

> Jennifer Pérez, Isidro Ramos, Javier Jaén, Patricio Letelier, PRISMA: Development of Software Architectures with an Aspect Oriented, Reflexive and Dynamic Approach, Dagstuhl Seminar N 03081 , Report N 36 "Objects, Agents and Features",Copyright (c) IBFI gem. GmbH, Schloss Dagstuhl, D-66687 Wadern, Germany . Eds.H.-D. Ehrich (Univ. Braunschweig, D), J.-J. Meyer (Utrecht, NL), M. Ryan (Univ. of Birmingham, GB), pp. 16, Germany, January, 2003. 
PRISMA: Aspect-Oriented Software Architectures

Jorge Ferrer, Ángeles Lorenzo, Isidro Ramos, José Ángel Carsí, Jennifer Pérez, Modeling Dynamic Aspects in Architectures and Multiagent Systems, Logic Programming and Software Engineering (CLPSE), pp. 1-13, Copenhagen, Denmark, affiliated with ICLP, july 2002.

\section{NATIONAL CONFERENCES}

> Nour Ali, Jennifer Pérez, Cristóbal Costa, Isidro Ramos, José Ángel Carsí, Distributed Replication in Aspect-Oriented Software Architectures using Ambients, XI Conference on Software Engineering and Databases (JISBD), pp. 379-388, ISBN: 84-95999-99-4, Sitges, Barcelona, October 2006. (In Spanish)

> Cristóbal Costa, Jennifer Pérez, Nour Ali, Jose Angel Carsi, Isidro Ramos, PRISMANET middleware: Support to the Dynamic Evolution of Aspect-Oriented Software Architectures, X Conference on Software Engineering and Databases (JISBD), pp. 27-34, ISBN: 84-9732-434-X, Granada, September, 2005. (In Spanish)

> Jennifer Pérez, Nour H. Ali, Isidro Ramos, Juan A. Pastor, Pedro Sánchez, Bárbara Álvarez, Development of a Tele-Operation System using the PRISMA Approach, VIII Conference on Software Engineering and Databases (JISBD), pp. 411-420, ISBN: 84688-3836-5, Alicante, November, 2003. (In Spanish)

> Jennifer Pérez, Isidro Ramos, Ángeles Lorenzo, Patricio Letelier, Javier Jaén, PRISMA: OASIS PlatfoRm for Architectural Models, VII Conference on Software Engineering and Databases (JISBD), pp. 349-360, ISBN: 84-688-0206-9, El Escorial (Madrid), November, 2002. (In Spanish) 


\section{NATIONAL WORKSHOPS}

> Cristóbal Costa, Jennifer Pérez, Jose Angel Carsí, Towards the Dynamic Configuration of Aspect-Oriented Software Architectures, IV Workshop on Aspect-Oriented Software Development (DSOA), XI Conference on Software Engineering and Databases (JISBD), Technical Report TR-24/06 of the Polytechic School of the University of Extremadura, pp.35-40, Sitges, Barcelona, Octubre, 2006. (In Spanish)

> Rafael Cabedo, Jennifer Pérez, Nour Ali, Isidro Ramos, Jose A. Carsí, Aspect-Oriented C\# Implementation of a Tele-Operated Robotic System, III Workshop on Aspect-Oriented Software Development (DSOA), X Conference on Software Engineering and Databases (JISBD), pp. 53-59, ISBN: 84-7723-670-4, Granada, September, 2005. (In Spanish)

> Jennifer Pérez,Nour H. Ali, Isidro Ramos, Jose A. Carsí, PRISMA: Aspect-Oriented and Component-Based Software Architectures, Workshop on Aspect-Oriented Software Development (DSOA), Conference on Software Engineering and Databases (JISBD), Technical Report TR-20/2003 of the Polytechic School of the University of Extremadura, pp. 27-36, Alicante, November, 2003. (In Spanish)

$>\mathrm{M}^{\mathrm{a}}$ Eugenia, Nour Ali, Jennifer Pérez, Isidro Ramos, Jose A. Carsí,DIAGMED: An Architectural model for a Medical Diagnosis, IV workshop DYNAMICA - DYNamic and Aspect-Oriented Modeling for Integrated Component-based Architectures, pp. 1-7, Archena, Murcia, November, 2005. (In Spanish)

> Rafael Cabedo, Jennifer Pérez, Jose A. Carsí, Isidro Ramos, Generation and Modelling of PRISMA Architecture using DSL Tools, IV Workshop DYNAMICA - DYNamic and Aspect-Oriented Modeling for Integrated Component-based Architectures, pp.79-86, Archena, Murcia, November, 2005. (In Spanish)

$>$ Nour Ali, Jennifer Pérez, Jose A. Carsí, Isidro Ramos, Mobility of Objects in the PRISMA Approach, II workshop DYNAMICA - DYNamic and Aspect-Oriented Modeling for Integrated Component-based Architectures, pp. 111-118, Almagro, Ciudad Real, April, 2005. 
PRISMA: Aspect-Oriented Software Architectures

> Jennifer Pérez, Rafael Cabedo, Pedro Sánchez, Jose A. Carsí, Juan A. Pastor, Isidro Ramos, Bárbara Álvarez, PRISMA Architecture of the Case Study: an Arm Robot, II workshop DYNAMICA - DYNamic and Aspect-Oriented Modeling for Integrated Component-based Architectures, Conference on Software Engineering and Databases (JISBD), pp. 119-127, Málaga, November 2004. (In Spanish)

> Nour Ali Jennifer Pérez, Cristobal Costa, Jose A. Carsí, Isidro Ramos, Implementation of the PRISMA Model in the .Net Platform,II workshop DYNAMICA - DYNamic and Aspect-Oriented Modeling for Integrated Component-based Architectures, Conference on Software Engineering and Databases (JISBD), pp. 119-127, Málaga, November, 2004.

> Nour H. Ali, Josep F. Silva, Javier Jaen, Isidro Ramos, Jose Á. Carsí, Jennifer Pérez, Distribution Patterns in Aspect-Oriented Component-Based Software Architectures, IV Workshop Distributed Objects, Languages, Methods and Environments (DOLMEN), pp.74-80, Alicante, November, 2003.

\section{TECHNICAL REPORTS}

> Rafael Cabedo, Jennifer Pérez, Isidro Ramos, The application of the PRISMA Architecture Description Language to an Industrial Robotic System, Technical Report, DSIC-II/11/05, pp.180, Polytechnic University of Valencia, September 2005. (In Spanish)

> Cristobal Costa, Jennifer Pérez, Jose Ángel Carsí, Study and Implementation of an Aspect-Oriented Component-Based Model in .NET technology, Technical Report, DSICII/12/05, pp. 198, Polytechnic University of Valencia, September, 2005. (In Spanish)

> Jennifer Pérez, Nour Ali, Jose A. Carsí, Isidro Ramos, PRISMA Architecture of the Robot 4U4 Case Study, Technical Report DSIC-II/13/04, pp. 72, Polytechnic University of Valencia, 2004. (In Spanish)

> Jennifer Pérez, Isidro Ramos, OASIS as a Formal Support for the Dynamic, Distributed and Evolutive Hypermedia Models, Technical Report DSIC-II/22/03, pp. 144, Polytechnic University of Valencia, October 2003. (In Spanish) 
Jennifer Pérez, Isidro Ramos, Jose A. Carsí, A Compiler to Automatically Generate the Metalevel of Specifications using Properties of the Base Level, Technical Report, DSICII/23/03, pp. 107, Polytechnic University of Valencia, October, 2003.(In Spanish)

\subsection{FURTHER RESEARCH}

PRISMA is a new approach that opens a perfect setting for further research. All the parts that the PRISMA approach is composed of can be extended in order to face new challenges.

PRISMA has been applied to both: the tele-operation domain and the electronic bank domain. However, other domains can have other specific properties that are not taken into account in PRISM. As a result, the application of the PRISMA model to other domains can assist us in defining new aspects that can introduce new properties of modelling and differences in aspect specifications. In fact, PRISMA only supports the definition of software architectures that are locally executed, despite the fact that most software architectures have a distributed nature. For this reason, we are currently working on introducing distribution and mobility properties in PRISMA using aspects. In addition, until now, PRISMA has been applied to software architectures that do not require persistence. However, information systems usually store their information in secondary memory. As a result, persistence is another important property that the model should support. There are other concepts from software architectures that PRISMA does not provide such as views and architectural patterns. In the long term, these concepts should also be defined in PRISMA.

The PRISMA model extensions imply modifications in the PRISMA AOADL at its different levels of abstraction (types and configuration) and at its different kinds of representation (textual and graphical). PRISMA AOADL supports cardinality constraints to define systems, but it should be extended to support other kinds of constraints as well.

Since PRISMA does not yet provide model checking mechanisms to check the properties of its architectural specifications such as reachability, deadlock detection and liveness, these model checking techniques should also be applied to PRISMA. 
PRISMA: Aspect-Oriented Software Architectures

Another important property of software systems is the continuous evolution that they undergo. Development frameworks must provide mechanisms to support evolution and to facilitate the software maintenance. As a result, PRISMA must be able to support the evolution of aspect-oriented software architectures. The division of the PRISMA architecture specifications into two levels of abstraction opens the opportunity to distinguish between the evolution of types and the evolution of a specific architecture. Despite the fact that the PRISMA evolution services have been identified and included in the PRISMA metamodel to modify software architectures, this only permits its modification at modelling time. However, since there are a lot of software systems that cannot stop their execution to be modified, runtime evolution must be provided. This run-time evolution is usually called dynamic configuration. Therefore, we are currently working on defining mechanisms to dynamically execute evolution services at run-time. Over the long term, our work with regard to software evolution will be related to the data evolution problem of software architectures, where we will apply our previous experience on data migration and data evolution of object-oriented conceptual schemas [Per02a], [Per02b], [Per02c].

For the application of PRISMANET, there are a lot of lacks that must be dealt with in the near future. The most important ones are the support of transactions and fault tolerance. In the long term, PRISMANET must also provide distributed and evolution mechanisms to the architectural elements. Automatic code generation of other programming languages and technologies is future work that could imply the implementation of other middlewares if required by the new technological platforms. Furthermore, an abstract middleware that would hide the differences between the different platforms could also be developed.

The PRISMA UML profile that is presented in this thesis allows us to export PRISMA specifications to other modelling tools and model management platforms in XMI format. Another further task is to automatically generate PRISMA XMI documents from PRISMA architectural specifications to be exported to other tools whose use could be advantageous.

The PRISMA methodology does not support the identification of architectural elements and aspects from the requirements specification. As a result, one future work is to integrate 
ATRIUM [Nav03]with PRISMA to provide complete support to every stage of the software life-cycle (from requirements to implementation).

In addition, the extension of PRISMA methodology also offers opportunities for future work. This extension can consist of providing mechanisms that will take into account product family modelling as well as the variability that software architectures of this kind would introduce at the PRISMA modelling stage. Yet another task is to create a repository with a query language and metadata description of the architectural elements and aspects to improve reusability even more. The incorporation of COTS introduces the possibility of importing web services in PRISMA, making the study of PRISMA as a Service-Oriented Architecture (SOA) necessary. Therefore, another interesting task is to analyze what implications the SOA support will have for the PRISMA MODEL and the PRISMANET implementation.

Finally, it is necessary to evaluate PRISMA using different applications and case studies in order to perform a quantified evaluation of the approach. A comparison of aspect-oriented and non-aspect oriented applications is necessary to be able to measure the advantages that PRISMA provides in comparison with other approaches. This measurement should be made taking into account different case studies and domains in order to have a wide sample that will allow us to get a set of well based conclusions. 
PRISMA: Aspect-Oriented Software Architectures 


\section{BIBLIOGRAPHY}

[Ald03] Aldawud O., Elrad T., Bader A., UML profile for Aspect Oriented Software Development. The Aspect-Oriented UML Workshop, International Conference on Aspect-Oriented Software Development, Boston, USA March 18, 2003.

[Ald01] Aldawud O., Elrad T., Bader A., A UML Profile for Aspect Oriented Modeling. Workshop on Advanced Separation of Concerns in Object- Oriented Systems, OOPSLA 2001, Tampa Bay, Florida, USA, 2001.

[Ali06] Ali N., Pérez J., Costa C., Ramos I., Carsí J.A., Mobile Ambients in Aspect-Oriented Software Architectures. IFIP Working Conference on Software Engineering Techniques, Warsaw, Poland, October, 2006.

[Ali05b] Ali N., Pérez J., Ramos I., Carsí J. A., Integrating Ambient Calculus in Mobile AspectOriented Software Architectures. Fifth Working IEEE/IFIP Conference on Software Architecture (WICSA), IEEE Computer Society Press, pp. 233-234, Pittsburgh, Pennsylvania, USA, 6-9 November, 2005 (position paper)

[Ali05a] Ali N., Ramos I., Carsí J.A., A Conceptual Model for Distributed Aspect-Oriented Software Architectures. International Symposium on Information Technology: Coding and Computing (ITCC), IEEE Computer Society, Vol. 2, Las Vegas, Nevada, USA, April, 2005.

[Ali03] Ali N., Silva J.F., Jaen J., Ramos I., Carsí J. A., Pérez J., Mobility and Replicability Patterns in Aspect-Oriented Component- Based Software Architectures. 15th IASTED International Conference, Parallel and Distributed Computing and Systems (PDCS),Marina del Rey, California, USA, 3-5, November 2003, ACTA Press, ISBN: 0-88986-392-X, ISSN: 1027-2658, pp. 820-826.

[Al198] Allen P., Frost S., Component-Based Development for Enterprise Systems. Applying the SELECT Perspective. Cambridge University Press, 1998. 
PRISMA: Aspect-Oriented Software Architectures

[All97a] Allen R., Garlan D., A Formal Basis for Architectural Connection. ACM Transactions on Software Engineering and Methodology, Vol. 6, No. 3, pp. 213-249, July 1997.

[All97b] Allen R., A Formal Approach to Software Architecture. Ph.D. Thesis, Carnegie Mellon University, CMU Technical Report CMUCS-97-144, Pittsburgh, Pennsylvania, USA, May, 1997.

[All94] Allen R., Garlan D., Formalizing Architectural Connection. The 16th International Conference on Software Engineering (ICSE), Sorrento, Italy, May, 1994.

[Ana03] V. Anaya, P. Letelier, Towards a method for developing UML Profile: a Case Study. VIII Jornadas de Ingeniería del Software y Bases de Datos, Alicante 2003 (In Spanish)

[And03] Andrade, L.F., Fiadeiro J. L., Architecture Based Evolution of Software Systems. Formal Methods for Software Architectures, Lecture Notes in Computer Science, Springer Verlang, Eds. Marco Bernardo and Paola Inverardi, LNCS 2804, September 2003.

[AO406] AO4BPEL Website, http://www.st.informatik.tu-darmstadt.de/static/pages/projects/AO4BPEL/index.html

[Ara03] Araujo J., Moreira A., An Aspectual Use Case Driven Approach. VIII conference on Software Engineering and Databases, Alicante, Spain, 2003.

[Ara02] Aráujo J., Moreira A., Brito I., Rashid A., Aspect-Oriented Requirements with UML. Workshop on Aspect-Oriented Modelling with UML, International Conference on Unified Modelling Language UML, 2002.

[ARG06] ArgoUML, http://argouml.tigris.org/

[ASP06a] The AspectJ Project Website, http://eclipse.org/aspectj/

[ASP06b] The AspectDNG Project Website, http://aspectdng.sourceforge.net/ 
[Bac00] Bachman F., Bass et al., The Architecture Based Design Method. Technical Report CMU/SEI-200-TR-001, Carnegie Mellon University, USA, January 2000.

[Bal85] Balzer, R., A 15 Year Perspective on Automatic Programming. IEEE. Transactions on Software Engineering, Vol.11, No.11, pp. 1257-1268, November 1985.

[Ban04] Baniassad E., Clarke S., Finding Aspects in Requirements with Theme/Doc. Workshop on Early Aspects, International Conference on Aspect-Oriented Software Development ( AOSD), Lancaster, UK, 2004.

[Bar04a] Barais O., Duchien L., Safarchie studio: Argouml extensions to build safe architectures. Workshop on Architecture Description Languages, IFIP WCC WorldComputer Congress, Toulouse, France, 2004.

[Bar04b] Barais, O., Cariou, E., Duchien, L., Pessemier, N., Seinturier L., Transat: A framework for the specification of software architecture evolution. The First International Workshop on Coordination and Adaptation Techniques for Software Entities (WCAT04), ECOOP, Oslo, Norway, June, 2004. http://wcat04.unex.es/bib

[Bar03] Barais O., Duchien L., Pawlak R., Separation of Concerns in Software Modeling: A Framework for Software Architecture Transformation. IASTED International Conference on Software Engineering Applications (SEA) ACTA Press, ISBN 088986-394-6, pp. 663-668, Los Angeles, California, USA, November 2003.

[Bar01] Barbacci M.R., Doubleday D., Weinstock C. B., Lichota R.W., DURRA: An Integrated Approach to Software Specification, Modeling and Rapid Prototyping. Technical Report CMU/SEI-91-TR-21, Software Engineering Institute (SEI), September, 1991.

[Bar95] Barrio M., Study of Dynamic Aspects in Object-Oriented Systems. PhD. Thesis, University of Valladolid, 1995. (In Spanish)

[Bass03] Bass L., Clements P., Kazman R, Software Architecture in Practice. Addison Wesley, $2^{\text {nd }}$ Edition, 2003. 
PRISMA: Aspect-Oriented Software Architectures

[Ber04] Bergmans L., Aksit M., Principles of Design Rationale of Composition Filters. In Aspect-Oriented Software Development, Addison-Wesley, Eds. Aksit M., Clarke S., Elrad T., and Filman R., 2004.

[Ber01] Bergmans L., Aksit M., Composing Multiple Concerns Using Composition Filters. Communications of the ACM, Vol. 44, No.10, pp. 51-57, October 2001.

[Ber99] Berkem B., Traceability management from business processes to use cases with UML. A proposal for extensions to the UML's activity diagram through goal-oriented objects. Journal of Object-Oriented Programming, pp. 29-34, 1.999.

[Ber94] Bergmans L., The Composition-Filters Object Model. PhD Thesis, Department of Computer Science, University of Twente, 1994.

[Bin96] Binns P., Engelhart M., Jackson M., Vestal S., Domain-Specific Software Architectures for Guidance, Navigation, and Control. International Journal of Software Engineering and Knowledge Engineering, Vol. 6, No. 2, 1996.

[Bra99] Bratthall L., Runeson P., A Taxonomy of Orthogonal Properties of Software Architecture. The Second Nordic Software Architecture Workshop (NOSA), Ronneby, Sweden, 12-13, August, 1999.

[Boo99] Booch, Rumbaugh and Jacobson, The UML Modeling Language User Guide. Addison-Wesley, 1999

[Bri05] Brichau J., Haupt M., Report synthesizing state-of-the-art in aspect-oriented languages and execution models. Lancaster University, Lancaster, AOSD-Europe Deliverable D12, pp. 1-259, AOSD-Europe-ULANC-9, 17 May 2005,

http://www.aosdeurope.net/

[Bri02] Brito I., Moreira A., Towards a Composition Process for Aspect-Oriented Requirements. Workshop on Early-Aspects, The 1st International Conference on Aspect-Oriented Software Development (AOSD), Twente, The Netherlands, April 2002. 
[Bru04] Bruns G., Jagadeesan R., Jeffrey A., Riely J., $\mu a b c$ : A Minimal Aspect Calculus. 15th International Conference on Concurrency Theory (CONCUR), Lecture Notes in Computer Science (LNCS), Springer-Verlag, Vol. 3170, London, UK, August 31 September 3, 2004.

[Bru02] Bruyninckx H., Konincks B., Soetens P., A Software Framework for Advanced Motion Control. Department of Mechanical Engineering, K.U. Leuven. OROCOS project inside EURON, Belgium, 2002.

[Cab05] Cabedo R., Pérez J., Ramos I.. The application of the PRISMA Architecture Description Language to an Industrial Robotic System. Techical Report, DSICII/11/05, Technical University of Valencia, September, 2005. (In Spanish)

[Can01] Canal C, Pimentel E., Troya J.M., Compatibility and Inheritance in Software Architectures. Science of Computer Programming, vol. 41, no. 2. 2001.

[Can00] Canal C., A Language for the Specification and Validation of Software Architectures. PhD. Thesis, The University of Malaga, 2000. (In Spanish)

[Can99] Canal C, Pimentel E., Troya J.M., Specification and Refinement of Dynamic Software Architectures. The First Working IFIP Conference on Software Architecture (WICSA), Kluwer Academic Publishing, pp. 107-126, San Antonio, Texas, USA, February, 1999.

[Car01] Caro P.S., Adding Systemic Crosscutting and Super-Imposition to Composition Filters. M.Sc. Thesis, Computer Science, Vrije Universiteit Brussel, Brussels, 2001.

[Car00] Carney, D., Long, F., What Do You Mean by COTS?. IEEE Software, March/April 2000, pp. 83-86. 
PRISMA: Aspect-Oriented Software Architectures

[Chi05] Chitchyan R., Rashid A., Sawyer P., Garcia A., Pinto M., Bakker J., Tekinerdogan B., Clarke S., Jackson A., Report synthesizing state-of-the-art in aspect-oriented requirements engineering, architectures and design. Lancaster University, Lancaster, AOSD-Europe Deliverable D11, , pp. 1-259, AOSD-Europe-ULANC-9, 18 May 2005, http://www.aosdeurope.net/

[Cle01] Cleaveland R., Lüettgen G., Natarajan V., Priority in Process Algebra, HandBook of Process Algebra, Part 4: extensions, Elsevier, 2001.

[Cle01] Clements, P., Northrop L., Software Product Lines: Practices and Patterns. Addison Wesley Longman, 2001.

[Cle00] Clements P., Active Reviews for Intermediate Designs (ARID). Technical Note CMU/SEI-2000-TN-009, August 2000.

[Coe04] Coelho W., Murphy G. C., Modeling Aspects: An Implementation-Driven Approach. Workshop on Best Practices for Model-Driven Software Development, OOPSLA, Vancouver, Canada, 2004.

[Col00] Colcombet T., Fradet P., Enforcing Trace Properties by Program Transformation. The $27^{\text {th }}$ ACM SIGPLAN-SIGACT symposium on Principles of Programming Languages (POPL), Communications of the ACM, pp. 54-66., 2000.

[Cos00] Coste-Manière E., Simmons R.., Architecture, the Backbone of Robotic System. IEEE International Conference on Robotics \& Automation, pps. 505-513, San Francisco, USA, April, 2000.

[Cue02] C.E. Cuesta Quintero, Dynamic Software Architecture based on Reflection. PhD. Thesis, Department of Computer Science, University of Valladolid, 2002. (In Spanish)

[Dan04] Dantas D., Walker D., Washburn G., Weirich S., Analyzing Polymorphic Advice. Technical Report TR-717-04, Princeton University, December 2004. 
[Dee05] Deelstra, S., Sinnema M., Bosch J., Product derivation in software product families: a case study. Journal of Systems and Software, Vol. 74, No. 2, pp. 173-194, 2005.

[Dij76] Dijkstra, E., A Discipline of Programming. Prentice-Hall, 1976.

[Dob02] Dobrica L., Niemela E., A survey on software architecture analysis methods. IEEE Transactions on Software Engineering, Vol. 28, No. 7, pp. 638 - 653 , july, 2002.

[Dou05] Douence R, Le Botlan D., Report Towards a Taxonomy of AOP Semantics. AOSDEurope Deliverable D11, AOSD-Europe-INRIA-1, INRIA, CNRS, 7 July 2005, pp. 1-13 http://www.comp.lancs.ac.uk:8080/c/portal/layout?p_ 1 id=1.94

[Dou04b] Douence R., Fradet P., Sudholt M., Trace-Based Aspects. Aspect-Oriented Software Development. Mehmet Aksit, Sioh' an Clarke, Tzilla Elrad, and Robert E. Filman, editors. Addison-Wesley, 2004.

[Dou04a] Douence R., Teboul L., A Crosscut Language for Control-Flow. The 3rd ACM SIGPLAN/SIGSOFT Conference on Generative Programming and Component Engineering (GPCE), Lecture Notes in Computer Science (LNCS), Springer-Verlag, Vol. 3286, Vancouver, Canada, October 2004.

[Dou02b] Douence R., Sudholt M., A Model and a Tool for Event-Based Aspect-Oriented Programming (EAOP). Technical Report 02/11/INFO, Ecole des mines de Nantes, 2nd edition, December 2002.

[Dou2a] Douence R., Fradet P., Sudholt M., A Framework for the Detection and Resolution of Aspect Interactions. Generative Programming and Component Engineering: ACM SIGPLAN/SIGSOFT Conference on Generative Programming and Component Engineering GPCE 2002, Lecture Notes in Computer Science (LNCS), SpringerVerlag. In D. Batory, C. Consel, and W. Taha, editors, Vol. 2487, pp. 173-188, Pittsburgh, PA, USA, October 2002. 
PRISMA: Aspect-Oriented Software Architectures

[Dou01] Douence R, Motelet O., Sudholt M., A Formal Definition of Crosscuts. Proceedings of the $3^{\text {rd }}$ International Conference on Reflection, Lecture Notes in Computer Science, Springer-Verlag, A. Yonezawa and S. Matsuoka eds., Vol. 2192, pp. 170-186, Kyoto, Japan, September 2001.

[DSL06] Domain-Specific Language (DSL) Tools.

http://ab.msdn.microsoft.com/teamsystem/workshop/dsltools/default.aspx

[DSo99] D’Souza D., Wills A., Objects, Components and Frameworks with UML. The Catalysis approach. Addison-Wesley 1.999.

[EAR06] Early Aspects: Aspect-Oriented Requirements Engineering and Architecture Design web site, http://www.early-aspects.net/

[ECL06] Eclipse Org, http://www.eclipse.org/

[EFT02] EFTCoR Project: Friendly and Cost-Effective Technology for Coating Removal. V Programa Marco, Subprograma Growth, G3RD-CT-2002-00794, 2002.

[Elr01] Elrald T., Filman R. E., Bader A., Aspect-Oriented Programming: An Introduction. Communication of the ACM, Vol. 44, No. 10, October 2001.

[Fer05] Fernández C., Pastor J.A., Sánchez P., Álvarez B., Iborra A., Co-operative Robots for Hull Blasting in European Shiprepair Industry. IEEE Robotics and Automation Magazine (RAM), September 2005.

[Fia04] Fiadeiro J.L., Lopes A., CommUnity on the Move: Architectures for Distribution and Mobility. FMCO 2003, Lecture Notes in Computer Science (LNCS), Springer-Verlag, Vol. 3188, pp. 177-196, F.S. de Boer et al. (Eds.), Heidelberg , Berlin, Germany, 2004.

[Fil00] Filman R., Friedman D., Aspect-Oriented Programming is Quantification and Obliviousness. Workshop on Advanced Separation of Concerns, OOPSLA, October, 2000. 
[Fow96] Fowler M., Analysis Patterns: Reusable Object Model. Addison Wesley, Reading, MA, 1996.

[Fra04] France R., Ray I., Georg G., Ghosh S., Aspect-Oriented Approach to Early Design Modeling. IEE Software, Vol. 151, pp. 173-186, 2004.

[Fue05] Fuentes L., Pinto M., Sánchez P., Dynamic Weaving in CAM/DAOP: An Application Architecture Drive Approach. Workshop on Dynamic Aspect, Aspect-Oriented Software Development, Chicago, Illinois, March, 14-18.

[Gam95] Gamma et al., Design Patterns: Elements of Reusable Object-Oriented Software. Addison Wesley, Reading, MA, 1995.

[Gar03] Garlan, D., Formal Modelling and Analysis of Software Architecture: Components, Connectors, and Events. Formal Methods for Software Architectures, Lecture Notes in Computer Science, Springer Verlang, Eds. Marco Bernardo and Paola Inverardi, LNCS 2804, September 2003

[Gar01] Garlan, D., Software Architecture, Wiley Encyclopedia of Software Engineering, J. Marciniak (Ed.), John Wiley \& Sons, 2001

[Gar00] Garlan D., Monroe R. T., Wile D., Acme: Architectural Description of ComponentBased Systems. Foundations of Component-Based Systems, Gary T. Leavens and Murali Sitaraman (eds), Cambridge University Press, pp. 47-68, 2000.

[Gar98] Garlan D., Higher-Order Connectors, Workshop on Compositional Software Architectures, Monterey, CA, January 6-7, 1998.

[Gar95b] Garlan, D., An Introduction to the Aesop System. July 1995. http://www.cs.cmu.edu/afs/cs/project/able/www/aesop/html/aesopoverview.ps

[Gar95a] Garlan, D., Perry D., Introduction to the Special Issue on Software Architecture. IEEE Transactions on Software Engineering, vol. 21 no. 4, April 1995. 
PRISMA: Aspect-Oriented Software Architectures

[Gar94] Garlan D., Allen R., Ockerbloom J., Exploiting Style in Architectural Design Environments. SIGSOFT'94: Foundations of Software Engineering, pp. 175-188, New Orleans, Louisiana, USA, December 1994.

[Gar93] Garlan, D., Shaw M., An Introduction to Software Architecture. Advances in Software Engineering and Knowledge Engineering, Vol. I. Eds. V. Ambriola and G. Tortora, World Scientific Publishing Company, New Jersey, 1993.

[Gor94] Gorlick M., Quilici A., Visual Programming in the Large versus Visual Programming in the Small. The 1994 IEEE Symposium on Visual Languages, pp. 137-144, St. Louis, Missouri, USA, October, 1994.

[Gor91] Gorlick M., Razouk R., Using Weaves for Software Construction and Analysis. The $13^{\text {th }}$ International Conference on Software Engineering (ICSE13), pp. 23-34, Austin, Texas, USA, May, 1991.

[Gre04] Greenfield J., Short K, Cook S., and Kent S. Software Factories. Wiley Publising Inc., 2004.

[Gru00] Grundy J., Multi-perspective specification, design and implementation of software components using aspects. International Journal of Software Engineering and Knowledge Engineering, vol. 20, 2000.

[Grun99] Grundy J., Aspect-Oriented Requirements Engineering for Component-based Software Systems. The 4th IEEE International Symposium on Requirements Engineering, Limerick, Ireland, 1999.

[Grun98] Grundy J.C., Mugridge W.B., Hosking J.G., Static and dynamic visualisation of component-based software architectures. The 10th International Conference on Software Engineering and Knowledge Engineering, KSI Press, San Francisco, California, USA, 18-20 June, 1998. 
[Ham05] Hammouda I., Hakala M., Pussinen M., Katara M., Mikkonen T., Concerni-Based Development of Pattern Systems. The $2^{\text {nd }}$ European Workshop on Software Architecture (EWSA), Lecture Notes on Computer Science, Springer Verlang, LNCS 3527, pp. 113-129, Pisa, Italy, June, 2005.

[Ham04] Hammouda I., koskinen J., Pussinen M., Katara M., Mikkonen T., Adaptable Concerni-Based Framework Specialization in UML,Automated Software Engineering, pp. 78-87, Linz, Austria, 2004.

[Har02] Harrison W., Harold L., Ossher H., Peri T., Asymmetrically vs. Symmetrically Organized Paradigms for Software Composition. IBM Research Report RC22685 (W0212-147), Thomas J. Watson Research Center, IBM, December, 2002.

[Har93] Harrison W., Ossher H., Subject-oriented programming (a critique of pure objects). Conference on Object-Oriented Programming: Systems, Languages and Applications, Communications of the ACM, pp. 411-428, September 1993.

[Hay95] Hay D., Data Model Patterns: Elements of Reusable Object-Oriented Software. Dorset House, NY, 1995.

[Her02] Herrmann S., Composable Designs with UFA. Workshop on Aspect-oriented Modelling, International Conference on Aspect-Oriented Software Development (AOSD), Enschede, The Netherlands, 2002.

[Hil04] Hilsdale E., Hugunin J., Advice Weaving in AspectJ. International Conference on Aspect-Oriented Software Development (AOSD), Lancaster, UK, 2004.

[Hin99] Hindriks K.V., Boer F. S., van der Hoek W., Meyer J.-J.Ch., Agent programming in 3APL. Autonomous Agents and Multi-Agent Systems, Vol. 2, No. 4, pp. 357-401, 1999.

[Hoa85] Hoare C. A. R., Communicating Sequential Processes. Electronic version of Communicating Sequential Processes, first published in 1985 by Prentice Hall International, 1985. 
PRISMA: Aspect-Oriented Software Architectures

[Hoo99] Hoover C. L., Khosla V, Analytical partition of software components for evolvable and reliable mems design tool. International Journal of Software Engineering and Knowledge Engineering (IJSEKE). Special Issue: High Assurance Systems, Vol. 9, No.2, pp.153-171, 1999.

[HYP06] Hyperspace web site, http://www.research.ibm.com/hyperspace

[IEE00] IEEE Recommended Practices for Architectural Description of Software-Intensive Systems. IEEE Std 1471-2000, Software Engineering Standards Committee of the IEEE Computer Society, 21 September 2000.

[Ira00] Irastorza D., Jaime A., Díaz O., Component-Based Design: alternatives for the partition step. Conference on Software Engineering and Databases (JISBD), Spain, 2000. (in Spanish)

[ISO01] ISO/IEC Standard 9126-1 Software Engineering- Product Quality-Part1: Quality Model, ISO Copyright Office, Geneva, June 2001.

[Jac05] Jacobson I., Ng P.-W., Aspect-Oriented Software Development with Use Cases: Addison Wesley Professional, 2005.

[Jac04] Jackson A., Clarke S., SourceWeave.NET: Cross-Language Aspect-Oriented Programming. Generative Programming and Component Engineering (GPCE), Vancouver, Canada, 2004.

[Jac03] Jacobson I., Use Cases and Aspects—Working Seamlessly Together. Journal of Object Technology, Vol. 2, pp. 7-28, 2003.

[Jac97] Jacobson I., Griss M., Jonsson P., Software Reuse. Architecture, Process and Organization for Business Success. Addison-Wesley 1997.

[Jae03] Jaen J., Ramos I., A Conceptual Model for Context-Aware Dynamic Architectures.The 23rd International Workshop on Distributed Auto-adaptive and Reconfigurable Systems, IEEE Computer Society Press, Rhodes Island, 2003. 
[Jag06a] Jagadeesan R., Jeffrey A., Riely J., Typed Parametric Polymorphism for Aspects. Science of Computer Programming, 2006.

[Jag06b] Jagadeesan R., Jeffrey A., Riely J., A Typed Calculus of Aspect-Oriented Programs. http://fpl.cs.depaul.edu/rjagadeesan/pubs.html, 2003.

[Jag03] Jagadeesan R., Jeffrey A., Riely J., A Calculus of Untyped Aspect-Oriented Programs. European Conference of Object-Oriented Programming (ECOOP), Lecture Notes in Computer Science. Springer-Verlag, pp. 54-73, 2003.

[Kan02b] Kande M. M., Kienzle J., Strohmeier A., From AOP to UML: Towards an AspectOriented Architectural Modeling Approach, Technical Reports in Computer and Communication Sciences, Faculté I\&C, École Polytechnique Fédérale de Lausanne, 2002.

[Kan02a] Kande M. M., Kienzle J., Strohmeier A., From AOP to UML - A Bottom-Up Approach. Workshop on Aspect-Oriented Modeling with UML, AOSD, Enschede, The Netherlands, 2002.

[Kan03] Kande M. M., A concern-oriented approach to software architecture. $\mathrm{PhD}$. Thesis, Lausanne, Switzerland: Swiss Federal Institute of Technology (EPFL), 2003.

[Kat03] Katara M., Katz S., Architectural Views of Aspects. The $2^{\text {nd }}$ International Conference on Aspect-Oriented Software Development (AOSD 2003), Boston, Massachusetts, USA,2003.

[Kat02] Katara M., Superposing UML Class Diagram. Workshop on Aspect-Oriented Modelling with UML, AOSD, Enschede, The Netherlands, 2002.

[Kaz98] Kazman R., Klein M., Barbacci M., Lipson H., Longstaff T., Carriere S., The Architecture Tradeoff Analysis Method. The Fourth International Conference on Engineering. of Complex Computer Systems, ICECCS, August, 1998.

[Kaz96] Kazman R., Abowd G., Bass L., Clements P., Scenario-Based Analysis of Software Architecture. IEEE Software, Vol. 13, No. 6, pp. 47-55, November, 1996. 
PRISMA: Aspect-Oriented Software Architectures

[Kaz94] Kazman R., Bass L., Abowd G., Webb M., SAAM: A Method for Analyzing the Properties Software Architectures. The 16th International Conference on Software Engineering (ICSE), pp. 81-90, Sorrento, Italy, May, 1994.

[Ken95] Kenney J.J., Executable Formal Models of Distributed Transaction Systems based on Even Processing. PhD. Thesis, University of Stanford, CSL, December, 1995.

[Kim02] Kim H., AspectC\#: an AOSD implementation for C\#. M. Sc. Thesis, Comp. Sci., Trinity College, Dublin, November, 2002.

[Kiz01] Kiczales G., Hilsdale E., Huguin J., Kersten M., Palm J., Griswold W.G., An Overview of AspectJ. The $15^{\text {th }}$ European Conference on Object-Oriented Programming, Lecture Notes in Computer Science (LNCS), Springer-Verlag, Vol.2072, Budapest, Hungary, June 18-22, 2001.

[Kiz97] Kizcales G., Lamping J., Mendhekar A., Maeda C., Aspect-Oriented Programming. The $11^{\text {th }}$ European Conference on Object-Oriented Programming (ECOOP), Lecture Notes in Computer Science (LNCS), Springer-Verlag, Vol. 1241, Jyväskylä, Finland, June 9-13, 1997.

[Kram85] Krammer J. Magee J., Dynamic Configuration for Distributed Systems. IEEE Transactions on Software Engineering, Vol. 11, No. 4, pp. 424-436, 1985.

[Kru95] Kruchten P., The 4+1 View Model of Architecture. IEEE Software, Vol. 12, no. 6, November, 1995.

[Laf03] Lafferty D., Cahill V., Language-Independent Aspect-Oriented Programming. In Proceedings of Object-Oriented Programming Systems, Languages and Applications (OOPSLA 2003). Anaheim, California, USA, 2003.

[Läm05] Lämmel R., Schutter K., What does aspect-oriented programming mean to Cobol?. International Conference on Aspect-Oriented Software Development, AOSD, March 22-26, Chicago, Illinois, 2005. 
[Läm02] Lämmel R., A Semantical Approach to Method-Call Interception. The 1st International Conference on Aspect-Oriented Software Development (AOSD), ACM Press, pp. 41-55, Twente, The Netherlands, April 2002.

[Lam03] Lamsweerde A.V., Goal-oriented requirements engineering: From system objectives to UML models to precise software specifications. International Conference on Software Engineerign (ICSE), pp. 744-745, 2003.

[Lar99] Larsen G., Designing component-based frameworks using patterns in the UML. Communications of the ACM, 42(10):38-45, 1999.

[Let98] Letelier, P., Sánchez, P., Ramos, I. and Pastor, O., OASIS 3.0: A formal language for the object oriented conceptual modeling, Polytechnic University of Valencia, SPUPV98.4011, ISBN 84-7721-663-0, 1998. (In Spanish)

[Lie99] Lieberherr K., Lorenz D., Mezini M., Programming with Aspectual Components. Technical Report NU-CCS-99-01, pp. 1-27, Northeastern University, Boston, Massachusetts, March 1999.

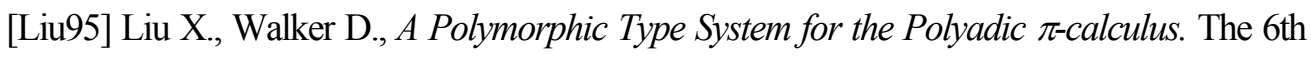
International Conference on Concurrency Theory, Lecture Notes in Computer Science, Springer, LNCS 962, pp. 103-116, Philadelphia, PA, USA, August 21-24, 1995.

[Lop05] Lopes A., Fiadeiro J. L., Context-Awareness in Software Architectures. The $2^{\text {nd }}$ European Workshop on Software Architecture (EWSA), Lecture Notes on Computer Science, Springer Verlang, LNCS 3527, pp. 146-161, Pisa Italy, June, 2005.

[Loq00] Loques O., Sztajnberg A., Leite J., Lobosco, M., On the Integration of Meta-Level Programming and Configuration Programming, In Reflection and Software Engineering (special edition), Editors: Walter Cazzola, Robert J. Stroud, Francesco Tisato, V. 1826, Lecture Notes in Computer Science, pp.191-210, Springer-Verlag, Heidelberg, Germany, June, 2000. 
PRISMA: Aspect-Oriented Software Architectures

[Lou05] Loughran N., Parlavantzas N., Pinto M., Fuentes L., Sánchez P., Webster M., Colyer A., Report synthesizing state-of-the-art in Aspect-Oriented Middlewares, Lancaster University, Lancaster, AOSD-Europe Deliverable D8, pp.1-114, AOSD-EuropeULANC-9, 14 June 2005, http://www.aosdeurope.net/

[Luc95b] Luckham D.C., Vera J., An Event-Based Architecture Definition Language. IEEE Transactions on Software Engineering, Vol. 21, No. 9, pp. 717-734, September, 1995.

[Luc95a] Luckham D.C., Kenney J.J., Augustine L.M., Vera J., Bryan D., Mann W., Specification and Analysis of Software Architecture using Rapide. IEEE Transactions on Software Engineering, Vol. 21, No. 4, pp. 336-355, April, 1995.

[Mag96] Magee J., Kramer J., Dynamic Structure in Software Architectures. ACM SIGSOFT'96: Fourth Symposium on the Foundations of Software Engineering (FSE4), pp. 3-14, San Francisco, CA, October 1996.

[Mag95] Magee J.N., Dulay N., Eisenbach S., Kramer J., Specifying Distributed Software Architectures. Fifth European Software Engineering Conference (ESEC), Barcelona, Spain, September, 1995.

[Mag89] Magee J., Krammer J., Sloman M., Constructing Distributed Systems in Conic. IEEE Transactions on Software Engineering, Vol. 15, No. 6, 1989.

[Mas03] Masuhara H., Kawauchi K., Dataflow Pointcut in Aspect-Oriented Programming. The First Asian Symposium on Programming Languages and Systems (APLAS'03), Lecture Notes in Computer Science, Springer-Verlag, vol. 2895, pp. 105-121, 2003.

[McD03] McDirmid S., Hsieh W.C., Aspect-Oriented Programming with Jiazzi. The $2^{\text {nd }}$ International Conference on Aspect-Oriented Software Development (AOSD), pp. 7079, Boston, Massachusetts, USA, march, 2003.

[MDA06] Object Management Group. Model Driven Architecture Guide, 2003 http://www.omg.org/docs/omg/03-06-01.pdf 
[Med00] Medvidovic N., Taylor R.N., A Classification and Comparison Framework for Software Architecture Description Languages. IEEE Transactions on Software Engineering,Vol. 26, No. 1, January, 2000.

[Med99] Medvidovic N., Rosenblum D. S., Taylor R. N., A Language and Environment for Architecture-Based Software Development and Evolution. The 21st International Conference on Software Engineering (ICSE’99), Los Angeles, California, May 1999.

[Med97] Medvidovic N., Taylor R.N., Architecture Description Languages. Software Engineering - ESEC/FSE, Lecture Notes in Computer Science, Springer Verlang, LNCS 1301, Zurich, Switzerland, September 1997.

[Med96] Medvidovic N., Oreizy P., Robbins J. E., Taylor R. N., Using Object-Oriented Typing to Support Architectural Design in the C2 Style. ACM SIGSOFT'96: Fourth Symposium on the Foundations of Software Engineering (FSE4), pp. 24-32, San Francisco, California, USA, October 1996.

[MEY] Meyer B., What to compose?. Eiffel Software, http://archive.eiffel.com/doc/manuals/technology/bmarticles/sd/compose.html

[Mey03] Meyer B., The Grand Challenge of Trusted Components. International Conference on Software Engineering (ICSE), IEEE Computer Press, Portland, Oregon, May 2003.

[Mez03] Mezini M., Ostermann K., Conquering Aspects with Caesar. International Conference on Aspect-Oriented Software Development (AOSD), ACM Press, pp. 90100, Boston, Massachusetts, USA, March, 2003.

[MIC05] Microsoft .Net Remoting: A Technical Overview, http://msdn.microsoft.com/library/default.asp?url=/library/en/dndotnet/html/hawkremoting.asp

[Mil99] Milner R., Communicating and Mobile Systems: The Pi-Calculus. Cambridge University Press, June, 1999. 
PRISMA: Aspect-Oriented Software Architectures

[Mil93] Milner R., The Polyadic $\pi$-Calculus: A Tutorial. Laboratory for Foundations of Computer Science Department, University of Edinburgh, October 1993.

[Mil80] Milner R., A Calculus of Communicating Systems. Lecture Notes in Computer Science, Volume 92, Springer Verlag 1980.

[Mon98] Monroe R.T., Capturing Software Architecture Design Expertise With Armani.Technical Report CMU-CS-98-163, Carnegie Mellon University School of Computer Science, October 1998.

[Mor05b] Moreira A., Araujo J., Rashid A., Multi-Dimensional Separation of Concerns in Requirements Engineering. Conference on Requirements Engineering (RE 05), Paris, France, 2005.

[Mor05a] Moreira A., Aráujo J., Rashid A., A Concern-Oriented Requirements Engineering Model. Conference on Advanced Information Systems Engineering (CAiSE'05), LNCS 3520, Porto, Portugal, 2005.

[Mor02] Moreira A., Araújo J., Brito I., Crosscutting Quality Attributes for Requirements Engineering. The $14^{\text {th }}$ International Conference on Software Engineering and Knowledge Engineering (SEKE 2002), Communications of the ACM, Italy, July 2002.

[Mor97] Moriconi M., Riemenschneider R. A., Introduction to SADL 1.0: A Language for Specifying Software Architecture Hierarchies. Technical Report SRI-CSL-97-01, SRI International, March, 1997.

[Mor95] Moriconi M., Qian X., Riemenschneider R. A., Correct Architecture Refinement. IEEE Transactions on Software Engineering, Vol. 21, No. 4, pp. 356-372, April 1995.

[Mos87] Moss J. E. B., Log-based Recovery for Nested Transactions. International Conference on Very Large Data Bases VLDB (1987), pp. 427-432.

[My199] Mylopoulos J., Chung, L. Yu E., From object-oriented to goal-oriented requirements analysis. Communications of the ACM, Vol. 42, No.1, pp. 31-37, January. 1999. 
[Nav05] Navasa A., Pérez M. A., Murillo J. M., Aspect Modelling at Architecture Design. $2^{\text {nd }}$ European Workshop on Software Architecture (EWSA), Lecture Notes on Computer Science, Springer Verlang, LNCS 3527, pp. 41-58, Pisa, Italy, June, 2005.

[Nav04a] Navarro E., Letelier P., Ramos I., Goals and Quality Characteristics: Separating Concerns, Early Aspects 2004: Aspect-Oriented Requirements Engineering and Architecture Design Workshop, OOPSLA, Monday, October 25, Vancouver, Canada, 2004.

[Nav04b] Navarro E., Letelier P., Ramos I., UML Visualization for an Aspect and GoalOriented Approach, The 5th Aspect-Oriented Modeling Workshop (AOM'04), UML 2004, Monday, October 11, 2004, Lisbon, Portugal

[Nav04c] Navarro E., Ramos I., Letelier P., Pérez J., Goals Model-Driving Software Architectur. The 2nd International Conference on Software Engineering Research, Management and Applications (SERA), May 5-8, 2004, Los Angeles, CA.

[Nav03] Navarro E., Ramos I., Pérez J., Software Requirements for Architectured Systems. The $11^{\text {th }}$ IEEE International Requirements Engineering Conference (RE'03), IEEE Computer Society, September 8-12, Monterey, California , 2003.

[Nie95] Nierstrasz O., Meijler T.D., Research Directions of Software Composition. ACM Computing Surveys (CSUR), Vol. 27, no. 2, June, 1995.

[Nor98] Noriega P., Sierra C., Auctions and Multi-Agents Systems. Latin American Joumal of Artificial Intelligence, no. 6, pp. 68-84, 1998.

[Obe97] Oberndorf T., COTS and Open Systems - An Overview, 1997, http://www.sei.cmu.edu/str/descriptions/cots.html\#ndi

[OCL06] OCL tools \& services http://www.klasse.nl/ocl/ocl-services.html

[Oli98] Oliva A., Garcia I.C., Buzato L.E., The Reflective Architecture of Guaraná, Technical Report IC-98-14. Instituto de computación, Universidad de Campiñas, April, 1998. 
PRISMA: Aspect-Oriented Software Architectures

[Oqu04a] Oquendo F., $\pi-A D L$ : An Architecture Description Language based on the HigherOrder Typed $\pi$-Calculus for Specifying Dynamic and Mobile Software Architectures. ACM Software Engineering Notes, Vol. 29, No. 3, May, 2004.

[Oqu04b] Oquendo F., Warboys B., Morrison R., Dindeleux R, Gallo F., Garavel H., Occhipinti C., ArchWARE: Architecting Evolvable Software. The 1st European Workshop in Software Achitecture (EWSA 2004), Lecture Notes in Computer Science LNCS 3047, St Andrews, UK, pp. 257-271. Springer, ISBN 3-540-22000-3. 2004

[Oss01] Ossher H., Tarr P., Multi-Dimensional Separation of Concerns and The Hyperspace Approach. The Symposium on Software Architectures and Component Technology: The state of the Art in Software Development, Kluwer, 2001.

[Oss00] Ossher H., Tarr P., Hyper/Jтм. Multi-Dimensional Separation of Concerns for $J_{a v a}{ }^{\mathrm{TM}}$. The International Conference on Software Engineering (ICSE). Communications of the ACM, pp. 734-737, Limerick, Ireland (2000) .

[Par78] Parnas D. L., Designing Software for Extension and Contraction. The 3rd International Conference on Software Engineering (ICSE), pp. 264-277, 1978.

[Par74] Parnas D. L., On a 'Buzzword': Hierarchical Structure. IFIP Congress 74, pp. 336-339, 1974.

[Par72] Parnas D. L., On the Criteria to be used in Decomposing Systems into Modules. Communications of the ACM, Vol 15, No.12, pp. 1053-1058, December 1972.

[Pas97] Pastor O. Et al, OO-METHOD: A Software Production Environment Combining Conventional and Formal Methods. The $9^{\text {th }}$ International Conference, CaiSE97, Barcelona, 1997.

[Paw04] Pawlak R., Seinturier L., Duchien L., Florin G., Legond-Aubry F., Martelli L., JAC: an Aspect-Based Distributed Dynamic Framework. Software - Practice and Experience, Vol. 34, pp. 1119-1148, 2004. 
[Paw02] Pawlak R., Duchien L., Florin G., Legond-Aubry F., Seinturier L., Martelli L., $A$ UML Notation for Aspect-Oriented Software Design. Workshop on Aspect-Oriented Modeling with UML, International Conference on Aspect-Oriented Software Development (AOSD), 2002.

[Paw01] Pawlak R., Seinturier L., Duchien L., Florin G.,. JAC: A Flexible Solution for AspectOriented Programming in Java. Reflection, Lecture Notes in Computer Science (LNCS), Springer-Verlag, Vol. 2192, pp. 1-24. September 2001.

[Per92] Perry, D., Wolf A., Foundations for the Study of Software Architecture. ACM Software Engineering Notes, Vol. 17, No. 4, pp. 40-52, October 1992.

[Per06a] Pérez J., Navarro E., Letelier P., Ramos I., Graphical Modelling Proposal For Aspect-Oriented SA. The $21^{\text {st }}$ Annual ACM Symposium on Applied Computing (SAC), ACM, Dijon, France, April 23-27, 2006. (short paper)

[Per06b] Pérez J., Navarro E., Letelier P., Ramos I., A Modelling Proposal For AspectOriented Software Architectures. The $13^{\text {th }}$ Annual IEEE International Conference and Workshop on the Engineering of Computer Based Systems (ECBS), IEEE Computer Society Press, Hasso-Plattner-Institute For Software Systems Engineering at the University Of Potsdam, Potsdam, Germany (Berlin Metropolitan Area), 27-30 March.

[Per02a] Pérez J., Carsí J A. and Ramos I., On the implication of application's requirements changes in the persistence layer: an automatic approach. Workshop on the Database Maintenance and Reengineering (DBMR'2002), IEEE International Conference of Software Maintenance, Montreal (Canada), October 1st, 2002, pp. 3-16, ISBN: 84-699$8920-0$.

[Per02b] Pérez J., Carsí J. A. and Ramos I., ADML: A Language for Automatic Generation of Migration Plans. The First Eurasian Conference on Advances in Information and Communication Technology, Tehran, Iran, October 2002 http://www.eurasia-ict.org/ (C) Springer LNCS vol n.2510 
PRISMA: Aspect-Oriented Software Architectures

[Per02c] Pérez J., Anaya V., Cubel J M., Domínguez F., Boronat A., Ramos I. and Carsí J A., Data Reverse Engineering of Legacy Databases to Object Oriented Conceptual Schemas. Software Evolution Through Transformations: Towards Uniform Support throughout the Software Life-Cycle Workshop (SET'02), First International Conference on Graph Transformation(ICGT2002), Barcelona (Spain), October, 2002 (C) ENTCS vol n. 72.4

[Pie00] Pierce B. C., Turner D.N., Pict: A Programming Language Based on the Pi-Calculus. In G. Plotkin, C. Stirling and M. Tofte, eds., "Proof, Language and Interaction: Essays in Honour of Robin Milner", MIT Press, 2000.

[Pin05] Pinto M., Fuentes L., Troya J.M., A Dynamic Component and Aspect Platform, The Computer Journal Vol. 48, No. 4, pp. 401-420, 2005.

[Pin03] Pinto M., Fuentes L., Troya J. M., DAOP-ADL: An Architecture Description Language for Dynamic Component and Aspect-Based Development. Generative Programming and Component Engineering: Second International Conference, GPCE Springer Verlag, Lecture Notes Computer Science, ISSN: 0302-9743, Erfurt, Germany, September 22-25.

[PRI06] PRISMA, http://prisma.dsic.upv.es

[Raj03] Rajan H., Sullivan K., Eos: Instance-Level Aspects for Integrated System Design. The 2003 Joint European Software Engineering Conference and ACM SIGSOFT Symposium on the Foundations of Software Engineering (ESEC/FSE 2003), Helsinki, Finland, September 2003.

[Ras02] Rashid A., Sawyer P., Moreira A., Araujo J., Early Aspects: a Model for AspectOriented Requirements Engineering. IEEE Joint Conference on Requirements Engineering, Essen, IEEE Computer Society, pp. 199-202, Germany, September 2002.

[RAT06] Rational Software, Rational Rose, http://www-306.ibm.com/software/rational/ 
[Rib02] J.M.Ribo, X. Franch, Una Metodología a dos Niveles para Extender el Metamodelo $U M L$, VII Jornadas de Ingeniería del Software y Bases de Datos, El Escorial (Madrid) 2002

[Rob03] Robinson S. et al. , Professional C\# $2^{\text {nd }}$ Edition, Wrox Programmer to Programmer.

[San01] Sangiorgi D., Walker D., The $\pi$-Calculus. A theory of Mobile Processes. Cambridge University Press, June 2001.

[Sch01] Scholl K.U., Albiez J., Gassmann B., MCA: An Expandable Modular Controller Architecture. The 3rd Real-Time Linux Workshop, Karlsruhe University, Milano, Italy, 2001.

[Sch03] Schult W., Polze A., Speed vs. Memory Usage - An Approach to Deal with Contrary Aspects. The 2nd Workshop on Aspects, Components, and Patterns for Infrastructure Software (ACP4IS), International Conference on Aspect-Oriented Software Development (AOSD), Boston, Massachusetts, 2003.

[Sch02] Schult W., Polze A., Aspect-Oriented Programming with C\# and .NET. The 5th IEEE International Symposium on Object-Oriented Real-time Distributed Computing, IEEE Computer Society Press, pp. 241-248, Washington, DC, 2002).

[Scü02] Schüpany M., Schwanninger C., Wuchner E., Aspect-Oriented Programming for .NET. Workshop on Aspects, Components, and Patterns for Infrastructure Software, International Conference on Aspect-Oriented Software Development, pp. 59-64, Enschede, The Netherlands, 2002.

[Ser94] Sernadas, A., Costa J.F., Sernadas C., Object Specifications Through Diagrams: OBLOG Approach. INESC Lisbon 1994.

[SET06] The SetPoint! Project Website, http://www.dc.uba.ar/people/proyinv/setpoint/

[Sha96] Shaw M., DeLine R., Zelesnik G., Abstractions and Implementations for Architectural Connections. The Third International Conference on Configurable Distributed Systems, May, 1996. 
PRISMA: Aspect-Oriented Software Architectures

[Sha95] Shaw M., DeLine R., Klein D. V., Ross T. L., Young D. M., Zelesnik G., Abstractions for Software Architecture and Tools to Support Them. IEEE Transactions on Software Engineering, Vol. 21, No. 4, pp. 314-335, April 1995.

[Sha94] Shaw M., Procedure Calls Are the Assembly Language of Software Interconnection: Connectors Deserve First-Class Status. Workshop on Studies of Software Design, January,1994.

[Sih03] Sihman M., Katz S., Superimpositions and Aspect-Oriented Programming. The Computer Journal, Vol 46, No. 5, pp. 529-541, September, 2003.

[Sou03] Sousa G. M. C., Castro J. B., Towards a Goal-Oriented Requirements Methodology Based on the Separation of Concerns Principle. Workshop on Requirements Engineering (WER), ISBN 8587926071, Piracicaba-SP, Brasil, November, 27-28, 2003.

[Spi03] Spitznagel B., Garlan D., A compositional Formalization of Connectors Wrappers. International Conference on Software Engineering (ICSE), 2003.

[Spi02] Spinczyk O., Gal A., Schröder-Preikschat W., AspectC++: An Aspect-Oriented Extension to $C++$. The $40^{\text {th }}$ International Conference on Technology of ObjectOriented Languages and Systems (TOOLS Pacific), Sydney, Australia, February 18$21,2002$.

[Spi01] Spitznagel B., Garlan D., A compositional Approach for Constructing Connectors. The Working IEEE/IFIP Conference on Software Architecture (WICSA), IEEE Computer Society, Amsterdam, The Netherlands, 28-31 August, 2001.

[Sti92] Stirling C., Modal and Temporal Logics. Handbook of Logic in Computer Science, vol II, Clarendon Press, Oxford, 1992. 
[Ste03] Stein D., Hanenberg S., Unland R., Aspect-Oriented Modeling: Issues on Representing Crosscutting Features. Workshop on Aspect-Oriented Modelling, International Conference on Aspect-Oriented Software Development (AOSD 2003), Boston, Massachusetts, USA, 2003.

[Ste02a] Stein D., Hanenberg S., Unland R., A UML-based Aspect-Oriented Design Notation For AspectJ. International Conference on Aspect-Oriented Software Development (AOSD), Enschede, The Netherlands, 2002.

[Ste02b] Stein D., Hanenberg S., Unland R., On Representing Join Points in the UML. Workshop on Aspect-Oriented Modelling with UML, UML 2002, Dresden, Germany, 2002.

[Sto02] Stojanovic Z., Dahanayak A., Components and Viewpoints as Integrated Separations of Concerns in System Designing, Workshop on Identifying, Separating and Verifying Concerns in the Design, AOSD, Enschede, The Netherlands, 2002.

[Sut02] Sutton S., Rouvellou I., Modeling of Software Concerns in Cosmos. The 1st International Conference. on Aspect-Oriented Software Development (AOSD),G. Kiczales, Eds., pp. 127-133, 2002.

[Suv05b] Suvée D., De Fraine B., Vanderperren W., FuseJ: An architectural description language for unifying aspects and components. Workshop on Software-engineering Properties of Languages and Aspect Technologies, 2005.

[Suv05a] Suvée D., Vanderperren W., Wagelaar D., Jonckers V., There Are No Aspects. Electronical Notes in Theoretical Computer Sciences (ENTCS), Special Issue on Software Composition, Vol. 114, pp. 153-174, January, 2005.

[Suv03] Suvée D., Vanderperren W., Jonckers V., JAsCo: an Aspect-Oriented Approach Tailored for Component-Based Software Development.The $2^{\text {nd }}$ International Conference on Aspect-Oriented Software Development (AOSD), ACM Press, pp. 2129, ISBN 1-58113-660-9, Boston, Massachusetts, March, 2003. 
PRISMA: Aspect-Oriented Software Architectures

[Suz99] Suzuki J., Yamamoto Y., Extending UML with Aspects: Aspect Support in the Design Phase. Workshop on Aspect-Oriented Programming, the European Conference on Object-Oriented Programming (ECOOP), 1999.

[Szy00] Szyperski C., Booch G., Meyer B., Beyond Objects. Software Development Magazine, March, 2000 (originally BrucePowel Douglass).

[Szy98] Szyperski C., Component software: beyond object-oriented programming. ACM Press and Addison Wesley, New York, USA (1998).

[SYS06] Telelogic System Architect.

http://www.telelogic.com/products/system architect/index.cfm

[Tar99] Tarr P., Ossher, H., Harrison, W., Sutton Jr., S. M., N Degrees of Separation: Multidimensional Separation of Concerns. The 21st International Conference on Software Engineering (ICSE), Communication of the ACM, pp. 107-119, New York, 1999.

[TEA06] The TeachMover Robot,

http://www.questechzone.com/microbot/teachmover.htm

[Tek05] Tekinerdogan B., Scholten F., ASAAM-T: A tool environment for identifying architectural aspects. Aspect-Oriented Software Development Conference (AOSD), March 18-19, Chicago, 2005.

[Tek04] Tekinerdogan B., ASAAM: Aspectual software architecture analysis method. The 4th Working IEEE/IFIP Conference on Software Architecture (WICSA), Oslo, Norway, 2004.

[THE06] The Theme: Aspect-Oriented Analysis and Design Website, http://www.dsg.cs.tcd.ie/index.php?category id $=353$

[TOG06] Together, Borland Software Corporation.

http://www.borland.com/us/products/together/index.html 
[UML06] The Unified Modeling Language Website, Object Management Group (OMG), http://www.uml.org/

[Van01] Vanderperren, W. and Wydaeghe, B. Towards a New Component Composition Process. The 8th Annual IEEE International Conference and Workshop on the Engineering of Computer Based Systems (ECBS), Washington DC, April 2001.

[Ver03] Verspecht D., Vanderperren W., Suvee D., Jonckers V., JAsCo.NET: Capturing Crosscutting Concerns in .NET Web Services. The Second Nordic Conference on Web Services NCWS'03, Vaxjo, Sweden. In "Mathematical modelling in Physics, Engineering and Cognitive Sciences", Vol. 8, November 2003.

[Ves96] Vestal S., MetaH Programmer's Manual, Version 1.09.. Technical Report, Honeywell Technology Center, April 1996.

[VIS06] Visio 2003, http://office.microsoft.com/es-es/FX010857983082.aspX

[Voa98] Voas J., Maintaining Component-Based Systems. IEEE Software, July/August, pp. 22-27, 1998.

[Vol01] Volpe R., Nesnas I., Estlin T., Mutz D., Petras R., Das H., The CLARAty architecture for robotic autonomy. IEEE Aerospace Conference. Vol. 1, pp. 121-132, Montana, USA, 2001.

[WAC06] Wacker Art, http://www.wackerart.de/

[Wag02] Wagelaar D., Bergmans L., Using a concept-based approach to aspect-oriented software design. Workshop on Aspect-Oriented Design, the International Conference on Aspect-Oriented Software Development, Twente, Enschede, The Netherlands, 2002.

[Wa103] Walker D., Zdancewic S., Ligatti J. A Theory of Aspects. International Conference on Functional Programming (ICFP), Communications of the ACM, pp. 127-139, 2003. 
PRISMA: Aspect-Oriented Software Architectures

[Wan04] Wand M., Kiczales G., Dutchyn C., A Semantics for Advice and Dynamic Join Points in Aspect-Oriented Programming. TOPLAS, Vol. 26, No. 5, pp. 890-910, September 2004. Earlier versions of this paper were presented at the 9th International Workshop on Foundations of Ob ject-Oriented Languages, January 19, 2002, and at the Workshop on Foundations of Aspect-Oriented Languages (FOAL), April 22, 2002.

[Whi03] Whittle J., Araujo J., Kim D.-K., Modeling and Validating Interaction Aspects in UML. Workshop on AOSD Modeling, UML 2003, San Francisco, USA, 2003.

[Wile99] Wile D., AML: an Architecture Meta-Language. Automated Software Engineering ASE, IEEE Computer Society, pp. 183-190, 1999.

[Wyd01] Wydaeghe B., Vanderperren W., Visual Component Composition Using Composition Pattems. Tools, Santa Barbara, California, July 2001.

[YuY04] Yu Y., Leite J. C. S. d. P., Mylopoulos J., From Goals to Aspects: Discovering Aspects from Requirements Goal Models, International Conference on Requirements Engineering (ICRE), Kyoto, Japan, 2004. 


\section{APPENDIX A}

\section{PRISMA AOADL SYNTAX}

This appendix presents the BNF of the PRISMA AOADL. The notation that has been used is the following:

$>$ bold term is a terminal term

$>$ 'bold term' is a terminal punctuation mark

$>$ [term] is an optional term

$>\quad$ is an alternative

Some simplifications are used in the BNF to define names, values, blocks, lists or sequences of terms. Let be $\mathrm{x}$ a term of the grammar. The simplifications are the following:

$>$ Names that are identifiers:

$<\mathrm{x} \_$name $>::=$is a terminal that corresponds to a string of characters.

$>$ Value of a property:

$<$ __value $>::=$ is a terminal that corresponds to a concordant constant value with the type of the property $\mathrm{x}$.

$>$ Blocks:

$<\mathrm{x}$ block $>::=<\mathrm{x}$ block $><\mathrm{x}>\mid<\mathrm{x}>$

$>$ Lists:

$<\mathrm{x}$ list $>::=<\mathrm{x}$ list $>$ ',${ }^{\prime}<\mathrm{x}>\mid<\mathrm{x}>$

$>$ Sequences:

$<$ __seq $>::=<$ X_seq $>^{6} ;{ }^{6}<\mathrm{x}>\mid<\mathrm{x}>^{6} ;^{6}$ 
PRISMA: Aspect-Oriented Software Architectures

\section{A.1. ARCHITECTURAL MODEL}

$<$ architectural_model $>::=$ Architectural_Model $<$ model_name $>$

$<$ interface block>

$<$ aspect_block $>$

$<$ component_block $>$

$<$ connector block $>$

$<$ attachments_block $>$

$[<$ system_block $>]$

End_Architectural_Model < model_name>';'

A.2.INTERFACES

$<$ interface $>:=$ Interface $<$ interface_name $>$

$<$ iservice_list $>$

End_Interface $<$ interface_name $>$;

$<$ iservice $>::=<$ service_name $>$ '(' $[<$ param_service_list $>]$ ')' ';'

\section{A.3. ASPECTS}

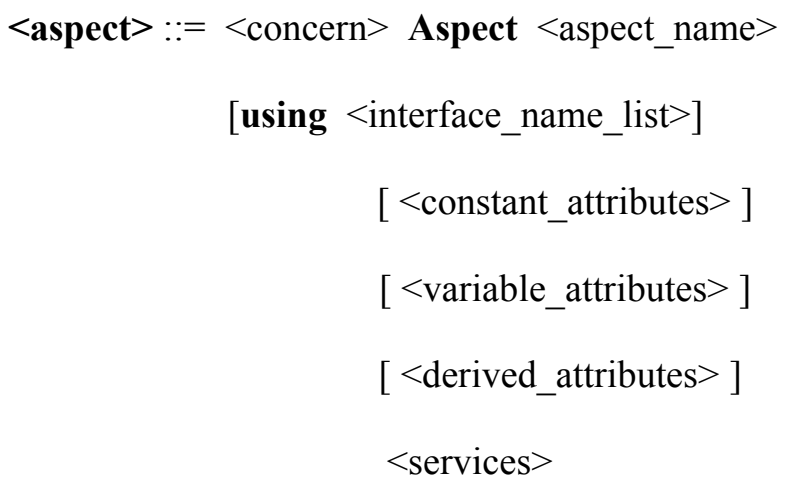




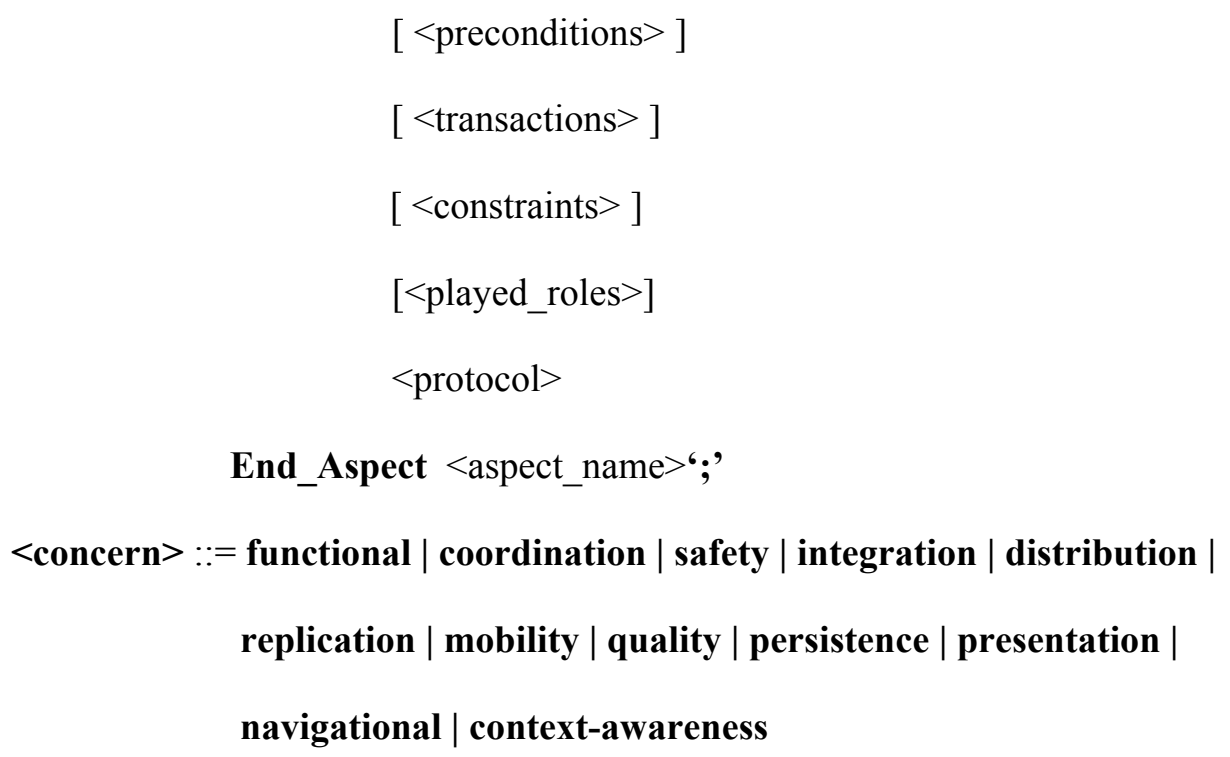

\section{A.3.1. Attributes}

$<$ constant_attributes $>::=$ Constant $<$ cons_attribute_seq $>$

$<$ variable_attributes $>::=$ Variable $<$ var_attribute_seq $>$

$<$ derived_attributes $>:=$ Derived $<$ der_attribute_seq $>$

$<$ cons_attribute $>:=<$ attribute_name $>$ ':' < data_type $>$ [','DEFAULT':’]

$<$ value $>$

$<$ var_attribute $>::=<$ attribute_name $>^{6}: '<$ data_type $>$ [', NOT NULL ]

[', DEFAULT':'] < value>

$<$ der_attribute $>::=<$ attribute_name $>$ ':' $<$ data_type $>$ ', derivation':'

$<$ formulae $>$ 
PRISMA: Aspect-Oriented Software Architectures

\section{A.3.2.Services}

$<$ services $>::=$ Services $<$ service_section_seq $>$

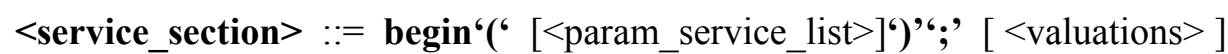

$<$ domain_services_seq $>$ end'(' ')';';

$<$ domain_services $>::=<$ service $>[$ as $<$ service_name $>][<$ valuations $>$ ]

$<$ service $>::=<$ service_type $><$ service_name $>^{\prime}\left({ }^{(}[<\text {param_service_list }>]^{\top}\right)^{\prime}$

$<$ service_type $>:=$ in $\mid$ out $\mid$ in/out

$<$ valuations $>::=$ Valuations $<$ valuation_seq $>$

$<$ valuation $\left.>::=\left[{ }^{\prime}\left\{{ }^{6}<\text { condition }>\right\}^{\prime}\right]\right]^{\prime}\left[{ }^{6}<\right.$ action $>$ ']' $<$ postcondition_list $>$

$<$ action $>::=<$ service_type $><$ service_name $>^{\text {'( }\left('[<\text { parameter_name_list }>]^{\top}\right)}$ '

$<$ postcondition $>::=<$ property $>$ ':=' <formulae $>$

$<$ property $>::=<$ attribute_name $>\mid<$ parameter_name $>$

\section{A.3.3.Preconditions}

$<$ preconditions $>::=$ Preconditions $<$ precondition_seq $>$

$<$ precondition $>::=<$ invocation $>$ if ' $\{$ ' $<$ condition $>$ ' $\}$ '

$<$ invocation $>::=<$ service_name $>$ '(' $\left.[<\text { parameter_name_list }>]^{6}\right)$ '

\section{A.3.4. Transactions}

$<$ transactions $>::=$ Transactions $<$ transaction_seq $>$

$<$ transaction $>::=<$ service_type $><$ transaction_name $>$ 


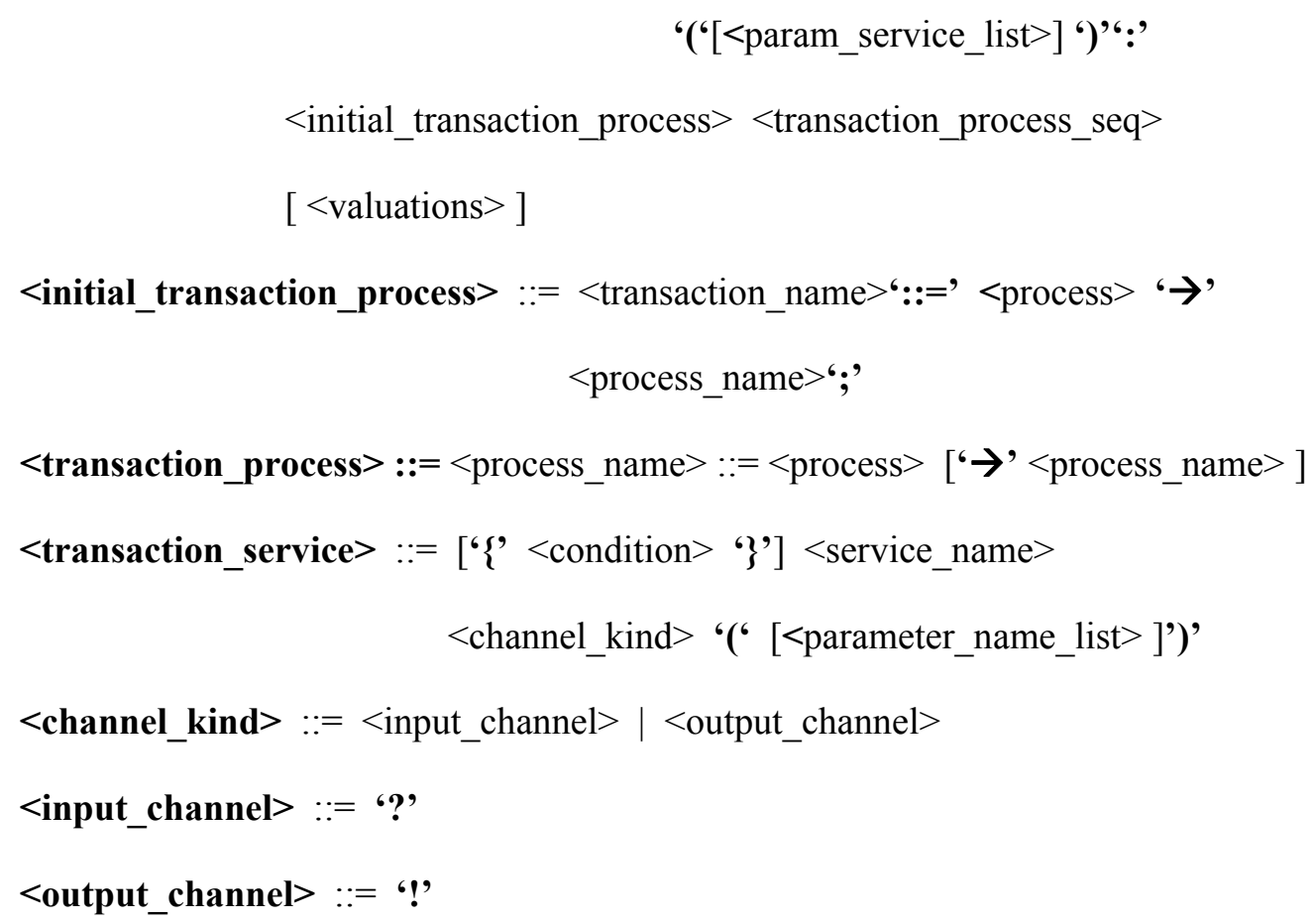

\section{A.3.5. Constraints}

$<$ constraints $>:=$ Constraints $<$ constraint_seq $>$

$<$ constraint $>::=$ always ' $\left\{{ }^{6}<\right.$ condition $\left.>6\right\}$ ' | never ' $\{6<$ condition $>$ ' $\}$ '

$<$ condition_before $>$ since $<$ condition_after $>$ |

$<$ condition_before $>$ until < condition_after $>$ |

always $<$ condition_before $>$ since $<$ condition_after $>$ |

always $<$ condition_before $>$ until $<$ condition_after $>$ |

never $<$ condition_before $>$ since $<$ condition_after $>$ |

never $<$ condition_before $>$ until $<$ condition_after $>$ |

$<$ condition_before $>::=<$ condition $>$ 
PRISMA: Aspect-Oriented Software Architectures

$<$ condition_after $>::=<$ condition $>$

\section{A.3.6.Played_Roles}

$<$ played_roles $>::=$ Played_Roles $<$ played_role_seq $>$

$<$ played_role $>::=<$ played_role_name $>$ for $<$ interface_name $>$ ' $=$, $<$ process $>$ ';'

$<$ played_role_service $>::=<$ service_name $><$ channel_kind $>$

$$
\text { '('[<parameter_name_list> }] \text { ')' }
$$

\footnotetext{
A.3.7.Protocol

$<$ protocol $>::=$ Protocol $<$ initial_process $><$ protocol_process_seq $>$

$<$ initial_process $>::=<$ aspect_name $>$ '::=' $<$ process $>$ ' $\rightarrow$ ' $<$ process_name $>$ ';'

$<$ protocol_process $>::=<$ process_name $>$ '::=' <process $>$

$\left[{ }^{6} \rightarrow\right.$ ' $<$ process_name $\left.>\right]$

$<$ protocol_service $>::=\left[{ }^{\prime}\left\{{ }^{\prime}<\right.\right.$ condition $>$ ' $\left.\}\right]<$ protocol_service_type $>$

$<$ channel_kind $>$ '(' $[<$ parameter_name_list $>]$ ')' :'

$<$ priority $>$

$<$ process_service_type $>::=<$ public_service $>\mid<$ private_service $>$

$<$ public_service $>::=<$ played_role_name $>$,', $<$ service_name $>$

$<$ private_service $>::=<$ service_name $>$

$<$ priority $>::=<$ priority_value $>$
} 


\section{A.4. COMPONENTS}

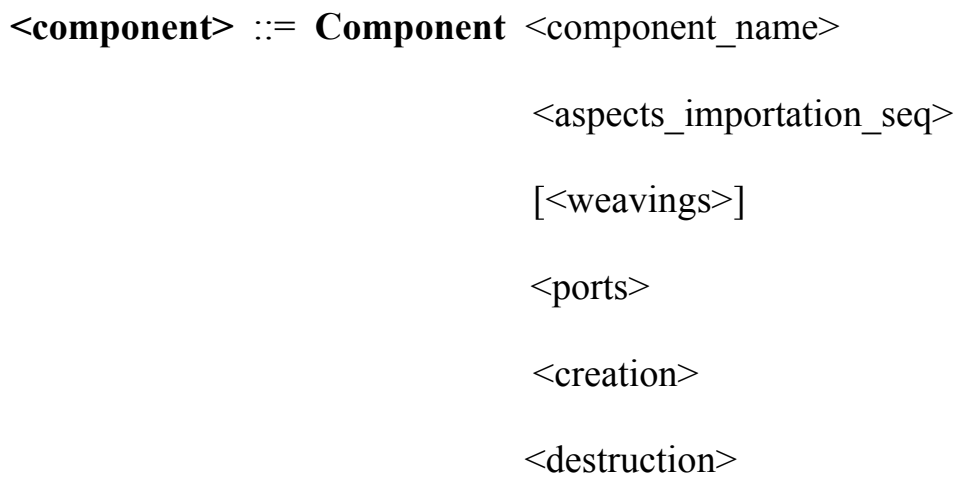

\section{A.5. WEAVINGS}

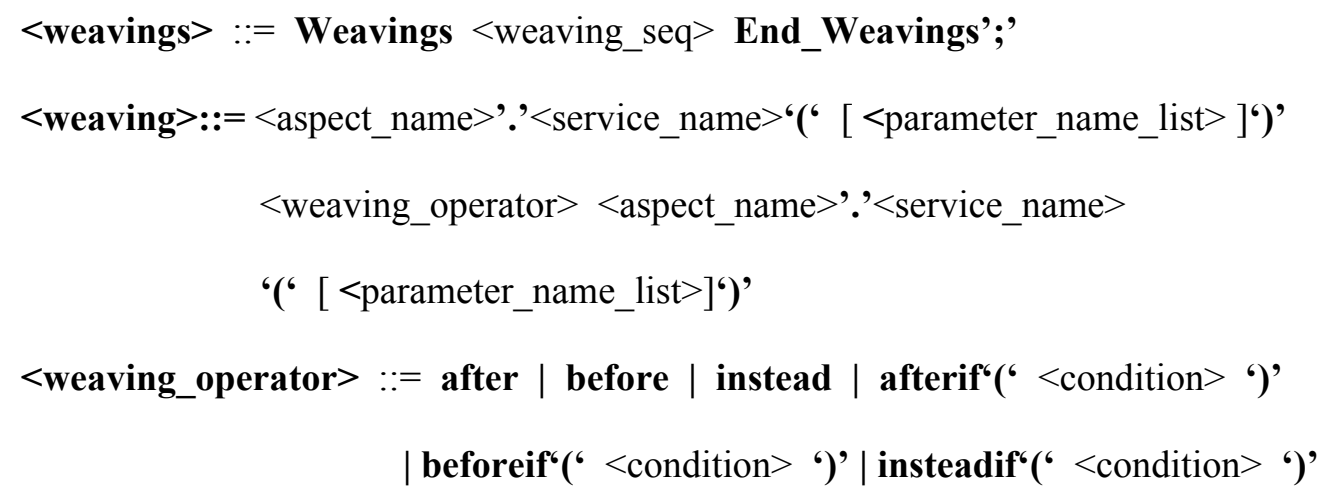


PRISMA: Aspect-Oriented Software Architectures

\section{A.6. PORTS}

$<$ ports $>::=$ Ports $<$ port_seq $>$ End_Ports';'

$<$ port $>::=<$ port_name>':' < interface_name>','

Played_Role $<$ aspect_name $>$ ' ', $<$ played_role_name $>$

\section{A.7. CONNECTORS}

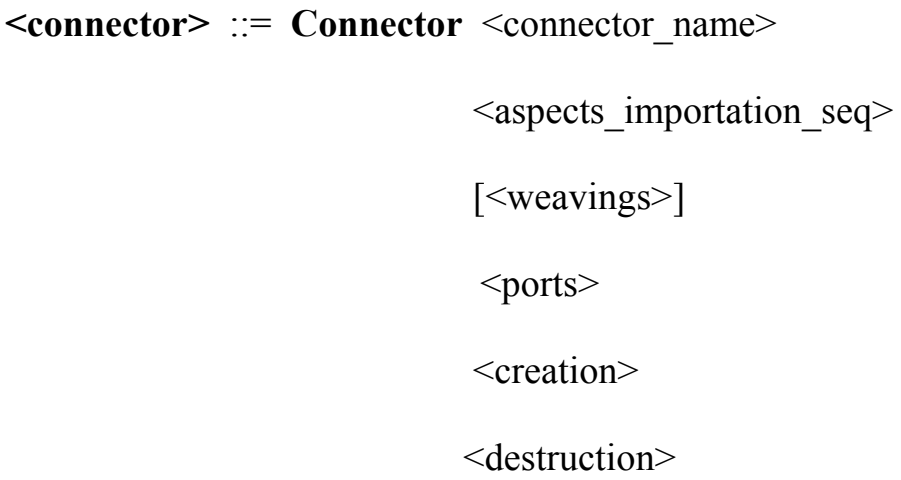

End_Connector < connector_name ${ }^{6}$;'

\section{A.8. ATTACHMENTS}

$<$ attachments $>::=$ Attachments $<$ attachment_seq $>$ End_Attachments';

$<$ attachment $>::=<$ attachment_name $>$ ':' $<$ connector_name $>$ ', $<$ port_name $>$

$$
\begin{aligned}
& \text { '('<card_min_value }>\text { ', }<\text { card_max_value }>\text { ') ' } \leftrightarrow \rightarrow \text { ' } \\
& <\text { component_name }>\text { ', }<\text { port_name }> \\
& \text { '('<card_min_value }>\text { ',' }<\text { card_max_value }>\text { ') })
\end{aligned}
$$

$<$ card_min $>:=<$ natural_value $>$

$<$ card_max $>::=<$ natural_value $>$ 


\section{A.9.SYSTEMS}

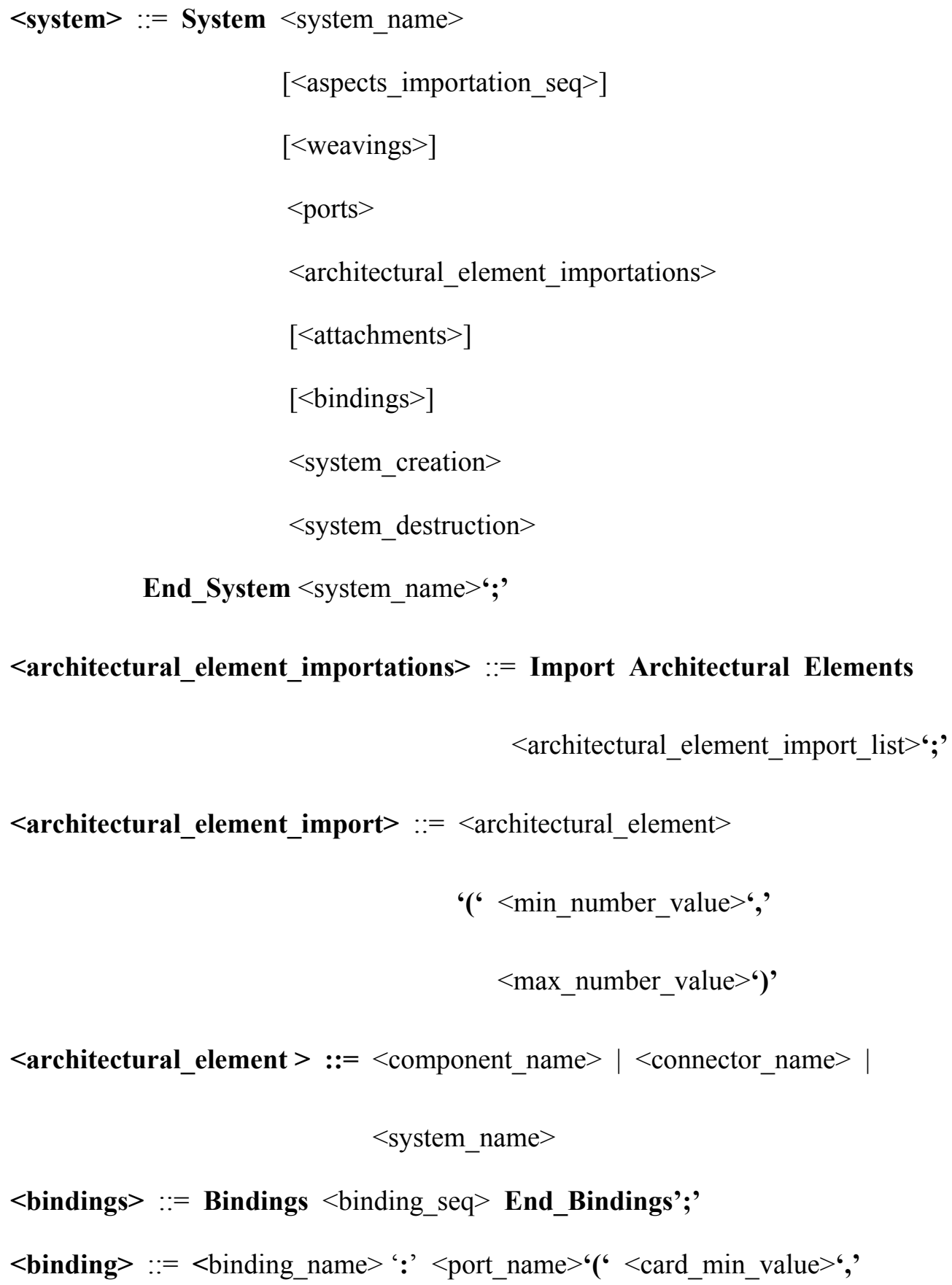


PRISMA: Aspect-Oriented Software Architectures

$$
\begin{aligned}
& \left.<\text { card_max_value }>^{6}\right) \text { ' ' } \leftarrow \rightarrow \text { ' } \\
& <\text { architectural_element }>\text { ',' }<\text { port_name }> \\
& \text { '('< <card_min_value } \left.>^{6}, \text { ' }<\text { card_max_value }>^{6}\right) \text { ' }
\end{aligned}
$$

$<$ system_creation $>:=$ new '(' $\left[<\right.$ param_service_list $>^{6}$, ']

$<$ architectural_element_number_list $>$,

$[<$ attachment_number_list $>$,

$<$ binding_number_list $>]^{6}$ )'

' $\{6[<$ start_aspects_seq $>]$

$<$ architectural_elements_creation_seq $>$

$[<$ attachments_creation_seq $>]$

[<bindings_creation_seq $>]$ ' $\}$ '

$<$ architectural_element_number $>:=$

input Num_<architectural_element_name>'‘'' natural

$<$ attachment_number $>::=$ input Num_<attachment_name $>^{6}: '$ natural

$<$ binding_number $>::=$ input Num_<binding_name $>^{6}: '$ natural

$<$ architectural_elements_creation $>::=$ new <architectural_element_name $>$

$$
\text { '(' } \left.[<\text { param_service_list }>]^{6}\right) \text { ' }
$$

$<$ attachments_creation $>::=$ new $<$ attachment_name $>$ '('<param_attachment $\left.>^{6}\right)$ '

$<$ param_attachment $>::=$ input ArgCnctName: string,

input ArgCnctPort: string,

input ArgCompName: string, 


\section{input ArgCompPort: string}

$<$ bindings_creation $>::=$ new $<$ binding_name $>$ '(' $<$ param_binding $\left.>{ }^{6}\right)$ '

$<$ param_binding $>::=$ input ArgSysPort: string, input ArgAEName: string,

input ArgAEPort: string

$<$ system_destruction $>::=\operatorname{destroy}$ '(6 6) ' $6\{6[<$ stop_aspects_seq $>]$

$<$ architectural_elements_destruction_seq $>$

$[<$ attachments_destruction_seq $>]$

[<bindings_creation_seq $>$ ] $\}$ '

$<$ architectural_elements_destruction $>::=$

<architectural_element_name>'.'destroy'( ${ }^{6} \quad 6$ ')'

$<$ attachments_destruction $>::=<$ attachment_name $>$ '.'destroy ${ }^{6}\left({ }^{6}\right.$ ' ${ }^{\prime}$ )'

$<$ bindings_destruction $>::=<$ binding_name $>$ '.'destroy ${ }^{6}\left({ }^{6}\right.$ ' $)$ '

\section{A.10. CONFIGURATION}

$<$ architectural_model_configuration $>::=$

Architectural_Model_Configuration $<$ configuration_name $>$ '=' new $<$ model_name $>$ ' $\{6<$ components_instantiation_seq $>\mid$

$[<$ systems_instantiation_seq $>]$

$<$ connectors_instantiation_seq $>$

$<$ attachments_intantiation_seq $>$ ' $\}$ ' 
PRISMA: Aspect-Oriented Software Architectures

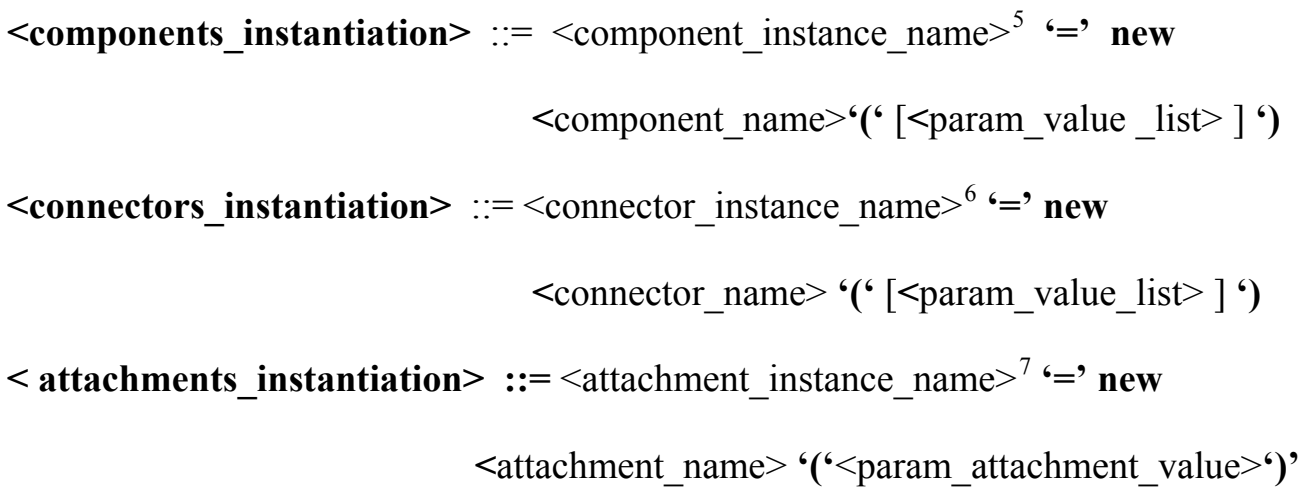

${ }^{5}$ component_instance_name is a simplification that represents the name of an instance of the component_name type

${ }^{6}$ connector_instance_name is a simplification that represents the name of an instance of the connector_name type

${ }^{7}$ attachment_instance_name is a simplification that represents the name of an instance of the attachment_name type

${ }^{8}$ system_instance_name is a simplification that represents the name of an instance of the system_name type 
$<$ bindings_instantiation $>::=<$ binding_instance_name $>^{9}{ }^{6}=$, new $<$ binding_name $>$ '(' < param_binding_value $>$ ')'

\section{A.11. COMMON ELEMENTS}

A.11.1. DataTypes

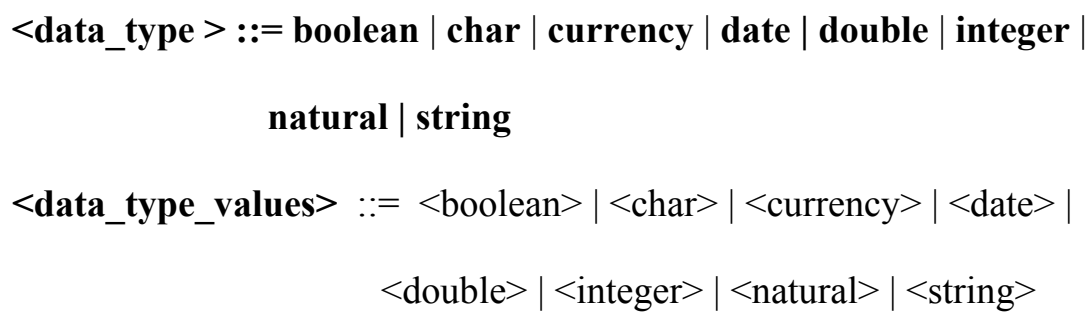

\section{A.11.2. Parameters}

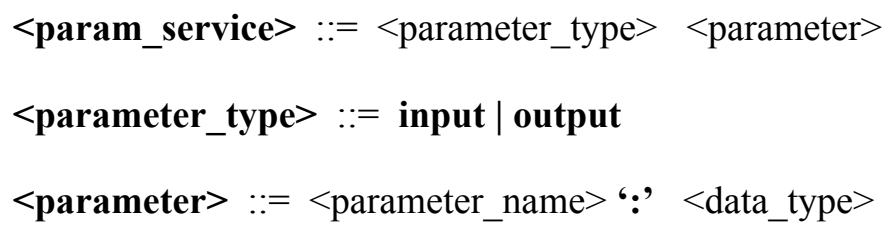

\section{A.11.3. Formulae}

$<$ formulae $>::=<$ condition $>\mid<$ arithmetic_expression $>\mid<$ function $>$

\footnotetext{
${ }^{9}$ binding_instance_name is a simplification that represents the name of an instance of the binding_name type
} 
PRISMA: Aspect-Oriented Software Architectures

\section{A.11.4. Conditions}

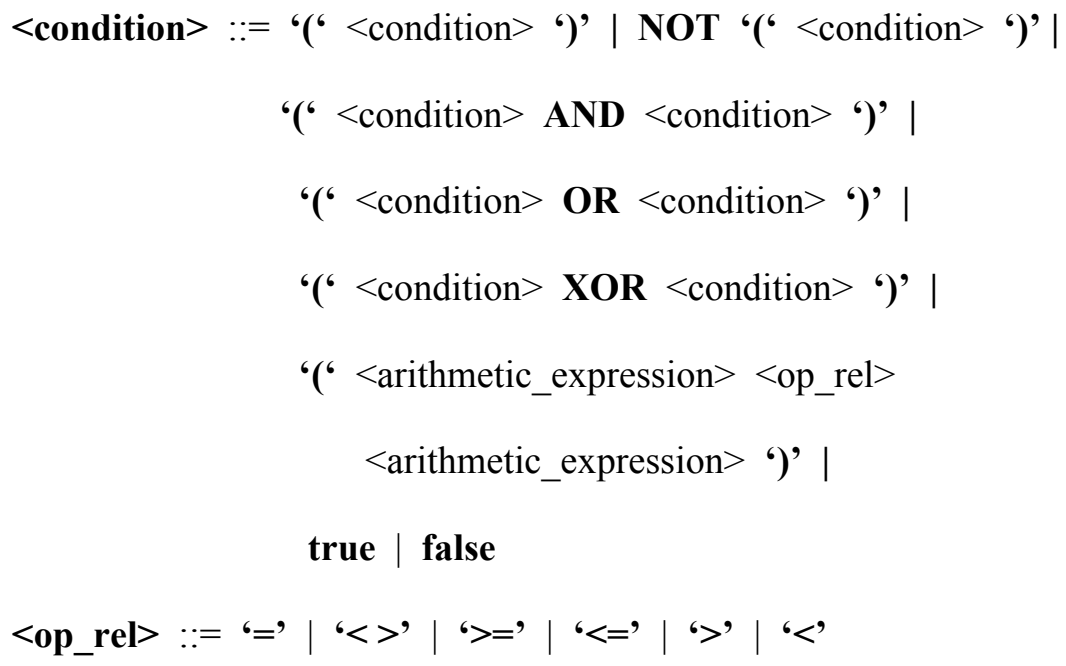

\section{A.11.5. Arithmetic Expressions}

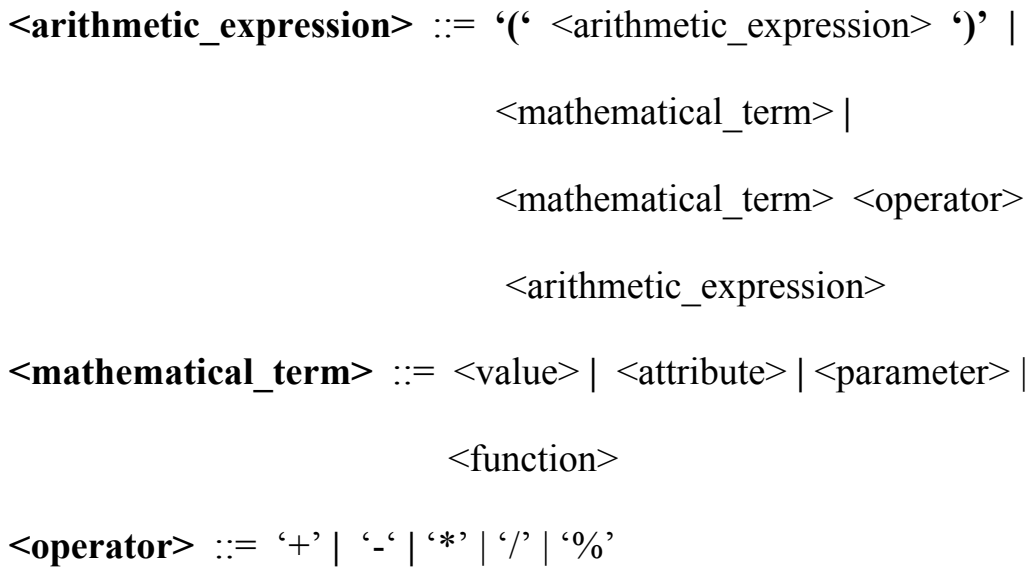


The PRISMA AOADL Syntax

\section{A.11.6. Functions}

$$
\begin{aligned}
<\text { function }>::= & <\text { data_type }><\text { function_name }> \\
& \text { '(' } \left.[<\text { param_service_list }>]^{\top}\right)
\end{aligned}
$$

\section{A.11.7. Processes}

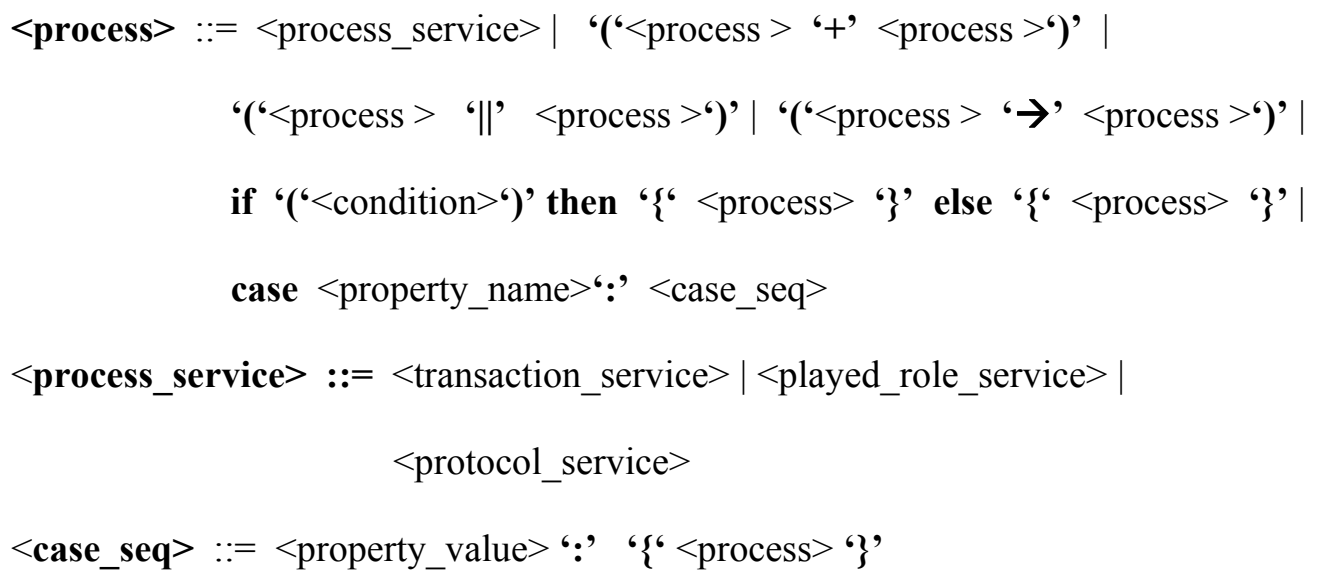


PRISMA: Aspect-Oriented Software Architectures 


\section{APPENDIX B}

\section{THE PRISMA UML PROFILE}

This appendix presents the PRISMA UML profile. The development of the profile has been realized following the steps proposed by the works [Ana03] and [Rib02]. These steps are the following:

1. To define a metamodel for a specific domain (see chapter 7).

2. To establish the correspondences between the metamodel concepts and the UML concepts.

3. To creation of a UML profile for the specific domain.

4. To complete the UML profile by textually describing the semantics of each concept and by introducing its OCL constraints.

Throughout the definition process of the PRISMA profile, especially for AOSD elements, it has been taken into account the satisfaction of the requirements that Aldawud et al stated for defining a UML profile for AOSD:

$>$ The Profile shall enable specifying, visualizing, and documenting the artifacts of software systems based on Aspect-Orientation. This requirement has been satisfied by means of the stereotypes and their visual representation as described below.

> The Profile shall be supported by UML (avoid "Heavy-weight" extension mechanisms), this allows a smooth integrating of existing CASE tools that support UML. This requirement has also been satisfied since our UML profile is defined according to the established construction rules for UML profiles. 
PRISMA: Aspect-Oriented Software Architectures

$>$ The Profile shall support the modular representation of crosscutting concern. The separation of concerns provided by PRISMA along with the defined weaving relationships allows us to identify and manage crosscutting efficiently.

$>$ The Profile shall not impose any behavioral implementation for AOSD, however it shall provide a complete set of model elements (or stereotypes) that enable representing the semantics of the system based on Aspect-Orientation. No constraint has been defined about the implementation, only a proper semantic that is related to the way we use the PRISMA elements has been defined.

This appendix presents a simplified version of the PRISMA UML profile with the main purpose of showing the extensions that have been done. As a result, this profile is presented without entering in details. The appendix presents the points 2, 3 and 4 of the PRISMA profile development without presenting the OCL constraints associated to the extension of each concept.

\section{B.1. CORRESPONDENCES BETWEEN PRISMA CONCEPTS AND UML CONCEPTS}

\begin{tabular}{|c|c|}
\hline PRISMA metamodel & Exact Correspondence in the UML metamodel \\
\hline Aspect & NO \\
\hline PRISMA Component & NO \\
\hline System & NO \\
\hline Connector & NO \\
\hline Attachment & NO \\
\hline Binding & NO \\
\hline Port & NO \\
\hline Weaving & UML Metaclass: Interface \\
\hline PRISMA Interface & NO \\
\hline Protocol & NO \\
\hline Table 5. & \\
\hline
\end{tabular}

Table 5. Correspondences between PRISMA and UML 


\section{B.2. PRISMA UML PROFILE}

\section{B.2.1.Aspect}

\begin{tabular}{|c|c|c|c|c|}
\hline Stereotype & Base Class & Parent & Tag Values & Description \\
\hline $\begin{array}{c}\text { Aspect } \\
<<\text { Aspect }>>\end{array}$ & Classifier & N/A & None & $\begin{array}{c}\text { An aspect defines the structure and the } \\
\text { behaviour of a specific concern } \text { of the } \\
\text { software system. }\end{array}$ \\
\hline
\end{tabular}

Table 6. Description of the stereotype Aspect

\begin{tabular}{|c|c|c|c|c|}
\hline Stereotype & Base Class & Parent & Tag Values & Description \\
\hline $\begin{array}{c}\text { Functional } \\
<<\text { Functional }>>\end{array}$ & & Aspect & None & $\begin{array}{c}\text { A functional aspect defines the structure } \\
\text { and the behaviour of the functional } \\
\text { concern of the software system. }\end{array}$ \\
\hline
\end{tabular}

Table 7. Description of the stereotype Functional Aspect

\begin{tabular}{|c|c|c|c|c|}
\hline $\begin{array}{c}\text { Stereotype } \\
\text { Coordination } \\
<<\text { Coordination }>>\end{array}$ & Base Class & Parent & Tag Values & Description \\
\hline & Aspect & None & $\begin{array}{c}\text { A coordination aspect defines the } \\
\text { structure and the behaviour of the } \\
\text { coordination concern of the software } \\
\text { system. }\end{array}$ \\
\hline
\end{tabular}

Table 8. Description of the stereotype Coordination Aspect

\begin{tabular}{|c|c|c|c|c|}
\hline Stereotype & Base Class & Parent & Tag Values & Description \\
\hline $\begin{array}{c}\text { Safety } \\
<\text { Safety }>>\end{array}$ & & Aspect & None & $\begin{array}{c}\text { A safety aspect defines the structure and the } \\
\text { behaviour of the safety concern of the } \\
\text { software system. }\end{array}$ \\
\hline
\end{tabular}

Table 9. Description of the stereotype Safety Aspect

\begin{tabular}{|c|c|c|c|c|}
\hline Stereotype & Base Class & Parent & Tag Values & Description \\
\hline $\begin{array}{c}\text { Integration } \\
<<\text { Integration }>>\end{array}$ & & Aspect & None & A integration aspect permits to wrap a \\
COT
\end{tabular}

Table 10. Description of the stereotype Integration Aspect 
PRISMA: Aspect-Oriented Software Architectures

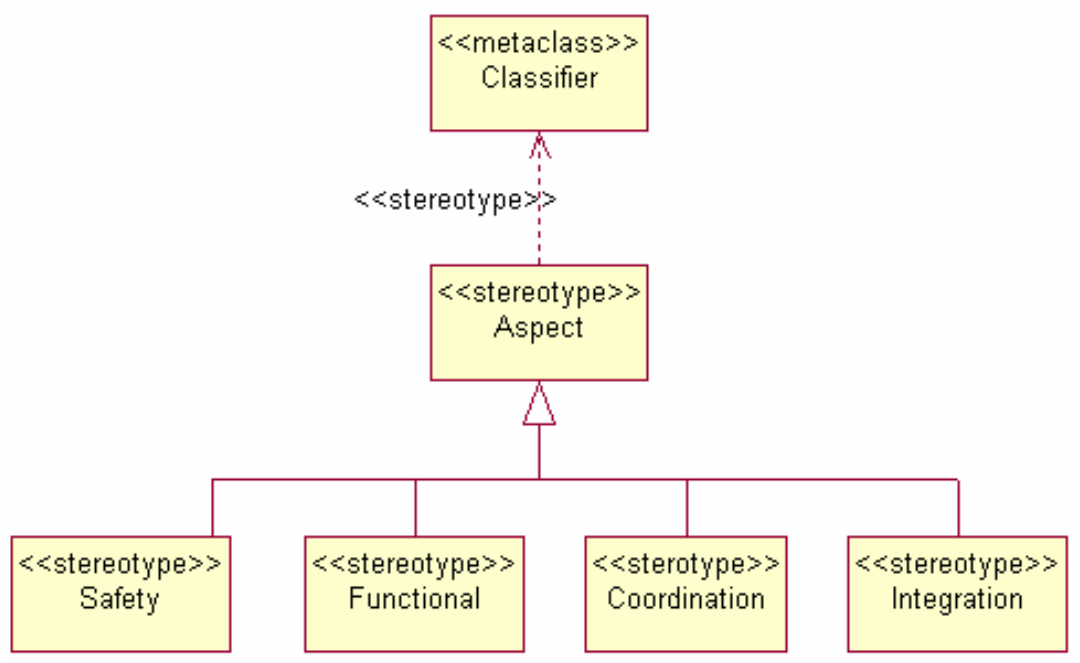

Figure 164. Modelling of the stereotype Aspect

\section{B.2.2. Component}

\begin{tabular}{|c|c|c|c|c|}
\hline Stereotype & Base Class & Parent & Tag Values & Description \\
\hline $\begin{array}{c}\text { Component } \\
<<\text { Component }>>\end{array}$ & Component & N/A & None & $\begin{array}{c}\text { A component is an architectural } \\
\text { element that captures a given } \\
\text { functionality of a software system. . } \\
\text { As such, components do not have a } \\
\text { coordination aspect. }\end{array}$ \\
\hline
\end{tabular}

Table 11. Description of the stereotype Component

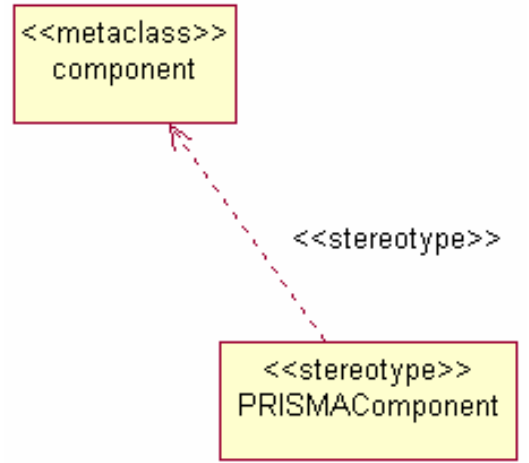

Figure 165. Modelling of the stereotype Component 
The PRISMA UML Profile

\begin{tabular}{|c|c|c|c|c|}
\hline Stereotype & Base Class & Parent & Tag Values & Description \\
\hline $\begin{array}{c}\text { Connector } \\
<\text { Connector }>>\end{array}$ & $\begin{array}{c}\text { PRISMA } \\
\text { Component }\end{array}$ & None & $\begin{array}{c}\text { A connector is an architectural } \\
\text { element that acts as a coordinator } \\
\text { between other architectural } \\
\text { elements. As such, connectors } \\
\text { have a coordination aspect. }\end{array}$ \\
\hline
\end{tabular}

Table 12. Description of the stereotype Connector

\begin{tabular}{|c|c|c|c|c|}
\hline Stereotype & Base Class & Parent & Tag Values & Description \\
\hline $\begin{array}{c}\text { System } \\
<\text { System }>>\end{array}$ & & $\begin{array}{c}\text { PRISMA } \\
\text { Component }\end{array}$ & None & $\begin{array}{c}\text { A PRISMA system is a } \\
\text { component that includes a set } \\
\text { of connectors, simple } \\
\text { components, and other } \\
\text { systems that are correctly } \\
\text { attached to one another. }\end{array}$ \\
\hline
\end{tabular}

Table 13. Description of the stereotype System

\section{B.2.3.Port}

\begin{tabular}{|c|c|c|c|c|}
\hline Stereotype & Base Class & Parent & Tag Values & Description \\
\hline $\begin{array}{c}\text { Port } \\
<<\text { Port }>>\end{array}$ & Interface & N/A & None & $\begin{array}{c}\text { A Port is an interaction point of an } \\
\text { architectural element (component or } \\
\text { connector). }\end{array}$ \\
\hline
\end{tabular}

Table 14. Description of the stereotype Port

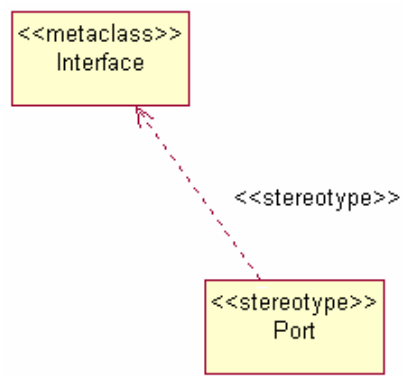

Figure 166. Modelling of the stereotype Port 
PRISMA: Aspect-Oriented Software Architectures

\section{B.2.4.Protocol}

\begin{tabular}{|c|c|c|c|c|}
\hline Stereotype & Base Class & Parent & Tag Values & Description \\
\hline $\begin{array}{c}\text { Protocol } \\
<<\text { Protocol }>>\end{array}$ & StateMachine & N/A & None & $\begin{array}{c}\text { APprotocol establishes how the services } \\
\text { of an aspect can be executed. }\end{array}$ \\
\hline
\end{tabular}

Table 15. Description of the stereotype Protocol

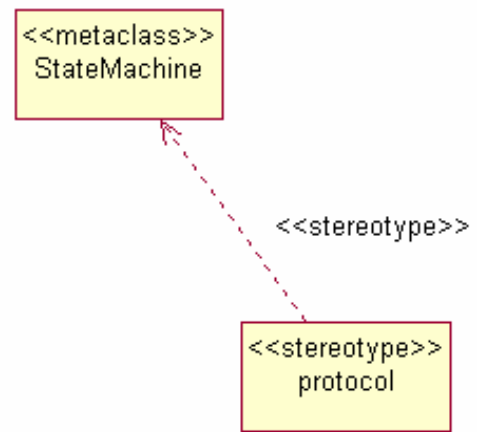

Figure 167. Modelling of the stereotype Protocol

B.2.5. Weaving

\begin{tabular}{|c|c|c|c|c|}
\hline Stereotype & Base Class & Parent & Tag Values & Description \\
\hline $\begin{array}{c}\text { Weaving } \\
<<\text { Weaving }>>\end{array}$ & Dependency & N/A & None & $\begin{array}{c}\text { A Weaving defines how the execution of a } \\
\text { service of an aspect can trigger the } \\
\text { execution of a service of another aspect. }\end{array}$ \\
\hline
\end{tabular}

Table 16. Description of the stereotype Weaving

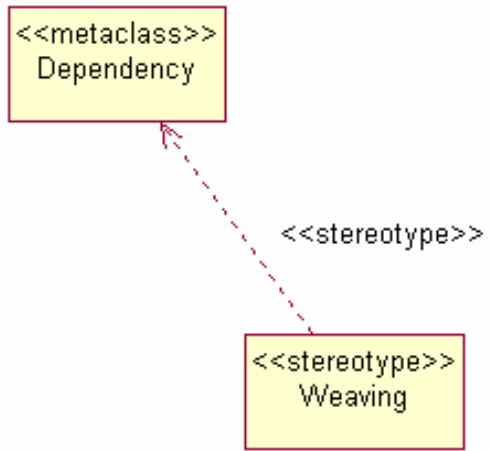

Figure 168. Modelling of the stereotype Weaving 


\section{B.2.6. Attachment}

\begin{tabular}{|c|c|c|c|c|}
\hline Stereotype & Base Class & Parent & Tag Values & Description \\
\hline $\begin{array}{c}\text { Attachment } \\
<\text { Attachment }>>\end{array}$ & Dependency & N/A & None & $\begin{array}{c}\text { An Attachment } \text { establishes a } \\
\text { connection between two } \\
\text { architectural elements, more } \\
\text { precisely between a port of a } \\
\text { component and a port of a connector }\end{array}$ \\
\hline
\end{tabular}

Table 17. Description of the stereotype Attachment

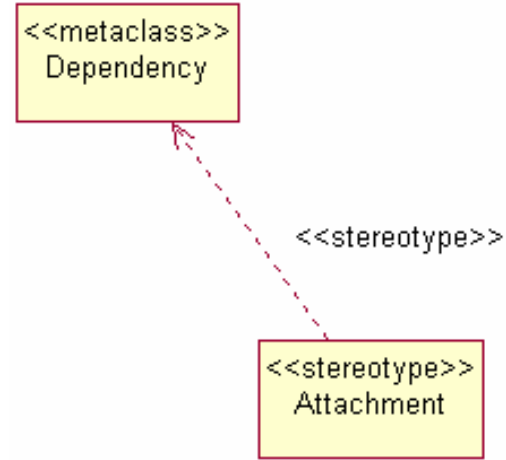

Figure 169. Modelling of the stereotype Attachment

\section{B.2.7.Binding}

\begin{tabular}{|c|c|c|c|c|}
\hline Stereotype & Base Class & Parent & Tag Values & Description \\
\hline $\begin{array}{c}\text { Binding } \\
<<\text { Binding }>>\end{array}$ & Dependency & N/A & None & $\begin{array}{c}\text { A Binding relationship defines the } \\
\text { composition between a complex } \\
\text { component and one of its architectural } \\
\text { elements, more precisely between a port } \\
\text { of a complex component and a port of } \\
\text { one of its architectural elements. }\end{array}$ \\
\hline
\end{tabular}

Table 18. Description of the stereotype Binding 
PRISMA: Aspect-Oriented Software Architectures

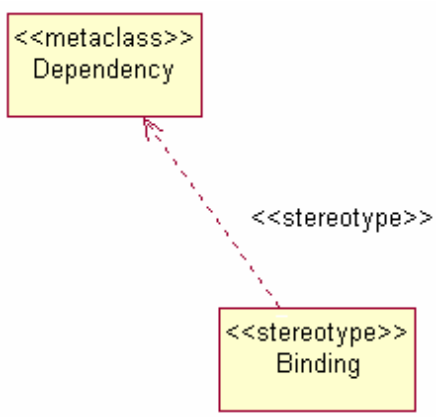

Figure 170. Modelling of the stereotype Binding 
APPENDIX C

PRISMA SOFTWARE ARCHITECTURE OF THE TEACHMOVER

This appendix presents the complete specification of the Joint system of the TeachMover robot using the PRISMA AOADL as well as the specification of its instances Base, Shoulder and Elbow. This specification has been automatically generated by the PRISMACASE. The complete specification of the TeachMover can be found in [PRI06].

\section{C.1. COMMON}

Domains

natural,

integer,

double,

char,

string,

boolean,

date,

End_Domains ;

Transformation_Functions

FTransHalfStepsToAngle (input Steps : integer, output RAngle : integer); End_Transformation_Functions ; 
PRISMA Software Architecture of the TeachMover

\section{C.3. ASPECTS}

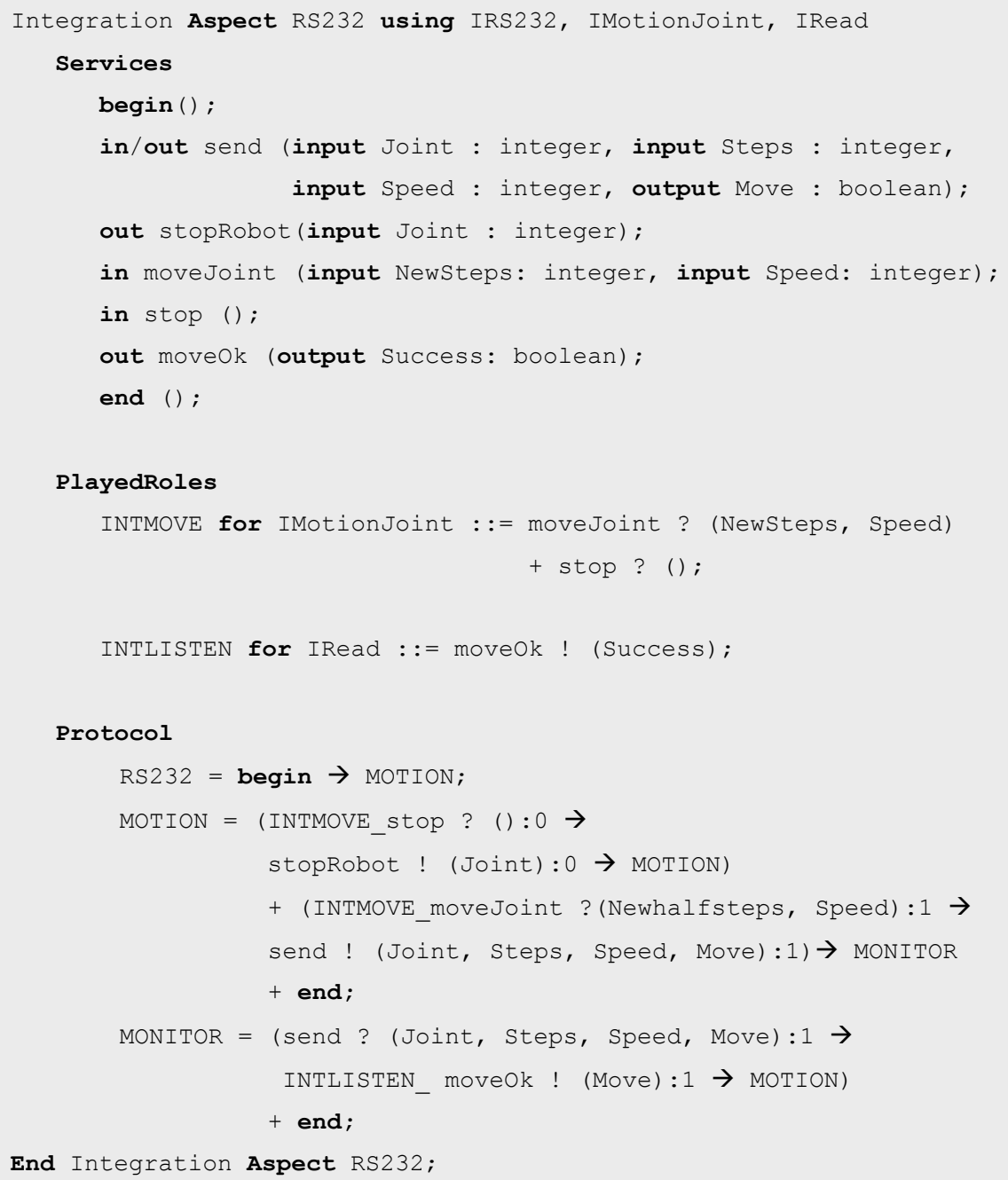


PRISMA: Aspect-Oriented Software Architectures

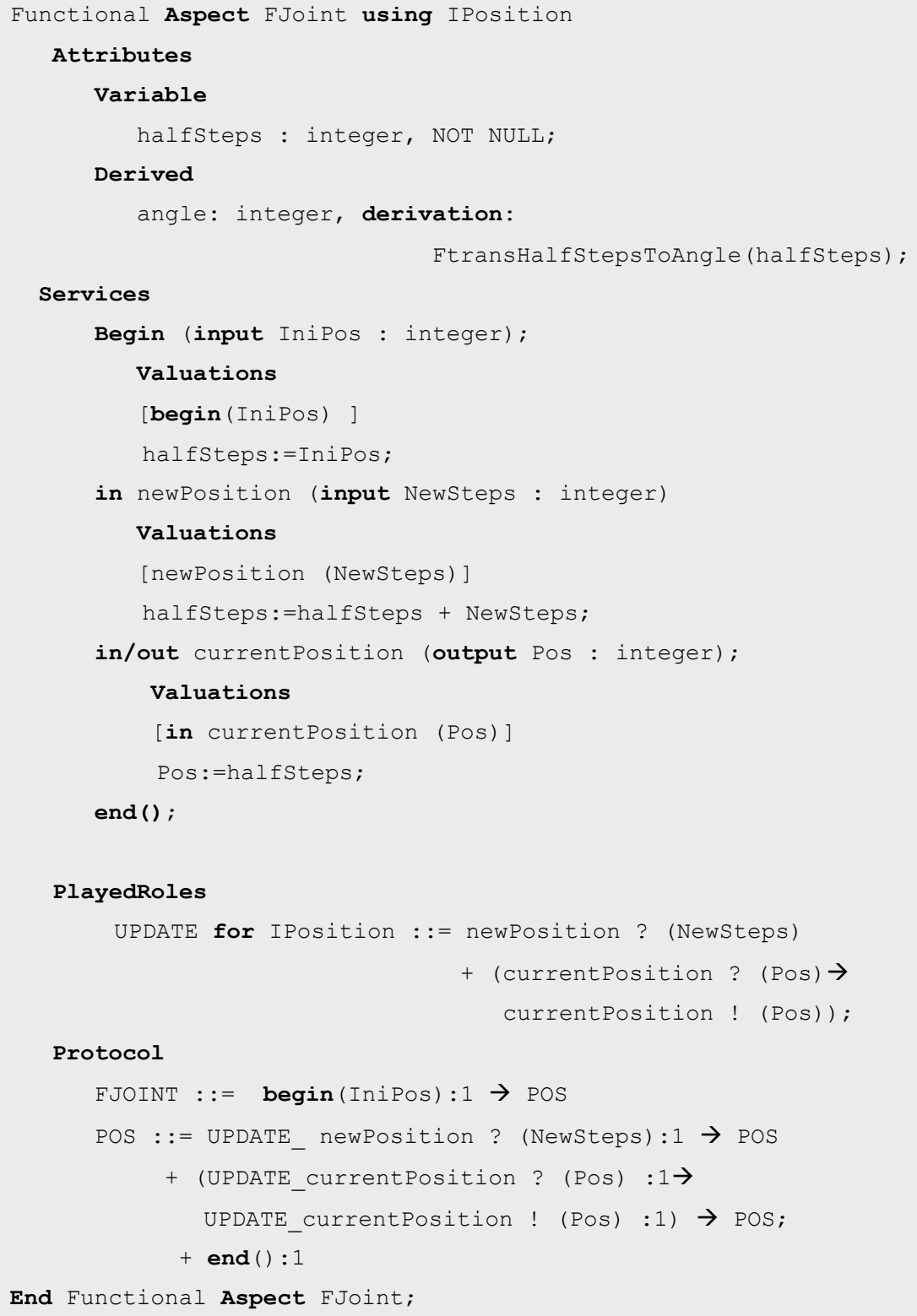


PRISMA Software Architecture of the TeachMover

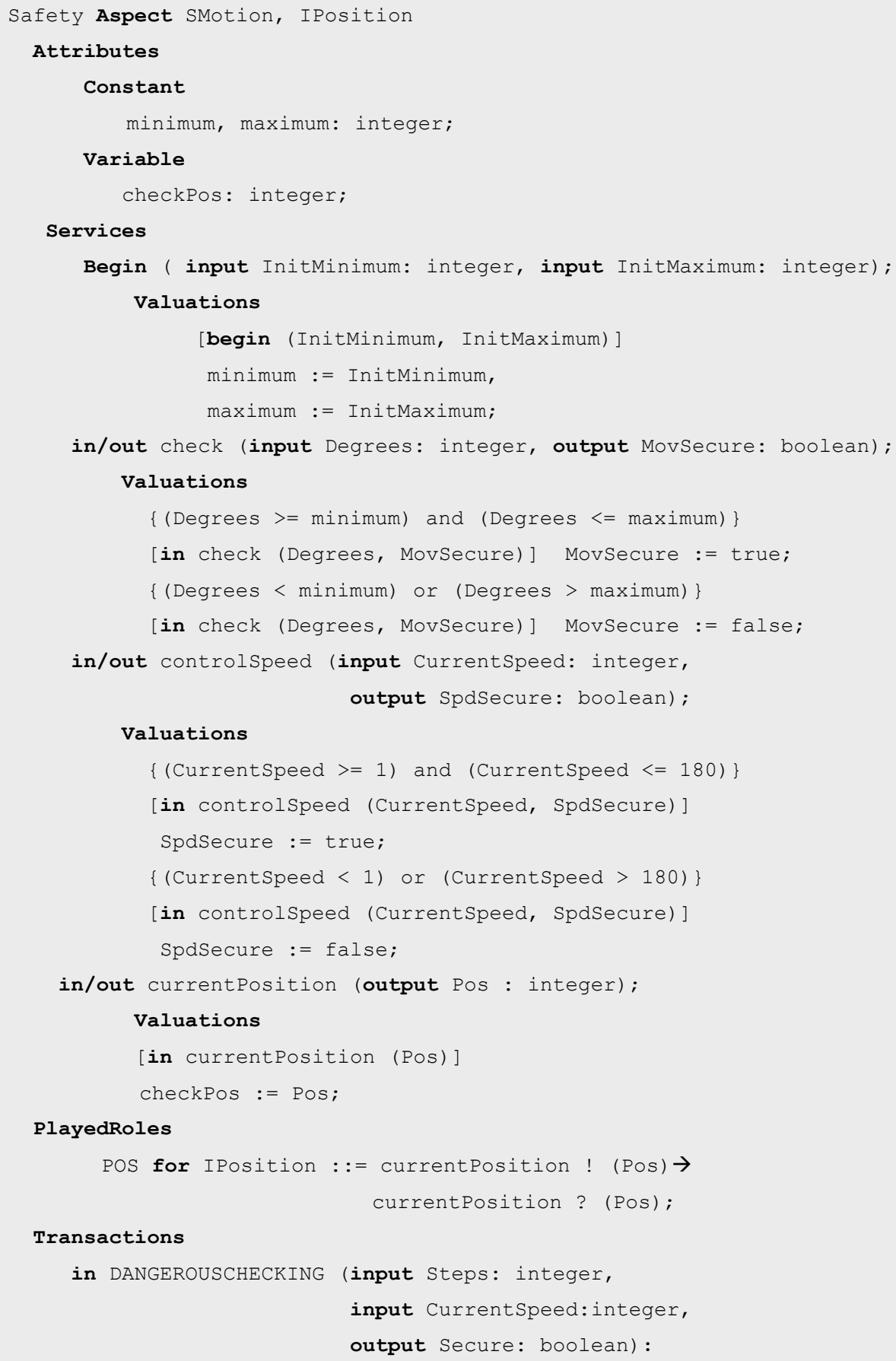

in/out check (input Degrees: integer, output MovSecure: boolean);

Valuations

$\{($ Degrees $>=$ minimum) and (Degrees $<=$ maximum)

[in check (Degrees, MovSecure)] Movsecure := true;

$\{$ (Degrees < minimum) or (Degrees > maximum) \}

[in check (Degrees, MovSecure)] MovSecure := false;

in/out controlspeed (input Currentspeed: integer,

output SpdSecure: boolean);

\section{Valuations}

$\{($ Currentspeed $>=1)$ and (Currentspeed $<=180)\}$

[in controlspeed (Currentspeed, SpdSecure)]

SpdSecure := true;

$\{($ Currentspeed < 1) or (Currentspeed > 180) \}

[in controlspeed (Currentspeed, SpdSecure)]

SpdSecure := false;

in/out currentPosition (output Pos : integer);

\section{Valuations}

[in currentPosition (Pos)]

checkPos := Pos;

\section{PlayedRoles}

POS for IPosition : := currentPosition ! (POS) $\rightarrow$ currentPosition ? (Pos);

\section{Transactions}

in DANGEROUSCHECKING (input Steps: integer, input Currentspeed:integer, output Secure: boolean): 
PRISMA Software Architecture of the TeachMover

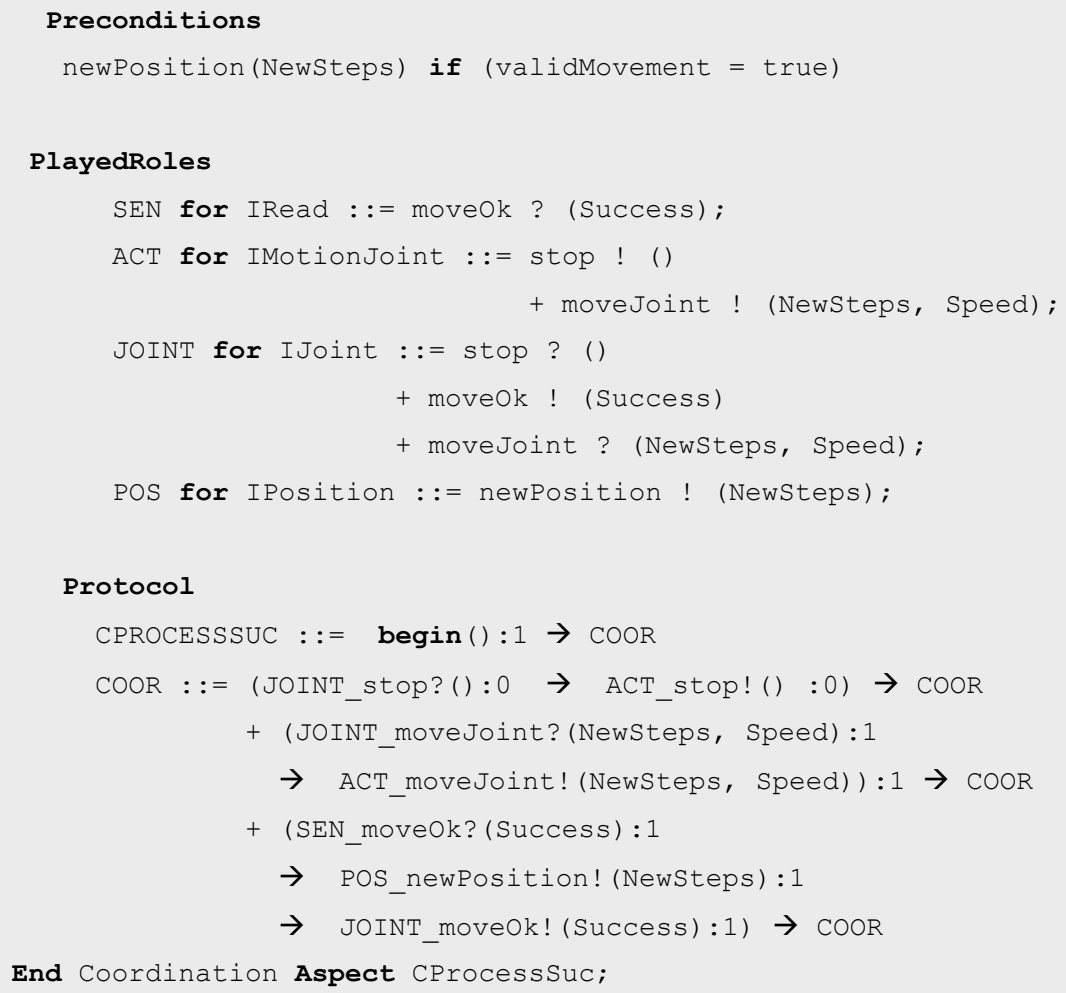

\section{C.4. ARCHITECTURAL ELEMENTS}

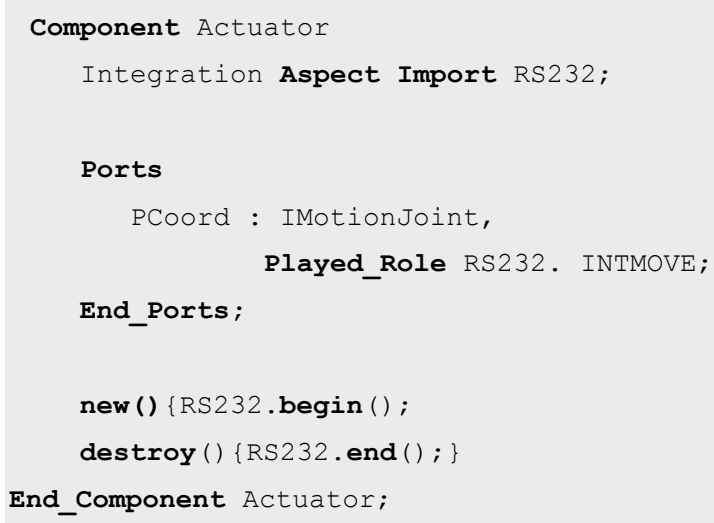


PRISMA: Aspect-Oriented Software Architectures

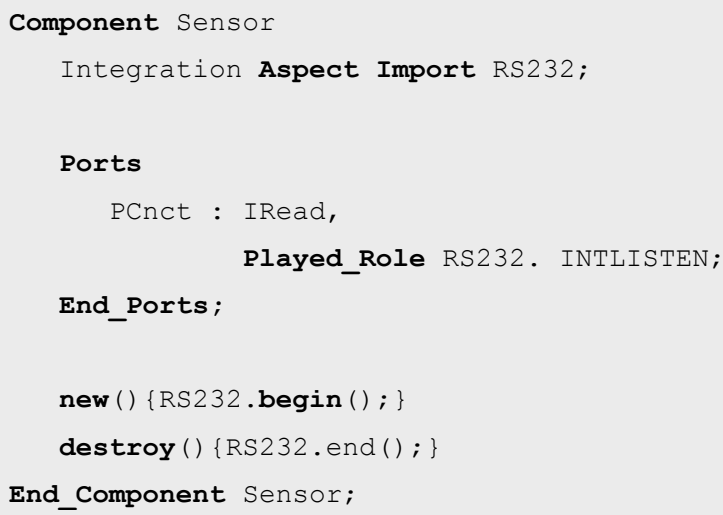


PRISMA Software Architecture of the TeachMover
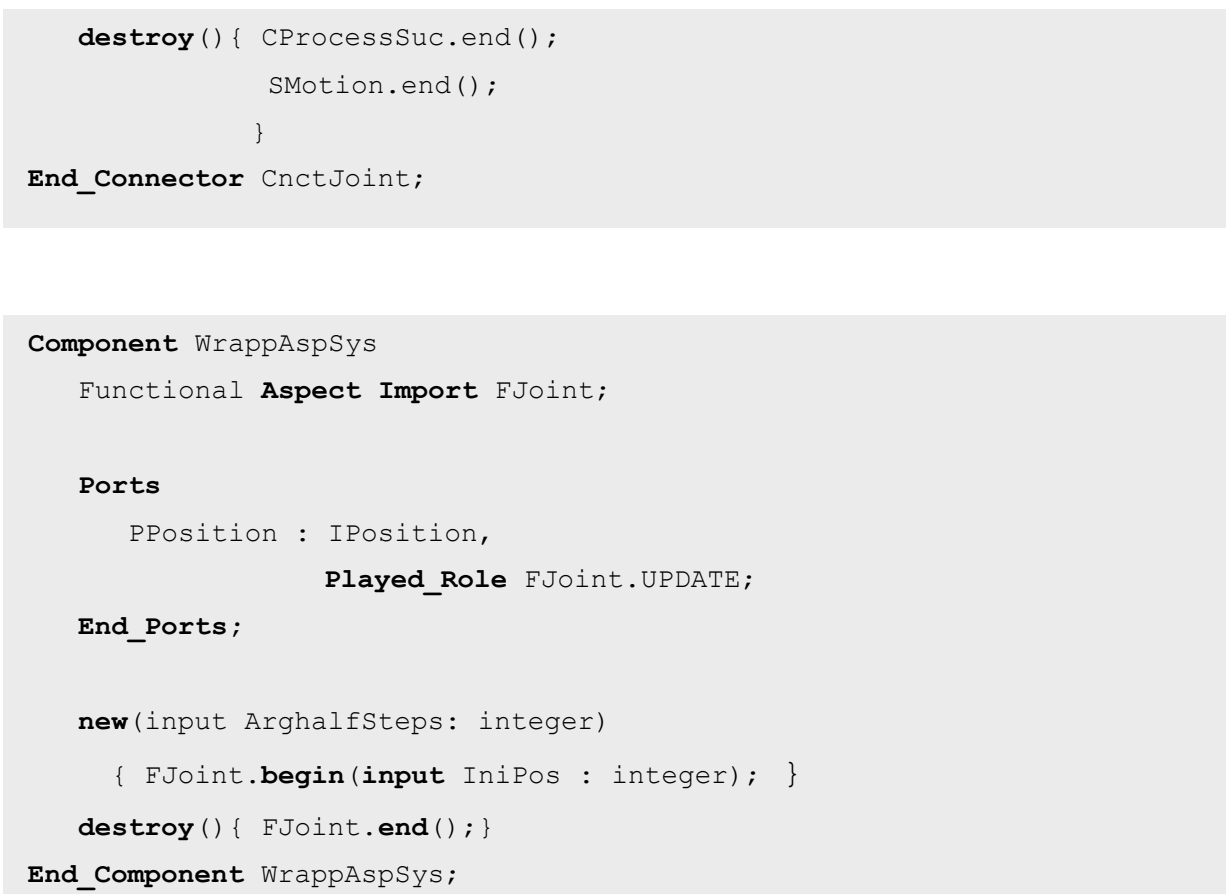
PRISMA: Aspect-Oriented Software Architectures

new (input ArghalfSteps, input num Actuator: integer,

input num_CnctJoint: integer, input num_Sensor: integer,

input num AttActCnct: integer, input num AttSenCnct: integer,

input num_BndJCnct: integer

new WrappAspSys (input ArghalfSteps: integer))

new Actuator ();

new CnctJoint new(input Argmin: integer, input Argmax:integer);

new Sensor ()

new AttActCnct (input ArgCnctName: string,

input ArgCnctPort: string,

input ArgCompName: string,

input ArgCompPort: string):

new AttSenCnct (input ArgCnctName: string,

input ArgCnctPort: string,

input ArgCompName: string,

input ArgCompPort: string);

new AttchPos (input ArgCnctName: string,

input ArgCnctPort: string,

input ArgCompName: string,

input ArgCompPort: string)

new BndJCnct (input ArgSysPort: integer,

input ArgAEName: integer,

\}

input ArgAEPort: integer);

destroy ()

destroy Actuator () ;

destroy CnctJoint ();

destroy Sensor();

destroy WrappAspSys ()

destroy AttActCnct ();

destroy AttSenCnct ();

destroy AttchPos ()

destroy BndJCnct();

End_System Joint;

\section{C.5. CONFIGURATION}

\section{Architectural_Model_Configuration}

TeachMoverJoints = new TeleoperationDomainJoints \{

$\operatorname{BASE}=$ new Joint $(0,1,1,1,1,1,1,1)$

$\{$ BaseWrappAspSys = new WrappAspSys (0);

BaseActuator= new Actuator ();

BaseConnector $=$ new $\operatorname{CnctJoint}(90,-90)$; 
PRISMA Software Architecture of the TeachMover

BaseSensor $=$ new Sensor ();

BaseAttActCnct $=$ new AttActCnct (BaseConnector, PAct, BaseActuator, PCoord);

BaseAt SenCnct= new AttSenCnct (BaseConnector, PSen, Basesensor, PCnct);

BaseAttchPos= new AttchPos(BaseConnector, PPos,

BaseWrappAspsys, PPosition);

BaseBndJCnct $=$ new BndJCnct (PJointSystem, PJoint, BaseConnector);

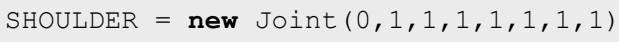

ShoulderAttSenCnct $=$ new AttSenCnct (ShoulderConnector, PSen, ShoulderSensor PCnct);

ShoulderAttchPos $=$ new AttchPos (ShoulderConnector, PPos, ShoulderWrappAspsys, PPosition);

ShoulderBndJCnct $=$ new BndJCnct (PJointSystem, PJoint, ShoulderConnector);

ELBOW $=$ new Joint $(0,1,1,1,1,1,1,1)$

\{ElbowWrappAspSys = new WrappAspSys (0);

ElbowActuator= new Actuator () ;

ElbowConnector $=$ new CnctJoint $(0,-149)$;

ElbowSensor= new Sensor ();

ElbowAttActCnct $=$ new AttActCnct (ElbowConnector, PAct, ElbowActuator, PCoord);

ElbowAttSenCnct $=$ new AttSenCnct (ElbowConnector, PSen, ElbowSensor, PCnct);

ElbowAttchPos= new AttchPos (ElbowConnector, PPos, ElbowWrappAspSys, PPosition);

ElbowBndJCnct= new BndJCnct (PJointSystem, PJoint, ElbowConnector); 
PRISMA: Aspect-Oriented Software Architectures 
ADL Architecture Description Language

AOADL Aspect-Oriented Architecture Description Language

AOP Aspect-Oriented Programming

AOSD Aspect-Oriented Software Development

CASE Computer-Aided Software Engineering

CBSD Component-Based Software Development

CCS Calculus of Communicating Systems

CF Composition Filters

CLR Common Language Runtime

CLS Common Language System

COTS Commercial Off-The-Shelf

CSP Communicating Sequential Processes

CTS Common Type System

DSL Domain-Specific Language

FIFO First In First Out

GUI Graphical User Interface 
PRISMA: Aspect-Oriented Software Architectures

MDA Model Driven Architecture

MDE Model-Driven Engineering

MDD Model-Driven Development

MDSOC Multi-Dimensional Separation of Concerns

MSIL MicroSoft Intermediate Language

MUC Mechanism Unit Controller

OCL Object Constraint Language

OOP Object-Oriented Programming

PC Personal Computer

RUC Robot Unit Controller

SEI Software Engineering Institute

SoC Separation of Concerns

STD State Transition Diagram

SUC Simple Unit Controller

UML Unified Modelling Language 
Index

\section{INDEX}

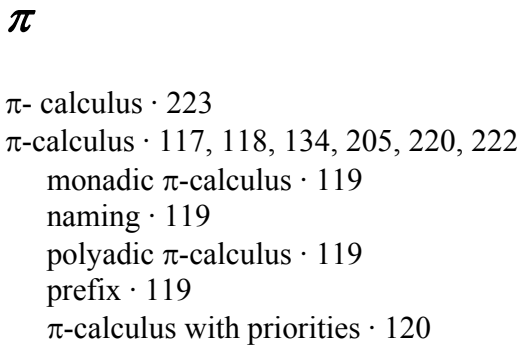

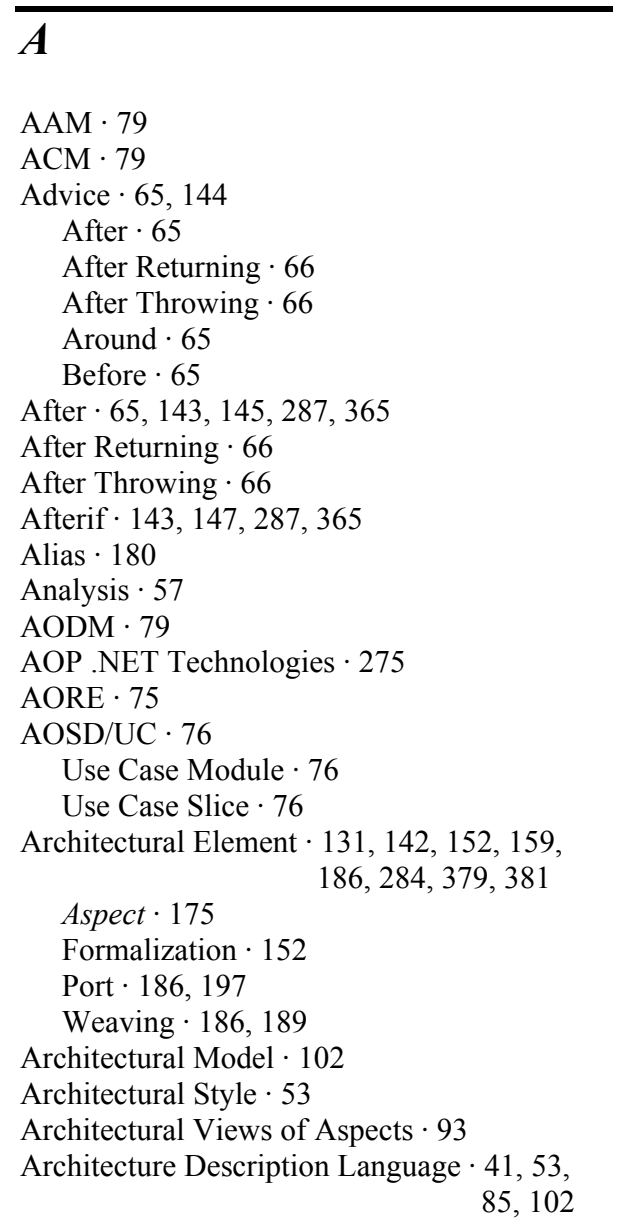

Architecture Specification $\cdot 198$

ARGM $\cdot 76$

Arithmetic Expression $\cdot 372$

Around $\cdot 65$

ASAAM $\cdot 92$

Aspect $\cdot 60,66,69,86,129,138,143,152$, $175,258,281,360,377,382$

Attribute $\cdot 138,176,178$

Behaviour $\cdot 139$

Changes of State $\cdot 138$

Composition $\cdot 68$

Constraint $\cdot 138,141,176,181$

Context-awareness $\cdot 140$

Coordination $\cdot 138,139,152,153,154$, $229,378,379$

Definition $\cdot 102$

Distribution $\cdot 139$

Formalization $\cdot 140$

Functional $\cdot 139$

Instantiation $\cdot 67$

Integration $\cdot 140$

Interface $\cdot 138$

Management $\cdot 68$

Mobility $\cdot 140$

Order $\cdot 68$

Played_role $\cdot 138$

Played_Role $\cdot 177,184$

Precondition $\cdot 138,141,177,182$

Private $\cdot 138,139$

Protocol $\cdot 177,185,380$

Public $\cdot 138,139$

Safety $\cdot 138,139,151,153,229$

Semantics $\cdot 138$

Service $\cdot 138,143,176,380$

Services $\cdot 138$

State $\cdot 66,138$

Structure $\cdot 138$

Valuation $\cdot 138,140,177,183$

Aspect Collaboration Interface $\cdot 97$

Aspect Component · 95, 96, 97, 100

Aspect Oriented Programming $\cdot 64$

AspectJ $\cdot 60$

AspectLeda $\cdot 94$

Aspect-Oriented Architecture Description Language ·

Aspect-Oriented Component Engineering · 95 Aspect-Oriented Evolution · 102 
PRISMA: Aspect-Oriented Software Architectures

Aspect-Oriented Model $\cdot 101$

Aspect-Oriented Programming $\cdot 59,62$

Data Type System $\cdot 67$

Properties $\cdot 66$

Semantics $\cdot 68$

Aspect-Oriented Software Development $\cdot 29$,

31,61

Asymmetric Model · 69

Symmetric Model $\cdot 69$

Asymmetric Model $\cdot 69$

Asynchronous $\cdot 280$

ATAM $\cdot 43$

ATRIUM $\cdot 77$

Attachment · 52, 133, 154, 192, 262, 290, 366, 381

AttachmentClientBase $\cdot 290$

AttachmentServerBase $\cdot 290$

Communication Pattern $\cdot 193$

Formalization $\cdot 155$

Attribute $\cdot 176,178$

Constant $\cdot 179,361$

Derived $\cdot 178,361$

Non-derived $\cdot 178$

Variable $\cdot 179,361$

Automatic Code Generation · 31

\section{$\bar{B}$}

\section{Base Code $\cdot 64$}

Before $\cdot 65,143,146,287,365$

Beforeif $\cdot 143,149,287,365$

Binding $\cdot 133,159,195,262,290,292,367$

Communication Pattern $\cdot 196$

ComponentBinding $\cdot 292$

Formalization $\cdot 159$

SystemBinding $\cdot 292$

Bindings $\cdot 53$

BNF PRISMA AOADL $\cdot 36,359$

Block $\cdot 359$

Instance $\cdot 370$

List $\cdot 359$

Name $\cdot 359$

Sequence $\cdot 359$

Value $\cdot 359$

$\bar{C}$

Caesar $\cdot 97$

Calculus of Communicating Systems (CCS) ·

CAM/DAOP $\cdot 88$

118

Class Diagram $\cdot 79$

Classifier $\cdot 80$

CLR $\cdot 276$

CLS $\cdot 277$

CoCompose $\cdot 80$

Code Generation Pattern $\cdot 266$

Aspect $\cdot 267$

Component $\cdot 266$

Collaboration Diagram $\cdot 79$

Communication $\cdot 57$

Complex Component $\cdot 53$

Component $\cdot 47,99$, 132, 152, 154, 164, 186, $190,261,285,365,378,381$

Component View $\cdot 96$

Component-Based Software Development .

Composition Filters $\cdot 72$

$$
\text { Filter } \cdot 72
$$

Superimposition $\cdot 73$

Composition Relationship · 53

Computer-Aided Software Engineering $\cdot 29$

Concern $\cdot 60,130,138,139,175,282,377$

Concern Space $\cdot 70$

Condition $\cdot 372$

Configuration Model $\cdot 271$

Modelling Tool $\cdot 271$

Specification $\cdot 369$

Connection $\cdot 52$

Connector $\cdot 49,88,98,132,152,154,186$,

$$
191,261,285,366,379
$$

Constraint $\cdot 56,176,181,363$

Dynamic $\cdot 181$

Static $\cdot 181$

CORE $\cdot 76$

Cosmos $\cdot 77$

COTS $\cdot 31,140,260$

Crosscutting-Concerns $\cdot 60,64,66,130$

CTS $\cdot 277$

D

DAOP $\cdot 88$

Data Type $\cdot 371$

Delegate $\cdot 277$

Domain Specific Language $\cdot 249$

Domain-Specific Model $\cdot 245,250$

DotNET Remoting $\cdot 277$

DotNET technology $\cdot 246$

DotNET Technology $\cdot 275$ 
Index

DSL Tools $\cdot 249$

Code Generation Designer · 252

Debugging Solution $\cdot 252$

Domain Model $\cdot 250,252$

Model Designer $\cdot 251,252,254$

Setup $\cdot 256$

Verification $\cdot 253,263$

Complete $\cdot 263$

Hardconstraint $\cdot 254$

Partial $\cdot 263$

Verification Rule $\cdot 254$

Dynamic Aspect Diagram $\cdot 80$

\section{$\bar{E}$}

Early Aspect $\cdot 75$

EFTCoR $\cdot 112$

\section{$\bar{F}$}

Filter $\cdot 72$

Formulae $\cdot 371$

Functional Decomposition · 61, 64, 69

Fuse J 99

\section{$\boldsymbol{G}$}

Goal-Oriented Requirements Engineering · 76 Graphical Modelling $\cdot 103$

GREMSoC $\cdot 76$

\section{$\boldsymbol{H}$}

Hyper/J $\cdot 70,77$

Concern Space $\cdot 70$

Encapsulation $\cdot 70,71$

Hyper/JTM $\cdot 70$

Hypermodule $\cdot 71$

Hyperslice $\cdot 71$

Hyperspace $\cdot 71$

Identification $\cdot 70$

Integration $\cdot 70,71$

Relationship Definition · 70, 71

Hypermodule $\cdot 71$

Hyperslice $\cdot 71$

Hyperspace $\cdot 71$
Hyperspaces $\cdot 70$

$\bar{I}$

InPorts $\cdot 286$

Instead $\cdot 143,147,287,365$

Insteadif $\cdot 144,150,287,365$

Interface $\cdot 47,131,132,134,137,138,142$, $164,173,206,257,360$

InterfaceService $\cdot 173$

\section{$\bar{J}$}

$\mathrm{JAC} \cdot 100$

Jiazzi $\cdot 100$

Linking Unit $\cdot 101$

Unit $\cdot 101$

Join Point $\cdot 64$

Joint $\cdot 113$

$\boldsymbol{K}$

Kinds of Aspects $\cdot 282$

Kripke Structure $\cdot 118$

$\bar{L}$

Language for PRISMA Protocols $\cdot 223$

$\log \cdot 299$

\section{$\boldsymbol{M}$}

Maintenance $\cdot 57$

MDSOC $\cdot 70,87$

Hyper/J $\cdot 70,77$

Hyperspaces $\cdot 70$

On-Demand Remodularization $\cdot 70$

Memory Persistence $\cdot 295$

Metaclass $\cdot 134,171$

Meta-level $\cdot 133$

Metamodel $\cdot 133,171$

Utilities $\cdot 200$

MiddlewareSystem $\cdot 295$

Modal Logic of Actions · 117, 134, 140, 205, 307 
PRISMA: Aspect-Oriented Software Architectures

Model-Driven Architecture · 249

Model-Driven Development · 29, 32, 245, 249, 300, 303

Model-Driven Engineering $\cdot 245$

MSIL $\cdot 276$

N

Non-UML language $\cdot 80$

$\boldsymbol{O}$

Object Constraint Language (OCL) $\cdot 171$

Object-Oriented Programming $\cdot 61$

Obliviousness $\cdot 62$

On-Demand Remodularization $\cdot 70$

OutPorts $\cdot 286$

\section{$\boldsymbol{P}$}

Package $\cdot 79$

Paradigm of Automatic Programming $\cdot 29,31$

Parameter $\cdot 371$

Perspectival Concern-Space $\cdot 87$

Played Role $\cdot 137,138,142,155,159,164$, $177,184,281,364$

Compatible $\cdot 154,159$

Formalization $\cdot 137$

Pointcut $\cdot 65,76,144$

Port $\cdot 52,131,142,152,154,155,159,160$, $164,186,197,261,284,285,366,381$

Formalization $\cdot 142$

Interface $\cdot 142$

Played_Role $\cdot 142$

Type $\cdot 142$

Precondition $\cdot 177,182,362$

Prism $\cdot 131$

PRISMA $\cdot 77,129$

AOADL $\cdot 246$

CASE $\cdot 245$

Methodology $\cdot 29,303$

PRISMA CASE $\cdot 29$

PRISMA Graphical AOADL $\cdot 257$

PRISMA model $\cdot 29$

PRISMA Model Compiler · 264

PRISMA Modelling tool $\cdot 256$

PRISMANET $\cdot 247$

Architecture $\cdot 275$

400 $\log \cdot 278$

Memory Persistence $\cdot 278$

PRISMA Execution Model · 277, 279

Communication Module $\cdot 279$

Communications Module $\cdot 277$

Type Module $\cdot 277,279$

Transaction Manager $\cdot 278$

Property $\cdot 56$

Protocol $\cdot 139,177,185,260,364,380$

$\boldsymbol{Q}$

Quantification $\cdot 62$

$\boldsymbol{R}$

Reflection $\cdot 277$

Reuse $\cdot 56$

$S$

\section{$\mathrm{SAAM} \cdot 43,92$}

Scenarios Model $\cdot 77$

Separation of Concerns $\cdot 59,85$

Serialization $\cdot 277$

Service $\cdot 134,135,137,176,180,282,362$

Alias $\cdot 180$

Begin $\cdot 362$

End $\cdot 362$

Formalization $\cdot 136,144$

Invocation $\cdot 135,144,155,156,160,161$

Private $\cdot 135,282$

Process $\cdot 136$

Public $\cdot 135,282$

Semantics $\cdot 135$

Signature $\cdot 134,174,206$

Transaction $\cdot 180$

Type $\cdot 180$

Software Architecture $\cdot 39,40,56,86$

Analysis $\cdot$ 43, 57, 92

Definition $\cdot 44$

Description $\cdot 54,55$

Evolution $\cdot 91$

Properties $\cdot 41,43,56$

Design $\cdot 42$

Quality Attributes $\cdot 43$

Software Architecture Evolution · 57

Software Architectures $\cdot 30$ 
Index

Software Factories $\cdot 249$

State $\cdot 260$

State Transition Diagram · 260

Substate $\cdot 260$

Superimposition $\cdot 73,90$

Symmetric Model $\cdot 69$

Symmetrical $\cdot 130$

System $\cdot 53,132,159,164,186,194,261$, 294, 367, 379

Binding $\cdot 195$

Composition $\cdot 165$

Inclusive $\cdot 165$

Weak $\cdot 165$

Emergent Behaviour $\cdot 164$

Formalization $\cdot 165$

Pattern $\cdot 164$

\begin{tabular}{l}
\hline $\boldsymbol{T}$ \\
Tangled Code $\cdot 60$ \\
TeachMover $\cdot 113$ \\
Actuator $\cdot 115$ \\
Base $\cdot 113$ \\
Elbow $\cdot 113$ \\
Mechanism Unit Controllers (MUCs) $\cdot 117$ \\
Robot Unit Controlers (RUCs) $\cdot 117$ \\
Sensor $\cdot 115$ \\
Shoulder $\cdot 113$ \\
Tool $\cdot 114$ \\
Wrist $\cdot 113$ \\
Tele-operation Domain $\cdot 111$ \\
Tele-operation Systems $\cdot 112$ \\
Theme $\cdot 77$ \\
Theme/Doc $\cdot 77$ \\
Theme/UML $\cdot 79$ \\
TOPSA $\cdot 43$ \\
Transaction $\cdot 180,296$ \\
Active $\cdot 298$ \\
Committed $\cdot 298$ \\
Precommitted $\cdot 298$ \\
Prerollback $\cdot 298$ \\
Rollbacked $\cdot 298$ \\
Transactional Context $\cdot 297$ \\
TransactionLog $\cdot 298$ \\
Transaction Manager $\cdot 296$ \\
Transat $\cdot 91$
\end{tabular}

\begin{tabular}{l}
\hline $\boldsymbol{U}$ \\
UFA $\cdot 79$ \\
UML $\cdot 78$ \\
$\quad$ Heavy Extension Mechanism $\cdot 79$ \\
Light Extension Mechanism $\cdot 79$ \\
Profile $\cdot 79$ \\
Understanding $\cdot 56$ \\
Unified Modelling Language $(\mathrm{UML}) \cdot 171$ \\
UXF $\cdot 80$ \\
\\
\hline $\boldsymbol{V}$ \\
Valuation $\cdot 177,183,362$ \\
$\quad$ Condition $\cdot 183$ \\
$\quad$ Postcondition $\cdot 183$ \\
Service $\cdot 183$ \\
Valuations $\cdot 137$ \\
V-Graph $\cdot 77$ \\
View $\cdot 93,96$ \\
Viewpoint $\cdot 75$ \\
Views $\cdot 42,55$ \\
\\
\hline $\boldsymbol{V}$
\end{tabular}

Weaving · 65, 66, 131, 143, 152, 164, 186, $189,262,284,287,365,380$

Advice Service $\cdot 189$

Definition $\cdot 102$

Dynamic $\cdot 66$

Formalization $\cdot 144$

Operator $\cdot 145,365$

After $\cdot 143,145,287,365$

Afterif $\cdot 143,147,287,365$

Before $\cdot 143,146,287,365$

Beforeif · 143, 149, 287, 365

Instead · 143, 147, 287, 365

Insteadif $\cdot 144,150,287,365$

Operators $\cdot 143$

Pointcut Service $\cdot 189$

Set $\cdot 151$

Static $\cdot 66$

Weaving Time $\cdot 66$

Weaving Manager $\cdot 287$ 
PRISMA: Aspect-Oriented Software Architectures 